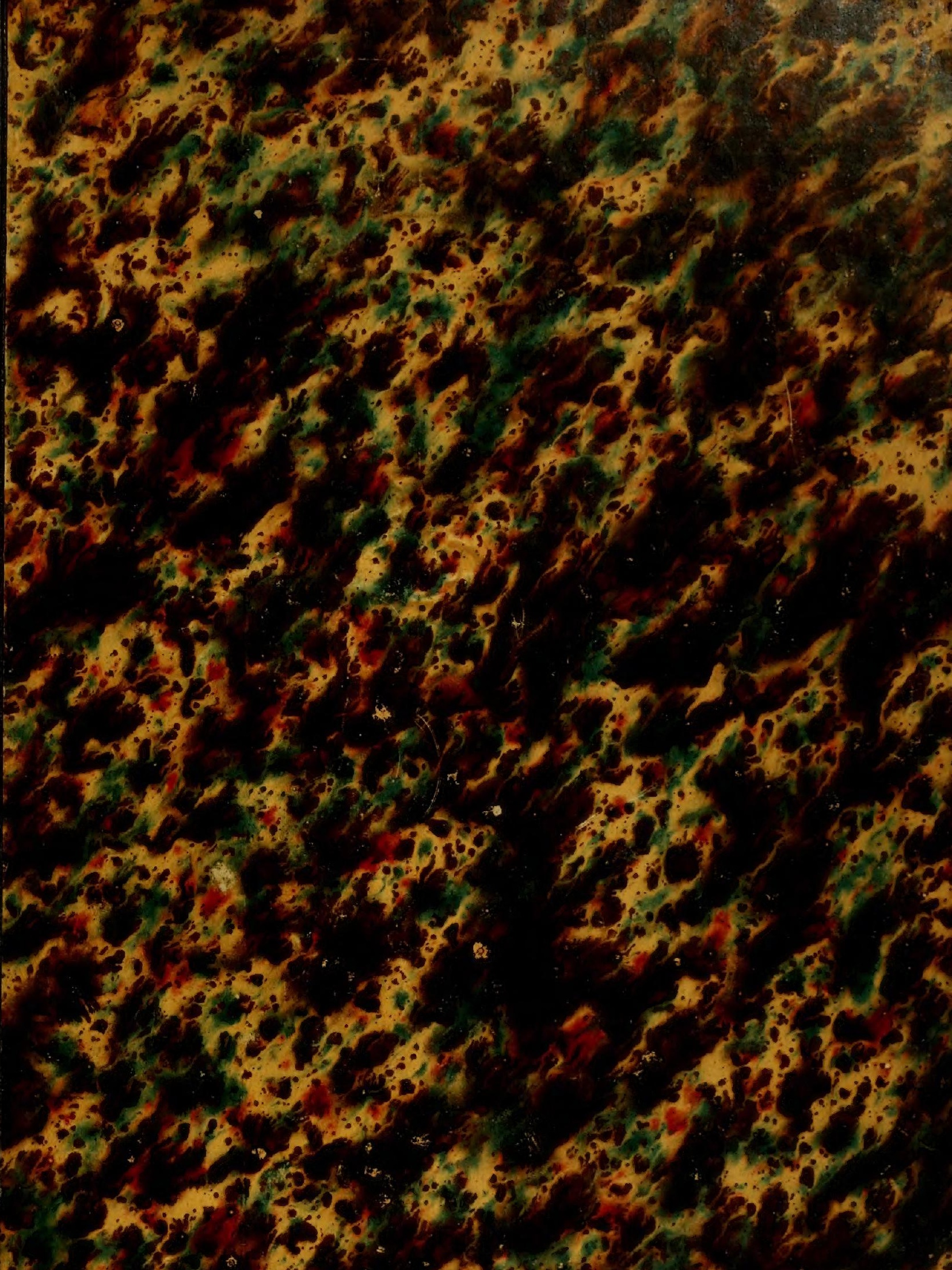

-

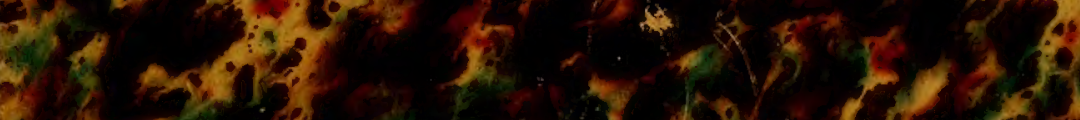

2.

20 20

क.

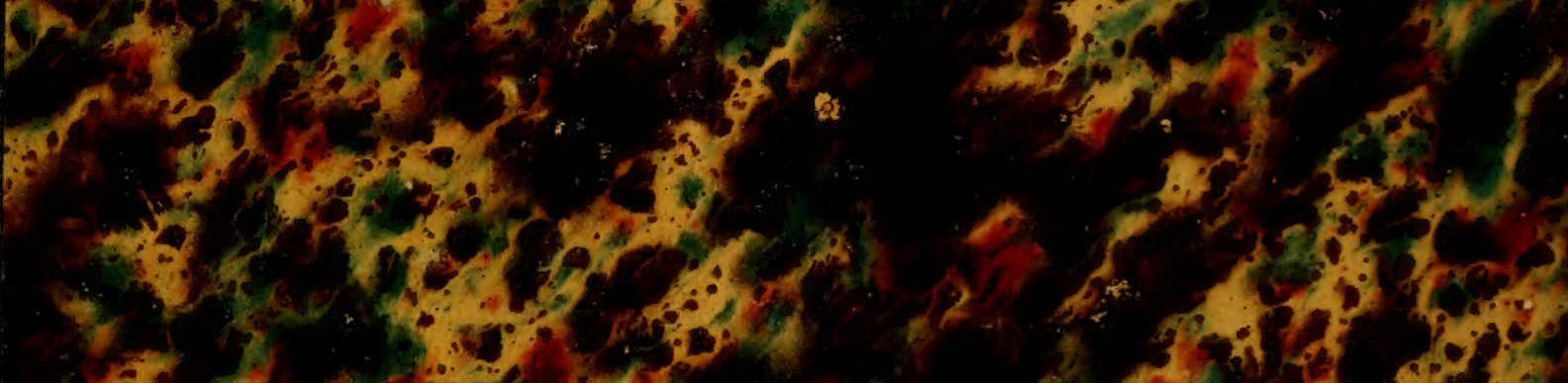

\title{
$58 x^{2}-1.6$
}

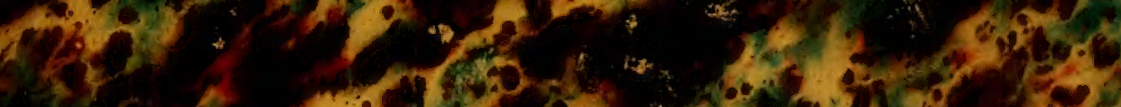

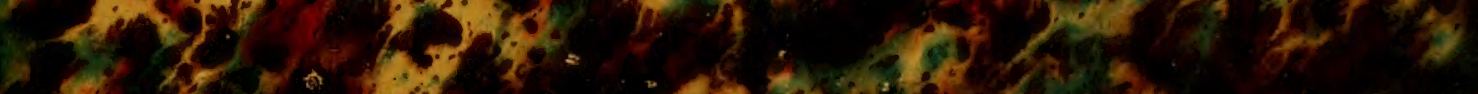

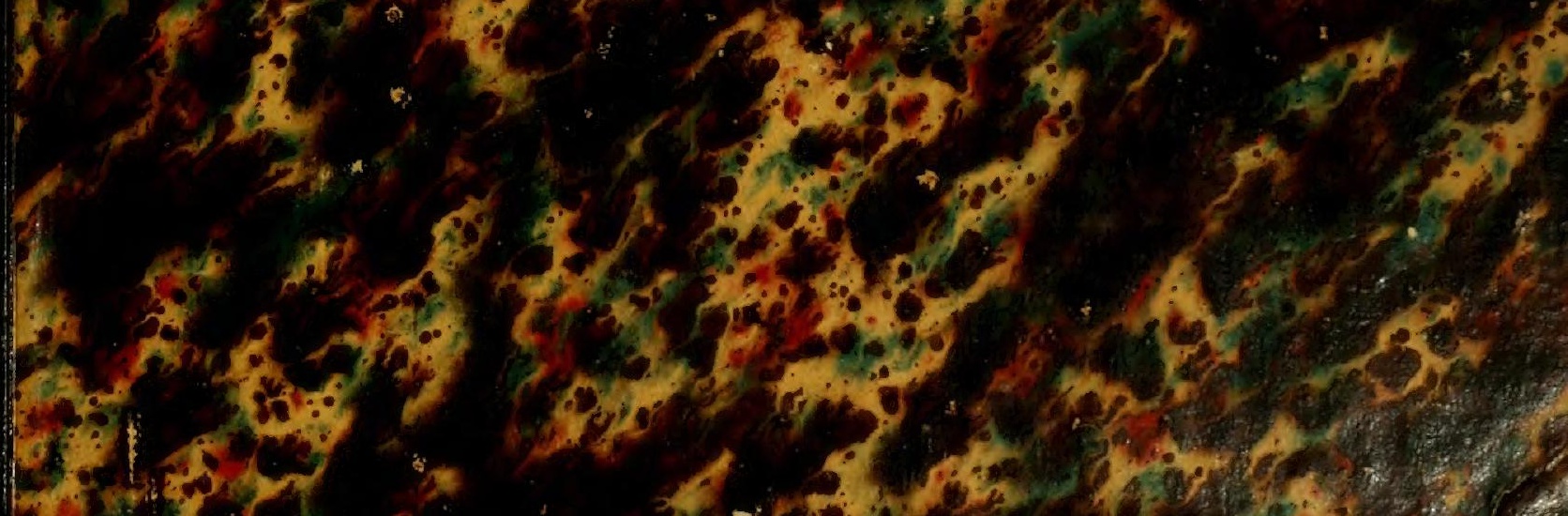




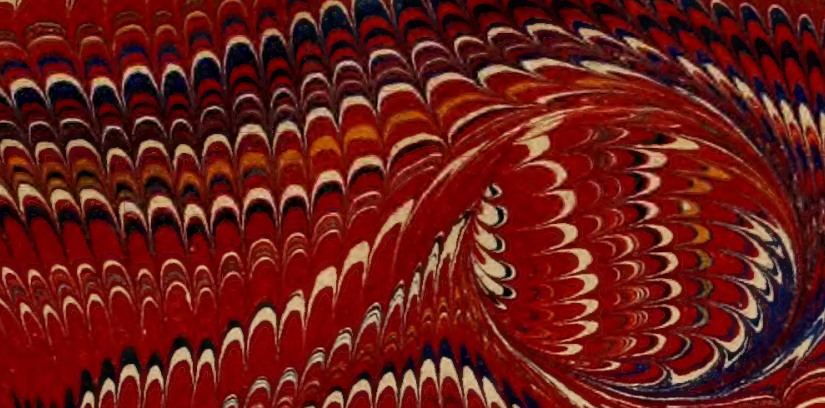

nanh amand 3.3

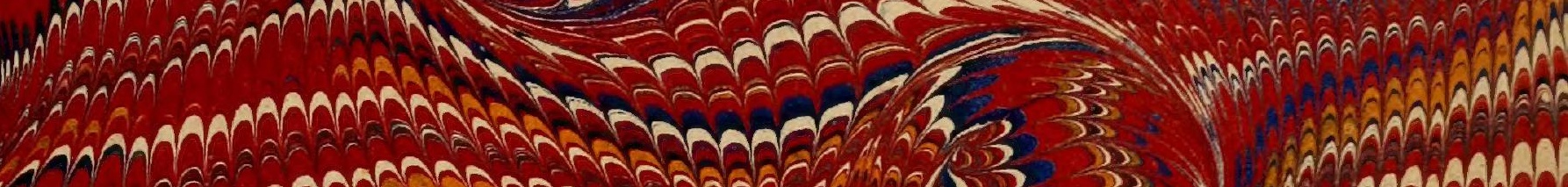

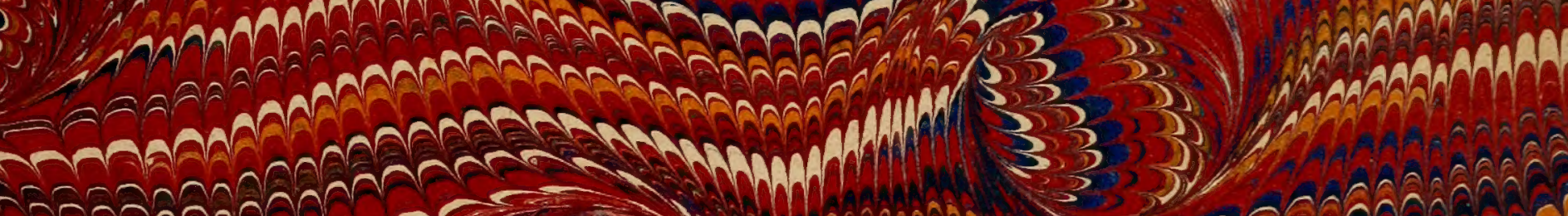

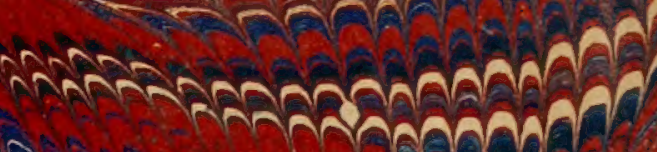

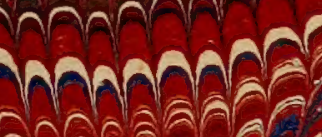

Manh naA?

A A $1 \mathrm{~N}, 3,3$

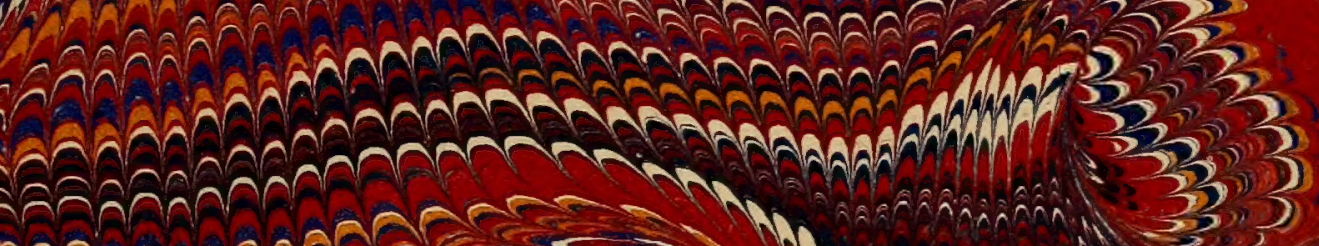

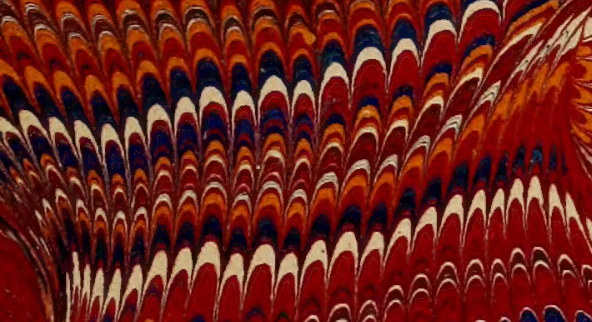

$3333^{3} 3^{3} 1$ Whmmhnth

3

3

a.

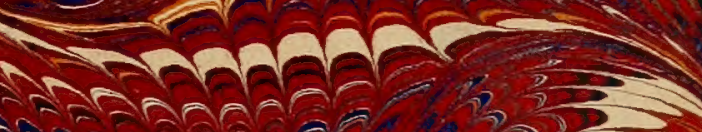

D

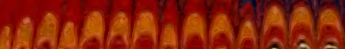

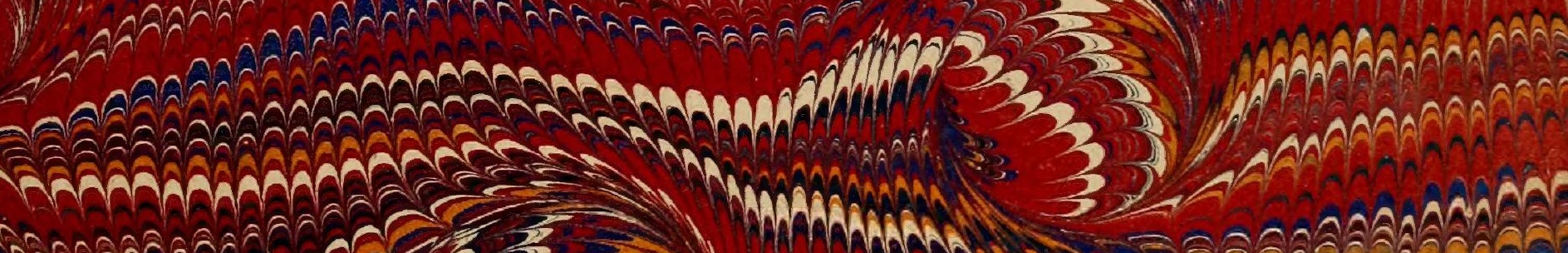

N.)

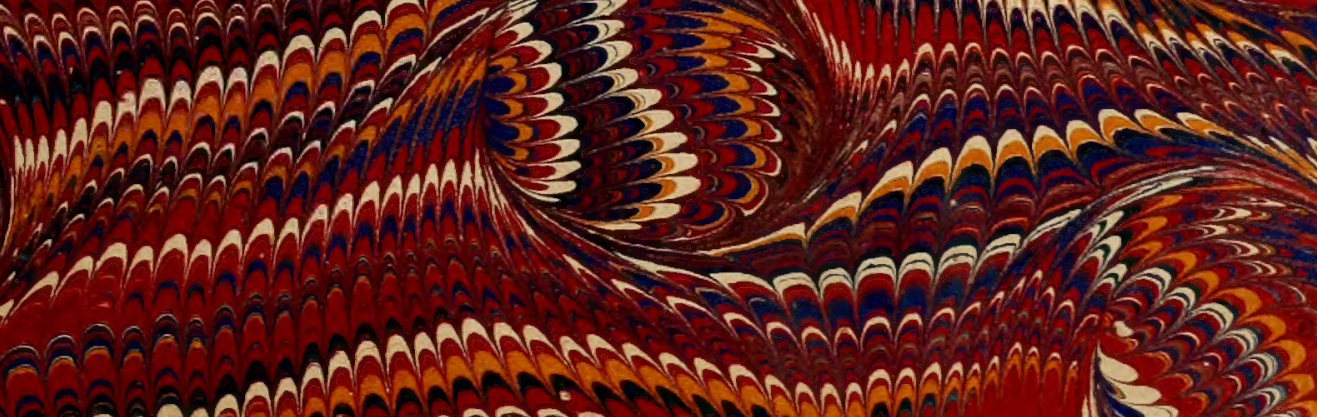

MNÃanâAล 




\title{
HISTOIRE NATURELLE
}

\author{
. \\ EQUISET U M \\ DE FRANGE \\ PAR

\section{J. DUVAL-JOUVE}

INSPECTEUR DE L'ACADÉMIE DE STRASBOURG, MEMrRe dE LA SOCIÉtÉ BOTANIQUE DE FRANCE.

\section{MÉMOIRE PRÉSENTÉ A L'ACADÉMIE DES SCIENCES}

ET ACCOMPAGNÉ

DU RAPPORT DE M. AD. BRONGNIART

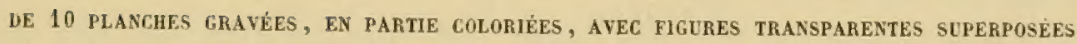
ET DE 33 FIgURES INTERCALÉES DANS LE TEXTE.

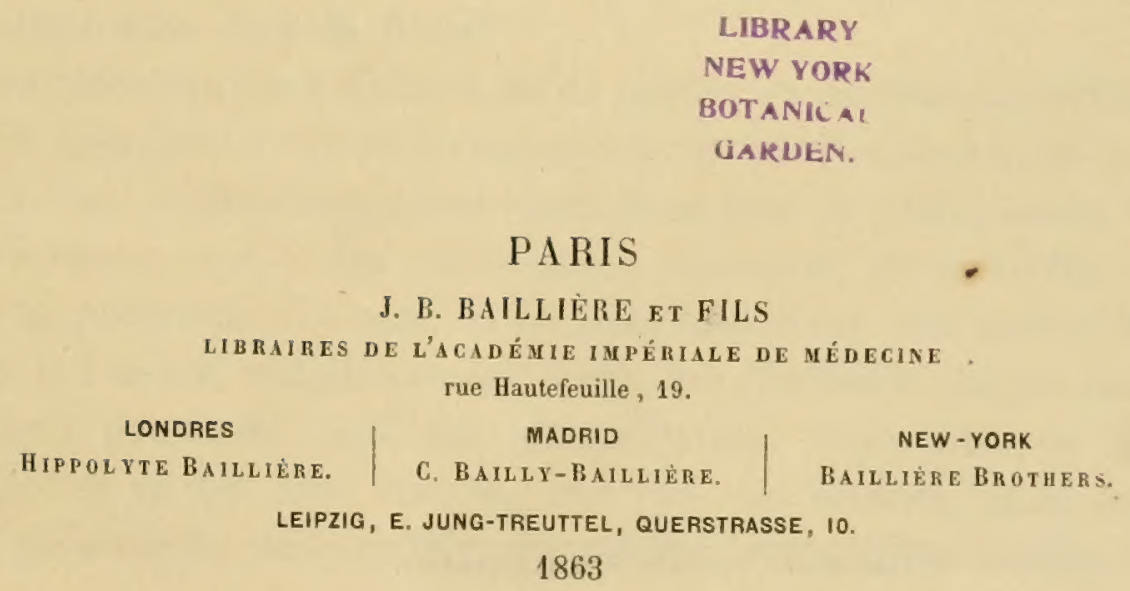




$$
\begin{aligned}
& Q<13 \\
& .087
\end{aligned}
$$


"Qui donc ne croit pas connaître l'Equisetum arvense?" se demande ironiquement M. J. Milde à la première ligne de l'un de ses importants travaux sur les Equisetum. S'il était permis de poser cette question en Allemagne, à combien plus forte raison ne le peut-on pas en France, où nous avons eu si peu d'études sur la famille des Equisétacées, et où sont en général trop peu répandus les nombreux travaux dont elle a été l'objet dans les Journaux botaniques, les Revues scientifiques, les Acta academica si multipliés au delà du Rhin?

Mon intention avait d'abord été de réunir, en me bornant à les résumer et à les coordonner, toutes les connaissances que l'on doit à ces documents si précieux, malheureusement épars dans tant de publications diverses; mais à mesure que je les soumettais à l'examen, de nouvelles observations se présentaient à moi, et de plus la culture par semis des Equisetum qui m'a si complétement réussi, me révélait à chaque instant soit des faits nouveaux, soit des interprétations différentes de faits déjù Saperçus. Il en est donc résulté, non plus un résumé, mais une ouvre toute personnelle. Je la publie aujourd'hui avec quelque confiance, parce $\rightarrow$ 
que je n'y ai rien dit, ni rien figuré que je ne l'aie vu. Cela ne signifie pas que j'ai la prétention d'avoir tout vu, ni celle d'avoir bien compris et bien interprété ce que j’ai vu. le sais au contraire qu'il reste encore beaucoup à voir, beaucoup à éclaircir; mais j’aurai atteint mon but et je m'estimerai heureux si par ce travail je contribue, soit directement, soit en provoquant de nouvelles observations, à une connaissance plus exacte et plus complète des Equisetum.

J'étais pour cette étude bien placé à Strasbourg. D'une part, toutes les espèces françaises d'Equisetum croissent en Alsace; et, de l'autre, je trouvais près de $\mathbf{M}$. W. Ph. Schimper et de M. Buchinger, l'aide et le concours que se devraient toujours prêter les amis d'une même science. Tous les deux se sont gracieusement empressés de m'être utiles; le premier, en me permettant de lui soumettre mes indécisions et en m'éclairant de son vaste savoir; le second, en mettant à ma disposition son précieux herbier, sa riche bibliothèque, ses vastes relations, et en consacrant de longs instants à m'aider dans l'interprétation des textes allemands qu'il connaît merveilleusement. Il m'est doux de prier ici ces deux amis de recevoir l'expression de ma reconnaissance.

J'ai donc essayé d'exposer l'organisation générale des Equisetum et les caractères spécifiques des espèces de France, en appuyant mes descriptions de très-nombreuses figures, toutes dessinées par moi, à la chambre claire, et avec les plus minutieuses précautions pour assurer l'évaluation exacte des grossissements.

J'ai suivi dans ce travail l'ordre suivant:

A la description d'un Equisetum adulte succède d'abord l'analyse des tissus des diverses parties, puis l'exposé de l'évolution de ces parties jusqu'à l'émission des spores. La série des phénomènes de la reproduction, depuis la germination d'une spore jusqu'au développement complet d'une nouvelle plante, nous ramène au point de départ; et un rapide examen des anomalies et de quelques opinions morphologiques termine la première partie.

La seconde, moins étendue, contient la description, la synonymie et l'iconographie des espèces; elle se complète par un aperçu historique sur 
les propriétés attribnées anx Equisetum, sur leurs usag̣es et sur leur composition chimique.

Ainsi, en tout, deux parties sous les litres et avec les divisions cidessons:

\section{PREMIÈ RE PATTIE. Anatomie. IReproduction.}

Coup d'EtL prélaninaine Sur LE GenRe EQuiseTUM ET SUR LES ESPÈCES FRANĢASES.

CHAPTTLE PIEMIER. Description exténieune D'UNE PLANTE ADULTE.

$\$ 1$. Du rhizome et de ses appendices.

\$2. De la tige stérile.

$\$ 3$. De la lige spicifère et de l'appareil de reproduction.

Cilap. II. Description analtrigue D'Une plante ADULTE.

$\$ 1$. De l'épiderme:

2. Des tissus du rhizome.

$\$ 3$. Des lissus de la tige.

\$4. De l'appareil de reproduction.

Cillap. III. Fommation et développement nes DIVERSES PARTIES.

$\$ 1$. De la tige et des gaines.
$\$ 2$. Des rameaux, des rejetons, des rhizomes et iles tubercules.

\$3. Des racines.

$\$ 4$. Des spores.

\$5. Anatomie des spores.

CilAP. IV. Reproduction.

§1. Développement des spores en sporophymes.

\$2. Des anthéridies et des spermatozoïdes.

\$3. Des archẻgones.

$\$ 4$. Du pseudembryon et de la jeune plante.

\$5. Mode de semis.

$\$ 6$. Ilistorique.

CHIAP. V. DE QUElques orinions morpholoGIQUES ET DES ANOMALIES.

§1. Morphologie.

2. Anomalies.

\section{DEUX IÈnE PATTE. Clasgifiention. Deseription.}

Cilapitre PREMier. Place de la familie.

CHAP. II. Examen des caractìnES SRÉCIFIQUES.

Cilap. III. Glassification et description.

\$1. Famille.

\$2. Genre.

\$3. Division et classification des espèces.

$\$ 4$. Description des espèces.
CIIAP. IV. IIrstorioue et SYNonyme.

\$1. Genre.

\$2. Espèces.

Cilap. V. IgonograpiIe.

CIIAPTTRE GONPLEMENTAIRE.

$\$ 1$. Noms.

9. Propriétés et usages.

\$3. Composilion chimique.

\section{Strasbourg, 7 août 1861 .}

Terminé à la date qui précède, ce Mémoire a élé, le 25 novembre 1861 , présenté à l'Académic des Sciences. Sur les conclusions du Rapport de 
II. Ad. Brongniarl, licadémie en avait ordonné l'jnserlion dans ses

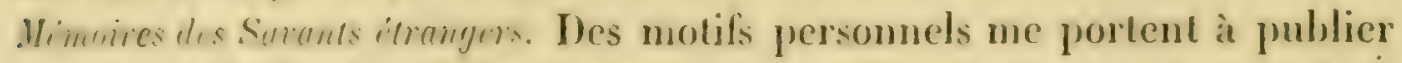
mun travail inmédiatement; $\mathrm{ct}$, si je raplefle ici l'insigne faveur dont jai été lumoré et dont je nai pas prolité, c'est afin de témoigner publiquenent à l'éminente Compagnie toute ma recommaissance pour cette marque si haute dapprobation domée à mes patientes études.

Strasbourg, 15 novembre 1863. 


\title{
INSTITUT IMPÉRIAL DE FRANCE
}

\author{
ACADÉMIE DES SCIENCES
}

\section{RAPP0RT}

\author{
Sur un Mémoire de M. Duval-Jouve, intitulé: Histoire naturelle des Equisetum \\ de France
}

Commissaires, MM. Decaisne, Tulasne, Brongniart rapporteur

(Le genre Prêle ou Equisetum constitue à lui seul une des familles les plus remarquables parmi les cryptogames supérieures ou vasculaires.

« La forme extérieure si particulière de ces végétaux, la nature et la disposition de leurs organes vígétatifs et les caractères de leurs organes de reproduction les isolent en apparence complétement des familles auprès desquelles cependant quelques points essentiels de leur structure et les pliénomènes les flus importants de leur reproduction doirent nécessairement les placer. Aussi, ces plantes ont-elles été de tout temps l'objet d'éludes spéciales, et, dans ces dernières années surtout, des découvertes importantes ont été faites sur leur mode de reproduction; car, grîce aux recherches de MN. Thuret, Hofmeister, Milde, dans l'espace de quelques années, de 1848 i 1852, le mystère de la fécondation a élé déroilé dans ces plantes aussi bien que dans les fougères, et a démontré l'intime affinité qui existe entre ces végétaux.

«Ces travaux récents auraient pu faire croire qu'il n'y avait plus lieu å de nouvelles études sur ces régétaux; aussi I. Duval-Jouve dit-il lui-même que d'ahord il n'avait eu l'intention que de résumer et de combiner les travaux des autres en en rérifiant les points les plus essentiels; mais l'intérêt du sujet, les résultals intéressants auxquels l'ont conduit ses propres observations, les points laissés obscurs el les contradictions de quelques observateurs l'ont engagé a approfondir et à étendre ses recherches, et il en est résulté, après plusieurs années d'études continuées avec persévérance, un travail original aussi complet qu'on peut le concevoir sur cette famille si remarquable.

"Sans doute, beaucoup des observations de M. Dural-Joure ne font que confirmer celles de ses deranciers, mais celte vérification même d'observations délicates et difficiles faite par la même nersonne pour tous les points de l'organisation de ces plantes, celte vérification, étendue à toutes les espèces de ce genre, donnerait à elle seule déjå une grande, valeur aux éludes de M. Duval-Joure.

«Il était impossible, cependant, que des recherches si bien dirigées ne lui fournissent l'occasion d'ajouter aux résultats obtenus par les sasants qui l'avaient précédé; en effet, cet excellent obserratcur a suivi la plupart des espèces d'Equisetum depuis leur premier déreloppement au sortir de la spore 
jusqu'il leur aceroissement complet et il la formation de ces mêmes spores, et par l'étude successire el

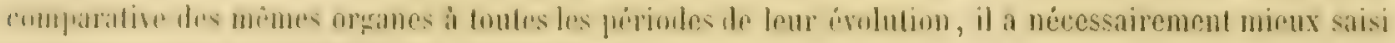
plusicurs points impiortants de leur organisation.

"Ainsi, il a étudié avec un soin tout particulier non-seulement la structure des tiges, des rameaux et des racines adultes dans les diverses especes, et il a montré les rapports qui existent entre les divers tissus qui les constituent, mais il a suivi le mode de développement de ces divers tissus, la formation et la multiplication des cellules qui au sommet du bourgeon déterminent la première évolution de la lige, la première apparition des gaines qui, dans ces plantes, remplacent les feuilles, la formation des stomates et des vaisseatux, et il a montré les changements qui s'opèrent dans ces organes aux divers âges

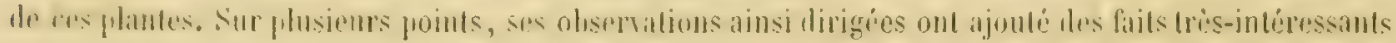
ì cenx déjì connus.

(Dans ces plantes, les gaines qui entourent de distance en distance les tiges el les rameaux sont

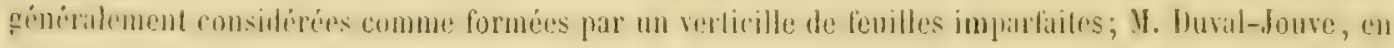
étudiant leur formation et en montrant qu'elles résultent, dés l'origine, d'un anneau ou bourrelet continu dont le bord libre ne se divise que plus tard pour former les dents de ces gaines, rend l'assimilation de ces organes arec les feuilles très-donteuse, et ce mode de formation, joint a la différence de position des dents de ces gaines, relativement aux rameaux, doit porter í considérer chacune des parties constituantes de ces gaines comme très-différente des véritables feuilles.

«L'étude de l'épiderme el des modifications accidentelles qqu'il peut éprouver l'a conduit à considérer la couche siticeuse gui le recouvre, et qui lui donne cette dureté remarquable rqui fait employer les liges de Prêles dans l'industrie, comme une sécrétion de la partie des cellules de l'épiderme qui est en contact arec l'air, et non pas comme entrant dans la constitution même de leurs membranes, ainsi que le pensent plusieurs auteurs. C'est, sans doute, un exemple très-remarquable d'une sécrétion de matière inorganique en dehors des cellules; sécrétion qui, malgré sa nature si différente, rappelle celle des matières cireuses qui recouvrent souvent la surface externe de l'épiderme des feuilles et des fruils.

"Ies détails pleins d’intérêt sur le déreloppement et la structure des stomates de ces végélaux, sur leur position toujours limitec aux parties de l'épiderme qui recourrent un parenchyme rempli de clılorophylle, sur leur perméabilité par l'air et sur leur occlusion dans d'autres circonslances, fournissent de nouvelles preuves du rûle de ces petits organes dans les fonctions respiratoires des plantes.

"Le système vasculaire des Prêles est trés-peu développé, mais il présente quelques faits intéressants mieux étudiés par .1. Duval-Jouve qu'ils ne l'avaient élé précédemment. Ce système vasculairc est constitué par un cylindre de faisceaux distincts très-réguliers composés de raisseaux annelés ou spiraux dont l'auteur du Némoire a suivi le mode de formation et de développement graduel avec beaucoup de prẻcision, grîce au mode d'accroissement de chacun des mérithalles. Mais il a constaté en outre que bientôt les plus internes des vaisseaux de chacun de ces faisceaux se détruisent, sont résorbés et

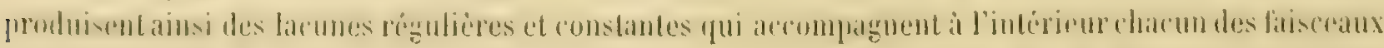
vasculaires dans la plante adulte. Celte existence temporaire de vaisseaux qqui se détruisent plus tarıl et dont les fonctions paraissent ainsi transitoires avait dejà élé signalée par M. Clatin et par quelques autres anatomistes, mais spécialement dans des plantes aquatiques dont les parties adultes en étaient complëtement dépourvues; mais les Equisetum foumissent peut-être le premier exemple de celto résorplion parmi des plantes non aqquatiques et qqui conservent un systeme vasculaire pendant tonte Ieur vie. 
('L'élude du mode de végélation des Prêles, de leurs rhizomes, des tubercules qui en naissent, de la multiplication de ces plantes par la division de ces rhizomes, mulliplication malteureusement trop lacile dans les terres cultivées, a été poursuivie avec une égale persévérance par M. Duval-Jouve pour toutes les espèces, soit en Alsace, soit en Provence, et on peut dire qu'aucun des phénomènes qui touchent ì la vie de ces plantes singulières n’a èté négligé par lui.

«Les organes de la reproduction méritaient une égale attention; ce ne sont pas en effet les parties les moins remarquables de ces curieuses cryptogames.

"Tout le monde connaît les épis qui terminent les tiges des l’rêles et qui laissent échapper de l'intérieur des conceptacles qu'ils supportent, lorsqu'ils sont arrivés à leur entier développement, une immense quantité de corpuscules sphériques accompagnés de filaments contournés en spirale el doués de mouvements hygroscopiques que détermine le moindre changement dans le degré d'humidité de l'air ambiant.

"Ces corps sont les spores des Equisetum, et les filaments auxquels on a voulu autrefois altribuer des fonctions fécondatrices ne sont destinés qu’à faciliter leur sortie et leur dissémination.

( M. Duval-Jouve a suivi arec le plus grand soin la formation des conceptacles ou sporanges et des spores qu'ils renferment; il a déterminé les divisions successives qu'éprouvent les cellules arant d'arriver à la produclion de la cellule-mẻre de chaque spore; il a constaté pour ces cryptogames, comme on l'avait déjả fait pour d'autres el pour les Equisetum eux-mêmes, l'analogie complète qui cxiste entre le mode de formation des spores et cclui des grains de pollen dans l'anthère; il a enfin parlaitement démoniré la manière dont se forment les filaments spiraux qui entourent chaque sppore et qui résultent de la division en une double bande spirale de la membrane épaissie de la cellule-mère, ou, si l'on veut, de la membrane la plus externe de la spore elle-même.

«La germination de ces spores donne naissance, comme dans les fougères, ả une petitefronde verte irrégulièrement lobée, qui n'est pas encore la nouvelle plante et qu'on a nommée proembryon ou prothallium, et que N. Duval-Jouve désigne par le nom de sporophyme.

«En suivant sur de nombreuses germinations des spores de la plupart des espèces d'Equisetum de France le développement de ces petites frondes, M. Duval-Jouve a pu constaler de nouveau et étudier dans leurs plus petits détails les phénomènes si singuliers signalés dans ces plantes pour la premièrc fois par M. Thuret et par M. Holmeister, et yui les rattachent si intimement aux fougères, chez lesquelles des phẻnomènes analogues avaient été observés depuis peu d'années.

«On sait en effet que dans les fougères, comme dans les Prêles, les spores donnent naissance a une petite fronde de quelques millimètres dont la croissance s'arrête bientôt, mais qui produit les anthéridies remplies d'anthérozoïdes et des archégones contenant clracun une cellule embryonnaire destinée, après la fécondation, ả devenir le germe de la nouvelle plante qui s'est ainsi formée librement al centre de l'archégone.

“Ces fails si inattendus, découverts dans les fougères el les Prêles de 1848 à 1852, àaient eu pour risultat non-seulement de faire rentrer toute cette grande classe de végétaux cryptogames sous les lois de plus en plus générales de la reproduction sexuelle, mais en outre de signaler un mode de fécondation dont on n'avait jusuu'alors aucune idée et dont les phénomènes se passaient, au moins en apparence, à une période de la vie de la plante pendant laquelle on ne pouvait penser à les chercher.

«M. Duval-Jouve, par les semis qu'il était parvenu à obtenir facilement et en abondance de toutes les espèces de Prêles, a pu suivre mieux rque ses prédécesseurs toules les circonstances de la production et de l'accroissement de ces organes; il a pu constater que, dans la majorité des cas, ces sporophymes ou prothallium étaient unisexués, que le développement des authéridies et des archégones sur une 
même fromde ètait un cas exceplionnel, et que, dans ce cas même, un de ces organes élail toujours

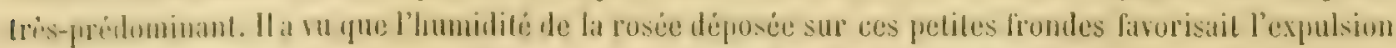

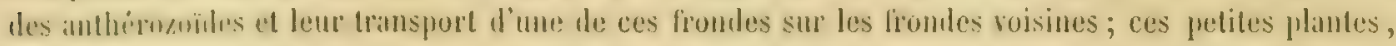
de 2 a 3 millimètres seulement, se développant en général en gazons serrés, de manière qu’il a pu voir sourent les animalcules sortis des anthéridies transportés jusqqu’à l'orifice des archégones.

a Toute la premierre partie du grand travail de M. Duval-Jouve, dont nous venons de faire comnattre quelquues-uns des résultats les plus saillants, est ainsi un exposé organograplique, anatomique et surtunt organorenique de lout ce qui concerne la structure et le développement des divers organes des Equisetım depuis leur première origine jusqu'à leur état adulte.

"Line seconde fartic est consacrie à l'élude farticulière des diverses expeces de Pröles, an nombre de onze, qui croissent en France, à leur distinction fondée également sur les caractères tirés de leurs furmes exterieures el de leur structure anatumiugne, enfin à l'examen de la variation des formes et du mode d'existence de chacune d'elles.

(Dans celte partie, qui n'est pas susceptible d'analyse, on reconnait également l'observateur scrupuleux qui a suivi sur le visant loutes ces plantes el qui les a étudiées dans toutes les périodes de leur vie.

Pour nous résumer, l'llistoire nuturelle des Equisetum de France, par X. Duval-Jouve, est un les travaux les plus complets qui aient jamajs été faits sur une famille naturelle, assez limitée, il est rrai,

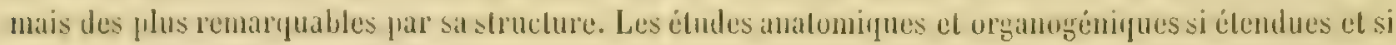
exactes que ce Mémoire comprend lui doment un caraclère plutôt pliysiologique que de botanique purement descriptive, et nous proposons à l'académie d'en ordonner l'insertion frarmi les démoires des Satants étrangers.

Les conclusions de ce liapport sont adoptées.

(Extrait des Comples rendus des séances de l'Académic des Sciences, tome.LVI, séance du 23 mars 1863.) 


\title{
HISTOIRE NATURELLE
}

\section{DES \\ EQUISET U M \\ DE FRANGE}

\section{PREMIĖRE PARTIE}

\author{
Anatomie Reproduction
}

\section{GOUP D'CEIL PRELIMINAIRE}

\author{
stik \\ LE GEXIE EQUISETUM ET SUR LES ESPECES FRANCAISES
}

Les Equisetum de France et d'Europe sont des plantes herbacées, terrestres ou aquatiques, vivaces, à rhizome souterrain, longuement traçant et dont l'organisalion est, dans son ensemble, analogue à celle des tiges. Celles-ci sont articulées el constituées par une suite d'entre-nœuds cylindriques. Ces entre-nœuds, plus ou moins profondément sillonnés et loujours d'une régularité parfaite, sont terminés à leur parlic supérieure par une gaine enveloppant la base de l'entre-nœud immédiatement superposé, et ils présentent dans leur longueur une cavilé centrale, fermée à la partic supérieure par un diaphragme qui correspond ainsi au point d'origine de chaque gaine. La partie solide de ces cylindres creux se décompose elle-même plus ou moins nettement en deux autres cylindres. Le cylindre externe ou cortical (pl. VI, fig. 9 a) est entièrement composé de lissu fibreux el cellulaire, dans lequel se présentent le plus souvent de grandes lacunes longitudinales répondant exactement aux sillons extéricurs, et qu'on appelle lacunes extérientes, valléculaircs, corticales (pl. VI, fig. $9 \mathrm{c}$ ). Le cylindre interne (pl. VI, fig. 9 b) renferme dans son tissu collulaire des 


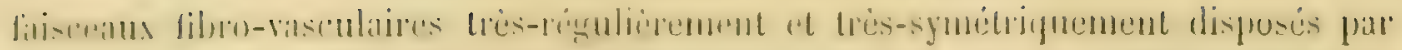

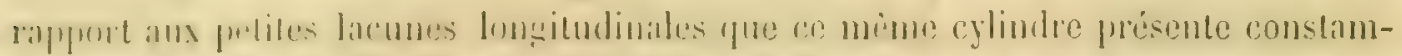

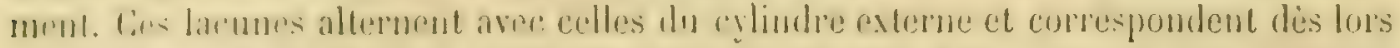

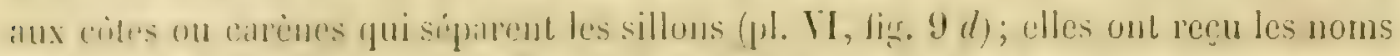

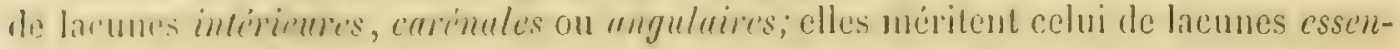
lirlles, pare qui elles ne manquent jamais, ni dans la tige, ni dans le rhizome, on les lacumes corticales el mime la cavité centrale manquent quelquefois. Les liges sont tonjums rigroureusement simples, en ce sens que leur axe continue jusquan point le

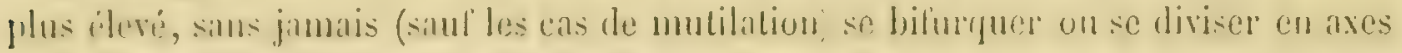
secondaires. Elles sont, suivant les espéces, nues ou pourves de rameaux régulièrement rerticillés et rigoureusement phacés ath-dessous des nudeuds el de la naissince des granes. Les rameaux et les ramuscules verticillés qüils supportent quelquefois, reproduisent sur une plus petite échelle l’organisation générale de la tig̨e; ils manquent souvent, sur certaines espéces, de cavité centrale el de lacunes corticales, mais ils uthent toujours lus lacunes intérieures on essentielles avec leur cortége de faisceaux filmo-sisculaires. Lépiderne des rameaux el des gaines est tonjumrs pourvu de slomater; quand ces organes existent sur les tiges, leur mode de distribution est, pour chargue espece, rigoureusement détermine, et lournit des caracteres excellents pour la distinction des groupes el des espèces.

Sur la plante adulte, l'appareil reproducteur est disposé en épi terminal. Cé épi est forme de plusieurs rerticilles de pédicelles horizontans, épanonis a lem extremite en une expansion verticale, peltiforne, dite clypéce (pl. VII, fig. 18 c), il la face intérieure duquel se trouvent cinq ou six sporanges, tous de même forme et de même

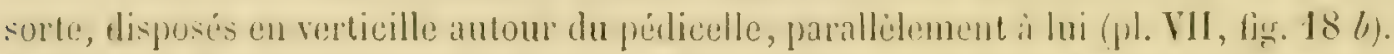
A leporgue de lis sporose", ees sporanges sourrent par une lente longitudinale sur le còté qui regarde le pédicelle.

Les sprues, toutes semblables, sont libres cutre elles, tris-nombrenses, splrérigues; elles portont deux appentices filiformes, dilatés a chacune de leurs extremites en une spatule applatie, tres-lyggroscopirque et se roulant en spire on se diroulant suiviml les altermatives de sécheresse on d'humidite (p). VIII, fig. $31 \mathrm{et} \mathrm{pl.} \mathrm{1X,} \mathrm{ligg.} \mathrm{S).}$

Ges spores se developpent en sporophymes" irregulierement lobules (p.l. IX, fig. 17), diö̈pues ou monö̈unes, portant a l'extrémite de leurs lobes des organes miles ou

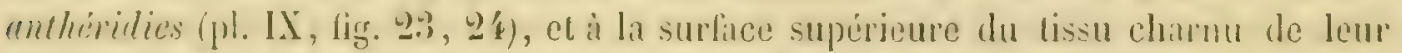
base des oreanes lemelles ou arehigones (pl. X, lig. 2, 3). Ceux-ci renferment un prent-

\footnotetext{
- J.es mots floraison, fruclificalion, maturite ne pouvant convenir pour désigner l'émission des spores des cryplo-

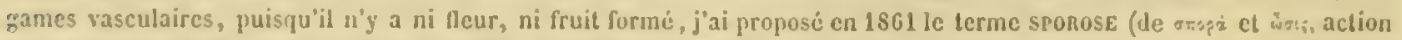
d'expulser, de chasser). Voir Lull. Soc. bol. de lirance, tome VIII, p. 36.

- Pour l'explication de ce terme, voy. ci-apres, p. 17, et aussi J. Duv. J., Orj. reprod. Eiquis., p. 701.
} 
embryon d'où résulte la nouvelle plante, en mème temps que se dessèche la plante intermédiaire ou sporophyme, qui a fourni et supporté les organes de fécondation et de reproduction (pl. $X$, fig. 1).

Ce mode de reproduction place les Equisetum parmi les Crxptoganes vasculaires; mais par l'ensemble de leurs formes, aussi bien que par les délails de leur organisation, les Equisetum se distinguent si profondément des autres vérgétaux de cel embranchement, que leur unique genre constitue la petile famille isolée des EquiséTaCÉES, ả laquelle il n'est pas très-facile d'assigner rigoureusement sa place, ainsi que le prouve la divergence des opinions sur ce point.

Les especes mentionnées jusqu'ì ce jour sur le territoire français, sont les suivantes:

$1^{\mathrm{er}}$ groupe. 1. E. maximum Lalr. (E. Telmatcia Ehrh.)

$2^{\circ}$ groupe. 2. E. sylvaticum L.

3. E. pratense EurH.?

4. E. arvense L.

$3^{\ominus}$ groupe. 5. E. littorale KüHLw.

6. E. limosum L.

7. E. palustre $\mathbf{L}$.

$4^{\mathrm{e}}$ groupe. 8. E. ramosissimum DESF.

9. E. variegatum Schleich.

10. E. trachyodon A. Braun.

11. E. hyemale $\mathrm{L}$.

\section{GHAPITRE PREMIER}

\section{Description extérieure d'une plante adulte}

\section{$\S 1^{\mathrm{er}}$. Du rhizome et de ses appendices}

La partie des Equisetum qui seule assure leur pérennité, et qui contribue le plus à leur propagation, est le rhizome. Il se compose d'articles séparés par des nouds solides, et terminés chacun par une gainne qui entoure la base de l'article immédiatement supérieur (pl. I, fig. 1, 2, 4, 5, 6). Ces articles, plus ou moins longs, ont toujours une forme régulièrement prismatique, it faces égales en largeur, un peu con- 
vexes ct dis lors a angles émoussés. Les gaines sont lobulées, et le nombre de leurs lobes ou divisions est exactement igal i celui des angles ou cotes, allendu que chaque graine eat la prolongation el la terminaison d'un entre-nerud, que la ligne mediane des lobes répond à une côte et leur sillon commissural à une face.

Sur quelques espèces (E. limosum, ramosissimum etc., j). V, fig. 14, 16..., les cntrenuruds ont une cavité centrale; sur d'autres (E. maximum, arvense cotc., pl. V, fig. 10 , 12...) ils en sont privés; mais, dans toutes nos especes, ils possident les deux cylimlres concentriques et les deux ordres de lacunes mentionnés précédemment. A chaque neud existe un diaphragme transversal, et, par la dislocation, ainsi que par l'analyse des entre-necuds, on constate que chaque diaphragme se trouve à la partie supérieure d'un entre-neud el correspond à peu près an point d'origine de la gaine terminale (pl. I, fig. 7 b). C'est vers la base de chaque gaine, dans la prolongation du sillon commissural de ses lobes, ou, en d'autres termes, sur la ligne médianc des faces de l'entre-neud qu'émergent, d'une part, les rhizomes lateraux et les tiges aíriemes, et, d'autre part, les verticilles des racines proprement dites (pl. I, figg. 1, $2, \quad k$ bis, $8 a, b)$. Sur cette région, aux faces qui n'ont encore ni racines ni thizomes lateraux, un examen attentif fait immédiatement reconnaitre l'existence de deux verticilles de petites saillies exactement superposées, et situces, comme les rhizomes laléraux el les racines, sur la ligne médiane des sillons ou des faces (pl. I, fig. $8 a, b)$. Une analyse, mème grossière, permet de voir que ces saillies résultent de la présence de deux verticilles presque contigus de petits bourgeons it létat expectant. Par leur développement, les bourgeons du verticille supérieur donnent des rhizomes et des tiges aériennes, et ceux du verticille inféricur des racines. Les rhizomes naissent loujours en très-petit nombre du même entre-nœud, un ou deux au plus, et sur les rhizomes profonds et horizontaux il n'y a des racines qu'au-dessous de leur point d'émersion (E. ramosissimum). Mais ordinairement sur les rhizomes latéraux et ascendants le verticille de racines est complet, et ces organes se montrent de préférence sur celle des faces oủ les bourgeons des rhizomes restent à l'état expectant. Lorsque, sur des entre-ncuds à côtes nombreuses, des rhizomes latéraux émergent de quelques fices et rute sur les faces voisines naissent des racines, comme ces dernières n'ont pas été fortement repoussées vers le bas par le développement du bourgeon supéricur, clles paraissent latérales aux rhizomes voisins et simulent un seul verticille sur un seul et même plan.

Lcs rhizomes latéraux ont la même organisation que celui dont ils émergent, el sont maintes fois plus vigoureux sur les especces dont les rhizomes rampent très-près de la surface du sol (E. limosum et ruriegutum). Il en est autrement sur les expéces dont les rhizomes principaux rampent à une grande profondeur $(\boldsymbol{E}$. artense, syliaticum, pulustre, rumosissimum etc.); ces rhizomes principaux sont beaucoup plus gros, 
et j'en ai vu dont les entre-nouds étaient longs de $0^{\mathrm{m}}, 18$. La longueur que les rhizomes eux-mêmes peuvent atteiudre, parait indéterminée et peut, en tout cas, devenir trìs-considérable. J'en ai obtenu des E. limosum, ramosissimum et hycmale qui dépassaient six mètres. Bischoff cite des faits analogues (Kiypt. (iew., p). 28), el Vaucher dit avoir observé que le plus souvent toutes les tiges qui remplissent une mare ou un fossé appartiennent à un même rhizome (10n. Prêl., p. 334 et 372).

Les racines naissent en verticilles, mais dans leur développement ultérieur elles se soustraient à la loi qui, sur les Equisetum, dispose tout par verticilles. Leurs divisions ne sont plus ni verticillées ni articulées, mais alternes et plusieurs fois répétées. Ces divisions commencent quelquefois dès le point d'origine de la racine, de telle sorte qu'il semble que deux ou trois racines sortent du même bourgeon ou qu'il y ait deux verticilles de racines. G'est probablement cette dernière disposition qui a porté M. Ad. Brongniart à dire « qu’on voil toujours deux radicelles placées "l'une au-dessus de l'autre et que quelquefois l'avortement de l'une d'elles les réduit "à une seule » (Hist. vég. foss., I, p. 100, pl. X, fig. 10). Toutes ces racines sont grêles et le plus souvent d'une longueur de $0^{\mathrm{m}}, 05$ à $0^{\mathrm{m}}, 10$, avec un diamètre inférieur ou à peine égal à $0^{\mathrm{m}}, 001$. Quelquefois aussi elles atteignent une longueur beaucoup plus grande ( $E$. arvense), presque sans augmenter en diamètre; mais sur les $E$. ramosissimum et hyemale elles prennent un tout autre développement. Des racines d'un mètre de long ne sont pas rares sur le dernier, et des pieds du second, arrachés à Rabet, près d'Arles, dans les berges du canal de Craponne, mont fourni des rhizomes horizontaux portant des racines de plus de 2 mètres de long, sur $0^{\mathrm{m}}, 004 \mathrm{de}$ diamètre au point d'émersion. Ces fortes racines émergeaient constamment isolées et chacune au-dessous d'un rhizome latéral à peine plus gros qu'elles.

A leur apparition, les racines sont très-tendres et d'un blanc jaunâtre; plus tard elles deviennent très-fermes, el leur surface, d'un brun foncé, est couverte d'une sorte de tomentum ou feutre. Les fibrilles qui constituent ce tomentum naissent directement d'une dilatation des cellules de l'épiderme, sans aucun diaphragme à leur base (pl. II, fig. 1-4), sans aucune cloison sur leur longueur, qui est quelquefois de cinq millimètres. Elles manquent sur les vieilles racines, ainsi qu’à la pointe des plus jeunes.

Ce tomentum existe aussi sur toute la surface des entre-nœuds de certaines espéces, ou seulement sur les gaînes et aux nœuds de certaines autres; d'autres espèces, enfiu, ont la surface des entre-nœuds glabre el luisante. Dans toutes les espèces, les rhizomes sont, au moment de leur évolution, d'un beau jaune citron; mais leur surface brunit très-vite et, avec l'àge, elle prend constamment une couleur foncée; sur les unes, mate et brune ( $E$. arvense etc.); sur d'autres, luisante et noire (E.palustre), ou d'un beau pourpre ( $E$. limosum). 
Souvent, à la place que devraient oceuper des rhizomes latéraux ou des rejetons alccendants, on wit apparaitre des tubercules (pl. I, fig. 1 - f). (duelquefois un seul et mime verticille de bourgeons donne naisance ì un rhizome latéral et à un ou deux tulurerules, mais le plus souvent ces derniers sont seuls et en petit nombre, comme les rhizomes. Ces tubereules sont simplement dus a une modification dans le développement des lourgeons qui devaient produire des tiges souterraines ou aériennes, et dont te tissu cellulaire, au lieu de s'allonger en entre-neends, s'est renflé en se chargreant de fúcule. Si on arrache, au mois de mai ou de juin, des rhizomes dE. urrense, on y trouve ordinairement des tubercules a tous les états de développement, depuis celui de simple bourgeon un peu gonflé, jusqu’à cclui de tubercule charnu ct complet. Sur ces tubercules on retrouve un peu modifiés toutes les parties et tons les organes qui existent sur un entre-nœud. A leur point d'émersion et d'adhérence ils ont, comme les rhizomes, une petite gaine, que leur développement en diamìtre a forcée a s’évaser en soucoupe. Au dernier terme de déreloppement, la grosseur de ces tubercules varie depuis celle d'un pois jusqu'à celle d'une petite noisette. Elle est mème beaucoup plus considérable sur l'E. maximum (pl. I, figg. 4). Ils sont en général de forme ellipsoïde, rarement subsphérique, on forme de poire sur l'E. maximum, toujours très-atténués à leur extrémité inféricure, ce qui fait qu'ils se détachent des thizomes arec une extrême facilité. Leur surface épidermique est semblable i celle des rhizomes sur lesquels ils naissent, et, comme elle, d'abord jaune, puis mate et feutrée, ou glabre et luisante. Ils sont dans le sens de la longueur marqués de petites cutes à peine prononcées, représentant celles des entre-nœuds et aboutissant, comme elles, aux côtes d'un petit appendice en forme de couronne courte et dentelie qui les termine constamment. Quand on examine cet appendice sur des tubercules trèsjeunes el qui sont encore d'un jaune citron, on reconnait très-aisément qu'il n'est qu'une gaîne à dents un peu charnues, courtes et formant une enveloppe unique à un petit bourgeon qui en occupe le centre. Souvent ce bourgeon avorte ou demenre à l'état expectant, et le tubercule reste isolé et terminé par un petit appendice conique; mais souvent aussi ce bourgeon se développe en un autre tubercule, sa gaine s'érase en soucoupe, et le second tubercule, terminé de la même manière, donne naissance i un troisième, et ainsi une suite de tubercules se disposent en chapelet, ayant chacun à leur base une gaine cn soucoupe, et le dernier étant couronné par sa gaine et son bourgeon (pl. I, fig. $1 d, e$ ). Ces tubercules ont tous la même forme et se détachent les uns des autres aussi facilement que les plus anciens se séparent du rhizome. Si on analyse la gaine et le tubercule, on reconnait que, malgré le rétrécissement de la régrion où la gaine prend naissance, il y a lì un no'ud et deux rerticilles de petits bourgeons. Linfërieur, qui doil donner des racines, les produit asse\% ordimairement; mais si l'on place dans l'eau, on dans une terre très-humide, des tuher- 
cules isolés ou accouplés en chapelet, au bout de quelques jours les bourgeons du verticille supérieur se développent, dans l'eau et. à la lumière, en petites tiges aériennes, et, dans la terre humide, en rhizomes grèles. C'est ainsi que ces tubercules servent de toute façon à la propagation de l'eśpèce'.

Il n'est pas très-rare de voir le petit bourgeon qui termine un tubercule, au lien de se renfler en un autre tubercule, s'allonger et se développer en entre-nœuds de forme normale; il en résulte un rhizome secondaire, qui a pour premicr entre-nœud un tubercule (pl. I, fig. $1 \mathrm{c}$ ). Enfin, j’ai trouvé, sur des rhizomes secondaires aussi bien que sur des rhizomes principaux d'E. arvense, certains entre-nouds renflés en tubercule (pl. I, fig. $2 b$ ).

Au moment de leur apparition, ces tubercules sont déjà entièrement solides, mais leur structure est molle; plus tard ils se durcissent et offrent sous leur épiderme une substance blanche, résistante et dure, d'une saveur un peu sucrée. Celte substance attire souvent les insectes ou les rongeurs, et il n'est pas rare de les trouver à moitié dévorés, ou ayant servi de retraite à des larves. Les tubercules si gros de l'É. maximum n'offrent presque aucune trace de fécule. La profondeur à laqquelle apparaissent les tubercules est très-variable. Sur de jeunes plantes d'E. arvense provenant de semis, j'en ai trouvé dès le premier entre-nœud, qui étaient presque à la surface du sol. Sur les anciennes plantes on ne les trouve ordinairement qu'à une grande profondeur.

C'est en 1712 que, pour la premiére fois, si je ne me trompe, les tubercules des Equiselum furent signalés par Ielwing; Haller (1768) les mentionne sur son $n^{\circ} 1676$ (E. arvense); il s'exprime en ces termes: «radicibus tamen gilandium simile aliquid "sape adharet, quod porcos credas requirere" (IIist. stipp. IIelw. III, p. 2). Un renvoi qui suit ce texte semble indiquer que l'auteur n'a pas vu le fait lui-même. $0 r$, en consultant l'ouvrage indiqué (Helwing, Flor.quasimod., p. 31), on y trouve ce qui suit: "LXXXVIII. Equisetum areense, glendiferum. IIujus radicibus glandes copiose ad"hærent, et quam maxime in agris arenosis effodinntur a suibus et pueris rusticis. "Grati et dulcis sunt saporis. Instinctu naturæ sues odoratu superficiem terre de"tegunt, et tam diu terram evolvint, quoad appropinquent ad glandes Nostratibus "Erl-Nüsse dictos, quod subulci animadvertentes statim accurrunt, et pedibus por«cos abigentes levissimo labore nucleos suos terrestres colligunt. Maturescunt circa (tempora autumni.) Le contraste entre la rareté des tubercules dans nos contrées et la fréquence que suppose ce passage, surprend d'abord, mais toutefois n'est pas un motif suffisant pour refuser de croire qu'il se rapporte à l'E. artense. Ruprecht a

\footnotetext{
1. Au jardin botanique de Breslau, dit M. J. Milde, de nombreuses plantes sont provenues de tubercules d'E. $T e l-$ mateia reçus par M. Gïppert de la Haute-Silésie» (Gef. Crypl. Schl., 1. 430).
} 
constatie en effit que dans le nord de la Russie ces tubercules se montrent en erande ahondance of it peu de profondeur sur certaines varietis de celte espèce: eTubra "E. arensis australionis profimdissime in terma latere, notum est; eo magis tubera

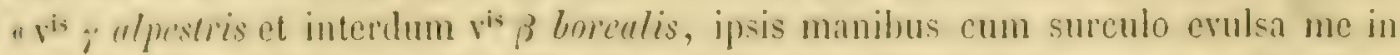
"admirationem converterunt" (Dist. crypt. lioss., p. 21). Si je conserve un tres-ligger doute sur l'assertion de Ifelwing, e'est uniquement parce que les floristes prussiens ne la mentionnent pas et me paraissent dies lor's ne pas avoir constaté la mème fréquence des tubercules.

Après Itewing et IIaller les tubercules furent remarqués par Smelowsky, ì qui Bischorr attribue i tort la priorite de leur mention (Krylt. Lew., p. 50 ). Cet auteur les avait également trouvés en si grande abondance sur l'E. artense gu'il proposait d'en tirer parti pour engraisser les pores (Mém. de l'Acal. de Pétersboury, vol. I, 18031806). En 1815 ils furent signalés par De Candolle sur l'E. palustre, et depuis ils lont étépar plusicurs auteurs sur les E. maximum, sylvaticum et artense. Je les ai recueillis sur toutes ces espèces et sur l'E. littorale. Ces organes ont été, en 185\%, l'oljet d'un travail spécial, où MI. E. Ramey a publié d'intéressantes observations el émis les deux assertions suivantes: "Les tubercules ne se trouvent que sur les espéces a tiges de "deux sortes oủ ils servent éridemment i l'alimentation des tiges fertiles » (Tub. Ë., p. 212), et que « sur les espèces dont les souches n'offrent pas de canal central » (o. c., p. 217). La première assertion est contredite par les tubercules si nombreux des E. littorale et palustre; la seconde, par ceux de l'E. littorale. Je crois que les tubercules nout pas encore été mentionnés, cl je ne les ai pas vus moi-même sur les E. prutense, limosum, ramosissimum', rariegutum et hycmale. On ne les trouve. pas d'ailleurs sur tous les pieds des autres espiecs; souvent on les y cherche en vain, si l'on ne pénetre it la profondeur considérable a laquelle ils se montrent en certains endroits sur quelques-unes d'entre elles, par exemple à I mètre pour les E. palustie, arrense. J'en ai trouvé sur ce dernier a $2 m, 50$ de profondeur dans les fortificilions de Strasbourg.

La profondeur it larquelle parviennent les rhizomes fut d'abord pour Vaucher le sujet d'un vif étonnement, ot, comme il n'avait point trouvé de rhizomes descendants, c'est-à-dire ayant les pointes des gainnes dirigées en bas, il avait posé, sans la résoudre, In question suivante: "Comment ces racines des Prìles pénètrent-elles ì une si "grande profondeur dans les terrains les plus argileux, el comment sont-elles ter--

\footnotetext{
' be Candolle (Fl. $r$ r., Yl, p. 215) semble vouloir rapporter son $E$, tuberosum à l'E'. elongatum Willd., e'est-it-tire ì I'E. ramosissimum Desf.; mais Vaucher (Mon. Prêl., p. 368) et Duby (Bot. gall., 1, p. 635) le rapportent trèsexpressément à l'E. palustre, ce que commande d'ailleurs le caraetère d'épis oblus cité par De Candolle. Cet auteur joute qiue llectot a trouvé son $\mathcal{E}$. luberosum à Nantes; scrait-ce sur cette autorité que M. Boreau attribue a l'E'. ramosissimm a de petits tubercules ovoïdes = (Wil. cent.)?
} 
"minées?» (Jon. Pril., p. 342). Après lui, Bischofl fit des remarqques analogues sur la profondeur à laquelle pénètrent el rampent les rhizomes (Krypt. Geu., p. 28); et après s'être posé la même question que Vaucher, il erut trouver des éléments de solution dans celte considération « que les Eiquisetum croissent toujours en des lieux oủ le sol s'élève constamment autour de leurs tiges, par de continuels dépôts aux bords des ruisseaux et des rivières, par de nouvelles couches d'humus dans les forèts, et enfin par les travaux annuels daus les terres cultivées „ (Krypt. Gew., p. 4t). Mais plus tard il reconnut lui-même l'insuffisance de celte solution et constata que, dans les premiers développements d'une jeune plante, il existe des rhizomes à direction descendante (Entw. Eq., p. 791). J'ai pu moi-même chaque année (voy. ch. IV, § 亻) constater l'existence de semblables rhizomes sur mes plantes provenant de semis. Ainsi, en général, ces jeunes plantes me donnaient après la première année quatre ou cinq rhizomes descendant verticalement, dont souvent quelques-uns, sortant par le trou du vase de semis, pénétraient dans le terrain enveloppant; ce que je voyais lorsqu'en mars je les plaçais dans un vase plus grand. Mais lorsqu'en juillet, pour les mettre en pleine terre, je les enlevais de ce nouveau vase, je trouvais qu'ils avaient pénétré la terre de nombreux rhizomes, lesquels venaient ramper contre le fond du vase. En juillet 1861 je suivis les travaux d'une tranchée ouverte à Bouxwiller dans une terre argileuse tonte couverte d'E. arvense et qui n'avait jamais été remuée. Je trouvai en élat de développement un grand nombre de rhizomes à gaînes descendantes, qui pénétraient verticalement à une profondeur de plus d'un mètre. J'ai constaté les mêmes faits sur des rhizomes d'E. palustre en faisant creuser dans les sables des bords du Rhin à Strasbourg, et sur l'E. ramosissimum en examinaut des tranchées failes aux digues du canal de Craponne, dans la propriété de M. Jouve, mon beaupère, à Rabet, près Arles (Bouches-du-Rhòne). La direction verticale descendante des rhizomes est un fait avéré qui explique suffisamment la profondeur i laquelle rampent certains rhizomes.

Les tiges qui, partant des rhizomes, s'élèvent à l'air libre, ressemblent entièrement aux thizomes dans toute leur partie souterraine, et les changements ne se font que vers la surface du sol. L'organisation des tiges répond d'ailleurs dans son ensemble à celle des rhizomes, et, pour les Equisetum, plus peut-être que pour toute autre plante, il est permis de dire que les rhizomes ne sont que des tiges souterraines. Les nœuds des tiges, même très-jeunes, offrent aussi à la base de leurs gaines denx verticilles de petites saillies ou bourgeons, dont l'un se développe en rameaux, et dont l'autre, l'inférieur', reste à l'état expectint, si les circonstances ne permettent pas le développement des racines. Mais si l'on entoure de mousse humide la partie inférieure d'une tige aérienue déjà munie de verticilles de rameaux, il se développe au bout de quelques jours un verlicille de racines au-dessous de chaque verticille de rameanx; ct 


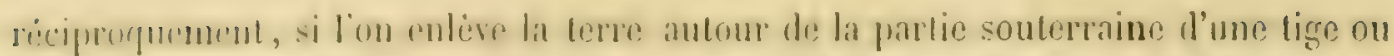

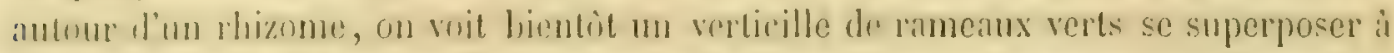

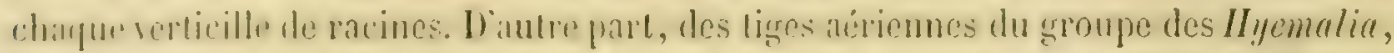

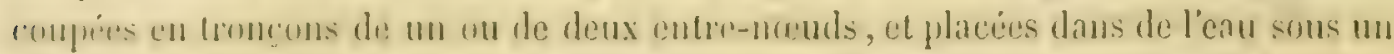
papier gris, on dans du terreau très-humide, ont produit des racines au bout de

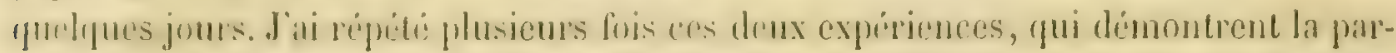
faite identite de nature et la double destination des rhizomes el des liges. M. J. Milde

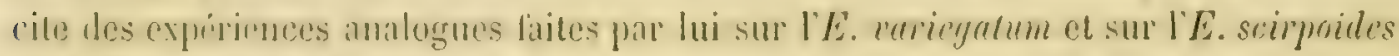
(Gief. Crypt. Sclll., p. 471').

Charpue article du rhizome et chaque tubercule, ajant au-dessous de leur nurd, comme nous l'avons vu, un verticille de bourgeons à rejetons aériens ou souterrains, et un verticille de bourgeons à racines, il s'ensuit qu'un nœud est un point vital, un centre de vigetation, et des lors qu'un seul article complet, on un scul de ces tubereules, sulfit pour reprorluire immédiatement lit plante. C'est, en effet, ce qui arrive. "L'E. arvense ne peut jamais être complétement extirpé des champs une fois qu'il les a envahis; car, à mesure qu'on coupe le rbizome avec la charrue, on lui donne l'oceasion de se multiplier. Remuer lit lerre, c'est augmenter la propagation de celte plinte cmbarrassante " IBischofl, Krypt. (iem., p. 4h). J'ai pu moi-mêtne vérifier la parfaite exactitude de cette assertion. Dans le courant de fürrier 1860, je vis linire sur la ligue de l'Est, près de Strasbourg, un remblai qui, en juin suivant, fut litteralement couvert de tiges d'E. arense. J'en arrachai phlusieurs, et toutes provenaient de fragnents de rhizomes dont quelques-uns étaient enfouis a plus d'un mètre de profondeur. In de ces rhizomes partait de l'extrémité d'un tubercule et se dirigenit en avant; ce tubercule n’était plus adhérent au rhizome mere, et il se trouvait a la base du rhizome anquel son bourgeon terminal avait domné naissance. Tuntes ces tiges etaient steriles; et je dois ajuuter que ju n’ai jamais rencontré, sur les E. muximum et arrense, de tiges spicilëres naissant directement des rhizomes frofonds et horizontaux; toules les tiges spiciferes mont paru naitre aux entre-nouds inférieurs d'une tige stérile et robuste de l'année précédente.

\section{\$2. De la tige stérilc}

Les rejetons du rhizome qui arrivent i l'air libre, constituent ec que l'on appelle,

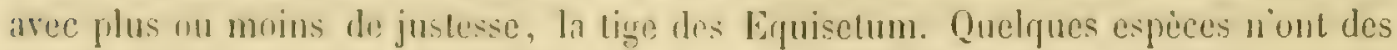
ligges que d'une forme, c'est-i-dire que les tiges stériles el les tiges spicifures ont ab-

\footnotetext{
'On comparera avec intérèt ce que dit M. A. Trécul sur e l’existence de racines latentes dans les tissus de certaines "plantes, et Jes racines rudimentaires dans l'écorce interne du Salix vitellinan (Urig. rac., p. 333).
} 
solument même apparence et même organisation; d'autres ont ordinairement des tiges spicifères différant de leurs tiges stériles par l'époque d'apparition, par la durẻe, par l'apparence extérieure et par quelques détails d'organisation. Je ne décrirai ici que les tiges stériles et les tiges spicifères conformes; et je réserverai ce gui est particulier aux tiges spiciêres non conformes pour le paragraphe suivant, oủ il sera question de l'appareil de reproduction.

Les tiges des Equisetum sont herbacées, grêles ( $E$. maximum excepté), cl leur développement en hauteur est généralement compris entre $0^{\mathrm{m}}, 15$ (E. variegatum) ot $1^{\mathrm{m}}, 50$ (E. maximum et ramosissimum); ce dernier devient même beancoup plus çrand dans les haies en Provence. Elles sont droites ou rarement ascendantes, et se composent d'articles cylindriques, creux, mais fermés à leur partic supéricure par un diaphragme, au delì duquel s'élève une gaine lobulée emboîtant l'article immédiatement supérieur.

Ces entre-nocuds sont de couleur verte, à l'exception de l'E. maximum, sur lequel ils sont d'un blane d'ivoirc. La surface en est plus ou moins rude au toucher, elle cric an contact d'un instrument tranchant, et un faible grossissement permet de voir que celte r'udesse est due à de petites saillies siliceuses de formes diverses selon les espèces et à peine sensibles sur les $E$. limosum et maximum. Cette surface n'est jamais rigourcusement cylindrique, mais plus ou moins prismatique et parcourue de côtes et de sillons parallèles et longitudinaux, quelquefois très-prononcés, mais à peine marqués sur les deux espèces citées en dernier lieu. Les côtes alternent sur deux entre-nœuds contigus, de telle facon que chaque fois les côtes d'un entre-ncud quelconque correspondent aux sillons de l'entre-nœud qui lui est inféricur et de celui qui le surmonte.

Comme les gaînes continuent régulièrement chaque entre-nœud, les côtes et les sillons des entre-nouds se prolongent sur les gaines, les sillons jusqu'au fond des sinus, et les côtes presque jusqu'à la pointe des lobes; côtes et sillons sont toujour's plus forternent marqués sur les gaines que sur les entre-noeuds. Par suite d'une particularité d'organisation qui sera décrite plus loin, les côtes des entre-nouds et des gaînes de certaines espèces ont sur leur ligne médiane ou carène une légère dépression qui a été appelée sillon carénal, le sillon de séparation des côtes des gaines ayant été appelé sillon commissural'. Les gaînes s'appliquent assez exactement sur la base de l'entrenoud supéricur et sont rarement un peu dilatées en cloche. Leur longueur totale, ainsi que celle de leurs lobes, varie considérablement selon les espèces, mais est assez constante sur une même espèce. Leur partie tubulaire présente d'ordinaire (cscepté

11. Däll fait remarquer que ales dents des gaînes ont encore souvent de chaque cóté une carène latérale, qui est séparée de la carène médiane par un sillon latéral. Chaque dent de gaîne peut, d'après cela, avoir trois carènes, et même quatre, lorsque la carène médiane est divisée par un sillon $(F l$. Bâl., I, p. 55, 1857). Le même autcur appelle "sillon marginal le sillon qui sépare chaquue dent" (ibid.). 
smr l'E. murimum la mime couleur ot la mime consistance que l'entre-naud qu'elle surmonte; mis les lohes, qui la terninent sur toutes les espèces, ont le plus souvent une, conleur et une consistance i eux particulières. Ils sont ici résistants et coriaces, de conlivur foncée ct à peine membraneux ì leurs bords (E. arense, palustre cle.), lit tont it firt membraneux (E. muximum, ruriegatum etc.), quelquefois même très-cadues (E. hyemale, voy. J. Dux. J. Ey. hycm., p. $165 \mathrm{ct}$ suiv.). Dans ce cas et après la chute des lobes, la gaine est toute cylindrique el terminée par de petites crenelures arronlies, un pen gibbenses, noires, dures el d'aspect corné. La forme des lobes, constante sur une mème espéce, est plus ou moins effilée, et quelquefois déliée comme une soie; les bords n'en sont jamais nettement terminés, mais toujours plus ou moins irrégulierement ondulés, comme les bords d'un tissu déchiré. En effet, comme on peut le remarquer sur les gros bourgeons et sur les tiges naissantes de toutes les especes, chaque gaine, pendant toute sa période d'évolution, constitue une coiffe entiere, à peine entrouverte et dentelée an sommet, mais non lobulée, et elle n'est plus lard divisée en lobes que par une cause toute mécanique, par la poussée des contri-neuds supérieur's qu'elle recoure. Ce déchirement se fait avee une apparence de grande régularité, parce que la moitié supéricure de la gaine présente des bandes longitudinales composées de nombreuses couches de cellules continuant les cótes de l'entre-nœud, el, en alternance arce les précédentes, des bandes composées d'une ou de deux couches de cellules très-délicates répondant aux sillons de l'entre-noud, de telle sorte que le déchirement a lieu là où les bandes membraneuses oflrent moins de résistance. Ilais, malgré celte apparence de régularite, les traces du déchirement sont toujours reconnaissables, même à un faible grossissement. D'ailleurs sur quelques individus, et constamment sur l'E. syluaticum, le déchirement ne se fait pas sur toutes les bandes minces, el trois, quatre et mème cinq̧ lobes continuent à demeurer unis (pl. III, fig. 16).

La lige des Éfuisetum est done dépourvue de fenilles proprement dites. Mirbel a considéré "la gaine comme formée de feuilles soudées et les dents comme les extrúmites libres de ces feuilles.» G'est en effet l'hypothèse qui se présente le plus naturellement. Nais, si l'on considere, d'une part, que les lobes ne sont point réellement des parties libres, el quils ne paraissent tels quä la suite d'un déchirement, d'autre part, que les feuilles sont des organes essentiellement aériens, et que les rhizomes sont munis de gaines aussi réggulières et aussi développées que celles les tiges, on sera moins porté a regrarder les divisions des gaines comme des feuilles; el, pour ma part, je me bornerai à les appeller divisions de la gaîne.

Lin rompant ou en tordant la tige d'un Eyulsetum adulte, surtout vers l'arrièresaison, on romargur qu'elle se sépare, comme les rhizomes, en deux cylindres, l'un isterne nu contical, lautre intrine. Ces deux cylindres ne sont que pen ou point du 
tout adhérents sur les E. maximum, sylvaticum, pratense, aricnse, palustrc; mais ils adhèrent plus fortement sur les autres espèces, attendu qqu’à leur surface de contact ils se pénètrent réciproquement par des ondulations, et dès lors on ne les dislingue bien qu’au moyen de coupes très-minces pratiquées tout contre les nœuds. Le cylindre interne est composé de tissu cellulaire incolore et de faisceaux fibro-vasculaires; il présente constamment la grande cavité centrale qui parcourt toute l'étendue de l'entre-nœud, et autour d'elle la série circulaire des lacunes essentielles. Le cylindre cortical, entièrement dépourvu de faisceaux fibro-rasculaires, présente, vers l'intéricur, du tissu cellulaire incolore, dans lequel sont crcusées les grandes lacunes extérieures en alternance avec les lacunes essentielles. La forme el les dimensions des lacunes extérieures sont assez constantes, mais toutefois ces lacunes manquent souvent sur l'E. limosun. Le long de chaque côte s'étend sous l'épiderme un faisceau de fibres très-longues et très-résistantes que je désignerai par le nom de filres corticales; autour ou à côté d'elles existent des groupes de cellules à chlorophylie dont l'arrangement et la forme sont constants sur chaque espèce; ils manquent sur l'E. maximum et sur les tiges spicifères non conformes.

La tige des Equisetum est rigoureusement simple et ne se bifurque ni ne se divise jamais (sauf les cas de mutilation) en axes secondaires; mais dans la plupart des espéces elle est pourvue de rameaux latéraux disposés en verticilles. Ces rameaux naissent de bourgeons adventifs situés à la base des gaînes, entre les còtes, daus la prolongation du sillon commissural des lobes; de sorte qu'ils alternent avec les divisions des gaînes. Ils sont constamment munis à leur point d'émersion d'une petite gaîne basilaire, fortement colorée et irrégulière, c'est-î-dire plus courte ou même presque nulle du côté de la tige, plus longue à l'extérieur. Comme les liges principales, les rameaux sont composés d'articles avec gaines régulières et lobulées. Sur quelques espèces ( $E$. limosum, palustre et le groupe des Hyemalia) ils apparaissent avec plus ou moins de constance et ne constituent que des verticilles incomplets, mais sur la plupart des autres ils forment des verticilles complets et d'une parfaite régularité. Ils sont d'ordinaire ascendants, quelquefois étalés ou mème arqués et infléchis vers l'extéricur. Relativement à la tige, les rameaux sont de longueur très-variable, mais sur un même verticille jls sont généralement d'égale longueur, ainsi que leur's entre-ncuds pris dans leur ordre de succession, ce qui revient à dire, par exemple, que tous les troisièmes ou tous les quatrièmes entre-nœuds d'un même verticille sont égaux entre eux. Le rapport qui existe entre la longueur du premier entre-nœud des ramenux et la longueur de la gaîne à la base de laquelle iłs émergent, fournit un caractère spécifique très-constant et très-facile à observer (roy. J. Duv. J., Eq. franc., p. 512). Le plus souvent les rameaux demeurent tout nus; sur quelques espèces cependant ils se revêtent de ramuscules disposés eux-mèmes cn verticilles. 
Les entre-nouds des rameaux el des ramuscules tont toujours verts, même sur l'E.

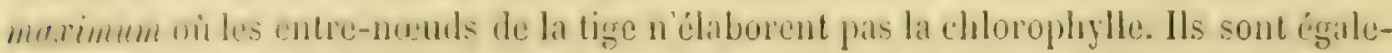
ment toujunts rudes et plus profondément sillomes que ceux de la tige, ee qui, par suite de leur moinde diamitre, les fait paraitre fortement anguleux (pl. VI, fig. 3, 6, 10); at ce nest que sur les especes ou ils acquierent une grossem considérable quils conservent divantige la forme prespue eylindrigue de la lige. La cavité centrale est ublitire sur quelques especes, aiusi que les lacunes corticales, mais les lacunes esseuticlles du cylindre interne existent constamment avec les faisceaux fibro-vasculaires qui les accompagnent.

Dans les trois premiers groupes, les liges nont qu'une dure rigoureusement annuelle; elles se développent an printempes et disparaissent aux premièes atteintes du froill, mime dans nos départements méridionaux. Les bourgeons qui doirent se développer l'année suivante se montrent sur les rhizomes et plus souvent sur la partic souterraine des tiges dis la fin de l'été, et restent en cet état pendant l'hiver; quelquesuns aussi apparaissent vers la fin du printemps et se développent dès l'été en liges tardives et stériles.

Les espèces du groupe IIyemalia peuvent persister pendant l'hiver, mais toutefois sous des condilions diflérentes. Ainsi, les tiges de l'E. rumosissimum se flétrissent et disparaissent aux premières alteintes du froid, non-seulement it Strasbourg mais dans le midi de la France. En Prorence, comme sur les bords du Rhin, elles ne persistent que dans les haies ties-fourrées où elles atteignent alor's, sans augmenter de diamètre, une longueur considerable. Dans la vallée des Baux, près d'Arles (Bouches-du-Rhòne), jail recueilli, au milieu de buissons épais et bien abrités, des liges de 3 métres de laaul el dont les rameaux araient $0^{\mathrm{m}}, 80 . \Lambda \Lambda$ Iger, dans les haies voisines du Jurdin l'essei, celte espece alteint une taille plus élevie encore, tandis que les pieds vérgélimt a decouvert sur les bords de l'Arach ont chaque année leurs tiges détruites par le froid. Il en est à peu près de mème pour l'E. variegulum; à découvert ses tiges sont brihlées par les premieres gelíes et se désarticulent aussilòt; mais si ses toulfes cespiteuses sont bien abritées par des buissons ou sous des l'enilles mortes, elles persistent et montrent leurs épis orangés dès le premier printemps.

L'E. hyemule el l'E. Irachyolon supportent admirablement les froids les plus rigroureux, el, il l'abri ou i dicouvert, une température de - $20^{\circ}$ paraitn'avoir sur eux d'autre rffet qu'un temps d’arrèt. Ils recommencent à pousser aussited que la gelée cesse. Leur noureau développement est lacile à constater i la partie inféricure de charque entre-nurud. En effet, il est d'un vert beaucoup plus clair que la partic superisure, i larpuelle te frobil a domus une couleur plus foncée ou quelquefois rougeatre. C.ue zone claire de croissune nouvelle est tres-apparente an mois d'avil; a peu pris mulle sur les entre-nouds inféricurs, parec que ces entre-nouds ont en général 
acquis tout leur développement dẻs la première annće, elle angmente progressivement de largeur en s'avancant vers l'extrémité, oir elle finit par oceuper toute l'étendue des derniers entre-nouds qui sont en entier de croissance récente.

\section{§3. De la tige spicifêre et de l'appareil de reproduction}

Les organes de reproduction sont disposés en un épi terminal, qui se trouve, selon les espèces, soit à l'extrémité des tiges ordinaires el de leurs rameaux, soit sur des tiges particulières d'un aspect tout différent de celui des tiges stériles de la même espèce. Divers auteurs ont donné le nom de hampes aux tiges spicifëres propres (Vaucher, Mon. Prêl., p. 353 et 359 ; Bischolf', Krypt. Gew., p. 31 etc.), voulant marquer par là les différences profondes qui les distinguent des autres liges de la même espèce.

Elles sont d'abord plus précoces, d'une évolution plus rapide, d'une plus courte durée, à tel point que deux ou trois semaines au plus des premiers jours de printemps

- leur suffisent pour se développer, accomplir leur fonction reproductrice et disparaître, alors que les autres ont à peine commencé à sortir de terre. Leur taille est d'ordinaire beaucoup moins élevée. Leurs entre-nœuds, au lieu d'ètre verts, ì tissus résistants, à surface marquéc de sillons profonds et de côtes rudes, ont une surface lisse et unie, d'une couleur rose pâle, tournant rapidement au brun, el des lissus mous, si chargés d'eau que la cavité centrale en est presque toujours à moitié remplie. Les gaines seules élaborent la matière verte, mais elles sont beaucoup plus longues, plus larges, plus colorées et plus membraneuses que celles des tiges stériles. Au-dessous des gaînes on reconuaît bien des rudiments de bourgeons, mais ordinairement ils ne se développent pas, et la tige reste nue. La cavité centrale, les deux sortes de lacunes, les faisceaux fibro-vasculaires n'offrent rien de particulier; mais la matière verte manque complétement sur les entre-nœuds.

Ce qui précède n'est exactement vrai que des E. maximum et arvense, et ne s'applique aux tiges spicifères des $E$. sylraticum et pratense que pendant la première période de leur développement. Après que l'épi de ces espèces a rempli ses fonctions, il se fane, mais seul, et la partie inférieure de la tige spicifère continue à végéter, élabore la matière verte et se couvre mème de rameaux plus forts que ceux des tiges stériles. Ces espèces sont done, à cet égard, comme des intermédiaires entre les espèces à tiges de deux sortes et les espèces à tiges toutes conformes.

Il est à remarquer que même sur ces dernières espèces, les liges vertes et conformes qui portent un épi se modifient notablement aux entre-nouds les plus rapprochés de l'épi terminal. Eu effel, ces entre-nouds perdent peu i peu l'intensité de leur couleur verte; ils deviennent moins résistants; leurs gaines se dilatent; celle qui est 
immediatement sun- lépi devient lange, Evasée, plus colorée el plus membrancuse, et,

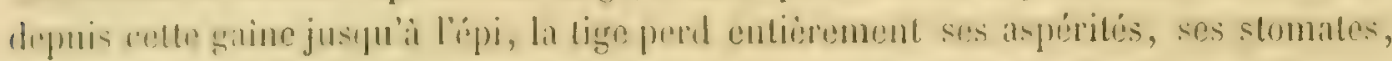

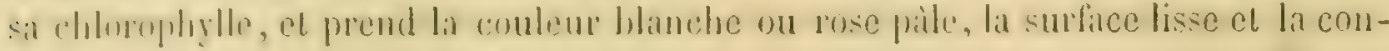
si-tance mulle des tiges spicileres propres. Il est impossible de ne pats recomiatre une amalngie évidente entre ces diveres moditications el celles qui, che\% centains phanirogames, existent vers les extrémités de leurs tiges, dans la forme et la couleur des fenilles voisines des rameaux floriferes. Lorganisation des liges spicilères propres, ainsi que celle des entre-nuends contigus a l'épi, est évidemment une préparation i Pappintion des or:anes reproducteurs; seulement, sur les premieres, cette constitution prefaratuire envahit tonte létendue de la tige, tandis que, sur les autres, clle so réduit aux cotre-noeuds supérietrirs.

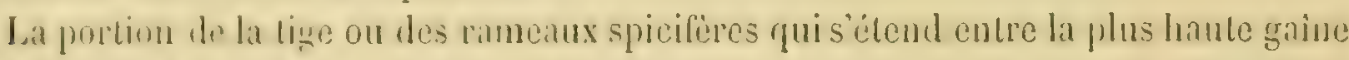
ct l'épi simule un predoncule et en a quelquefois improprement reen le nom. Cutle frartie qui, je le répetere, est toujours de consistance molle el de couleur prile, est quelquefois tres-longue sur les liges spicilëres propres, el d'autres lois, sur les tiges spicifies confurmes, tellement courte qu'elle reste comme cachée dans la dernicre gaine. Sur cutte uème partie, au-dessous de l'épi, et à une distance variable, se trouve me saillic circulaire, et qui semble les derniers vestiges d'une gaine modifié on l'appelle l'améu. Au moment où l'épi sort de la dernicre gaine, l'annean estétroitement applingué contre lui, et son tissu de consistance molle présente des saillics monlées sur les inegalités du verticille inférieur de l'épi, et simulant des lobes qui s'eflacent bientôt. Souvent sur' l'E. maximum, et quelquefois sur l'E. arvense, il y a deux anneaux. Lépi est subeylindrique, un peu atlénué aux deux extrémités el quelquefois

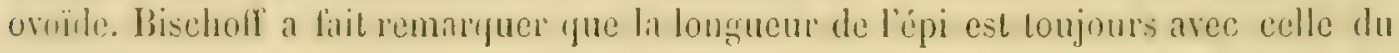
pédoncule dans une relation telle que si l'un s'étend ou se réduit, l'autre s'allonge ou se raceourcit de même, et que, plus le pédoncule est long, plus il est de consistance molle et prompt it se faner (Krypt. Gew., p. 31). Sur les trois premiers groupes, l'épi est obtus; sur le groupe des IIyemaliu il est apiculé.

Ce point excepté, les épis, quels que soient les tiges ou les rameaux qui les suppuntent, ollient sur toutes tes expeces la mème organisation essenticlle et ne diflërent Enicr que par les dimensions. Ils at composent done invariablement de plusieurs verticilles lapprochés et superposés de pédicelles horizontaux, épanouis a leur extré-

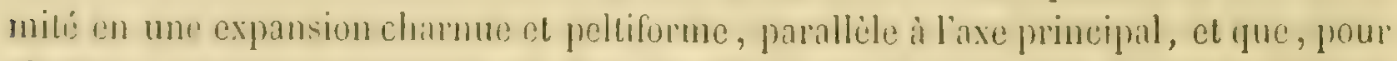

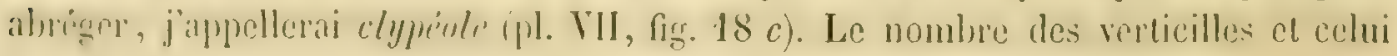
des pédicelles est un peu variable sur une même espèce et très-variable d'une espèce à l'autre; sur l' $E$. maximum on compte jusqu'à 35 verticilles et une moyenne

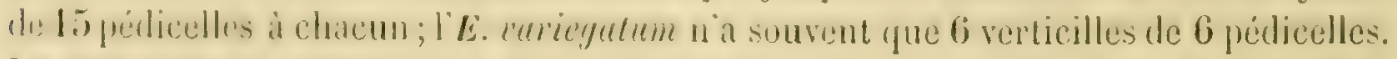
le pridicelle est, comme lase principal de lépi, diun blane pale ou rose; la surface 
externe du clypéole est de couleur très-variable, rose, verditre, brune ou d'un noir mat; et, par suite de la compression réciproque, elle est le plus souvent de forme hexagonale. $\Lambda$ la face interne se trouvent disposés en verticille et parallìlement aux pédicelles einq ou six petits sacs, deux ou trois fois plus longs yne larges, ayant la forme de la dernière phalange d'un doigt; ce sont les sporanges (pl. VII, fig. 18 b). Ils sont tous semblables entre eux. Leur tissu est simple, incolore, un peu transparent et formé de cellules fibro-spiralées très-ćlégantes. Ces sporanges s'ouvrent par une fente longitudinale sur le còté qui regarde le pédicelle, et laissent échapper, ou plutòt lancent au loin, sous forme de poussière verte, des spores très-nombreuses. Cies spores, toutes semblables entre elles, consistent en une petite sphère verte, à laquelle adhèrent deux longs appendices filiformes, aplatis et spatulés à leurs extrémités; on les a appelés élatères (pl. VIII, fig. 31 , et pl. IX, fig. 8). Comme ils sont contigus entre eux à leur point d'adhérence à la spore, ils simulent quatre branches qui se réuniraient sous la spore. Souvent, lors de la sporose, les élatères s'entortillent entre eux, et les spores restent suspendues contre les sporanges en petits paquets qui présentent l'aspect d'un feutre gris verdàtre. Ces élatères sont éminemment hygroscopiques; avec l'humidité ils s'enroulent étroitement autour de la spore en une double spirale; mais, en se desséchant, ils se déroulent brusquement, et sont ainsi lancés au loin et la spore avec cux. Observé au microscope sur plusieurs spores ì la fois, ce phénomène offre à l'œil un spectacle ravissant. Sous le souftle le plus léger de l'observateur, les élatères, par la succession de leurs contractions et de leurs extensions, exécutent les mouvements les plus gracieux, et toute la masse des spores parait animée.

De ces spores proviennent les sporophymes ', sur' lesquels apparaissent d'abord les véritables organes de fécondation, savoir les anthéridies avec leurs spermatozoïdes, et les archégones avec la cellule reproductive, et enfin les nouvelles plantes, comme il sera exposé avec détails au ch. IV.

\footnotetext{
1 Dans un mémoire publié en 1859 (Bull. Soc. bol. $F r$., VI, p. 701) j'ai adopté et proposé le nom de sporophyme, pour désigner la petite plante qui résulte immédiatement du développement de la spore. Il m'a paru remplacer avec avantage ceux de proembryon, de prothalle, qui manquent entièrement de juslesse, puisqu'il n'y a chez les Lquisetum ni thalle ni embryon proprement dit. On peut voir dans le même Bull., VI, p. 212, les justes observations de 3 . Clos sur ces diverses dénominations. M. W. Ph. Schimper donne le nom de "prolonema à celle végétation intermédiaire entre la sporule et la jeune plante" (Sphag., p. 17 et 18). Parfaitement juste pour les Sphagnum, oủ cette végétation intermédiaire n'est "destinée qu'd̀ nourrir le jeune individu ", cetle expression cesserait de l'ètre pour les Equisetum, où le végétal de transition doit donner naissance aux organes de la fécondation avant le supporter et de nourrir les plantes nouvelles.
} 


\title{
CHAPITRE II
}

\section{Description analytique d'une plante adulte}

\begin{abstract}
$\S 1 \mathrm{er}$. De l'épiderme
De lifriderune des rhizont's. - L'épiderme des rhizomes se compose invariablement d'un seule couch de cellules allongées, présentant généralement dans claque crouple dis caractives essentiels communs, et, sur chaque espece, des différences de détail très-constantes.

Ainsi, les espreces des deux premicrs groupes (E. maximum, syleaticum, arrense) allient des cellules épidermiques à parois tres-minces, presque toutes pourrues de fibrilles, uqui cumrent la surfice du rhizome d'un tomentum plus ou moins serré.

Les espreces du troisieme groupe, toutes plus ou moins aquatigues (E. littorale, limosum, julustri') et, de plus l'E. jrutense', ont des cellules épidermiques ì parois tri-tipaises, entièrement dépourvues de tomentum (les gaines exceptées), et la surface des entre-noends du rhizome est lisse et luisante.

Les cellules du quatriène groupe (Iyemuliu) ont des parois très-épaisses, revètues al l'extéricur d’une croùte siliceuse el rugueuse. Elles ne sont tomenteuses que sur les gaines, et la surface des rhizomes est d'un noir mat.

Les rhimones de l'E. maximum (pl. II, fig. 1, 2) ont des cellu'es épidermiques transparentes, peu colorées, presque cylindriques, tout unies; seulement, nu point où duit paraitre une fibrille, la paroi extéricure se soulève en un petit mamelon hémisphririque, dont le milieu se prolonge en fibrille à base dilatée, sims aucun diaphragme ni à l'origine ni sur sa longueur.

Ici, comme dans les autres especes, ces fibrilles, toujours simples et sans ancune arliculation, deviennent sourent très-longues et atteignent jusqu’a cing millimètres. Elles sunt exessivement hygrométriques, et sur le porte-objet le souffe de l'observileur sultil pour les faire se tordre el sautiller. Le peu d'epaisseur de leurs parois, liriblement colures en roux poile, permet de voir s'arancer dans leur intérieur le liquide arec lequel on les mel en contace, après les en avoir privées pendant quelques instants. Ges filnrilles sont assuz caduques; apres leur chute, le mamelon qui les sup)porlait oflie all summet une ouverture circulare. Arec l'ige el sur les vieux rhizomes, toutes les fibrilles se délachent et sutuvent avec eltes les collules minees de l'épiderme;

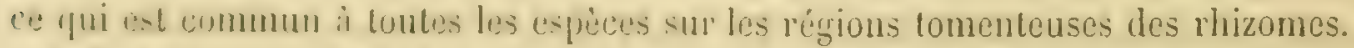

Lil. syluticum ofliw des cellules i parois encore plus minces et moins coloréc;
\end{abstract}


mais plusieurs rangs des cellules sous-jacentes ont des parois très-épaisses et fortement colorées, dont l'épaisseur ct la coloration vont en diminuant de la périphérie vers le centre. Les dilatations de la base des fibrilles sont très-faibles, et assez ordinairement plusieurs sont placées transversalement ì côté les unes des autres. Les fibrilles ne persistent pas aussi longtemps sur la partie moyenne des entre-nœuds que vers leurs extrémités.

Les cellules de l'E. arvense (pl. II, fig. 3-5) sont également transparentes et minces, avec des cellules sous-jacentes à parois très-épaisses et très-fortement colorées en roux brun. Les fibrilles sont plus nombreuses et plus persistantes que sur l'espèce précédente, el les dilatations de leur base sont beaucoup plus élevées et plus grosses; elles sont aussi toujours plus étendues dans le sens de la longueur que dans celui de la largeur et placées en grand nombre à côté les unes des autres, le plus souvent vers l'extrémité des cellules, mais fréquemment aussi vers leur milieu.

L'épiderme du rhizome de l'E. pratense (pl. II, fig. 6) fail exception dans ce groupe : il ne présente ni grandes cellules extérieures à parois minces, ni tomentum, mais une surface lisse et des cellules très-petites, à parois très-épaisses, à cavité presque nulle. Les trois ou quatre couches sous-jacentes sont également composées de cellules à parois très-épaisses et à cavité très-petite.

L'E. littorale (pl. II, fig. 7) est le plus caractérisé des trois espèces du troisième groupe. Les cellules épidermiques de ses rhizomes horizontaux et de la partie souterraine de ses tiges sont simples, cylindriques et d'une dureté qui égale au moins celle de la corne. Leurs parois deviennent si épaisses par l'addition successive de couches intérieures colorées, que c'est à peine s'il subsiste à leur intérieur une petite cavité longitudinale presque imperceptible; le plus souvent, pour ne pas dire toujours, les cellules de la seconde couche se sont également remplies par des dépôts successifs, et celte épaisseur va en diminuant à mesure que les cellules se rapprochent du centre. Les parois latérales sont à peine ondulées, et les extérieures, constituant la surface de l'épiderme, sont d'un noir trẻs-foncé, parfaitement lisses et luisantes.

Les cellules épidermiques de l'E. limosum (pl. II, fig. 8,9) sont d'une extrême simplicité, à peu près cylindriques, avec une cavité longitudinale tout unie et des parois épaisses, fortement colorées en pourpre noir et, malgré cela, assez transparentes. Les parois latérales sont un peu ondulées. Les cellules immédiatement sous-jacentes sont à peine colorées et encore à parois épaisses; la troisième couche est incolore et à parois minces.

Les parois des cellules épidermiques de l'E. palustre (pl. II, fig. 10-12) sont trèsépaisses, très-fortement colorées en roux noir, dailleurs entièrement opaques et presque aussi dures que celles de l'E. littorale. Des coupes transversales et longitu- 


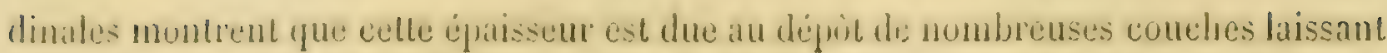

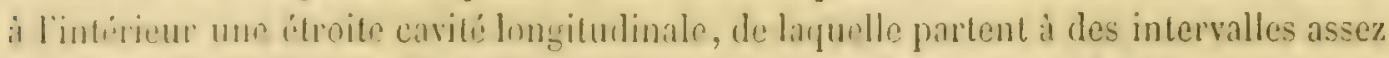
fartux de petiles cavités allant perpendicularement vors la surface de lépiderme dont . lles ne sont sépares yue par une membrane tris-mince. Vues de face et du dehors res putites cavile paraisent sous forme de petits points brillants distribués tout le long des cellules; et la tinuité de la membrane, qui les empèche de communiquer arec l'exterieur, semble leur permettre de remplir les fonctions des fibrilles dont ces hizomes sont dípnurve. Les couches sous-jacentes sont très-peu colorées et à parois épaisses.

baus re groupe el dans le sroupe suivant, l'épiderme des gaines des rhizomes ne ressemble en rien i l'üpiderme des entre-ncends, comme celui des gaines des tiges áriennes, il n’a de ecllules longues que sur le dos des cótes el des lobes; dans les silluns commissuraux il se compose de cellules courtes, plus ou moins ovales, trèsirrécrulieres el disposées obliquement de chaque côté du sillon (pl. II, figg. 19). Ces diverspe cellules portent des fibrilles et sont i parois minces, tout à fait comme celles des deux premiers groupes. Tout le tissu de la gaine est lache et mou, de telle sorte qüil se pourrit très-vite; tandis que l’épiderme des entre-nneuds semble se conserver indífiniment, ol apress un an de macération dans l'eau il a encore toute sa fermeté et loule sa fraîcheur.

L.es caracteres communs aux espèces du groupe des IIyemalia ont été énoncés plus haut. L'E. ímosissimum présente des cellules épidermiques plus longues que celles des especes roisines a parois fortrment ondulies. Sur les jeunes rhizomes, les parois cxtérieures ile ces cellules offrent vers leurs extrémités, el quelquefois vers leur milien, un space demi-circulaire on eirculaire tres-transparent (pl. II, fig. 17 a), ce glitimlique que rers res points la paroi extérieure devient plus mince et la cavité intirienre plus grande, comme le montre d'ailleurs une coupe longitudinale. Les encroultements siliceux qui avec l'ige recourrent l'épiderme des rhizomes de ce groupe et s'y prisentent sous forme de mamelons, on plutùt de saillies transversales, sont moins prononcés sur celle espece que sur l'E. liyemale, mais beaucoup plus forts que sur l'E. variegalum.

Sur retle dernière espèce, en effet, ces rugosités transversales sont si petiles, si rapprochices et i nombreuses, qu'i la vue simple lépiderme parail uni et presque brillant. I a cavite intérieure dés cellules se rapproche considérablement de celle de l'E. pulustie, en ce quelle présente à des intervalles à pel près égaux de petites cavités transversales dirigies vers le dehors (pl. II, fig. 18); de sorte que, rues de face, ces cellules montrent parmi leurs rugosités de petits points lumineux disposés en lignes regulieres. Les paruis sont d’ailleurs très-épaisses, très-colorées; les latérales sont pen ondulées. 
$L^{\prime} E$. hyemale, type de ce groupe, a des cellules épidermiques à parois trèsépaisses, très-colorées en roux noir et dès lors très-opaques (pl. II, fig. 13-15). Leur paroi extérieure est très-dure, très-rude et tout encroûtée de rugosités siliceuses transversales et allant d'une cellule sur l'autre :(pl. II, fig. 15 c); l'épaisscur de ces encroûtements s'ajoute à celle des parois, contribue à augmenter la dimension dans le sens du rayon, et fait paraître les cellules comprimées par les côtés et plus profondes que larges. Les cellules s'unissent latéralement par des parois profondément sinueuses. Leur cavité, presque régulière dans le jeune àge, devient plus tard trèsirrégulière; petite et cylindrique vers le milieu de la longueur, elle se dilate vers les extrémités en cavités sinueuses (pl. II, fig. 14 k), qui, vues du dehors, rendent la paroi extérieure plus transparente (pl. II, fig. 16 a) sur des espaces circulaires irréguliers. Les cellules immédiatement sous-jacentes sont très-petites, à parois épaisses et peu colorées. La couleur appartient aux parois des cellules; les rugosités siliceuses sont de silice pure parfaitement translucide.

L'épiderme de l'E. trachyodon ne diffëre pas notablement de celui de l'E. hyemale.

L'épiderme des tubercules est dans son ensemble semblable à celui des entrenœuds du rhizome de la même espèce; comme lui pourvu ou dépourvu de fibrilles, luisant ou non, avec celte différence constante que les cellules sont plus grosses, beaucoup plus courtes et d'une longueur à peine double de la largeur.

L'épiderme des racines (pl. II, fig. 25) est à peu près le mème sur toutes les espèces; il présente constamment des cellules longues, simples, à parois minces avec fibrilles, ct il se détruit assez rapidement. Les cellules de la pointe des racines seront décrites an chapitre suivant.

De l'épidcrme des tiges. - Ge qui frappe tout d'abord dans l'examen de l'épiderme des tiges, c'est le remplacement de la cuticule par un encroûtement transparent, plus ou moins épais et plus ou moins rude.

Cet encroûtement est de la silice parfaitement transparente et qui m’a constamment paru être à l'état amorphe. M. Edw. Newman dit très-expressément en parlant de l'E. Inyemale: "La silice s'y montre sous forme de petits cristaux, disposés arec une régularité admirable et parfaite. Dans le volume sur l'optique, faisant partie du Cabinet Cyclopedia de Lardner, le $\mathrm{D}^{\mathrm{r}}$ Brewster a fait comnaitre qu'il a trouré chaque molécule de cetle silice possédant un axe de double réfraction, that he found each "particle of silex to possess an axis of double refraction (Brit. ferns, p. 22). Je n'ai pu voir rien de semblable, ni, je le répète, distinguer ancun signe de cristallisation. La silice m’a paru être très-pure. Quand on traite l'épiderme intact par l'acide sulfurique, il se produit un petit dégagement de gaz, annonçant la présence du carbonate de chaux; mais, comme le fait remarquer M. C. Sanio dans ses savantes recherches, il est difficile de déterminer si cette dernière substance se trouve a dans les cellules de 
lépiderme on dans la carapace avec l'acide silicique o (Epid.u. Spalt. Eq. p. 397). Il se pourrait aussi que l'effet observé fìt dù simplement à la présence sur la surface rugneuse des Equisetum de quelques grains de poussière calcaire ; car l'effervescence, quand elle a lieu, cesse de suite, ct, d'autre part, il m'a été impossible d'en constater la moindre trace sur l'épiderme de jeunes sujets élevés sous cloche.

Get encroûtement siliceux n'est jamais, comme la cuticule, d'une épaisseur uniforme; néanmoins je n'ai pu y découvrir aucun signe de structure organique proprement dite. Quand on l'isole par une longue macération, par l'incinération de l'épiderme, ou par l'abullition dans l'acide sulfurique, on y constate les modifications suivantes. La surface intérienre porte le plus souvent, mais non toujours, l'empreinte des parois ondulees des cellules et des stomates. La surface extérieure n'est que rarement unie; le plus ordinairement elle est toute parseméc de petits mamelons obtus repandus sans ordre sur toute la surface ( $E$. areense), ou situés au-dessus des sinuosilés des parois latirales des cellules (E. arvense, littorale, limosum, palustre; pl. III, fig. 9, 7). Ils forment aussi des lignes transversales peu régulières ( $E$. arvense, palustre); ou hien ils sont disposés concentriquement soit autour de plus gros mamelons très-saillants (E. syluaticum, pl. III, fig. 7), soit autour de certains espaces circulaires dépourvus de points (E. arvense, pl. III, fig. 3, 15); soil autour des stomates et sur cux (pl. III, fig. 3); ou bien encore il n'y a presque pas de points en saillie et ces espaces circulaires sont si minces et si transparents qu'ils simulent des lacunes (E. artense, pulustre, limosum, littorale etc.). Ces espaces circulaires sont toujours vers les extrémité des cellules épidermiques et exactement au-dessus de leur surface externe, jamais au-dessus des cloisons de séparation (E. arvense, pl. III, fig. 3, 15).

Il est difficile de ne pas recounaitre dans ces mamelons arrondis et dans leur dispasition en cercles concentriques la tendance de la silice à se déposer en zones concentriques. On trouve des dispositions tout à fait analogues dans les couches siliceuses qui ont pénétré le test des coquilles fossiles 1.

Les Equisetum du troisième groupe ont souvent sur les côtes des saillies silicenses contiguës disposées en lignes transversales; mais c’est surtout dans le groupe des IIyemalia que ces saillies transrersales prennent sur les côtes une épaisseur très-considérable. Elles y constituent de véritables gibbosités coniques, à sommet émoussé (pl. III , fig. 10 bis), visibles à l'wil nu, et elles donnent aux espèces de ce groupe cclie rudesse toute particuliere qui les rend un objet de commerce. Souvent sur les E. hyemale et trachyodon cel encroùtement n'est pas sculcment superficiel, mais à rertains points il pénètre à l'intéricur de la tige, cntre les tissus. Ces points sont tou-

\footnotetext{
' Yoy. Alex. Brongniart, arł. Silex du Dict. sc, nal, de Levrault, tom. XLIX, p. 169 cl suiv.; pl. VI, VIl d. minéral., 590 cah., et aussi Ann. sc. nat., tom. XXIII, p. 166, pl. VI, C. 1-3; pl. VI, fig. 1, 2, 1; J. Duval-Jouve, Bélemniles iles Llasses-Alper, p. 23.
} 
jours reconnaissables sur les vicilles tiges à ce quilits sont noirs a l'extérieur et indiquent une lésion. Si sur ces points on dissèque le sujet, on y trouve un cône siliceux, irrégulier, dont la base adhère à la croùte antéricure et dont la pointe est dirigée vers l'intérieur. Il paraît qu'à la suite d'une lésion les parois cellulaires disloqućes ou mises à $n u$ ont été le siége d'une sécrétion plus abondante, mais que l'induration de la croûte siliceuse précédemment sécrétée n'a pas permis l'issue à l'cxtérieur' de la nouvelle sécrétion, refoulée ainsi vers l'intérieur entre les cellules. J'ai fréquemment obtenu de ces cònes siliceux ayant plus d'un millimètre et demi dans chacune de leurs dimensions. Ce fait si constant et si facile à reproduire ì rolonté, en meurtrissant sur un point les cellules de l'épiderme, ou en les disloquant par une brusque courbure, ne m’a pas permis un doute sur la nature de cet encrontement, comme dépôt de substance sécrétée par les cellules épidermiques. Je crois done que la silice entre dans la plante à l'état d'acide libre, dissoute dans l'eau de végétation; qu'elle se dépose à la surface externe des cellules de l'épiderme et s'y accumule avec l'âge, par suite de l'évaporation dont l'épiderme est le siége. M. C. Sanio, après avoir rappelé quelques-unes des particularités d'aspect que présente la «carapace siliccuse », ainsi que les empreintes des cellules épidermiques sur cette carapace, ajoute: «Tout cela paraît prouver que l'acide silicique n'est pas simplement sécrété à la surface des cellules, mais qu'au contraire cette substance s'est déposée dans la paroi la plus extérieure des cellules de l'épiderme, après quoi la substance organique paraît avoir disparu plus tard » (Epid. 11. Spalt. Eq., p. 400). Il me semble que les empreintes des cellules sont une simple conséquence de ce fait que l'acide silicique s'est moulé sur les cellules en se solidifiant. D'autre part, les innombrables modifications que présente cet encroûtement, variables avec son épaisseur, mais toujours en rapport avec les formes générales qu'affectent les dépôts de silice, semblent prouver arec la plus complète évidence qu'il y a là simple sécrétion, encroûtement avec modifications se rattachant aux lois générales des dépôts siliceux.

Un motif de croire que celle croùte siliceuse n'est qu'une sécrétion qui se dépose et s'accumule par effet d'évaporation à la surface libre des cellules, se trouve pour moi dans le fait suivant. Les cellules des stomates n'ont que leur partie centrale à l'air libre; à leur pourtour elles s'enfoncent plus ou moins sous les cellules épidermiques contiguës. Or' la croûte siliceuse, qui recouvre les stomates, n'en recoụre que la partie libre et ne pénètre point entre cux et les cellules contiguës ${ }^{1}$; mais elle s'étend, sous forme de plaque mince hérissée de saillies plus ou moins fortes, dans la fente longitudinale qui constitue l'ostiole des stomates. Ce fait, constaté par M. C.

\footnotetext{
' $A$ moins que, par exception, les cellules épidermiques ne se soulévent un peu et ne laissent de l'air entre elles et les cellules stomatiques, comme dans Ies cas que M. C. Sanio cite sur l'E. pratense (Epid. $u$. Spalt. Eq., p. 405).
} 


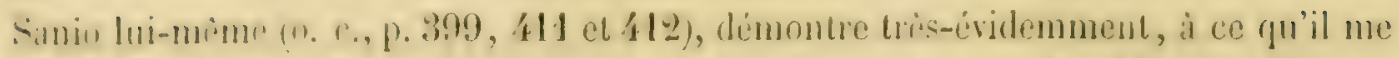
semble, que lis collules stomaliques, comme lis autres, sécrètent l'acide silicique disons dans lenu de veretation, mais seulement sur la partie de leur surface qui est libre et à l'air; tandis que la partie des mimes collules qui n'est pas à l'air libre ne sécrite pas entle subtanere. Je dois aroir la franchise d'ajouter que M. C. Sanio tire du mème lait une conclusinn directement opposée i la micnne (o. c., p. 402).

Les modifications superficielles de cet encroutement varient extrêmement aver. l'isce, la force, la station, on la région de la tige des individus d'une même espèce. Ainsi, sur les especes aquatiques du troisième groupe, les entre-nocuds submergés ont la cronte siliceuse trés-minee ot presque nulle, ou au contraire épaisse et couverte de trì-grosses saillies disposées en lignes transversales, si la submersion a été longtempls interrompue. C'est pourcquoi il convient, à mon avis, de ne pas trop s'arrêter à ces pelites diflérences aflectant la surface d'un dépôt dont les modifications ultérieures deviennent étrangères à l'organisme végétal qui l'a sécrété.

Apris la croùte siliceuse, ce qui frappe l'attention dans l'étude de l'épiderme des tiges, c'est que sur les espèecs du deuxième et du troisième groupe, au-dessous de la rouche exlérieure, notamment dans le roisinge des stomates, il semble y aroir une seconde conche de cellules, qui par leur position el leurs dimensions correspondent aux premieres presque cellule ì cellule (pl.IV, fig. 2, 4, 6, 8, 9, 15). MI. W. IJofmeister mentunnne ces deux couches, ajoutant que "les cellules qui les composent n'ont en grosscur que le 18 de celles de la couche voisine intérieure, et sont huit fois plus nombreuses (que ces dernières (Yergl. Cuters., p. 92). .) Cette expression est trop al)solue; au-dessous de ces deux couches, quand elles existent, sont ou des fibres longues et étroites, nu des cellules à chlorophyile dont la différence de grandeur est loin d'ètre aussi considérable et souvent à peine appréciable (pl. III, fig. 1).

Sur tous les rhizomes d'une mème espèce l'épiderme est constamment semblable, parce que les tiges stériles el les tignes spicifëres s’èlèvent indifféremment des mèmes rlizomes. Jlais il nen est pas ainsi de l'épiderme des tiges; et aux différences générales d'aspect qui existent entre les tiges spiciferes et les tiges stériles de certaines esperes, répondent sur l'épiderme des différences tout aussi remarquables el aussi profondes.

L'épiderme des tiges spiciféres propres de l'E. maximum est composé sur toute la surfice, de l'nntre-nmud d'une seule sorte de cellules très-étroites, très-longues, trèsrésistantes, sans stmmates. Locurs parnis latérates sont pen ondulées et leur surface cxtérieure est wrêtue d'un encroûtement siliceux un peu pointillé (pl. III, figg. 5).

Sur lF. arrense les cellules épidermiques de la lige spicifére propre sont également d'une scule sorte et sans stomates, mais elles ont le double de la largeur et soulement la moitio de la lonęueur des précédentes. Leurs parois latérales sont beau- 
coup plus ondulies; l'extérieure est très-délicate et la cronte silicense n'est souvent représentée que par quelques petites saillies qui, sur les sujets les plus vigoureux, se montrent comme des points brillants vers les sinuosités latirales (pl. III, fig. 2).

Si sur les tiges spicifères de ces deux espèces l'épiderme des entre-nourls est entièrement dépourvu de stomates, composé d'une seule sorte de cellules, et si les entre-nœuds sont privés de matière verte et, à ce double titre, diffèrent essentiellement des entre-nœuds des tiges stériles, il n'en est pas de même des gaînes de ces mêmes - tiges. Elles ont des cellules épidermiques de deux sortes, des stomates; elles élaborent la chlorophylle et, malgré l'ampleur de leurs dimensions et la couleur particulière qui les distinguent de celles des tiges stériles, elles en contiennent dans leur ensemble tous les éléments (pl. III, fig. 11-15); j’en parlerai avec plus de détails en traitant des gaînes des tiges stériles, p. 27-29.

Les rapports généraux de ressemblance entre les tiges stériles el les liges spicifëres des $E$. sylvaticum et pratense se reproduisent sur leur épiderme; et celui des tiges spicifêres est tout à fait conforme à celui des tiges stériles jeunes, dont la description viendra en son lieu.

L'épiderme des liges stériles de l'E. maximum offre une particularité qui n'appartient qu'à cette espèce. Il ne présente que des cellules d'une seule sorte, avec absence complète de stomates et de chlorophylle. Ces cellules sont à peu près de même forme que celles des tiges spicifères; toutefois un peu moins longues et plus ondulées sur leurs parois latérales. Leur cavité parait unie et en même temps un peu dilatée à l'extérieur vers les extrémités'. La croute siliceuse est faible et marquée de petits points mamelonnés disposés le plus souvent en lignes transversales (pl. III , fig. 6). Les cellules épidermiques de la gaine de ces tiges, comme celles des tiges spicifères, sont de deux sortes, avec des stomates et de la chlorophylle (pl. III, fig. 11, 12); il en sera question p. 27-29.

L'épiderme des tiges stériles de l'E. arvense et celui des tiges spicifères ou stériles de toutes les autres espèces offre comme caractères communs: $1^{\circ}$ deux sortes de cellules: les unes plus longues, plus étroites et très-régulières, supperposées aux faisceaux de fibres corticales; les autres plus courtes, plus larges et moins régulières, superposées aux bandes de cellules à chlorophylle; 20 parmi ces dernières des stomates (pl. III, fig. 4); $3^{\circ}$ à la surface du tout absence complète de la cuticule, remplacée par une croûte siliceuse.

Les cellules de l'épiderme des entre-nœuds sont donc de deux sortes, ou, si l'on aime mieux, présentent les deux aspects qui viennent d'être signalés et auxquels

\footnotetext{
- C'est à tort que M. C. Sauio a nié ces dilatations des cellules épidermiques (o, c., p. 400); elles existent dans celte espèce, aussi bien que sur les cellules épidermiques de certains rhizomes précédemınent décrites.
} 
se réduit presune hat diflérence qui les distingue. Les unes et les autres ont leur paroi intérieure constamment lisse sur ses deux surfaces. Leurs parois de contact, aux côtés comme aux extrémités, sont plus ou moins ondulies, of elles adhèrent les unes aux autres par un engrénement de saillies el d'échancrures qui rend teur union très-solide et très-résistante. Ces sinuosités ondulées n'existent point sur toute l'útendue de la paroi latírale, mais plus particulièrement vers l'angle extérieur, et elles diminuent et disparaissent presque en allant vers l'intérieur. La surface interne de ces parois latérales est quelquefois presque unic (E. limosum, littorale), d'aitres fois elle suit les ondulations de la surface interne, et alors la cavite de la cellule s'enfonce dlans les simuosités en les pénétrant par de petites cavités coniquues (E. arrense, palustre et IIyemalia); la fig. 16 de la pl. II donne une idte des sinuosités de la surface interne des cellules. Mais il n'y a rien de bien constant dans ces légères différences. La paroi extérieure offre souvent des ondulations et des soulevements vers ses extrémités et quelquefois en mème temps sur la région médiane, soulèvements qui répondent toujours exactement à quelques-uns de ces espaces circulaires mentionnés plus haut, et oủ l'encroûtement siliceux présente soit une moindre épaisseur, soit des cereles concentriques de petites saillics. L'épaisseur des parois est très-variable aree l'igge el mème très-variable sur des individus de mème âge. Des coupes transversales de l'épiderme non préparé permettent déjà de reconnaitre les couches qui constituent celte épaisseur; on les distingue arec une extrême facilité en traitant les mêmes coupes par l'ébullition dans une solution de potasse caustique et par la macération dans le chlorate de potasse et l'acide azotique. Mais par ce traitement, les parois se gonftent extrêmement, se déforment et ne donnent plus leur apparence réelle et normale.

Les cellules courtes et les cellules longues sont, comme nous l'avons vi, invariablement superposées, les unes, aux faisceaux de fibres corticales, les autres, aux bandes de cellules à chlorophylle; leur distribution suit dès lors toutes les différences que présente, d'une espèce à l'autre, la répartition générale de ces bandes très-constante sur tous les sujets d'une même espèce.

Ainsi l'E. sylvaticum porte sur ses cótes dix-sept ou dix-huit rangs de cellules lonģues, puis un seul rang, rarement deux, de cellules courtes à stomates; et enfin au fond du sillon une vingtaine de cellules longues. Sur le fond des sillons ct sur la ligne médiane des crites, ces demières cellules n'offrent d'autre particularité que leur etroitesse extrème el l'abondance des petites éminences de la croûte siliceuse précédemment décrites, mais de chaque côté des côles, vers le sixième rang i partir des cellules à stomates, au point oì un angle très-saillant marque la séparation des cites el des sillons, il y a une ligne de cellules un peu plus larges et plus courtes, Insquelles émettent de leur surface exterieure une saillie creuse et sans diaphragme 
en forme de longue papille ou de poil (pl. III, lig. 7). C'est ce qui a fail dire les tiges " finement hérissées sur les angles 》(Grenier). L'épiderme des tiges spicifères offre les mêmes caractères; seulement dans le jeune ìge les rangs des cellules à stomates sont moins marqués et moins nombreux que sur les sujets adultes.

L'E. pratense offre à peu près les mêmes faits sur de moindres dimensions. Une douzaine de longues cellules occupent le fond des sillons, puis vient un rang de cellules courtes à stomates, sur chaque flanc des côtes cinq ou six cellules longues; mais le "dos de la côte est occupé par trois à. six rangs de cellules larges et courtes, à parois épaisses, portant les longues papilles piliformes décrites ci-dessus (pl. III, fig. 8), non, comme l'E. sylvaticum, seulement sur les bords des côtes, mais jusque sur leur dos, ce qui rend les tiges stériles assez rudes.

$L^{\prime} E$. arvense présente une répartition analogue de ses deux espèces de cellules; sur les côtes une vingtaine de longues cellules, parmi lesquelles quelques-unes s'élèvent un peu au-dessus des autres; puis de chaque côté quatre rangs de cellules courtes à stomates, et au fond de chaque sillon six ou huit rangs seulement de cellules longues.

Sur les espèces du troisième groupe le mode de répartition change entièrement; il n'y a plus de cellules longues au fond des sillons et il ne s'en trouve que sur les côtes (pl. III, fig. 9,10$) ;$ l'E. littorale en a une quinzaine, les deux autres au maximum douze. Les sillons sont entièrement occupés par de larges cellules, sur tous les rangs desquelles de très-nombreux stomates sont disséminés sans ordre nettement déterminé; l'E. palustre en a une vingtaine de rangs; le limosum jusqu'à trente; le littorale de quinze à vingt; il n'est pas rare que sur un même entre-nœud de cette espèce quelques rangs du fond du sillon soient dépourvus de stomates.

Dans le groupe des Hyemalia, la répartition est encore différente: un seul rang de cellules à stomates se trouve de chaque côté du fond des sillons et tout le reste est de cellules longues superposées à des fibres corticales. L'E. variegatum n'a guère que cinq rangs au fond du sillon et seize sur les côtes. Les E. ramosissimum, trachyodon et lyyemale en ont de sept à dix au fond des sillons et une vingtaine sur les côtes. Ces nombres sont sujets à varier avec la force des pieds, mais la relation qu'ils expriment demeure constante.

L'épiderme des gaines mérile une mention spéciale. Les particularités qu'il présente sont communes à toutes les espèces et consistent en ce que:

$1^{\circ}$ Quelle que soit la répartition des deux sortes de cellules sur les gaînes, les cellules de l'épiderme et les cellules sous-jacentes, à mesure qu'elles se rapprochent du milieu d'un sillon, deviennent de plus en plus irrégulières, obliques et finalement transversales (pl. III, fig. 11-18).

$2 \circ$ Vers la moitié supérieure des lobes des gaines et sur leur ligne médiane, les cellules de l'épiderme présentent, par leur mode d'articulation, des aspérilés remar- 
Ifuahlir. An linu de sarticuler simplement par li juxta-position de leurs extrémités, les cellules s̈̈mbriquent de bas en haut, de facon que, deux cellules étant domnées, l'unférieure s'avance sur la supérieure immidiate ol ne s'articule avec olle qu’après avoir liat un pli el par sa face inféricure, comme le montre le profil de la fig. 19 a, 11. III. Sur l'E. truchyodon, ce mode d'articulation en crémaillère est i son développement extrême el constitue res aspérités que M. Al. Braun a voulu rappeler par le nom qu'il a imposé à cet Equisetum.

30 Eufn, quelle que soit la différence qui, sur certaines espèces, existe entre l'épi-" derme des entre-neuds de lcurs tiges spicifères et celui de leurs tiges stériles, sur les mèmes especes, les graines des tiges vernales spicifëres ont leur épiderme tout à fait semblable i celui des gaines des tiges plus tardives el stériles. Et sur toutes les espèes, les unes el les autres portent des stomates au moins sur leurs deux tiers inférieurs, c'est-à-dire sur toute la région non déchirée en lobes. Dans le roisinage des stomates des gaines, les petites saillies de la croûte siliceuse se disposent plus particulierement et plus constamment qu’ailleurs en cercles concentriques très-élégants (pl. III, fig. 11-18, et particulièrement fig. 15).

L'E. maximum oflie sur les còtes de ses graines douze à quatorze rangées dle cellules épidermiques très-allongrées, très-étroites, il bords trẻs-élégamment ondulés; les cellules à stomates occupent le dernier rang, plus rarement les deux derniers, de chaque còlé de cette bande médiane; après elles il y a encore un ou deux rangs de cellules longues; au delì, ces cellules deviennent courtes, irrégulières, puis obliques et tout à fait transversales dans le sillon commissural (pl. III, fig. 11, 12). Vers la pointe des lobes, les cellules épidermiques sont moins distinctes, il n'y a plus qu'une couche de cellules démesurément longrues et étroites; et je ne sais pas si on doit les rapporter a l'épiderme plutit qu'ì la couche sous-jacente qui aurait dépassé l'épiderme et n'ausait pas été recouverte par lui.

Lä́piderme des tiges spiciferes de l'E. arvense change subitement à la base des gaines et devient identique à celui des gaines des tiges stériles; sur les unes et sur les autres, vingt rangs de cellules longues occupent le dos de la còte; viennent ensuite de chargue ritie cing rangs de cellules courtes avec stomates; puis cinq on six rangs de cellules longues, eufin les cellules obliques et transversales du fond du sillon (pl. III, fig. 13-15).

L'E. sylraticum at l'E. pratense ne conservent pas sur leurs gaines les papilles que prisutent certaines cellules de l'épiderme de leur's entre-nœuds. A la base de lia gaine, les rangs de cellules qui portaınt ces papilles deviennent semblables à leurs voisins; du reste les autres cellules présentent les mèmes nombres et le même ordre que sur le; entre-nceuds. Les cellules transwersales du fond des sillons sont quelquefois cordiformez, et toujnurs mnins longrues et moins nettement transwersales que dans les deux 
espèces précédentes (pl. III, fig. 16-18). C'est sur les gaines de l'E. sylvaticum que l'encroûtement siliceux montre avec le plus de netteté et d'élégance toutes les dispositions précédemment décrites.

Sur les autres espèces, l'épiderme des gaînes offre vers leur base le même nombre et la même clisposition de cellules que sur l'entre-nœud. Les modifications particulières que présentent les gaînes de l'E. hyemale après la chute de leurs lobes seront mentionnées dans la descriplion de cette espèce.

De l'épiderme des rameaux. — Sur toutes les espèces, la gaîne basilaire des rameanx offre des cellules épidermiques peu allongées, à parois très-fortement ondulées, entièrement dépourvues de stomates (pl. III, fig. 20, 21). Sur la ligne de séparation des dents elles prennent une direction oblique ou mème transversale, comme les cellules de la même région sur les grandes gaines (pl. III , fig. 20). Au moment oủ les gaînes basilaires sortent de la tige en recouvrant le jeune rameau, leurs cellules sont entièrement incolores; elles prennent avec le temps une couleur brune ou noire, et sont mates ou luisantes selon les espèces.

Sur l'E. maximum, l'épiderme des entre-nœuds des rameaux n'a aucun rapport avec celui des entre-nœuds des tiges, et il diffère tellement de l'épiderme des gaines qu'au premier coup-d'œil il est permis de croire qu'on examine le rameau d'une autre espèce. La surface rentrante des côtes (pl. III, fig. 22 e et $23 c$ ) offre environ dix rangs de cellules très-longues, à parois latérales peu ondulées (pl. III, fig. $23 \mathrm{c}$ ); elles recouvrent une bande de fibres corticales. Sur les arêtes qui s'élèvent à chaque bord de celte surface (pl. III, fig. 22 b et 23 b) et la séparent des sillons, sont étendues des cellules épidermiques articulées en crémaillère, comme celles de la ligne médiane des dents des gaînes (voy. p. 27, 28 et pl. III, fig. 19); leurs aspérités rendent les rameaux «rudes de haut en bas » (Grenier). Enfin, toute la surface du sillon (pl. III, fig. 22 a et 23 a) est occupée par douze à quinze rangs de cellules à stomates. Sur ces cellules, les ornements circulaires de la croûte siliceuse sont très-nombreux et très-variés.

L'E. arvense offre moins de différence entre l'épiderme de ses rameaux el celui de ses tiges stériles. Sur la ligne médiane des côtes on voit deux ou trois rangs de cellules qui se dilatent à leurs extrémités articulaires de manière à former une série d'aspérités saillantes alternant avec des dépressions. La jonction de deux cellules est au milieu d'une élévation (pl. III, fig. 24). Viennent ensuite douze à quatorze rangs de cellules peu longues à parois très-ondulées, recouvrant des cellules à chlorophylle, puis deux ou trois rangs de cellules à stomates, et enfin an fond du sillon trois ou quatre rangs de cellules longues sans stomates.

L'E. pratense n'a sur les côtes que deux ou trois rangs de cellules longues, puis ces côtes sont bordéss de courtes cellules ì papilles, et après cinq ou six rings de cellules longues viennent trois à cinq rangs de stomates; puis tout le fond du sillon est 
occupe par six ou huit rangs de cellules longues, sur lesquelles les ornements circulaires siliceux sont répartis en immense qquantité.

L.E. sylvaticum offre encore moins de difference. Le faisceau fibreux du dos de lat cilte est recouvert de sept ou huit rangs de cellules longues, et sur l'angle de séparation des cùtes et des sillons seétend une ligne de cellules à papilles en forme de poils; puis viennent trois ou quatre rangs de cellules courtes avec une ligne de stomates, ct sur le fond du sillon s'étendent cing ou six rangs de cellules longues recourrant le faisceau fibreux cortical (pl. III, fig. 25).

On voit ainsi que, sur cette dernière espèce, les cellules épidermiques des rameanx représentent celles des entre-nauds des tiges, sans autre différence que le nombre des rangs. Il en est de mème sur les espèces des deux autres groupes; seulement les cellules des cùtes des rameaux des $E$. littorale, limosum et palustre présentent quelques légères aspérités analogues à celles qui ont été signalées sur les rameaux de l'E. artense.

De l'épiderme desépis. - Les cellules épidermiques du dernier entre-nœud, depuis la gaine supérieure jusqu'a l'anneau, ainsi que celles qui plus haut recourrent l'axe de l'épi et les pédicelles des sporanges, ressemblent à celles des tiges vernales spicifères de l'E. arvense (pl. III, figg. 2); elles sont, comme elles, extrêmement délicates, i parois minces et très-finement ondulées, un peu courtes, sans stomates et sans encroûtement siliceux. Les espèces ne présentent que de très-légères différences qu'il serait superflu de noter.

L'épiderme des clypúoles se compose de cellules courtes, assez grandes, à parois épaisses et plus ou moins furtement ondulées, sans forme générale régulière et déterminée. Par leur direction elles semblent irradier du centre à la circonférence (pl. III, fịc. 26-30\%. Elles contiennent d'abord une assez grande quantité de granules amylacés (IE. maximum excepté), et plus tard de la chlorophylle en grains. Leur encroutement siliceux est très-faible et ce n'est que sur le groupe des Ilyemalia qu'il présente de petites saillies mamelonnées. J'ai trouvé quelquefois sur l'E. limosum la surface extérieure de ces cellules toute hérissée de papilles courtes et obtuses (pl. III, fig. 26 et 27). Il est très-fréquent de voir les parois se colorer en brun foncé presque noir; les cellules sont alors très-dures.

Toutes les espèces portent sur les clypéoles quelques stomates semblables à ceux de la tige, mais un peu plus petits et loujours largement recouverts aux bords par les angles extérieur's des cellules voisines. Ils y sont épars sans ordre déterminé et en nombre trìs-rariable sur le mème épi (de quatre à douze). Quclquefois ils m'ont paru manquer sur des clypéoles de l'E. arvense.

Des stomate's. - Dans la description de Pépiderne des Equisetum, celle des stomates mérite à tous égards une mention particulière. 


\section{\$1. - DE L'ÉPIDERME.}

Ces organes occupent une position invariable et rigoureusement déterminée. Sur les tiges, ainsi que sur les gaînes et sur les rameaux, ils sont constamment situés dans les sillons qui alternent avec les còtes et jamais sur le dos des côtes.

Si la position des stomates dans les sillons est commune à toutes nos espèces, leur répartition sur ces mêmes sillons présente de notables différences selon les groupes, et des différences moindres selon les espèces. Ainsi, dans toul le groupe des IIyemalia, les stomates sont régulièrement disposés sur deux lignes parallèles, une ligne de chaque côté du sillon au point oủ commence l'élévation des côtes (pl. V, fig. 4). Ils sont, de plus, assez régulièrement espacés sur cette ligne, où ils ne sont séparés que par une seule cellule très-courte (pl. V, fig. 3,4 ). A la vue simple, ou avec un faible grossissement, ils simulent sur la plante fraîche deux lignes blanchâtres très-facilement reconnaissables.

Les espèces du troisième groupe ont leurs stomates disséminés sans ordre, mais en rangs très-nombreux sur toute la largeur des sillons (pl. III, fig. 10). Toutefois la partie submergée de leur tige en est dépourvue. Ce n'est que vers la surface de l'eau que ces organes se montrent d'abord peu nombreux sur les côtés des sillons, puis leur nombre augmente à mesure qu'on s'élève, et sur la région tout à fait aérienne ils occupent toute la largeur des sillons.

Les espèces du second groupe n'ont point de stomates au fond des sillons; elles en ont seulement de chaque côté un petit nombre disposés sans beaucoup de régularité sur l'E. sylvaticum et sur l'E. pratense en un rang (rarement deux) (pl. III, fig. 7), et sur les tiges stériles de l' $E$. arvense en deux ou trois rangs (pl. III, fig. 4).

L'E. maximum n'a de stomates que sur ses gaînes; ils y sont peu nombreux et presque de moitié plus grands que sur les autres espèces (pl. IV, fig. 1, 2). Les entrenœends de ses tiges stériles et ceux de ses rameaux forment une exceplion complète; ainsi, les premiers sont entièrement dépourvus de stomates, et, par une sorte de compensation, les sillons des seconds en sont entièrement couverts (pl. III, fig. 23), comme cela a lieu dans le troisième groupe. Rappelons que les tiges spicifères vernales des $E$. maximum et arvense ne portent des stomates que sur leurs gaines et que leurs entre-nœuds en sont absolument dépourvus.

Ces dispositions des stomates sont si parfaitement constantes qu'elles ont pu servir pour l'établissement de groupes très-naturels. Mais c'est surlout par leur structure spéciale que les stomates des Equisetum méritent notre attention.

Dans cette famille, ces organes se composent de deux paires de cellules, superposées de telle sorte que la paire extérieure (pl. IV, fig. 5 et $6 u$ ), qui est la plus large, se courbe sur la paire intérieure ( $\mathrm{gl}$. IV, fig. 5 et 6 b), la recourre et l'emboite. La paroi intérieure des deux cellules extérieures est marquée à sa surface interne, c'est- 


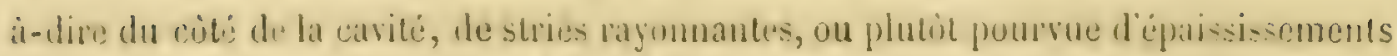
Ini, en divergent, sétendent de lat fente longitudinale, ot ostiole, vers la péri-

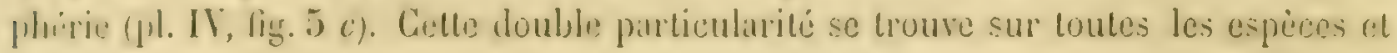
jusqu'ici parait n'appartenir qu'aux stomates de celte famille!.

Il importe de rendre comple des liverses apparences que presente successisement un stomate d'Éguisetum place sur le porte-objet. A mesure qu'on éleve alu foyer l'épiderme d'une espece des trois premiers groupes, on roit d'abord l'ostiole long et trés-étróit, bordé de petites saillies mamelonnées appartenant ì l'encroùtement siliceux, et au-dessus de li surfice du stomate de scmblables saillies disposées le plus souvent en lignes concentriques ou presque paralleles aux bords de l'ostiole (pl. IV, ligr. 7). Si on rapproche dasantage le porte-objet, l'encroûtement siliceux disparait, mais sa transparence laisse voir au-dessous de lui le commencement des stries rayonnantes dans le voisinage de l'ostiole, et les contour's des deux cellules supérieures; ces contuurs paraissent mal définis et mal limités parce ruils ne sont pas sur le mème plan que les bords de l'ustiole. Un nouseau rapprochement laisse voir le prolongement extrème des mèmes stries simples ou bifides, el les contours des cellules de la seconde paire, qui reproduisent en petit dans leur ensemble les contours de la paire extérieure, arec celte diflérence qu'entre ces cellules l'ostiole est plus court et beaucoup plus large. On reconnait en même temps que les cellules extérieures sont i leur pourtour et surtout vers leurs pointes recouvertes par un avancement de l'angle supérieur des cellules ippidermiques contiguës (pl. IV, fig. 1-4, 7, 8 elc.); la paroi qui forme cet arancement est ordinairement marquée de fortes ondula!ions en forme de dent. Sur les E. littorale et palustre la paire extérieure ne s'enfonce que très-peu sous le bord des cellules voisines; mais, dans le trís-jeune àge de toutes les especes et en particulier a l'épiderme des clypéoles, les cellules stomatiques continuent assez longtemps à ètre presque entièrement recouvertes par les cellules épidermiques extérieures, lesquelles ne s'écartent que successivement, ainsi que nous le verrons plus loin en traitant du mode d'évolution des stomates.

Mais si l'on examine un stomate du groupe des IIycmaliu, la premiere apparence est grandement modifiée. On remarque tout d'abord que la surface des stomates, au lien d'être i peu pres de nivean avee celle des cellules épidermiq̨ues, est située au fond d'une dépresion cratérilorne que l'on dirait furmée par l'écartement des cellules ¿́pidermiques aroisinant les stomates (pl. V, fig. 1-3, 5). Cette cavité, dija mentionnéc par II. C. Sanio, a reçu de cel auteur le nom de cavité respiratoire extérieure 10. c., f. $\{10 \%$, par opposition sans doute à celui de carilé respiratoire intérieure

' Y. C. Sanio (p. 389 , note) indique chez les Protéacées une disposition qui se rapproche de la double paire de cellules; et $\mathrm{M}$. H. Schacht (P/lansenselle, p. 232 , et pl. X, fig. 11 e) indique et figure les stamates du Dasylirium acrotrichum Zuccar. comme ayant deux paires de cellules superposies. 
(o. c., p. 397) qu'il donne aux pelits espaces qui, dans les Equiselum comme dans les autres plantes, se trourent au-dessous des stomates, cutre cux et les cellules i chlorophylle (pl. IV, fig. 2, 4, 8, 11 etc.). On voit d'autre part que la croûte siliceuse, au lieu de suivre la surface el les contours de cétte cavité el de s'étendre jusqu'a l'ostiole en s'appliquant sur la lace du stomate, s'avance en voûte au deli du bord supérieur et inférieur de la cavité, comme si elle allait s'étendre an-dessus d'elle et la fermer (pl. V, fig. 2, 5), mais elle laisse en son centre une lacune irréguliere i bords déchirés, toujours plus large que haute of transrersale sur l'ostiole (pl. V, figg. 1,3, 价. En meltant le stomate lui-mème an foyer, on constate quelques légères différences de forme avec les precédents; les stries sont moins irradiantes et presqque perpendiculaires à la fente longitudinale; de plus, elles ne paraissent pas s'étendre sans inter. ruption de l'ostiole à la périphérie, et le plus souvent on les voit comme interrompues vers le milieu de leur longueur (pl. V, fig. 1).

Sous d'autres aspects, des coupes transversales permettent de constater les mèmes faits. Elies montrent très-clairement que la paire extérieure de cellules stomaliquez est disposée en calotte sphérique, qu'elle dépasse les cellules intérieures en les recouvrant et les emboitant à la façon d'un verre de montre; que les stries irradiantes sont dues à des épaississements existant du còté de la cavité sur la paroi intérieure de ces mêmes cellules; tandis que les cellules de la paire intérieure sont plus écartées à leur ostiole, ont des parois minces, d'une épaisseur à peu près uniforme et à surface tout unie (pl. IV, fig. $2,4,6,8,9,11,13,14,15$, et pl. V, fig. $2,5,6,9$ ). Les mêmes coupes laissent voir que, dans les trois premier's groupes, les stomales sont à peu près de niveau arec les cellules épidermiques contiguës, sous l'angle cxtérieur desquelles ils s'enfoncent un peu seulement à leur pourtour splsérique (pl. IV, fig. 2, 4, 6, 8, 9, 11, 13, 14, 15), mais que, dans le groupe des IIyemalia, les stomates sont dans un enfoncement et bien au-dessous du niveau des cellules épidermiques (pl. V, fig. 2, 2 bis, 5). Et si ces coupes ne sont pas failes exactement sur le milieu du stomate et sur la lacune de l'encroùtement siliceux, elles montrent que cet encroûtement s'avance en forme de pont vers les extrémités de la cavité et semble la couvrir et la fermer (pl. V, fig. 2, 5). En coupant longitudinalement les stomates, ou plutòt en séparant leurs cellules selon la ligne de l'ostiole, on voil arec facilité les cellules inférieures abiitées sous les supéricures; chez certaines espèces, l'E. palustre par exemple, on reconnait que les petites saillies siliceuses du pourtour de l'ustiole pénètrent dans l'ostiole mème, et, sous forme de saillies bacillaires, en tapissent les côtés jusque vers la cavitẻ des cellules supérieures (pl. IV, fig. 19).

Sur les sujets jeunes, le contenu des cellules extéricures est hyalin el se colore en jaune par l'iode; celui de la paire intérieure renferme des granules dont la nature amylacée est indiquée par leur coloration en bleu sous l'action de l'iode. Sur de vieilles 
tiges mutilées de l'É. hycmale el ramosissimum j'ai souvent trouvé dans les deux

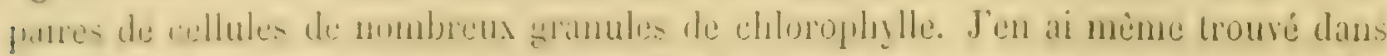

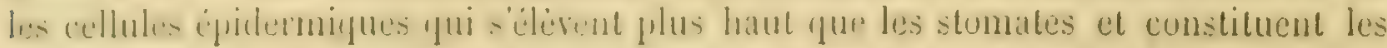

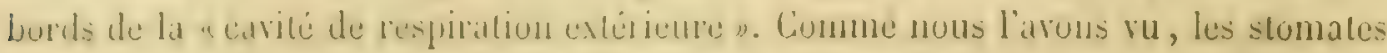
sunt foujumrs sitmés sur les bandes de ceilules a chlorophylle, et il n'y a de cellules à chlorephylle que lit on il y a des stumates. Toutefois il est it remarquer que, sur les especes du troisieme groupe, aux cutre-neuds submergés où manquent les stomates, lis cellules de lépiderme conliennent exceptionnellement de la chlorophylle. Cette matière y devient plus rare i mesure que les stomales apparaissent, cl elle ne se montre phus dims lépiderme aux entre-neuds régulierement pourvus de stomates.

A prat la dinterence profunde qui existe entre les stomates du groupe des IIycmalia et ceux des antres groupes, les stumates des diverses espèces rus de l'extéricur ont un grand nombre de traits communs. Leurs coupes transversales offrent des diffirences dans linelinaison et la courbure des cellules extérieures, dans la largeur de la ciritie de ces eellules, dans les contours de celle carité plus ou moins accidentés par des saillies lungitudinales on renflements des farois vers l'intérieur. Jaai dessiné ces différences; ulles sunt, dans chaque espece, ilssez constantes sur les sujets de mème àge; mais, cumme tous les accidents de l'épiderme, elles se modifient notablement avec l'àge et l'épaississcment des parois des cellules (pl. V, figg. 5 ì 9 ); elles ne sont donc, an double point de rue organique et spécifique, que d'une importance secondaire.

Les tiges des Equisetum sont merveilleusement propres ì démontrer la perméabilité des canaux aériferes et leur connexion immédiate avec les stomates. Si l'on prend une lige fraiche, bien intacte et non mouillic de l'E. limosum, de l'E. liyemale ou de tont autre, et si, apres aroir plongé la partic supérieure sous l'eau, on soulle fortement par l'autre extrémité, on verta de petites bulles dair sortir des parties de la surlice munies de stomates. Lexpérience est un peu plus pénible, mais plus belle encore, aree les liges stériles de l'E. maximem, parce quon ne voit sortir des bulles diair que de la surface des gaines el des rameaux ou il existe des stomates. Le phénomène na plus lieu lorsque ces plantes restent dans l'ean pendant quelque temps arant d'üre mises en expérience. It parait que l'humidité détermine la turgescence des cellules stunatiques et des cellules épidermiques et par suite l'occlusion de l'os-

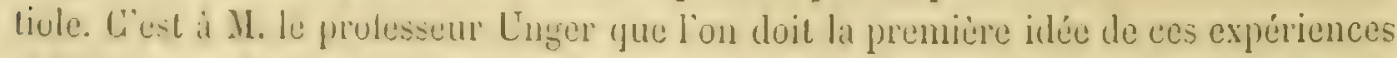
si belles el si licita. Les détails en sont eonsignés et fingurés dans la troisieme partie

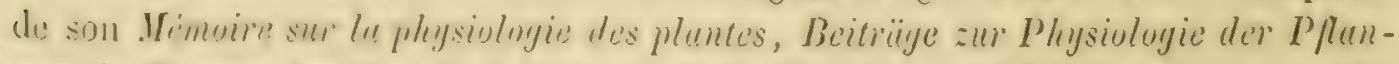

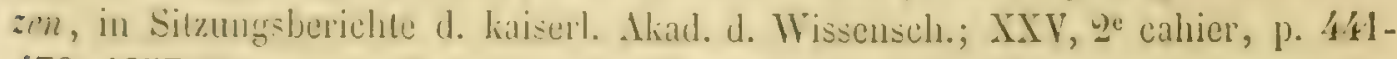
470; 1857. Voyez aussi Bull. Soc. bot. France, VI, p. 157 et 158.

IIstorique. - Viucher a été le premier a reconnaitre et à signaler l'importance de 
l'examen des stomates qüil nomme "glandes corticales" 》. Il a appelé l'ittention sur "leur position dans les enfoncements des stries (les sillons), sur leur nombre, sur "leur disposition éparse ou régulière, sur leur forme el leurs contours 》) (Mon. Prél., p. 34t, 1822); et enfin il a figuré le mode de répartition des stomates de toutes les espèces observées et décrites par lui, sans donner toutefois une analyse des stomates eux-mêmes.

G. W. Bischoff, dans son important travail Dic liryptogamischen Gcwüchse, donnait, dès 1828, d'excellents détails sur l'épiderme des rhizomes des Equisetum (p. 33 et 34), sur celui des tiges el sur les stomates. Il mentionna, comme Vaucher, et figura très-exactement les divers modes de répartition des stomates dans les sillons (p. 35, fig. 19, 23, 24, 26); il signala la forme tonte particulière des stomates de l'E. liyemale (p. 35, fig. 23 a), des deux sortes de cellules de l'épiderme, et enfin l'absence des stomates «sur les tiges qui ont une autre coulcur que la verte, comme l' $E$. Telmateia (fig. 25); tandis que les branches toujours vertes de la mème plante cn sont, comme les autres espèces, richement pourvues» (fig. 26, p. 36).

En 1833, dans une Thèse inaugurale soutenue à Breslau De plantarum epidermide obscrítiones, M. H. Krocker fils, exposa que les cellules épidermiques des Equisetum s'éloignent par leur forme de celles des autres cryptogames rasculaires, "in(fimoque plantarum Ionocolyledonearum, ordini Graminearum accedunt» (p. 3); ct plus loin il appelle de nouveau l'altention sur les stomates de l'E. arvense, et attribue les stries rayonnantes à la paroi supérieure des cellules, «cellularum paries superior «rugas præbet, radiorum instar versus rimam concurrentes» (p. 11 et pl. I, fig. 5 , vue extéricure d'un stomate de l'E. arvense, assez bonne; fig. 6 , coupe transversale du même, très-mauvaise).

La mème année, M. Unger rappelle la « disposition des stomales sur des lignes déterminées dans les sillons de la tige des Equisetum »; et il mentionnne «les stries qui s'étendent en rayonnant et souvent en se ramifiant du bord in téricur vers l'extérieur', et qui, vues de còté, se montrent comme des aspérités de la membrane cellulaire» (Die Exantheme der Pfanzen elc., p. 48; 1833). Cet auteur, ainsi que II. II. Krocker, ne paraît pas avoir soupçonné l'existence de deux paires de cellules superposées.

Struve soutint à Berlin, en 1835, une thèse de chimie, De silicia in plantis nonmullis, dans laquelle il établit que la croûte siliceuse qui recouvre l'épiderme des

\footnotetext{
'Malpighi (1675, Anat. plant., Edit. de Leyde, p. 52, lig. 106, 107 male) et N. Grew (1682, Anal. of plants, p. 153, t. 48) figurèrent grossièrement des stomates, en les mentionnant sous le nom de " hiatus quidam». J. E. Guettard, qui fut, je crois, le premier à leur donner un nom, les appela "glandes miliaires " (Mém. de l'Acad. de Paris, 1745, p. 377, t. 6). Mirbel leur donnait encore ce nom en 1815 (Élém. de physiol. végét., 1815, 1, p. 36); De Candolle les appelait "pores corticaux" $(F l, f r,, 1, p, 67)$. La dénomination de Vaucher réunit celles de Guettard et de De Candolle.
} 
Equisolun est constitue par de lacide silicinge pur, mon par un silicate. L'auteur signale of figure les aspéritis des cites de l'E. hyemule; il expose avec raison que,

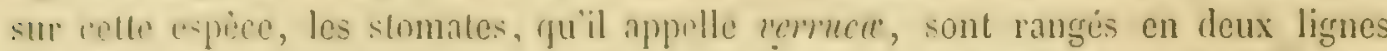
dans les sillons longiludinanx; mais, apres les travaux de Vancher, de Bischoff, de. X: Unger, il a tout is filit tort dajouter que "sur l'E. limosum ils sont répandus sur toutes les partic de la lige, sur les inférieures plus que sur les supérieures» (p. 14-22 et spécinlement p. 19). T,es figures qu’il donne des stomates sont trèsdéfectuenses.

F. Heven, dans son Kenes System der Pflanien-Physioloyie (vol. II, p. 537 et $538 ; 1838$ ), fit porter aussi ses recherches sur la croùte siliceuse el la considéra comme un déprit homogìne pénétrant la cuticule, se moulant sur toutes les inégalites de l'épiderme, et les reproduisant, tout en pourant prendre avec läge certains rentlements particuliers dont l'E. hyemale offre des exemples. Cet auteur constata aussi la présence du dépòt siliceux sur la membrane des cellules stomatiques. Ses firg. 15-17 de la pl. V, représentant les cellules des stomates, sont de médiocre valeur.

En 1843, M. . N. Braun, appliquant, pour ordonner les espéces, les observations de Vaucher et de Bischofi sur la répartition des stomates, établit les groupes Sperropora, i slomates «épar's 》 (Vaucher), el Stichopora, il stonates «arrangés dans un ordre régulier » (Vaucher); (Silliman's umer. Journ. of Scienc. and Arts, nos d'octobre et de déccmbre).

II. W. Hofmeister mentionna briexement la double couche de cellules épidermiques, ajoutant qute c'est "une cellule de la seconde couche qui dans les rejetons aériens devient la cellule-mere de denx cellules de stomates: die aüssersten wandeln sich a zur Epidermis un ; je die zweite Zelle der Oberhaut überirdischer Sprossen wird zur "Multerzelle zweier Spaltulhumgrzellen » (Veryl. Luters., p. 92). Dans cette expression du savant mierographe je n’ai pas su reconnaitre sil avait constaté l'existence dans les stomates de deux paires de cellules superposées.

Gomme nous l'avons vu plus haut, p. 34, II. Unger constata par des expériences sur l' $E$. limosum la connexion des stomates avec les canaux aériferres.

Enfin daus son très-savant travail daté du 1'́ aonit 1858, el publié dans le $29^{\circ}$ tome du Linnéa, en 1859. .1. le doeteur Carl Sanio, abordant spécialement l'étude de l'épiderme et dris stomates des Equisetum, par ses excellentes deseriptions, par le soin consciencienx quil met a indiquer le détail de ses préparations, par ses betles figures, a ripandu la plus vive lumiere sur la comnaissance de ces organes. Lauteur u’a point fiut mention de l'eppilerme des rhizomes, et ses recherches n’ont purlé que sur l'ipiderme des liges steriles des six especes suivantes: E. limosum, palustre, artens", fratense, sughrolicum et hyemule. 11 en a donné des descriptions com- 
plètes et a signalé les crreurs de détail commises par ses devanciers. Ce beau mémoire m'a rendu les plus grands services el je me plais a consigner ici l'expression de ma gratitude.

\section{§2. Des lissus du rhizome}

Il a déjả été dit, p. 1 et 12, que les entre-nœuds aériens et souterrains de nos espèces se décomposent naturellement en deux cylindres conecntriques, un interne et un externe ou cortical. Sur les liges aériennes et sur les rhizomes de plusieurs espèces (E. maximum, s!llvaticum, pratense, arvense, palustie), ces cylindres se séparent avec une extrême facilité, soit par torsion, soit par rupture des entre-nœuds, et une coupe transversale de ces espèces montre que la circonférence du cylindre interne est occupée par un rang de cellules plus petites que les plus intérieures du cylindre cortical avec lesquelles elles sont en contaet. Ce rang de cellules est toujours reconnaissable à ce qu'il constitue, sur une coupe transversale, une sorle de guirlande circulaire dont les ondulations reproduisent symétriqnement en petit le contour extérieur de la section (pl. V, fig. 10, 13 etc.; pl. VI, fig. 1, 2 etc.; pl. VII, fig. 11 c). Sur les tiges des autres espèces, ainsi que sur la plupart de leur's r'hizomes, l'adhérence entre les deux cylindres est plus considérable; les tissus du cylindre interne pénètrent dans les tissus de l'autre par de profondes ondulations, et son contour est moins nettement dessiné par un rang de petites cellules. Hais si on pratique des coupes transversales dans le voisinage immédiat du diaphragme des nouds, comme sur celle région tous les tissus du cylindre interne sont plus développés et plus apparents, on y reconnaît avec facilité que les deux cylindres sont distincts et même séparables.

Au premier coup-d'œil, le cylindre cortical d'un rhizome parait sur une section transversale entièrement composé d'un tissu cellulaire uniforme, dans lequel sont creusées les grandes lacunes; ce tissu, incolore ou blanchàtre, ne prend la couleur roux brun que vers la circonférence. L'épiderme, qui en est la couche extérieure, a dû, à cause des importantes particularités de son organisation, ètre d'abord décrit à part. Immédiatement au-dessous de lui, tous les rhizomes présentent des couches compactes de cellules très-longues (six à dix lois plus longues que larges), à parois épaisses formées de plusieur's membranes superposées, très-résistintes et fortement colorées en roux brun ( $p l$. VII, fig. 1). Le nombre de ces conches, la longueur de leurs cellules, l'intensité de leur coloration et l'épaisseur de leurs parvis varient avee les espèces, mais, sur toutes, ces différences dans la couleur et l'éparsseur des parois et dans la longueur des cellules diminuent en s'éloignant de la périphérie, et, à l’entour des grandes lacunes il n'existe plus que du tissu làche el incolore, de grosses 


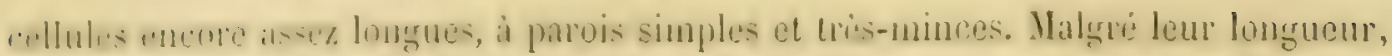

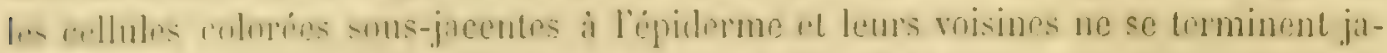

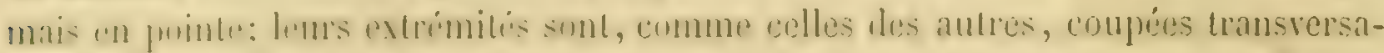

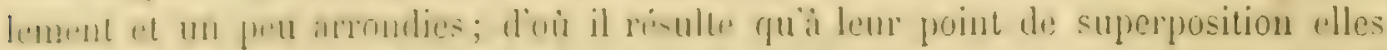

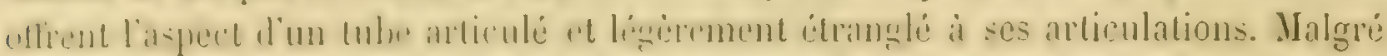

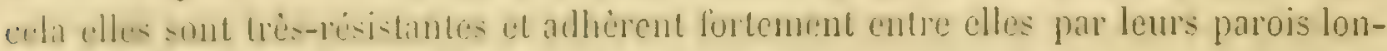
qutudinales. sur ees parois on observe de nombreux petits conaux transwersaux, tres-

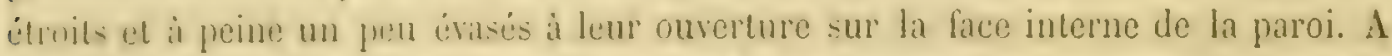
chacun de cre canaux parichux cortespond exactement un canal scmblable de la collule entigü; mais il arrive rarement que ces deux canaux correspondants soient en communication directe. Chasun d'enx est ordinairement fermé par lá membrane exteriente on primitive, laquelle est tres-mince el oceupe le milieu de la cloison com-

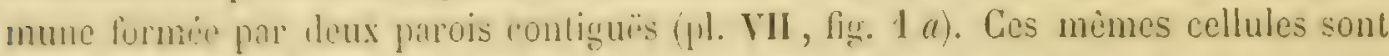

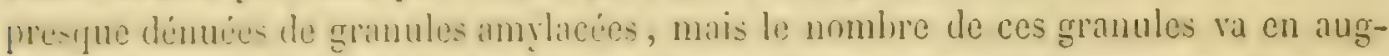
nentant vers le contre de la tire, el les autres cellules en sont tellement remplies que les cloisons sunt imposibles i dislinguer sur le porte-oljet, si on n'a pas la précantion de les presser et de les laver plusieurs fois.

Ciest dans ae lissu liuche du cylindre externe que se trowrent les grandes lacunes; elles ne manfuent jamais sur les rhizumes, mime sur ceux de l'E. limosum, dont les tiges en sont souvent privées (pl. V, fig. 10 à 20). Ici, comme sur les liges, ces lacunes, ausi que les autres et la cavité centrale, nont point de parois propres. fien que lis lormes et les dimensions relatives des unes at des autres soient assez constantes sur ume meme espece, elles napparaissent toutes que comme des intervalles, des lacumes, dans le tissu ambiant, et sumvent lon voit des lambeaux de ce

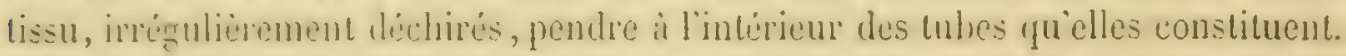

La composition du cylindre inteme est un peu moins simple: à lexamen d'une coupe transwersale on est tout d'abord frappé par lit.distinction de deux lissus. L'un con-litue la masse grénérale; il col biche el formé de cellules larges toutes pleines de granules anylacés; lantre, régulierement reparti en faisceaux dans la masse du premier, est line lexture plus serrée el en meme temps plus transparent, parce qu'il est presque entièrement dépoursu de granules amylacés.

Ln examen plus attentil du premier tissu lait voir quil cet a l'extéricur circonscrit par un: lisn circulaire ol ondulep de petites cellules mentionnées ci-dessus, lesquelhs sont flus ou moins distinctes; que les antres cellules, toujours chargées de lesule, ansmentent de diametre en se rapprochant du centre, et latteignent meme yur. Iquefuis sinn y laisser de cavile (E. maximum ete.); et rue, quand la cavilé cenllatu: criste, a ses parois toijours mal limitess sont suspeudus des lambeaux de ce li--n direhire. Du resto rien de particulier a faire remarguer dans son organisation, si 
ce n'est que ses cellules sont toujours assez allongées, comme dans tout le système cellulaire d'un Equiselum adulte.

Les faisceaux du second tissu sont rigoureusement situés sur des rayons qui partiraient de la carène ou ligne médiane des côtes. C'est au point le plus interne de ces faisceaux que se trouvent les petites lacunes, dites lacunes carinales par suite de leur position. ou lacunes essentielles parce qu'elles ne manquent jamais. Ces firisceaux sont assez nettement limités, et des sections transversales nous les montrent constamment comme de gros cordons qui, selon les espèces, sont ou subcylindriques, ou comprimés dans le sens du rayon, ou déprimés transversalement, et, dans tous les cas, échancrés du côté interne par la lacune essentielle (pl. V, fig. $20 e, f$ ).

Leur tissu est principalement constitué par des fibres étroites, à parois peu épaisses, mais néanmoins très-résistantes. Ces fibres sont si longues qu’elles semblent quelquefois ne pas s'articuler sur toute la longueur de l'entre-noud; leurs cxtrémités, au point d'articulation. sont relativement peu obliques (pl. VII, fig. 2); dans ce tissu fibreux se trouvent aussi des vaisseaux proprement dits, déjà reconnaissables sur une coupe transversale à leurs parois plus épaisses et à leur ourerture circulaire. Ils so séparent très-facilement des fibres et se présentent presque indifféremment sous la forme de tubes très-longs, très-effilés aux extrémités, à parois incomplétement échancrées, ou tout à fait divisées on anneaux, ou enfin roulées en spirale (pl. VII, fig. 3 et 10). Ces divers états se succèdent à de très-petits intervalles et offrent toutes les transitions possibles entre la simple échancrure, les anneaux isolés et la spirale. Quelques-uns de ces vaisscaux se trouvent, sans grande régularité, au pourtour des lacunes essentielles (pl. VII, fig. 4 ct 9), le plus souvent contre la paroi la plus éloignée du centre de la tige; mais il n'y en a guère qu'un ou deux et ils ne s'étendent pas dans toute la longueur de lentre-nerud. Sur plusieurs points ils manquent, et sur d'autres ils ne sont représentés que par des anneaux isolés et souvent même couchés à plat sur la paroi, comme des restes d'un faiscean vasculaire plus considérable en partie résorbé. Ils manquent sur les rhizomes très-vieux. Les autres vaisseaux sont au contraire disposés avec une régularité parfaite, et dans chaque faisceau fibreux ils constituent, au nombre de trois à cinq, deux petits cordons vasculaires, plats, situés en avant des lacunes essenticlles, un peu de côlé et assez exactement sur une ligne menée du centre de ces lacunes rers le milieu des sillons ou des faces extérieures les plus voisines (pl. V, fig. 10-20). Ces vaisseaux sont moins gros que les précédents. On trouve encore quelquefi is de très-petits vaisseaux, spiralés et parfaitement déroulables, le long des parois des lacunes essentielles; ils sont d'une extrême ténuité et leur diamètre attcint à peine le quart de celui des autres (pl. VII, fig. 5).

Sur les plus gros de ces vaisseaux, c'est-à-dire sur ceux qui sont épars au pourtour des lacunes essentielles, on distingue avec facilité que les fils de leurs anneaux 


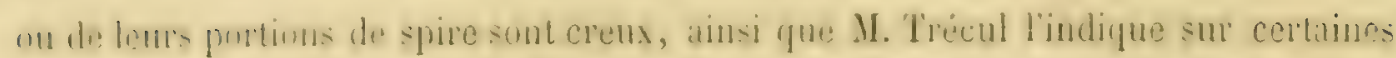
plantes (Form. sec. cell., p. 317 et suiv.). Le plus souvent ces fils sont plals sur leur

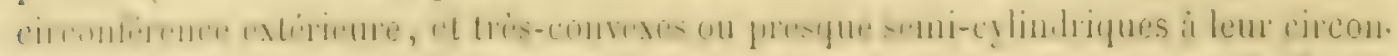

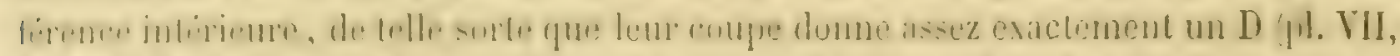

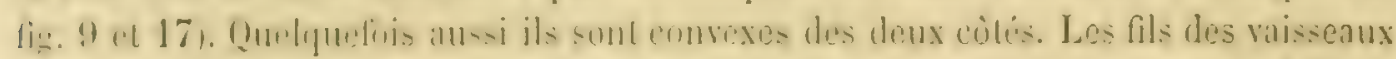

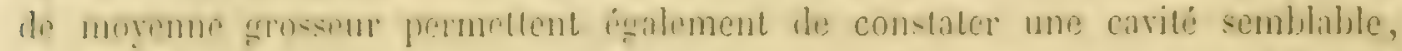
mais olle ret hien moins applarente, pare que ces fils sont prespue plats ipl. Yll, liz. 10, Hans les rhizmes prinement developpes, ni les anueaux, ni les parties spi-

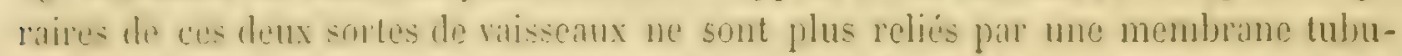
hare, mais - -ur les rhizums tri-jeunes of en voie de formation, comme sur les liges, on constate lies-liteilement liexistence de ce tube, ainsi que nous le verrons au clsap. III.

Ces faisenux du cylindre interne, ofirant des fibres et des vaisseaux, doivent ètre appelés faisceaux fibro-tasculaires; el c'est par ce nom que je les désignerai.

Bischoll a fli le premier, je crois, a signalur l'existence des deux cylindres si dis-

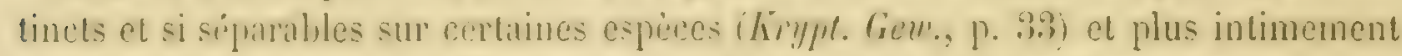
unis sur d'autres (o. c., p. 37); il a aussi très-bien distingué les divers tissus du cy-

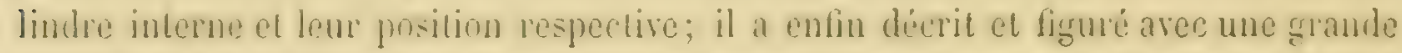
cxactitule les diversitis de forme que presentrut les vassenus; mais il sest tout a

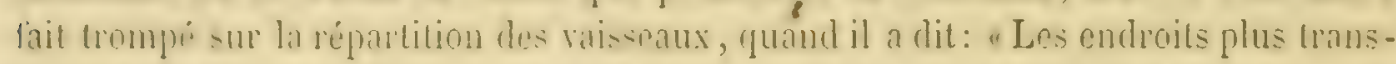
pareuts les faisceaux filmo-risculanest, atmsi que les lacunes intérieures, sont entourés

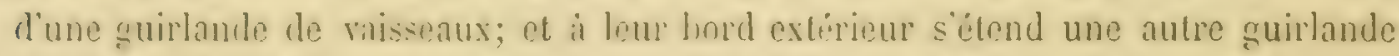
de vaisecaux qui forme un polygone parallibe i la circonférence de la seclion transversale, et qui sipare la section en deus ronches dont lintérienre represente, pour

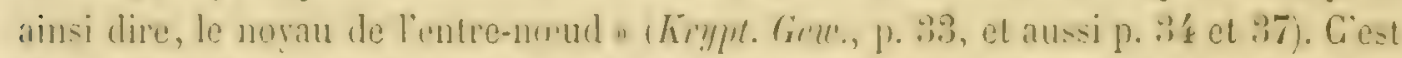
la wne double erreur. Il ni a proint de cerele on de fruirande de vasseaux a la surfice du rylindre interne; il ne sy trouve que les pelites cellules sus-mentionnes. qui, sur une coupe transversile, auront sans doute fit illusion a cel observateur si

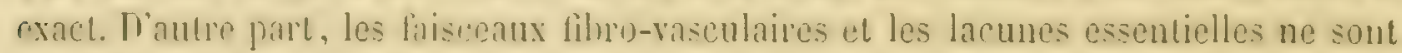

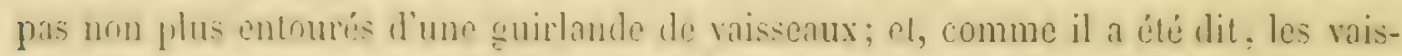

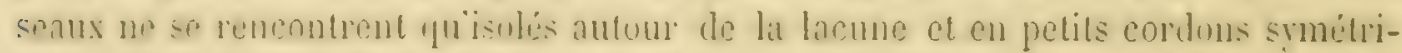

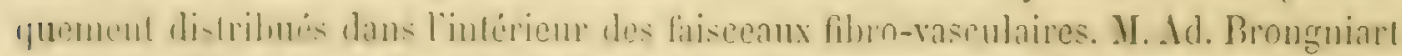

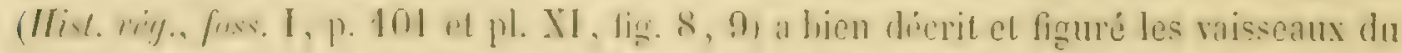
fourlour des lammes il na pas mentiomme les antres, el il ajoule meme: "Les raisa soux placis sur las parois de ces lacumes sont los seuls quion observe dans ces (plantes. )

Li:- tubercules qui naisent aux arliculations du rhizome ne se prêtent pars a la

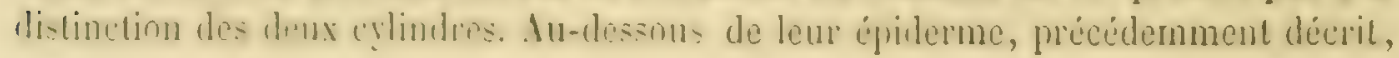


se trouvent des cellules à parois épaisses el culorées, formant une enveloppe trèsdure, puis le reste se compose d'une masse compacte de lissu cellulaire, dans lerguel sont répartis huit i dix faisceaux fibro-vasculaires, que leur conleur moins blanche et leur disposition en cercle font facilement reconnaitre it l'wil nu sur une coupe transversale (pl. I, fig. 3). Ces faisceaux sont fort petits, et ne se composent que d'un petit nombre de fibres courtes contenant parfois des granules amylacés, et de quelques vaisseaux spiro-amnulaires assez semblables à ceux du rhizome (pl. I, fig. 13). Yers la base du lubercule ces faisceaux sonl fort rapprochés, souvent mème contigus, puis ils se séparent en divergeant fortement, pour se réunir de nouveau vers l'autre extrémité. On n'observe dans les tubercules ni lacunes, ni carité centrale régulière. Toutefois les cellules du centre se détruisent assez facilement, particulièrement sur l'E. maximum; il en résulte une cavité plus ou moins prononcée avec l'àge, mais toujours mal terminée et irrégulière. Les faisceaux fibro-rasculaires et les cellules qui les aroisinent persistent beaucoup plus longtemps sous forme de saillies latérales simulant des rudiments de cloisons. La masse du tissu cellulaire se compose uniformément de cellules assez grosses, beaucoup plus courtes que dans loute autre partie du rhizome ou de la tige, et contenant une grande quantité de granules amylacés et quelques traces de sucre'. Ces granules, tout à fait semblables à ceux dont est chargé le tissu cellulaire du rhizome, sont généralement de forme ovoïde et de grosseur fort variable $\left(0^{\mathrm{mm}}, 015\right.$ à $\left.0^{\mathrm{mm}}, 030\right)$; mais les plus gros sont encore fort petits, comparativement aux grains de fécule des pommes de terre, et ceux de l' $F$. sylvaticum sont les seuls qui s'en rapprochent un peu. Ils m’ont généralement paru tout unis; ceux de l'E. syluaticum m'ont offert quelques petites ondulations, et sur ceux de l'E. liyemale j’ai pu distinguer quelques stries concentriques à un point presque central. Eclairés at microscope avec de la lumière polarisée, ils présentent une croix très-nettement dessinée (pl. I, fig. 9 à 12). Rappelons ici que ces granules manquent presque absolument dans les rhizomes, comme dans les tubercules de l'E. maximum. Ceux qui s'y trouvent, et dans le jeune àge seulement, sont en très-petit nombre, irrégulièrement mamelonnés et diformés à tel point qu'il n'y en a pas deux de mème dimension au de mème forme (pl. I, figg. 12). A la lumière polarisie ils ne m’ont présenté aucun effet appréciable. Il y a done sur cetle espéce absence, ou formation très-incomplète des granules amylacés.

Une racine bien développée, mais encore jeune, se montre recouverte à son extrémité d'une petile coiffe cellulaire, qui se soulève et s'exfolie irrégulièrement à une petite distance de la pointe (pl. II, fig. $21 b, c, c^{\prime}$ ); quelques-unes de ces cellules

\footnotetext{
' M. W. Hofmeister dit au contraire : a Le tissu cellulaire des tubercules (de l'E. artense) contient un peu de fícule et beaucoup de sucre, non cristallisable à ce qu'il m'a semblé.... Das Zellgewebe der hnolle enthält etwas Stärkemeh 1 " und vielen Zucker (wie mir schien, nicht krystallisisbaren)" (Vergl. Linters., p. 91).
} 
-r: ditarlunt même vers la pointe et sont retennes dans une matiere mucilaginense, de consistance visqueuse. Ce mucilage et eelte coiffe constituent l'extrémité absolue de la rarine, souvent appelie spongiole el plus réecmment piltiorhize par M. Trécul (Orig. rac., p. 310). Au-dessous de la pilćorhize, ou, pour mieux dire, at l'extérieur de la racine, existent doux ou trois couches de cellules prismatiques hexagonales, longues el colories; l'externe fait fonction d'épiderme, c'est d'elle que naissent les fibrilles du tomentum. Sous ces longues cellules une couche de cellules courtes, d'aspect mídullaire et sourent remplies de granules amylacés, entoure un faisceau central fibro-rasculaire. Les vaisscaux sont ammulaires et inigaux en grosseur; le plus gros est au centre, et autour de lui sont répartis trois groupes de deux ou trois raisscaux plus petits (pl. II, fig. 20 et 26). Quand les racines sont très-vieilles, il arrive d'abord que les grosses cellules de la couche moyenne se disloquent et disparaissent, ce qui constitue un vide cylindrique antour du faiscean fibro-vasculaire; bientôt la destruction atteint les couches extérieures, et ainsi la racine ne se compose plus que du faisceau central fibro-vasculaire.

Il y aurait encore à décrire les tissus des gaînes et ceux des diaphragmes des nœuds; mais, comme l'analyse de ces parties ne présente rien de bien particulier sur les rhizomes, je n'en parlerai qu'une fois dans la description des tiges.

\section{§ 3. Des tissus de la tige}

La structure de la tige est tnut à fait digne d'attention, d'abord parce qu’elle diffère notablement de celle du rhizome, ensuite et surtout en ce que les variations qui, selon les espéces, s’offrent dans la répartition symétrique des organes élémentaires, sont trìs-considérables et peut-être même plus considérables que daus toute autre famille de plantes, les Fougères exceptées (voy. J. Dux. J., Pétiol. Foury.). Or, comme ces diverses répartitions sont parfaitement constantes sur une mème espèce, el trèsfaciles à distinguer, elles fournissent des caractères spécifiques thune très-haute valeur.

Dans les entre-nouds de la tige, arons-nous déji dit p. 12 et 13 , on retroure avec plus ou moins de nettelé et de facilité les deux cylindres mentionnés sur le rhizome.

L.e cylindre entical du rhizome ne nous a présenté, au-dessous de l'épiderme, que des cellules longues, a parois épaisses et colorées, passant par degrés aux cellules à parnis minces et incolores entourant les grandes lacunes. Or le coup-d'mil le plus superficiel jete sur la section transversale d'une tige de couleur verte, nous y fait reconnaître, en allant de l'extéricur à l'intérieur:

$1^{\circ}$ Des groupes de fibres à parois très-épaisses et à cavité très-petite; 
$2^{\circ}$ Des groupes de cellules remplies de chlorophylle;

$3^{\circ}$ Du tissu cellulaire lâche et incolore (pl. VI, fig. 5, 9, 12-15, 17-20).

Les tiges vertes, stériles ou non, nous offrent donc, au-dessous de l'épiderme, le long des côtes saillantes et quelquefois au fond des sillons, des faisceaux l'un tissu particulier et qu'on ne rencontre que sur ces points. Il consiste en cellules extrêmement longues et étroites, à parois très-épaisses, longuement atténuées en pointe à leurs extrémités (pl. VII, fig. 6 el pl. III, figg. 10 bis). Ces cellules adhèrent fortement cntre elles et contre l'épiderme; et les faisceaux qu'elles constituent sont si résistants que, quand on rompt un entre-nœud, ils pendent en cordons blanchâtres au-dessous du point de rupture. En plaçant un de ces cordons sous la loupe i dissection, on le sépare alors assez bien de l'épiderme auquel il adhère. Une coupe transversale montre que ces longues cellules ont des parois formées de plusieurs membranes superposées, qu'elles sont prismatiques et à contour plus ou moins hexagonal, que leur cavité longitudinale est extrêmement réduite, assez régulière dans le voisinage immédiat de l'épiderme, et de plus en plus large et irrégulière en allant vers le centre; enfin qu'elles communiquent par des canaux pariétaux aboulissant sur la ligne médiane de leurs faces (pl. VII, fig. 7, 8). L'examen d'une coupe longitudinale de ces faisceaux fait voir en outre que quelques-unes de ces cellules sont parcourues dans leur longueur par d'épaisses stries spiralées d'un aspect plus transparent que le reste (pl. VII, fig. 8); de place en place on distingue des canaux pariétaux, qui se montrent comme des points ou comme des lignes plus transparentes, selon la position. Si la plante est jeune, on trouve souvent dans la cavité de ces cellules, et plus particulièrement vers leurs extrémités, de nombreux granules très-petits, un peu leintés de vert (pl. VII, fig. 6). Tous ces caractères sont ceux des tissus fibreux, et si particulièrement ceux du liber que plusicurs auteurs compétents n'ont pas hésité à en domner le nom à ces faisceaux (Bischoff, Livpt. Gew., p. 37; J. Milde, Gef. Crypt. Schl., p. 414́, 472, 473 etc.). Au même lieu Bischoff les désigne aussi sous les noms de faisceanx fibreux, de faisceanx vasculaires sévenx, « Faser-oder Saftröhren-Bündel; Faserbündel » (o. c., p. 36 et 37). M. C. Sanio les dit seulement semblables an liber, "bastähnliche Zellen » (Epid. u. Spalt, p. 390) et aussi "bast«artige Zellen » (o. c., p. 404, 405 etc.). Mais M. Schacht dit très-expressément: «Les véritables cellules du liber manquent aux cryptogames... Den kryptogamen Ge( wächsen fehlen die eigentlichen Bastzellen » (Pfanzenzelle, p. 209); et «quant aux cryptogames les plus élevés, je n’y connais aucune espèce de cellules que maintenant je puisse avee quelque droit appeler cellules de liber. Für die höheren Kryp« togamen kenne ich lieine Zellenart, welche ich nur mit einigem Recht als Bastzelle «ansprechen dürfte»(0. c., p. 221).

Il ne m'est pas permis de méconnaitre les analogies de forme qui justifient la déno- 
mination de liber, jy ajunterai mème une analegie de position en rappelant que dans lese dimotylinlonées annuelles à lige anguleuse (Labiées et Rubiacées), le tissu fibreux de Pérorere se comporte comme dans les Equisetum et se réunit ver's les angles en épais faisceanx (roy. A. Kirchhof, Lab. org., p. 8-13). Mais, comme l'identité de nom entraine lidece de la parfaite identité de nature et de fonction, et cun'il s'agit de cryptogames, où la présence du liber est contesté, je préfére, malgré ma conviction, ícarter un nom qui prüjuge la ruestion, me borner à indiquer les analogies et m'en tenir aux dénominations plus générales de fuisecanx fitrenx on de filmes corlicales pour designer ce tissu el ses éléments. Elles sulfisent d'ailleurs pour le distinguer des faisceaux fibro-vasculaires précédemment décrits.

La position générale de ces faisceaux est parfictement invariable dans tout le genre; ils sont toujours, je le répète, sous-jacents it l'épiderme et situés soit seulement sous la saillie des còtes (E. limosum, pl. VI, fig. 14), soit en mème temps au fond des sillons (E. ure'nse', ramosissimun clc., pl. VI, figg. 9, 17 etc.). Leur's contour's et les détails de leur répartition sunt tont aussi invariables sur les individus d'une mème espèce, mais ils varient notablement d'une espèce à l'autre; ces différences seront décrites et ligurecs plus loin comme caractères diagnostiques. Ces faisceaux manquent complétement sur les tiges spicilëres non conformes des E. maximum et artense (pl. VI, fig. tet 8$)$.

Autour ou de chaque crité de ces faisceaux fibreux, el toujours vers l'intérieur, sont groupees des cellules toutes remplies de chlorophylle et rui sétendent en cordons verts tout lo long de chaque entre-nocud; aux extrémités, et surtout à l'extrémité inférieure, elles sunt moins chargées de chlorophylle quau milieu. Comme les filmes corlieales, ces cellules sont disposies avee une symétrie parfaite, et, bien ugu ayant toujours la mème position géuérale, elles présentent sur chaque espèce un mode de distribution parliculier et très-constant (pl. VI, figg. 5, 9, 12, 14, 15, 21). Elles sont en général séparées de lépiderme par les fibres corticales, mais toutefois il y a constamment certains points ou clles paraissent en contact arec l'ipiderme, et ces points correspondent constamment à la position des stomates. Là nii les stomates sont ripartis sur toute la largeur du sillon, les bandes rertes sont, sur toute celte largeur, sous-jacentes el contiguës à l’épiderme (E. limosum); là oì il ny a qu'une ligne de stomates de chaque crite des coiles (E. syleuticum, hyemule ete.), les groupes de cellules vertes ne viennent toncher l'épiderme que le long de cette ligne. Telle est enfin la relation entre la matière verte el les stomates que là nii manquent ceux-ci, celle-li manque absolument dans les cellules'. Ainsi les sto-

\footnotetext{
- En 1828, Dischofi signalait "l’absence des stomates sur les tiges qui ont une autre coulcur que la verte" (Kirypt. 6"ek., p. 36). Ach. Richard dit jus explicitement encore: - Dans tous les points de la lige qui offrent une coloration
} 
mates manquent sur les entre-nœuds des tiges spicifires de l'E. arvense et de toutes les tiges de l'E. maximum, et ces entre-nceuds n'ont pas de chlorophylle, tandis que les gaines des mêmes tiges de ces deux espèces, et les rameaux de la dernière, sont pourvus de stomales et en même temps de chlorophylle (pl. VI, fig. \& el 11). Or, comme tontes les parlies de ces tiges sont également exposées à l'action de la lumière et que cependant celles de ces parties qui sont munies de stomates sont les seules à élaborer la matière verte, il faut bien en conclure que la production de la chlorophylle n'est pas due seulement à l'action de la lumière, mais aussi à une cause particulière dont les stomates permettent l'action". Rappelons cependant que sur les entrenouds submergés de l'E. limosum, ct dépourvus de stomates, les cellules de l'épiderme renferment quelques granules verts qu'on ne trouve plus dans ces mêmes cellules aux entre-nœuds oủ il existe des stomates et de viaies cellules à chlorophylle.

Au-dessous des stomates, entre eux et les cellules à chlorophylle, existent de petits méats aériens, qui quelquefois mème sont assez considérables sur les jeunes tiges de l'E. hycmale (voy. ci-dessus p. 33; pl. IV, fig. 2, 4, 6, 8, 9, 13, 14, 17, et pl. V, fig. 2,5).

Comme il a été dit précédemment, le système cellulaire des Equiselum bien développés présente des cellules très-allongées et dont le grand axe est vertical; les cellules à chlorophylle font exception: elles sont ovoïdes et le grand axe en est horizontal (pl. VI, fig. 16 et 21).

Le reste du cylindre cortical se compose de tissu cellulaire làche, incolore, dont les cellules, à parois minces, augmentent de diamètre à mesure qu'elles s'éloignent de la circonférence. Les lacunes qu'il circonscrit ont toujours une forme et une grandeur relative constantes, surtout si on les observe vers le milieu de la tige. Sur l'E. limosum ces lacunes s'oblitèrent souvent, parce que, les stomates étant répandus sur toute la largeur du sillon, les couches de cellules à chlorophylle ne sont point interrompues sur la ligne médiane du sillon, et pour peu qu'elles se développent sur le cylindre cortical, qui est très-peu épais, elles occupent la place de ces lacunes.

Les tissus du cylindre intérieur des tiges, plus distincts encore que ceux du rhizome, sont absolument de même nature; la disposition générale est la même, je He m'y arrêterai donc point. Après la diminution ou la disparution des granules anylacés, la seule différence générale qu'on puisse signaler consiste en ce que la couche de tissu cellulaire la plus intérieure et dans laquelle est creusée la cavité centrale est moins épaisse sur les tiges que sur les rhizomes. Quelquefois même sur ces derniers

"verte, on aperçoit des stomates disposés par liggnes longuitudinales " (Ẻlém. bol. et phys., p. 611). Si la seconde partie de cette observation n'est pas d'une rigoureuse exactitude, la première est parfaitement vraie.

1 M. Sachs a exposé dans le Chemische Centralblath, 1859 , no 10, p. 145, que, pour que la leucophylle se transforme en chlorophylle, il suffit qu'elle se rencontre avec de l'oxygène actif ou ozone en quantité suflisante. 
elle demeure si développée qu'il n'y a plus de cavité centrale (E'. maximum,

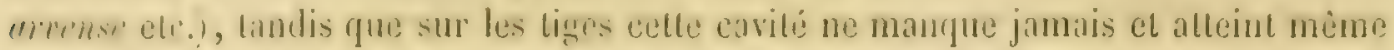
des proportions tris-consideralles et assez constantes pour fournir des caractires spuedifipues. Il en sera fait mention it la deseription des especes, ainsi que des différences de détail que l'on remaryue dans les dimensions el la position relatives des faisceaux fibro-rilsculaires, et dans celles de lenrs petits cordons de raisseaux.

Les tiges spiciferes non conformes se distinguent tout d'abord des autres par leur précocití, leur coulcur el leur prompte caducité. Leurs tissus sont aussi moins résistants, moins fermes et toujours chargés d'une grande quantité d'eau. En outre, leur composition présente quelques diffírences qui ont déji été signalées dans tout ce quui précède et que je me bornerai à résumer ici:

$1^{\circ}$ Côtes à peine saillantes;

20 Point de fibres corticales; elles sont remplacées par des cellules un pen plus étroites que les autres et tenant le milieu entre les grandes cellules et les fibres corticales;

$3^{\circ}$ Absence de stomates sur l'épiderme des entre-nouds;

$4^{\mathrm{n}}$. Absence de chlorophytle aux entre-nouds (p). VI, fig. 1, 8, et. pl. VII, fig. 11).

Ces dernières différences ne sont tout it fait exactes que pour les tiges non conlormes caduques ( $E$. muximum el urvense); car sur les liges spicifères persistantes de I'E. syleaticum et prutense on trouve dejjà les stomates qui leur permeltront d'élaborer la matiere verte a leurs entre-nouds; et les faisceaux fibreux s'y montrent presque en même lemps que la chlorophylle.

Le cylindre interne ne présente aucune différence de composition; seulement la couche intérieure des cellules est plus considérable. Il m’est done impossible de comprendre ce qui a pu porter M. L. Reichenbach ì dire que les tiges de l'E. maximum et les hampes (scapus) de l'E. areense sont dépourvues de vaisscaux "vasis spiralibus "carentes» (Flur. excurs., p. 15\%); quand ces organes y sont développés plus peutêtre qu’ailleurs.

Voici, également en résumé, les différences qui existent entre les entre-nœuds des rhizomes el ceux des tiges:

10 Angles moins nombreux;

$2^{\circ}$ Faces planes remplaçant les sillons;

$3 \circ$ Absence de faisceaux fibreux corticaux;

$4^{\circ}$ Cellules chargées de granules amylacés;

$5^{\circ}$ Absence de stomates et de chlorophylle;

$6^{\circ}$ Couche cellulaire interieure du cylindre interne plus épaisse, et, sur quelques espèces, sans cavité centrale.

Iais quetles que soient les differences entre le rhizome et les deux sortes de liges, 
elles s'efficent graduellement i mesure que la partie souterraine se rapproche de la surface du sol. La cavité centrale apparait el grandit; les granules amylacés deviennent de moins en moins nombreux, puis disparaissent; les cellules sous-jacentes i l'épiderme s'allongent plus encore, perdent leur couleur brune et se groupent vers les côtes ou au fond des sillons; enfin l'apparition successive des stomates et de la chlorophylle vient compléter le changement.

Les divers tissus des deux cylindres des entre-nœuds sont tous représentés dans les gaînes, mais ils y sont plus intimement unis et non séparables. Ils y occupent la même position relative. Ainsi, la carène des divisions offre sous l'épiderme un large faisceau de fibres corlicales (pl. VI, fig. 4, 7, 11), qui se prolonge jusqu'au deli du milieu des lobes. Le même tissu se montre quelquefois aussi en très-pelite quantité au fond des sillons, mais toujours séparé en deux cordons par la ligne commissurale (pl. VI, fig. 11). Comme ce tissu, en se desséchant, diminue moins de volume que le tissu cellulaire ambiant, il constitue de chaque côté des sillons commissuraux de petites saillies longitudinales qui ont été appelées carènes latérales, et on a appelé sillon latéral la petite dépression qui sépare ces carènes de la carène médliane. Et, comme sur la ligne médiane de cetle dernière le faisceau fibreux se déprime quelquefois, celte dépression a été appelée "sillon carénal» (voy. ci-dessus p. 11, et Al. Braun, Sillim. Journ., p. 81).

Au-dessous du faiscean carénal s'étend un dépôt de cellules à chlorophylle occupant toujours une portion relativement considérable; sa distribution varie avec les espèces et les détails s'en trouveront plus loin. Rappelons seulement que les faisceaux fibreux corticaux et la chlorophylle se trouvent sur toutes les gaines de toutes les tiges, même des tiges dont les entre-nœuds sont privés de matière verte et de fibres corticales ( $E$. arvense et maximum).

Enfin toute la région interne de la gaine est occupée par une large bande de tissu cellulaire làche et incolore, au milieu duquel se trouve un faisceau fibro-vasculaire continuant en partie celui de l'entre-nœud. Nous verrons bientôt comment il pénètre dans la gaîne. On constate quelquefois ( $E$. maximum, arvense elc.) contre ce faisceau une petite lacune, mais le plus souvent elle est à peine visible et oblitérée (pl. VI, fig. 4, 11).

Les divers tissus qui composent la gaine diminuent graduellement à partir de la naissance des lobes et disparaissent avant d'en atteindre l'extrémité. Les vaisseaux, par exemple, diminuent d'abord de diamètre et ne sont plus spiralés; puis, enfin, ils ne sont plus représentés que par quelques anneaux isolés et très-espacés. Au sommet et sur le bord des lobes il ne se trouve plus que des cellules minces, membraneuses, à cloisons ondulées, à direction transversale; elles appartiennent à l'épiderme et ont été décrites p. 28. 


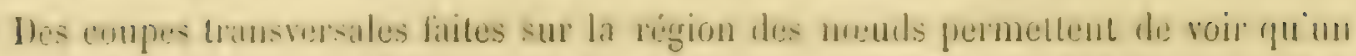

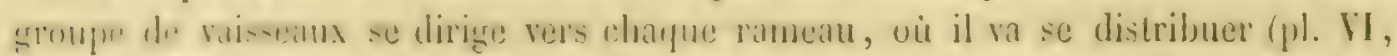

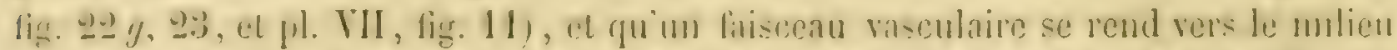

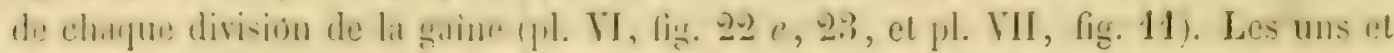

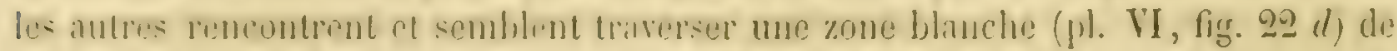

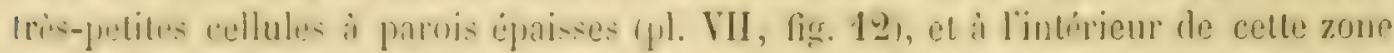

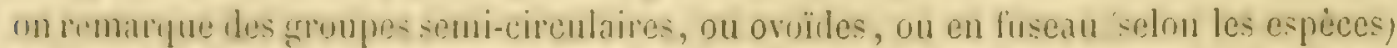

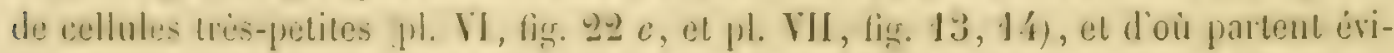

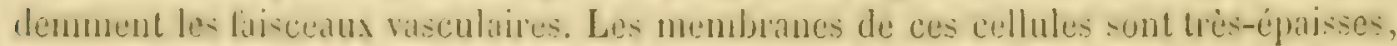

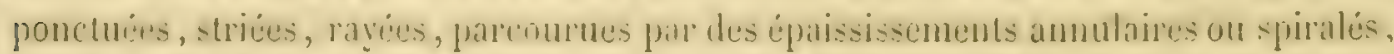
en 10 mot présentant toules les apparences possibles depuis la simple poncuntion jusrguaux grandes onvertures, aux anneanx, aux spirales, le tout sourent mele sur

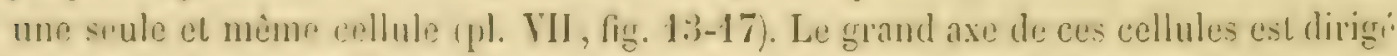

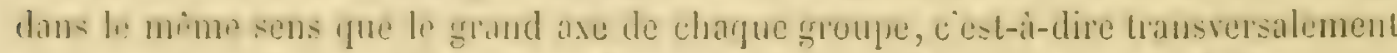

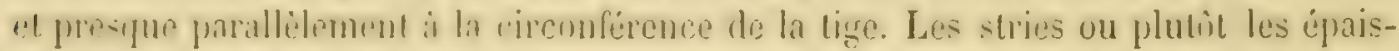
sissments de res cellules ont une direction gencrale perpendiculaire i leur grand axe (fl. VII, fig. 13, 14). Le diaplıragme proprement dit est composé uniquement de trois ou quatre couches de cellules simples, plus pelites gur les cellules les plus intérieures des entre-nœuds (pl. VII, fig. 11).

Si lon rent maintenant se rendre comple du rapport de ces diver's lissus avec ceu. dont nous nvons reonmu lexistence dans les entre-nauds, l'examen simultane el

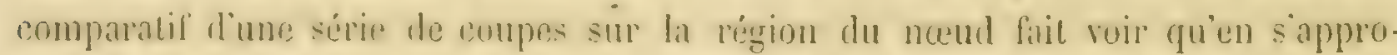

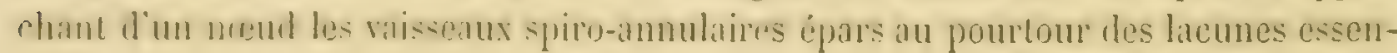

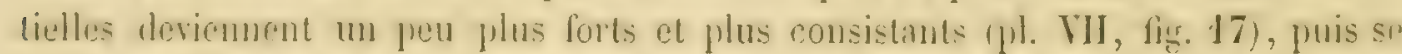
rourbent horizontalement pur aller rejoindre les cordons vasculaires qui se trourent en arant et de charfue cote de ces latunes. En se courbant ansi, ils repoussent vers lextericur la partic fibreuse du linseran fibro-rasculaice. Un pen atu-dessous du

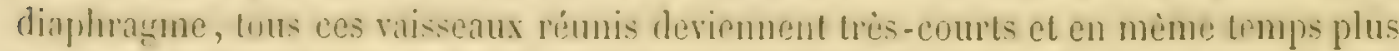
gros al plus nombreux, el finalement par cette transformation ils conslituent en avant

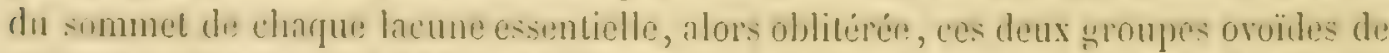

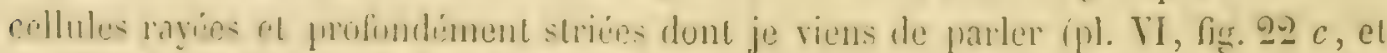

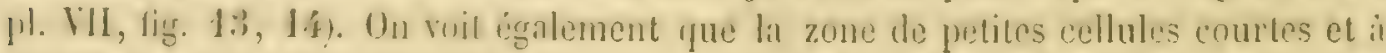
flaruis equases entourant ces grompes correspond aux filnes qui sont en avant de rharue cellule esenticle. En approchant du noud ces cordons de fibres angmentent de volume ct so transforment en une ceinture de cellules pelites, courtes, a parois ipgai-ses (pl. VI, lig. 2-2 d, et fll. VII, fig. 12), lout comme les vaisscaux spiro-

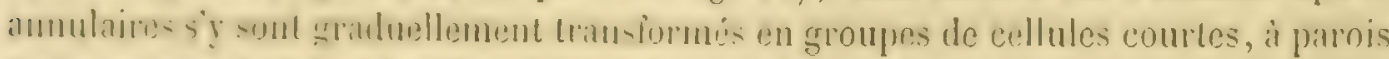

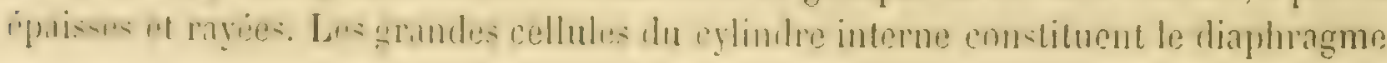


en se modifiant d'une manière analogue dians leur longucur ot dans l'épaisscur de leurs parois. Cette disposition générale se montre dans toutes les espieces avec une parfaite régularité. Il me semble done que M. Nägeli n’a pas donné une idée suffisamment cxacte de cette organisation en disant simplement que les faisceaux vasculaires de la tige se réunissent dans chaque nœud en un tissu embrouillé (Zcitschr. F. Botanik, IIefl 3 u. 4, Seite 143).

Revenons aux groupes de cellules rayées, correspondant aux cordons vasculaires. Nous avons vu qu'il y en a un de chaque côté du sommet d'une lacune essentielle; or, de chacun de ces deux groupes et de son extrémité la plus rapprochéc de cette lacune se détache horizontalement et vers la périphérie un faisceau vasculaire, et ces deux faisceaux se réunissent bientôt en un seul, qui passe à travers les grandes cellules séparant les lacunes corticales, et monte brusquement dans le milicu de la division de la gaine à son point d'origine (pl. VI, fig. $22 c$ ). De l'autre extrémité de ces groupes, c'est-à-dire de l'extrémité la plus éloignée des lacunes essentielles, partent aussi des faisceaux vasculaires, mais pour suivre des directions très-différentes. D'une part, les uns se réunissent deux à deux pour constituer un faisceau unique, lequel passe horizontalement, comme le précédent, mais au-dessus même des grandes lacunes corticales et se rend dans les rameaux (pl. VI, fig. 22g). Il est dès lors facile de voir que le faisceau vasculaire qui entre dans un rameau se compose de deux faisceaux ayant appartenu dans l'entre-nœud chacun à un faisceau fibro-vasculaire différent. Ces faisceaux manquent ou ne sont qu'i l'état rudimentaire dans la plupart des tiges spicifères des E. maximum et arvense. D'autre part, de la même extrémité s'élève un cordon vasculaire qui se rend dans l'entre-nœud supérieur et devient le cordon latéral d'une lacune essentielle (pl. VII, fig. 11). Par suite de cette disposition il y a alternance, c'est-ì-dire que le cordon issu du groupe de droite d'une lacune essentielle devient, en pénétrant dans l'entre-nœud supérieur, le cordon latéral gauche de la lacune qu'il avoisine.

La composition des nouds du rhizome est absolument identique, ct toul s'y passe de la même manière, avec cetle circonstance en plus que du nouveau faisceau vasculaire destiné au rhizome latéral (qui remplace le rameau) il se détache un petit cordon vasculaire qui se rend dans la racine infraposée (pl. I, fig. 7 h).

Les rameaux sont composés des mêmes tissus que les tiges, toutefois il y a lieu à distinction. Dans les deux derniers groupes les tissus des rameaux offrent, sur une plus petite échelle, une disposilion identique a celle des tiges, avec leurs lacunes et leur cavité centrale, et, sauf peut-être une proportion plus considérable des cellules à chlorophylle, et des còtes plus saillantes par suite de la réduction du diamétre, il n’y a rien de spécial à y remarquer et rien à ajouter à ce qui a été dit précédemment. Mais, dans les deux premiers groupes, les rameaux diffërent notablement des tiges. 
A part quelques rares exceptions, les entre-nouds présentent quatre côtes, mais les

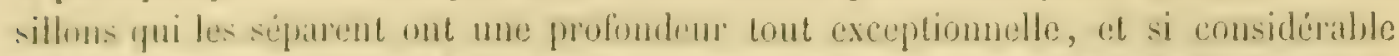

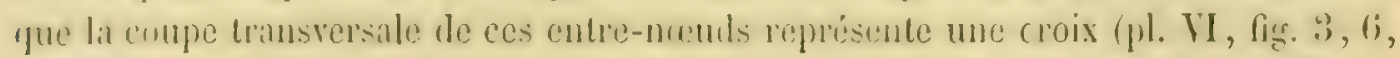

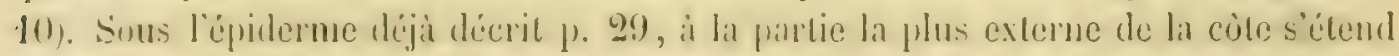
un linscean fibreux cortical, et lout le reste de la prartie sillante est occupé par des collules a chlorophylle. Au fond du sillon et de chayue cite on pent constíter encore la presence de petits cordoms de fitres corticales; puis viemone quelques grandes cellules incolores, répondant a celles qui entururent les lacunes corticales, lespuelles cxistent sur les rameaux de l'E. maximum (pl. VI, fig. 3), mais non sur les autres espéces (p). YI, fig. 6, 10). La gamirande de petites cellules qui distingue et sépare les deux cylindres se montre tres-apparente, et, i son interiem, les lissus du cylindre interne et des lacmes essentielles sont disposés comme sur lit tige, mais sans jamaits oftrir de cavité centrale. I ces traits généraux sajoutent sur chaque espree des diftérences tres-considérables, et qui, par leur invariabilité, fommissent des caraclires spécifigues d'une valem d'antant plus grande qu'une simple coupe transiersale permet de les constater avec une extrême facilité.

Sur une forme décombante de l'E. arvense il survient vers le bas de la tige des verticilles de tiges secondares, simulant des rameanx et dime longueur sument plus considérable que celle de la tige princjpale; la disposition des lissus y est la meme que sur les tiges.

Sur toutes les especes, la gaine basilaire est d'apparence tout ì fait membraneuse; incolore en paraissant, clle ne brunit que plus tard. Elle se compose de deux conches de cellules, dont les extériemres sont, sur les cótes, longues el a parois ondulies, et, dans les sillons, obligues el transwersales. Les intirienres ont une forme analogue avec des parois tout unies (pl. III, fig. 21). Nulle trace de stomates, ni de chlorophylle, ui de missean. '. Lorganisation des autres gannes n'est que la contimuation des entre-nududs quelles couromnent; elles sont loujours sur les ranteax d'un aspect moins membraneux que les gaînes des liges.

\section{\$. De l'apparcil de reproduction}

L'annenu offie les apparences d'une gaine non developpe, el son analyse ne présente qu'un lissu cellulaire oủ les vaisseaux ne pénètrent qu'imparfaitement.

La partic de la tige qui va de la dernière gaîne à l'épi, el qui a reçu le nom de pédoncule, ressemble aux entre-ncuds des liges spicifüres propres, non-seulement par lexti-

\footnotetext{
- Il est impossible de ne pas être frapné de la ressemblance qui exisie cntre ces petites gaines membrancuses ef les membranes qui entourent la base des feuilles dans certains Conifères.
} 
rieur, mais encore par la nature de ses tissus, et sur cette région toutes les espèces perdent les stomates, la chlorophylle et les fibres corticales. Bientòt les lacunes ellesmèmes s'oblitèrent et les sections de l'axe de l'épi n'en offrent plus de traces. La cavité centrale persiste ou disparail selon les espéces; en tout cas, il n'y a aucune trace de diaphragme vis-à-vis des verticilles de sporanges. Entre ces verticilles les faisecaux fibrovasculaires sont disposés en cercle interrompu, el alternent avee les pédicelles du verticille inférieur. En s'apprechant d'un verticille les faisceaux fibro-vasculaires aboutissent, comme aux nouds des articulations, ì des gronpes de cellules rayces et spiralées, disposés en cercle continu, et desquels il se délache vis-à-vis de chaque pédicelle un faisceau fibro-vasculaire. Ces petits faisceaux, composés de deux ou trois raisscaux qu'entourent quelques fibres, occupent le centre du tissu cellulaire qui forme les pédicelles et vont se rendre dans les clypéoles. La ils se ramifient et leurs divisions irradient ver's les sporanges, au-dessus desquels elles expirent (pl. VII, fig. 18). La surface externe des clypéoles, ordinairement hexagonale, est occupée par une couche épidermique précédemment décrite (voir p. 30 et pl. III, fig. 26 a 30 ); le tissu sousjacent est très-lìche et se compose de cellules grandes, allongées, à parois minces, renfermant quelques granules amylacés, dont le nombre diminue en s'éloignant de la surface.

A la face interne des clypéoles sont suspendus les sporanges. Ils ont la forme d'un sac membraneux, fermé, un peu courbé vers le dehors comme la dernière phalange de l'index (pl. VII, fig. 18 b) et se composent d'une seule couche de cellules filrcuses, c'est-i-dire de cellules ayant contre la surface interne de leurs parois de petites lames filiformes disposées en spirale (pl. VII, figg. 19). Ce tissu, vu au microscope, est d'une incomparable élégance; les parois des cellules sont d'une extrême ténuité, et d'une transparence si parfaite que le tissu ne semble formé que de fibres spiralées. Bischoff lui-mème y a été trompé; il dil du sac des sporanges : «aucune trace de tissu cellulaire proprement dit ne s'y laisse reconnaitre; il se compose entièrement de fibres en spirale, très-rapprochées, paraissant enfermées entre deux eouches d'une membrane uniforme et très-délicate» (Krypt. Gew., p. 39). Une goutte de solution d'iode colore en brun les parois des cellules et les rend très-facilement visibles. Le fil qui court en spirale à leur intérieur est creux dans toute sa longueur (pl. VII, fig. 20). Les spirales qu'il forme sont dextres, ou, si l'on veut, tournent dans le même sens qu'une vis ordinaire"; c'est aussi la direction des élatères. Les cellules sont en général disposées en groupes de quatre ou cinq, qui semblent provenir de la dernière multiplication des cellules-mères. Sur le dos du sporange, c’est-i-dire sur la ligne médiane externe et

\footnotetext{
' Nees v. Esenbeck (Form. l. Nat., ]. 43) ol plusicurs autres auteurs entendent autrement le sens dextre; pour cux, ces spirales seraient sćnestres.
} 


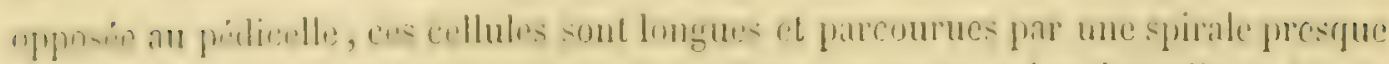

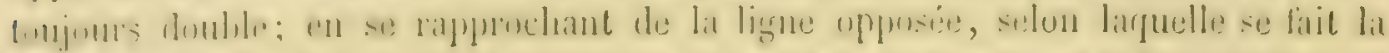

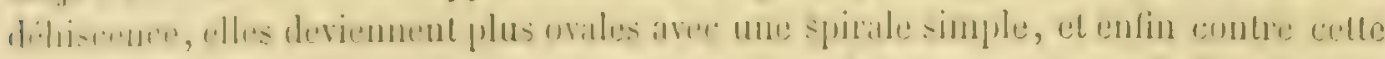

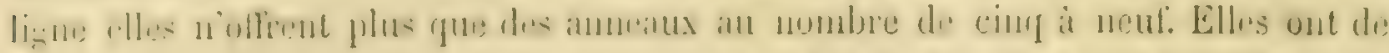

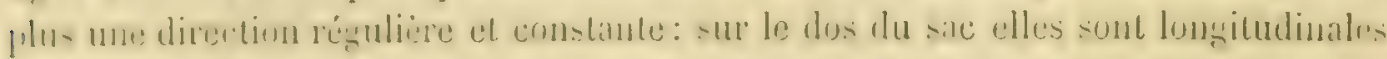

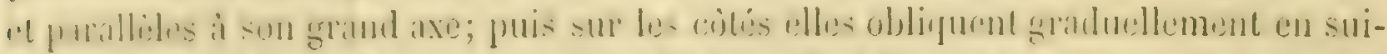

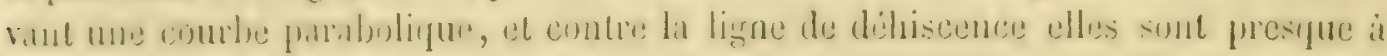

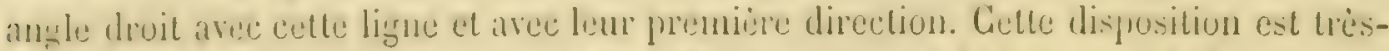
livorable a la disperson des spores. En etled, an moment de la maturite, la contrac-

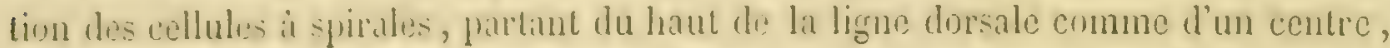

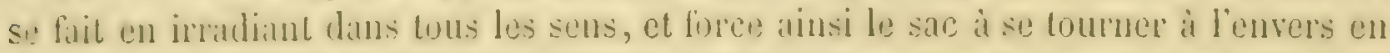
sinurant at il lancer an loin son contenu. Aplis la lispersion des spores, les sporinges

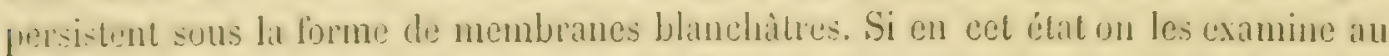

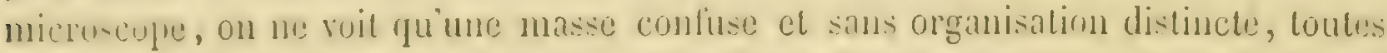

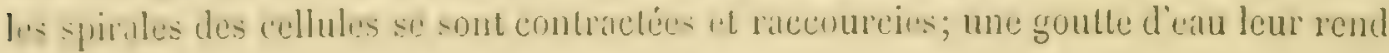
aussitôt leur élasticité, et elles reprennent leur disposition première.

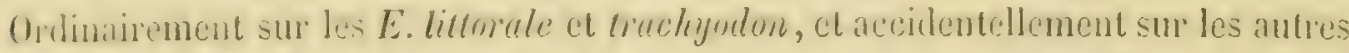
aprees, on trume des speranges dont les cellules sont simples et non fibro-spiraleus.

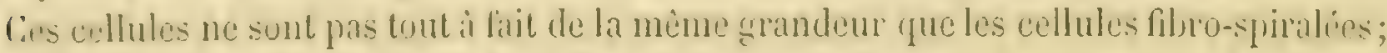
Hes sont, comme elles, el méme plus visiblement, disposces par groupes de quatle, el, eomme elles encure, longitudinales sur la ligne dorsile du sac, de plus en plus

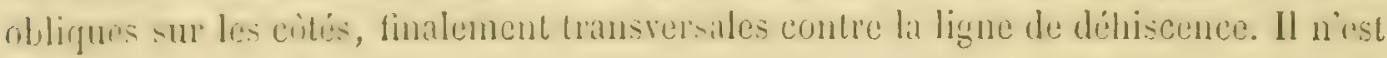
pas rare de voir, dans des sporanges de cetle sorte, au-dessous de la membrane du

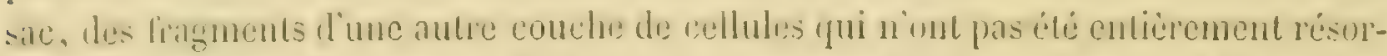

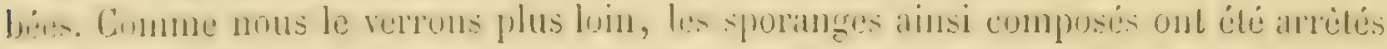

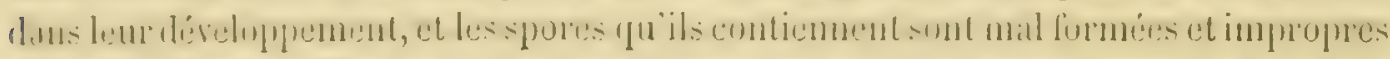

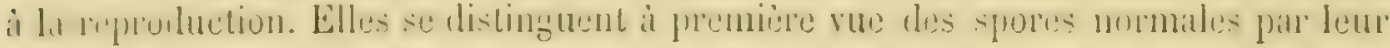

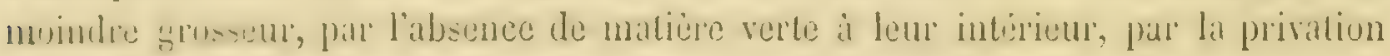
totale ou particlle d'élatères etc.

\section{CHAPITRE III}

\section{Formation et développement des diverses parties}

\section{$\S 1^{\mathrm{er}}$. De la tige el des gaines}

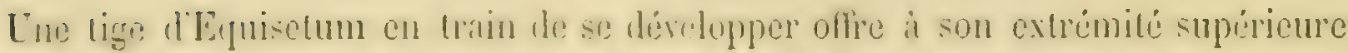

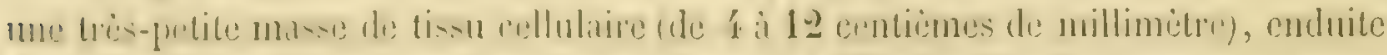

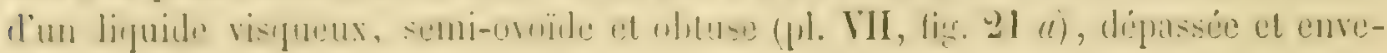


loppée par les gaines des entre-nouls inférieurs Ub, mais dépassant à son tour le point d'origine de la plus jeune gaine $c$. Celle masse se termine par une cellule dont la multiplication répétée est le point de départ du développement en longueur, et, en définitive, de toutes les cellules de la plante; elle en constitue le sommet végétatif (pl. VII, fig. 21-25, 27).

L'examen extérieur, aussi bien que celui des coupes, montre avec la plus grande netteté que cette cellule terminale a la forme d'une lentille plus ou moins aplatie, quelquefois même presque sphérique, qu'clle est parfaitement symétrique et que son axe est exaclement dans la continuation de celui de la lige (pl. VII, fig. 22-25, 27). Cela ne peut être que si de sa multiplication il résulte deux cellules séparées par une cloison conduite dans un plan perpendiculaire ì l'axe du rejeton en évolution. Cette direction de la première division, si rare dans le règne végétal, paraît tout d'abord nécessaire dans une famille où tous les organes constituent des verticilles situés dans des plans perpendiculaires ả l'axe, et ensuite l'observation la plus minutieuse et la plus souvent répétée montre qu'il en est ainsi en effet. Très-rarement la collule terminale semble occupée et presque remplie par un très-grand nucléus ou plutòt par une grande vésicule nucléaire, plus souvent elle en offre deux exactement superposés (pl. VII, fig. 25 a), à l'apparition desquels succèdent deux cellules superposées, chacun des nucléus étant passé à l'état de cellule, et la membrane de leur cellulemère ayant élé résorbée. Ces deux cellules sont inégales; la supérieure est d'abord plus petite et ne se développera que longtemps après que l'autre se sera mullipliée. Leur cloison de séparation est d'abord presque plane, puis ensuite relevée vers ses bords en forme de verre de montre renversé. Cette cloison et celles qui ont paru avant elle dans la masse cellulaire terminale, sont d'une extrême téuluité; et, comme la moindre coupe et la plus légère compression les déforment, je conseillerais toujours d'étudier celte organisation plutôt intacte que coupée. Sa transparence s'y prête d'ailleurs convenablement, malgré l'immense quantité de petits granules qui sont en suspension dans les jeunes cellules. En observant ces cellules terminales, il faut se tenir en garde contre une apparence qui peut et qui a dủ induire en erreur. Dans le déreloppement ultérieur de cette masse, les cellules du pourtonr s'accroissent en extension et en nombre plus rapidement que celles du centre; d'où jl résulte qu'elles se relèvent toutes vers la périphérie el s'abaissent du côté de l'axe (pl. VII, fig. 24). Cette disposition peut porter à croire, quand on n'en voit qu'une partie, que la pre. mière cloison de séparation a été elle-même inclinée sur l'axe.

Y a-t-il un moment oủ la cellule terminale déjà formée n’a qu'un grand nucléus, auquel succèdent deux autres nucléus, ou bien la présence d'un nucléus indique-t-elle seulement que les deux vésicules nucléaires de la cellule primordiale viement de se développer en cellules et que la cellule-mère n'est pas encore entièrement résorluéc? 


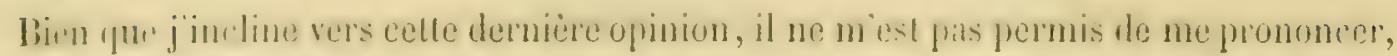

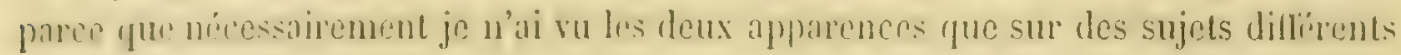
ct sans pouvoir constater avec certitude la succession des phẻnomènes.

Ouni qüil en soit, deux cellules ayant rísulté de la multiplication de la cellule terminale, celle des deux qui est in linupér montre bientob, au milien des granules qu'elle tient en suspension, d'ahord des agghomérations, puis de grandes vésicules nucléares plus transparentes, au nombro de deux larement, plus frépuemment an nombre de trois el de quatre. Ces vésicules ne sont jamais superposies, mais elles sont placies dans un plan perpendiculitre il laxe. A leur apparition sucecide tressapidement la division de la cellule, soit rn deux par une division diametrale, soit plus sonvent ('n trois nu quatre par des cloisons dont la ligne de contact est dans l'axe (pl. VII, fig. 22). Ainsi au-dessous de la cellule terminale qui s'agrandit un peu, se troure un plan de trois, qunatre on mime ring cellules, daus chacune desquelles apparaissent deux muclós passant i l'útat de vésicules muclénires, et, comme ces cellules sont un peu oblipquement disposées el relevées vers la púriphéric, ees vésicules sont elles-mèmes dispories selon une ligne oblique, l’une, l'extérieure, vers le point le plus élevé, l'autre, lintérieure, vers la basc contre laxe commun (pl. VII, fig. 27); de lit il résulte lientot que chacune de ces cellules se multiplie en deux aules separies par une cloisou, s’élevant presque du milieu de la paroi inférieure et rejoignant la paroi supérieure en s'inclinant vers laxe et presque parallelement a la surface du bourgeon (p). VII, fig. 23). Il suit de cette disposition et de la compression réciproque des nouvelles cellules que les extérieures se rapprochent de la forme d'un prisme létragonal et que les intérieures sont des pyramides triangulaires trongueses. Ces dernières, sitnécs du ribe de laxe, deviennent les cellules génératrices du tissu intérieur ou médullare; dres cellules situes a la périphérie proviendrunt les autres lissus de la tige el ceux des gaines. I partir de ce moment le nombre des cellules exterieures s'aceroit inrompirablement plus vite gue celui des intéricures; la lenteur de ces dernieres i se multiplier aceuse dijai la future impuissance de la masse cellulaire intérieure i suivre, frup son díveloppement, echi du tissu extérieur, el la nécessité pour elle de se disjoindre et le donner ainsi naissance à la cavité centrale. Ainsi ces cellules restent en retard tandis que celles de la préphérie se multiplient par une division verticale radiale el par une autre division parallete i celle de la cellule termiuale (pl. VII, fig. 24). II rósulte de cette inegalití de croissance entre les cellules extérieures el les autres que les exlépures proment la position tres-oblique ascendante vers la périphérie, que

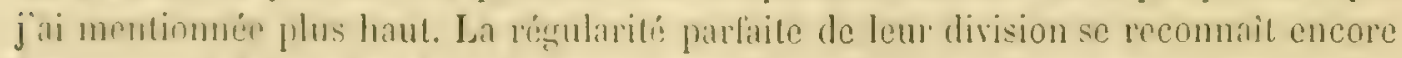
lunglamps it li surface de colle masce, sur laquelle on distingrue constamment des

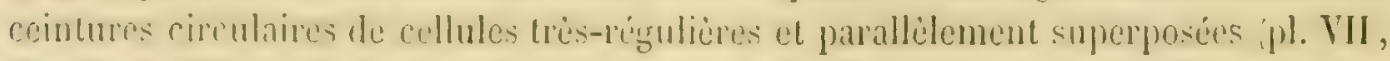
fig. 25 ct 27 ) 
Quand on observe la petite masse cellulaire terminale sans l'aroir déforméc par une section, et seulement après avoir enlevé les gaines qui la recouvrent, on voit que les cellules de chaque còté présentent presque toutes deux nucléus, tandis que celles du milieu paraissent n'en aroir qu'un; cela vient: de ce qu'on voit les prernières selon leur longueur, au lien que celles du milien se présentent par le bout et qu'alors les deux nuclées se trouvent à peu près sur un même rayon visuel et se confondent (pl. VII, fig. 25 et 27). Ces grands nucléus plus transparents qui précèdent toujours la multiplication des cellules et la déterminent, sont limités el circonscrits par une membrane mince qui en fait de vraies vésicules nucléaires. En même temps que celte membrane se solidifie, celle des cellules génératrices s'amincit. Cette dernière est d'ailleurs d'une extrême ténuité et présente si peu de consistance qu'à la moindre compression entre la lame mince et le porte-objet elle se rompt et les nucléus en sortent bien entiers. J'ai pu presque à chaque observation en dessiner que j’arais ainsi obtenues et fait sortir de leur cellule génératrice (pl. VII, fig. 26).

La multiplication des cellules intérieures ou médullaires se fait par divisions horizontales, puis par divisions longitudinales. Mais par une conséquence de l'inégalité de développement entre l'extérieur et l'intérieur, les premières de ces divisions prennent bientôt une direction oblique, surtout ver's les diaphragmes. L'accroissement de la lige en grosseur se produit dans les cellules de la périphérie par la répétition continuelle de divisions longitudinales alternativement irradiant de l'axe ou parallèles à la paroi extérieure. $\Lambda$ mesure que la tige devient plus grosse, les divisions par cloisons radiales succèdent moins régulièrement aux autres, et ce n'est qu'après l'apparition de plusicurs cloisons parallèles à la paroi extérieure qu'il en survient une radiale. La division transversale est toujours la moins fréquente.

Le volume de la petite masse cellulaire terminale, ou, ce qui revient au mème, le nombre de ses cellules intérieures ou extérieures varie considérablement selon les espèces, et sur une même espèce selon la force des rejetons, ou sur un même individu selon que l'on observe la formation d'un entre-nœud inférieur ou d'un entre-nœud supérieur, mais, quoi qu'il en soit, on voit constamment se produire ce qui suit.

A une certaine distance au-dessous du sommet végétatif, les cellules d'un cercle parallèle (du $5^{\mathrm{e}}$ au $8^{\mathrm{e}}$ sur les $E$. arvense et pulustre; du $9^{\mathrm{e}}$ au $12^{\circ}$ sur les $E$. limosum et hyemale etc.) prennent un développement tout exceptionnel; elles font un peu saillie, et à l'apparition de deux grands nucléus succède leur division par une cloison inclinée à lhorizon en sens inverse de leur direction générale (pl. VIl, fig. 25 b). Des cellules qui en résultent, celles qui sont supérieures et en même temps extéricures sont le point de départ des jeunes gaines, et les intérieures, qui sont comme la base 
des gaimes, donmeront par leurs multiplications ultericures naissance aux tissus des deux cylindres de la tige. La multiplication des premieres se fait ainsi qu’il suit: dans chacune d’olles (pl. VII, figg 2 \& bis a) apparaissent deux nucléus superposés presyne verticalement, auxquels succède la division on deux cellules superposées. La superieure on terminale continue i se multiplier de la mème manière; dans celle qui est aur-dessous (pl. VII, fig. 24 bis $b$ ) apparaissent deux nucléus, non plus superposés, mais placés a cóté l'un de l'autre dans le sens du rayon et presque horizontalement, auxpuels succide la division en deux cellules inégales (la plus grande en dehors), séparées par une cloison un peu oblique. L'obliquité de cette cloison est telle que sa partie inféricure (même fis. $c$ ), au lieu de correspondre à la partie supérieure de celle fu'elle surmonte (mème fig. d) et de la continuer, se trouve toujours en dehors d'elle. Liensemble, ru dans une coupe de profil ( $\mathrm{pl}$. VII, figg. 21 et 2't bis), figure done une sorte d'alteruance de cloisons; alternance qui devient plus scnsible encore par l'allongement ultirieur des tissus. Cette alternance des cloisons n'est pourtant qu'apparente et rísulte d'un seul mode de multiplication toujours le même, savoir: de charque cellule terminale ou plutit marginale en deux cellules superposíes, une supéricure el une inférieure, et de cette dernière en deux autres juxtaposées, une intérienre et une extéricure. Jamais je n'ai pu roir cette division s’établir "par des cloisons de séparation alternativement inclinées vers l'axe ou vers le dehors, » comme le dit M. Hofmeister (Vergl. Unters., p. 90).

Cette multiplication de cellules constitue au-dessous du bourgeon final un bourrelet annulaire gui est le commencement el la première forme d'une gaine (pl. VII, fig. 256 6). Immédiatement après son apparition ct après les premières multiplications mentionnées ci-dessus, la gaine augmente rapidement en épaisseur vers sa base par la multiplication répétée des cellules de ectte partie au moyen de cloisons parallètes i la lace extérieure, el comme dans les plus extérieures de ces nouvelles cellules la multiplication se répète dans l'autre sens, il arrive que la poussée des cellules extírieures de la gaine prédomine et force son bord libre à se courber ver's l'axe et finaIement la gaine ì s'b́lever presque parallèlement à lui (pl. VII, fig. 2I b). Cette multipliration de cellules se produit seulement vers la base, et le sommet de la gaine reste plus longtemps composé des deux couches de cellules provenant de sa seconde division.

Au moment où se dessine nettement le bourrelet d'une gaîne naissante, on voit, vis-i-vis du point d'origine de cette gaine, les cellules de la région centrale se multiplier par division transversale, se charger de granules et dessiner ainsi tris-netlement la place des futurs diaphragmes de cliaque noud.

C'est ì ce mème moment que j'ai constamment ru deux nucléus apparaître dans la cr.lluke du sommet et en préparcer la division (pl. VII, fig. 25 a). Tant que la masse cel- 
Iulaire terminale s'est montrée plus petite et sans les cellules rudimentaires d'une nouvelle gaîne, la cellule du sommet ne m’a offert que des granules très-nombreux el très-petits en suspension dans un liquide assez transparent; mais aussitût que j’ai pu constater l'apparition du premier rang des cellules de la gaine, j’ai trouvé dans la cellule terminale deux nucléus transparents, repoussant vers les parois un liquide plus trouble et plus chargé de granules. Je ne veux pas établir en principe que la cellule terminale ne se multiplie qu'ì des intervalles très-éloignés, répondant exactement à l'apparition d'une gaine à la base du bourgeon terminal, je veux dire seulement que c'est là ce que j’ai vu constamment.

Bientôt au bord libre, qui est le sommet circulaire de la gaîne, il se produit, sur quatre points opposés, une inégalité de développement, consistant en ce que, lỉ, deux cellules se multiplient plus vite que les autres par le mode de division ci-dessus mentionné. La multiplication ne s'opère qu'un peu plus tard dans leurs voisines immédiates el plus tard encore dans les plus éloignées: De lil résultent quatre pointes courtes et obtuses, qui sont les premières apparitions des lobes ou divisions de la gaine. Le développement de ces divisions en largeur provient, comme celle de la gaine, d'une multiplication longitudinale répétée. Peu après, sur quelques-unes des pointes élargies on voit les premières traces d'une bifurcation qui, s'avançant et se répétant rapidement, mais plus ou moins souvent selon les espèces et la vigueur du rejeton, détermine le nombre des lobes futurs (pl. VIII, fig. 1).

Les cercles d'origine des cellules-mères des gaînes sont très-rapprochés entre eux et à peine séparés; et, par suite, les jeunes gaînes sont étroitement superposées et appliquées les unes sur les autres. Or, comme il y a alternanee et que la ligne médiane des divisions d'une gaine s'appuie sur la ligne commissurale des divisions de la gaine supérieure, comme en même temps cette partie médiane est de beaucoup la plus épaisse, elle exerce sur les lignes commissurales de la gaîne supérieure une compression qui s'ajoute à l'arrêt de développement dont les cellules de cette région sont frappées (pl. VIII, fig. 1). Il résulte donc de tout cela qu'elles sont tout à fait arrêtées dans leur développement tant en épaisseur qu'en largeur; que celles de la ligne commissurale, sollicitées par le développenent général en grosseur, prennent de trèsbonne heure une direction transversale, et que leurs voisines, entraînées par la croissance en longueur de celles du milicu des divisions, prennent successivement et par degrés la direction verticale, ainsi que je l'ai signalé el figuré précédemment (p. 27 et 28, et pl. III, fig. 11-18).

Sur la troisième ou quatrıème gaine, en partant du sommet végétatif, il se produit subitement une très-considérable extension en longueur des cellules de la pointe de chaque lobe; et, sur l'E. maximum en particulier, on voit ces jeunes lobes se terminer par des cellules qui ont jusqu'à dix fois la longueur de celles de la base des gaines 


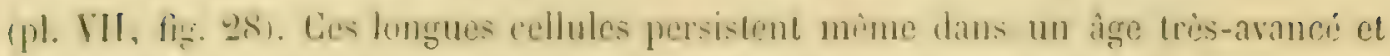
font paraitre les lobes de cette espèce terminés par d'assez longues soies.

Guelle que suil la longuem apparente sous le michoscope des lobes ou divisions du

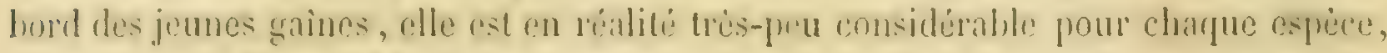
relativement i l'ensemble diune gaine complete, altendn que cette longucur re-te ce

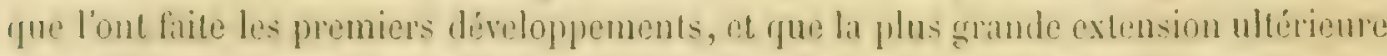

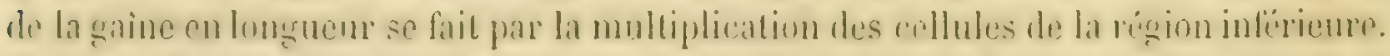
Commu, d'autre part, la multiplication des cellules vers la fareo cxterioure lemporte

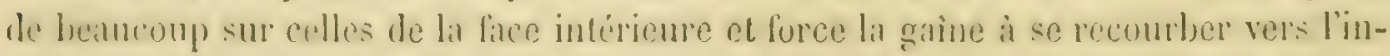
lérieur, il śensuit que l'ensmble d'une gane complétement develuppie est rommu une coille ovoüde, presque fermé el a peine entrouverte a sa partie superieure; quine gaine mvelople el recomre ainsi toutes celles qui sont plus jeunes quelle, on, en d'autres termes, foules celles des entre-nouds supérieurs, et que sa divisinn en lolies un dents bien prononcres nest que l'edee du développement ultérieur el de la poussée des entre-nœuds qu'elle recouvre et enveloppe.

Laceroissement en longueur des gaines a lieu ultirieurement par la multiplication des rellules do leur région inférieure, et les F. maximum el hycmale montrent diune manière frappante el ircécusable que cotte multiplieation continue a la hase longtemps aprie quielle a déjil ecssé sur toute la partie supérieure. En clfet, sur le premier les longrues cellules terminales des très-jemes lohes sont frappies de mort et dessichies de tres-bonne heure, presque anssitòt apres leur apparition, et, conme je l’ai dejai dit, ne persistent que sous forme de soies déliées. Néanmoins le reste de la gaîne diemple sa longuen depuis ce monent jusqu'i sa croissance. Sur l'E. hyemale ce me sont pas seulement quelques collules teminales qui se desechent et se fletrissent; toules celles qui constituent la moitié suprérieure de la jeune gaine sont arreties dans leur développement, et toute cette moitic, non-seulement se dessieche, mais le plus souvent elle se détache entièrement, comme je l'ai exposé en 1860 (Eq. hyem., 1). 10.5 el suiv.). Or la partie restante, fui forme une gaine cylindrique non hobulée a son bord, n’a guere à celle éporgue qu'un ou deux millimitres de long, el apres fuplyues semaines elle en aura atteint dix, et fuatorye au bout d'un an. Lorsçu'i lil

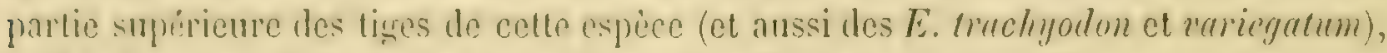
le dévelupmenent des gannes, arrité par les fortes gelées de l'hiver, recommence lans les premiers jours du printempis, on voit vers la base de chaque gaine une zone flus claire qui indigue les lissus de nouvelle croissance. En disséquant cetle zour, on en trouve les tissus, cellules, fibres, vaisseaux, stomates, a tous les ćtats de développement. En outre, les gaînes de l'E. hyemale ne peuvent point prendre leur

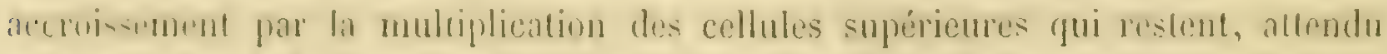
yuins citot apris la dunte des cellules de la pointe, les cellules qui lorment le bord 


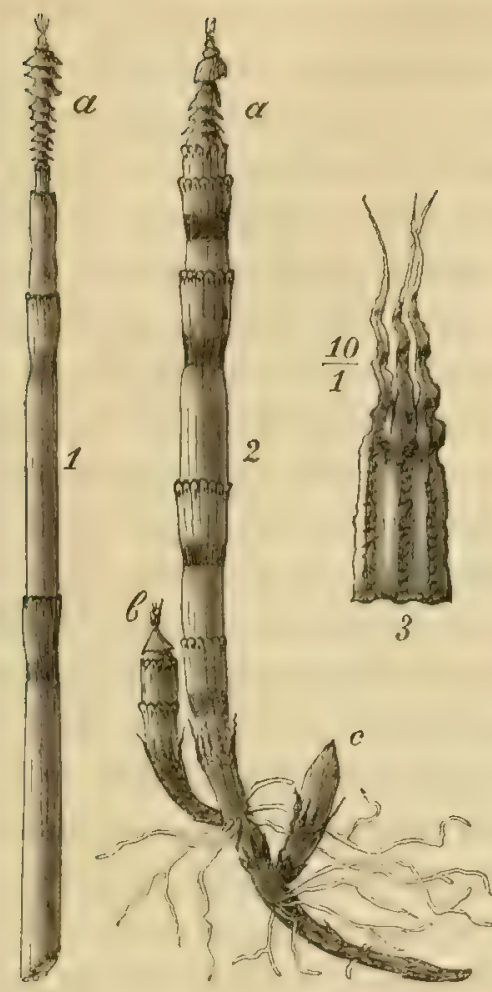

se chargent d'une matiẻre colorée qui augmente leur volume et constitue comme de petits mamelons charnus à l'extrémité de chaque côte (fig. 1 ci-contre); caractère déjà signalé en ces termes par Linné: «denticulis obsoletis atris giblis ${ }^{1}$. M. IIofmeister a également établi l'aceroissement de la gaine par la multiplication des cellules des régions inférieures, en s'appuyant avec beaucoup de force sur des considéralions d'un autre ordre (Vergl. Unters., p. 91 et 92).

Revenons au développement de la tige. Les diverses multiplications décrites plus haut avant celles de la gaine, ne sont que celles du bourgeon terminal. Or la masse cellulaire qui en constitue la partie centrale ne donnera, par ses multiplications ultérieures, que des cellules médullaires occupant le centre de la jeune tige avant l'apparition de la cavité centrale. Tout le tissu du cylindre intérieur et du cylindre extérieur et cortical résulte, comme je l'ai indiqué plus haut, de la multiplication de la deuxième cellule, sœur de la cellule-mère des gaînes.

Les cellules qui en proviennent forment une masse intérieure à la base des jeunes gaines, et c'est sur la multiplication en longueur et en largeur de cette masse cellulaire que repose exclusivement tout le développement ultérieur des entre-nouds ${ }^{2}$.

Dans le tissu provenant ainsi de la multiplication des cellules de cette région, c'est ordinairement vers le quatrième ou au plus vers le cinçuième verticille ou næud qune commencent à se montrer des vaisseaux; leur apparition dans le tissu de la gaîne est en retard d'un verticille. Nous avons vu précédemment que les faisceaux fibro-vasculaires sont distribués en avant des lacunes intérieures ou essentielles, et que celles-ci sont situées dans un plan mené du centre de la tige au milieu des côtes d'un entrenœud ou de la gaine qui les termine. Sur les points oủ doivent se trouver les vaisseaux, le tissu cellulaire commence de bonne heure (déji au-dessous de la deuxième gaine) à montrer des cordons verticaux distincts et toujours reconnaissables par de trèsgrands nucléus uniques dans chaqne cellule, puis, après la prompte disparition de

\footnotetext{
- Sur le feuillet intercalaire (p. 1517) de l'exemplaire original du Species plantarum conservé à Londres avec l'herbier de Jinné. Voyez aussi Mant. II, p. 504.

\& II est facile d'étudier en un mème jour et de bien comparer la série des dévelopnements d'une gaine (ou plus tard celle des rameaux), attendu qu'il y a toujours sur un mime bourgeon quinze ou vingh gaines fou sur une mime tige dix ou quinze verticilles de rameaux) ì tous les états de développement, et qu'on peut ainsi suivre toute la série des transformations; ce qui donne une certitude complète.
} 
ces nuclius, par une plus grande épaisseur et par une transparenee toute particuliere. A ret aspect, el vers la quatrieme on la cinquieme graine, succide en allant du milien anx extremites de elaque cellule, l’apparition, contre les parois, d’épaisseurs trinsersales tres-lénues en lorme d’anneau. Le nombre de ces épaisscurs ou saillies anmulares ast dejic de ciuf ou six que tontes les parois des cellules sont encore dislinctes; mais bientot les parois horizontales (on des extrémités) se résorbent, el vers la place quielles oecupaient il se forme aussi des anneaux. I ce moment un vaisseau est réellement constitué par un lube continu, ì l'intérieur duquel cxistent des épaisseurs anmulaires assez rigulierement espacécs. Mais a mesure que la tige s'aceroil cn longueur, il apparail de nouveaux anneaux qui semblent résulter de la formation de nouvelles saillies annulaires entre les premières ou pius fréruemment (i ce qu'il m’a semblé sur l'E. urénse) du dédonblement des premiers anneaux. Si le dédoublement est comple sur loute la circonférence, le vaisseau montre sur une ćlendue plus on moins grande des anneaux régulièrement espacés; si le dédoublement n'est gue partiel, les ammeaux restent unis sur une partic de leur pourtour, et offent lit ou ils se sont dédoublés des ouvertures transversales plus ou moins grandes (pl. VII, fig. 3, 4, 9). A mesure que s'arance celte multiplication, on voil diminuer l'üpaisseur des parois du tube contre lesquelles apparaissent les anneaux, et finalement, mais beaucoup plus tard, il n'en reste plus aucune trace, et les vaisseaux sont alors réclement conslitués par des anneaux libres et non unis par une enveloppe commune. Ce n'est qu'i celte éporue que j’ai pu constater dans les anneaux un commencement de cavilé Lubulaire (roir p. 39 et 40); jusqu’alors ils paraissent composés d'une matière homogine. La cavité se montre d'abord lenticulaire et tres-aplatie et ne devient circulaire que sur les sujets trés-adultes. Elle demeure toujours aplatic lorsque les baudes annulaires sont très-larges.

$\Lambda$ la continuilé du vaisseau correspondent dans les cellules voisines des divisions longitudinales qui en multiplient le nombre et en changent complétement la forme; elles deviennent tris-longues el fibreuses et perdent entièrement les granules verditres et mucilacrineux qui les remplissaient. On voit aussi apparaître parmi les vaisseaux anmulaires quelyues vaisscaux spiralés beaucoup plus petits, ct dont le mode de formation est anilogue, avec cette seule différence que les saillies sont spiralées au lieu dètre anmulaires. La transformation de certains groupes de cellules dans la région du diaphragme en cellules striées et rayées, et en petites cellules ì parois épaisses (pl. VII, fig. 12, 13, 14), précide un peu ou accompagne l'apparition des vaisseaux el des fibres dans la partic supéricure de l'entre-nœud.

Ciest, en eflet, i la partie supérienre de chaque entre-noud que se forment d'abord lus vaisseaux, les fibres a (comme nous le verrons plus tard) les stomates. Sur toute la region de l'entre-nurul qui est hors des gaines el mime sur les neul dixiènes de 
celle qui en est entourée, les cordons vasculaires offrent des vaisscaux bien formés, parfaitement transparents, sans aucune trace de la membrane de leurs cellules génératrices. Vers la partic tout ì fait inféricure du même entre-neud on voit, aulour des vaisseaux, des traces de celte membrane devenue trés-mince el très-diaphane, puis on voit les vaisseaux s'obscurcir de plus en plus par la présence de la matière granuleuse gris verdatre; en même temps on reconnail qu’aux vaisseaux déji bien constitués succèdent, en allant vers le bas, des cellules longues, à bouts arrondis, et qu'à l'intérieur de leur membrane des lignes trùs-ténues dessinent soit des anneaux espacés, soit des fragments de spirales à tours écartés, et enfin tout contre le diaphragme il n’y a plus que des cellules longues à extrémités arrondies, avec abondance de matière granuleuse, mais sans aucune trace d'inneaux ni de spirales. L'étude du développement des vaisseaux est peut-être plus facile encore sur cette région des entre-nocuds déjả assez longs que sur les entre-nœuds dans leur première jeunesse.

La démonstration que le développement en longueur des entre-nouds a licu presque. exclusivement par la multiplication des cellules de leur base est, sans aucune observalion microscopique, parfaitement évidente sur les espèces des groupes des Iyycmalia. En effet, les tiges de ces espèces, qui n'ont acquis pendant l'èté que la moitić de leur longueur normale, cessent de s'accroître pendant les froids de liner, et par l'effet du froid la partic de chaque entre-nocud qui est hors de la gaine prend une teinte verte très-foncée ou souvent rougeâtre. Le développement recommence dès les premiers jour's de printemps, mais alor's la partie nouvellement formée se distingue de l'ancienne par sa couleur d'un vert tendre et très-clair, et on la voit sortir des gaînes à la base de chaque entre-noud. De plus on remarque un léger rétrécissement de diamètre au point de démarcation entre la nouvelle el l'ancienne région. Si l'on mesure la longueur de la partie coloréc par le froid, on voil qu'elle s'allonge à peine, tandis que l'ensemble s'allonge considérablement par le dévcloppement de la partic inférieure. Ainsi, pour citer un exemple, des tiges d'E. trachyodon, mesurées par moi le $1^{\mathrm{er}}$ mars 1861, offraient vers leur milicu des entre-nouds dont la partic foncéc et ancienne avait au-dessus de la gainne 20 millimètres de longueur. Le 25 mai suivant, les mêmes tiges avaient doublé leur longueur totale, mais la partie foncée de chaque entre-noud ne s'était allongée que d'un millimètre, tandis que la partic nouvelle et d'un vert tendre, qui s'était formée au-dessous d'elle, s'était élevée audessus de la gaîne de 18 millimètres. Ce développement noureau à la base des entrenouds n'est pas de lá même longueur sur chaque entre-nceud d'une même tige. Presque nul aux entre-nouds inférienrs, il suil une progression croissante jus(ju'au sommet, où le plus souvent les derniers entre-nouds sont en entier de développement récent. J'ai observé aussi ces faits sur des tiges d'E. v'arieyatum que l'eau avait noircies pendant l'hiver. On peut les constater sur tous les Equisetum, et en particulicr 
sur l'E. limosum, en faisant sur une de ces jeunes tiges, dans le courant d'avril ou de mat, de margues aux points des entre-numb superieurs qui correspondent sont anx sims suit aux sommets des lobes; on voit apres quelques jours que la croissance ulteriene a lien soulement an-dessous des points marques, lespucls se sont élevés au-dessus des gaines ${ }^{2}$.

I puelque moment que lon observe le diveloppement d'une lige, tris-jeune nu tris-avancie, on ne trouve jamais que les vaisseaux furment une rireonférence conlime dans le tissu cellulare; ils comstituent toujours des groupes isules placés comme nous l'arous dija ra². Mais dans le tris-jeune àge ces groupes sont composés diun nombre de vaisseaux bien plus considerable que plus lard (p). VIII, fig. 2). C"est dans Jintérieur de chacun de ces groupes el vers leur centre que commence i apparaitre Ja lacune esicnticlle, par écartement el par résorption, a ce quil m'a semble, dabord des vaissnaux du centre de chaque groupe, puis de cenx (qui se rapprochent du centre de ha tige, cnsuite de ceux qui sont placés vers l'extériem, et funalement il ne subsiste de chaque còté de chayue lacune et un peu en avant que deux ou trois gros vaissenux continus, et un assez grimel nombre d'anncaux qui n’ont pas été résorbés et sunt épars sans ordre et quelquefois mème couchés contre le tissu cellulare environnint. Les vaisseaux un peu plus petits qui sont placés en avant et de charue cóté des licunes essentielles, dans le tissu fibreux, napparaissent que très-postérieurement à ceux que je viens de décrire.

Lapparition des cellules-mires des hourgeons latéraux est à peu prés contemporaine de celle des vaisseaux. Nous en suivrons plus loin l'évolution complète.

La formation de l'epiderme des entre-nududs el des gaines suit de très-prés l'apparilion des vaisseanx; clle a lieu aussitit que la jeune gaine se trouve, par lécartement de celles qui l'ont précédée et qui l'enveloppent, un peu en contact avec l'air. Les cellules de la surface extérieure se multiplient (deux fois selon MI. IIofmeister, I eryl. linters., p. 92) par divisions transrersales, puis deux fois par divisions longiludinales

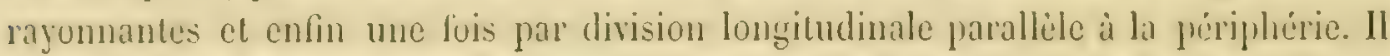
résulte de celte derniere division une double couche de cellules longues, et tellement

\footnotetext{
- Comparez ce que dit M. Unger de l'accroisscment des entre-nocuds : "L'extension cesse d'abord de se faire à la - hasc, tandis que les autres parties des entre-nœuds continuent a s'allongern ('nger, Acer. enlren., p. 194, trad.). II. Fermond, ayant constate aque chez les Pulygoníes, les Graminées et quelques Caryoplyllées l'accroissement du " mérilhalle a lieu plus fréquemment par le bas, "considère comme cause déterminante de ce fait "ou l'ochrea, ou Ia gaine, ou mème la base des feuilles opposées, qui enveloppe la base du mérithalie, et par li s'oppose à l'évaporation de ses liquides, entretient la mollesse de ses tissus et farorise ainsi l’élongation s (Bull. Soc. bot., 1, p. 309.311) Si celle opinion est applicable ì la croissance par le bas des entre-nœuds des Equisclum, elle l'est moins au dérelop pement des gaines qui a licu aussi par la base. Il est vrai qu'il est moins considérable que celui des entre-noeuds.

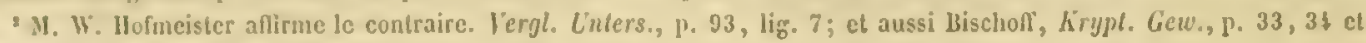
39 (voy. supra, chap. II, P. 40).
} 
disposées que leurs circonscriptions se correspondent à peu de chose près. L'interne a les parois latérales presque lisses; l'externe, qui est l'épiderne proprement dit, les a très-fortement ondulées, surtout vers ses angles extéricurs, et sur sa face libre on voit se former successivement des encroûtements siliceux qui y déterminent des saillies plus ou moins régulières, selon les espèces.

Au moment où les cellules de la surface vont se multiplier par une division longiuudinale parallèle à la périphérie, une différence se fait remarquer daus quelques cellules situces aux points ou doivent apparaitre des stomates '. Iu lieu des deux grands nucléus que les autres cellules montrent dans le sens de leur rayon, les cellulesmères des stomates n'en présentent qu'un seul (pl. VIII, fig. 6), dont la vue distincte est quelquefois rendue très-difficile par la quantité considérable de granules verditres accumulés dans ces mêmes cellules. Peu à peu ce nucléus s'agrandit el s'etrace, et ì lui et à sa cellule-mère succède une nouvelle cellule un peu ovoïde, dont la pointe, tris-obtuse d'ailleurs, est dirigée vers l'intérieur. Comme celte nouvelle cellule ne suit point ses voisines dans leur développement en longueur et qu'elle demeure tonjours beaucoup plus courte, il résulte de sa forme arrondie que ses voisines la recouvrent presque en entier par l'avancement de leur angle extérieur. Alors apparaissent très-rapidement aux extrémités de son diamètre horizontal deux nucléus latéraux (pl. VIII, fig. 3, 7). D’abord sphériques ou discoïdes, ils prennent à mesure qu'ils se développent un aspect réniforme, et les granules accumulés entre eux deux ct qu'ils refoulent vers la ligne médiane simulent un ostiole qui n'existe pas encore (pl. VIII, fig. 4, 8). Enfm ils aboulissent à la division de la cellule-mère en deux cellules longitudinales, contiguës seulement vers l’angle extérieur et aux extrémilés de leurs cloisons de séparation, et un peu séparées à l'intérieur vers le milieu de la ligne de contact, ce qui continue à simuler un ostiole. Vers le milieu de chacune d'elles se montrent deux nucléus très-inégaux situés dans un mème plan horizontal, mais à des profondeurs différentes; le plus grand est contre la paroi extérieure à l'opposé de la cloison commune; le plus petit vers la cloison intérieure (pl. VIII, figc. 5). Leur développement aboutit à la division de chaque cellule en deux autres trés-inégales, superposées et séparées par une cloison en quart de sphère et dont la convexité est tournée

\footnotetext{
" Je n'ai étudié avec suite le développement des stomates que sur les $E$. arvense, limosum et ramosissimum. On peut l'observer avec facilité sur les jeunes plantes provenant de semis ou sur de trẻs-jeunes rejetons que l'on obtient quelques jours après avoir placé des rhizomes dans un vase rempli de terre et d'eau. Mais cetle étude est plus facile encore sur de jeunes tiges des E. limosum et ramosissimum en ćtat de développenent. Si l'on prend alors tout à fait à la base d'un entre-nœud un petit lambeau d’épiderme de 1 à 2 millimètres de lonğ, on y recra des stomates à tous les ćtats, et on en suivra la formation successive avec autant le facilité qu'on suit le développement des vaisseaux dans les tissus de la même régíun. Il sera bon de comparer ce qui suit avec ce qui a été dit sur le mode de formation et d'érolution des stomates par Mirbel, March. polym. Mém. Inst., XIII, 1836; Mohl, Ueb. Enlw. Spall., p. 54 t el suiv.; Trúcul, Nuph. lut., p. 308 et 309 , et Garreau, Form. stom., p. 215 et suiv.
} 
vers le delors. Le stomate se trouve alors comstilue par quatre cellules, dont deux estirioures superposées à deux intérieures qu'elles dépassent et quelles emboitent. I re moment les gramules verts sont tellement accumulés contre la ligne médiane qu ilk sopposent entièrement an passage des rayous luminemx el y forment une large bande oparfue. Bientit se montrent entre les deux cellules quelyues points ronds et brillants, annongant lappration des saillics siliceuses qui bordent les livres de l'ostiole, et peu ì peu de semblables points, apparaissant successivement de la ligne médiane au pourtour extérieur, révilent la formation d'asperités analogues à la surlice du stomale. I mesure que ee développement a lieu, les cellules se disjoignent el lostiole apparait nettement. Les deux eellules extérieures se disjoignent peu, mais daus leur développement vers les còtés el vers le dedans elles semblent entrainer aree clles les cellules inlérienres dejii non contiguës vers leur centre, et entre lesfuelles lourreme devient du double plus large, sans que pourtant clles se séparent tout i farit il leur extrémité supérienre et infërieure. Jusqualors les cellules extérienres paraissent lises i leur parni interne, mais les stries rayonnantes apparaissent si subitement rüil m’a toujours été impqossible de suive les détails du pliénomiene, dont la vue est constamment obscurcie par nne énorme quantile de granules accumulés vers les livres de la paire extérieure de cellules. Par le développenent du stomite, les cellules épidermiques roisines sont refoulées dans lous les sens, mais clles ne cessent jamais de le recourrir par lavancement ondulé de leur angle exterieur, ainsi que nous l'avons exposé précédemment p. 32 el 33.

Tous ces détails ne sont rigoureusement exacts qu'en ce qui concerne les stomates des trois premiers sroupes; ceux des IIyemalia oftrenl quelques dillërences. Ainsi, avint la prenuere division de lit cellule-mère en deux cellules longitudinales (pl. VIII, lis. 7,8$)$, el mème encore aprés celte division, le grand diametre de la cellule-mere el du groupe des deux cellules qui résultent de sa division est transversal (j)l. VIII, lig. 7, 8). La sécrétion de la croùte siliceuse commence déjil sur les cellules roisines, mais non sur les stomates; la sierétion des cellules à grand diametre transversal, qui séparcul les stomates (pl. VIII, fig. 7, 8, et pl. V, fig. 2 bis), s'arance un peu sur ces dernicrs sans les recouvir en entier ef en laissant vers leur centre une lacune transversale irregulière. Un peu plus tard, quand les cellules voisines grandissent en tout sens et diterminent l'aceroissument de la lige en longueur et en diamitre, il arrive, d'une part, que celte lacune transversale ne se comble pas; d'autre part, que le stomate ne saceroit guire qu'en longueur et ne suit pas l'extension en diametre radial des cellules voisines, el quainsi il se troure placé en retrait par rapport a elles el an-desous de la cronte silicuse qui, en s'en détachant, forme vers les cxtrúmités l'avancement décril p. 32 el 33 el figuré pl. V, fig. 2, 2 bis ctc.

Jhus les cellules qui aroisinent la surtace épidermique des liges, il y a des granules 
légèrement colorées en vert avant l'apparition des stomates, mais la chlorophylle, bien formée dans ses grandes cellules transversales, ne se montre que tris-postéricurement à la formation complete des stomates. Celle double formalion des stomates et de la chlorophylle a toujours lieu dé très-bonne heure daus les gaines de toutes les cepèces, et précède de beaucoup la même formation dans les entre-nocuds. Il y a même des espèces oì elle n'apparait jamais dans cette dernière partie, par exemple sur les tiges spicifères de l'E. arvense et sur toutes les tiges de l'E. maximum.

Nous arons vu précédemment qu'il se présente dans chaque entre-nceud une cavité centrale et deux cercles concentriques de cavités plus petites, l'un intérieur de lacunes essenticlles, et l'antre extérieur de lasunes corticales. La première provient de ce que les cellules médullaires ou de la partie centrale de l'entre-noud ne peurent point suivre les cellules du tissu environnant dans leur extension en longueur et leur multiplication en diamètre. Elles se déchirent donc et se désunissent, se crispent et disparaissent, ou pendent en pelits lambeaux desséchés le long des parois de la cavité que leur disparition engendre. Celte cavité est très-régulière d'ailleurs, et d'une grandeur relative assez nellement déterminée sur les tiges principales de chaque espèce, parce qu'elle est une conséquence du rapport numérique qui existe d'une manière générale entre les cellules médullaires et celles du cylindre interne et du cylindre cortical de ces liges. Mais toutes nos espèces, ainsi que nous l'avons vu, n'offient pas cette cavité sur leurs rhizomes et sur leurs rameaux. Les lacunes extérieures ou corlicales, crensées dans un tissu à grandes cellules analogues à celles de la moelle, doivent leur existence exactement à la même cause. Leur dimension relative est moins constante sur les tiges que celle de la cavité centrale; il y a même une de nos espèces, sur les tiges de laquelle ces lacunes, immédialement après leur apparition, s'oblitẻrent le plus souvent par l'extension en épaisseur que prennent vers le milieu des sillons les bandes continues de cellules à chlorophylle (roy. p. 45). La même cause les empêche aussi de se montrer dans les rameaux de plusieurs de nos espèces. L'existence des lacunes essentielles est due à une autre cause, à l’écartement et à la résorption des vaisseaux mentionnés p. 62. Ces lacunes ne manquent ni dans les rhizomes ni dans les rameaux d'aucune espèce de nos climats.

\section{\$2. Des ramcaux, des rejetons, des rhizomes ot des tubercules}

Les verticilles des bourgeons à rameaux sont situés un peu en dessous du diaphragme. Ges bourgeons prennent naissance à la surface du cylindre interne, vis-ivis d'un sillon, c'est-à-dire entre deux divisions de la gaine et dans un plan vertical qui passerait par le milieu des grandes lacunes corticales. Mais ils se montrent bien DEvAL-SOUve 
avant l'applution de ces lacunes; et, quand plus tard ils s'arancent ver's l'extérieur, il reste entre eux et la lacune une couche de deux ou trois rangs de cellules guils reponsent en saillie rentrante au sommet et sur la ligne médiane de la lacune (pl. I, fig. $7 \mathrm{~g})$.

Lapparition contre le cylindre interne de la masse cellulaire gui est le point de départ d’un bourgeon i rameau, m’a paru être en gienéral contemporaine de l'évolution iniliale des tiges, meme sur les liges des especes qui, comme l\%. hyemale, sont i feur état nomal dépourvues de rameaux; et, de plus, clle cxiste i léelat trés-rudimentaire, sinon sur tous les verticilles, au moins sur les inferrieur's des tiges spiciforres de l'E. maximum et de l'E. areense, lesquelles périssent aussitót après la sporose. Il n'y a done rien d'étonnant à ce que, les circonstances aidant, ces tiges doment quelques rameaux tardils, et la lígère variation qu'elles en regoivent dans leur forme extérieure ne me parait pas mériter les honneurs des dínominations qu'on leur a octroyées ${ }^{1}$.

L'apparilion du rudiment d'un bourgeon a done licu de tres-bonne heure; clle est a peu pres contemporaine de celle des raisseaux dans la jeune tige. M. Hofmeister la dit mème de beaucoup) antéricure (Térgl. Luters., p. 94); je n’ai pu constater celte

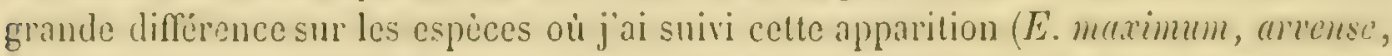
limasum, matense, remosissimum, hyemale). Au point designé plus haut, sur la ligne de division de deux lobes et un peu au-dessous de leur base, dans la couche la plus externe des cellules devant constituer le cylindre interne, une cellule se distingue des autres par son contenu incolore et plus mucilagineux, et surtout par ses plus grandes dimensions, sa forme ovoüde transversale et non allongée de bas en haut (pl. VIII, fig. 9). Au lieu de suive les autres dans leur extension longitudinale, celte cellule s'aecroit en rayonnant vers l'extérieur et fail saillic en dehor's d’elles. Elle ne tarde pas a se dédoubler par une cloison perpendiculare a son axe de production. Des deux cellules qui résultent de celte division, la cellule non terminale se multiplic en deux cellules latérales an moyen d'une division médiane verticale; bientôt après, siuon simultanement, une multiplication par division horizontale double ces deux cellules, el en fait un petit verticille de quatre cellules rectangulaires à leur ligne commune de contacl. Par suile d'une nouvelle division qui, presque parallè à la surfice cxtéricure de ce bourgeon naissant, s'opère dans chacunc de ces cellules, leur nombre est double; les nouvelles cellules intérieures sont les cellules génératrices du tissu médullaire du rameau; les plus extérieures domneront naissance aux gaînes et aux tissus interne et cortical. Enfu il s'opere dans ces dernicres d'abord une division

\footnotetext{
I Comme nous le verrons plus loin, on produil ces rameaux ì volonté par la mutilation de la partic supéricure des tiges
} 
\$ 2. - DES RAME.UX, DES REJETONS, DES RHZOMES ET DES TUBERCULES.

longitudinale et rayonmante, puis une division transversale parallèle a la division initiale. C'est a ce degrí, ou au degré précéclent, que le bourgeon s'arrête et demeure ì l'élat expectant sous les cellules corticales des tiges spicifères des E. maximum et arvense.

Par celte disposition, quui répond entièrement au mode de formation de la ligge, le bourgeon d'un futur rameau se compose donc de verticilles cellulaires superposés. Au moment que je viens de décrire, les cellules extérieures du verlicille contigu au cylindre interne se multiplient au mojen d'une division divergente par rapport à l'axe de production, é la inouvelle cellule extérieure, se développan l plus vite que les autres, fait saillie et dessine le bourrelet d'où proviendra la gaine basilaire. Pendant que le sommet végétatif accomplit son développement ultérieur, en suivant la loi préécdemment constatée sur la tige, ce bourrelet marche plus rapidement que lui, en suivant constamment le premier mode de multiplication que nous avons sigualé dans l'évolution d'une gaine caulinaire. Il en résulte que, comme une gainc naissante, il se compose de deux couches concentriques de eellules. Le nombre de ces cellules se multiplie ensuite par des segmentations radiales, aprés quoi, sur quatre points opposés, des cellules s'avancent plus que les autres et marquent par leur's inégalités la place des quatre dents de la gaine basilaire. Ces ruatre inégalités ou fulures dents sont disposées sur deux lignes horizontales et forment les coins d'un carré, de telle sorte que leurs tissus de séparation seront l'un en haut, entre le futur rameau et la lige, l'autre opposé en bas, et les deux autres latéraux (pl. VIII, fig. 11). Le développement de cette gaîne est trèsrapide, beaucoup plus rapide que celui du sommet végétatif, mais il n’est pas égal sur tout son pourtour. A sa partie supérieure, comprise entre le bourgeon et la lige, il est moindre que sur ses côtés, mais il est très-prononcé à la partie inféricure qui donne même quelquefois naissance it une dent de plus. De cette inégalité de líveloppement il résulte, d'une part, que le bourgeon prend une direction un peu oblique ascendante, ct, de l'autre, que la gaine qui l'enveloppe perd sa forme conique symétrique et devient ventrue vers l'extérieur (pl. VIII, fig. 12). Le développement ultírieur de celle gaine demeure toujour's celui de son apparition, de telle sorte que sur loute son étendue et dans son plus complet achèvement elle ne présente jamais de vaisseaux et ne se compose que de deux couches superposées de cellules allongées, presque rectangulaires. Les faces extéricures de ces cellules sont lisses, mais leurs parois de contact sont très-fortement ondulées, particulièrement à la couche externe (E. maximum, arvense, palustre, pl. III, fig. 21).

Lu gaine basilaire s"accroît done très-rapidement et de manière à enrelopper complétement le jeune bourgeon. Aussitôt qu'elle l'a recouvert, ce qui se fait trèsrapidement, on roit apparaître le bourrelet de la première gaîne; et c’est ì ce degré de développement que le bourgeon demeure sur' les tiges de l'E. higemale, où il n'ip)- 


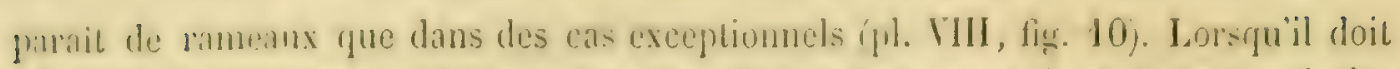
imerger, il andive qune, par laceroissement de toule sil masse, les cellules corticales placies sous lépiderme de la tige sont écartees; celles de lépinterne sont dialord poussiés en araut, puis aplaties et comme écrasié, enfin elles cedent a la pression et se séparent en une petite fente longitudinale it travers larpuelle passe le bourgeon tmijours entierement recouvert de sa gaine basilaire; ensuite elles se colorent fortement en noir de chanue cúté de la lente, comme il arrive partout où lépgiderme est meurtri et liappé de mort. P'ar suite de la disposition oblique que nous avons constatéc ci-dessus, le bourgeon et sa gaîne, dans la pousséc qu'ils ont à exercer, ne sortent pas par lit pojute et dans la direction horizontale quaura plus tard le rameau, mais, obliquement dressés, ils exercent leur poussée par leur cité extérieur et par le seul fail de leur divelopjement en dianètre. C'est done par le flane que le hourgeon se présente a lanir libre, toujours étroitement enveloppé et prutigé par sa gaine basilaire'; ce n'est quaprès étre complétement sorli rüil s’en dégage, et alors le développement de celle-ci sarrète. Elle persiste bien à la base du rameau, mais desséchée et plus ou moins fortement coloríe cn noir, conservant toujours l'inégalité de son premier développement. Elle a rempli le ròle des écailles des bourgeons des phanérogames; comme eux, elle n'est pas arrivic a l'état de feuille parfaite, et par sa coloralion, comme par sa lexture, elle offre les plus grandes analogies arec ces organes prolecteurs. Celte anilogie avait été remarquée par Yaucher, el mème elle avait induit en erreur cet excellent observateur. It avait pris les lobes de la gaine pour de véritables écailles, sans remarquer que ces prétendues écailles forment à leur base une cnveloppe continue: "Charque rameau est entouré ì sa base de quatre ou cinq petiles "écailles qui ont été considérées quelquefois comme des feuilles \#. (Mon. Pril., p. 313).

Le déreloppement ultérieur du raneau et de ses gaines suit la mème loi que celui de la tige. Il est pourtant a remarquer que les gaines des rameaux sont des le principe beaucoup plus profondément fendues que celles des tiges. Le rüle protecteur de ces derniers est rempli sur les rameaux par la gaine basilaire, et mème, sur l'E. maximum, par la seconte gaine, qui est très-ample et recourre pendant trìs-longtemps le jeune rameau (pl. VIII, fig. 13).

Dis le milicu du printemps, ou avant cette éporpue, on roit sur la plupart de nos espices se développer an-dessous des gaines de nombreux rameaux, souvent mème aussi nombreux que les lobes de ces groines. Les $E$. areense, pialustre, limosum portent tris-souvent vers leur partie infericure de très-gros rameaux chargés de ramuscules, et presigue intermédiaires entre les rameaux et les rejetons ordinairement appelés

\footnotetext{
' C'est a tort que $\mathbf{M}$. Ilufmeister (Vergl. L'ulers., 6. XIX, fig. 18) a représenté le mamelon terminal en delors de la saine hasilaire. Cet élat n’existe jamais, tant que le bourgeon n'est pas à l'alr libre.
} 
tiges. Mais les bourgeons inférieurs, destinés a la propagation ultérieure du sujet, demeurent bien plus longtemps plongés dans leur repos expectant. Ils n'entrent en développement que lorsque les tiges, sur li partic souterraine desquelles ils apparaissent, ont accompli en entier leur déreloppement aérien. Ce n'est que rers la fin de l'été qu'ils commencent à apparailre sur l'E. ariensc; en automne sur l'E. limosum où ils sont très-gros et très-beaux; au commencement du printemps sur l'E. palustre, et seulement vers la fin d'avril sur l'E. liyemale. Il ne se développe guère, de ces noeuds souterains, qu'un seul ou qu'un très-petil nombre des bourgeons, mais en revanche ils ont dans toutes leurs parties une force et une vigueur luxuriante qui laisse bien loin le développement des faibles rameaux aériens. C'est à peu près toute la différence qui distingue leur premier développement de celui des rameaux; le développement ultéricur des tiges stériles a été précédemment décrit; ce qu'il y a de parliculier dans celui des tiges spicifères sera décrit plus loin, $§ 4$.

Les rudiments des bourgeons destinés à donner des rhizomes existent à l'état expectant sur les rhizomes, et loujours, comme ceux des rameaux, un peu on arrière du plan formé par le diaphragme d'un noud. Du verticille qu'ils forment, il ne sort qu'un rhizome, rarement deux, et toujours sur le côté. Ainsi que la gaine basilaire d'une tige, celle d'un rhizome est plus grande que celle d'un rameau, mais d'ailleurs identique. Les bourgeons souterrains qui doivent produire des rhizomes ne se dislinguent de ceux qui donnent des tiges que par une moindre grosseur et une pointe plus effilée; leur développement ultérieur est analogue à celui des tiges. L'extrémité d'un rhizome en voic de développement se termine en pointe acérée constituée par plusicurs gaines superposćes, et dont les plus grandes et les plus âgées sont à l'extérieur. Comme il a été dil précédemment, ces gaînes s'accroissent en longueur par la multiplication des cellules de leur base, et leurs cellules terminales, meurtries, noircies et devenues impropres ả tout déreloppement ultéricur, constituent une pointe de lissus solides el durcis, qui pénètre à travers les résistances du milieu, et sous l'abri protecteur de laquelle s'opère la multiplication des cellules si délicates du sommet végétatif. Nous avons déjà vu comment la gaine basilaire prolége le rameau naissant; nous verrons bientôt par quelle autre combinaison il a été pourvu à la protection du point végétatif des racines, lesquelles sont dépourvues de gaines, et combien est harmonicux l'ensemble des dispositions concourant à la protection des organes en état de développement.

G'est en général à la fin de mai ou au commencement de juin que se développent les nouveaux rhizomes. C'est également à la même époque que commencent à apparaitre les tubercules. Aux points oủ devraient se montrer des rhizomes, on roit saillir une masse cellulaire d'un beau jaune, qui, au lieu d'avoir, comme le bourgeon d'un rhizome, son extrémité recourerte par la série des gaines emboiltées les unes sur les 
antres, ne présente que trois ou quatre dents courtes et repliées contre un petit

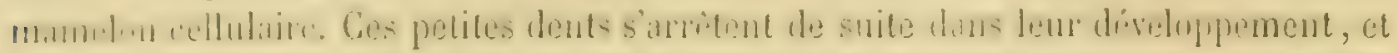

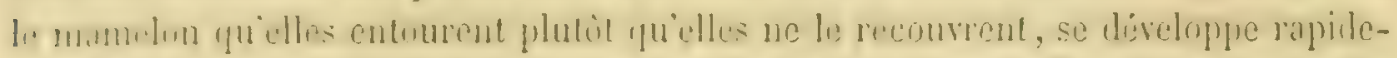

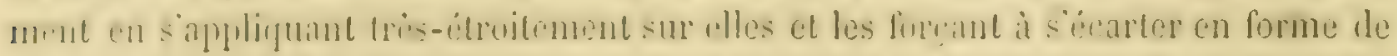

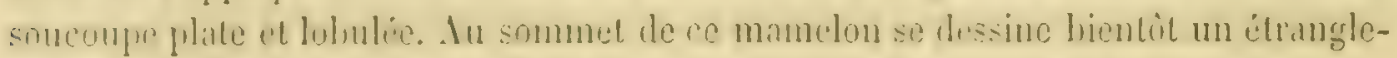
ment mirmonte diune petite masse a pen puris conique, fui consiste en une couronne de trois a six dents cutourant un petit mamelon cellulaire, dont le devoloppement a lieu comme ci-desus. Ters l'automme, la coloration en roux brun des cellules épidermiques devient tres-prononcée. Les modifications des cellules sont réduites dans les Intercules a leur plus simple expression: point de lacunes, point de distinction tran-

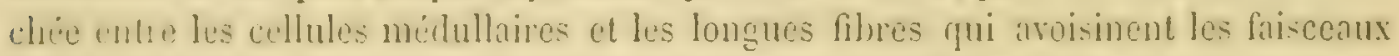
vasculaires; tout est presque semblable el consiste en cellules orides, peu allongres et complitement sonflées de erranules amylacés. I peine les plus voisines des groupes de vaisseanx anmulaires sont-elles un peu plus allongeses. Les gaines rudimentaires sont cntierentent composés de tissu cellulaire fortement coloré et tres-durci; les vaisseaux ny puétrent point, et cost à peine si l'on trouve quelques cellules rayies et striées vers l'étranglement qui répond au diaphragme.

\section{§. Des racines}

Jail signale plus haut, p. 4, la position rigourensement invariable des racines et des bourecens qui y donnent naissance, après étre restés quelquefois très-longtemps a létat expectant. Ciest dans les sillons, presque vis-it-vis du diaphragme, immediatement all-desious des bourgeons it rameaux ou it ihizomes, el sans jamais alterner avec eux, que se montrent les bourgeons it racincs. Il ne s'en produit jamais ailleurs. Ils commencent it se former sulus le cylindre cortical quils ont a traverser, en passant, comme nous l'avons vu p. 65 , au-dessus des lacunes corlicales.

Pour mieux faire comprendre ce que jai pu saisir sur te mode de développement des racines, je dois en rappeler brièvement l'organisation.

Lne racine dlÉguisctun bien developpee el quon étudie en allant du point d'origine a l'entrimite, présente ce qui suit: I" a lextéricur une cunche de deux ou trois rangs de crllules longues, prismalipues, assez régulierement hexagonales ol d'une

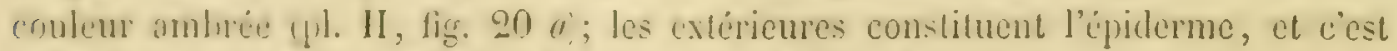
thelles que nairsent les fibrilles du tomentum; 3o an-dessons une couche simple de

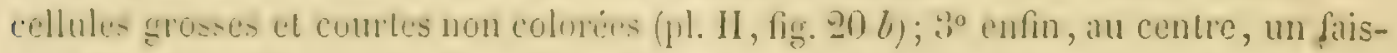
ceau fibro-vasulaire, dins lequel sont épars quelyues vaisseaux anumlaires de diverses gronsurs; un plus gros est central, ct autour de lui dautres plus petits sont groupés 
par deux ou par trois (pl. II, fig. $20 c$ et $21 \mathrm{l}$ ). Mais ì mesure que l'on s'avance vers la pointe, on roit cette organisation se modifier. Le faisceau fibro-vasculaire s'efface peu a peu (pl. II, fig. 21 i); les anneaux des fiisceaux deviennent plus espacés, plus indislincts; la forme des fibres radicellaires se dégrade également, et ce faisceau est ainsi remplacé par' un cordon central de cellules qui, à mesure qu'elles se rapprochent de la pointe, deviennent de moins en moins grandes et de plus en plus indistinctes, par suite de la quantité de granules qu'elles contiennent. Ces granules sont tris-petits, de coulcur verdàtre, de forme indéterminée et constamment agités du mouvement brownien. Sur l'épiderme on remarque des modifications analogues. Très-longues, trèsnombreuses et très-transparentes vers l'origine de la racine, les fibrilles radicellaires deviennent, en allant vers son extrémité, de plus en plus courtes, rares et verdàtres (pl.II, fig. $21 h, g$ ); bientòt elles ne sont plus que rudimentaires et alors très-chargées d'une matière finement granuleuse jaune verdâtre (fig. 21 f); enfin de pelits amas de la même matière se forment sous la paroi des cellules, en la soulevant un peu aux points oủ doivent apparaitre des fibrilles, puis ces amas eux-mêmes diminuent et finalement disparaissent. Un peu au delà du point oủ cesse toute trace de fibrilles développées ou rudimentaires, on voit qu'à son extérieur l'extrémité de la racine est tout entourće d'une matière incolore, mucilagineuse, de consistance un peu visqueuse, se diluant facilement dans l'eau (pl. II, fig. 21 a). Dans cetle matière, de la pointe de la racine à un millimètre cnviron en remontant, on voit des cellules plus ou moins déformées, entièrement ou i demi-détachées d'une petite coiffe qui recouvre complétement le bout de la racine (pl. II, fig 21 b). Cette petite coiffe est très-délicate, mal terminée à sa pointe, mal terminée à ses bords, qui se soulèvent et s'exfolient sa n régularité; ellc adhère d’ailleurs intimement à la pointe de la racine, el ce n'est qu'en arrière et à un millimètre environ (pl. II, fig. 21 c) qu'elle se détache en s'exfoliant. Elle est composée de deux ou trois couches de cellules, ou vers la pointe d'un plus grand nombre, dont les extérieures, longues et hyalines, portent vers leur milieu un pelit renflement ovale, je n'ose dire un nucléus, à granulations verdàtres, et trèsélégant (pl. II, fig. $23 a, b, c)$. Cette petite coiffe (́pl. II, fig. $21 c, c^{\prime}$ ), avec le mucilage qui l'enveloppe (id. a), constitue l'extrémité absolue de la racine, qui avait reçu le nom de spongiole, parce qu'on arait cru que cet ensemble terminal se gonflait à la manière d'une éponge en s'imbibant des liquides ambiants, et qu'il était ainsi le principal organe d'absorption. M. Trécul (Orig. rac., p. 310) a donné à la petite coiffe le nom de piléorhize, qu'on doil lui conserver comme plus convenable en ce qu'il ne préjuge rien sur la fonction.

Telle est l'organisation de l'extrémité d'une racine, arrachée arec toutes les précautions nécessaires, encore jeune et dans son état de développement normal. Les vaisseaux bien formés, les fibrilles radicellaires s'effacent et s’arrêtent, avons-nous 
dit, smultincumblers te meme point et it une distince relativement assez considé-

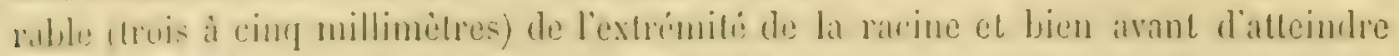

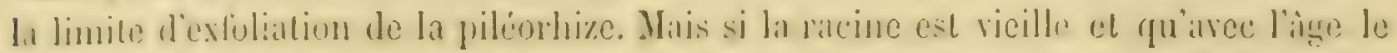
ti:-u liche de la pultiorhize se soit ditruit, le faisesu filmo-vasculatire el les fibrilles malicellaires arrivent alors tout contre cette extrumite dénulie (p). II, fig. 2h). Cette correblation intime démontre une fuis de plus le rapport itroit que Link avait supposé entre les fonctions des vaisenux et eolles des fibrilles, orsanes didsorption. On voit en méme temps que l'extrémite de la racine ne devient propre a labsorption que quand elle est déponille de lil piléurhize, el que cette function ne peut s'exercer, lorsgue celte région est recourerte par un tissu dont les couches extérieures sont vicillies, disloquées et enduites du mucilage visqueux qui semble résulter de leur decomposition et, en tout cas, s'oppose í tout acte d'absorption par la révion qu'il recourre. Les viritables organes d'absorption sont les fibrilles, d'autant plus nombreuses, plus courtes et plus fortes, que lespice croit dans un terrain moins hunide; dimintumt en nombre et s’allongeant aux dépens do la force sur les racines des espéces arguatiques (E. limosum, pl. II, fig. 25, Je n’en ai point vu sur les cellules exlérieures de la pilcorhize comme MII. Garreau el Brauwer's l'ont vu sur la couche exfoliable du Sinapis nigra L. (Ace. real., p. 189). La piléorhize parait n'ìtre qu'un organe protecteur, suppléant, sur ces racines, les gaines qui, sur les rhizomes, recouvrent et protégent les cellules délicates du sommet végétatif.

La dissection d'un bourgeon de racine naissant el réduit à ses premières cellules ne m’a permis de roir qu'un amas conlus de cellules, dans lequel il m’a été impossible de me reconnaître. $\Lambda$ un état plus avancé, alors que la pointe du bourgeon a déjả soulevé lépiderme du rhizome sans l'avoir disloqué, j’ai pu distinguer nettement une pileorhize bien constituée, mais je ne lai point vue entource de mätiere mucilagineuse, non plus que sur des lacines ayant quelques millimetres de long et dont la piléorhize ne commence pas encore à s'exfolier. L'apparition de ce mucilage accompagne done l'exfoliation des premières cellules sur des racines ayant au moins six millimitres. Il contient toujours des fragments de cellules et de tris-petits granules mis en liberti par la désorganisation des cellules. Il résulte d'abord de tout cela que cette matiere mucilagineuse, regardée par plusicurs auteur's comme une excrétion, parait provenir de la décomposition des cellules extéricures de la piléorhize; que, au lieu de considérer arec Link les cellules isolées quielle contient comme des organes en voie de furmation, il convient de les regander comme des collules désagrégíes en voie de décomprosition, et ru'enfin ce n’est point à l'extrémité alssolue de la racine quäl faut chercher le point de formation et de multiplication de ses tissus.

Mais, par cela send rue ce proint de végidution n'est pas terminal comme à l'extrémité d'une ligre, sa determination exacte el celle du mode de multiplication derient d'une 
difficulté cxtrême. Les cellules de cette région sont très-petiles, très-serries, à parois confuses et comme mal déterminées, tontes chargées ile granules amylacés soit uniformément épars, soit plus souvent agglomérés en plusieur's amas et qui, en tout cas, interceptent le passage de la lumière. Elles renferment rle grandes vésicules nucléaires, ou quelquefois des espaces sans granules, qui simulent des nucléus; et jamais je n’ai pu parvenir à voir distinctement dans la cellule ou les cellules centrales, une disposition déterminée des vésicules nucléaires qui en commencent la multiplication. Aussi me bornerai-je à décrire et à figurer les apparences qu'offrent les coupes d'une extrémité de racine bien développée, à constater ainsi ce qui est fait, sans aroir vu comment cela s'est fait.

Les fig. 21 et 22 de la pl. II, très-fidèlement copiées au moyen de la chambre claire, nous offrent deux séries de couches cellulaires bien distinctes en ce que les courbes qu'elles forment sont en sens opposé : les unes, plus intérieures, ayant leur convexité vers lés tissus du corps de la racine; les autres, plus extérieures, vers la pointe. Ces dernières, par leur aspect hyalin, leurs plus grandes dimensions surtout cn longueur, se distinguent au premier coup d'œil des couches plus intérieures; toutefois cela n'est exactement vrai que pour les plus extéricures; à mesure qu'elles se rapprochent de l'intérieur, leur dimension et leur transparence diminuent, elles ressemblent aux autres, et la différence dans la direction des couches permet seule de les dislinguer. De plus, sur une racine en bon état de développement, l'union du tissu intérieur et de la piléorhize est si intime que celle-ci ne saurail se détacher com. plétement et nettement, sans enlever quclques cellules de l'extrémité sous-jacente ou sans y laisser adhérentes quelques-unes de ses plus intérieures. C'cst donc incontestablement à ce point d'union intime, centre des courbes opposées, que se trouve le foyer te multiplication, le point végétatif, oủ prennent naissance, pour se développer en sens inverse, d'une part, les tissus permanents de la racine, d'autre part, l'enveloppe qui les protége en se renouvelant constamment.

Les cellules qui naissent vers la racine constituent son accroissement en longueur par leur multiplication ultérieure et par des modifications analogues à celles que nous arons constatées ailleurs; elles aboutissent à constituer les tissus de l'épiderme et ceux du faisceau fibro-vasculaire.

Celles qui naissent vers l'extérieur, ou mieux vers la pointe, sont refoulées en avant et latéralement par les multiplications qui s'opèrent incessamment au centre végétatif. $\Lambda$ mesure qu'elles s'en éloignent, elles s'accroissent en tout sens; impropres à tirer du dehors leurs éléments nutritifs, elles absorbent les granules qui les remplissaient et deviennent plus transparentes. Celles d'entre elles (qui sont latérales et appliquées contre l'épiderme (pl. II, fig. 22 c') s'allongent considérablement, et d'autant plus qu'elles sont plus éloignées de l'extrémité absolue, soit par l'effet de leur propre 
développement, soit parce que, encore adhérentes à la racine qui croît en longueur, elles cident it re mourement dextension. Enfm, pendint que de nourelles cellules se forment incesamment au centre virgétatif, les plus ancicnnes, el dis lors les plus extérieures, sappaurrisent, ne penvent plus suive la racine dans son extension, se neitrisent, sexfulient et laissent ainsi l'épiderme furlifié, et qu'elles ont d'abord prolégé, produire ses fibrilles el accomplir ses fonctions.

L'isolement par exfoliation des cellules de la pilćorhize montre trés-bien que la mulliplication des cellules n'a pas lieu par l'extension d'une paroi simple dans les cellules-mires, paroi qui serait alors mitoyenne i deux cellules et n'en permettrait pas lisolement; mais bien par le développement en cellules des vésicules nucléaires el par résorplion des cellules-mères. Chaque cellule a ainsi sa paroi propre, et s"isole de sa roisine par simple dédoublement de la cloison commune.

Miris maintenant d'après quel mode s'opère la multiplication des cellules au centre vigétatif pour donner naissance ì deux déreloppements en sens opposé? C'est ce qu'il ne ma pas îte permis de voir et jai constamment échoué dans mes recherches sur ce point.

Dins leurs savants travaux sur l'origine et le développement des racines, MM. Ohlert (Limnea, 1837, p. 609), Unger (Ann. sc.nat., 3e sér., bot., XVII, p. 128 et suir.), Trécul (Ann. sc. nat., 3 sér., bot., IV, p. 293 et suiv.; VI, p. 312 et suiv.; $4^{\circ}$ sér., bot., I, pl. XIV, fig. 12, 13), Link (Bibl.univ. Genève, XI, p. 355, 1819; Anm. sc. nat., $3^{\circ}$ sür, bot., XIT, p. 10), Gasparini, Succ. rarl., Naples 1856), Garreau et Brauwers (Amm.se.nat., fe sér., bot., X, p. 181 et suiv.) ne s’en sont pas occupés. M. Hoimeister s'est expliqué à deux reprises sur la formation des extrémitís radicellaires des cryptugames rasculaires. Dans la premiere explication, qui date de 1851 , cet habile et savant phytotomiste altribue i la racine des Equisetum et des Fougeres le mode de formation suivant:

"La cellule de premier degré, dont la segmentation permanente contribue le plus a l'extension de la racine, se trouve à l'intérieur du tissu, très-prés au-dessous du sommet de la racine. Sa forme est celle d'une lentille (tab. XVIII, 3; XIX, 14). Au moyen de cloisons dont la convexité est altermativement dirigée vers le haut et vers le bas, elle se partage en une nouvelle cellule de premier degré, encore de la forme diune lentille, et en une cellule de deuxieme degré de la forme d'un ménisque. Dans chacune de ces dernières il se forme bientòt après leur apparition quatre cellules disposies en quadrant et résultant d'ume double segmentation par parois perpendiculaires. Des cellules qui proviennent de la multiplication diue cellule de second degré, celles qui sont dirigées vers le bas se partagent aussi dorénarant au moyen de cloisons perpendiculaires au plan de la base de la cellule-mere, ou quelquefois à la partie la flus voisine de la surfice de la racine. Toutes les cellules-filles d'une de ces cellules 
de second degré sont situées en un seul plan (courbé en parabole), et y forment un fuseau creux el obtus cntourant les pointes des racines autant de fois qu'il a paru de cellules de second degré à leur point végétatif. La plus ancienne et la plus extéricure des couches s'élend jusqu'au point d'origine de la racine, les plus jeunes à l'intérieur s'étendent, selon leur àge, graducllement moins haut.

«Dans les cellules de second degré dirigées vers le haut, la multiplication tend ù augmenter le nombre des cellules plutòt dans le sens de la longueur que dans celui de la largeur etc. ) (Vergl. Unters., p. 96).

Je n'ai rien pu distinguer qui fùt en accord ou en contradiclion avec la théoric exposće ci-dessus. Et quand le savant micrographe, négligeant de mentionner les exfoliations de la piléorhize, expose que «les cellules-filles des cellules de second degré forment un fuseau creux et obtus entourant les pointes des racines autant de fois qu'il a paru de cellules de second degré à leur point végétatif..., ) il me devient impossible de concilier ce nombre croissant de couches arec la grosseur uniforme de la racine et le nombre uniforme de ses couches cellulaires extérieures. Sans doute je n'ai pas saisi Ja pensée de l'habile observateur, dont la figure schématique (tab. XIX, 14) est reproduite ici pl. II, fig. 27.

Voici la seconde explication (1852), dans laquelle il est fait mention de la piléorhize: (....La première racine (de l'Isoetes lacustris) est semblable à loutes les racines des cryptogames vasculaires. Son développement commence avec la multiplication d'une cellule du tissu intérieur de l'embryon... Cotte cellule se divise d'une manière durable et répétée, par des cloisons transversales opposées les unes aux autres, et elle forme des cellules de second degré, alternativement placées en dessus et en dessous de la cellule primaire. Les inférieures résultent de l'apparition d'une cloison faiblement convexe vers le bas; clles ont done en apparaissant la forme de ménisques. Leur multiplication ne se fait que suivant deux directions; toutes les cloisons de séparation qui s'y forment sont situées perpendiculairement sur la face voûtée supérieure et inférieure de la cellule, dont toute la postérité représente parfois une des couches cellulaires emboîtées les unes dans les autres en forme de bonnet, couches qui enveloppent la pointe extérieure de la racine et s'exfolient lentement du côté extérieur pendant le développement de cette racine» (tab. III, fig. 13; tab. IV, fig. 1, 2; Kemntn. Gef. Krypt., I, p. 136 et 137).

Par la seule raison que M. IIofmeister donne cette explication, je la tiens pour très-probable; et, fidèle à ma résolution de ne rien figurer et de ne rien décrire sans lavoir vu distinctement, je dois me borner à citer l'opinion d'un des plus habiles phytotomistes de notre temps.

Dans sa thèse de botanique (Études sur le rôle des racines dans l'absorption et l'excrélion; Strasbourg, 1861), M. le docteur Cauvet émet sur' les rapports de l'épi- 


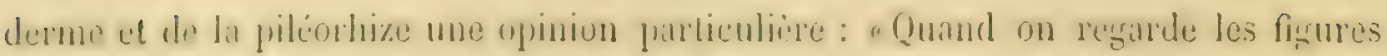

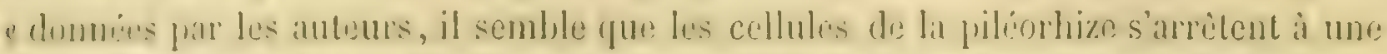

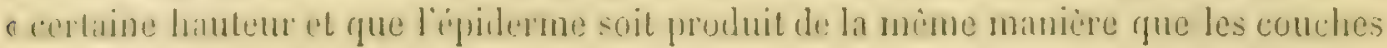
«internes. Un examen un peu attentif démontre qu'il n'en est pas ainsi, et que la

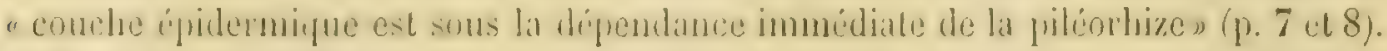

En ce qui concerne les Equisctum, cette assertion me parait au moins douteuse; mais comme, diune part, son anteur ne done point de fignres, et fue, dlautre part, il ne dit point sur quelles plantes il a observé les pilćorhizes, qui présentent de grandes modifications "suivant les plantes, la temperature et l'humidite du milicu " (Garreau et Brauwers, Ace. rad., 1). 191), il est difficile de discuter son opinion et j'ai dû me borner à la mentionner.

\section{$\S$ 4. Des spores}

J'ai suivi la formation et le développement des spores tout particulièrement sur les E. arrense, limosum, palustre, et ensuite sur les E. maximum, syleaticum et rerieyatum. Ces espicers étaient les phlus communes autour de moi et celles que je pourais me procurer le plus ficilement à tout instant, el, d'autre part, les deux premières sont les plus avantageuses it étudier attendu que ce sont deux types extrèmes, tant pour la diflërence des tigres spicifëres que pour celle des époques oir se développe l'appareil de reproduction. Ce que j'ai vu d'ailleurs sur toutes les autres especes m’a permis de recomnaitre avec certitude que la lui générale constatée sur ces types est la même sur les autres.

Pour faire avee quelque clance de succes des recherches sur la première apparition et sur lévolution de l'appareil reproducteur des E. maximum, syleuticum et "rrense, il faut les commencer des le mois de juillet pour les épis qui doivent répandro leurs spores at mois d'arril suivant. Aux nouds inféricurs des rejetons robustes on trouve alor's des bourgeons gros et ventrus (pl. I, fig. 1 a) destinés à douner des tiges spicilères ${ }^{1}$. En les disséquant à cette époque, on peut suive le déreloppement de la

\footnotetext{
' Je les ai vus apparaitre dès le 13 juin sur l'E. artense, et pl. 1 , fig. 1 a j’ai représente un bourgeon de tige spicifere, arraché et dessiné le 25 septembre; il ne devrait sortir de terre qu'en mars ou avril suivant. Ceux de l'E. maximum ne commencent qu'en juillet, et ceux de l'Ji. syltaticum en août. Ėn septembre l'épí est tout a fait formí dans ces gros bourgeons. Your ne point perdre son temp̧s à arracher des tiges qui n'auraient à la baso que des bourgeans de tiges stériles, on devra choisir parmi les tigges stériles celles qui, quoique étant les plus robustes et ayant toutes les apparences de la vignueur, sont néanmoins les premières à jaunir et à se fétrir. On sera sùr de trouver à leur base, ả un ou deux centimètres au-dessous de la surface du sol, de magnillques bourgeons à épi, dans lesquuels est atliréce el se concentre la force de végétation, qui manque de bonne lieure ì ces tiges. Quant à l'E. limosum et à l'E. palustre, il suffit, pour voir l'apparition de l'épi, de prendre vers la fin de mars une tize nouvelle ayant dejà qquelques centimètres de long. Ces deux espéces prołluisent d’ailleurs des épis tout l’été. L'É. variegatum en donne presque toute l'année. Les $E$. ramosissimum et liyemale portent de très-bonne lieure des épis, qui sont en état de sporuse au mois de mai.
} 
partie inférieure el des gaines de la tige spicifère, tel que je l'ai décrit au $\S \mathcal{1}^{\text {er }}$ de ce chapitre. La gaine la plus élevée, celle qui doil être l'anneau, offre les mêmes commencements de formation qu'une gaine ordinaire; seulement elle s'arrête à l'étal de bourrelet uniforme, court et charnu, tout composé de cellules et sans vaisseaux. Les pelites saillies supérieures, qui plus tard simulent des dents avortées, ne sont que le résultat de la pression contre le verticille inférieur de l'épi.

Le groupe de cellules constituant le sommet végétatif d'un épi en fonction de développement présente la mème structure et le même mode de multiplication que celui d'une tige quelconque. Au-dessous de lui, le premier étal d'un verticille sporangifère naissant se montre sous la forme d'un bourrelet annulaire, beaucoup plus large, plus massif et en même temps moins élevé que celui d'une gaîne naissante. Ce bourrelet n'est point non plus uniforme et continu comme celui d'une gaine, mais il offre un ensemble très-serré et très-compact de saillies tout à fait analogues à celles que font les bourgeons naissants. On a quelque peine à distinguer cela sur les deux verticilles les plus voisins du sommet et dès lors les plus récents, parce que les mucilages et les granules qui en remplissent les cellules, en rendent les parois peu distinctes et les font ressembler a des bourrelets annulaires tout unis. Hais déji le quatrième verticille, en comptant du sommet végétatif, et mème quelquefois le troisième sur l'E. limosum, montrent nettement des saillies hémisphériques, dont le nombre est sur chaque espèce dans un rapport exact avec celui des pédicelles sporangifères qu'elle présente à chaque verticille. On reconnait ensuite avec évidence que le développement de ces bourrelets n'est point conforme à celui des gaines (p. 56-58), mais que les bourgeons qui les constituent par leur réunion se comportent dans leur apparition et leur développement tout comme des bourgeons à rameau (p. 66,67). Chacun de ces bourgeons commence donc par une cellule qui, après s'être développée plus que les autres, s'est multipliée en deux cellules séparées par une cloison transversale à leur axe de développement (pl. VIII, fig. 14). Des deux cellules qui résultent de cette multiplication, la plus rapprochée de l'axe principal se multiplie en un plan de deux, trois ou quatre cellules, dont la ligne commune de contact est au centre du plan et presque perpendiculaire à l'axe principal. La multiplication ultérieure a lieu comme sur les rameaux, en constituant un axe secondaire, avec centre distinct, comme un rameau (pl. VIII, fig. $15 \mathrm{ct}$ aussi pl. VII, fig. 29), et non un appendice latéral et plat comme un lobe de gaine (pl. VII, fig. 21,24 bis). La cellule terminale m'a paru s'arrêter très-rite dans sa multiplication; et, sur la partie de ces saillies ou bourgeons qui est contiguë à l'axe de l'épi, la multiplication s'arrête aussi bicntùt, tandis que sur le pourtonr de la région terminale et extérieure, la multiplication est extrême. Il suit de là que les bourgeons sont à leur base amincis en pédicclles, ct à leur extrémité dilatés en bourrelets circulaires qui, par suite de leur pression réci- 
proque, affectent, presque dis leur apparition, des formes hexagonales. La cellute inrminale, qui a servi de point de déprat el qui oceupe la partic contrale de crs dilatations en burrelel, s'est, comme nous venons de le dire, arrètée dans son développement, et il semble que les cellules qui l'avoisinent aient subi la mème influence, car on remargue tonjours une légere depression vers le centre. Bientót an bord interieur et vers le pourtour de ces dilatations, sur cing ou presque toujours sur six prints, apparaissent de petites protuléranees oltuses, dues à ce que sur ces points une collule se multiplie plusieurs fois, et forme ainsi les premiers rudiments des sporanges. Ils consistent en une masse presque sphérique, ayant it lat surface une couche simple de cellules et ruelques-unes seulement an centre. Bientiot une de ces dernieres saceroil vivement el de manière à dépasser de beaucoup toutes ses cungéneres. Ceet d'elle que proviendra toute la masse des spores; celles qui l'cutourent, apres certaines transformations, conslitueront le sac des sporanges (pl. VIII, figr. 16). A ce moment de l'évolution correspond l'apparition des vaisseaux au pourtour de l'axe de l'épi.

La cellule centrale se multiplie d'abord transversalement à l'axe du sporange (pl. VIIl, fig. 17), ensuite chacune des cellules provensat de ce dédoublement se dédonble elle-mème, et la multiplication continue ainsi par de nouveanx dédoublements. En mème tempse et parallélement les cellules du sac se multiplient par des cloisons perpendiculaires à leur fice extérieure, et leur nombre angmente rapidement; mais l'enveloppe cxtéricure qu'elles constituent est encore simple. Ensuite, au moyen d'une cloison parallite à la face extérieure, chacune de ces cellules se dédouble ol le sac offre ainsi deux conches de cellules, et eufun, par une seconde division dans le mime sens, ie sac se troure composé de trois conches cellulaires. Peudint ce temps, le groupe des cellules génératrices a augmenté, et, par des multiplications rípondant i chaque acte dévolution du sae, le nombre de ces cellules dépasse 120; toutes paraissent renfermer un ou deux grands nucléus (pl. VIII, fig. 18)'.

Il se passe alors plusieurs phénomènes qui se compliquent et sont difficiles it saisir, paree quil y a dans les cellules une grande quantité de granules qui troublent le liquide, et fuien mème temps le nombre et la granden des nucléus rend les parois moins faciles ì distinguer. W'une part, dans le pédicelle s'avance l'apparition des vaisseaux spiro-ammulaires, en même temps rque la partic tout it fait extérieure de lit dila-

\footnotetext{
I In moyen aussi sûr que simple de bien distinguer à celle époque les cellules génératriecs de celles de leur enveloppe, consiste à couper un épi par le milieu dans le sens de sa longueur et d̀ le laisser macérer trois ou quatre jours lans l'eau. La masse des cellules génératrices se colore en roux et devient merveilleusement distincte. line coupe, mème faile grossic̀rement, permet de distinguer et de compter les cellules de chan̨ue sorte. On arrive assez heurcusement à isoler les cellules de ces jeunes tissus, en les faisant macérer quelques heures dans une cau légèrement acidulée arce l'acide azotique, ou chaufer quelques instants dans une solution potassiqque très-ćtendue.
} 
tation s'organise en clypéole, c'cst-i-dire s'épaissil, se durcit, se colore et montre les commencements des stomates; d'autre part, deux des couches du sac, l'intérieure d'abord et ensuite la moyenne, semblent se liquéfier pour être résorbées; d'autre part enfin, les cellules génératrices s’isolent les unes des autres, soit entierrement, soit par groupes de deux ou de quatre au plus, qui restent encore unies quelque temps. Les sporanges ont alors la forme qu'ils conserveront définitivement, malgré les modifications qui s'accompliront à leur intérieur (pl. VIII, fig. 19).

Daus les cellules génératrices isolées complétement (pl. VIII, figg. 20) ou encore réunies par groupes (fig. 21) on voit bientòt apparaitre deux nucléus qui les occupent presque en entier (fig. 22), ct auxquels succède la division de la cellule en deux autres étroitement accolées (fig. 23). Dans chacune des deux nouvelles cellules il apparait de nouveau deux nucléus; ils se disposent vers les extrémités de chaque cellule, en chevauchant, et comme sur deux lignes qui se croiseraient pour atteindre les quatre angles d'un tétraèdre (fig. 94). Il m’est arrivé assez fréquemment de voir les quatre nucléus disposés comme il vient d'ètre dit, sans qu'aucune cloison indiquât que leur cellule-mère eùt été au préalable dédoublée. Quoi qu'il en soit, à ces nucléus succèdent de nouvelles cloisons, par suite de quoi la cellule génératrice est multipliée en un groupe de quatre cellules, qui sont définitivement les vraies collules-mères des spores.

A leur tour ces quatre cellules s'isolent entre elles'. De la cellule commune qui les contenait on ne voit plus d'autre trace qu'une sorte d'atmosphère mucilagineuse et granuleuse que quelques-unes de ces cellules entraînent autour d'elles el qui disparaît bientôt. Très-souvent, et particulièrement sur l'E. limosum, immédiatement après l'isolement de ces cellules, on voit suspendus dans ces mucilages des corps sphériques transparents et ressemblant entièrement à des nucléus qui, au lieu d'aboulir, auraient survécu ả la résorption de leur cellule.

A la première division des cellules génératrices et à l'isolement des cellules-mères a correspondu la dernière double multiplication des cellules du sac. Elle a eu lieu dans le sens de la longueur, et elle demeure reconnaissable jusqu'ì la fin, parce que ces cellules sont disposées par groupes de quatre, autour desquels les parois de circonscription sont très-saillantes. Celte disposition est très-apparente sur les cellules encore dépourvites de fibres spiralées (pl. VIII, fig. IS); et, avec un pen d'attention, elle est encore reconnaissable mème aprés l'apparition de ces fibres, qui n'a lieu que plus tard.

Les nouvelles et dernières cellules-mères des spores sont alors assez régulière-

\footnotetext{
'Voy. Ad. de Jussicu (Cours élém. de bol., 1848, p. 425) et Payer (Bot. crypt., p. 312) „Il me semble que ces savants botanistes, trompés sans doute par les assertions de M. H. Mohl (Bemerk. Spor.), ont eu le fort de nier d'une manière absolue l'apparition de quatre spores dans les cellules génératrices, et de contester la résorption de ces cellules pour la formation des spores des Eqquisetum:
} 
mont spheriques. Leur membrane délicate hasse diahond voir contre la paroi un murbir aplati et de forme peu réguliere; puis hientot apres, ce nuclieus grandit, disparait, el au-dessous de la surface on distingue une zone très-étroite, trèstransparente et une membrane tres-fine, separint celle zone diune masse sphirique plus colorie, plus dense el plus charece de gramules moleculaires. Celle masse sphirigue est la spore proprement dite, et la membrane minec sura la membrane extivicure de la spore. Presgue en mème temps on distingue daus la spore, inmédiatement sous la surface de sa membrane, une couche mucilagineuse arec des granules relts tres-lénus, el dans celle conche un espace sphérique oecupé yra un liquidesans sranules, d'une couleur un peu dillérente et comme tres-légtement ro-éc (pl. VIII, lig. 25. Le plus sourent mime il commence par y avoir plusieurs de ces espaces (fir. 25 u), mais l’un d'eux est beaucoup plus grand que les autres, qui finissent tonjours el assez vite par se rémir a lui et se fondre en un seul. A mesure que cel espace sacrandit, il perd sa forme sphérique sur un de ses cólés, qui est refoulé en calolle concive par la matiere verte. Lin mème temps el assez rapidenent la membrane de la spore se sulidifie el se durcit, et sur la membrane de la cellule-mere commencent i se montrer des saillies intérieures ou épáississements disposés en lignes spirales; ce sont les premiers ruliments des fils élastiques ou élatéres de la spore. Cies saillies se montrent d'abord vers l'équateur de la cellule-mère, el après avoir commencé par paraitre à l'intérieur, elles deviennent sensibles à l'extérieur, el clles débordent dans les deux sens la membrane primilive aux dépens de laquelle elles semblent se former (pl. VIII, fig. 26). Celle membrane samincil done de plus en plus el funil par ètre résorlece entre les spires qui, sans ètre contignës (sauf en m point où elles adhèrent i la spore), sont tris-peu séparíes. Vers les pôles, ou sisolent en dernier lieu les dilatations spatulées des élateres, cette membrane s'écarte davantage de la spore et, de sphérique qu'elle élait d’abord, elle devient un peu ovol̈le (pl. VIII, fig. 26). On иe voit jamais vers les poiles les deux petites saillies globuleuses que M. Ilenderson y a repreisentées (j)l. VIII, fig. 97, copie de celle de M. IIenderson, Leprod. Ey., pl. :39), mais on voit quelquefois se placer, contre un point queleonque de la surface, un de ces nucléus isolés dont jai parlé plus haut (pl. VIII, figg. 26 u). A ce moment, où les diateres ne sont isolés que vers l’éfuateur, et non encore vers les pòles, on distingue encore a l"intériem de chaque spore l’espace sphérique sans granules, avec une partic de sa surface concave (pl. VIII, fig. 26). I sa surface se forme une membrane diunc entrume lénuli; ce sera la seconde membrane ou membrane intéricure de la spore. Linfin, ces deu membranes deriennent contiguës el les fils blastiques s’isolent completement (pl. IX, fig. \&) par la résorplion du reste des parois de la cellule-mere!.

1 M. H. Mohl ne mentionne point la formation des saillies à l'intérieur de la cellule-mère, et pour ce savant a lez élatères 
Ce n'est qu'un peu plus tard que commence la transformation des cellules simples du sac en cellules fibro-spiralées. Celte transformation est précédée de l'apparition dans chaque cellule d'un très-grand nucléus, qui s'y développe un peu après leur dernière multiplication (pl. VIII, fig. 28), en occupe presque toute la largeur et disparaît ensuite. On voit lui succéder une assez grande quantité de matière verte et de granules moléculaires, et les parois des cellules paraissent épaisses et comme doubles. A l'époque qui suit l'isolement complet des élatères, la matière verte fait place à un liquide mucilagineux, semi-transparent et un peu opalin; puis bientôt sous la paroi des cellules apparaissent des saillies très-minces, spiralées sur la région médiane du sac, et annulaires vers la ligne où se fera la déhiscence. Sur l'E. limosum ces saillies ne se forment que la veille ou l'avant-veille de l'émission des spores, et elles se développent avec une incroyable rapidité. Un épi très-avancé, qui en montre à peine quelques traces, laissé à sec et à la chaleur pendant quelques heures, a bientôt toutes ses cellules spiralées; et même, si l'on a soin d'entretenir l'humidité du porte-objet, on y voit, au bout de une ou deux heures, devenir plus distinctes celles qui se montraient à peine. On constate ainsi avec une parfaite certitude que les spirales ou les anneaux prennent dès les premiers moments de leur apparition la disposition qu'ils conserve. ront ultérieurement. La spirale une fois formée, les cellules sont d'une parfaite transparence et si minces qu'on a quelque peine à les distinguer; chacune d'elles a conservé exactement sa grandeur et sa position antérieures (pl. VII, fig. 19).

Après cette transformation des cellules du sac, qui est la dernière de toules, le contenu des spores devient plus épais, moins soluble dans l'eau, et se charge de granules moléculaires nombreuses; enfin il prend une belle couleur vert intense. $\mathrm{L} c$ fluide, qui précédemment baignait toutes ces cellules et qui a été graduellement résorbé, laisse après lui des granules moléculaires qui, isolés ou par groupes, s'altachent à la surface de la spore et à celle des élatères. Ce sont ces granules que pendant longtemps on a pris pour des grains polliniques.

Sur les $E_{\text {s }}$ arvense, maximun et sylvaticum les choses restent en cet état depuis le commencement de l'automne jusqu'aux premiers jours du printemps, oủ, par la déhiscence du sac des sporanges, les spores sont disséminées avec leurs élatères. Vers la mi-août on peut quelquefois observer, du sommet à la base d'un mème épi de ces trois espèces, presque toute la série des développements que nous venons d'exposer 
(j). VIII, fig. :30). Sur l'E. limosum la dlíhiscence succède sans intervalle au dernier acte d'ivolution; ct sur un mème épi de cette espèce les divers phénomènes sont moins successits et moins éloignés (pl. VIll, figg. 29); mais pendant tout le printemps, el souvent cncore pendant l'été, on trouve dans un mème fossé des sujets à tous les degrés de développement!

Les premiers développements de l'épi se font régulièrement de bas en haut, mais il n'en est plus de mème pour les dernier's, c'est-i-dire pour la formation des spirales dans les cellules du sac. Gelle formation commence sur le cùté de l'épi le mieux exposé au soleil, el le plus souvent de haut en las. Sur les E. maximum, areense et palustre la sporose marche ordinairement de bas en haut; souvent cependant les verticilles infërieurs, enccre enveloppés par la gaîne, n’émettent les spores qu’après ceux du milieu, mieux exposés à l'action de la lumière et de la chaleur.

Il est facile de remarquer qu'ì chacune des évolutions du sac répond parallèlement une évolution des cellules génératrices, et dès lors on peut se rendre approximativement compte des rapports numériques que présentent successivement ces dernières cellules dans leurs diverses évolutions, et finalement les spores qui en proviennent.

Ainsi pendant les premières et rapides multiplications des cellules du sac, la cellule génératrice se multiplie par ses deux premiers dédoublements en quatre cellules, que deux nouvelles multiplications portent à seize, nombre maximum que renferme le sae simple? 2 partir de ce moment il y a une corrélation parfaite et on ne peut plus facile à saisir. Ainsi à l'apparition des divisions qui multiplient le nombre des cellules du sac et a la première des divisions transversales qui rend l'enveloppe double, répondent deux divisions successives qui font des 16 cellules génératrices d'abord :2, puis 64. Au dernier dédoublement, qui domne trois couches a l'enveloppe, et i la liquéliction des deux intérieures répondent encore deux nouvelles multiplications qui élevent les 64 cellules a 128 , puis à 256 ; ol à la dernière quadripartition, dont les cellules du sac conservent toujours la trace, répond la dernière quadripartition des cellules grénératrices en cellules-mères proprement dites, au nombre de 102', , qui est le même que celui des spores. En effet si, sur un porte-objet divisé en

\footnotetext{
1. Les degrès de développement de la cellule-mère des spores sont parcourus en très-peu de temps. Dans les mêmes sporanges de l'E. palustre se trouvent des cellules-mères arec des noyaux primaires en train de se dissoudre, d'autres avec deux noyaux-filles aplatis, d'autres enfin arec quatre de ces noyaux sphériques; on y trouve des cellules-lilles tétraédriques unics et isolées, et enfin on y voit des cellules qui montrent un peu leur aréole transparente p (Ilofmeister, Vergl. Enlers, elc., p. 99). En citant cet ouvrage de M. Hofmeister, je dois dire que, quoique sur plusieurs points je me sois ćcarte de la manière de voir du savant et habile phytotomiste, son admirable travail m"a rendu de très-grands services pour l'étude qui précède. Dans le Flora de $1833, \mathrm{p} .45$ et $46, \mathrm{M}$. H. Mohl a exposé les déveluppements de l'E. variegatum; en 1842, M. Henderson a exposé ceux de l'E. hyemale dans Ies Transactions of Linn. Soc. (heprod. Eiq. ctc.).

- Il y a une multiplication de plus dans l'E. maximum el quelquefois, mais rarement, dans l'E. artense; il paraft y en avoir souvent une de moins dans I' $E$. variegatum et sur les épis des rameaux de l'E. paluslre.
} 
petits carrés d'un millimètre de côté, on épand dans une goulte d'eau le contenu d'un sporange mûr mais non ouvert d'un $E$. arvense, et qu'au moyen d'une loupe montée (ou microscope Raspail) on compte ce qu'il y a sur chaque petit carré, on trouvera presque toujours un total de 900 à 950 spores, ce qui correspond assez bien au dernier nombre trouvé, si l'on tient compte de quelques cellules abortives, dans lesquelles la multiplication aurait subi un temps d'arrêt ${ }^{1}$. Enfin aux dernières modifications de la cellule-mère el à sa transformation en élatères spiralés succèdent les dernières modifications des cellules du sac el leur passage de l'état de cellules simples à celui de cellules fibro-spiralées. Dans les épis qui restent ordinairement ou exceptionnellement stériles, ces deux dernières modifications manquent parallèlement sur les cellules du sac des sporanges, comme sur les spores, qui ne contiennent pas non plus de matière verte.

Il semble donc permis d'établir le tableau de corrélation et le résumé suivants:

$1^{\circ}$ Apparition d'un bourrelet circulaire de cellules au-dessous du sommet végétatif. $2^{\circ}$ Apparition sur ce bourrelet d'une rangée de 10 à 12 saillies se développant ultérieurement en dilatations clypéolaires à leur extrémité.

$3^{\circ}$ Apparition, au pourtour de la face intérieure de ces dilatations, d'un cercle de 5 ou 6 saillies devant constituer les sporanges.

$4^{\circ}$ Apparition au centre de ces saillies d'une cellule génératrice; les vaisseaux se montrent dans l'axe de l'épi.

$5^{\circ}$ Les cellules du sac augmentent en nombre.

$6^{\circ}$ Les cellules du sac se dédoublent pour former deux couches.

$7^{\circ}$ Une nouvelle multiplication donne trois couches à l'enveloppe; liquéfaction et résorption des deux couches intérieures.

Apparition de vaisseaux dans le pédicelle et de stomates sur l'épiderme du clypéole; enfin isolement des cellules génératrices.

$8^{\circ}$ Dernière double multiplication des $\quad 8^{\circ}$ Division des cellules génératrices d'acellules du sac. bord en deux cellules (512), puis de ces deux en deux autres (1024), constituant des groupes de quatre cellules se croisant deux à deux.

$9^{\circ}$ Isolement de ces dernières cellules qui sont les cellules-mères des spores.

\footnotetext{
'Ces nombres sont ceux que j’ai régulièrement trouvés sur les $E$. arvense, sylvaticum, limosum, palustre, ranio. sissimum, hyemale; mais le nombre des spores est beaucoup plus élevé dans l'E. maximum et moins élevé dans l'E. variegatum et sur quelques épis latéraux de l'E. palustre. Voir la note 2 de la page précédente.
} 
$10^{\circ}$ Apparition des membranes propres de la spore et des saillies spiralées sur la cellule-mère.

$11^{\circ}$ (Un peu plus tard) Transformation des cellules simples du sae en cellules fibro-spiralées.

$11^{\circ}$ Solidification des membranes; transformation définitive de la cellulemère en fils spiralés ou élatères.

120 Rupture du sac des sporanges et dispersion des spores.

\section{§5. Anatomie des spores}

Les spores des Equisetum et le gracieux appareil de leurs élatìres ont été un objet favori d'éludes prour les micrographes; aussi ont-clles donné lieu à un grand nombre de figures, de descriptions et d'opinions différentes.

L'aspect qu'i première vue présente une spore complètc hors du sporange, encore fraiche et bien vivante, mais à l'état sec, est celui d'une sphère posće au point de réunion de quatre fils plus ou moins contournés et constamment aplatis et élargis ell spatule à leur extrémité (pl. VIII, fig. 31). C'est a tort que l'on a dit que celle extrémité est claviforme ${ }^{1}$; clle est plate, d'une épaisseur ì peine appréciable, dilatée en spatule non symétrique, et offrant un de ses còtés beaucoup moins dilaté et moins courbé que l'autre (pl. VIII, fig. 32). Cet aspect général avait encore fait croire aux premiers observateurs que ces fils ou élatères sont au nombre de quatre ${ }^{2}$; il est facile de s'assurer qu'il n’y en a rue deux, soit en pressant légèrement les spores entre deux lames de verre que l'on fait un peu glisser l'une sur l'autre, soit en les faisant passer brusquement de l'élat de sécheresse et de dilatation à celui d'humidité et de contraction. Dans l'un comme dans l'autre cas, les élatères se détachent presque constamment, et l'on roil qu'ils consistent en deux fils aplatis et dilatés en spatule ì chacune de leurs extrémités. Il est moins facile de sassurer de la manière dont ces fils adhèrent ì la spore. En effet, sur le porte-objel, les spores se présentent constamment à l'observateur avec leur point d’adhérence placé en bas. Lì est évidemment leur centre de gravité. Car; si on prend des spores dont les élatères ne se soient pas

\footnotetext{
1. Richard (Précis de bol., vol. II, p. 51, 1852), B. Grenier (Fl. Fr., vol. IIl, P. 642, 1856), M. Cosson (H'l. ent'. Paris, 20 ed., p. 876, 1861) disent seulement: "quatre appendices renflés au sommet... N. Henderson (Reprod. Eq., p. 569) dit plus explicitement: "...the clavate ends of the filaments... Et pourtant dès 1827 Bischoff avait dit trèsexactement: "dilatés a leurs deux bouts non en massue, mais au contraire en forme de spatule: ...an ihren beiden a Enden nicht kolbig, sondern vielmelır spatelfürmig erweitert "(kirypt. Gew., p. 32). Malgré cela, en 1830, M. L. Reichenbach disait encore de ces élatères : "staminodia bina, cruciatim afixa, polline repleta , ( $F l$. excur., p. 15\$).

2Les floristes en général (non Koch); Duvernoy, De Salvinia natante, p. 10, 1825; IIenderson, Keprod. Eq., p. 567, 1842; Ad. de Jıssieu, Cours élém. Ue bot., p. 425, 1848; J. Payer, Bol. crypt., p. 214, 1850; A. Richard, Précis bol., rol. II, p. $31,1852$.
} 
cncore déroulés, et qu'on les jette sur un verre sec ou sur une goutte d'eau, on en verra quelques-unes qui présenteront en dessus leur point d'adhérence aux élatères, toujours reconnaissable à ce que les élatères adhẻrent entre eux sur ce mème point; mais aussitôt on voit ces mèmes spores se tourner en oscillant, puis s'arrêter définitivement sur ce point. Les spores plus avancées et dont les élatères se sont déjá étalés se placent de suite en équilibre sur leur point d'adhérence, qu'il est dès lors très-difficile sinon impossible de voir. Pour y parvenir, j'avais d'abord essayé d'une plaque de verre que je plaçais sur le porte-objet et je retournais le tout rapidement. Mas il arrivail presque toujours que le frottement des deux plaques, si faible qu'il fùt, détachait les élatères ou écrasait les spores, et si quelques spores, restées libres et complètes, étaient renversées et montraient leur point d'adhérence, ce n'était que rarement et pour un instant très-court; elles se mettaient aussitôt à osciller et se retournaient. Une observation durable ne m’est devenue possible que par les procédés suivants. Sur une de ces petites lames de verre dont on recouvre le porte-objet j'étends une couche très-mince d'unc solution gommeuse très-étendue et je secoue dessus des spores encore entourées de leurs élatẻres. Après qu'elles y ont pris leur centre de gravité, je laisse le tout sécher un peu, et au bout de quelques instants les spores sont assez fixées pour qu'en retournant la petite lame je puisse en voir tout à l'aise le point d'adhérence. Pour les spores dont les élatères sont déjà étalés, je les mets à sec sur un verre très-légèrement concave, puis j'applique par dessus une lame de verre enduite d'une solution gommeuse presque sèche. Les élatères, en se redressant par l'effet de l'humidité, se prennent à la gomme, s'y fixent dans toutes sortes de positions, et toujours un certain nombre d'entre elles me présentent leur point d'adhérence, quand je prends pour porte-objet cette lame renversée.

J'ai pu dès lors m'assurer avec la plus complète évidence, et sur des milliers de spores, que les deux fils des élatères sont adhérents entre eux au point où ils adhèrent encore à la spore, et adhérents en restant parallèles et par union latérale, non par entre-croisement, comme on l'a souvent affirmé. Mais alors même qu'ils sont encore adhérents entre eux et à la spore, on voit le plus souvent sur la région d'adhérence une ligne que le jeu du miroir réflecteur rend plus ou moins apparente, et qui indique une moindre épaisseur suivant laquelle aura lieu la séparation définitive. Cette apparence me parait être la cause de l'opinion émise par M. Pringsheim « que les élatères ne sont jamais contigus à leur point d'adhérence à la spore» (Schl. Eq., p. 244). Ce savant botaniste va même jusqu'à dire que souvent les deux fils ont des points d'adhérence très-éloignés l'un de l'autre «et même diamétralement opposés. 》 Je démontrerai plus loin l'impossibilité d'une telle disposition.

L'épaisseur des fils m'a paru dans la jeunesse un peu moindre que leur largeur. Le diamètre des spores (E. maximum, arvonse, palustre, variegatum) m'a paru mesurer 
trois centiemes et demi de millimètre, soit $0^{\mathrm{m}}, 000036$; la dilatation spatuléc de l'élatere égale en largeur à peu pres un demi-diamètre de la spore, el la longueur totale d'un clatère contient neuf fois et demi environ ce même diamètre". La spirale des élatères autour de la spore est dexire.

Par leur disposition en spirale, les deux élatires constituent une sphère autour de la spore. Leur point commun d'adhérence est placé sur l'équateur de cette sphère et leurs dilatations spatulées ver's les pôles. Or, si on considere une sphère (pl. VIII, fig. 33) et qu'on suppose que d'un point 0, placé sur l'équateur de celte sphère, deux bandes a et $b$, siluées chacune d'un cóté de l'équateur, aient à se rendre au pôle $P$ en deux spirales parallèles, on remarquera que la bande $b$, la plus éloignée du púle $P$, a nécessairement à parcourir un chemin plus long que celui de la bande a pour arriver à ce pôle, el que la différence entre les deux chemins est égale à la moitié de léquateur, puisque ce n'est qu'au point diamétralement opposé à 0 que la bande $b$ sera par rapport au póle $P$ à la distance où se trouvait primitivement la bande $a$. Par la même raison la bande a a un demi-équateur de plus que la bande $b$ à parcourir pour se rendre au pôle P'. Ainsi en supposant des bandes soudées seulement au point 0 et ensuite étendues, la bande $b$ du côté du pûle $P$ dépasserait la bande a d'un demiéquateur, et réciproquement, vers le pôle opposé P', la bande a dépasserait de la même quantité la bande $b$; de telle sorte que, les deux bandes étant d'une même longueur totale, seraient réciproquement partagées à leur point d'union en deux parlies inégales comme l'indique la pl. VIII, fig. 34². Or c'est précisément ce que l'on observe sur les élateres; les quatre bras apparents qu'ils montrent, pris deux à deux, de quelque faron que ce soit, présenteront toujours une inégalité répondant à un demi-équateur ou à un diamètre el demi de la spore. C'est le résultat que l'on obtient en traçant, sur une sphère creuse de caoutchouc, la forme et les spirales des élatères et en découpant celte sphère selon les lignes tracées, sauf en un point d'union sur l'équateur. Ccla démontre donc géométriquement l'unité du point d'adhérence des élatires aux spores et entre cux. On concoit bien d'ailleurs qu'une spore sphérique adhère par un seul point à sa cellule-mère également sphérique; mais on concoit moins facilement la possibilité entre deux sphères de deux points d'adhérence séparés ou opposés, comme l'a dit MI. Pringsheim (Schl. Eq., p. 244), qui d'ailleurs avail très-nettement distingué el figuré l'inégalité des bras apparents des élatères.

\footnotetext{
- Dans les mesures que donne M. Pringsheim (Schl. Eq., p. 212) el qui sont à peu près les mémes (savoir: diamètre des spores $2 / 53,1 / 25,1 / 28$ de millimètres; longueur des ćlatères de 10/32 à 10/35 de millimètres), et dans les conclusions qu'il en tire, il me semble que ce savant observateur n'a pas sufisamment tenu compte de la grande surface des dilatations terminales spatuliformes.

'Il est bon de se prémunir contre une illusion qui porte à voir la bande b' comme une continuation de $a$ (pl. VIII, lig. 31), et a croire qu'un des deux clatères est plus court que l'autre.
} 
Il est absolument impossible de concevoir les élatères comme se croisant au point d'union. Deux lignes spirales, qui se croiseraient en un point, se couperaient nécessairement à chaque tour de spire et ne pourraient en aucun cas courir parallèlement; et de plus, pour cette disposition il faudrait concevoir la cellule génératrice des lignes spirales double aux points de croisement, et simple aux autres points; ce qui est absurde. Bischoff (K'ypt. Gev., p. 32, 1828) avait bien reconnu qu'il n'y avait que deux élatéres, mais trompé par une disposition accidentelle que les élatères prennent assez souvent en se détachant de la spore, il les avait considérés comme se croisant a leur point d'union, «die sich in ihrem Befestigungspunkte durchkreuzen ${ }^{1}$. » Aussi plus tard, et pour rester autant que possible conséquent à sa première idće, ce botaniste affirma que les élatères constituent deux enveloppes (Lehrb. $d$. Bot., I, p. 443 et 455, 1834). M. II. Mohl (Morph. sporang., p. 8, en note) réfuta en 1837 l'assertion de Bischoff; mais elle avait été répétée par M. L. Reichenbach, el on la trouve encore dans des flores toutes récentes.

Ordinairement, pour ne pas dire toujours, la surface des élatères est parsemée de petits granules moléculaires, que tous les auteur's ont mentionnés et que l'autorité imposante de Hedwig a fait si souvent regarder comme des grains polliniques. Je ne sais ce qui a pu porter Bischoff à dire «qu'à cause de la finesse des fils des élatères on ne peut établir avec certitude si ces petits grains sont réellement épars à la surface ou contenus dans l'intérieur » (Krypt. Gew., p. 32), car le moindre lavage suffit pour les faire tomber en totalité ou en grande partie, et alors on les voit dans le liquide exécuter avec assez de vivacité ce mouvement spontané d'oscillation appelé mouvement brownien ou moléculaire. On rencontre ces mêmes granules, et en même quantité, sur la surface des spores elles-mêmes, ainsi que contre les cellules fibro-spiralées du sac des sporanges, et, comme on en constate la présence dans presque toutes les cellules de la tige des Equisetum ${ }^{2}$, il nous est impossible d'y voir autre chose que les granules restées libres après la résorption de tant de cellules, et surtout d'y voir avec M. Pringsheim «des points correspondant aux dessins cuticulaires et aux formes qu'on reconnaît sur la membrane extérieure de certains pollens et des spores des Fougères, des Mousses... ete.» (Schl. Eq., p. 244).

Lor'sque, avec un fort grossissement, on cxamine des élatères à l'élat sec et distendus, on remarque encore trois choses: $1^{\circ}$ la partie spatulée est marquée, ver's le commencement de sa dilatation, de lignes ou plutut de rides dirigées dans le sens de la longueur (pl. VIII, fig. 31 et 32); 20 ver's le même point cette partie est un peu con-

\footnotetext{
'M. Raspail a aftirmé la même erreur, en la doninant de plus comme une preuve d'un de ses principes de physiologie végétale : "Les organes reproducteurs des Equiselum sont de gros grains polliniques, nés sur l'entre-croisement de "deux spires" (Physiol. végét., 1837, vol. II, p. 4i6).

${ }^{2}$ Ils ont été, dès 1842, mentionnés dans les spores, jiar M. Henderson (Reprod. Eq., p. \$68).
} 
tournée en spirale; $3^{\circ}$ sur les fils on distingue des lignes i peu près parallèles entre elles, mais obliques el comme contournées en spirale autour du fil. Cette triple apparence s'explique par ce seul fait : $1^{\circ}$ que les fils qui, á l'état frais et d'enroulement, sont un peu plus larges qu’épais, se contractent suivant leur largeur en se desséchant, ce qui forme comme une ou deux rides sur la longueur et un plus grand nombre à la naissance de leur spatule; $2^{\circ}$ que pour se distendre en ligne plus ou moins droite, ils sont obligés, attendu leur nature de bandes originairement spiralies, de se tordre sur eux-mêmes, et alor's ces rides simulent des lignes spirales courant le long du fil'. Celte torsion s'arrête au commencement de la spatule, qui montre ainsi en plus grand nombre et en lignes parallèles la continuation des rides. Quelquefois à ce point la spatule se fend elle-mème suivant un de ces plis, quand la dilatation ou la contraction a élé trop subite.

II. Pringsheim qui, dans la notice précitée, a étudié tout spécialement ces apparences, les attribue à un autre ensemble de causes. Ce savant croit que les renflements en rubans spiralés, qui se forment dans l'intérieur de la cellule-mère, ne s'isolent pas par résorption des parois primitives de celte cellule, mais bien «que lorsque, a la maturité, la membrane de cette cellule se divise dans la direction et le long de ces renflements, ses lambeaux se déploient en prenant la forme de deux rubans spiralés, sur lesquels l'un des bords est épaissi el l'autre composé d'une lame mince; et qu'alors cette lame mince se replie spiralement autour de la partie épaissic... So schlägt « sich zugleich der unverdickte Theil dieser Bänder um den verdickten spiralig a herum, » et, d'autre part, "que cette partie mince du ruban est, comme la dilatation spatulée, marquée de stries, mais suivant une direction oblique à sa longueur» (Schl. Eq., p. 243). Ajoutons que, ainsi qu'il l'avait fait pour les granules moléculaires, M. Pringsheim considère ces stries comme analogues aux dessins cuticulaires de quelques pollens et de certaines spores.

On conçoit à la rigueur que si l'assertion précédente était admise, à savoir que la lame mince füt «marquée de stries obliques à sa longueur, » et qu’elle se repliât sur la partie épaissie, le fil total parût strié; mais alors on ne conçoit plus l'assertion principale que acette lame se replie spiralement autour de la partie épaissie. » Se replier sur une autre partie comme une moitié de feuille de papier sur l'autre moitié n’est pas «se replier spiralement.» Pour cela, il faudrait que la moitié mince ne fùt ni égale en longueur à la partie épaissie, ni à elle adhérente, mais au moins moitié plus longue et détachíe, car une bande, contiguë à une autre, ne peut, en se repliant, entourer spiralement cette dernière.

D'ailleurs l'examen le plus attentif des spores à tous les àges (mème avec un gros-

\footnotetext{
- Cette disposition se voil admirablement q̨uand on élire les laniẻres d'une sphère de caoulchouc découpée en élatères.
} 
sissement de 1100 diamètres) ne montre rien de semblable. Ces stries ou plutôt ces rides, visibles à l'état sec, disparaissent par un séjour de quelques instants dans l'eau, et plus vite et plus complétement dans une solution potassique très-étendue. Comme les fils s'y gonflent un peu, ils montreraient nécessairement alors leur partie latérale mince; ou bien il arriverait, si elle est enroulée en spirale scrrée, qu'elle se relàcherait sur quelque point, ce qui ne se voit jamais. M. Pringsheim semble avoir voulu prévenir cette objection en ajoutant: «On reconnaît encore que l'élatère est composé d'un renflement et d'une lame mince contournée autour de la première... Endlich a crkennt man die Zusammensetzung des Schleuderers aus einem verdickten und « einem um diesen sich windenden unverdickten Theil noch dadurch, dass... par cela que très-souvent, en suite de cet enroulement de la lame mince, celle-ci vers son extrémité se détache du cordon renflé» (pl. VIII, fig. 35 , copiée de M. Pringsheim). Cet effet est dû au déchirement de la partie spatulée, déchirement que l'on produit presque à volontẻ, en se servant pour porte-objet d'une plaque de mica très-mince et un peu humide, sous laquelle on fait passer un gros fil de fer chauffé très-fortement. Les élatères se déroulent alors et se contournent sur eux-mêmes avec une telle violence que le plus souvent ils se brisent en morceaux, et comme leur partie spatulée est une portion considérable de sphère, et par cela résiste à la torsion en spirale, elle se fend longitudinalement, comme une hart se fendille quand on la tord. Ce petit lambeau latéral figuré par M. Pringsheim, ou des lambeaux plus grands, ne sont donc point les excédants d'une lame mince qui s'enroulerait en spirale autour des fils épaissis, parce qu'il n'y a point de semblable lame; parce que ces fils n'ont aucun appendice latéral, et que, s'ils en avaient, on les verrait aussi bien que la petite irrégularité, saillie ou échancrure qu'on reconnaît parfois vers leur point d'adhérence; parce que rien ne s'entortille autour d'eux, ni ne se replie sur eux, attendu qu'ils sont simples, très-netlement limités et déterminés, sans aucune trace d'une cellule-mère qu'ils. auraient déchirée, et cela par la raison qu'ils ne la déchirent pas, mais, comme je l'ai vu avec la plus grande netteté, qu'après avoir commencé par des renflements ou lignes saillantes à l'intérieur de la cellule-mère et s'être épaissis, ces fils s'isolent par suite de la rẹsorption des parois de cette cellule.

Ces fils ont-ils, comme ceux des anneaux ou des spirales des vaisseaux, une cavité intérieure longitudinale? Pour ma part, je le crois, en m'appuyant sur ce que, à un fort grossissement, leurs bords présentent toutes les apparences d'un tube de verre éclairé par dessous et jamais celle d'un cylindre plein. Mais bien qu'ils se cassent fréquemment sur le porte-objet et offrent bien à l'observation leur bout cassé, la cassure en est très-inégale, et je n'ai jamais pu, comme sur les gros vaisseaux, distinguer l'orifice de la cavité de manière à pouvoir' en affirmer l'existence avec certitude.

A l'état de complet développement, les spores m'ont paru parfaitement sphériques. 
Elles ont le plus souvent la surface parsemée de ces granules moléculaires mentionnés tant de fois. M. Hofmeister dit que leur surface est fincment granulée, «feingekörnelt» (Vergl. Unters., p. 99); malgré mes efforts je n’ai rien pu voir de semblable. Ainsi que le fait observer M. J. Ilenderson (Reprod. Eq., p. 571), les spores trèsmùres et non humides ont la surface comme un peu ridée; lumectée d'une goutte d'eau, la spore redevient lisse et unie, et, en même lemps, se gonfle jusqu'à dépasser d'un quart son diamètre à l'état sec.

Hedwig non-seulement avait affirmé que les spores ont un stigmate en forme de papille «stigmate apiculata, » mais il avait figuré ce prétendu stigmate (Theor. gen., p. 33, tab. 1). Bischofi reproduisit la même assertion et le même dessin (Krypt. Gicw., p. 32; pl. IV, fig. 16, 1828), tout en faisant remarquer aque ce petit bouton obtus est « ì peine sensible, mit einem kaum bemerkbaren stumpfen línöpfchen.» Plus tard, dans ses Remarques sur le développement des Equisetum (1853), ce consciencieux savant exposa que, par suite de la faiblesse de son microscope, il s'était fié aux figures données par Iledwig, mais que depuis, avec un excellent microscope moderne, it avait reconnu que les spores sont exactement globuleuses et ne présentent pas d'éminence (Bemerl. Eq., p. 105).

Le même botaniste avait également décrit el figuré «l'épiderme des spores écrasces comme se déchirant suivant la direction d'une ou de plusieurs stries qui aboutissent à une tache transparente, limitée par des lignes sombres; ces stries paraissaient dessiner les parois des cellules dont l'épiderme des spores est composé » (Krypt. Gew., p. 40 , pl. IV, fig. 18); mais dans son travail de 1853 , cité plus haut, il reconnaît encore que l'épiderme des spores n'est pas composé de cellules comme il l'avait d'abord pensé'.

En effet, la membrane de la spore est, d'une part, sans aucune éminence, et de l'autre, non composée de cellules; mais elle n'est pas unique, et il y a, au contraire, deux membranes superposées. Comme toutes deux sont parfaitement unies et incolores, il est impossible d'en constater l'existence par le simple écrasement d'une spore. Pour y arriver avec certitude, j'emploie les moyens suivants. Sur une plaque très-mince de mica jétends une goutte de solution potassique (une partie de potasse caustique, cing parties d'eau) et j'y secoue le contenu d'un sporange. Aussitòt chaque spore prend une teinte bistrée unilorme, sans que les deux membranes se distinguent bien nettement. J'ajoute alors deux ou trois gouttes de la solution potassique et je place la plaque de mica au-dessus de la flamme d'une lampe à alcool, de manière à déterminer une légère ébullition. J'ajoute ensuite de l'eau sur la plaque de mica, et

\footnotetext{
1 Les firnures qque M. K. Müller a donuces des spores des Eịuisetum et de leur premier développenent (Der P/lansenslaat, p. 33t, 1861) sont tout å fait fautives. Qulques-unes $(b, c, d, e)$ représentent des spores hérissées! La figg. $h$ doit même ge pas appartenir aux Eqquisetum.
} 
la masse des spores agitée dans cette eau reprend aussitôt, à la vue simple, sa couleur verte; au microscope on voil la membrane extérieure très-distendue, formant une zone d'un rose pâle et un peu bistré, au dedans de laquelle nage très-distinctement la membrane intérieure enveloppant la matière verte (pl. IX, fig. 1). Quelques-unes des spores se gonflent si prodigieusement que leur diamètre est plus que doublé (pl. IX, fig. 2). Il n'est pas rare de voir, après quelques minutes, la membrane intérieure se fendre et répandre son contenu vert (pl. IX, fig. 3), ou la membrane extérieure se briser et se détacher en fragments ou calottes sphériques (pl. IX, fig. 4); on reconnaît alors avec la plus parfaite netteté que la surface en est absolument lisse. Si parmi les spores ainsi traitées il s'en trouve d'abortives ou d'infécondes, sans matière verte à l'intérieur, elles demeurent plus petites dans leur ensemble. On voit trèsneltement qu'elles se composent d'une membrane extérieure, qui se distend beaucoup moins autour de la sphère intérieure, et que celle-ci présente à sa périphérie deux cercles concentriques, ou, pour parler plus exactement, une membrane double (pl. IX, fig. 5).

Lorsqu'on traite les spores par l'acide sulfurique concentré, et comme je vais le dire, la distinction des membranes se manifeste, non avec plus d'évidence, mais d'une manière plus saisissante.

Une goutte de cet acide étant placée sur une lame mince de mica, on met des spores sur une autre lame que l'on renverse sur la première, et on place le tout rapidement au foyer du microscope. On ne voit déjà plus d'élatères; ils ont été brisés en fragments infiniment petits, qui se sont rangés vers les bords de la goutte d'acide, mais on voit la couleur des spores changer en allant de la périphérie au centre, de telle sorte que chaque spore présente un disque central, terminé par une ligne irisée, se rétrécissant assez rapidement jusqu'à ce que toute la spore soit d'un vert jaunâtre obscur. Alors, sur un point, ou sur tout le pourtour, on voit la membrane extérieure se dilater et former autour de la membrane intérieure une auréole faiblement bistrée, unie, si elle se dilate en liberté, mais plissée et irrégulière, si elle est comprimée par la plaque supérieure de mica (pl. IX, fig. 6). Après l'introduction d'un peu d'eau entre les deux plaques, et sans doute par l'effet de la chaleur qui en résulte, on voit au bout de quelques instants les membranes se briser et se détacher en calottes sphériques; et l'effet est général, si on le facilite en pressant légèrement avec un objet obtus les plaques de mica et en les faisant un peu glisser l'une sur l'autre. Toutes les membranes extérieures se brisent alors (pl. IX, fig. 4) et laissent échapper les sphères intérieures; celles-ci se colorent subitement en vert tendre un peu azuré, puis bientôt après en rouge brun.

Le contenu des spores est un liquide d'un beau vert jaunâtre, sur le bord duquel sc montrent souvent quelques gouttelettes d'huile, quand les 'spores se brisent dans 
l'eau on micux dans l'acide sulfurigue. Les spores des Equisetum ne s'enflamment point avec la même vivacité que celles des Lycopodes; elles pétillent un peu. Dès avant la sporove on y trouve des grains de chlorophylle assez nombreux et de grosseur trèsviniable. Ces grains augmentent en nombre et en volume avec une incroyalble rapiditi, si on laisse séjourner les spores à la surfice de l'ea!n. II. Nägeli parail avoir été le premier i y affirmer la présence d'un nucléus ou cytoblaste ${ }^{3}$; M. Hofmeister l'a mentionne et lui a attribué une forme sphérigue (Vergl. Unters., p. 99). M. Milde le décrit ainsi: "Ce cytoblaste a une structure en forme de disque; il est pourvu de deux on trois grains posés excentriquement. Il est transparent, sans couleur, et est suspendu au milieu de la spore par des fils mucilagineux » (Entu. Ey., p. 620, et aussi Ge'. Crypt. Schl., p. 414). Quelque peine que je me sois donnée, il m’a éte impossible do rien voir de semblable; et je n’ai pu constater la présence d'un nucléus que dans les spores commeneant à végéter après quelques heures de séjour sur la terre humide ou dans l'eau. M. Milde ajoute que ce cytoblaste est «la tache claire » que Bischoff menlionne. Je ne puis partager cette opinion; “la tache claire» de Bischoff n'a rien de commun avec le cytoblaste des éminents observateurs précités. D'abord, comme nous l'avons vu précédemment, Bischoff lui-même a reconnu son erreur au sujet de su lache cluire et des rayons qui en partaiont, non dans l'intérieur, mais sur l'épilerme des spores, et ì la p. 32, où il est encore question de ce point lumineux, Bischoff se borne à dire: "Sous le microscope les spores se montrent plus transparentes en leur milieu, «sie zeigen sich in der Mitte mehr durchscheinend. „ Or cette diffẻrence dans la transparence n'est due ni à une différence dans le contenu ni à une différence d'organisation dans la texture de l'épiderme. Il n'existe point en eflet sur les spores de région plus transparente que le reste. Leur enveloppe membraneuse díchirée, étendue autant qu'on peut le faire, ne présente aucun point de la surface différent des autres. Le contenu ne m’a point montré de nucléus, tant que les spores n'ont pas séjourné dans l'eau. Cependant il arrive fréquemment qu'en observant des spores au microscope, on voit exactement dans la direction du centre une région parfaitement circulaire, neltement limitée, trés-rivement lumineuse et dès lor's paraissant plus transparente que le reste. Mais si l'on place les spores sur une goulte d'eau étendue, el qu'on incline un peu le microscope, on voit bientòt l'eau se déplacer, et les spores qu'elle entrante traverser le champ en roulant, et quelte que soit leur position, conlinuer à offrir ce petit cercle virement éclairé et comme transparent; d'où cette première conclusion que ce cercle lumineux ne peut appartenir à un point spécial et déterminé de l'enveloppe de la spore. Reste que re point circulaire brillant révèle

\footnotetext{
' Schleiden u. Nägeli, Wachsthumsgeschichte der Laub-u. Lebermoose, dans Zeitschrift für wissensch. Bofan., ques Meft, Zürich $18+5$.
} 
l'existence d'un espace ou d'un organe central plus transparent; mais on devra mieux voir cet organe, lorsqu'on placera au foyer de l'instrument le grand cercle qui forme l'horizon des spores observées. Or, à mesure qu'on rapproche les spores, le cercle lumineux s'élargit en diminuant d'éclat et finit par disparaitre quand leur centre est au foyer. On ne l'aperçoit que lorsqu'on éloigne les spores et qu'on place au foyer l'extrémité supérieure de leur diamètre vertical; d'où il suit, comme seconde conclusion, que ce point brillant n'est pas un phénomène d'organisation, mais un simple effet d'optique. La spore étant un corps sphérique, diaphane, plus dense que le milieu ambiant, réfracte les rayons lumineux qui lui viennent du miroir, et les fait converger vers l'extrémité supérieure de son diamètre vertical. Ils y forment alors un petit cercle de vive lumière, que Bischoff et d'autres après lui ont pris pour un espace circulaire plus transparent que le reste de la surface. Voici une autre preuve très-concluante de la vérité de cette assertion. Si l'on graisse légèrement la surface du verre porteobjet (par exemple en la frottant contre les cheveux) et qu'avec un pinceau on y projette de loin et doucement de très-petites gouttelettes d'eau, celles-ci prendront la forme sphérique et offriront exactement les mêmes phénomènes de la présence ou de la disparition d'un cercle lumineux central, lorsqu'on rapprochera ou qu'on éloignera du foyer leur partie supérieure. M. G. Thuret dit également des spores des Fucacées: «Les spores sont d'un jaune olivâtre; elles renferment chacune un globule central plus clair, qui est problement un nucléus, mais que je n'ai pu parvenir à isoler (Fécond. Fuc., p. 202). Celte assertion d'un observateur si habile et si consciencieux m'a fait recommencer bien des fois l'examen de la tache claire de Bischoff et la recherche d'un nucléus; mais le résultat a toujours été le même.

J'ai dit plus haut, p. 91, que les spores abortives et infécondes, qui se trouvent parmi les spores normales, sont toujours plus petites et demeurent telles sous l'action des réactifs, et qu'en outre elles présentent une membrane intérieure double (pl. IX, fig. 5). Comme à ces spores n'adhèrent jamais des élatères, ce qui semble être leur membrane extérieure est la membrane de la cellule-mère qui devait, par sa division en une double spirale, donner naissance aux élatères, et les deux membranes intérieures sont les deux membranes ordinaires des spores. Ge qui exclut tout doute sur ce point, c'est que parmi ces spores j'en ai maintes fois observé qui, à l'état naturel ou traitées par la solution polassique, présentaient encore sur la sphère intérieure celte dépression mentionnée plus haut, vis-à-vis de laquelle on voyait très-nettement deux membranes: l'une plus épaisse, à courbure convexe et continuant la forme sphérique, c'était le sporoderme extérieur; l'autre plus mince, à courbure concave et limitant la dépression (pl. IX, fig. 7), exactement comme on le remarque sur l'arantdernier état des spores.

Les spores infécondes ci-dessus décrites se montrent par exception, et toujours rela- 
tivement en très-petit nombre dans les épis vraiment fertiles et bien constitués; j’ajouterai mìme quion ne les rencontre guère que dans les sporanges des épis les plus développés el sur les liges les plus vigoureuses des $E$. arense, maximum, ramosissimmm. Or on troure des spores abortives d'une tout autre forme sur d'autres Equisetum, par exemple sur les E. litlorale, trachyodon, el sur la plupart des épis trèstardifs de l'E. limosum. Mais si, dans le premier cas, les spores infécondes sont l'exception, ici elles constituent la totalité et ne sont accompagnées d'aucune spore bien constituée.

Comme les premières, et plus qu'elles encore, elles restent fort au-dessous de leur grosseur normale; quelques-unes sculement ont les deux tiers du diamètre, peu de matière verte, des élatères ébauchès et incomplets dont elles ne sauraient sortir; les autres, et ce sont les plus nombreuses, beaucoup plus petites et réduites à un tiers ou mème à un cinquième du diamètre normal, sont irrégulièrement sphériques avec un côté plus saillant; forme qui atteste le défaut d'élasticité, et paraît résulter de leur compression réciproque quand elles étaient par quatre en groupes tétraédriques. Elles présentent assez neltement deux membranes et une sphère centrale remplie de granules presque incolores (pl. IX, fig. 9-10); d'autres sont encore unies deux à deux, d'autres quatre à quatre; et enfin on y trouve des cellules génératrices arec une cloison de séparation et deux nucléus (pl. IX, fig. 11). Le tout nage dans un liquide un peu visqueux, lentement et difficilement soluble dans l'eau et pas du tout dans la solution potassique. Trailies par celte solution, ces spores ne se comportent pas comme les spores normales: leur membrane extérieure ne se dilate pas, ne se colore pas; seulement l'intérieur parait un peu plus vert.

Sur tous les épis qui contiennent ces spores, le sac des sporanges, au lieu d’être gonflé et vert par transparence, est fiasque et blanc, attendu l'absence presque complète de liquide vert dans les spores. Ce même sac, au lieu de s'offrir sous la forme définitive et dernière, c'est-ḋ-dire avec des cellules fibro-spiralées, s'offre avec l'apparence qui précède, sous son arant-dernière forme, c'est-à-dire avec des cellules simples, comme je les ai décrites et figurées plus haut, mais sans aucun nucléus (pl. VIII, figg. 28, abstraction faite des nucléus). Ces cellules ont d'ailleur's la mème disposition relative que les cellules fibro-spiralées (pl. VII, fig. 19), et seulement un peu plus de largeur, comme si la spirale interne avait forcé les cellules à se contracter un peu. Il n'est pas rare de voir au-dessous de cette couche de cellules simples des fraggments d'une autre couche, qui était la couche moyenne et n'a pas été entièrement liquéfiée et rẻsorbẻe.

Il est dés lors évident quill y a eu dans tout l'appareil fructifère un temps d'arrêt, qui n’a pas permis aux divers organes d'atteindre leur dernier développement. Or, si l'on remarque que ces sortes de spores abortives ne se présentent que sur des espèces qui 
§ 1. - DÉVELOPPEMENT DES SPORES EN SPOROPIIYMES.

ont des rhizomes d'un extrême développement, ou sur des pieds tardifs ou appauvris par des conditions défavorables, tandis que les autres spores abortives s'offrent sur les sujets les plus vigoureux, on est naturellement porté à conclure qu'il y a à ces états abortifs deux causes très-diffẻrentes. Les dernières spores décrites sont abortives par arrêt et par défaut d'évolution, soit que la force vitale s'épuise dans les rhizomes ', soit que l'épuisement provienne des circonstances défavorables; les autres, celles des sujets les plus vigoureux, sont abortives par excès d'évolution, parce que, dans cette violence de développement et de division des cellules génératrices, il y a eu une multiplication de trop, un dédoublement en excès, et qu'alors la force a manqué après ce dédoublement pour que ces spores arrivassent à leur dernier terme en même temps que leurs congénères, ayant eu à franchir un degré de plus.

\section{GHAPITRE IV}

\section{Reproduction}

\section{$\S 1^{\mathrm{er}}$. Développement des spores en sporophymes}

Les spores des Equisetum ne paraissent pas conserver indéfiniment, comme les spores des Fougères, leur faculté végétative, et même elles la perdent quelques jours seulement après la sporose. Sur aucune espèce je n'ai pu la voir se prolonger au delà du dixième jour. M. Milde l'a constatée jusqu'au quatorzième (Entw. Eq., p. 621).

On reconnaît de suite qu'une spore est devenue incapable de développement à ce qu'elle ne présente plus le petit cercle lumineux mentionné ci-dessus p. 92. Elle offre, comme les spores anciennes et desséchées, l'aspect d'une masse sphérique, grisâtre, opaque, à surface finement chagrinée ou plutòt ridée. Après quelques minutes de séjour dans l'eau, ces spores redeviennent diaphanes, et laissent voir de nouveau avec une grande netteté le petit cercle d'un vert jaunâtre très-brillant. $\mathrm{A}$ mesure qu'elles reprennent leur transparence, on parvient à distinguer que leur membrane intérieure s'était contractée et tellement séparée du sporoderme extérieur qu'elle y était flottante, comme dans un sac trop large.

\footnotetext{
- La plupart des graminées à rhizomes traçants demeurent constamment stériles quand l'élongation de ce rhizome est extrême (exemple: Agrostis alba, Cynodon Dactylon, Phragmites communis etc.). Il en est do même sur beaucoup d'autres plantes, ainsi que sur les Mousses. Un moyen de propagation semble suppléer l'autre (roy. Annotations a la $A$. d. Fr, et d'All., p. 112).
} 
On peut appliquer ici ce que M. Alph. De Candolle dit des graines en général: "La durée de la faculté de germer est le plus souvent en raison inverse de la faculté "de germer vite» (Ann.sc. nat., 3 e sér. bot., VI, p. 382); car si, d'une part, les spores des Ėquisetum perdent vite leur faculté ségétative, d'autre part cette faculté entre trèspromptement el très-facilement en exercice. Dans le papier gris de dessiccation, les spores qui se répandent à còlé d'un épi un peu gros, trouvent là assez d'cspace et assez d'humidité pour commencer à régéter et à émettre leurs premières cellules. Ce développement s'arrête bientôt, comme on peut le penser; les traces s'en retrouvent à côté des épis desséchés, sous la forme de petites masses pulvérulentes, grisàtres. $\mathrm{Si}$, après un séjour de quelques minutes dans l'eau tiède, ces masses informes sont placées sur le porte-objet, elles laissent voir soit les premières cellules des spores qui ont commencé i se développer, soit d'autres spores qui n’ont pas végété, soil enfin des élatères plus ou moins brisés qui feutraient le tout.

Des spores fraiches, prises au moment de la sporose et répandues sur l'eau ou sur la terre humide, doment après quelques heures les premiers signes de développement. Elles se gonflent notablement, et les granulations vertes augmentent trèsrapidement en nombre et en volume. Un nucléus devient très-visible et présente le plus souvent deux nucléoles; puis au nucléus primitif il en succède ordinairement deux, dont la position est indiquée par les nombreux grains de chlorophylle qui s'amassent à leur circonférence. Au bout d'un jour, la spore se dédouble en deux cellules trèsinégales et très-différentes (pl. IX, fig. 12). L'une, de beaucoup la plus grande, contient presque tous les grains de chlorophylle; l'autre, beaucoup plus réduite, n'en renferme qu'un petit nombre, réunis près de la paroi de séparation; le reste de son contenu ne parait ètre qu'un mucilage à grains fins. C'est celle-ci qui donnera la première radicelle. Le plus souvent les nucléus sont nettement visibles et se montrent indifféremment contre les parois ou vers le centre; mais souvent aussi je les ai cherchés sans en pouvoir découvrir la plus légère apparence.

Pendant ces premiers développements le sporoderme extérieur, qui s'était un peu élargi d'abord, se brise tout à fait et le jeune sporophyme s'en débarrasse (pl. IX, fig. 14 $(t)$. Ses débris incolores et ceux des élatères ne sont facilement visibles que lorsquu'ils sont retenus dans des groupes de spores répandues très-dru et se développant très-serrées. Le rôle des élatères paraît être nul dans le développement des spores et se borner à en favoriser la sortie du sporange et la dispersion.

Il est assez ordinaire de voir, dis la fin du premier jour ou dès le second, lapparition de la première radicelle (pl. IX, fig. 12). Elle se montre d'abord comme une grosse papille, puis comme une extension filiforme de la cellule la plus petite; elle est incolore, diaphane, arrondie à son extrémité inférieure, et offre quelques grains d'apparence mucilagineuse accumulés vers ses deux extrémités. Quoiqu'elle naisse 
directement, et sans cloison aucune, de la cellule inférieure, dont elle n'est qu'une extension, la chlorophylle de cette cellule n'y pénètre pas, et, d'abord accumulćc contre la paroi de séparation, elle finit par disparaître. La radicclle, évaséc à son point d'origine, se rélrécit subitement, et, ainsi que les radicelles qui la suivront, elle est sur toute sa longueur sans aucun diaphragme et d'une grosseur égale. Toutefois j'en ai vu, à des sujets semés sur l'eau, qui présentaient vers leur tiers inférieur des renflements d'un diamètre plus que double, renfermant une matière verdâtre et comme des grains de chlorophylle en voie de formation.

Les premières cellules du sporophyme sont très-riches en chlorophylle, et la parfaite transparence de leurs parois rend très-facile l'observation des granules de cette substance. Dans les premiers jours ils ont ordinairement une forme elliptique, et plusieurs, après qu'on les a fait sortir des cellules et qu'on les a écrasés, laissent voir très-nettement de deux à cinq nucléoles peu transparents, dont la teinture d'iode révèle la nature amylacée. On voit tout aussi nettement une cloison transversale de séparation apparaître entre ces deux nucléoles, et partager ainsi les granules de chlorophylle comme en deux petites cellules, contenant chacune un nucléole. Ce fait a déjả été très-exactement observé et décrit par M. Milde, en 1852 (Entw. Eq., p. 624 et 625). Des faits analogues sont mentionnés par MM. Nägeli (Zeitschr. f. Botanik, Heft III, S. 110), Göppert et Cohn (Bot. Zeit., 1849, p. 665), et enfin par M. Hofmeister, qui cite même la chlorophylle des Equisetum (Vergl. Unters., p. 10 et 11, note).

L'apparition des cellules ultérieures manifeste dès le principe une assez grande variété dans la forme de l'ensemble (pl. IX, fig. 13, 14, 15, 16). Elle a lieu, sans ordre bien déterminé, par multiplication le plus souvent transversale d'une ou de plusieurs cellules du sommet, et il n'est pas rare de trouver jusqu'à sept cellules superposées et dues uniquement à ce mode de multiplication. La multiplication longitudinale est moins fréquente et n'a guère lieu que pour les cellules de second degré; mais les cloisons transversales de séparation sont souvent si obliques qu'elles simulent des cloisons longitudinales. Les bifurcations et les ramifications latérales se produisent bientôt et en très-grand nombre. Les premières sont dues ordinairement à la multiplication de deux cellules de l'extrémité, divariquées à leur origine. Les secondes paraissent dues à un dédoublement, qui a lieu tardivement et sur le côté d'une cellule marginale déjà ancienne et surmontée d'autres cellules. La nouvelle cellule latérale est le point de départ d'une expansion en forme de rameau, laquelle suit dans son développement ultérieur le même mode de multiplication que les expansions primitives, qu'elle dépasse souvent en vigueur et en dimension. En général le dédoublement d'une cellule quelconque est précédé, à l'endroit et dans la direction où doit se montrer la cloison de séparation, d'une agglomération de grains de chlorophylle, dis- 
posés en forme de bande, comme s'ils étaient repoussés de part et d'autre, bien qu'il soil souvent impossible d'y distinguer des nucléus.

La production des sept ou huit premières cellules est trìs-rapide, mais il y a ensuite constamment un temps d'arrèt, qui dure presque une huitaine de jours.

A mesure que le sporophyme se développe, de nombreuses radicelles apparaissent sur les cellules les plus rapprochies du sol. Elles ressemblent à la première et sont, comme elle, sans diaphragme. Comme la première encore, chacune de ces radicelles n'est que l'expansion filiforme de la paroi libre d'une cellule. M. Milde, après avoir exposé que les radicelles paraissent d'abord sous forme de papille incolore, puis s'allongent en fil rempli de mucilage, répète, à quatre reprises différentes, "qu'il se forme, entre la radicelle et la cellule qui lui a donné naissance, une cloison de séparation 》 (Entw. Eq., p. 624, 626, 628, 629). Je n'ai pu rien voir de semblable, comme je l'ai déja indiqué p. 79, en parlant de la première radicelle. Celle-ci provient de ce que, après le dédoublement de la spore en deux cellules, la paroi libre de la cellule inférieure s'étend successivement en papille, puis en radicelle. Or il en est exactement de même pour les autres radicelles; ce qui est d'autant plus facile à constater que les cellules d'oủ elles naissent sont plus grosses. J'ai vu sur la paroi libre de ces cellules s'élever une papille incolore et sans chlorophylle, puis celle papille s'allonger en radicelle; j'ai vu en mème temps que la cellule d'oủ elle part perd sa chlorophylle, diminue de largeur à mesure que la radicelle angmente; j'ai souvent constaté que la partie supérieure et dilatée de la radicelle est constituéc par le reste de la cellule-mère un peu plus saillante, et tout aussi souvent que cette cellule persisle sous sa forme première à peine altérée; mais je n'ai jamais pu voir se produire une cloison de séparation entre la radicelle et sa cellule-mẻre, et celle-ci subsister séparée de l'autre, comme cellule devenue indépendante.

La masse du tissu cellulaire du sporophyme ne dépasse point, même sur les plus complets et les micux développés, quatre millimètres en longucur, et n'offre guère une forme déterminée et définie. Tantôt les sporophymes sont constitués par une expansion multilobulée, plus ou moins digitée et ramifiée, étroite à la base et se dilatant en éventail arec des radicelles sur la moitié de la face inférieure (pl. IX, fig. 17); tantùt cette expansion se relère par les côtés de telle sorte que les lobes et leurs ramifications sont comme latéraux ả une région médiane contiguë au sol dans sa moitié inférieure, d'où naissent les radicelles. Ce sont là les formes les plus ordinaires; mais on troure toutes les variations possibles, avec des radicelles sur tout un côté, ou ả la base sculement, ou jusque vers le sommet. On ne trouve donc point dans les sporophỳmes des Equisetum l'élégante symétrie et les formes déterminées que présentent ordinairement ceux des Fougères. Il y a bien une apparence de symétrie, en ce que sur les deux formes les plus ordinaires et décrites plus haut, on peut remarquer une 
ligne médiane, de chaque côté de laquelle s'élèvent les lobes ramifiés de manière à simuler une feuille très-multifide pliée en deux, par exemple une feuille de chicorée frisée (Cichorium Endivia L., vas crispum); mais il arrive aussi que l'un des nombreux lobules se développe seul dans toutes les dimensions el sans aucune symétrie.

Les différences que présentent les sporophymes suivant les espèces sont si faibles qu'elles ne méritent pas une mention particulière. Je n'ai pu en saisir aucune entre les sporophymes des $E$. arvense, limosum et palustre. Les sporophymes des $E$. maximum et sylvaticum présentent des expansions plus larges et moins divisées; ceux des E. ramosissimum et variegatum m'ont paru au contraire plus grêles et plus allongés dans toutes leurs parties.

\section{Des anthéridies et des spermatozoïdes}

Cinq semaines environ après les premiers développements des spores, les sporophymes ont alteint une longueur de deux à trois millimètres; et alors, à la pointe terminale des lobules, ou aux pointes latérales voisines on voit apparaître des renflements d'un vert très-intense.

Ces renflements ou épaississements précurseurs des anthéridies sont des agglomérations de plus petites cellules (pl. IX, fig. $20 a, b, c$ ), dues à la division plusieurs fois répétée d'une des cellules du bord du lobule. Cette cellule, qui est la cellule-mère des anthéridies, se distingue tout d'abord par sa forme plus ovoïde et moins allongée, et surtout par sa couleur plus uniformément verte, due à une grande quantité de mucilage avec granulations vertes très-fines. Elle se multiplie en premier lieu par deux divisions longitudinales presque simultanées en quatre cellules se rencontrant à angle droit sur son grand axe (pl. IX, fig. 20a)'. Bientôt ces nouvelles cellules se dédoublent transversalement ( $\mathrm{pl}$. IX, fig. 20 b). Des divisions dans le sens de la longueur sẻtendent de nouveau presque du centre à la périphérie (fig. $20 c^{\prime}$ ). Toutes ces cellules en forme de petits prismes, soit triangulaires, soit quadrangulaires, dont la face la plus large est à la périphérie, ne tardent pas à se diviser parallèlement à cette face en deux cellules très-différentes. L'une, située vers le centre, est remplie d'un liquide mucilagineux à grains très-fins, l'autre extérieure, large et plate (pl. IX, fig. 21). L'ensemble de ces dernières constitue la couche extérieure ou l'enveloppe de la jeune anthéridie. Elles offrent d'abord quelques grains de chlorophylle, et sont toujours un peu plus tard remplies d'un liquide diaphane faiblement coloré en vert jaunâtre. Celles du sommet, au nombre de six le plus ordinairement, ne sont remplies que de ce liquide. Quand on observe une anthéridie en cet état ou plus avancée, les cellules extérieures occupant le point le plus élevé et placées pour l'observateur

\footnotetext{
1 Je n’ai pu reconnaître le mode de seymentation indiqué par M. Hofmeister (Vergl. Unters, p. 100).
} 
i) lit région centrale de cet organe couché sur le porte-objel, présentent nécessairement leur surface la plus large el leur moindre épaisscur; les cellules des côtés et du sommet présentent au contraire leur face étroite et latérale, et sont vues dans le sens de leur plus grande épaisseur; d'où vient qu'elles paraissent plus itroites et plus colorées rque les autres, et qu'elles sinuulent une zone ou a anneau plus brillant qui entoure l'anthéridie» (Milde, Spor. Eq., p. 15, et Entw. Eq., p. 631). Mais comme celle apparence persiste, dans quelque sens qu'on tourne l'anthéridic couchée, et comme on voit par une coupe transversale que les cellules forment une enveloppe continue et uniforme (pl. IX, fig. 22), il s'ensuit que ce prétendu anneau n'est qu'une apparence résultant de la position des cellules et non la manifestation d'un organe spécial, d'un anneau latéral, qui entourerait l'anthéridie, ainsi que M. Milde l'a cru et figuré 1.

Revenons i notre description. Nous avons vu que l'anthéridie naissante se présente sous la forme d'un renflement ovoïde un peu comprimé, ayant une enveloppe de cellules larges, faiblement colorées en vert, et au centre un groupe de cellules en forme de prismes triangulaires el contenant un liquide peu coloré (pl. IX, fig. 21). Cet état ne dure que peu de temps, un jour ou deux, et bientôt le groupe central se multiplie, par des divisions en tout sens, en une multitude de très-petites cellules, presque cubiques, très-intimement unies, et dans chacune desquelles on voit un globule ellipsoïde, aplati, à contenu liquide (pl. IX, fig. 22). Peu après, les parois de ces cellules, si solidement unies d'abord, disparaissent comme si elles se liquéfiaient; on n'en voit plus aucune trace, et les globules sont isolés en liberté, sans qu'on remarque en eux de changement notable. Ce n'est qu'au bout de quelques jour's qu'on voit se dessiner à l'intérieur de ces globules une zone très-transparente, incolore, fixée contre la circonférence du disque, en occupant les trois quarts et y formant un anneau incomplet, dont les cxtrémités sont un peu et inégalement renfées. L'espace restant est occupé par une masse mucilagineuse, dans laquelle apparaissent des granulations tris-fines et d'autant plus nombreuses qu'elles se rapprochent davantage de la zone t'ansparente ct incolore (pl. IX, fig. 25). C'est lả la première apparence sous laquelle se montrent les spermatozoïdes. Le groupe ovoïde que les cellules-mères forment au centre de l'anthéridie paraît quelquefois sous le microscope comme coloré en rouge tendre, et d'un rouge plus brun à la simple loupe. Cette couleur m'a paru inhérente à la paroi interne de l'enveloppe de l'anthéridie et non aux spermatozoïdes, ainsi que je l'expliquerai plus loin.

\footnotetext{
- Dic ersten Anfinge der Antheridien zeigen sich uns in Gestalt von grünen Kugeln, um welche man einen helle- ren, aus melireren tafelformigen Zellen gebildelen Ring herumgelegt sieht. Entw. Eq., p. 631; tab. LVIII, fig. 38 et 39). It. W. Ph. Schimper a fait voir dès 1857 qu'on avait eu tort d'attribuer un semblable anneau aux anthéridies des Sphagnum (Sphag., p. 41).
} 
Presque simultanément, ou un jour ou deux après l'apparition de ces phénomènes, les cellules terminales de l'anthéridie se disjoignent au centre du sommet et s'écartent en simulant une couronne ouverte (pl. IX, fig. 23 b). C'est li le signe évident de la maturité de l'anthẻridie, mais ce n'est pas celui de la sortie des spermatozoïdes. Si un sporophyme, chargé d'anthéridies arrivées à ce point, est mis à sec sur le porteobjet, on n'en voit rien sortir, à moins de provoquer par une pression l'expulsion du contenu des anthéridies; mais si on le recouvre d'une goutte d'eau, et surtout si c'est le matin ou le soir, on en verra presque immédiatement les globules sortir par jets régulièrement intermittents (pl. IX, fig. $23 c$ et $24 c$ ). Ces globules sont assez gros (pl. IX, fig. 25), et leur diamètre peut être évalué à un centième et demi de millimètre. Leur nombre est très-considérable, et, comme M. Milde, j'en ai trouvé plus de cent cinquante dans chacune des anthéridies oủ je les ai comptés. Ils sont ả peine sortis qu'on les voit frémir, s'agiter et osciller à la façon d'un balancier de montre. La cause de ces mouvements paraît résider dans la zone incolore qui alternativement s'étend et se replie sur elle-même. On a à peine quelques instants pour observer ces oscillations, ct l'on voit aussitôt des'spermatozoïdes à la place des globules. Telle est, d'une part, la rapidité et, de l'autre, la variété de leurs mouvements qu'il est trèsdifficile de les suivre et plus facile de dire en détail ce que l'on voit que de décrire d'une manière exacte leurs mouvements et leur véritable forme. Les uns ressemblent à une ammonite en rotation dans un plan horizontal, d'autres à un croissant qui tournerait en rapprochant et étendant alternativement ses deux extrémités. D'autres reproduisent la forme serpentante d'un fouet et s'avancent obliquement en tournoyant (pl. IX, fig. 26, 27). On ne peut distinguer en eux de forme saisissable et déterminée que quand ils s'attachent par une extrémité à quelque point du sporophyme, ce qu’ils font d'ailleurs assez fréquemment. On reconnaît alors les formes que leur ont attribuées d'abord M. G. Thuret, puis MMI. Hofmeister et Milde; ce sont celles d'une bande vermiforme tordue en spirale senestre ${ }^{1}$. Le nombre des tours de spire est le plus souvent de deux et demi à quatre; un des tours est très-lâche, et la partie de la bande qui le forme est nue, plus large, mais plus mince; les antres sont extérieurement munis de cils nombreux, courts et forts, qui se remuent avec une extrême vitesse pendant la vie et surtout pendant le mouvement. On ne distingue aucune autre trace d'organisation dans ces petits êtres, dont la substance paraîtêtre mucilagineuse et comme visqueuse. M. G. Thuret représente la partie la plus ténue comme pourvue du côté intérieur d'une membrane mucilagineuse mal terminée (Zoosp. Alg., p. 79,

\footnotetext{
' Senestre signifie pour moi le sens opposé à celui du filet d'une vis ordinaire. M. G. Thuret a figure les spermatozoides avec une spirale senestre, M. Milde avec une dextre, et M. Hofmeister avec une senestre (Vcrgl. Uniers., tab. XX, fig. 52, 53, 56), et avec une dextre, ט. c., fig. 54, 55; et hennln. Gef. Crypt. les fig. 1, 6 sont dextres ef les $f_{g} .2,3,4, b$ sont senestres.
} 
pl. XV, fig. 2); et M. Ilofmeister dit expressément: "l’extrémité du fil séminal porte au côté intérieur de la partie hélicoïde un large appendice en forme de nageoire, membrane délicate qui pendant le tournoiement du fil vibre vivement, semblable aux membranes ondulantes que portent les fils séminaux des crapauds et des tritons. Pendant un mouvement tant soit peu rapide des fils séminaux, cette bordure membraneuse est, comme les cils de l'extrémité antérieure, tout à fait invisible; ce n'est que par la paralysie du mouvement vital du fil séminal que l'apparition en devient distincte » (Keim. Eq., p. 385). J'ai bien vu, et cela très-souvent, que, à son tour de spire le plus lìche, la bande du spermatozoïde est plus large et plus mince vers l'intéricur, mais je n'ai pu reconnaìtre sur cette membrane une forme arrêtéc et définie. Les cils sont tout à fait invisibles pendant le mouvement; on les devine, il est vrai, à une sorte de frémissement du liquide autour du spermatozoïde; mais on ne les distingue bien que lorsque celui-ci est tué par l'iode ou qu'il s'attache à un objet par son extrémité non ciliée, laquelle devient alors la plus déliée. Dans le premier cas, le spermatozoïde se roule sur lui-même en forme d'escargot, et les cils paraissent à l'extéricur en irradiant obliquement. Le phénomène est tout autre quand les spermatozoïdes s'attachent par leur extrémité à un point du sporophyme; on voit alors cette cxtrémité se terminer en un long fil d'une ténuité extrême (pl. IX, fig. 28), et que je n'ai jamais pu voir dans aucune autre position, ni pendant leur vie ni après lcur mort. $\Lambda$ u premier moment de l'adhérence, cet appendice filiforme paraît assez court; on dirait qu'il s'allonge par suite des mouvements violents auxquels le spermatozoïde se livre comme pour se détacher. On a sous les yeux un effet analogue à ce qui se passe quand on met en contact avec un objet de la cire à cacheter ramollie et qu'on la retire en la faisant filer. De là je conclurais volontiers, avec M. IIofmeister, "que l'extrémité postérieure du fil séminal consiste en une masse molle et sêmi-fluide, qui se colle facilement en un endroit quelconque et s'étire en long fil „ (Keim. Eq., p. 386), mais je préfère me borner à signaler ce que j'ai pu voir.

Les organes de locomotion sont les cils vibratiles, et peut-être cette membrane qui, d'après M. IIofmeister, «vibre si vivement pendant le mouvement, mais ces cils ne sont point les organes directs de la translation. Ils n'impriment aux spermatozoïdes qu'un mourement de rolation, que leur forme en hélice change nécessairement dans le liquide en un mouvement de translation, dont la vitesse est en rapport direct avec celle de la rotation. Laquelle des deux extrémités est en avant dans le mouvement? C'est ce que je n'ai pu déterminer avec une parfaite certitude. Comme M. IIofmeister (Keim. Eq., p. 385, et Kemutn. Gcf. Crypt., p. 169), j’ai vu fréquemment en avant la partic ciliée et étroitement spiralée, mais presque aussi souvent jai cru voir la translation s'opérer latéralement ou même dans le sens directement opposé, comme le dil .I. Milde, qui appelle « tète la partie dépourvue de cils » (Entw. Eq., p. 633). 
Toutes les différences de mouvement et de forme décrites plus haut sont, pour la plupart, des apparences résultant de ce que la translation du spermatozoïle est verticale, ou horizontale, ou oblique. Beaucoup d'autres très-variées sont dues à ce que les spermatozoïdes ne parviennent pas toujours à se débarrasser complétement du globule discoïde qui les contient. Tantôt ils ne sortent que leur moitié la plus large, entrainent avec eux le reste du globule et ressemblent à des lézards à queue spiralée; lantôt c'est l'autre moitié qui sort avec un ou deux tours de spire; d'autres fois, et très-fréquemment, les cils seuls sortent d'une fente des bords et fonctionnent comme des rames pour imprimer au globule un rapide mouvement de rotation sur lui-même (pl. IX, fig. 28 b). Enfin, si à la forme qui semble la forme normale de ces êtres l'on veut rattacher l'apparence qu'ils offrent quand ils sont encore dans le globule, c'està-dire celle d'un anneau diaphane étroit et ouvert, avec ses deux extrémités renflées, mais sans aucun indice de cils ou de spirale, on le peut en se représentant le spermatozoïde étendu contre la circonférence du globule discoïde et les cils vibratiles couchés contre la paroi marginale. L'extrémilé dénuée de cils et un peu plus large constitue le moindre renflement, et la partie opposée, ultérieurement roulée en spirale serrée et alors repliée vers l'intérieur, constitue la plus renflée des deux extrémités. Le reste de la cellule serait alors occupé par la masse mucilagineuse et à grains fins des figures de M. G. Thuret, qui est la nageoire de M. Hofmeister.

Après la mort, les spermatozoïdes se contractent et reprennent une forme assez analogue à celle qu'ils présentaient dans leur globule. On ne voit plus de spirale, mais un anneau ouvert, obtus à ses extrémités, avec cils à l'extérieur et membrane mal définie à l'intérieur; le tout est coloré en roux clair. Gette couleur est aussi celle que prennent les cellules-mères et leur contenu, si, avant l'émission de ce dernier, elles sont frappées de mort dans l'anthéridie. Comme je n'ai jamais pu trouver aucune couleur au spermatozoìde vivant, et que cette couleur roux clair est généralement celle qui, aussitôt après la sortie des globules à spermatozoïdes, affecte la paroi interne des cellules formant l'enveloppe de l'anthéridie, j'incline à croire que la faible couleur rouge que l'on voit à la masse des spermatozoïdes avant leur sortie ne leur appartient pas, mais est due à un commencement d'altération dans la couleur de cette même paroi. Après l'émission de son contenu, l'anthéridie revêt une couleur de plus en plus foncée, et finit par former, au sommet des lobules, de petites boules de la couleur brune des feuilles mortes. Cette couleur se communique rapidement aux cellules voisines, et, comme les sporophymes anthéridiferes ne se composent que d'une ou de deux expansions de tissu cellulaire et que leur chlorophylle tire un peu sur le jaune, ils ont très-vite un aspect maladif.

Quant à ce que deviennent les cellules ou plutôt les globules, après que les spermatozoides s'en sont dégagés d'une manière normale et complète, je l'ignore; je n'ai 
jamais vu la moindre trace de ces cellules vides, soit que ce qui en subsiste se fonde dans l'eau, soil que la parfaite transparence et l'extrême ténuité de leurs restes les dérobent à notre vuc, soit enfin qu'il n'y ait pas de restes appréciables et que la cellule soit résorbée à mesure que se forme le spermatozoïde. J'incline d'autant plus vers celte opinion, qu'au moment de leur apparition en liberté, je n’ai jamais vu les spermatozoïdes sortir de leur cellule et la laisser, mais ces cellules semblent s'currir et se transformer en spermatozoïdes. Ceux qui restent engagés en partie dans leur cellule seraient alors ceux qui sont incomplets et qui ne l'ont pas résorbée tout entière. L'action de l'eau, qui facilite le dégagement des spermatozoïdes, pourrait aider à cette résorption. Les globules à spermatozoïdes sont au premier moment de leur sortie de l'anthéridie entourés d'une atmosphère mucilagineuse, car des infusoires que je placais ì dessein sur le porte-objet, en passant à distance, les entrainaient dans leurs mouvements. Mais au bout de quelques minules il n'en était plus ainsi, et cetle sorte d'atmosphère visqueuse s'était dissoute.

L'émission des globules hors de l'anthéridie est accompagnée de celle de granulalions opaques, comme grisàtres, très-fines et douées d'un mouvement moléculaire très-rapide. Ce mouvement paraît durer indéfiniment; il résiste à l'action des acides, de l'iode, de l'alcool etc.; s'il cesse avec l'évaporation de l'eau, il reparait avec la présence d'un liquide etc. Il n'en est pas du tout ainsi du mourement des spermatozoïdes; il n'a qu'une durcée limitée, et, avec tous les soins possibles, je ne l'ai pas vu durer au delà de huil heures. Il cesse avec leur vie, et leur vie cesse à la première atteinte de dessiccation ou au moindre contact d'un des réactifs cilés plus haut, et une fois que ce mouvement s'est arrèté sur des spermatozoïdes morts ou tués, il ne reparaît jamais. Il n'est donc point un simple mouvement moléculaire, mais bien un vrai mouvement vital. C'est pourquoi, malgré des autorités imposantes', j'ai conservé très-expressément le nom de sPEnMATozö̈dé, voulant marquer par là la parfaite identité de fonctions entre les êtres fécondateurs des végétaux et les êtres fécondateurs des animaux. La vie est parlout la vie, toujour's identique à elle-même; les différences ne sont que de degré, non de principe et d'essence.

\footnotetext{
- En 1822, Fréd. Nees von Esenbeck décrivit le premier le mouvement hélicoïde des spermatozoïdes des Sphagnum. On a successivement proposé pour ces petits êtres les noms de bryozoaires et de phytozoaires. MM. Derbès et Solier, qui avaient adopté d’abord le nom de spermatosö̈de, l'ont ensuite, "pour se conformer au vœu de l'Académie des - sciences, changé en celui d'anthérozoüde" (Phys. Alg., p. 3, note); nom qui parait plus convenable i M. G. Thuret - en ce qu'il ne préjuge rien sur le rapport de ces corpuscules avec les spermatozoildes des animaux * (Zoosp. Alg., p. 76). M. W. Ph. Schimper, après avoir parlé des mouvements des " anthérozoïdes s des Sphagnum, ajoute: - Je ne - veux pas dire par lì que j’attribue à ces êtres une vic animale, ni que je les considère mème comme des animale cules; jai renoncé expressément a la dénomination de spermalosoïde, dont je m’ćtais servi autrefois, pour éviter "lonte éguivoque. La vie végétale peut avoir aussi ses mouvements! " (Sphag., p. 45).
} 


\section{§3. Des archégones}

Au temps où des anthéridies bien développées couronnent l'extrémité des lobules des sporophymes, on peut remarquer que quelques-uns de ceux-ci, d'un aspect plus vert et plus vigoureux, en sont presque constamment privés. En examinant ces sujets qui semblent stériles, on trouve que la partie supérieure de leur expansion foliacée est très-chargée de chlorophylle, plus dilatée, plus ramifiée et plus lobulée que sur les autres, et qu'elle offre plus particulièrement l'aspect cité plus haut d'une feuille de chicorée frisée pliée en deux; la partie médiane inférieure est très-épaisse, comme charnue, et composée de cellules plus petites que celles des ramifications latérales. Cette épaisseur atteint même quelquefois la partie inférieure de ces ramifications (pl. X, fig. $1 b$ ).

$\mathrm{Si}$, au moyen d'un grossissement médiocre, on explore avec un peu d'attention loute cette région épaissie vers la base des ramifications lobulées, on sera tout d'abord frappé par la vue de plusieurs petits matras, à ventricule globuleux, à col allongé et étroit, terminé par un large évasement quadrilobé d'une charmante élégance. Le tout est coloré en roux clair, ce qui le fait trancher sur le reste et permet de le distinguer avec une parfaite netteté $(\mathrm{pl} . \mathrm{X}$, fig. $1 \mathrm{l}$ ). Le ventricule est entièrement engagé dans le tissu à petites cellules, et il contient le plus souvent un corps plus ou moins sphérique, qui le remplit presque en entier (pl. X, fig. 2, 3). Le col, qui n'est qu'une colonne creuse composée de quatre rangées longitudinales de cellules un peu plus grosses, offrant rarement quelques granulations vertes, est également vers sa base engagé dans le même tissu, et sa zone supérieure est la seule à s'élever au-dessus. Les cellules du ventricule semblent donc faire partie du tissu enveloppant; sur celle de leurs faces qui constitue la paroi du ventricule et qui est pentagonale, elles sont colorées en roux ( $\mathrm{pl} . \mathrm{X}$, fig. $3 \mathrm{e}$ ). Il en est de même des cellules du col; elles ne sont colorées en roux que sur la face longitudinale très-étroite, qui n'est point en contact avec les autres, et forme le canal du col (pl. X, fig. $3 \mathrm{f}$ ); les supérieures seules sont en même temps colorées sur leur face supérieure et tout à fait libre; ce qui simule le gracieux évasement quadrilobé dont j'ai parlé (pl. X, fig. $3 a$ et $a^{\prime}$ ). Le plus souvent cependant la partie supérieure de ces cellules, au lieu d'être colorée et de former une rosace roux brun, est surmontée par des appendices aplatis, repliés en deliors, allongés en forme de petites feuilles, et quelquefois si grands qu'un seul égale tout l'appareil coloré (pl. X, fig. 2). Leur nombre varie de un à quatre. Un peu d'attention les fait reconnaître pour des cellules longues, à parois minces et flasques, et leur présence au-dessus des parois le plus souvent colorées indique de suite que 
cette coloration en roux n’est point due à une membrane spéciale tapissant les parois du col el du ventricule. On voil d'ailleurs cette coloration s'étendre assez, avant jusque dans les commissures des cellules du col et du ventricule, ce que ne ferait pas une membrane.

Ces petits matrus, avec toutes ces apparences, ne sont autre chose que les archégones. Il me reste a en exposer le mode d'apparition, et a suivre dans ses diverses phases le développement qui précède et amène les apparences ci-dessus décrites, et sous lesquelles les archégones se révèlent tout d'abord au regard de l'observateur. Ils indiquent ainsi la région où l'on doit en chercher la première apparition.

Si done, à l'époque du complet développement des premières anthéridies, dont l'apparition est toujours plus précoce que celle des archégones, on explore avec un fort grossissement la surface de la région charnue des sporophymes les plus vigoureux, vers la base des ramifications lobulées, on y verra une cellule qui se distingue de ses voisines par sa forme plus sphérique el surtout par son contenu, qui, bien que renfermant des granules de chlorophylle, est très-riche en matière mucilagineuse à granulations très-fines ( $\mathrm{pl}$. X, fig. 4). Cette cellule est une cellule-mère d'archégone. Il y en a ordinairement sur un mème pied un nombre assez grand (environ une vingtaine), à divers degrés de développement, pour que leur étude comparée révèle la série de leurs modifications successives, ainsi qu'il suit.

La face extérieure et libre de cette cellule s'élève au-dessus des autres en formant une saillie considérable, et bientôt deux forts nucléus amènent son partage en deux cellules superposées ( $\mathrm{pl}$. X, fig. 4). L'inférieure, engagée dans le tissu du sporophyme, deviendra la cellule ventrale de l'archégone, et la supérieure en constituera le col par ses divisions successives.

La première de ces divisions est verticale, et chacune des deux cellules qu'elle détermine est immédiatement doublée par une division à angle droit avec la précédente (pl. X, fig. 5). Et comme en même temps la cellule inférieure ou ventrale s'est développée en hauteur, aussi bien que les quatre nouvelles cellules qui la recouvrent, cet ensemble constitue alors des élévations faciles à reconnaître et à distinguer, tant par la forme spéciale que par l'aspect hyalin des quatre cellules supérieures, contrastant par l'absence de chlorophylle avec le contenu plus foncé de la cellule ventrale. Bientòt chacune des quatre cellules se dédouble deux fois de suite par division horizontale, de manière qu'il s'élève au-dessus de la cellule ventrale une colonne de quatre couches de cellules superposées, chaque couche composée de quatre cellules se rencontrant à angle droit sur une ligne centrale (pl. X, fig. 6 et 7). Les quatre terminales ou supérieures ne se divisent plus, mais s'étendent si considérablement en longueur qu'elles égalent à elles seules tout l'ensemble des autres, et constituent les longs appendices cilés précédemment; les trois couches qui sont au-dessous ne se subdivisent plus et 
s'étendent aussi en longueur, mais de moins en moins, et celle qui recouvre la cellule ventrale non-seulement reste plus petite, mais est atteinte par le dédoublement qui divise les cellules environnant immédiatement la cellule ventrale. Elle se confond donc avec ces cellules, qui, déjả petites, sont par leur subdivision réduites à n'avoir plus que le sixième ou le huitième du diamètre des cellules ordinaires; ainsi, d'une part, est constitué autour de la cellule ventrale un tissu d'une ou de deux couches de petites cellules, et, d'autre part, le col de l'archégone semble presque toujours composé seulement de trois couches de quatre cellules (pl. X, fig. 7).

Pendant la formation du col, la cellule ventrale se modifie elle-même, et aux premières divisions du col correspond dans cette cellule l'apparition d'abord de deux nucléus (pl. X, fig. 5), puis d'une cellule à la place du nucléus supérieur, qui occupe la partie fortement voûtée ( $\mathrm{pl}$. X, fig. 6). Cette nouvelle cellule est la cellule reproductive et le point de départ du pseudembryon et de la nouvelle plante. Peu à peu elle se développe en refoulant et comprimant le reste du contenu de la cellule et le nucléus inférieur; de l'un et de l'autre il ne reste bientôt plus qu'une mucosité granuleuse déposée ả l'extérieur de cette cellule (pl. X, fig. 7).

C'est à ce moment que les quatre rangées longitudinales de cellules formant le col se séparent un peu à leur angle commun de contact, et leur écartement constitue ainsi, suivant l'axe du col, un canal étroit à quatre faces conduisant à la cellule reproductive (pl. X, fig. $2 a$ ' et 3). En même temps les quatre longues cellules terminales se courbent plus ou moins en dehors ( $\mathrm{pl}$. X, fig. 2 , et 7 bis); elles sont alors entièrement incolores, hyalines et dénuées de chlorophylle, ainsi que les autres cellules du col et que les petites cellules qui entourent la cellule reproductive à la manière d'un épithélium.

C'est sous cette forme que l'archégone est définitivement constitué et prêt à la fécondation. Je parlerai des modifications qui suivent, après avoir dit quelques mots sur le sexe des sporophymes.

Des observations très-nombreuses et très-souvent répétées sur des sporophymes provenant des semis des E. maximum, sylvaticum, arvense, limosum, palustre, ramosissimum et variegatum, et un certain nombre faites sur des sporophymes spontanés de l'E. arvense ${ }^{1}$, m'ont permis de reconnaître qu'en général les sporophymes qui portent des archégones nombreux et bien développés ne portent point d’anthéridies, et que, sur plusieurs centaines de pareils sujets, à peine en trouve-t-on un ou deux portant des anthéridies. La tendance à la diœcie n'est pas réciproque pour les sporophymes anthéridifères, et il n'est pas rare de trouver des archégones vers la base de

\footnotetext{
'J'en ai pour la première fois trouvé des milliers dans une fourbière près de Haguenau, le 22 juillet 1860 , cn com. pagnie de mon excellent ami, M. Billot.
} 
leurs rameaux; mais ces archegones m'ont tous paru steriles. MI. le docteur Milde a constaté aussi que les cas de dirncie sont les plus ordinaires (Entw. Eq., p. 638). II. Hofmeister, qui avait remarqué des archégones sur un sujet chargé d'anthéridies, a constaté et expliqué le fait en ces termes : "Les prothallium de l'E. arvense montrent une tendance tout a fait marquée vers la dicecie. Les individus qui portent des anthéridies en sont richement pourvus; ils n'ont point d'archégones ou n'en ont que trèstard et isolément sur des rejetons qui paraissent après coup sur les vieilles parties du prothallium, et qu'il est permis de considérer comme des individus propres „ (Kicim. Eq., 1852, p. 387). J'ai pu moi-mème constater plusieur's fois cette différence d'àge évidente entre l'ensemble d'un sporophyme et un rameau de mème sexe ou de sexe différent. Ces rameaux, qui naissent tardivement sur de vieux sujets dont la partie inférieure et contiguë au sol est à moitié détruite, sont tout à fait analogues à ces expansions cellulaires qui naissent aux bords des vieux sporophymes de Fougères. On peut bien "les considérer comme des individus nouveaux" (Hofmeister, Kemntn. Gef. Crypt., p. 170); mais il est moins facile de s'expliquer comment ils sont d'un sexe différent:

Le voisinage immédiat ou l'entrelacement des rameaux de sporophymes de sexe différent, conséquence de la réunion ordinaire des spores par l'enchevêtrement de leurs élatères, remédie à l'obstacle que la dicecie semblerait apporter à la fécondation des archégones par les spermatozoïdes. Grâce à cette intimité de voisinage, une goulte de pluie ou de rosée permet aux spermatozoïdes de gagner par leur mouvement de natation les archégones qu'ils out à féconder', et le rapprochement est encore facilité par la force d'éjaculation qui les lance, même sur le porte-objet, jusqu’ả deux millimètres de l'anthéridie.

Maintenant comment les spermatozoïdes pénètrent-ils dans l'archégone pour y exercer la mystérieuse influence fécondatrice? C'est ce que je n’ai pu voir. Je les ai bien vus sortir des anthéridies avec plus de vigueur et de spontanéité le matin et le soir, aux heures de la rosée nécessaire à leurs mouvements de translation, rosée que je remplaçais par une goutte d'eau; j'en ai vu un grand nombre se présenter à l'orifice ćvasé de l'archégone et s'y attacher par leur extrémité filiforme; j'en ai trouvé de morts

\footnotetext{
- M. Ilofmeister, après avoir constaté les différences de vigueur ct de couleur qui permettent à un ail exercé de dislinguer du premier coup les sporophymes mảles des sp̣orophymes à archégones, exp̧ose qu'il "y a identité de grosseur entre les spores d’où naissent des prothallium mảles et celles qui se développent en prothallium femelles. " Puis Ic mème savant ajoute: - Les influences extérieures me paraissent avoir une certaine action sur le sexe du prothallium en voie de germination. Un lieu plus sec, plus éclairé parait sur $l^{\prime} E$. arcense favoriser le développement d'anthéridies, (Kenntn. Gef. Crypt., p. 171). L'observation ne m'a rien appris sur ce point; jincline volontiers d croire qque l'action des circonstances extérieures se borne à arrêter ou à favoriser le développement des sporophymes de l'un ou de l'autre sexe, dont la vigueur est d'ailleurs si différente, sans aller jusqu'à une infuence de détermination de sexe sur un sporophyme en végétation.
} 
dans le canal du col, mais je n'en ai jamais vu au moment de leur entrée ni observé de vivants dans le canal qui conduit à la cellule reproductive.

Les premières modifications qui suivent la fécondation consistent: $1^{\circ} \mathrm{en}$ une augmentation du volume de la cellule reproductive; 20 dans la disparition de son nucléus; $3^{\circ}$ dans la multiplication des cellules dı tissu qui l'enveloppe, multiplication qui rend ce tissu plus serré el amène l'occlusion de la base du canal du col. A ces changements succèdent ceux de la cellule elle-même. Elle contient trois ou quatre nucléus (pl. X, fig. 7), qui aboutissent presque simultanément à sa division en trois ou quatre cellules, dont une supérieure séparée par une cloison qui paraît plus ou moins oblique sur l'axe de l'archégone, et deux ou trois inférieures séparées au moyen de cloisons presque perpendiculaires à la première (pl. X, fig. 8 el 9 ).

Quand le développement de la cellule reproductive est normalement établi, le col de l'archégone paraît frappé de mort, et les parois de son canal prennent la couleur feuille-morte ou roux brun qui distingue sur les sporophymes les organes qui ne fonctionnent plus, comme les anthéridies vides et toutes les cellules oủ la vie s'est arrêtée. Les quatre cellules terminales, longues et recourbées, deviennent flasques, se détachent des autres et tombent ${ }^{1}$; le col entier s'affaisse, se déforme plus ou moins et se colore légèrement en brun aux commissures de ses cellules.

Les archégones non fécondés, et ce sont de beaucoup les plus nombreux, se déforment moins, mais ils subissent dans leur aspect des changements de coloration plus prononcés encore. La cellule ventrale et son contenu, la paroi des cellules du tissu ambiant qui forme le ventricule, le canal du col se colorent en roux très-foncé; les longues cellules terminales tombent l'une après l'autre, et les faces supérieures des quatre cellules du col qui les supportaient, se colorant en roux très-foncé, offrent ainsi l'aspect d'une rosace quadrilobée. Cet ensemble de parties colorées constitue ces petits matras si reconnaissables que j'ai dû commencer par décrire, parce que ce sont eux que l'on voit d'abord le plus facilement, et que, je le répète, ils indiquent la région oủ l'on doit chercher les archégones en voie de formation, toujours beaucoup moins apparents.

Dans sa Description des archégones de l'E. Telmateia, M. Milde place ces organes «su le bord d'une excroissance à petites cellules, moins divisée, mais plus foliacée que le proembryon à anthéridies, 》 et les décrit comme composés «à l'état parfail de huit cellules» (Arch. Eq. Telm., p. 497 el suiv.). Ces expressions et les figures à l'appui pourraient faire croire d'abord que la forme des archégones varie notablement

\footnotetext{
- La chute de ces cellules terminales offre une remarquable analogie avec ce qui se passe sur les anthéridies et sur les archégones d'autres cryptogames. "Au moment où l'ovule (de l'anthéridie) se rompt pour chasser son contenu, on "voit des cellules se détacher de l'orifice nouvellement formé . etc. (W. Ph. Schimper, Sphag., p. 42).... "Les cellules - du sommet (des archégones) se gonflent.... quelques-unes se détachent complétement ê tombent s $\left(0, c_{0}, p .48\right)$.
} 
selon les especes; mais l'observation des archégones de l'E. maximum el des autres, a leurs divers états de développenent, permet de reconnaitre que le savant botaniste de Breslau avait vu et décrit des archégones encore non complétement développés, el que leur position et leurs formes sont identiques sur toules nos espèces.

\section{§4. Du pseudembryon et de la jeune plante}

J'ai mentionné ci-dessus, p. 107 et 109, l'apparition et les premières divisions de la cellule dont le développement constitue successivement le pseudembryon et la nouvelle plante.

Une suite de divisions, commençant à la partie inférieure et s'opérant au moven de cloisons longitudinales et transversales toutes perpendiculaires aux faces extéricures, constitue l'axe primaire du pseudembryon, ainsi composé d'abord d'un petit nombre de cellules aboutissant toutes à la périphérie (pl. X, fig. 9). Un peu plus tard il apparait un groupe de cellules internes par la formation de cloisons paralleles à la périphérie (pl. X, fig. 9). Aussitôt que ce groupe a paru, une de ses cellules latérales (pl. $\mathrm{X}$, fig. $9 \mathrm{t}$ ) devient, par une suite de divisions analogues aux premières ci-dessus décrites, le siége d'une rapide multiplication de cellules; c'est le point de départ d'un axe secondaire, du premier rejeton latéral du pseudembryon, rejeton qui arrivera i l'air libre et produira des gaines, tandis que l'axe primaire du pseudembryon s'arrêtera dans son déreloppement. La multiplication des cellules de ce rejeton marche arec rapidité; clles poussent d'abord en avant les cellules de la périphérie du pseudembryon, puis les écartent'. A partir de ce moment les cellules terminales supérieures du pseudembryon se développent peu ou point du tout, tandis qu'au contraire la partic de l'axe primaire siluée au-dessous du point d'origine de l'axe secondaire ou rejeton latéral s'accroit considérablement en grosseur, el que l'axe secondaire luimême se développe avec une force et une rapidité extrême. Il en résulte que le rejeton

\footnotetext{
1 M. Hofmeister fait naître le rejeton latéral non point d'une des cellules du groupe central, mais d'une des cellules de la périphérie, alors que le pseudembryon n'a point encore de groupe central, mais seulement quatre cellules, et it lit très-expressément: "Une multiplication de cellules suivant une loi analogue commence dans l'une des cellules latérales du rudiment d'embryon à quatre cellules... II en résulte un rejeton latéral o etc. Eane ähnlichen llegeln a folgeade Zellenvermehrung beginnt in der einen seitlichen Zelle des vierzelligen Embryo-Rudiments.... Es wird - dadurch cine seitliche Sprossung des Embryo-Kügelchens gebildet.... (Kiennln. Gef. Grypt., p. 17\$ et 175).

Ce texte d'un éminent observateur m'a suscité bien des doutes sur ce que je croýais voir et m’a fait bien souvent recommencer mes observations; mais j’ai ru avec tant de clarté et si constamment les choses se passer comme je les ai exposées que j’ai da les dire comme je les voyais. Sur des sporophymes de semis et sur d'autres spontanés et trèsvigoureux, recueillis à Haguenau, j’ai pu reconnaitre avec une complète éridence que les cellules de la périphéric étaient repoussées en dehors par la multiplication d'une des cellules sous-jacentes du groupe central.
} 
secondaire repousse de côté la partie supérieure de l'axe primaire du pseudembryon, prend de plus en plus la direction verticale, attcint presque la base oblitérée du canal du col, et finit ainsi par occuper lui-même le sommet de la masse cellulaire à peu près sphérique, dont il constitue presque touta la moitié supérieure, tandis que la partie inférieure appartient encore au pseudembryon ou axe primaire.

A ce moment, au point opposé et un peu inféricur à celui oủ avait commencé l'axe secondaire, une des cellules du groupe interne du pseudembryon se multiplie et se constitue en une petile bosse demi-sphérique, qui repousse el sépare bientôt les cellules de la périphérie. C'est la première racine latérale répondant au premier rejeton latéral (pl. X, fig. $9 r$ ).

A mesure que la masse totale se développe, elle rejelte de côté tout le col de l'archégone devenu flasque et déformé (pl. X, fig. 10); puis elle distend tout cet espace, que j'ai appelé le ventricule de l'archégone, et repousse les cellules ambiantes du sporophyme, lesquelles continuent encore souvent à se diviser. Soumises à cetle double pression, les cellules du sommet primitif de l'axe primaire perdent successivement leur matière verte et leur forme, et finissent sans doute par être liquéfices et résorbées, car elles disparaissent entièrement. Les autres cellules de la périphérie du pseudembryon semblent d'abord, par l'effet de la même pression, se coller et s'unir à celles du sporophyme, puis elles cessent d'être distinctes. Le pseudembryon ou l'axe primaire paraîl alors avoir terminé son développement; le rejeton latéral et la racine latérale constituent à eux deux un nouvel axe, axe secondaire qui est la nouvelle plante. Ainsi, on ne peut point dire du premier développement des Equisetum ce qui nous est enseigné de celui des végétaux dicotylédonés, qu'ils ont un axe unique, « dont les deux extrémités sont entraînées par un double mouvement de polarité dans «une direction opposée, la tigelle et la gemmule vers le zénith, et la radicule vers le «centre de la terre» (A. Richard, Préc. bot., p. 291, 1852). Dès les premiers développements de la cellule reproductive ou pseudembryon, les deux extrémités opposées de l'axe primaire s'arrêtent dans leur croissance, et ce sont des appendices latéraux ou axes secondaires qui remplacent, l'un la tigelle, l'autre la radicule. Il nous reste à en suivre le développement ultérieur.

Bien que celui de la petite racine ne soit ni le premier ni le plus rapide, c'est de lui que je parlerai d'abord, parce que, ce développement étant une fois mentionné, sa complète uniformité et sa parfaite identité avec celui des plantes adultes me dispenseront d'y revenir.

La racine croît donc en longueur exactement de la même façon que les racines de la plante adulte; elle s'avance directement dans le tissu du sporophyme, le disloque et pénètre dans le sol, oủ elle s'enfonce assez rapidement. Par sa force et par sa composition cellulaire, qui la rend opaque, elle se distingue au premier coup-d'œil des 
radicelles simples et transparentes du sporophyme. Comme les racines ordinaires des rhizomes, elle se compose d'un épiderme très-distinct, à cellules longues, tétragonales, à parois lisses et brunâtres, donnant naissance à des radicelles plus nombreuses et plus petites que les radicelles ordinaires des sporophymes (pl. X, fig. $1 d$ ). Au-dessous de l'épiderme est un cylindre de cellules moins fortes, moins longues, au milieu desquelles s'étend, mais non jusqu'à l'extrémité, un faisceau de vaisseaux spiro-amnulaires, toujours incomplétement formés, dont les anneaux vont en s'espaçant de plus en plus et finissent par disparaître en se rapprochant de l'extrémité inférieure, laquelle est recouverte d'une piléorhize. Il y a done, it tous égards, identité entre cetle racine et les racines ordinaires des rhizomes.

Le sommet végétatif du rejeton latéral ressemble également de très-bonne heure, tant par sa forme que par sa loi de multiplication, au sommel végétatil d'une tige d'Equisetum adulte. On y distingue avec netteté la grande cellule terminale et le mode de division qui détermine l'agencement des cellules de second degré en ceintures circulaires superposées. Et aussi de la même manière que sur les bourgeons naissants des plantes adultes, il apparaît au-dessous de la petite masse cellulaire terminale un bourrelet annulaire de deux couches de cellules, et sur ce bourrelet se montrent trois inégalités, lesquelles sont le point de départ des dents de la première gaine dont le bourrelet était la première apparition (pl. X, fig. 10 b). Cette première gaîne n'est qu'une gaîne basilaire, ainsi qu'on peut le reconnaître plus tard à ses dimensions définitives, à sa contexture et à sa couleur ( $\mathrm{pl} . \mathrm{X}$, fig. 1 f). Et enfin les mêmes lois d'évolution président à l'apparition des autres entre-nœuds de la jeune plante.

Il est très-facile de suivre sur les jeunes plantes de quelques millimètres de longr la formation des vaisseaux, et de voir dans les cellules apparaitre des mneaux isolés et espacés, entre lesquels il s'en développe d'autres, jusqu'à ce qu’après la résorption des extrémités des cellules ils constituent un tout régulièrement espacé. La petite tige, constamment formée d'entre-nouds à trois côtes et dès lors de gaines à trois dents, contient trois faisceaux vasculaires, dont la position répond à celle qu'ils occupent dans les plantes adultes. La jeune racine, comme la racine des sujets adultes, n'en présente qu'un seul, central et composé de deux ou trois vaisseaux inégaux en grosseur.

Il m’a paru important d'opérer des coupes pour reconnaître le mode de transition des faiscenux vasculaires de la jeune tige au faisceau de la racine, et ces coupes m'ont permis de constater qu'à la région où cessent les faisceaux de la tige et oủ commence celui de la racine, il n'y a pas continuité des premiers au dernier, mais bien une interruption constituce, comme dans les nwuds d'une plante adulte, par un petit plan de courtes cellules rayées ou spiralées (pl. X, fig. 12). Ce plan est oblique, et son obliquité répond à celle qu'a dû prendre le pseudembryon. 
L'ensemble de ces faits nous révèle:

$1^{\circ}$ Que la disparition du pseudembryon ou axe primitif n'est pas si complète qu'elle le semble d'abord;

2. Que ce qui a complétement cessé d'cxister est seulement le sommet végétatif de cet axe primitif, mais que le développeneni de sa masse cellulaire centrale et de sa moitié inférieure a constitué un véritable nneud, offrant, comme ceux d'une plante adulte, de courtes cellules rayées;

$3^{\circ}$ Que de ce noud persistant sont issus deux appendices latéraux : d'une part, un rameau qui a été la première tige, el, de l'autre, la première racine;

$4^{\circ}$ Que ces deux parties, la pelite tige et la première racine, ne sont pas des parties d'un seul et même verticille.

En effet, comme nous l'avons vu p. 4, 10 et 70, les racines de la plante adulte, quelle que soit la région où elles apparaissent, rhizome ou partie inférieure des tiges aériennes, n'occupent jamais la place des rameaux articulés ni celle des tubercules, mais elles émergent un peu au-dessous, constituant ainsi aux nouds un double verticille d'appendices latéraux. La première petite lige n'est qu'un des rameaux du premier verticille, qui doit se développer au noud primaire que constitue la partie centrale du pseudembryon, et la racine, en apparence opposée, est la première du verticille de racines infraposé. Comme la première tige prend la direction verticale ascendante, en même temps que la première racine prend la direction verticale descendante, la masse du pseudembryon, cédant à celte double traction, s'incline presque horizontalement, et son noud central prend une direction rapprochée de la verticale. Il suit de là:

$1^{\circ}$ Que lorsque plus tard naîtront du même nœud des bourgeons adventifs, appartenant au même verticille, ceux qui apparaîtront ả côté de la première tige seront forcés de prendre une direction plus ou moins horizontale;

$2^{\circ}$ Que celui qui sera opposé à la première tige et superposé à la première racine sera forcé de prendre sa direction et son extension plus ou moins au-dessous de la surface du sol, sinon tout droit en bas. Il formera ainsi le premier rhizome qui assure la pérennité de la plante. C'est aussi ce qui arrive; et tout rejeton latéral, souterrain ou aérien, qui succède au premier, est ordinairement plus vigoureux que lui.

Bischoff, dans son excellent mémoire Sur le développement des Equisetum provenant des spores, avait remarqué la production des tiges nouvelles autour de la première tige, et la différence de grosseur a entre ces nouveaux rejetons qui se dirigent vers le sol et la première lige qui reste toujours grêle dans son développement) (Enhw. Eq., p. 790). Mais de ce que ces tiges prennent quelquefois une direction assez inclinée pour paraitre s'enfoncer verticalement dans le 
sol ', il await tiré la conclusion suivante : "Il paraît done que les Prêles ne montrent ru'une scule fois dans leur vie une croissance dans deux directions opposées;.... il n'y a dans ces plantes de polarité de croissance, bien manifeste sur deux organes principaux, que dans la première période du développement, el elle disparaît ensuite pour toujours dans la végétation ultérieure » (o. c., p. 795).

Nous croyons cette conclusion inexacte au moins dans les termes, en ce qu'elle ferait supposer l'existence d'un «caudex» articulé faisant suite immédiate à une tige d'axe primitif, et n'étant que le prolongement descendant de l'axe de cette lige.

Il nous semble aussi que N. le docteur Milde n'a pas été plus exact en qualifiant la première racine de véritable racine principale : «Diese Wurzel, eine wahre IIaupt(wurzel....) (Enu. Eq., p. 638); et en terminant ses nouvelles observations par ces mots : «'ai observé aussi la véritable racine pivotante de la jeune plante » (Arch. Eq. Telm., p. 500, 1852, et aussi Gef. Crypt. Schl, p. 415, 1857). M. Milde ajoute chaque fois que cette racine pivotante périt bientôt après l'apparition de jeunes rhizomes qui la remplacent dans ses fonctions de nutrition (Entw. Eq., p. 638, et Gcf. Crypt. Schl., p. 415). J'ai pu constater qu'elle dure aussi longtemps que les autres et mème se ramifie souvent ${ }^{2}$.

Il est juste d'ajouter que ces deux excellents observateurs, à l'époque oủ ils émirent ces assertions, n'avaient pas encore été assez heureux pour rencontrer un pseudembryon dans sa première période de développement. Ils n'avaient vu que la jeune plante déjà assez avancée. Comme c'est à cet état qu'il est le plus ordinaire et le plus facile de la trouver, je crois qu'il peut être utile de donner ici une description de la jeune plante telle qu'elle est quand elle se montre distinctement sur le sporophyme, et de faire voir comment chacune de ses apparences et de ses parties se raltache, en le continuant, au développement ci-dessus décrit.

Si, après la mi-juillet pour les E. arvense, maximum, sylvaticum, et sept ou huit

\footnotetext{
1 - Bientủt à la base se produit une seconde petite tige qui, en s'élevant au-dessus du coussinet (sporophyme), prend dès le commencement une direction presque horizontale et s'étend tout près de la surface du sol, fig. 13. Cette seconde liģe est bientôt suivie de plusieurs autres, se développant toujours autour du point du coussinet, qui est le premier noud de la petite plante de germination, fig. 14 (p.789)... La tendance de ces nouvelles tiges à prendre une direction rampante s'accroît au point que l'extrémité d'une ou de plusieurs se dirige directement en bas et s'enfonce dans le sol pour former le caudex souterrain articulé, fig. $14 a$; fig. $15,(0, c ., p .790)$.

* Au mème lieu le mème savant décrit et figure, pl. 59 , figh. 48 , le premier développement de la jeune plante connme " un cylindre creux, vert, divisé en six dents, et se dilatant vers le bas en forme de bulbe." La mème planche représente, fig. 51 et 52 , au bas des jeunes tiges des sporophymes de forme sphérique avec des couches concentriques de folioles, sans aucune radicelle, forme el disposition qui n'existent jamais. Enthn la fig. $5 \downarrow$ représente un jeune Equisetum impossible, en ce sens qu'elle lui attribue des rameaux épars et alternes; les deux rameaux de gauche sortent du milieu d'un entre-noud. Il y a sur toute cette planche des lapsus qui m'ont tourmenté, parce que je croyais d'abord i l'exactilude des figures; ils résultent sans aucun doute de l'inattention du graveur, car cette planche porte, comme les autres, la signature de M. Milde.
} 
semaines après le semis pour les autres espèces, on arrache un de ces sporoplymes sans anthéridies, plus verts et plus vigotreux que les autres, on voit au-dessous de lui et au milieu de ses radicelles descendre une racine brune, composée de nombreuses cellules et de vaisseaux, et, si on étale le sporophyme, on voit presque directement au-dessus de la racine s'élever un corps cylindrique, un peu courbé, creux, dilaté et partagé en trois dents à son bord supérieur ( $\mathrm{pl}$. $\mathrm{X}$, fig. $1 \mathrm{f}$ ).

Dans son intérieur, un peu plus bas que la naissance des dents, on distingue une colonne à sommet trilobulé, d'un vert intense (pl. X, fig. $1 \mathrm{~g}$ ), et au-dessus du milicu de cette colonne une masse de forme globuleuse et d'une couleur verte encore plus intense ( $\mathrm{pl} . \mathrm{X}, \mathrm{fig} .1 \%$ ).

$\Lambda u$ point oủ cesse la racine et où semble commencer la petite tige, on voit constamment une zone interrompue et irrégulière de cellules brunes, flétries et déchirées (pl. X, fig. 1 i).

La présence de vaisseaux dans son tissu cellulaire ne permet pas de confondre la première racine avec les radicelles du sporophyme, et fait de suite reconnaître en elle la première racine issue du pseudembryon, cette racine sans articulations que Vaucher avait entrevue, mais mal décrite et plus mal figurée encore (Fruct. Prêl., pl. XXVII, fig. 4, 5), et que plus tard Bischoff avait mieux observée (Entw. Eq., p. 788 et suiv.). Mais cette racine n'a point l'importance que lui attribuait ce savant. Elle peut manquer; elle manque même souvent sur des sujets oủ le tissu cellulaire inférieur du sporophyme a été trop résistant pour qu'elle le traversât. Il n'est pas rare de trouver sans aucune racine, ou seulement avec une racine rudimentaire, de jeunes plantes qui, en taille et en vigueur, ne le cèdent en rien à leurs voisines du mème îge. Dans ces cas on trouve presque toujours des radicelles naissant des cellules de la gaîne basilaire et même du premier entre-nœud de la jeune plante, qui d'ailleurs continue pendant longtemps encore à tirer sa nourriture du sporophyme.

Les restes de cellules brunes et déchirées que l'on remarque presque toujours entre la racine et la petite tige, sont évidemment les restes des cellules qui avaient formé l'enveloppe archégoniale du pseudembryon.

La courbure qui s'observe constamment au point d'où partent en sens contraire la racine et la petite tige fait encore reconnaître avec évidence que ces deux parties ne sont point la continuation l'une de l'autre en sens directement inverse, qu'elles n'appartiennent point dès lor's à l'axe primitif, mais qu'elles ne sont que des appendices latéraux simulant, au moyen d'une inflexion, un axe primitif.

Le tube cylindrique extérieur, qui se réduit à une sorte de plateau surmonté d'une gaîne tridentée, se compose de deux couches de cellules à parois très-ondulées; ver's la pointe des dents, sur leurs bords et au fond des sinus de séparation, ces cellules ne forment plus qu'une couche; elles sont alors d'une ténuité extrême et sans chloro- 
l'hylle; les alutres en oflient assez abondamment. Cette gaine n'est atutre chose que la gaime basilaire, naissint directement mais latéralement du pseudembryon. Elle n'est fas noire comme celle des rameaus, parce qu'elle u’a pas eu la même compression à supporter, ni la même résislance à vaincre.

La colonne verte qu'elle contient est le premier entre-nueud, devant succéder, comme sur les rameaux de la plante adulte, à la gaine basilaire et se prolongeant en une gaine tridentie. Sa structure est identique i celle des entre-nauds d'une tige en évolution, seulement il s'allonge proportionnellement davantage à la manière des entre-ncends des rameaux. An-dessous de la gaine quelques anneaux isolés indiquent la place des raisseaux spiro-annulaires moins formés encore ver's la base de l'entre-nceud, oủ une masse de cellules courtes et rayées les sépare des vaisseaux de la racine.

Le corps plus vert, subglobuleux, qui se voit vers la base de la gaine de cet entre-nœud, se compose d'une masse de tissu cellulaire, qu'entoure un bourrelet circulaire, dont le bord libre présente trois saillies; et ce corps n'est autre chose que le sommet régétatif de la jeune plante, oủ le second entre-nueud montre déja le commencement de la fulure gaine autour de la masse cellulaire qui doit se développer ultérieurement.

Tels sont les détails de l'aspect reproduit pl. X, fig 1, et sous lequel se présente ordinairement une jeune plante d'Equisetum définitirement constituée.

Je dois signaler ici la différence qui existe entre le développement de la gaine sur les jeunes plantes et celui de la même partie sur les tiges des plantes anciennes. Nous arons vu précédemment p. 58, comme quoi, sur ces dernières, chacune des gaines, pendant toute sa période d’érolution, constitue une coiffe entière, à peine dentelée à sa pointe. $\mathrm{Ol}^{\prime}$, sur les jeunes tiges d'un nouvel Equisetum, la masse cellulaire constituant le bourgeon terminal, ofire it sa base un bourrelet annulaire entierement semblable à celui qu'on trouve autour du bourgeon terminal d'une plante adulte. Au bord libre supérieur de ce bourrelet il se produit semblablement une inégalité de développement, mais sur trois points seulement et une seule fois. Les trois pointes libres qui en résultent continuent a se développer isolément par multiplication de leur's cellules; d'oủ il suit que, dans la jeune plante, les divisions de la gaine, à leur extrémité supérieure, se déreloppent dés le principe en courtes dents isolées et un peu étalées, sans former, comme dans les plantes adultes, une enveloppe continue à bandes d'inégale épaisseur qui se transforment en lobes en cédant i la poussée des entre-ncuds supérieurs.

La première jeune tige, après aroir alteint trois ou quatre centimètres de longueur, nia tonjours paru subir un assez long temps d'arrit (deux a trois semaines); ensuite, it cùté d'elle naissent d'autres tiges, suivant le mode et la direction que jai indiqués p. 113, c'est-i-dire qu'elles naissent du nwud primitif épaissiet développé (pl. X, fig. 12), 
du næud de l'axe primaire ou pseudembryon'. Il en paraît d'abord une à côté de la première petite tige ( $\mathrm{pl}$. $\mathrm{X}$, fig. 12 et 13). La direction en est d'abord presque horizontale, et quelquefois mềme elle ne se relève qu'après s'être un peu enfoncée dans le sol. Mais ce n'est ordinairement qu'un peu plus tard que se montrera, à l'opposé de ces tiges aériennes, une vraie tige souterraine, un rhizome à direction descendante. L'apparition d'une nouvelle tige est presque toujours accompagnée de celle d'une racine infraposėe; ces racines sont ordinairement ramifiées (pl. X, fig. 13).

Pendant la période d'arrêt que je viens de signaler, les nouvelles tiges sont à l'état de bourgeons blancs composés d'une gaîne à trois dents autour d'une masse cellulaire destinée au développement ultérieur. Le tout est très-chargé ou plutôt gonflé de grains amylacés, ce qui donne à la base de la nouvelle plante une apparence charnue. Les tiges souterraines ou rhizomes ont un développement très-lent, que la saison d'automne ralentit encore. D'abord blanches, elles prennent bientôt la couleur brun rougeâtre particulière à l'épiderme des rhizomes. $A$ la base de leurs gaines on voit le double rang de petites saillies deslinées à donner, les unes des rejetons articulés, les autres des racines. Ces dernières apparaissent vite et en grand nombre. Des rejetons que produisent les jeunes rhizomes, les uns, se dirigeant vers la surface du sol, deviennent de nouvelles tiges aériennes; les autres s'enfoncent perpendiculairement à une grande profondeur. Ils se présentent tous d'abord sous la forme des bourgeons blancs et charnus que l'on trouve en hiver et au printemps sur les rhizomes des plantes adultes.

Assez souvent, mais non toujours, ces bourgeons se gonflent en tubercules au lieu de se développer en rhizomes, et vers l'automne de la première année il n'est pas rare de trouver presque à la surface du sol un ou deux tubercules sur les jeunes plantes. M. Milde a aussi déjà constaté l'existence des tubercules sur de jeunes rhizomes (Gef. Crypt. Schl., p. 415, et Entw. Eq., p. 639, pl. LIX, fig. 53-56).

Le développement ultérieur est assez conforme à celui des plantes adultes décrit

1. Ilofmeister s'explique ainsi qu'il suit sur l'apparition des nouvelles tiges : "Lorsque la première tigge feuillée a atteint un certain degré de développement, il se forme à l'intérieur de son tissu cortical un bourgeon adventif par suite de la multiplication d'une cellule cambiale de sa base, à la hauteur de la masse ligneuse solide du premier nœud. Ce bourgeon est situé sur le côté du rejeton feuillé, qui est tourné vers l'axe primaire de la plante de germination, sous l'enfoncement entre deux lobes de la première gaine, tab. XIX, fig. 3n (Kenntn. Gef. Krypt., p. 176).

J'ai pu faire sur l'apparition des nouvelles tiges de très-nombreuses observations, ģrûce aux sujets que m'ont fournis mes semis et surtout aux milliers de jeunes plantes spontanées que j'ai trouvées dans les tourbières de Haguenau, et j’ai vu constamment avec une parfaite évidence les choses se passer comme je les décris. La figure que donne M. Hormeister est très-bonne, quoique la masse des cellules rayées constituant le noud primaire du pseudembryon y soit trop peu marquée, et cette figure confirme pleinement ce que je dis, et fait voir que le nouveau rejeton sort du nœud primaire du pseudembryon.

Au même lieu, M. Hofmeister ajoute: "Le nouveau rejeton se distingue du premicr axe feuillé par des gancs d 'quatre dents....) Je n'ai vu que très-rarement sur l' $E$. arvense les rejetons de première Ynée avec des gaaines à quuatro lents; ils n'en ont ordinairement que trois. 
aux $\$$ I $I^{\text {er }} \mathrm{et}$ g du chap. III. Toutefois les premières tiges ne produisent que très-peu de rameaux (pl. X, fig. 13). LiE. arense, dont les tiges adultes sont si richement ramifiées, dome le premier été jusqu'i huit tiges aériennes, mais ces tiges grêles, bien qu’atteignant une longueur de vingt centimètres, demeurent en général simples; à peine deux ou trois présentent-clles, à un ou deux de leurs entre-nœuds, un ou deux rameaux.

Dès la fin d'octobre les tiges de première année sont flétries par les gelées. La vígétation recommence de très-bonne heure sur les rhizomes. En effet, les plantes provenant de mes semis ne m'ont guère montré à l'automne que trois ou quatre rhizomes deseendants; mais quand du $1^{\text {er }}$ au 15 avril je jes enlevais des petits vases du semis pour les meltre dans de plus grands, je trouvais constamment un nombre déja considérable de rhizomes qui pénétraient la terre du vase en tout sens, rampaicnt contre les parois et sortaient par le trou du fond. La denxième année m'a toujours donné en avril et mai des tiges grêles, très-nombreuses, étalćes en éventail, trèsirrégulièrement ramifiées, à verticilles incomplets, en un mot ayant toutes les apparences d'un rameau. Mais vers la mi-juin il sortait tout contre les parois du nouveau vase de nouvelles tiges, vraies tiges stériles, bien régulièrement pourvues de verticilles complets de rameaux; c'est alors que je les mettais en pleine terre. Ce n'est qu'après la quatrième année et plus souvent après la cinquième que j'ai vu apparaitre quelques tiges spicifères peu robustes.

La direction verticale descendante des rhizomes, si facile à constater en dépotant les jeunes plantes, fournit l'explication suffisante de la marche et de la propagation des rhizomes au-dessous de la surface du sol, ainsi que Bischoff l'avait très-justement remarqué (Entw. Eq., p. 792 et 793), après que Vaucher (1Lon. Prêl., p. 342) et Bischoff lui-mème (Krypt. Gew., p. 28 et 44 ) avaient exprimé combien difficile à expliquer leur paraissait la profondeur à laquelle rampent certains rhizomes (roir plus haut p. 8 et 9). Si l'on ne trouve plus les premiers rhizomes qui ont conduit les autres à celte profondeur, c'est que ces premiers rhizomes plus grêles et plus délicats sont bientôt détruits, et que d'ailleurs la propagation par semis est incontestablement celle qui se rencontre le moins souvent (Milde, Gef. Crypt. Schl., p. 385 et 415). Elle est pourtant moins rare que Vaucher ne l'avait cru d'abord (Ion. Prế., p. 351, el Fructif. Prél., p. 424) et que MI. IIofmeister paraît encore le croire (Vergl. Unters., p. 102). Il est mème probable que tous ceux qui chercheront de jeunes Equisetum en trouveront; car en juin 1825 Requien en trouva près d'Avignon et les adressa ì Vaucher (Vaucher, Germ. Prếl.); en 1827 Bischoff en a trouvé près de Deux-Ponts (Entw. Eq., p. 785); en 1850 M. Milde sur le bord de l'Oder, en face de Masselwitz (Entu. Eq., p. 637); cn 1852 M. IIofmeister a vu l'E. varicyatum, cultivé dans un jardin, se reproduire par semis (Kenntu. Tief. Krypl., I, p. 171, note); moi-mème, en 1860, j’ai 


\section{\$5. - MODE DE SEMIS.}

trouvé à Haguenau d'innombrables jeunes plantes d'E. arvense, que j’ai pu suivre depuis leur apparition sur les sporophymes jusqu'au dernier terme de leur développement. En outre j'ai presque constamment réussi dans mes semis des $E$. maximum, sylvaticum, arvense, limosum, palustre, ramasissimum et varicgatum.

\section{$\S 5$. Mode de semis}

La série des divers phénomènes qui précèdent ne s'accomplit sur les sujets provenant de semis que s'ils ont été entourés de précautions et de soins.

Si l'on ne veut qu'observer les premiers développements des spores en sporophymes, on peut se contenter de jeter des spores à la surface de l'eau, dans un vase recouvert d'une plaque de verre, et de soustraire le tout à l'action directe de la chaleur solaire, en l'exposant toutefois à une belle lumière. Les spores commencent immédiatement à se développer en cellules, les unes au fond de l'eau, les autres à la surface. Ces dernières vont le plus souvent jusqu'à la production des anthéridies; celles du fond périssent d'assez bonne heure. Cependant j’ai vu, au fond d'un vase en verre, des sporophymes des $E$. palustre et arvense durer du 15 avril au 3 octobre 1860. Simples ou rarement bilobés, avec une ou deux radicelles, mais sans anthéridies, ils étaient encore bien vivants quand, par erreur, on enleva l'eau du verre qui les contenait. Ce mode de semis a l'avantage de rendre les observations trés-promples, et de donner de suite des sujets débarrassés de sable et de parcelles terreuses.

Le moyen qui m'a constamment réussi pour amener mes semis jusqu’à la reproduction de jeunes plantes, est le suivant:

Un pot de vingt centimètres de diamètre et de hauteur est rempli aux trois quarts de terre ordinaire; un autre pot d'un décimètre de diamètre et dont le fond est percé de grands trous est placé dans le premier, de manière à ce que le niveau de sa terre soit d'un centimètre plus élevé que la terre du premier. Ce pot est rempli de terre prise dans un endroit quelconque où l'espèce à semer croît en abondance; au-dessus de cette terre je répands une couche de deux millimètres de sable siliceux, très-fin, également pris dans quelque lieu affectionné par les Equisetum. Ce sable est au préalable lavé à l'eau bouillante et sêché sur une plaque de fer portée au rouge, à l'effet d'éviter l'apparition des divers cryptogames qui étouffent les jeunes sporophymes. Les spores sont semées au-dessus de ce sable humecté par infiltration; le pot qui les contient est recouvert d'une cloche de verre, et le tout est placé en un lieu très-bien éclairé et à l'abri des coups de chaleur solaire. Le vase intérieur n'est jamais arrosé directement pendant le premier mois; mais matin et soir le vase extéricur cst arrosé, et la terre de celui de l'intérieur s'imbibe suffisamment par infiltration. Lorsque les 
fremitres anthéridies ont paru, il convient d'arroser tris-léserement matin et soir le vase intérirur, afin de remplacer l'effet de la rosie qui permet aux spermatozoides de gagner par leurs mouvements de natation les archégones fu’ils ont à féconder. Commu it partir de l'arrosage direct les cryptogames paraitront en abondance, il est bon de les enlever de temps on temps au mojen d'une loupe et de pinces très-fines, en ayant soin de ne pas ébranler les sporophymes, car il vaut mieux laisser les plantes étrangìres que de déraciner les sporophymes en enlevant le reste. Mr. Hofmeister fail contnaitre qu'il a employé avec succés des arrosages d'eau froide longtemps prolongés pour débarrasser ses semis de l'envahissement des algues (Konntn. Gef. Krypt., p. 169).

Il est bon de semer les spores assez dru. Semées clair, elles se développent bien, mais des sporophymes isolés risquent de ne pas être fécondés, parce que les spermatozoïdes ne peuvent pas aller sur des pieds que ne couvrirait pas une mème goutte de rosée. Le rapprochement forcé des spores par l'enchevêtrement de leurs élatères au moment de l'émission indique assez d'ailleurs qu'il est dans leur nature d'être semées dru.

Il faut faire remarquer ici quelques différences entre le développement des spores semées sur l'eau et celui des spores semées sur la terre. Le développement des premières est plus rapide, et des spores d' $E$. palustre, semées sur l'eau le 8 septembre 1859 , avajent déja vingt-guatre heures après une radicelle égalant la longueur des cellules. Le troisieme jour, le plus grand nombre des sporophymes offraient deux cellules presque côte à còte (pl. IX, fig. 14 bis), et une très-longue radicelle; ceux qui s'étaient céveloppés au fond de l'eau, ne se distinguaient des autres que par la direction oblique de leur longue radicelle. Les spores du même épi, semées sur terre au même moment, se développèrent moins rapidement, et le plus souvent elles se multipliaient en deux ou trois cellules arant d'émettre une seule radicelle. Rarement elles débutent par la forme citéc ci-dessus, mais elles offrent des cellules disposées en ligne les unes au-dessus des autres (pl. IX, fig. 14 $a, b, c, d$ ). La seconde cellule, je veux dire celle superposée à la cellule d'où sort la radicelle, est ordinairement plus longue que les autres et moins chargée de chlorophylle.

Le développement ultérieur présente des différences plus considérables, en ce sens gue les semis sur terre produisent des sporophymes it expansions foliacées décrits p. 96 i 99 et figurés pl. IX, fig. 17; tandis que les semis sur l'eau ne donnent guère que des sporophymes grêles, filiformes, à ramifications composées de un ou de deux rangs de cellules. Ils sont aussi très-irréguliers, couchés, courbés ì angle droit elc. (roy. pl. IX, fig. 18).

Lorsque mes jeunes plantes ont acquis deux ou trois centimètres de long, je les transplante dans un petit pot que je renferme toujours dans un plus grand, et je les

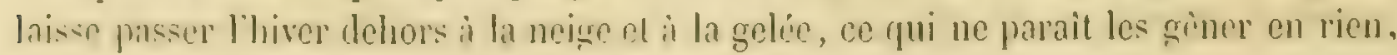


§6.- - HISTOHQVE.

car dès le mois de février les jeunes pousses se montrent déjà. Du 15 avril au $1^{\text {er }}$ mai je les mets dans des vases plus grands et plus profonds, de manière à ce que plus tard, en enlevant un peu de terre sur les còtés de la masse isulée par le renversement du vase, on puisse observer à l'aise la direction descendante des tiges souterraines ou rhizomes, la production des tubercules, l'extrémité des racines etc. Au mois de juillet ils ont des centaines de petites et de grosses tiges, je les mets alors en pleine terre.

Je donne ci-dessous les dates auxquelles mont apparu les principales phases d'érolution, à Strasboury, en 1859. Les dates des autres années ne diffèrent pas sensiblement.

$1^{\text {er }}$ mai. Semis des spores de l' $E$. arvense.

5 juin. Apparition de grosses cellules terminales.

8 》Division en cellules longitudinales.

16 Apparition de petites cellules cubiques.

20 „Des globules libres apparaissent dans les anthéridies.

25 „Vu une émission de spermatozoïdes.

Jusqu'au 13 août revu sans interruption la reproduction des phénomènes précédents.

6 juillet. Vu le premier archégone de ce semis; il était déjả trop avancé.

18 Du la première jeune plante.

Jusqu'au septembre revu des archégones.

22 et 23 septembre. Revu des spermatozoïdes sur un sporophyme tardif du même semis.

Les jeunes tiges se sont fanées le 8 novembre; elles avaient de huit à douze centimètres de long.

\section{§6. Historique}

11 est inutile de chercher les traces d'une théorie de la reproduction des Équisetum dans les ouvrages des anciens botanistes. Ne connaissant pas encore le mode de fécondation des végétaux phanérogames, ils se préoccupaient peu de la génération de ceux que Linné appelait plus tard cryptogames, par cette seule raison, toujours valable, que «corum fructificationes oculis nostris se substrahunt et structura ab aliis diversa «gaudent» (Gen. plant. post titulum clas. XXIV).

Cependant dès 1583, Cesalpino, dans son traité De plantis, avait écrit les lignes suivantes en tête du chapitre consacré au genre Equisetum : Equiseti genera, quam ( vulgo Asprellam vocant, peculiarem constitutionem sortita sunt... Pleraque sine "fructu sunt.... quæ fructum ferunt, in cacumine capitulis oblongis congestum "habent, granis nigris, asperis et larclis constantem, sine ullo flore» (lib. XVI, cap. 15, p. 598). 
Dans son Epistolu ad Valentimum de sexu planterum (25 août 169:4) R. J. Camerarius, mentionnant chez les Equisetum un "semen masculinum quod more solito dif(llatur in auras, ") fail observer que "credibile non videtur omissas esse in quadam "plantarum specie femellas...., nee Equisetum illo pulvere seri putem...; ; egre tamen "sententia nostra patitur tantam scminalis aure copiam frustra dispergi; mais, malgré ce regret, il a la sagesse de n'émettre aucune opinion sur la reproduction de ces plantes, «quarum ortus et propagatio obscurior» (p. 265).

La phrase précitée de Cesalpino, où, remarquons-le bien, cel auteur ne disait pas que les Equisetum ont des fleurs sur certaines tiges ct sur d'autres des fruits, mais seulement que plusieurs liges ne portent pas de fruit, et que celles qui en portent n'ont pas de fleur's, la phrase de Cesalpino, mal comprise, fut reproduite sans examen par les botanistes qui le suivirent, et, cent vingt ans encore après lui, Tournefort (Inst. rei herb., 1700) place le genre Equisetum dans sa quinzième classe: «IIerbø Ruribus apetulis, "à la sixième section: "De herbis flore apetalo, quarum alic in codem genere floribus, alice vero fructibus plerumque donantur, ) c'est-it-dire dans ses Herbes apétales divïques, à côté du Chanvre, de la Mercuriale et du Iloublon, par cette seule raison que: «fructus Equiseti speciebus innascuntur, quæ floribus carent "grana nempe, auctore Cesalpino, nigra, aspera et farcta.»

J. Ray subit la même influence. Comme Tournefort, il sépara les Equisctum de ses Capillaires (Fousgères) et les plara dans la classe suivante à côté du Chanrre et de l'Ortie, en faisant remarquer toutefois qu'il n'était pas bien établi « an semen in eadem "cum flore planta nascatur necne. » Puis il mentionne les observations du docteur Ch. Preston, suivant lesquelles « Equisetum sylvaticum tchuissimis setis, C. B. Pin., a 16 , semina profert rolunda, alba, musci cujusdam seminum remula, pediculo "semunciali tenuissimo et plane capillaceo, e foliorum geniculis exeunte, sustentata; et, sans, élever aucun doute sur l'observation elle-même ni sur l'existence de cet organe, il ajoute: "verum an semina fuerint hee corpora, an vascula seminalia, «dubito (Meth.pl. emend., p. 20, 1703).

Dans les deux Dissertationes epistolares de plantarum propagatione qu'il adressa en 1717 à l'Académie des Curieux de la nature, Dillenius réfuta vivement la place assignée aux Equisctum par Tournefort et par Ray, ainsi que les prétendues observations de Ch. Preston, et il exposa les rapports d'identité qu'il voyait entre la fructification des Equisetum el celle des Capillaires (p. 58). L'année suivante, dans son Nov. plent. genera, il plaça les Equisetum dans ses Capillaires: "Equisetum autem "vera herba capillaris est, non minus ac Osmunda et Ophioglossum, nec quisquam "de eorum seminibus certi quid adhue afferre potuit; unde, cum seminibus, stami«nibus el reliquo apparatı florido destituantur, forma vero florida et propagandi "modo Capillaribus respondeant, co omnino referenda sunt. Differt Equisetum ab 
§ 6. - HISTORYUE.

"aliis quod utriculi farinam continentes in capitatum clavum disponantur, quorum «plurimis junctis clava vel capitulum exsurgit conicum » (p. 88).

Le «propagandi modus, » auquel Dillenius fait allusion, est exposé dans les deux ouvrages précités; suivant lui, les Capillaires et les Equisetum, aquæ quidem Capil"laribus omnino annumeranda sunt» (Nov. plant. gen., p. 81), manquant de vraies fleurs et de véritables semences, se propagent par rameaux souterrains, auxquels les granules farineux de leur fructification donnent la faculté reproductive. «Nobis ergo "probabilissima videtur opinio Capillarium genus non seminibus, non stolonibus, « ut nec alia adhuc nota et observata propagandi aut multiplicandi ratione, sed radi"cibus propagari; ita tamen ut novellie plantæ in his actu formentur, sed assurgere «nequeunt, ni pulvere foliorum foccundentur» (Diss. epist., p. 55). "Non diversa " fructificatione gaudere nobis videntur Equiseta » (ibid., p. 58; voy. Diss. epist., p. 71, 86, 87; et Nov. plant. gen., p. 77, 82, 83, 84). Comme le pollen d'un grand nombre de phanérogames a la propriété de s'enflammer en pétillant lorsqu'on le projette sur la flamme d'une bougie, et que les spores (farina) des Equisetum, des Fougères et des Lycopodes offrent la même propriété, Dillenius en avait conclu qu'ils n'étaient point une semence, mais une poussière fécondante de la nature du pollen. La même observation induisit Linné en erreur sur la nature des spores des Mousses et des Lycopodes (Sem. musc. in Am. acad., II, p. 284; 25 mai 1750). N'oublions pas de dire en faveur de Dillenius qu'il fut le premier à séparer nettement les Chara des Equisetum (Nov.pl. gen., p. 88, et Diss. epist., p. 58).

L'opinion de Dillenius paraît avoir été peu connue ou peu adoplée, car je ne la retrouve citée qu'une fois, sans adoption, et toujours à côté de celle de Cesalpino, dans Mappus (Hist. plant. Als., p. 98 et 99), en 1742, c'est-à-dire cinq ans après l'apparition du Genera plantarum de Linné.

Il est vrai que dans cet ouvrage, Linné lui-même paraît n'avoir voulu admettre aucune opinion hasardée sur la fructification des Equisetum, et il se borne à formuler en ces mots une description tout extérieure: « Spica fructificationibus peltatis, basi dehiscentibus multivalvis. » On est porté à croire que Linné n'avait jamais examiné des spores d'Equisetum, lorsqu'on lit la phrase suivante, ajoutée par lui à la description de l'E. arvense dans la deuxième édition du Flora suecica: «Pollen, quum «primum e spica excutitur, saltat ac si viveret, observante Stæhelino, quod olim in (Filicibus vidit Borellus» (p. 367). Ce que contient le Gen. plant., édition Schreber (1789) est emprunté à Hedwig, comme l'éditeur lui-même le mentionne.

En 1738, Duhamel du Monceau examina au microscope el dessina les spores des Equisetum (Phys. des arbres, II, p. 288, pl. X, fig. 277).

Haller vit dans les spores des grains verts de pollen : «ipsa natura elastica, quæ in "antheris plantarum admodum multarum observatur, demonstrat masculas has 
"Equiseti particulas esse; semina vero feminina desiderantur" (Enum. stirp. Ilch", p. $143 ; 1742$ ).

Adanson, réformateur et innovaleur quand même, place sans hésitation les Equisetum dans sa cinquante-septième famille, Les Pins. Comme Linné et Haller, il assigne atux spores la fonction du pollen, et, prenant ainsi les épis jour l'appareil des fleurs màles, il chercha les parties femelles dans la gaine des jeunes ponsses. "Les piés qui "sortent en forme de còne auprès des piés mâles contiennent dans chaque gaine des "branches plusicurs embryons de rameaux qui tiennent licu des ovaires et sont ter(minés par quatre stiles veloutés » (Fam. d. plant., II, p. 477; 1763). Il conjecture que ces ovaires ont besoin d'être fécondés par la poussière des fleurs mâles.

Gleichen prit les stomates des Fougères pour les organes mîles de ces plantes (Neuest. Unters., p. 24-30; tab. III, fig. 6 , bona; 1764).

Dans les intéressantes observations qu'il publia sous ce titre: Das cntdeckte Geheimniss der Kryplogamie (Le myslère de la cryplogamie déroilé, Carlsruhe 1777), Kölreuter exposa que les loges qui entourent les grains sont la partie mâle et que les grains sont les semences; il comparait les filaments (élatères) aux fils élastiques des Jungermannes, avouant en même temps que, dans ses expériences sur l'E. fluviutile, il n’arait pu réussir à faire germer les grains qu'il croyait être des semences.

De Lamarck prend de nouveau les spores pour du pollen : \& La face intéricure des "écailles est garnie de cellules qui contiennent une poussière assez abondante; ces "parties sont regardées comme des fleurs màles; les fleur's femelles, en ce eas, " sont encore inconnues》(Fl. fr., I, p. 5, 1778 et 1793).

Dès 1782, J. Hedwig avait décrit les organes générateurs des Mousses (Fund. musc. etc.). Il en avait représenté les anthéridies avec le mode d'émission de leur contenu, et enfin il avait constaté par des semis la nature reproductive de leurs spores. Mais, daus la famille des Mousses, les organes de fécondation, anthéridies et archégones, se trouvent sur les plantes adultes; ils précẻdent les spores, qui sont des lors fécondées, el la végétation passagère qui résulte du déreloppement de la spore n'a qu'une fonction à remplir, celle de supporter et de nourrir le jeune individu, d'en ètre, comme on l'a dit, le protunema. Dans les familles des Fougères et des Equisetum il n'en est pas ainsi; la végétation intermédiaire, née du développement des spores non encore fécondées, doit, avant de supporter et de nourrir la jeune plante, produire les organes de fécondation, anthéridies et archégones. Cette circonstance parait avoir induit Iledwig en erreur; cẹt éminent observateur voulut, pour les Fougères el les Equisetum, trouver les organes fécondateurs sur les plantes adultes, comme il les y trouvait pour les Mousses, et dans sa Théorie de la génération des cryplogames, il considéra les spores et les sporanges des Fougères comme des organes femelles fécondés par des étamines placées sur la face non fructifère de la fronde ct consistant en petits 
poils claviformes glanduleux (pilos glutinosos et capitellatos). Ces prétendues étamines se trouvaient dans un contact fécondateur avec l'organe femelle de la face opposée pendant le premier âge de la fronde et alor's que son enroulement tenait les deux faces en contact (Theor. gen., $1^{\text {ro }}$ édit., p. 41 et suiv., tab. 4-7). Quant aux Equisetum, il regarda chaque spore comme une fleur hermaphrodite; les élatères furent pour lui les quatre filaments de l'organe mâle, dépourvus d'anthères, il est vrai, mais portant à la surface de leur's extrémités spatulées du pollen en granules pulvérulents; la spore elle-même devint un germe muni d'un stigmate en forme de papille (o. c., p. 33, tab. 1, 2).

Dans son Genera pluntarum, 1789, A. L. de Jussien, après avoir exposé qu'il y a deux sortes de tiges, les unes vernales et conifères mais éphémères, les autres dépourvues de cônes mais durables, se demande «an priores masculæ, posteriores foemi«neæ sexu nondum detecto?» et il se borne ensuite à rappeler que : "sphærulas Hed( wigius stigmati assimilat, et setas pulvisculo aspersas, apice cochleariformi dilatatas, " conniventes in humido, in sicco patulas, microscopio manifestas pro filamentis ( antheriferis habet» (p. 17).

Ce fut le dernier écho de la doctrine attribuée à Cesalpino et qui avait retenti pendant deux siècles, et le premier écho de celle de Hedwig qui devait retentir moins longtemps, mais avec plus d'éclat.

En 1791 Schreber l'introduit, sans aucune remarque, dans son édition du Genera plantarum de Linné; comme en 1795 Hoffmann la répète simplement dans la partie cryptogamique du Deutschlands Flora (clas. XXIV; II, Peltata).

Roth l'admet également sans observation dans son Tent. fl. germ., IV, p. 3, 1800; mais ce qui surprend, c'est de voir cet auteur combattre énergiquement, p. 29, l'opinion de Hedwig sur la fécondation des Fougères et revenir au bas de cette même page à l'opinion de Hedwig sur les Equisetum, en la proposant comme l'expression typique de ce qu'il y aura à découvrir sur la fécondation des Fougères : «Vero similius videtur, "quod in Filicibus, uti in Equiseto, Pilularia et nonnullis aliis plantis cryptogamicis e spermatis masculini officina non extra uterum muliebrem, sed intra ovarium "quærenda sit, licet ob teneritatem partium in oculos non incidat etiam optime ( armatos. 》

Willdenow fournit occasion à une remarque analogue. Dans l'introduction du t. V de son édition du Species plantarum, 1809, il établit par les semis de Lindsay, par ceux d'Ehrhart, par les siens propres, que les spores des Fougères et des Lycopodes possèdent la faculté reproductive (p. viI et virr); puis arrivé aux Equisetum, il semble oublier et ce qu'il a dit précédemment et les lois de l'analogie, il revient à l'opinion de Hedwig et fait de leurs spores des «flores hermaphroditi ex filamentis "quatuor, quorum bini semper basi cohærent, apice spathulatis absque antheris et 
"polline tantum conspersis, atque ex germine stigmate instructu compositi „ (p. xxrx).

De Candolle (Fl. fr., เ. II, p. 580, 1805) reproduit sans modification l'opinion de Iledwig, seulement il range les I rềles et les autres cryptogames vasculaires dans les Monocotylédones, sur cette considération que les graines de Fongères, «semées avec «soin, lèvent accompagnées d'un cotylédon latéral, étalé, membraneux, large et eréniformen ( $F l$. fr., II , p. $546 \mathrm{ct} 5$ 47). On verra plus loin, p. 130, la modification des idées de cet auteur.

Mirbel (Pliys. vég., I, p. 387, 1815) répéta simplement l'opinion et les expressions de Hedwig.

Il est facile de comprendre comment, après avoir méconnu la nature reproductive des spores des Equisetum, en les assimilant au pollen ou à la fleur des autres plantes, on dut rester si longtemps sans songer à les semer, et tourner tous les efforts vers la recherche de l'organe femelle.

Enfin l'auteur de la Monographie des Prêles indiqua et ouvrit la route à suivre pour orriver à la solution scientifique du problème. Après avoir rapporté l'opinion de Hedwig, il ajonte: "Mais toutes probables que fussent ces conjectures, il fallait les a vérifier par des expériences. C'est ce que j'ai fail dés l'annéc 1817. J'ai mis ces graines «supposées des Prêles les unes dans l'eau et d'autres dans des rases remplis d'un «terreau très-fin. C'était le 3 arril, à l'époque de l'apparition des Prèles a hampes. a Au bout de deux jours, les grains plongés dans l'eau montraicnt une petite pointe à "peu près du tiers de leur diamètre. Le troisième jour celte pointe s'était allongéc; a elle était transparente et ressemblait à un tube vide. Au bout d'un mois les grains «semés sur terre avaient poussé d'un côté une ou deux radicules simples et blan«châtres, et de l'autre ils s'étaient renflés et divisés d'abord en deux, ensuite en «trois et quatre lobes. Ils continuèrent à croître pendant tout le mois de mai, en se "ramifiant considérablement. $\Lambda$ la loupe, ct surtout au microscope, c'étaient des tiges «cylindriques, nombreuses, articulées et ramassées en faisceau.

«J'ai mis successivement en expérience les grains de la Prêle fluviatile (E. maxi( $m u m)$, de la Prêle des marais (E. palustre) et de la limoneuse (E. limosum). Ils se sont «agrandis de la même manière et ont présenté les mêmes apparences".

a Mais ils se sont arrêtés à ce terme, malgré toutes les précautions. Dans les «années qui ont suivi 1817, je n'ai pas été plus heureux; mais je recommencerai mes (expériences avec une nouvelle attention dès le printemps de cette année (1822).

«Cependant ce que j'ai vu tant de fois suffit, je pense, pour constater que le grain (vert est bien la semence des Prèles, qui sont des plantes acotylédones, puisque ce

\footnotetext{
${ }^{2}$ L'auteur a représente les sporophymes de l'E. arvense, pl. 1, fig. 5 et 6 ; ceux de l'E. maximum, pl. II, fig. 3 et 4 ; ceux de l'E. palustre, pl. V, fig. 5 et 6 , et ceux de l'E. limosum, pl. VIII, fig. 5 . Ces figures sont mal faites et simulent des cotylédons.
} 


\section{§ 6. - Historique.}

"grain tout entier se développe sans qu’on puisse y apercevoir aucun corps étranger à "l'embryon ou à la plantule. Je ne pense pas que l'on puisse s'assurer par des expé« riences directes que les quatre filets soient des étamines, et que les pulviscules qui les «recouvrent soient un véritable pollen. Cependant l'analogie est fortement prononcée " pour celle opinion, surtout si l'on convient qu'il ne faut pas chercher ici des anthères «et des loges semblables à celles des plantes phanérogames, et il faut avouer que les «mouvements élastiques des lames qui entourent le grain vert sont merveilleusement "propres à secouer la poussière fécondante, et à favoriser la dissémination des graines "fécondées. Mais si l'on ne peut douter que les grains verts ne soient de véritables «semences, on doit s'étonner de ce qu'elles remplissent si mal leur destination; car je «n’ai jamais trouvé une seule Prêle qui ne me parât pas fort ancienne et dont la «racine ne se prolongeât pas indéfiniment dans la terre. Je crois que les autres bota“nistes n'ont pas été plus heureux. Il faut donc convenir, au moins jusqu'à présent, "que les Prêles sont des plantes dont les mêmes individus subsistent depuis un temps «dont on ne saurait assigner le terme, et qui répandent chaque année des myriades (de graines autrefois fertiles, aujourd'hui infécondes» (p. 347 à 351).

a Malgrẻ mes efforts, je n'ai point trouvé l'extrémité des tiges souterraines. La Prêle "est donc une plante dont jusqu’à présent la durée doit être considérée comme indé«finie, et qui, du moins à ma connaissance, ne fournit plus de nouveaux individus, «quoique ses graines soient susceptibles de développement 》 (p. 390).

Dans la même année G. A. Agardh, professeur d'histoire naturelle à Lund (Suède), publia dans les Mémoires du Muséum d'histoire naturelle (t. IX, p. 283-292) des a0bser"vations sur la germination des Prêles.» Après avoir brièvement rappelé les principales opinions émises sur ce point par Linné, Adanson. Hedwig, il expose que « dans « les derniers jours d'avril 1822, il fit des semis de la poussière de l'E. arvense sur plu« sieurs pots, placés an soleil, à différents degrés de lumière, de chaleur et d'humidité. «Le 10 de mai un petit bouton vert et bifide avait paru et une petite radicule pel" lucide était descendue dans le terreau. Je ne doutai plus que les deux lobes du bou«ton ne fussent des cotylédons, et que la plante ne fût dicotylédone et plus voisine « des Casuarina qu'on n'aurait osé le penser.

"Le 19 de mai je fus fort étonné de trouver, au lieu d'une jeune Prêle que je sup" posais devoir s'élever entre les deux cotylédons, une petite conferve verte qui n'était «autre chose que le développement de ce que j'avais pris pour les cotylédons; les « filaments étaient articulés. Aucun rudiment d'une plante plus parfaite ne se mona trait encore, et je ne saurais concevoir comme une Prêle pourrait se développer "des organes qui s'étaient déjà montrés. Je craignis de m’ètre mépris ct que les plan"tules observées ne fussent autre chose qu'une de ces conferves menues qui naissent «si facilement sur le terreau.» 
Il répéta donc ses expériences sur "la poussière de l'E. pulustre et de l'E. limosum;" et comme il vit ades plantules exactement de même nature que les précédentes,» il en conclut "que les poussières des Prêles sont des semences qui les reproduisent «(p. 290). Mais ce fail, quelque important qüil soit, rend la question sur la germi«nation des Prêles plus embarrassante encore. Comment concevoir en effet que les "grains semés germent, mais donnent des plantes d'une autre famille, qui restent ades mois entiers dans cet état étrange? On reconnaitra du moins que dans certaines "plantes, comme dans certains animaux, il y a un état intermédiaire entre la semence « ou l'ouf et la parfaite évolution. Les lois de celte transformation sont encore incon«nues, mais le fait ne l'est plus.»

L'auteur rappelle ensuite le mode de reproduction des mousses, ajoutant que, a i ( en juger par la germination, les Prêles sont voisines des. Mousses.» Puis, sur des considérations très-élevées, il établit que « les prétendus cotylédons des cryptogames "sont d'une tout autre nature que ceux des plantes plus parfaites, qu'ils ne sont "point, comme ces derniers, déjà formés dans la graine, mais qu’ils sont le produit « de la végétation. Ainsi ils ne sont pas de vrais cotylédons, mais un premier àge ou "un premier état de la plante.

Malgré tous ses soins, ses semis périrent dès les premiers jours de juillet; il the put donc entrevoir aucune trace d’anthéridie. Cet important mémoire est accompagné d'une planche dont les figures sont, comme celles de Vaucher, peu satisfaisantes; les cellules sont trop semblables à des cotylédons.

Fidèle à la promesse faite par lui (IOn. Prêl., p. 350), Vaucher répéta ses essais en 1822, mais sans succès. Il les recommença en 1823 sur les E. maximum et palustre, et la mème année en consigna l'heureux résultat dans son .Hémoire sur la fructification des Prêles. "Tout s'est passę́ d'abord cornme je l'avais déjà annoncé dans mon "Histoire des Prèles, et enfin (le 12 et le 21 septembre) il s'est élevé du centre du "gazon un point vert qui, en grandissant, a laissé voir à sa base une collerette ì quatre a divisions, puis une seconde, puis successivement une troisième, d'oủ sortait le som« met de la jeune tige.

«Les grains verts renfermés dans les loges dont l'assemblage forme l’épi de la "Prêle sont en conséquence de véritables semences acotylédonées, dépourvues non« senlement de cotylédons proprement dits, mais encore d'albumen et d'enveloppes; "elles sont réduites au seul embryon. Mais cet organe ne ressemble point à ccux que «nous connaissons. Il n'est pas composé d'une radicule et d'une plumule....

«Ce mode de germination présente la circonstance remarquable de deux espèces de aracines. Les unes appartiennent au grain vert dans sa première érolution; les autres "dépendent de la tige mème de la jeune Prèle; celles-ci se réduisent à une racine " nnique, fort apparente et qui s'enfonce perpendiculairement dans le sol : celles-li 
(au contraire sont multiples, mais laibles et délicates: elles se délruisent prompatement.

“La racine propre de la Prêle, examinéc au microscope, ne m’a pas paru articu«lée; elle est simple, continue, pivotante et semblable aux racines des autres plantes. «Comment devient-elle articulée? c'est ce que j’ignore.

" J'ai une douzaine de jeunes Prêles qui proviennent de la Prêle fluviatile ( $E$. maxi( mum) et cing ou six de celle des marais. Jusqu’à présent elles se ressemblent beau«coup, et je ne serais pas en état d'y reconnaître la moindre différence.

" Je m’étais trompé en imaginant que les Prêles pouvaient être considérées comme "des plantes dont les semences élaient actuellement infécondes. Je crois aujourd'hui "que les Prêles, comme les Fougères, se reproduisent toutes les fois qu'elles sont "placées dans des circonstances convenables, mais je persiste à pensel que ces cir«constances sont fort rares» (p. 429-434).

Ce mémoire est accompagné d'une planche représentant la première évolution de la jeune tige. La fig. 5 est mauraise en ce qu'elle simule sur la racine des sillons qui ne s'y trouvent pas. La fig. 4 a élé reproduite par. Bischoff; toutes les autres ont été copiées en sens inverse par Payer, qui les attribue à tort à l'E. ar'ense (Bot. crypt., p. 244).

Les expériences et les observations de Vaucher et d'Agardh avaient eu ce premier et immense résultat d'établir que les spores possèdent à elles seules la faculté reproductive. Aussi est-ce avee un profond étonnement qu'on voit, en 1824, H. E. Link reproduire sur la fleur des Equisetum l'opinion de Iledwig (El. phil. bot., p. 301, $\$ 173)$, et en décrire le fruil dans les termes suivants : « Equisetina habent capsulam «absque pistillo viridem, semina minutissima grumosa continentem. Fila clastica «capsulam cingunt, quam dehiscentem non vidi. Amentum gerunt squamis peltatis asubtus folliculis membranaceis obsitum capsulas includentibus. De germinatione "Agardhi habemus observationes, quæ vero rem non expediunt) (u. c., p. 355 , \$ 191).

En 1825 G. L. Duvernoy, dans une thèse botanique : De Salvinia natante, mentionna, p. 10, le premier développement des spores des Equisetum, constata l'absence du style et des grains polliniques affirmés par Iledwig; mais il ne fournit d'ailleurs aucune vue nouvelle.

En 1826 Vaucher ajouta à la fin de sa Yonograplice des Prêles (dans les exemplaires du tirage à part) une longue Note sur la germination des Préles. Il y reproduil, arec de légères modifications de style, son mémoire de 1823 Sur la fiructificalion de's Prêles, et il la termine par l'importante constatation qui suit: :Tontefois je n'arais «jamais aperçu de jeunes Prêles, germant et se développant naturellement, lorsque «l'annie dernière (1825) M. Requien, cet excellent botaniste d'Arignon, m'a fait par- 
a venir des tapis de jennes Prûles, quill arail trouxées la mème année an mois de a juin, croissant el se développant sans eflort dans un lieu inondi l'hiver et le princtemps. Elles avaient germe plus tit que les miennes; mais elles leur ressemblent "entierement par leur tige télragone, leurs involucres el leur port. Elles pourraient "bien appartenir à la Prêle des Limons.»

Hans sun Orycunographie régctule (t. II, p. 126 et 127, 1827), De Candolle modifia un pen son opinion; il mentionna les semis de Vaucher et d'igardh, en reconnaissant (que le flobule vert (la spore) est un organe consistant en un tubercule analogue "aux bulbilles, el que les lames élastiques ne peuvent être des étamines.»

Le 20 mars 1827 Bischofi publia le premier cahier des Végélaux cryplogames d’Allemagne et de Suisse. Après avoir exposé l'organisation générale des Equiselum d'une maniere beaucoup plus complete et plus savante qu'on ne l'avait fait avant lui (Krypt. Gev., p. 28-40), il donna des spores une description longuement détaillée (p. 32 et 40, mais toutefois pen exacte, et it rendit compte des semis tentés par lui en 1826 (p. $41 \mathrm{et}$ suiv.). Les premiers développements de la spore en sporophyme avaient été assez lien observés, et l'auteur, après aroir réfuté (p. 42 et 43) la comparaison faite. par Vaucher entre les premières cellules du sporophyme el l'embryon des phanérogames, exposa arec une grande clarté que "le déreloppement des spores n'est pas celui d'un embryon formé d'arance, mais que dans les Prèles, comme daus les autres cryplogames et en particulier dans les Fougèes, la germination passe d'abord par l'état intermédiaire d'un embryon incomplet avant darriver à celui de la nouvelle. petite plante, et que ce proembryon, composé sculement de tissu cellulaire, ne dome. pour ainsi dire que le fond sur lequel doit se développer la véritable plante nouvelle, pourvue de vaisseaux $\gg$ (p. 43).

Les obserrations de Bischoff sur le sporophyme se bornent done à le consilérer "comme fournissant la première nourriture it la plantule» (p. 43). Il ne parait pas avoir soupconné l'acte de fécondation dout le sporophyme doit porter les organes, bien qu'il ait dit: "Dans l'acte de la germination des cryptogames, le proembryon cet en soi un organe plus important que les cotylédons de la graine, parce qu'il est obligé de donner l'existence a l'embryon proprement dit, avant de pousuir remplit à son égard la fonction habituelle des lobes séminaux, laquelle parait se réduire i la nutrition de l'embryon préexistant en mème temps qu'eux dans la graine » (p. 43). Mais, je le répète, nul soupron de la présence d'organes de fécondation. Ses semis de 1826 p.rirent dailleurs de très-bonne heure et probablement avant l'apparition de cess organes.

Dans l'automne suivant, Bischoff trouva prés de Deux-P'onts un grand nombre de jeunes plantes d'E. paluslie, et il publia la ilescription et les figures de ces jeunes plantes et de leur spornphỹme; mais rette fois ce dernier était trop avancé (Eutu. Eq., 
p. 787) pour suggérer a cet excellent observatemr lidcic dly chereher les organes de fécondation. A part quelques erreurs d'appréciation presque inévitables à celle époque, ce mémoire se recommande par une exactitude parfaite dans la description et dans les figures.

M. Ad. Brongniart (Ilist. vég. foss, I, p. 10 ' et 105, 1828), après avoir rappelé que "Hedwig considère le globule central comme un ovaire surmonté d'un stigmate très"court et les filaments comme quatre anthères portées sur leurs filets, » ajoute: "Peut-être serait-il plus naturel, d'après ce que nous connaissons actuellement sur "la structure des organes reproducteurs des plantes Phanérogames, de considérer "le globule comme un ovule surmonté de son mamelon d'imprégnation, et les quatre «filaments renflés comme quatre grains de pollen réunis deux à deux à la base de "l'ovule. »

Avec M. L. Reichenbach l'hypothèse prend une autre forme: elle affirme absolument et sans hésitation. Après avoir démenti les expériences de Willdenow, et formellement refusé aux spores des Lycopodiacées la faculté reproductive (Flor. cxcurs., p. 152), cet auteur dit des Equisetum: "Flores insunt præformato-hermaphroditi numerosi, «conglobati. Gyncec. ovarium minimum globulosum vel subturbinatum. Androc. « staminodia bina basi cruciatim affixa, elongata, utrinque spathulata, polline repleta? " elastice ovarium circumvolventia. E germinatione potius Cerioblastce, cotyledonibus «demum verticillatis 》(Flor. excurs., p: 154).

En 1833 M. H. Mohl publia dans le Flora des Observations sur la formation et l'organisation des spores. Il exposa que les élatères ne sont que les restes de la cellulemère, et il émit l'idée que les cellules génératrices ne donnent naissance qu’à une spore au lieu de quatre, et qu'elles persistent au lieu d'être résorbées. Mais il ne dit rien de la reproduction (Bemerli. Spor., p. 33-46).

Après ces travaux, les résultats des recherches sur la reproduction des cryptogames subirent un long arrêt de presque vingt ans, pendant lequel il se produisit pourtant encore une nouvelle opinion sur la fécondation des Equisétacées. Ce fut celle que M. Raspail émit dans son Nonveau système de physiologie végélale et de botanique (novembre 1836), et qui n'est qu'un emprunt, avec application aux Equisetum, des idées de Hedwig sur les organes mâles des Fougères. Suivant M. Raspail, « oủ cher" cher l'appareil mâle des Equisetum, si ce n'est dans chacune des dents de la gaine, "qui recouvre immédiatement le chaton femclle encore rudimentairc? Or, si l'on cxaa mine celle gaîne à celte époque, ou même après les premiers instants de sa déhis"cence, on ne manque jamais de lui trouver une turgescence résineuse el dorée, " laquelle rappelle l'aspect et le facics des organes males des plantes, qui en pos" sèdent sous des formes mieux caractérisées. Le chaton imprègne, en passanl, cha"cune de ses écailles ovariennes, avec l'aura seminalis, que recèlent les dents dorées 
a de la gaine quil lend; et après celte fécondation, chaque dent dureit et noircit, a rumme les dents des gaines infërieures qui avaint servi il féconder les entre-neuds "de diveloppement. I celle éporque, on les trouve bordées d'une membrane blanche, "qui rappelle le lissu de certains thece épuisés de pollen» (t. II, p. 417, §1905, Observ.) $)^{\prime}$

Le mourement hélicoïde les spermatozoïdes avait élé des 1822 constaté sur les Sphagmum par Fréd. Nees d'Esenberk et décrit dans le Flora de la même annéc; mais celte découverte semble avoir plutut relardé qu'avancé celle des organes de fécondation des cryplogames vasculares. La plupart des observateurs, à l'exemple de Hedwig, croynient que sur ces deruieres plantes, comme sur les Mousses, les spores ¿́taient déji fécondécs. Ils śubstinaient done à chercher les organes de fécondation sur le vigutal adulte, el les plus importantes découvertes sur la génération des Fougeres ne furent laites quen 184' el 1847 par M. Nägeli, en 1848 par M. LeszezycSuminsli. Ce fut également en 1848 que les anthéridies et les spermatozö̈des des Equisetum fureut obscrvés poun LA pnemene fols par M. Gustave Thuret et brièvement décrits par lui dans sa Note sur les anthéridies des Fougères.

A celte époque, les recherches sur la génération des cryptogames vasculaires se poursuivaient avec une grande aclivité. Le 6 novembre 1850 M. Mille présentait ì l'Université de Breslau une thèse pour le doctorat, dont le sujet était De sporamm Equisetomm germinatione. Le jenne docteur mentionnail la découverte de M. G. Thuret, decrivait longuement et figurait la germination des spores (p. 8-14), la position des anthériclies el l'émission des spermatozoïdes (p. 14-18). A part l'anneau altribué aux anthéridies (p. 15) et dont nous arons parlé p. 99 el 100 , tout ee qui est dit sur la position des authéridies au sommet des lobes (p. 1/1), sur leur grandeur comparée i celle des anthéridies des Fougères (p. 15), sur leur mode de déhiscence (p. 15) est tout à fait exact. Les semis de M. Milde périrent avant de lui laisser roir des archégones. Liannée suivante M. G. Thuret décrivit avec quelques détails et figura les anthéridies des Equisetum daus ses admirables Recherches sur les aoospores des Algues et les anthéridies de's Fougieres. Au même moment et dans le $n^{\circ} / \mathbf{t}$ du Flora de 1851 ,

\footnotetext{
- Le même ouvrage contient sur les Equisetum unc foule d'assertions qui ne semblent résulter ni d'observations exactes ni de démonstrations rigoureuses. Il suffira de citer les suivantes:

* La tize porte en spirale des écailles hexagonales peltées n (I, p. 590, 81231). "Elles (les spores) se composent d'une "Vésicule transparente, cuntre les parois de laquelle serpentent deux spires en sens contraire l'une de l'autre, plus - un tissu cellulaire interne $(I, p .591, \% 1232)$. Tout ce paragraphe est de la meme force, et on y lit que ule gros " Globule jaune (la spore) est sans doule le produit de l'accouplement des deux spires " *Nous placeruns les Équisétacées "dans les monocolylédones, parce que dans l'acle de leur geermination, et par leur structure générale, ils se comportent - de la mème manière que les monocolylédones. (I, p. 596, Z 1238). - L'organe des Equisetum, unique organe sexuel «q que l'on connaisse à ce genre, n’est qu'une spore emportant ses spires fécondatrices avec elle s (1, p. 398, 8 1244). "Les organes reproducteurs sont des gros grains polliniformes, nés sur l'entrecroisement de deux spires " (11, p. 446, \% 1903). "1 nimalcules spernatiques des végétaux; grossières illusions, \& $1666=$ (II, p. 633 à la table des matières).
} 
M. Hofmeister, rendant comple de la thèse de MI. le docteur Mrilde, annonçait qunit avait fait lui-même avec un plein succès des recherches sur la fécondation des Equisetum.

En effet, le savant micrographe de Leipzig publiait quelques mois plus tard son important Examen de la germination, du développement et de la fructification des cryptogames les plus élevés (Mousses, Fougères, Équisétacíes, Rhizocarpées et Lycopodiacées) comparés à la fécondation des Conifères; et là il décrivit l'évolution des tiges, des gaines, de l'épiderme, des vaisseaux, des rameaux et des racines, de l'appareil fructifère, des spores, des sporophymes, des anthéridies et des spermatozoïles. Ce beau travail, avec les excellentes figures qui l'accompagnent, est incontestablement jusqu’ici ce qui a été publié de plus complet sur cette matière. L'auteur le termine par l'expression du regret de n'avoir pas vu les archégones.

Toujours dans celte mème année 1851 (le 25 aoûl) M. Milde communiqua à l'Académie des Curieux de la nature un Némoire pour servir à l'histoire du développement des Equisetum, lequel ne parut qu'en 1852 dans les Acta de celte Académie. Ce tritvail, reproduction développée de la thèse précitée, contient d'abord, comme cette thèse, l'histoire des recherches relatives à la germination des spores des Equisetum, et ensuite l'organisation des spores et les divers états de leur développement en proembryon (sporophyme). La description des anthéridies et de leur contenu est moins complète que celle de M. Hofmeister, et ne contient pas l'histoire de la formation et de l'évolution de cet organe, auquel l'auteur continue à altribuer un anneau, qui n'existe pas. Les spermatozoïdes sont décrits avec détails, et, comme II. Hofmeister, M. Milde termine en exprimant ses regrets de n'avoir pas vu d'archégones.

A ce mémoire se joint un Supplément (p. 637-641), où l'auteur décrit de jeunes plantes d'Equisetum trouvées par lui sur les bords de l'Oder. Malgré quelques imperfections déjả signalées p. 114, ce Némoire et son Supplément sont deux travaux très-importants et qui m'ont été particulièrement utiles.

Dans le Flora de 1852, no 25, p. 385, M. Hofmeister pullia ses excellentes obselvations Sur la germination des Equisetum, et décrivit, sans les figurer, les archégones de l'E. arvense et les premiers développements du pseudembryon et de la jeune plante.

La même année et dans le même journal (n 32, noût, p. 497), N. Nilde fit paraître ses observations Sur les archégones de l'E. Telmateia; une planche est jointe au texte. Ces observations, avec quelques changements de rédactiou, paraissaient simultanément dans le numéro du même mois du Botanische Zeinung (n 32, août, 1852, p. 537). Deux figures sont intercalées dans le texte, et, comme la planche du Flor elles représentent des archégones. Le savant botaniste de Breslau ne dit rien sur le dẻveloppement de la jeune plante qui provient de l'archégone. 


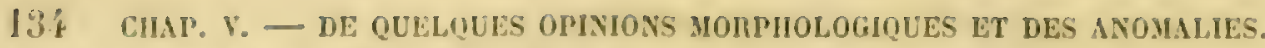

Celle lacune fut en partie comblée vers la fun de la mème annce 1852 par .H. IIolmeister, yui, dans les Mémoires de la Socirile des sciences de Saxe, vol. IV, exjnosa et fiscura le déveluppement de lanchégone, celui de la cellule reproductive el de li jeune plante qui en provient.

Liannee suivante, Bischofl fit parailte dans le Botunische' Zeilung des Remarques sur lihistoire du développement des Equisetum. Aprés un historique de la question, ce savant exposait quelques observations failes par lui en 18.18 sur des sporophymes d'E. sylenticum; puis il criliquait les dénominations d'archingune's, de spermatozanires, d'anthérozoüles, de prothallium ele., ainsi que quelques détails des travaux de M. Milde. Ces remarques ne contiennent d'ailleurs rien de noureau. Elles ont été reproduites en parlie, avec les figures, dans les Amnales des sciences naturelle's, III ${ }^{\circ}$ série., bot., t. XIX, p. 232 el suiv.

La Monographie des cryptogames rasculaires de la Silésie, communiquée par II. Milde à l'Académie des Curieux de la nature le 2 janvier 1852 , el publiée l'année suivante dans les Nova acta, ne contient qu'un énoncé général sur la génération des Equisetum.

\section{GHAPITRE V}

\section{De quelques opinions morphologiques et des anomalies}

\section{$\S 1^{\mathrm{er}}$. Morphologie}

Mirbel a été le premier ou un des premiers à dire que al'on peut considerer lit "gaine comme formée par la réunion de feuilles verticillées ") (Phys. vigit., I, 1. 387,. Celle opinion a été successivenent aloptée; et la plupart des auteurs qui se sont prononcés sur la nature des gaines, les ont considérées comme des feuilles soudécs et libres seulement a leurs extrémités ${ }^{1}$. S'il y a des dissidences sur ce point, je les jgnore. Je mentionne done celte opinion simplement pour memoire, sans la disculer, sans y rien objecter; me bornant, ainsi que je l'ai déjà fait p. 12 et 58 , ì représenter, d'une part, que les parties dont la réunion constitue la gaine, ne sont point à leur extrémili aussi essentiellement libres qu’on pourrait le croire el qu'elles ne s'isulent que par division mécanique; et, d'autre part, qu'il n'y a peut-ètre pas rigueur logique à refuser le nom de feuilles aux expansions aériennes des Fougires et à le domner à

' Ad. Brong., Jist. t'ég. foss., I, P. 102; Al. Braun, Sillim. Journ., p. 81; Koch, Syn., 963; A. Richard, Dréc. bol., II, P. 30 ; 10ill, l\%. Bad., 1) 53; Bernoulli, Gel, Kirypt. Schu., p. 64 ; elc. 
des organes qui, sur les rhizomes les plus profonds des Equisetum, sont aussi contplétement développés que sur leurs tiges.

II. Döll a émis sur les a verticilles de feuilles y une opinion que je dois rapporter et examiner, d'abord parce que toule opinion du savant bolaniste de Carlsruhe mérite d’ètre citée, el ensuite parce que l'auteur lui-mème provoque l'examen à ce sujet: "Mes recherches mont presque conduit à la certitude que les rerticilles de feuilles d'Equisetum ne se composent pas seulement des granes libres, mais que clacun d'eux commence déjà à se développer au noud précédenl de la lige ou du ramean, couvre ensuite la tige tout le long d'un entre-nœud, el que ce n'est qu'au nœud suivant qu'il se détache comme gaine libre en embrassant la base dı nouvel entrenoud et se terminant dans les pointes des dents. D’après cela, les tiges, aussi bien que les rhizomes, seraient recouverts, sur toute la surface et par entre-nouds, d'un verticille foliaire soudé, et la base des entre-nozuds serait en outre entourée de l'extrémité libre du verticille foliaire précédent, c'est-à-dire de la gaine libre.

aEn faveur de cette opinion milite cette circonstance que, entre l'entre-noud el la gaine libre qui s'en détache à la partie supérieure, il ne se trouve aucune ligne de démarcation qui indiquerait que là est le point d'origine des feuilles. A l'extrémité de l'entre-nœud on voit alterner avec les feuilles la disposilion des faisceaux vasculaires et des parlies cellulaires, et, avec celles-là, les lacunes à air. Les canaux de l'entre-nœud inférieur cessent, et, un peu plus vers l'intérieur, entre les extrémités des canaux inférieurs, commence, en alternant, un nouveau cercle de pareils canaux. Au contraire, la masse cellulaire qui constitue l'enveloppe extérieure el qui entoure le canal angulaire souvent dilaté, se transforme, ainsi que les faisceaux vasculaires des angles, en la partie libre de la gaine; celle-ci a donc son origine non à la base de celte partie libre, mais bien à la base de l'entre-nœud infraposé.

"En harmonie avec cette opinion se trouve la distribution égale des stomates sur les entre-nœuds des rejetons et sur leurs gaînes libres; et de plus il serail difficile de prouver que dans une autre famille quelconque les stomates se trouvent en aussi grand nombre sur les tiges des plantes » ( $F l . B a d .$, p. 55 el 56 ).

Le fait cité en dernier lieu à l'appui de l'opinion de M. Döll, savoir l'égalité de répartition des stomates sur les entre-nœuds el sur les gaines, n'est point d'une exactitude générale; ainsi les tiges spicifères de l'E. ar'cnse, et loutes celles re l'E. maximum, spicifères ou stériles, n'ont point de stomates aux cntre-nouds, tandis que leurs gaines en sont richement pourvues. De plus la chlorophylle et les faisceaux de fibres corticales (liber de quelques auteurs, faiscenux rasculaires des angles de M. Döll) manquent absolument aux entre-nœuds des mèmes tiges, tandis qu'jls se trourent à leur's gaînes. Ainsi donc il existe déjà, sur ces espèces, une ligne de démarcation indiquant le point oủ commencent les gaines. 
Si les autres cepèces ne présentent pas a l'extérieur des différences ausi frappantes, clles uffrent, ainsi que les premieres, dans leur structure interienre des differences tres-considerables, qui mirrguent nettement que la gaine nest pas simplement la prolongation du revitement extérjeur des entre-nmuds. Ėn eflet, comme nous l'avons vu p. 48 el 49 , les faisceaux fibro-vasculaires ne passent point sans modification de l'interieur d'un entre-nerud dans la ganine qui le surmonte; mais vers chargue unud il existe des groupes de cellules courtes, striées ou spiralée, d'on ils sortent, ou, si l'on veut, auxquels ils aboutissent en suivant des directions tris-differentes pour se rendre soit dans la gaine, soil dans l'entre-naud inféricur ou supérienr. Il y a done lit, à l'intérieur, une ligne, ou plutùt un plan de différences indiquant une modification profonde et l'origine d'un organe.

Si l'opinion de MI. Döll était complétement exacte, c'est-à-dire si les gaines com mençaient dès la base de chaque entre-noud, en formaient le revêtement extérieur et s'en détachaient au nœud suivant, il s'ensuivait que les crites des entre-nouds seraient en quelque sorte les pétioles décurrents des « Peuilles; que le eylindre externe serait constitué par leur réunion et que des lor's ce cylindre derrait renfermer tous les tissus élémentaires des gaines. Or il n'en est rien. Tous les éléments des deux cylindres d'un entre-noud se retrourent dans sa gaine, et la lacune qui y existe quelquefois est celle du cylindre interne. Mais, comme nous l'avons vu, les éléments de ce cylindre se modifient tous entre l'entre-nœud et la gaîne. Donc il n'est pas exact de dire que la gaine "a son origine à la base de l'entre-noud, qu'elle en forme l'enveloppe extérieure (ïusscre Ilaut), et sc détache ensuite comme simple continuation de celte enreloppe, sans ligne de démarcation et sans différence d'organisation.» Liassertion n’aurait pas mème une exactitude générale, si on la réduisail à l'épiderme, car sur certaines espèces, l'épiderme des gaines n'est pas identique a celui des entrenœuds (roy. p. 28).

Puisque, d'une part, la gaine renferme tous les tissus des deux eylindres de l'entrenoud presque semblablement disposés, puisque, d'autre part, l'apparition et surtout l'évolution de la gaine précède celle de l'entre-ncud, et que c'est de la base de la gaine que naissent d'abord les tissus de l'entre-neud infraposé, il semble quau lieu de faire de la gaine une simple prolongation de l'enveloppe des entre-nouds, il serait plus naturel de dire que l'entre-noud est la continnation inférieure de la gaine. Chacune des divisions de l'entre-neud répondant ì une division de la gaine, serait alors consitérée comme le pittiole de cette expansion foliacie; el de même que le slipe ou le rhizome des Fongères est formé par la réunion, autour d'un centre médullaire, des pétioles qui le parcourent bien au-dessons de leur point d'émersion, un entre-noud d’Equiselum scrait constitué par la réunion de pétioles régulièrement verticillés, fuu murent sur toute sa long̨ueur. Mais cotte manière de considérer l'entre-nend et la 
gaine comme Is développement et la continuation l'un de l'autre, au-dessus et audessous du noud, n'est pas mieux en harmonie avec ce que nous avons vu p. 58 , 59 et 61, de l'évolution de la gaine et de celle de l'entre-noud, puisque la gaîne se développe par sa base contre le nend adjacent, cl que l'entre-noud quielle surmonte se développe par sa base contre le nœud inférieur. Il me parait donc plus sage de me borner à constater' ce qui est, et de m'abstenir de tou'c hypothèse morphologique.

Les rameaux naissent immédiatement au-dessous des gaines. Celte loi est si absoluc que ni les mutilations, ni les déformations, ni les «monstruosités » n'amènent jamais une exception ". En même temps ces rameaux émergent, comme nous l'avons vu p. 13 et 65, dans les sillons, c'est-à-dire sur la ligne commissurale des divisions de la gaîne ou «feuilles, de Mirbel; de telle sorle que, au lieu d'être supérieurs el axillaires à ces divisions, ils leur sont latéraux et inférieurs. $\Lambda$ la sortie de terre des tiges stériles de l'E. maximum, les entre-nœuds, qui plus tard seront très-longs, sont alors extrêmement courts, et par conséquent les nœuds sont presque contigus. Les gaînes, qui sont déjả complétement développées ou à peu près, se recourrent les unes les autres. A ce moment les verticilles des jeunes rameaux commencent déjả à se montrer, et, par suite du rapprochement des nœuds et de l'alternance des parties, chacun de ces rameaux parait naître exactement à l'aisselle d'une des divisions de la gaîne inférjeure qui les recouvre. La concordance est tellement parfaite que, si les choses restaient en cet état, on n'hésiterait pas un instant à dire les rameaux axillaires aux divisions de la gaine. C'est peut-être cette circonstance, on la constatation de quelque circonstance analogue, qui a porté MI. Döll à essayer de faire rentrer la ramification des Equisetum sous la grande loi de l'axillarité des bourgeons, et à dire que les rameaux des Equisetum sont axillaires, non à la gaîne qu'ils touchent, mais à celle qui recouvre la base de leurs entre-nœuds (Flor. Bard., p. 56). Pour que les rameaux de la partie supérieure d'un entre-nœud fussent axillaires aux divisions de la gaine qui est à la base du même entre-noud, il faudrait que ces rameaux el cette gaîne, malgré la distance qui les sépare, provinssent d'un mème nœud et appartinssent au mème verticille. Dans ce cas leurs vaisseaux auraient une origine commune. Or il

\footnotetext{
' Rien n'est plus facile que de constater la position relative des rameaux. C'est done avec surprise qu'on lit dans Payer: "Immédiatement aut-dessus des gaines naissent souvent des rameaux verticillés " (Bot. Crypt., p. 213; 1850). L'auteur attribue cette opinion à Mirbel, sans citer toutefois l'ouvrage où il l'a prise. Or, dans les Élém. de phys. t'ég. et de bot., I, p. 387, à l'article ÉcusÉTACÉEs, on trouve en effet à peu près cette phrase, mais avec le mot dessous. On croirait à une faute d'impression si l'auteur n'avait ajouté : "Cette organisation ne diffère point de celle du Casua"rina que l'on prendrait volontiers, si l'on ne connaissait sa fleur et son fruit, pour une Prèle en arbre. "Comme les rameaux verticillés des Casuarina naissent au-dessus des gaines, on voit qu’il y a eu méprise. M. Raspail dit aussi : "Les clıatons du Taxus ont exactement la structure de l'épi des Equisetum..... Les genres Ephedra et Casuarina ont "exactement le port et la structure caulinaire d'un Equisetum arborescent. (Phys. l'ég., 11, r. \$56). D'une vague ressemblance dans l'aspect extérieur à un rapnort essentiel de structure, il y a loin.
} 
est loin d'en étre ainsi. L'examen analyglique des tissus nous a montré que le latiscean vasculaire qui se rend a la lase d'un ramean provient de la réunion de deux petits

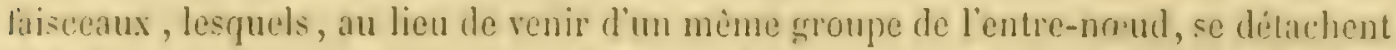
lateralement chacun d'un groupe diflérent. Au contraire, le faisceau vascularie qui parcourt la ligne médiane de la division de la ginine infraposée à un samean, et i laquelle ce rameau serait axillaire, se ratlache a un seul el meme faisceau viscularie

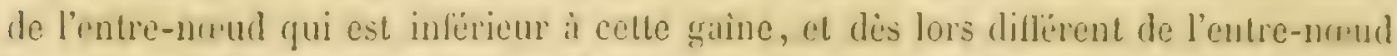
supportant le rumean gue l'on voudrait dire axillaire à ectle saine. Lélude de la premiere apparition et de l'érolution d’un bourgeon a ramean sajonte a ce qui pricede pour dimontrer peremptoirement que la formation du ranean a lieu au neend contre lequel il émerge, qüil n’y monte pas de l’aisselle des "fenilles du norud inférieur, que rien ne le relie a ce verticille de "leuilles, ) el que les faisceaux vasculaires de lun et de lautre se rattachent à ceux de deux entre-nouds différents (vor. p. 49 et 66 ).

Je dois me hàter d'ajouter que M. Düll cite au même lieu ce fait que "les Lygcopodiucées, les Marsilea et les Pilulariu ont des rameaux lateraux el non axillaires i leur's leuilles; ce qui diminue l'importancè de la question. s Comme la loi de l'axillarité des bourgeous n'est pas applicable aux cryplogames, sur lesugels un bourgeon peut naitre au-dessus, au-dessous, à cuté d'un pétiole ou mème sur un péliole (roy. Ad. Brongrniart, Ilist. véy. foss., II, p. 30; Ilofmeister, hénntn., Gef. liryll., p. 630, 649; Nettenius, Seitenlin. Form., p. 611, 627; J. Dural-Jouve, Pél. Lülg., p. 3, 4, 19; pl. I, fig. 16, et pl. II, fig. 21 B), il n'y a pas lieu d'essayer de rattacher les Equisetum a celle loi, et il sulfit de constater exactement comment les parties sont disposies el comment s'en fait l'évolution.

Par sa position et par ses formes, l'anncau se montre comme un intermédiaire entre les gaines el les verticilles sporingifères; il représente assez bien les feuilles supérieures d'un grand nombre de phanéroganes, lesquelles ne sont pas encore des enveloppes florales, mais ne sont plus des feuilles proprement dites. L'anneau est sujet it présenter des irrégrularités qui sont en mème temps la conséquence el la manifestation de sa nature intermédiaire. Tantót il conserve encore quelques traces d'expansion fuliacée, qui simulent une gaine incomplete, el mème au-dessous de lui peuvent apparaitre des rameaux comme sur l'E. syleaticum; lantil il porte deja sur ses bords quelques sporanges plus ou moins réguliers, arrivant ainsi par anticipation à celle fonction suprème de reproduction vers laquelle toute la plante aspire el qui est réservéc aux verticilles supérieurs. En cilant des firts semblables, M. Milde sexprime ainsi : "Jai souvent observé sur les $E$. Thlmuteia el areense comment cet organe (l'anneau) se développe peu à peu (allmählis hölser wirl) et se transforme, soit partiellement, soit complotement, en une gaine; souvent lous les passages de l'anneau a la gaine 
complète se montrent sur un seul el mème individu. L'anneau se trouve parfois aussi au milieu de la tige, ce qui est la preuve la plus lorte de sa nature. J'ai constaté aussi, sur les deux mèmes espèces, que l’anneau le plus rapproché de l’épi portait à quelques Jobes isolés des sporanges ovales qui étaient remplies de spores. Quelques-uns de ces lobes montraient déjä leur transformation en réceptacles, en ce qu’ils se séparaient de l'anneau sous forme de petites feuilles isolées.»

"L'anneau peut done se transformer tantôt en une gaîne, tantòt en un verticille de réceptacles, et peut, en conséquence, ètre regardé, avec un droil égal, comme une gaîne avortée, ou comme un verticille de réceptacles avortés; il est pour ainsi dire un intermédiaire qui, selon les circonstances, peut se transformer en l'un ou cn l'autre » (Kenntu. Eq., p. 608 et 586).

Entièrement d'accord avec ce savant botaniste sur la nature intermédiaire de l'anneau, je ne peux néanmoins adopter ni sa théorie ni ses expressions, quand il dit qu'un « anneau se développe (höher wird) 》 en se montrant à l'état de gaîne.

La gaine ou, pour parler avec plus de généralité, les feuilles des végétaux sont des appendices, des organes accessoires, utiles sans doute, et quelquefois même indispensables comme organes de protection el de nutrition, mais enfin ce ne sont que des organes accessoires qui peuvent manquer, qui manquent même très-souvent dans presque toutes les familles, sans que le végétal en soit moins ce qu'il est. Les organes reproducteurs, au contraire, sont les organes essentiels, essentiels au premier chef, parce qu'ils assurent la propagation de l'espèce, parce que sans eux le végétal ne continuerait pas à être. Ils remplissent la fonction la plus élevée, celle qui résume toutes les autres et qui en est le terme et la fin suprême, celle à laquelle toutes les autres fonctions et tous les actes de la vie végétale ne semblent qu'une préparation et une aspiration. Les organes reproducleurs sont donc les organes les plus élevés, les plus parfaits, ceux qui renferment en eux la vie ultérieure du végétal, le végétal futur, réduit à ses éléments essentiels, mais complet. S'ils sont les plus élevés, ce sont ceux que l'on doit prendre pour terme final de comparaison dans l'appréciation du développement successif des organes préparatoires et accessoires. La feuille se déreloppe et s'élève à mesure qu'elle cesse d'ètre feuille pour devenir enveloppe florale. L'étamine est d'un degré supérieur au pétale; et, si par les manœuvres habiles de nos jardiniers la plante est arrètée dans son développement final el demeure à un degré inférieur, il ne faut pas voir, par exemple, dans le remplacement des étamines par des pétales, une transformation, une élévation, un développement, mais bien plutòt un arrèt, une dégradation, un avortement si l'on veut. La feuille est plus apparente sans doute, plus grande dans l'espace, mais non dans l'ordre des fonctions; clle s'élève el se déreloppe vraiment en derenant bractée ou enreloppe florale; mais si l'enveloppe florale se montre sous forme de bractéc ou de feuille, il n'est plus permis de dire qu'elle s'est 
développie, uque les sépales, par exemple, se sont déreloppés en feuilles, mais bien

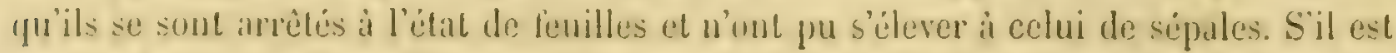
permis de dire que l'anneau ou les lubes de l'anneau se développent en sporanges et śétent par anticipation a un degré supérieur, il ne peut etre permis de dire que ces parties se diveloppent en gaine, quand elles s'arrètent par anomalie à ce degré inférieus.

Dire que "l'annean peut, arec le meme druil, être considéré comme une gaine "rorléc ou comme un verticille de sporanges urortés, c'est exclure tonte rigueur de langage des considérations morphologiques. L'anneau est déji plus qu'une gaine; il n'est pas encore un rerticille d'organes reproducteurs; il est au-dessus de l'un et un ćtat de transition vers l'autre; il n'est ni l'un ni l'autre à l'élat d'avortement. Si un annean se trouve au milieu d'une lige, e'est par anticipation et développenent prématuré, ou encore parce que les verticilles supraposés, au lieu de s'ilever au degré supéricur d'organes reproducteurs, se sont arrètés à létat de gaines par délaut d’ac. tion vitale.

En botanique, comme partout, un organe avorté est celui qui, après avoir commencé a se développer, s'arrête, s'atrophie et péril, comme, par exemple, les spores qui s'arrètent à l'arant-dernier degré d'évolution el demeurent impropres à la reproduction (p. 93-95;. Dans la comparaison des organes d'un rang supérienr, les sporanges, avec l'anneau, le terme "avorté» ne pourrait leur être appliqué (et encore sans grande rigueur de langage) que si, à la place normale d'un verticille de sporanges, se trouvait un anneau. Mais dans la comparaison de l'anneau arec les gaines qui sont d'un rang inférieur, dire de hui qun'il est une gaine avortée, sera tonjours et absolument une expression impropre et fausse. Comme les feuilles en général, les gaines des Equisetum résultent de la simple multiplication du tissu celluloso-vasculaire; elles s’arrètent à la première période d’évolution. L'organe reproducteur résulte, non plus d'une multiplication de tissus toujours identique à clle-mème, mais de la série des modifications qui alleignent ces tissus; ainsi dans l'évolution d'un sporange et de son contenu, on peut compler jusqua anze périodes successives, toutes différentes (p. 76-84). Or un organe dont les parties élémentaires ont passé par toutes les phases de l'ívolution normale, et qui, dans la série des fonctions, est des lors plus rapproché de la fonction finale et suprème, ne saurait être considéré comme un avortement ou une déformation de l'organe inférieur dans celte mème séric. Si l'anneau, supérieur à la gaine, est une gaine avortée, les sporanges, supéricur's à l'anneau, sont des anneaux avorlés ou déformés, et les spores, supérieures aux sporanges, sont des sporanges défurmés ou arortés. It ne saurait en itte ainsi. L'organe reproducteur, spore, cmbryon, graine, n’est point un organe asorlé, déformé, transformé. Il est ce qu’il doit être; il est arrivé a ce quall decait ètre, et chacun des autres organes intermé- 
diaires s'est arrêté i sun point dans la série des actes d'érolution et dans la suite des fonctions. Il n'y a donc point, à mon avis, exactitude et rigueur logique, à prendre un organe accessoire et inférieur, la feuille, le plus éloigné peut-être du terme, comme celui auquel on rapporte tout dans les considérations morphologiques, el j'aimerais mieux renverser les rapports, partir de l'organe final et considérer la feuille comme organe incomplet, inachevé, arrêté aux premières modifications cellulaires, que de considérer les organes reproducteurs comme des déformations ou des transformations de la feuille. J'insiste trop longuement sur ce point; je le reconnais. Nais je vois dans les expressions qui ont cours le reflet d'une idée inexacte qui fausse toutes les considérations morphologiques.

A mesure que les organes accessoires ou foliacés se rapprochent des organes essentiels ou reproducleurs, nous les voyons, sur les tiges des phanérogames, se réduire dans toutes leurs dimensions, se modifier dans leur forme et souvent mème dans leur coloration. A la feuille succède la bractée, à celle-ci l'écaille, puis apparait le pédoncule. Celui-ci, considérć avec raison comme un rameau ou axe d'un autre ordre, supporte d'autres verticilles d'expansions foliacées arrivées à l'état d'enreloppes florales, et enfin relie les organes reproducteurs à l'axe principal de l'inflorescence. La réduction des parties de l'anneau nous indique qu'il en est de même sur les Equisetum, el que les expansions foliacées vont disparaittre et faire place aux rameaux supportant les organes reproducteurs. Les verticilles des pédicelles sporangifères me paraissent en effet répondre exactement à des rerticilles de rameaux, dépourvus de gaine caulinaire et de gaîne basilaire, comme sur tant de phanérogames les expansions foliacées «manquent soit à l'origine des inflorescences géné"rales ou partielles, soit à celle de chaque fleur en particulier» (Ad. Juss., Ėlém. bot., p. 225).

Mais cette manière de considérer les pédicelles n'est peut-être pas aujourd'hui généralement partagée. Je mentionnerai, sans les discuter, les opinions de \. Lindley el de Bischoff, qui ont regardé chaque sporange comme correspondant à une seule loge de l'anthère (Lindl., Introd. nat. syst., p. 311) ou à une anthère complète (Bischoff, Lehrb. Bot., p. 44I), et l'opinion émise par. II. II. Mohl "que le réceptacle des Equisetum provient d'une feuille de la hampe mème, et représente en quelque sorte le connectif d'une anthère démesurément grrossi, et que les sporanges situés à sal face inférieure correspondent aux loges isolées d'une anthẻre „ (.Morph. Spor., p. 10). Plus récemment M. Ulidde s'est aiusi exprimé sur ce point: "Les carpophores (Fruchtträger) sont disposés, comme les rameaux, en verticilles superposés, el chacun d'eux, ainsi que nous l'apprennent de nombreuses monstruosités, peut ètre considéré comme une feuille métamorphosée (Gef. Cirypt. Schl., p. 413). Dans tous ses travaux sur les Equisetum, ce savant botaniste a émis la même opinion el s'est complu a accumuler 


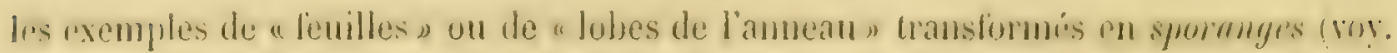
particulièrement Gef. Crypt. Schl., p. 475).

J'ai été moi-mème issez leureux pour rencontrer des exemplus de presque foutes les monstrusites cities; cat ces anomalies par anticination ne sont pas rares sur les E. maximum, arecuse, lithrule el limosum. J'ai pu en outre constaler que guand les divisions de la gaine se chargent de sporanges, ceux-ci sont furlquefois disposís avee une certaine symetrib, m rers la pointe el un de chaque coté, mais le plus somvent ils sont irrégulièrement placés.

Linsi pour moi, comme pour M. Milde, le passage des divisions de la gaine ou de l'amneau i l'étal de sporanges est un fait aequis. Mais jamais je n'ai vu la "fenille» passer à l'état de pédicelle terminé par un clypéole et supportant un verticille régulice de sporanges. M. Milde n'en rapporıe pas d'exenıple, et les anomalies qüil cite en si grand nombre n'élablissent, je le répète, que le passage des divisions de l'anneau ou des divisions de la ggaîne à l'état de sporanges.

Les considerations suivantes me portent à penser que les pédicelles répondent plutút à des rameaux qu'ì des parties de gaînes.

Le mode d'apparition el d'évolution d'un verticille de púdicelles sporangiferes ressemble, ainsi que nous l'avons vu p. 77, non à celui d'une gaine dont les partics sont soudées, mais bien à celui d'un rerticille de rameaux dont les parties sont isolées.

Je possede une tige d'E. palustre vas polystachyon, sur laquelle chacun des rameaux d'un verticille placé au-dlessous d'une gaine est directement passé à l'état de pédicelle. terminé par un clypéole régulier et un verticille de sporanges ${ }^{1}$.

Les púdicelles sporangifères des Equisctum, ainsi que les pédoncules chez les phanérogames, sont des axes de second ordre, ayant un centre comme une lige, comme un rameau, et l'analogie générale commande d'abord, et presque avant tout cxamen, de les rapporter aux rameaux, comme on y rapporte les pédoncules des phanérogames. Si le pédicelle répondait à une «fenille ordre, mais simplement un appendice latéral, avec des faces dissemblables, ainsi que le sont les sporanges et les divisions des clypéoles. On conçoit bien, et c'est d'ailleurs un fait avéré, que l’anneau et la gaine puissent passer a l’élat de sporanges, parce que les sporanges sont des appendices latéraux comme les feuilles; mais on ne conpoit pas, el les faits manquent pour létablir, qu’ils puissent passer a celui de pédicelle, parce que le pédicelle est un axe de second ordre. Lorsgue les parties de l'amean ou des gaines s’élèrent par anticipation à l'état de sporanges, elles ne se changent point

\footnotetext{
'Il me semble que les fig. 27 et 28 de la pl. XXXII, Gef. Crypt. Schl, de M. Milde, représentent le même fait; mais l'imperfeclion du dessin des sporanges ne me permet pas de l'affirmer.
} 
en pédicelles avec verticille de sporanges terminal, mais directement el sans intermédiaire en sporanges dirigés presque parallìlement i l'axe de la tige. Ces sporanges occupent toujours ainsi la face extérieure de la gaine ou de l'anneau, et ne constituent jamais de verticilles complets, comme ceux que l'on voil sous les clypéoles, mais seulement des fragments de verticilles, qui n'ont d'autre axe et d'autre centre commun que le centre et l'axe de la tige. De telle façon que si la tige était coupée immédiatement au-dessus d'un anneau ou d'une gaine sporangifère et qu'on rapprochatt un peu tous ces fragments de verticilles, on aurait un verticille de sporanges complet, sous un vaste clypéole dont la tige serait le pédicelle.

Sur les espèces dont l'épi est obtus, cet épi se termine par un clypéole plus ou moins régulier, à la face inféricure duquel sont suspendus trois ou quatre sporanges. Le pédicelle de ce clypéole n'est plus un axe de second ordre; il est la prolongation de la tige elle-même, et pour celui-ci le doute n'est vraiment pas possible.

Il me semble que ces faits nous révèlent suffisamment que les choses ne se passent pas autrement pour les pédicelles, les clypéoles et les sporanges ordinaires. Le pédicelle étant considéré comme un rameau, son expansion terminale, le clypéole, peut être considéré comme son premier et unique verticille foliaire dont les divisions seraient encore soudées à leur base. Et si maintenant on veut bien se rappeler que dans chaque division de la gaime ou «feuille» la face supérieure est celle qui est appliquée contre la tige, et l'inférieurc celle qui est extérieure, on verra de suite que les sporanges el leur contenu sont disposés à la face inférieure de cette expansion foliaire, et que la face supérieure est sans organes reproducteurs, absolument comme sur les Fougères. Cette analogie nouvelle vient it l'appui de l'opinion suivant laquelle je considère les pédicelles comme répondant à des rameaux sans gaine basilaire, el les sporanges comme répondant aux divisions de l'expansion foliaire.

Je viens de dire que le clypéole terminal des épis obtus supporte trois ou quatre sporanges. Le plus ordinairement ces sporanges sont mal conformés, el les spores qu'ils contiennent sont dénuées de matière verte et dès lors stériles. Il est évident qu'il y a eu là arrêt par épuisement, comme au sommet des épis d'un grand nombre de phanérogames. Souvent ce délaut d'action se manifeste d'une autre manière sur ce mème clypéole terminal. Ses sporanges s'arrêtent à un degré rudimentaire à peine reconnaissable, et les divisions de l'expansion foliaire, au lieu de s'étaler en clypéole, demeurent rapprochées, se soudent entre elles, se durcissent et se prolongent en une pointe centrale. Les stries qui la sillonnent en révèlent d'abord la nature, que la plus simple analyse fait ensuite reconnaître avec certitude. Cet acumen ne se rencontre qu'exceptionnellement sur les E. arvense, pratense, sylvaticum et palustre; il est, au contraire, sur le groupe des IIyemalia assez constant pour constituer un caractère distinctif. C'est ainsi que sur certaines Labiées on voit l'épi se terminer constamment par un bouquet 


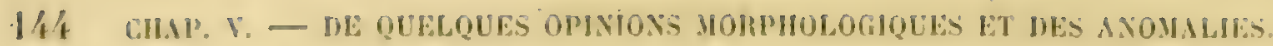

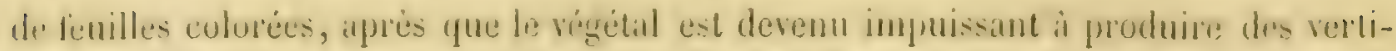

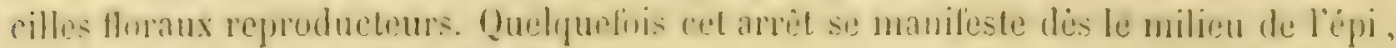

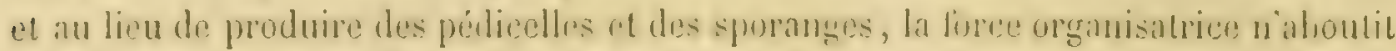
plus qüi des verticilles foliares ou ganes qui partois interrompent seulement lieju, ou d'autres fois en remplacent completement la partie supérieure. Ges dernier's cas sont fiéquents sur les épis qui terminent les rameaux allongés de l\%. arentere, forma serolina polystuchyon. Cesont des cas d’anomalies par rélrosradalion, ou plublot par artèt, a opposer aux cas d'anticipation. Cest ainsi encore que sur de nombreux phanérogames, el en particulier sur les Labiós, on voit l'ejpi s̈intermure el les verticilles de pédicelles florigeres étre remplacés par des verticilles d'expansions foliacées. Cus verlicilles foliaires avaient disparu aux approches de leppi, ils reparidsent par suite d'un arrét qui abaisse tout d'un degré el ne permet plus l'apparition des rameaux floriferes. El de même que sur les phanérogames il nest pas pour cela permis d’ilentifier les rameaux floriferes avec les bractées qui paraissent les remplacer, il me semble quiil ne l'est pas darantage de rapporter les rerticilles de pédicelles sporangifüres aux verticilles foliaires ou gaines qui, dans les anomalies par arrèt, semblent également en occuper la place.

Je n'insiste pas davantage. $\Lambda$ mon avis, l'important n'est pas de savoir si tel organe ripond ou non à tel autre, mais ljen de connaitre exactement lérolution, la structure el la fonction des divers organes. Et ce n'est quaprès la parlaite acquisition de ces connaissances pour les principales familles, que deviendront possibles des considérations morphologiques rigoureuses. Avant ce moment, appuyécs sur des connaissauces incompletes, clles sont prématurées; el, si quelquefors el par lasard elles peuvent jeter un peu de lumiere sur un point, elles ne cessent pas pour cela d'ètre plus conjecturales que seientifiques, et des lor's elles nous exposent le plus sourent it faire fausse roule. Lhistoire des opinions sur la reproduction des cryptogames, et particulièrement sur celle des Equisetum, en est une preuve remarquable. Tant qu'on a essayé de résoudre la question par des analogies, on s'est plus éloigné que rapproché de la sulution. Ce sont des considérations morphologiques qui ont égaré Iledwigr, cel éminent observateur, el gui cnsuite ont inspiré a MII. L. Reichenbach et Raspail les affirmations les plus erronices. Des connaissances positives et raiment scientifipuos nont été obtenues quà mesure que Wrilldenow, Vaucher', M. Niggeli, M. G. Thuret ont etudie sur des semis la reproduction des cryptogames, sans se préoceuper du ripport que leurs organes reproducteurs pumaient presenter are ceux des autres plantes. Voir venir les choses est le meilleur moyen de les expliquer (Turpin).

\footnotetext{
' Malgré les expériences qui lui avaient démontré aque le grain vert est bien la semence des Préles, "Vaucher était tellement dominé par les analogies qu’avait indiquées lledwig. qu'il se demande encore -si les quatre filets ne seraient pas des étamines (Mon. Prél., p. 22). Celle page est très-curicuse à lire.
} 


\section{\$2. Anomalies}

Ce que je viens de dire des considérations morphologiques, je le peuse it plus forte raison des opinions tératologiques. Il me semble qu’elles n’auront une valeur raiment scientifique que lorsque l'on commaitra à fond les lois de l'évolution élémentaire de chaque organe. Alors seulement l'on pourra rapporter les anomalies aux lois générales, et reconnaitre avec certitude comment dans chaque anomalic l'application de ces lois a été modifiée par défaut ou par excès, par simultanéité accidentelle remplaçant la succession ordinaire ou par succession substituée à la simultanćitć normale. L'étude des anomalies ne se bornera plus à citer et à décrire des faits plus ou moins isolés ou cn apparence contradictoires, à les qualifier de « dégénérescences ou d'ennoblissements, d'écarts de la régularité habiluelle ou de retours accidentels au type régulier, " mais celte étude pourra être soumise à une direction philosophique et aboutir à une savante unité. En l'état je ne trouve point en moi le degré de savoir nécessaire pour domıer à des considérations tératologiques une valeur autre que celle d'une pure hypothèse. C'est pourquoi je m'abstiendrai arec tout le soin possible de ces considérations, apportant le mème soin à noter les diverses anomalies qu'il m'a été donné de constater.

J'essaicrai seulement de les citer arec ordre ou, si l'on veul, de les classer, et cela simplement afin d'en faciliter la comparaison par ce rapprochement, et afin surtout de pouroir les rappeler par leur numéro d'ordre ou leur désignation, et de ne pas répéter la description d'une même anomalie après celle de chaque espèce sur laquelle elle peut se présenter. Je parlerai d'abord des anomalies qui changent l'aspect ordinaire des individus, puis de celles qui modifient seulcment la forme de quelque partie, et en dernier lieu de celles qui troublent les fonctions de reproduction.

$1^{\circ}$ Racines naissant sur des tiges enterrées. - J'ai vu et j’ai pu obtenir cette anomalie sur les tiges des E. arvense, littorale, limosum, ramosissimum, variegatum, trachyodon et hycmale. Je n’ai pu la déterminer sur l'E.pulustre, et n’ai point expérimenté sur les autres espèces (roir p. 9 et 10).

20 Rameaux naissant sur des rhizomes déterrés. - J'ai constaté et déterminé la présence de celle anomalie sur les $E$. arense, ramosissimum, variegatum et lyycmale (voir p. 9 et 10).

$3^{0}$ Tiges ordinairement mes devenant rameuses après une mutation. - Les tiges de l'E. hycmale sont à l'état normal entièrement nues, et sur d'innombrables quantités que j'en ai vues à Strasbourg, oủ cette espèce est un objet de commerce, je n’en ai pas vu une scule qui eût des rameaux. Mais si une tige est mutilée, les bourgeon: 


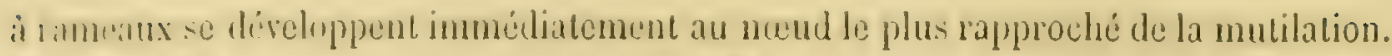

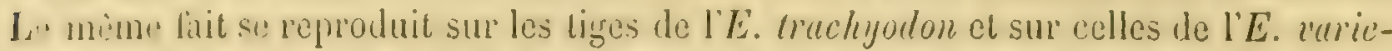
yulum. Si ces dernieres sont alteintes il leur sommet par une geléc tardive, clles produisent immédiatement quclques rameaux. Les tiges d'E. rumosissimum coupés dans l's prés au mois de mai produisent, des le mois suivant, aux nouds inferieurs subsistints une grande quantilé de rameaux longs et grêles, qui ont un aspect cespiteux el ont élé pris plus d'une fois pour l'E. v'urie'yalum ${ }^{1}$. Sur ces espices ces rameaux tardifs portent quelquefois des épis.

4. Tiges spicitives propres devenant rameuses vers le bas. - Ordinairement apres Lit sporose les tiges spicilüres propres des E. aruense et maximum ne tardent pas à se flétrir et à disparaître. Mais il arrive aussi que dans des lienx qui ont été inondés pendent le premier printemps, la partie supérieure des liges de la première espéce est seule i se faner un peu; la moitié inféricure persiste en se courbant quelquefois légèrement. Alors les bourseons à rameaux qui, ì l'ćtat cxpectant, existaient aux nouds inféricurs se developpent en rameaux de quelques centimètres de lonģ. En même temps les cútes deviennent plus prononcées, et les stomates se développent dans les sillons qui se colorent en vert. Les stomates sont disposés comme sur les tiges stériles ordinaires, mais les cellules a chlorophylle ne conservent pas exactement la position qu'elles occupent sur ces tiges, et elles pénètrent irrégulièrement et quelquefois très-profondément vers l'intérieur. J'ai déterminé artificiellement la production de cetle anomalie", et je l'ai trouvéc quelquefois aussi sur l'E. arvense dans des prés d'Alsace; elle est très-commune dans certaines localités d'Europe (Milde, Gef. Crypt. Schl., 1. 429), mais comme elle dépend des circonstances extérieures, elle ne se produit pas tous les aus dans les mêmes localités. Quelquefois mème elle cxiste sur un même rhizome ì còté d’une tige spieifére normale. Bischofr, qui, le premier, je crois? a nettement signalé et décrit cette forme (Krypt. Geu., p. 16), avait done eu raison de ne li considérer que comme un état exceptionnel et accidentel. M. Milde (o. c., p. 421) ne la regarde également que comme une forme passagìe el non comme une variété.

\footnotetext{
- On produit le fait a volonté sur toutes les especes du groupe Ilyemalia, en coupant la partic supérieure de tiges placées dans un vase rempli d'eau. Ln 1856 j'en avais mis dans une boite d'herborisation avec de la mousse lumide el, après en avoir étudié quelques-unes, j'oubliai les autres. Du 2 f février au 27 mars elles produisirent des rameaux de vingt à vingt-deux millimètres. Je les mis alors dans un vase avec de l'eau, et en julı ces rameaux atteignaient quinze à dix-huit centimètres.

- Je prenais de bonne heure, et arant l'emission des spores, une tige spicifere d'E. artense avec son rlizome, el, après avoir enlevé l'épi avec précaution et sans meurtrir les tissus de la partie inféricure, jo plaçais celte partic et le rhizome indifféremment dans de la mousse mouillée, ou dans de la terre très-fumeclée, ou dans un vase ayant quatre ou cinq centimétres d'cau, et au bout de vingt at vingt-cinq jours des rameaux se montraicnt aux nocuds inféricurs. L'expérience n'a jamais manqué̉.

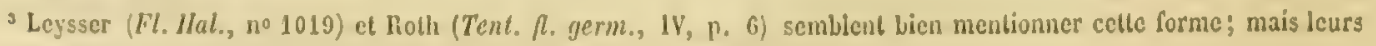
expressions ne sont pas assez précises pour permettre une aflirmation.
} 
Elle a pourtant figuré comme telle plus d'une fois, sous les noms d'irrigum et de frondescens. C'est à celle forme que M. Milde, après comparaison des échantillons originaux (Gef. Crypt. Schl.., p. 422), rapporte l'E. viparium Fries (voy. Döll, Fl. Baul., p. 58).

Sur l'E. maximum la même anomalic se réduit à l'apparition des rameaux, et les entre-nouds de la tige demeurent toujours sans stomates et sans chlorophylle. Eille est très-rare sur celte espèce, du moins en France, car dans quelques contrées d'outre-Rhin elle est si fréquente qu'elle semble la forme normale, et que c'est elle que Roth et Schreber ont déerite sous le nom d'E. eburncum. M. Al. Braun l'a signalée en 1843 comme variété qu'il a nommée frondescens.

Gette anomalie se complique, mais très-rarement, d'une autre, consistant en ce que ces rameaux anormaux se terminent eux-mêmes par de pelits épis.

On conçoil facilement que cette anomalie ne se constate d'une manière bien tranchée que sur les tiges spicifères propres des deux espieces précitées, attendu que ces tiges sont ordinairement nues; mais le principe auquel elle se rattache, savoir le dércloppement des bourgeons qui se trouvent à l'état expectant sous tous les nauds, occasionne à tout instant, sans qu'on les remarque, les mêmes fails sur les $E$. limosum, palustre et ramosissimum.

Ce développement est même un fait constant et une loi sur deux de nos cspèces, E. sylvaticum et E. pratense; et, malgré cela, il détermine encore une anomalie sur la promière des deux, savoir l'apparition de rameaux immédiatement au-dessous de l'anneau. Anomalie assez rare en France, mais qui doit être bien plus fréquente en Suisse, puisque M. Bernoulli, toujours si exact, mentionne la présence de rameaux au-dessous de l'amneau comme un caractère distinctif de l'E. syltuticum (Gc). Kingt. Schw., p. 69).

$5^{0}$ Tiges ordinairement stériles devenant spicifires. - L'anomalic précédente consiste en ce que les tiges spicifẻres propres des $E$. arvense et maximum se revêtent, contre l'ordinaire, de verticilles de rameaux. Celle-ci est précisément l'inverse, e'est-ildire que quelques-unes des tiges stériles des mêmes espèces, vertes, richement ramifiées et d'une structure tout à fait normale, au lieu de demeurer stériles comme ì l'ordinaire «sont terminées par des épis très-bien formés qui donnent des graines "fécondes.... Quelquefois elles ont de plus leur's rameaux pourrus d'épis plus petits (et fructifères» (Vaucher, Mon. Prêl., p. 364). Cet auteur n'avail vu cetle anomalie que sur des tiges d'E. maximum recueillies près de Berne. Je l'ai trouvée sur l'E. arvense près de Schlestadt et de Strasbourg, et sur l'E. maximum près de Lisicux. Elle ne me paraît pas très-fréquente; quand clle se montre, elle est rarement aussi simple que dans la description qui précède, et le plus souvent l'épi de la tige et ceux des rameaux présentent cncore les anomalies suivantes $n^{05} 6$ et 7 . 


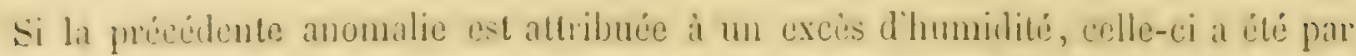
plusicurs butanistes considire comme un elfel de la sichresco. M. Francis dil mène

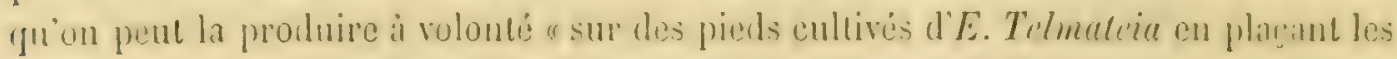
pots dans un licu sec (Anal. brit. ferns, p. 76); il cite MI. Wilson comme élant de

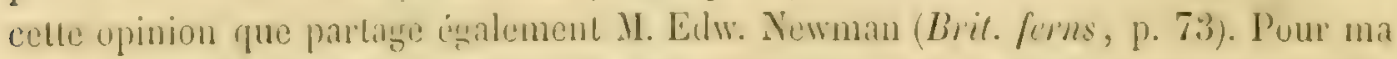
part, je ne l'ai trourée que dans des endroils secs, sur le haut de digues dont les tilus étaient alor's (ourerts de longues tiges toutes stériles. Les épis me portaient presque que des spores imparfaites.

Celle anomalie, plus constante que la précédente, ne se reproduit pas cependant arec rigularite sur les mèmes rhizomes, ni dans les mémes lieux. Elle n'en a pas moins élí élevéc à la dignité d'espece par K. F. Schultz (1819), qui en a fail son $E$. cempestre, puis ramence au rang de rariété par G. F. WV. Mejer (1836), E. arrense $v^{\text {als }}$ scrolinum.

Cette dernière dénomination, assez gúnéralement arloplée pon désigner cette anomalie sur les E. maximum, syluaticum, matense el avense (roy. Milde, Gef. Crypt. Schl., p. 122, 128, 133 et 139) lait allusion a l'apparition lurdie des épis de celle forme, et, à ce titre, me paraît devoir donner lieu à une observation. Il est d'abord évident que cette anomalie ne peut se conslater que sur les quatre espèces précilées, à liges spicifères non conformes el rernales. La plupart des autres espéces sont en sporose a peu pres pendant toute l'année, je reux dire pendant tonte la belle saison depuis la mi-arril jusqu’a la fin de septembre, et dis lors leurs tiges ue peurent se distinguer en precoces et serotince. A mesure que leurs bourgeons apparaissent sur les rhizomes, ils sortent et se développent en tiges stériles ou spicifëres. Il n'en est point de mème des premières especes, E. maximum, syleaticum et artense. Lours bourgeons de liges stériles ou spiciferres apparaissent sur les rhizomes des le mois de juin: les spicifères sont, en aout el septembre, si complétement lurmées que leurs épis ont les sporanges parfaits el des spores vertes avec des élitires; néamoins, dans les cas ordinaires, les liges provenant de ces bourgeons ne sortent pas de terre à celte éporjue, et elles ne se montrent quau printemps suivant. Comme alors elles sont en retard presque d'un an sur les tiges spicilëres de l'E. palustre, par exemple, qui, apres avoir commencé en même temps ì l'etat de bourgeon, auraient répandu leurs spores en anut ou septembre, elles méritent plutot le nom de serutince que celui de presences. El si, comme cela arrive en effet, quelque pied des E. urense el maximure, lirrorisé par les circonstances, vient a produire ses tiges spicilères daus le courant de lautomne', c'est prématuré et jrecon qüil fiudrail le dire et non en relard el seroli-

\footnotetext{
2N. Milde a vu des a lampes o d'E. Telmatei sortir de terre en oclobre; e elles provenaient, suivant ce savant botaniste, de bourgeons dont le développement avait été favorisé par un sol très-gras " (liennln. Eq., p. 587). J'ai vu le mème fait prendant un automne trés-chaud of liumide se produire cn Alsace sur les $E$. maximum el arense.
} 
mum ${ }^{2}$; à moins qu’il ne füt bien constatí que les bourreons de ces tiges, dẹji formés pendant l'été précédent, ne sortent et ne se développent récllement qu’après les nutres.

$6^{\circ}$ Interruption des épis. - Au-dessus des verticilles inféricurs de l'épi apparaissent une ou plusieurs gaines, soit régulières, soit en partie passíes it l'état d’anncaux ou de sporanges, soit mème quelquefois accompagnées de courts rameaux. Ces gaines sont ou rapprochées ou un peu espacées, et au-dessus d'elles l'épi recommence arec ou sans anneau et se termine normalement. De telle sorte que la tige offre comme deux épis placés l'un au-dessus de l'autre. J'ai vu sur l'E. litlorale une double interruption qui simulait trois épis superposés. Ordinairement les gaînes de l'interruption sont un peu plus petites que les autres, et un peu moins vertes ainsi que les entre-nceuds qui les séparent. Quelquefois encore l'épi est interrompu par' un anneau plus ou moins régulier ou chargé sur ses lobes de quelques sporanges.

$7^{\circ}$ Terminaison irrégulière des épis. - Les gaines qui viennent remplacer les verticilles de sporanges ne cèdent pas toujours devant un retour de ces verticilles, et sonvent elles les remplacent définitivement; ce qui a lieu de deux manières. Ou bien elles sont espacées par des entre-nœuds, et, avec ou sans rameaux, elles constituent audessus de l'épi une extrémité de tige stérile tout à fait semblable aux extrémités normales. On a appelé, à tort selon moi, cette forme proliferum. Ou bien quelques gaines en très-petit nombre sont très-rapprochées el terminent l'épi par une houppe foliacée; on a appelé cette forme comosum (voy. ci-dessus p. 144). Dans l'un comme dans l'autre cas, quelques-unes des divisions des gaines portent parfois des sporanges plus ou moins mal formés. Celte anomalie et la précédente accompagnent assez solivent l'anomalie $n^{0} 5$, el elles sont toutes deux assez fréquentes sur' les $E$. pratense et littorale. MI. Milde a décrit et figuré celte double anomalie sur l'E. artense (Komntn. Eq., p. 571, pl. LIV, fig. 3-4).

Quelquefois encore le clypéole aplati qui constitue le sommet obtus de lépi de certaines espèces est remplacé par un acumen tout à fait analogue à celıi qui termine normalement les épis du groupe des Inycmulia. Je renvoie à ce qui a été dit p. 143 de cette anomalie constatée par M. Milde sur les E. syltaticum et palustre (Gef. Criypt. Schl., p. 434 el 462), par M. Cosson sur l'E. palustre (Flore envir. Paris, 2e éd., p. 880 , et Bull. Soc. bot. Fr., VIII, p. 297 et 368), et par moi sur les E. artense, mr(ttense, sylvaticum et palustre. Comme je l'ai trouvée sur des échantillons recueillis au hasard ou envoyés par des correspondants, j'ai lieu de coire que cette anomalie se

\footnotetext{
1 Par la même raison le Narcissus serotinus, qui forme ses bourgeons en étó et produit des fleurs de suite, mérite le nom de precox, car il est en avance sur les plantes qui, comme le Lencoium vernum, ont leurs fleurs, leurs étamines, leur pollen complétement formés avant l'hiver, mais ne sortent qu'au printemps, et sont dès lors en retard de toute la durée de l'hiver, serotince.
} 


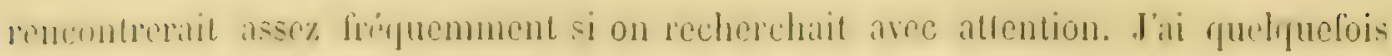
trouvé des épis d'E. ramosissimum tcrminés par un clypéole obtus.

8o Anneau un milicu des tiges. - Jo n'ai vu qu'un seul cas de cette anomalie sur

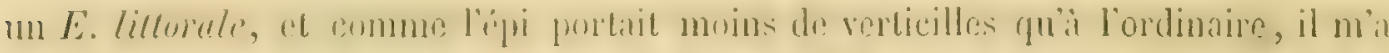
paru que celte anomalie peut rentrer dans le $n^{0} 6$, Interruption des épis, el que des

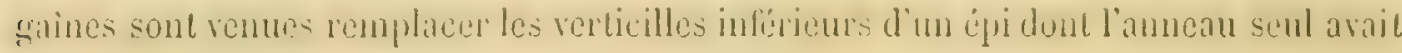
alouti.

$9{ }^{\circ}$ Pluralité des anneaux el amneanx incomplets. - La pluralité des anneaux est tris-friquente sur les liges spiefferes propres de IE. mucimum. Jen ai vu juspu'i trois asce distanls les uns des autres et parfaitement riguliers. Il. Milde fait mention

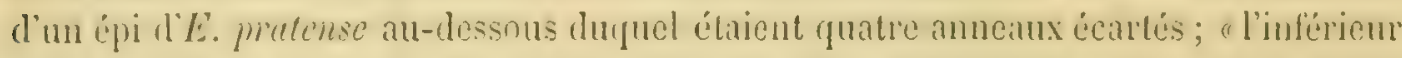
et lesupericur araient la forme normale; les deux intermédiaires étaient it moitie

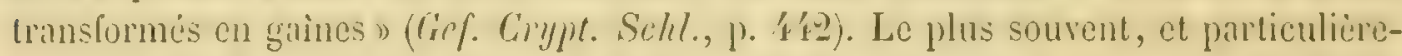
ment sur les E. arrense et litlorale', la pluralite des annenux est accompagnée d"irrégularitís consistant en ce qu'une partie des lohes de lanneau s'est élevéc a l'élat de sporanges. Alors la gaine immédiatement inférieure passe à l'état d'anneau, mais seulement du mème citté el sur une largeur correspundant exactement is celle de la partie de l'anneau chargée de sporanges (pl. X, fig. 14). Entre cette partie et le premier verticille de sporanges, d'une part, el la gaine infraposie, d'autre part, les entre-nesuds ne se sont pas allongés. Du côté opposé, et sur lequel chaque verticille est resté à l'état normal, les entre-neuds se sont allongés ì peu près comme it l'ordinaire; il en résulte que le "pérloneule se courbe el que l'épi s'incline. C'est un signe qui permet de reconnaitre facilement et de loin les sujets sur lesquels existe une de ces anomalics.

$10^{\circ}$ Entre-nouds du vhizome ronfés en lubercules (roy. p. 6 et 7, et pl. I, fig. 1, 2, 4, 5). - La tuberculisation qui, chez certaines plantes, est un état habituel, n'a pas étí observée sur toutes les espèces d'Equicetum, el n’est pas igalement constante sur tous les sujets des espèces oủ clle a été constatćc.

$11^{\circ}$ Gumes dispusies en crite. $-\Lambda$ la suite d'une lísion ou d'une giène qui a cxisté pendant l'érolution des organes, un ou deux entre-nnuds demeurent tris-courts. Sur lit mime région la tige se coutbe el se tord; les gaines se détachent el sout refunlies sur un des côtés en forme de crête ondulée. M. Moquin-Tandon cile des faits semblables sur des Galium, des Mentha el des Valeriana (T'́rat. vég., p. 181 el 182).

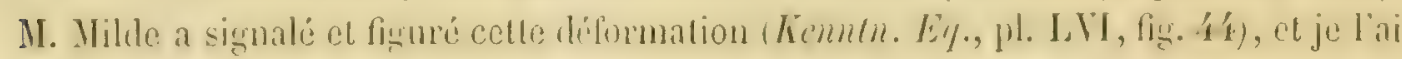
moi-même assez fréquemment rencontrée sur les E. arvense, limosum, palustre.

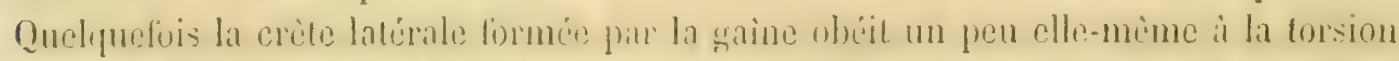
el forme ainsi un passigne à liblat anmmal suivant. I) autres fois les divisions de ces gaines refoulews latéralement sont síparies parr des dichirures si profondes quielles sont 
presque isolées jusqu'à leur basc. M. Milde, après avoir cité ét figuré (Kemmln. Eq., p. 608, pl. LV) une déformation de l'E. littorale sur laguelle les divisions de la gaine étaient isolées, ajoute: "Cette monstruosité montre très-bien que la gaîne en général peut être considérée comme une soudure (Gomplex) de petites feuilles réunies par la croissance et dont les bouts libres forment les dents.) En matière d'interprétation d'une anomalie, j'avoue la faiblesse de mon esprit et son impuissance absolue à comprendre qu'une déformation accidentelle el sans permanence nous montre comment nous pouvons considérer l'état ordinaire et permanent des choses. En suivant l'évolulion des gaînes, je vois leur's divisions se développer en restant unies el se déchirer ensuite mécaniquement, et je ne puis les considérer comme cssentiellement soumises à une autre loi. L'isolement accidentel des divisions de la gainc, leur disposition anomale en ligne latérale ou en spirale ne peuvent me porter à soustrairc par la penséc les organes foliaires des Equisetum à la loi constante qui les force à se développer unis et à se disposer en verticilles, pour les considérer comme soumis à la loi de la disposition en spirale. Une bifurcation accidentelle ne peut me faire regarder les tiges des Equisetum comme sounises à une loi de dichotomie. Je ne puis considérer les choses autrement qu'elles se montrent, une anomalic autrement que comme une anomalie, c'est-à-dire comme une déviation dans l'application de la loi permanente, par défaut, par excès, ou par intervention de toute autre circonstance accidentelle, et non comme la révélation d'une loi autre que la loi permanente.

$12^{\circ}$ Gaines disposées en spirale. - Vaucher, qui paraît avoir le premier constaté celte anomalie sur une tige stérile de l'E. maximum recueillie près de Thun, l'a décrite en ces termes: «Les verticilles sont contournés en spirale depuis la base de (la plante jusqu'à son sommet» (Mon. Prêl., p. 364, pl. II A). M. Moquin-Tandon mentionne rla même anomalie retrouvée à Meudon par $\Lambda$. de Jussieu (Térat. rég., p. 181). M. Milde a mentionné et représenté à plusieurs reprises celte anomalie, notamment Kemntn. Eq., p. 593, pl. LVI, fig. 40, 41 (E.Tclmatcia); fig. 43 (E. pratense); fig. 44 (E. palustre); p. 601, fig. 45 et p. 602 (E. limosum); Gef. Crypt. Schl., pl. XXXVI, fig. 55 (E. limosum). Dans le Florn de 1858 (nº 5, 4 février, p. 69; ct fig. 3 et 4 de la pl. II) M. P. Reinsch publia une notice Sur le passage des verticilles d'une tige estivale d'E. Telmateia en une spirale non interrompue, avec des consilérations mathématiques sur ce phénomène. Cette notice peut se résumer en ce qui suit. Après le douzième entre-noeud se trouvait une spirale continue, occupant li place de plusieurs entre-nœuds et au-dessus de laquelle les verticilles recommençaient. Le verticille immédiatement inférieur à la spirale comptait vingt-huit parties ou divisions, et celui qui lui était immédiatement supérieur en présentait trente. La spirale en portait deux cent trois. En divisant ce nombre par vingt-neuf, moyenne des deux verticilles extrêmes, M. Reinsch montre que la spirale représentait sept verticilles. 


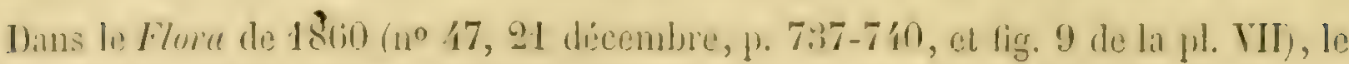
mime anteur publia un complément diuferration. Il cite un nouvel exemple de rertirilles déroulés en spirale, different du premier en ce clue la spirale terminait la tige stérile, et il s'cn sert pour confirmer ses considérations el en même temps pour corriger ce qu'il y arait de trop absulu dans sa maniere de regarder la súrie numérique des rameaux comme une progression arithmélique.

$13^{\circ}$ Bifurcation des liges. - Cette anomalie n'est pas trìs-rare sur les E. arrense et palustre, et elle se présente sous deux formes différentes. $1^{\text {ro }}$ forme. La tige s'aplatit el s'élargit on véritable fascic sur trois ou quatre entre-nceuds, et, à partir d'une gaine, la division a lien. Sur une tige d'E. palustre recueillie a IItguenau, chacune des tiges résultant de la division demeure fasciée, et se bifurque de noureau au quatrieme entre-noud au-dessus de la première bifureation. Sur une autre tige de la mème espèce, recueillic au même lieu el sur le mème rhizome, l’expansion fasciée se termine par une division en trois petites tiges situées dans un mème plan. 2c forme. La division commence immédiatement dans une gaine et sans être précédée d'aucune apparence de fasciation; c'est a peine si la gaine ou apparait la division est it sa partic inféricure un peu élargie sur les cótés. Vaucher mentionne celle anomalie sur l'E. maximum: "la hampe était divisée jusqu’a la base en guatre ou cincj épis particls" (Mon. Prêl., p. 364). M. Milde cite une tige de la mème espèce, bifurquée vers son milieu, avec l'une des tiges de la bifurcation disposée en spirale (Komntn. E\%., pl. LVI, fig. 40), ct une tige d'E. pratense sur laquelle cing petites liges jaillissaient d'une gaîne commune (Gef. Crypt. Schl., p. 443, pl. XXXV, fig. 52) et plusieurs autres exemples.

$14^{\circ}$ Bifurcation des épis. - $1^{\text {re }}$ forme. L'épi terminal se divise en deux sur un point quelconque de son étendue, au-desus de l'anneau. 2e furme. Lal division commence dans la dernière gaine el chaque épi a un "pédoneule » et un anneau. M. Milde a figuré plusieurs fois celte anomalic (Gef. Crypt. Schl., pl. XXXII, XXXIV et XXXV, el Kenntn. Eq., pl. LIV, fig. 2).

$15^{\circ}$ Pluralité d'épis sur un épi mutilé. - Je dois à II. le docteur Warion une lige spicifere d'E. maximum recueillie par lui près de Itetz le 28 avril 1859 , sur laquelle l'épi, tout à fait normal ì sa partie inférieure, arait été coupé au-dessus du sixième verticille; sa cavité intéricure était béante et sur les bords s̈älevaient verticalement et en cercle sept épis; chacun d'eux avait nenf rerticilles et la grosseur d'un épi terminal d'E. palustre. Gette anomalie, figurée pl. VIII, fig. 36, n'est qu'un cas particulier, mass fort curieux, de l'anomalic no 3.

$16^{\circ}$ Graciliti. - Plusicurs espices el notamment les E. palustic, limosum, ramosissimum, rariegatum et liycmule, ofrrent des sujets à formes si grêles et si chétires qu'on peut être tenté de les prendre et qu'on les a pris souvent pour des variélés ou 


\section{§. - ANOMALIES.}

même pour des espèces. Ainsi les formes grèles de l'E. palustre ont fourni l'E. prostratum IIoppe, la v' mudum Duby, la v' teme Döll; celles de l'E. limosum ont occasionné l'E. uliginosum Mühlb., qui, pour M. Al. Braun, est la ví minus; celles de I'E. ramosissimum ont produit l'E. clongatum ctc. Sur ces individus ainsi réduits il n'est pas rare de rencontrer des sporanges et dés spores mal conformés.

Il est incontestable que, dans la plupart des cas, il n'y a que simple appaurrissement et que les influences locales suffisent pour l'explication de ces anomalies; ainsi j’ai trouvé la forme constituant la vé minus de l'E. limosum à celle des extrémités de rhizomes qui était hors de l'eau, tandis que sur le reste des rhizomes, dans le fossé, les tiges atteignaient $1^{\mathrm{m}}, 70$, et des faits analogues sont fréquents sur les E. littorale, palustre, ramosissimum.

Mais dans beaucoup d'autres cas on voit ces différences de taille exister et se perpétuer dans la même localité sur des groupes de rhizomes s'étendant à côté de sujets vigoureux; elles ne peuvent alors être attribuées ni aux influences du sol ni à celles de l'altitude et de la température etc. J'ai remarqué que ces différences, qui vont jusqu'à l'anomalie, n'existent à ce degré que sur les espèces qui émettent des spores pendant toute la belle saison, et particulièrement sur les espèces portant à leurs rameaux des épis plus tardifs que celui de la tige principale. El alors je me suis posé cette question : ne serait-il pas possible que les spores des épis des rameaux, provenant d'une plante déjả fatiguée, donnassent naissance à des sujets plus faibles, qui, à côtẻ des sujets forts, sembleraient constituer des variétés?

$17^{\circ}$ Infécondité des spores. - On a dejả observé et constaté depuis plusieurs années que les spores de l'E. littorale sont en général mal formées et impropres à la reproduction. M. Milde, à diverses reprises (Ge\%. Grypt. Schl., p. 457; Eq. litt., p. 30 elc.), a tiré de cette circonstance et de plusieurs autres, il est vrai, la conclusion que cette plante n'est qu'un hybride. Je ne veux rien objecter à l'opinion de ce savant botaniste; mais comme j'ai trouvé assez fréquemment des spores bien constituées sur celle plante, et que, d'autre part, j’ai très-fréquomment trouvé des spores infécondes el des sporanges mal conformés sur des individus à constitution appauvrie appartenant à toules les espèces, je me suis demandé si les E. littorale et trachyodum, dont les rhizomes rampent d'une manière démesurée, ne devraient pas à cette circonstance l'infécondité de leurs spores. La fructification de la plupart des graminées el des autres plantes stolonifères avorte constamment quand leurs stolons sont très-étendus, et n'aboutit que si les stolons sont supprimés ou seulement arrètés par un obstacle (voir ci-dessus p. 94 et 95, et Annotations ì la fl. de Fr. et d'All., p. 112).

J'ai déjà mentionné p. 93 et 94 comment, sur certains pieds à développement luxuriant, la mauvaise conformation et l'infécondité des spores peuvent aussi bien résulter d'un excès que d'un arrêt dans la multiplication des cellules-mères. 


\section{TABLEAU RÉSUMÉ DES ANOMALIES}

ET DES ESPĖCES SUR LESQUELLE ELLES ONT ĠTÉ OBSERVÉES

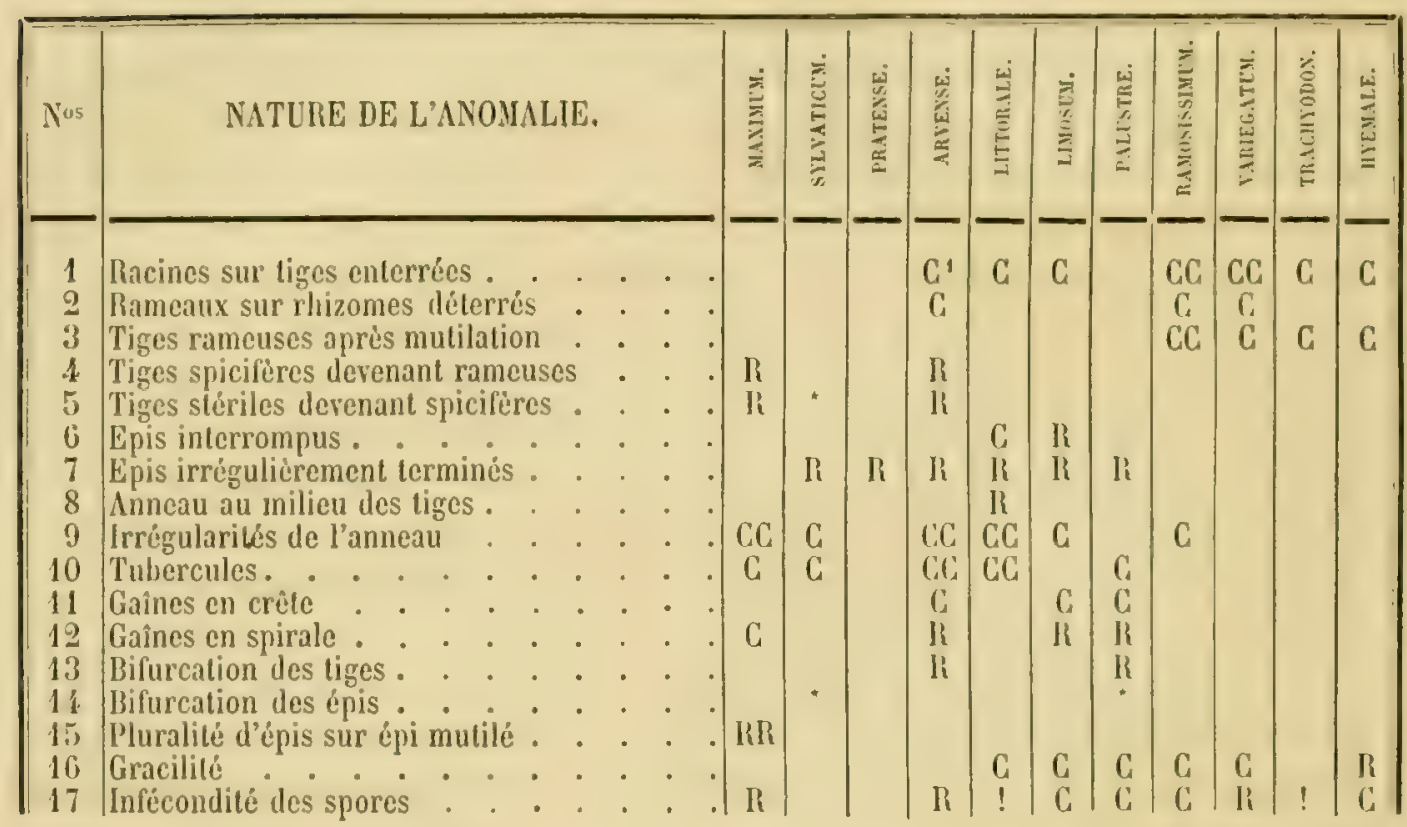

- $\mathrm{C}$ signifie commun. $-\mathrm{CC}=$ très commun. $-\mathrm{R}=$ rare. $-\mathrm{R} \mathrm{R}=$ très-rare. $-{ }^{\circ} \Rightarrow$ Non trouvé en France $!=$ Presque constant sur l'espicec. 


\title{
DEUXIËME PARTIE
}

\author{
Glassification. - Description
}

\section{GHAPITRE PREMIER}

\section{Place de la famille}

Les Equisetum ont été très-anciennement connus, comme l'attestera l'histoire des vertus qu'on leur attribuait, mais ce n'est que dans des temps relativement très-récents qu'on s'est occupé sérieusement de la place à leur assigner dans l'ensemble des végétaux.

G. Bauhin les jette, sans dire pourquoi, dans la seconde section de son livre premier, avec les Juncus et les Cyporus, au beau milieu des Graminées. Je ne cite ce grand botaniste que parce que je retrouve comme un écho de son opinion dans Mirbel, (Anat. Prêl., p. 445), dans De Candolle (Org. vég., I, p. 230), cl dans Bischoff (Krypt., Gew., p. 28), lorsque ces auteurs font mention du rapport que la gaîne des Equisetum leur paraît établir entre ces plantes et les Graminées.

Nous avons vu p. 122 que Tournefort (1700) les plaça dans ses plantes dioïques à côté du Iloublon et de la Mercuriale, et que J. Ray leur assigna la même place, quoiqu'avec doute (Meth. plant., p. 20, 1703).

Dillenius (1717) fut le premier, je crois, à signaler les rapports des Equisetum avec les Capillaires ou Fougères (Diss. epist., p. 58); il les plaça donc parmi les Fougères, en justifiant ce rapprochement et en combattant l'erreur commise par Tournefort (Nov. gen., p. 88). 


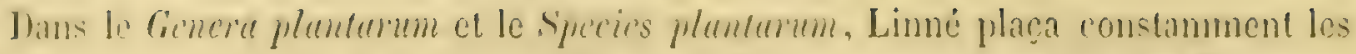
Equisctum dans sa vingt-quatrieme Classe, lil Gryptrastmie, et en léte des Fongeres.

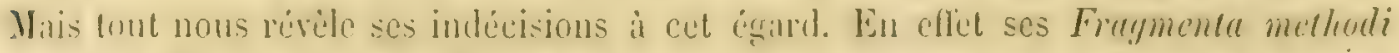
nuluralis (Philos. bot., p. 27 et suiv.) ne contiennent aucune mention de ce gentre. Inas les Ordin's nuturales qui terminent les idtitions du Genera pluntaruin postérinures à la sixieme, on voit le gente Equise'tum mentionné le dernier du LI Ordo, Coxifere, ainsi qu'il suit:

«? Equisetum (filicis pollen).»

Il parail toutefuis que si Limné avait été un instant entrainé par l'analogie des formes cxterieures, qui existe, d'une part, entre l'ípi des Equiselum et l'inflorescence des fleurs miles de quelques Conifëres, el, d’autre part, entre les articulations des Equisctum et celles des Ephedra (Precl. ord. nat., p. 592), il n'avait jamais cessé de reconnaitre les rapports p'us essentiels qui les unissent aux Fougères. En effet, daus ce que Fabricius el Giseke nous ont conservé des Leçons de Linué, on troure les explications suivantes:

«LV ordo, FILICES.

(I. Filices pructificationibus spicalis.

"Eọcisetum ritione floris cum Taro et Ephedra fere convenit, el tamen hue pera tinel, quod sane singulare. TounNefont illud a Filicibus separavil, el unam peltam " fructum, alteram florem masculinum proferre credidit. Fructificatio enim ex peltis (c in spicam positis constat, quie figuram habent subrotundam el sub valvulis inflexis "pulverem continont. Equisetum articulatum est, et omnes verticilli prxidatim se «invicem excipiunt» (Prel. ord. nat., p. 597).

Les botanistes qui adopterent le systeme sexuel de Linné continuèrent it comprendre les Liquisetum dans le premier Ordre de la Cryptoyamic, c'est-i-dire dans les Fougères. Scheber, dans l'édition du Genera plunturum qu il donna en 1791, les en sépara, et les rangea, avec les Lycopodes et les Rhizocarpées, dans son premier Ordre qu'il appela Hiscellenere. Roth adopta ce nom et celte classification. Willdenow fut le premier it les considerer comme constituant à cux sculs un ordre particulier, quil nomma Goxopterners, Gliederfarn, Fongires arliculées; il le plaça en lite de la Cryplogamic, à cülé des Stachyopterides ou Fongères en épi, et rejela ses IIydropteride's (Rhizocarpées) i l'autre extrémité des Fougères Grundr. d. Krïuterliunde', 1792; et Sp.pl., V, p. xxxxi, xxxxy et 1, 1810). G. F. IIoffmann en fit à son tour ses

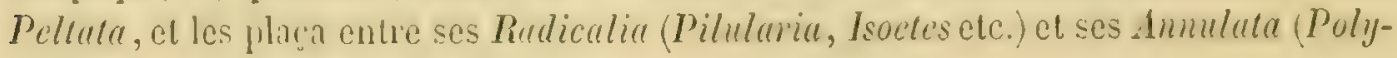
podiume etc.) (Deutschl. Fl., 1795).

Les auteurs de systemes naturels ont été moins d'accord. Adanson, ne tenant pas comple de la structure anatomique, ne commaissant pas encore le mode de reproduction des Équisetum, les classa, comme les autres vérgétaux, en considérant le nombre 
des ressemblances extérieures, et fut ainsi nécessairement amené i les rapprocher des Coniferes. Il les plaça done dans sa cinquante-septième Famille, les Prxs, ì lia suite du genre Casuarina (Fam. Plant., II, 1) 477, 1763). De Lamarek les comprit dans ses Fougères sous le nom de Fougères fausses (Fl. fr., I, p. 3, 1778).

Le Genera plentarum de A. L. de Jussicu (1789) les mentionna dans la premiere Classe et le cinquième Ordre, à la suite des Fougères, à côté des Salvinia et de l'Isoctes, sous la rubrique: «V. Fructificatio minus nota. Planta filicibus affines.»

De Candolle en fit, d'après L. Cl. Richard, une famille particulière, sous le nom de Equisctacece, Ordo X (Fl. Pr., p. 580, 1805 , et Syn.pl.,p. 118, 1806); et il les sépara des Fougères par les Liycopodiucew et les Rhizosporme. Sprengel, qui les avait d’abord rangés parmi ses Pteroides (Anleit. z. Kénntn. d. Gew., III part., 1804), les en ćloigna dans la deuxième édition du mème ouvrage (1817), et les plaça comme genre isolé en supplément après les Rhizospermes. Oken les comprit avec les Chara dans la XIe Tribu de sa $3^{\circ}$ Classe, parmi les Pfaumen-Drossler, dans le voisinage de l'Ephedra et du Casuarina (Lelwb. Natury., $2^{2}$ part., 1825). El. Fries les classa d'abord parmi les Fougères (Syst. orl. veget., 1825); mais vingt ans plus tard il les mit comme appendix, tout en les notant «Acotyledones, dans sa XI ${ }^{\circ}$ Classe Iuliflore, ả la suite de sa LXXXII Famille, Conifore, et tout à côté des Callitriche el des Chara (Sum. veget. Scand., p. 59). Bischoff, qui mentionna la plupart des opinions précédentes, repoussa « la réunion des Equisetum aux Fougères, à cause des particularités de leur structure et surtout de leurs organes reproducteurs. » Il les considéra « comme un sous-ordre à part, occupant un rang systématique égal à celui des Fougères propres ) (Krypt. Gew., p. 55), et il plaça ses Enuisete. entre les Characer el les Rhizocarpece. M. Lindley rangea les Equisetum, comme famille, dans sa grande Classe des Gymnospermes, auprès des Conifères (Nat. syst. bot., p. 317, 1826).

M. L. Reichenbach place les Salviniacea parmi les IIusci, conserve les .Harsileaceie parmi les Fougères, qu'il ne décrit pas, place les Isoetes avec les Potamogeton, puis viennent les Glumacées, les Iridíes, les Liliacées etc., et enfin les Lycopodiacece el les Eqquisctacece réunies aux Conifëres el aux Thyméléacécs elc. (Flor. excurs., p. xiv ct 153), en appuyant toute celte complication sur des considérations physiologiques absolument hypothétiques, sur celle-ci, par exemple, que les Equisctum Telmaleia et arrense sont dépourvus de vaisscaux spiralés (roir ci-dessus p. 46 ct 84, note); ce qui, à son avis, «refutat systema quod plantas in cellulosas et vasculosas distinguit» (p. 154).

La plupart des hésitations et des contradictions qui préeedent étaient sans doute permises à une époque oủ l'on ne comnaissait ni les organes reproducteurs, ni le mode de fécondation, ni les premiers développements des Equisctum, mais il me semble que M. IIenderson allail beaucoup trop loin quand il disait encore en 18k2 : "La place des Equisetum est si douteuse que de très-éminents botanistes ne paraissent pas 


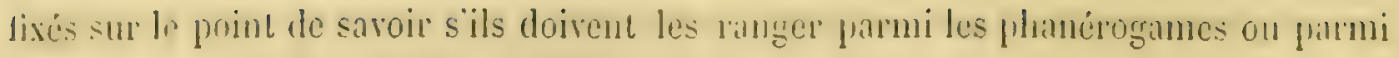
les cryplogames (Rieprod.Eq., p. 567).

nepuis cette époque les doutes ont bien diminue, si non cessé, cl les Equisetum se flacent au nombre des Cryptogames vasculaires, et, comme famille a part, dans le roisinage des Fouseres. Koch (Sym. fl. germ.) ct M. Ad. Brongniart (Ëmu. gen., p. 51, 1850) les en séparent par les Marsileacee el les Lycopudiucea'; M. Grenier $(F / . F \%$.) et M. Itolmeister (Veryl. Unters., p. wi ct 89) les placent eutre les Fougìres et les Rhizocarpées; Ach. Richard (Préc. bot., II, p. 50), M. Bernoulli Gref. Krypt. Schw., p. 64), MI. Döll (Fl. Butl., p. 53), ainsi (que M. Milde (Gef. Crypl. Schl., p. 386), entre les Fougères et les Lycopodiacées.

On semble done d'accord aujourd'hui pour placer celte famille à còté des Fougères, et, à mon avis, avec pleine raison. Le mode général de reproduction s'opérant, comme celui des Fongères, au moyen de spores qui se développent en sporophymes et sur lesquels apparaissent les deux organes de fécondation, ainsi que le pseudembryon ou nouvelle plante, on ne voit pas bien ce qui peut sopposer an rapprochement des Equisetum et des Fougères.

Si l'on compare une tige d'Equisetum avec une fronde de Fougère, on trouvera à première vue que l'apparence extérieure, aussi bien que la struclure intéricure, scmblent ne présenter que des différences. En effet, $1^{\circ}$ une fronde de fougère est un appendice latéral dont le pétiole et l'expansion foliacée, symétriques, il cst vrai, par rapport à leur ligne médiane, ont une face supéricure et une inférieure très-dissemblables, et elle ne présente point, comme les Equisctum, un axe central autour duquel toules les partics de l'organisation s'ordonnent circulairement; de plus on remarque que tout est verticillé sur les Equisetum, disposition rui semble ne pas appartenir aux Fougères; $2^{0}$ enfin, les sporanges sont ordinairement placés i la face inlérieure des frondes de Fougères, el, sur les Equisetum, rien ne semble rappeler celle disposilion.

Mais ces différences sont-elles aussi réelles et aussi essentielles qu'clles paraisscnt d'abord évidentes?

$1^{0}$ Si la fronde d'une Fougère est évidemment un organe latéral, il ne convient point de la comparer avec la tige complète d'un Equisetum; la comparaison n'est permise qu'entre deux organes latíraux, par exemple entre une fronde et l'une des divisions de la gaine, ou entre deux tiges on axes principaux, par exemple cutre une tige d'Equisetum el une lige complete de Fungère. Or, en faisant cette dernière comparaison, on est trop porté, ce semble, à altribner aux Fougères un rhizome trasant ou rampant. Nème sous notre climat de France, vingt-neul especes sur un total de quarante-trois mentionnées dans la Flore de MII. Grenier et Godron, c'est-i-dire une trés-forte majorité de vingt-neul contre quatorze, n'ont point de rhizome tracant is 
pétioles simplement alternes et esparés, mais bien des tiges déterminées, oủ les pétioles sont, avec un ordre rigoureux et selon des nombres invariables, régulièrement disposés en lignes spirales, lesquelles ne sont après tout que des verticilles étendus en spires (voir G. Ogilvie, Struct. fern., p. 401 et suiv.). Ces tiges sont courtes, il est vrai, bien que sur les Polystichum filix mas, spinulosum, Oreopteris, elles alleignent quelquefois vingt à vingt-cinq centimètres au-dessus de la surface du sol; mais l'organisation est indépendante des dimensions. Il serait donc plus exact d'attribuer aux Fougères de notre climat des tiges verticales, et, par exception, des rhizomes traçants, que de leur attribuer uniquement des rhizomes traçants, et aux exotiques, par exception encore, une tige verticale. Le rhizome lui-même est une tige autour de laquelle les cycles ou verticilles ne peuvent se développer en spires régulières à cause de la contiguité du sol, et, pour cette raison, ils s'y espacent en alternant avec plus ou moins de régularité, suirant que le rhizome est tout à fait contigu au sol ou tout a fait souterrain. Nous en voyons des exemples sur les Rubiacées, les Labiées etc., oủ les stolons ont des feuilles alternes, et les tiges des feuilles verticillées; et, pour les Fougères, le Struthiopteris germanica nous en fournit un exemple remarquable. En effet, sur les tiges de cette admirable espèce nous voyons se détacher des rhizomes traçants, des stolons, qui demeurent grêles, et portent latéralement des rudiments de pétioles alternes, tant qu'ils ne trouvent près de la tige-mère qu'un terrain épuisé ou appaurri par elle, mais qui, à une distance propice, s'arrêtent et s'élèvent en une nouvelle tige dépassant souvent vingt-cinq centimètres de long, et sur laquelle les pétioles s'ordonnent en belles spirales ${ }^{1}$. Il y a plus: on trouve même sur des Fougères exotiques « des pétioles ordonnés en cercles superposés et des stipes creux à leur partie (centrale» (Ach. Richard, Préc. bot., p. 71, 1852; Ad. Brongniart, Hist. víg. foss., I, pl. XLIII, fig. 1 et 2 , et pl. XLIV, fig. 5 et 6). Sur nos Fougères indigènes la tige n'est pas creuse; mais on peut voir déjà, dans les portions les plus vieilles, que le tissu cellulaire central est le premier à disparaître (G. Ogilvie, Struct. fern., p. 409). Ainsi il n'y a point entre les Fougères et les Equisetum cette différence fondamentale que ceux-ci auraient un axe principal régulier avec des verticilles d'organes latéraux, et que celles-là n'offriraient point celte disposition.

D'oủ cette conséquence immédiate que si l'on veut opérer des coupes de comparaison entre les Equisetum et les Fougères, c'est sur les tiges de ces dernières qu'il faut opérer ces coupes, et non sur des appendices latéraux, sur les pétioles, que la négligence ou la paresse introduit seuls dans nos herbiers, en les mutilant même le plus souvent.

Or, sur de semblables sections on remarque au premier coup d'œil qu'au licu d'of-

1 J. Duv. J., Pél. Foug., p. 19, pl. Il, fig. 21, et Mett., Seitenkn. Farn., ए. 628. 
frir, comme les Equisetum, une serie de cereles concentriques, sur charun desquels on relrouve les mimes organes ílumentaires, cellules ou vaissenux, semblathlement disposís à des intervalles rigoureusement égaux, les tiges de l'ougères indigènes ou exotigues offient un eylindre central de tissu cellukire, antour duguel "se voient un " grand nombre de lignes noires, diversement contournées, formant des figures irréa gulieres et bizares, qui eependant se reproduisent aree une sorte de régularite. Ges "figures, rapprochécs les unes contre les autres, forment par leur rémion 14 corcle "on une conche circulaire a la partic externe de la tige " (1ch. Richard, Príc. but., I, 1. 72). Comme ces lignes sont constituées par du tissu fibru-vasculaire, ce quu préceide revient it dire que, sur une conpe horizontale d'une tige de Fougiere, le tissu fibrovasculaire apparait inégalement et irrégulièrement distribué, tandis que c'est le contraire chez les Equisetum.

IIais cette irrógularité n’est qu'apparente; elle est la conséquence rigoureuse de la disposition spiralie des eycles de pétioles. Quand on coupe transversalement une lige d'Equisetum, on coupe toute la série des organes à une même distance de leur point d'origine, par cela seul que sur les Equiselum tout apparait en verticilles horizontaux et non en cycles déroulés spiralement. On ne contestera pas la parfaite régularité des cyeles d'un cûne de Pin; or, si sur un point quelconque on fail une coupe perpendiculaire à l'axe, on n'obtient qu'une série de figures plus ou moins dissemblables et bizarres. Pour obtenir une coupe présentant la mème régularité que celle des Equisetum, il faudrait, sur ce cône comme sur une tige de Fougère, la pratiquer, non suivant un plan perpendiculaire à l'axe, mais suivant une spirale qui couperait tous les appendices latéraux à une mème distance au-dessus ou au-dessous de leur point d'émersion. Une coupe transversale symétriyue el régrulière ne s'obtiendrail que sur to slipe des espèces dont les pétioles sont ordonnés en verticilles superposés.

Comme, dans la grande fimille des Coniféres, la disposition des rameaux en verticilles ou en spirales ne constitue nullement un caraclère d'exclusion ou d'admission, il est permis d'en faire autant ici et de regarder comme purement secondaires et plus apparentes que réclles les premières différences sigualées entre les Equisetum et les Fougères.

Si lon voulait comparer un appendice latéral des Equisctum arec un appendice latéral des Fongères, il fiudrait comparer une des divisions de l'entre-noud arec un pétiole, ou micux, une des divisions de la gaine avec une fronde, et l'on tronverait alors de très-nombreux points de ressemblance dans la distribution des vaisseaux par groupes latéraux symétriques".

20 Si maintenant on se rappetle ce rui a été dil de la disposition des sporanges par

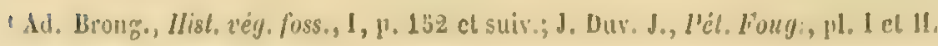


rapport à ces mèmes expansions foliacées, on verra qu'elle n'est pas aussi éloignée de celle des Fougères qu'on le pourrait croire au premier abord. En eflet, les pédicelles sporangifëres étant considérés comme de véritables rameaux privés de gaine basilaire, le clypúole qui les termine répondra très-exactement à un verticille d’expartsions foliacécs, qui, au lien de se prolonger en gaine, sont ćpanouies et réfléchies to long du pédicelle. Ét comme pour chaque «feuille» ou division de la gaine, la face supéricure est celle qui cst appliquće contre la tige, et linféricure celle qui cst extérieure, ces divisions, cn s'épanouissant el se réfléchissant au sommet du pédicelle, présentent à l'extérieur leur face supérieure, supportent ì leur face inférieure l'appíreil reproducteur, et dès lors se comportent comme des feuilles qui se seraient rerversées et pliées selon leur ligne médiane pont former à leur face inférieure une cavité longitudinale arec déhiscence parallèle au pédicelle. Il suil de là que, puisque sur les Equisetum, comme sur les Fongères, la fructification est à la page inférieure des expansions fuliacćes, il n’y a aucune raison sérieuse pour éloigner les Equisetum des Fougères.

\section{CHAPITRE II -}

\section{Examen des caractères spécifiques}

Le premier caractère que j’ai considéré pour la division en sections a été la conformité ou la non-conformité des tiges spiciféres. Et cela d’abord parce que ce caractère, ficile à saisir et constant, ne consiste pas en une simple différence superfieielle, mais qu'il entraine arec lui des différences considérables dans la constitution el dans les fonctions (roir ci-dessus p. $24,95,44,46$ ), qu'il a ainsi en même lemps une valeur organique et une valeur physiologique, et enfin parce que les espèces qu'il rémnit ont dans tout leur ensemble plus de ressemblance entre elles qu'avec aucune de celles qu'il en sépare.

Les deux grandes sections ainsi obtenues se divisent chacune en deux groupes; si l'on considère, pour la première, l'absence ou la présence, et, pour la seconde, la forme et la répartition des stomates. Ces caractères, qui semblent d'abord d'une constatation difficile à l'œil nu, sont, par une très-heureuse coïncidence, toujours accompagnés d'autres caractères très-faciles à saisir. Ainsi, dans la première section, la présence ou l'absence des stomates est nécessairement signalée par la présence ou l'absence de la couleur verte sur les entre-nœuds (p. 44); et, dans la seconde, la dispersion des stomates sur les sillons de l'entre-nœud on leur ordonnance rigoureuse cn deux lignes parallèles (p. 31), la situation de l'ostiole des stomates au niveau de l'épi- 


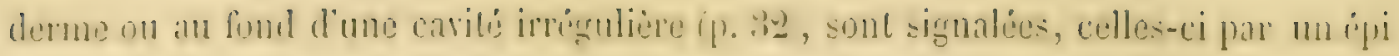
acuminé, celles-là par un épi obtus.

Linsi se trouvent obtenus yuatre groupes, établis sur des différences de structure.

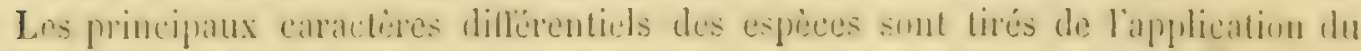
mème principe. Autant que possible, ch sans une rigueur absolue qui risque die tont fausere, jai placé au premier rang ceux que fournisent les différences de la constitution organique, par exemple, dans le second groupe, les modifications, pour ne pas dire les transfurmations, que subisent les liges spicileres apres la sporue ep. 15, 46); dans tous les groupes, la section transersale des tises el des rameaux. Elle permet de sisir les divers détails de la constitution, la grandeur relative de la cavité centrate "l des lacunes, ainsi que la disposition de ces dernieres, et elle funrnit ainsi des dif-

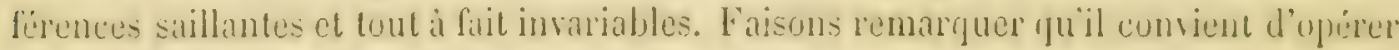
les eoupes vers le milieu de la tige, région ou tous les caracteres sont te mienx develuppés. Aux entre-nuends inférieurs, l'organisation se rapproche de celle des rhizomes, tandis quanx derniers entre-nueds des tiges stériles clle devient presque identique a celle des rameaux. Viennent ensuite les caracteres si précieux tirés du rapport qui existe entre la longucur d'une gaine de la tigne el la longueur du premier entre-nemel

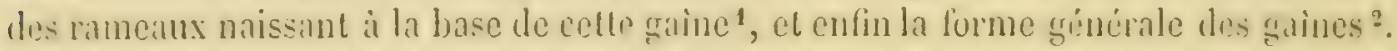
Ce n'est qu'en dernier lieu que j'arrive à considérer le nombre des divisions des gaines. On a plusicurs fois employé comme caractere distinctif d'une certaine valun lil prisence sur les divisions des gaines de certains sillons (sillon carétal, laléral); ot en eflet ces sillons ripondent a la constitution intérieure, en ce sens qüils sont dus au retriat plus considéraljle de certains tissus. Mnis, comme ils ne se montrent guere qu'ipprés la desiceation, et que, d'une part, ils sont plus ou moins apparents selon liage de lindividu dessiché, tandis que, d'autre part, la pression eu altere toujours la rigularite, jérite de les emploser, et jo prefere indiquer sur des cuupes at l'etat vivant le cartetire riel auquel ils correspondent. Je suis convaincu que, pour arriver i la connaissince sérieuse d'une csprece, l'étude de sujets en herbiers cst insulfisante, et qui un ny parvient aree certitude que par lousservation fréquemment répétée de la phinte vivaule, à ses divers étals de développement ot dans des lieux diflérents. sur le

\footnotetext{
I Par memier entre-noud j'entends celui qui, quelle qu'en soit la lonģueur, succèle immédiatement ì la gailnc basilaire. Si donc on compare ce premier cntre-nocul, y compris la gaine qui le termine, a la longueur de la gaine caulinaire, on trouve que, sur l'E. arcense par cxemple, cet entre-noud est toujours plus long qque la gaine caulinaire et qu'il en est souvent lo double. Sur l'E'. palus/re il atteint à peine la moitié de celte mìme gaine elc. 11 importe encore de faire cette comparaison vers le milieu te la tige, frarce que quelquefois les verticilles inféricurs ont été gènés et arités dans leur déveloprement.

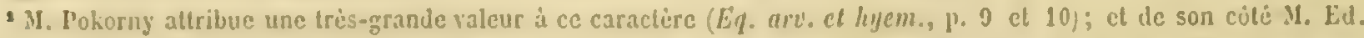
Kewman dit que "la forme des gaines des rameaux fournit des caracteres si importants que l'inspeclion d'une seule es

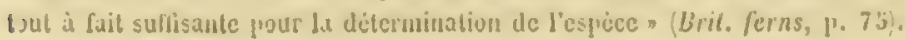


sec on est toujours exposé i prendre un état internídiaire de développement pour une forme définitive el distincte (roy. J. Duv. J., Eq. hyem., p. 164 el suiv.).

On a encore assez sourent altribué une certaine valeur différentielle aux taches, aux zones, aux bordures colorées des gaines. Irais la couleur noire est celle que sur les Equisetum prennent les cellules qui ne fonctionnent plus ou qui ont été lésées; elle se produit sur les gaînes sans grande constance et de toute façon par suite de déchirure, de compression, d’atteinte du froid etc. Je n'ai done en général tenu ancun compte de ces différences de coloration, non plus que des différences de taille, parce que ces caractères, sans fixité et sans permanence, sont plus propres ì induire en erreur qu’à éclairer. On peut sans doute, et j’ai ainsi fail, énumérer ces différences dans les détails d'une description étendue, mais ils ne doivent point, à mon avis, être pris en considération pour la distinction el l'établissement des espèces. Je crois que la structure anatomique des Equisetum est set'Le d'une haute importance pour celle distinction et cet établissement, el que le principe posé par Linné devrait dominer toute distinction d'espèces: "Ergo species tot sunt quot diver'se " formx seu STRCCTUR.e Plantarum, rejectis istis, quas locus vel casus parum diffe«rentes (Varictates) exhibuit, hodienum occurrunt» (Gon. plant., rat. op., §5). Le plus grand des classificateurs avait ainsi prévu et rejeté à l'arance ces incualifiables espèces, dont les caractères purement comparatils se réduisent invariablement à ceci: "Cette espèce a le port de .....; mais elle s'en distingute neltement par une taille moins "élevée, des poils plus nombreux, et surtout par son habitat! Elle est propre en effet «aux terrains secs des côtes de la Méditerranée, tandis que.... habite les coteaux de «l'ouest.» Une différence d'aspect est la conséquence naturelle et nécessaire d'une diffërence dans l'habitat et la station, et il faudrait en vérité un miracle pour que d'autres conditions de sol, d'humidité, de température, de lumière etc. ne produisissent aucune modification dans les détails extéricurs d'un type dont les éléments essentiels persistent identiques. Si des différences de taille, de ramifieation, de coloration étaient admises comme caractères spécifiques suffisants, il fuudrait créer aux dépens des E. maximum, arvonse, limosum, palustre, ramosissimum et de tous les autres, autant d'espèces que ces plantes peurent présenter de formes.... sur un mème rhizome. Les espèces ne sont pas sculement des apparences ou formes différentes, mais des types compris entre certaines limites, pourant varier entre ces limites selon les circoustances. C'est pourquoi, dans la distinction des espèces, jai cru deroir laisser de còté tout ce qui est purement extérieur et dépendant des eirconstances, pour mattacher de préférence aux caractères essentiels, si intimement dépendants de l'organisation qu'ils ne disparaissent qu'avec la destruction de l'être lui-même.

La coupe transtersale des liges fournit des caracteres anatomiques excellents, et

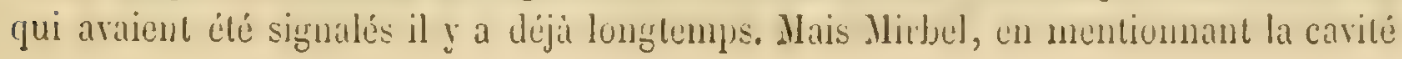




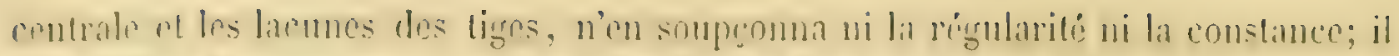
I: attribuat it une circonstance accidentelle, "i la retraite des celtules qui occupent a la partic intéricure des tiges» (Anat. Prêl., p. 4k6), el il en fit ainsi méconnaitre tuate l'importance. Yancher, énumcrant el subordomant les caracteres pu'il emploie:

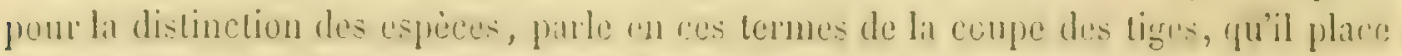
an dernier rang: "Independanment de ces caracteres principanx, il en est dintres

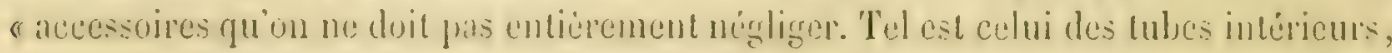
«qui varient pour le nombre des rangs et pour la forme. Quelgues Prêles ont deux "rangs de tubes, tandis que d'auties en ont trois: la plupart sont cylindriques, "ceprendant eeux de la l'ride des limons sont allongrís dans le sens de la circonfërence,

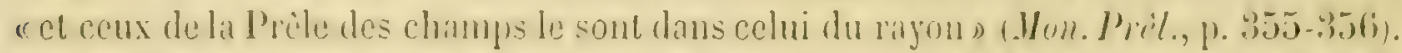

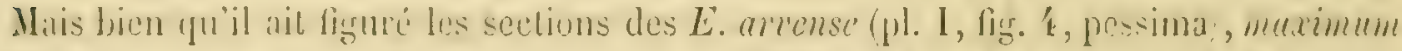
(II, 6, mala), pelustre (V, .4, mala), limosum (VIII, 6, bona), hygenuele (IX, 3), bona), 1. meme auter néglige entierement ee caractere dans la description de ces espees. Il ne dit rien non plus de la situation des groupes de cellules i chtorophylle, ni de celle des filures corticales elc.

Ces caractères furent micux appréciés par Bischofr, qui donna avec de grands détails les coupes des $E$. arvense, maximum, limosum, hycmale, ramosissimum.

M. Ed. Newman, alnès avoir figuré au simple trait quelques sections, ajoute arrec beaucoup de raison: "Je recommande létude comparative de ces sections à loule lattention des botanistes. Ce n'est pas, il est rai, un mojen de listinction tout a fait neul', mais je crois quion n'a justu'a présent donné à cette élude qu'une trop faible importance» (Brit. ferns, p. 50).

II. Nilde a figuré les sections de plusieur's espèces et a même dressẻ un tableau comparatif des coupes des espèces européennes (Gef. Crypt. Schlo, p. 472-474).

De mon coté joi essayé d'appeler l'altention des botanistes français sur la valeur de

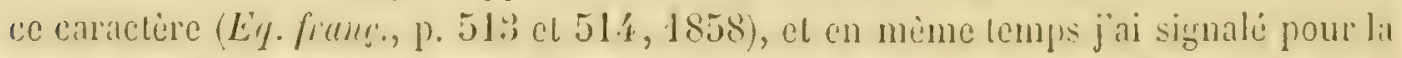
premiere fois l'importance des earactères que fournit la structure des rameaux. Vilucher avitit détourne l'altention des botanistes de l'étude de celte structure, ch disant: "lorganisation des ramenux est la mème que celle des tiges. 1ls ont extéricurement "leurs divers rangs de cylindres concentrijues" (Mon. Ireil., p. 392). Cest une ermur. Sur tuntes les especes, la dieposition des fibres corticales el des cellules a chlorophille presente des diflërences particulieres, tout a lait invariables, tris-saillantes, el quane? section transversale revele aree une extreme facilité? Dans los deux premin's groupes

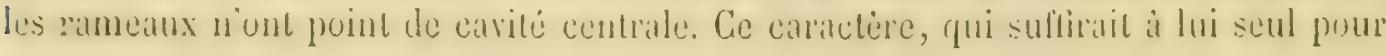

\footnotetext{
- l'our avoir des points de comparaison déterminés, jl convient d'opérer les sections sur le méme entre-nocud. J'ai chuisi le deuxicme au-lessus de la gaine basilaire, et c'est au milicu de cel entre-noud que j’ai fait toutes mes coupes comparalives de raneaux.
} 
établir la division proposée, non-seulement la confirme licureusement, mais cncore, en s'adjoignant au caractire sur lequel elle repose, il lui cnlève ce qu’il y a toujous d'un peu trop absolu dans une division établie sur un seul caractere, quelle qu'cu soit la valeur. Vaucher avait pris pour point de: départ de sa division la présence ou l'absence de hampes spicifères, mais en ne considérant que ce caractère et en lui attribuant une valeur absolue, cet habile observatcur fut nécessairement conduit it rattacher comme variélé a l'E. palustre une forme anomale de l'E. arvense, dont la lige spicifère se revêt de rameaux (voy. Bernoulli, Gef. Kmypt. Schw., p. 66, et cídessous la synonymie). M. Al. Braun a reproduit la division principale de Vaucher, en variant l'expression: $1^{0}$ "IIETEromiyadica, caules fertiles a sterilibus diversi; illi prececiores, discolores; 200 IIonorny.ura, caules fertiles et steriles conformes, herbacei et cocetanci » (Sill. Journ.). Or celte division, ainsi que la subdivision de la première section en Ametabola et Metabola, ne s'appuyant que sur un seul caractère, cessent d'être exactes et se trouvent même en contradiction avec les termes que leur éminent auteur comploie pour caractériser les deux principales anomalies des E. maximum et arvense : " $1^{\circ}$ Frondescens: caulis fertilis non marcescens, sed ramos proferens; (20 Serotinum: caulis sterilis spicam proferens») (o.c.). Avec l'adjonction du caraclìre liré de la structure des rameaux, il sera impossible de se méprendre et de placer daus un groupe auquel elle n'appartient pas une forme quelconque, anomale ou non, présentant des rameaux.

En considérant bien le caractère qui a servi de point de départ à la division de Vaucher, à celle de M. Al. Braun et ả la nôtre, on voit de suite que, malgré sa haute valenr, il est loin d'être aussi absolu qu'il le parait tout d'abord. L'organisation qui règne sur tout l'ensemble des tiges spicifẹres propres (tissu mou, absence de stomates, de chlorophylle et de fibres corticales) se retroure en définitive sur le pédoncule des tiges spicifères de loutes les espices. Sur les unes comme sur les autres, clle est évidemment une modification préparatoire à la constitution commune et invariable de tout l'appareil reproducteur' seulement sur les unes cette modification préparatoire n'atteint que les deux entre-nneuds supérieurs, qui se flétrissent après l'émission des spores, tandis que sur les intres elle envahit toute la tige, qui alors est aussi molle et aussi caduqque que le pédoncule lui-même. Mais néanmoins certaines liges, au début très-distinctes, deviennent plus tard semblables aux tiges stériles, ct en prennent graduellement l'organisation an lieu de se flétrir et de périr. Cet ítat, qui est une anomalie sur les E. maximum et arvense, est l'état normal sur les E. sylvaticum et pratense, qui forment ainsi une transition d'un groupe à l'autre.

La terminaison de l’épi par un acumen, cmployée par quelques auteurs modernes (Koch, Grenier et Godron) pour séparer le groupe des IIyemalia, se retrouve quelquefois sur les E. arrense, matense, syllaticum et palustre; des lors elle n'a point i clle 


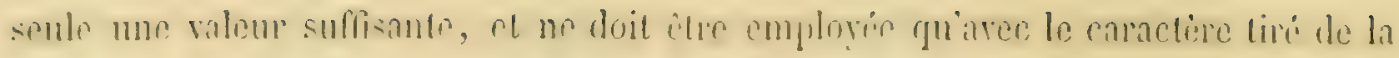
disposition et de la forme des stomates.

Ancun caractẻre, réduit à lui seul, n'a une valeur absolument constante. C'est pour cela que je ñi voulu imposer aucun nom caractéristique à mes groupes, parce que, dans fous les cas diannmalic, ces noms dericment juexacts of capables d'induire en erreur. Sil marrive ruelquefois de désigner le quatrieme groupe par le nom Iyyemaliu, c'est simplement paree gue la principale espèce de ce groupe est IF. hygemule, et que les esprices qui le composent jonissent seules de la propriaté de pouvoir passer l'hiver, quelques-unes sans abri, d'autres un peu abritées.

Je n’ai pu adoptre te principe de disision d'apres lequel M. II. Braun partage encore les Equiscum en seetions principales: $1^{\circ}$ Sren:opori, stomates dissémines duns les

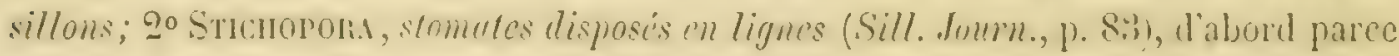
que, comme l'ont lait remarguer MII. Diill (F. Ball., p. 57, note), Bernoulli (Gef.

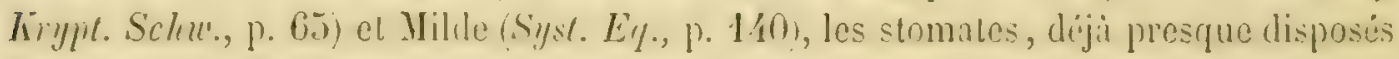
en lignes sur l' $E$. arvense, le sont tout à fait sur les E. sylvaticum et pratense, et surtout, à mon avis, parce que dans ce principe de division ne rentre pas l'E. maximum, dont les entre-nœuds ne présentent point de stomates.

En comparant les espries du genre Erfuisctum et la valeur des caractères qui ont servi à les étahlir, il est impossible de ne pas être frappé de ce fait, que ecs especes n'ont pas toutes la même valeur, ou, si l'on veut, le même degré de spécićité.

L'E. maximum, par labsence de stomates el de chlorophylle sur les cutre-ncends, par la gaine du premier entre-noud des rameaux, par la structure el la forme de ses rameaux cux-memes, est un type en dehors des autres et sans intermédiaire qui le rattache a cux. Ses tiges steriles offrent si completement la structure des entre-naends des tiges spicifires propres, quion serail lacilement porté il les considérer comme des tiges cssentiellement spicifures qui accidentellement nont pas abouti; et la prísence. d'épis sur ces liges ramenses, lapparition de ramenux sur les tiges spieifëres, si fréguentes dans certaines contrices, viendraient a l'appui de cette hypothese. Quoi runil en soit, et sans rien préjuger, il est permis de placer celte espece dans une section it part et en avant de toutes les autres.

L'E. syltaticum est un type tris-distinet: mais un intermédiaire, l'E. pratense, le relie à l'E. arvense.

Celui-ci, à son tour, est une cspece de premicr ordre, mais non isolée, et qui s’allie dans un sens a l'E. sylenticum par l'E. pratense, el, dans l'autre, par l'E. littorale i l'E. limosum, lequel est lui-même un type très-beau et très-distinct.

Arrivé là, on retrouve à l'extrémité de cette série d'espèces l'E. palustre, type presque égal à l'E. maximum et presque aussi isolé que lui.

Le (fuatrieme groupe, IIychuliu, se distingue des autres par des caracleres si esecn- 
licls ct si tranchis, qu'il est sans intermediaire arec eux el sen tient it grande distance. Mais les especes qui le composent, comparées les unes aux autre's, sont extrêmement voisines el si intimement unies qu'elles ne se distinguent plus que par des caractères de second ordre. Eilles ne sont évidemnent que des modifieations d'un type primitif, modifications devenues permanentes par la continuté d'action des causes qui les ont déterminées. Si nous ne pouvons pas nier la persistance de différences qui les distinguent suffisamment, si nous ne pourons pas par nos cxpériences effacer, ni même notablement affaiblir ces caractères différentiels consolidés par la force invétítée de l'atavisme et corroborés par le nombre prodigicux de générations qui se sont écoulées depuis deur apparition, il n'en est pas moins vrai que fréquemment nous pouvons reconnaitre à l'état spontané des furmes moins tranchées et presque indécises, attestant des modifications relativement récentes, produites dans un sens ou dans un autre, par des circonstances longtemps répétées. Ainsi nous trourons des variations qui rattachent la forme clongatum simplex de l'E. ramosissimum, d'un côté, à l'E. trachyodon, ct par lui à l'E. liycmale, et, d'un autre còté, aux formes luxuriantes de l'E. varicgatum vas Wilsoni. Ainsi encore les formes rabougries et cespiteuses de l'E. ramosissimum rappellent si bien celles de l'E. v'tricyutum des licux herbeux et ombragús, que plus d'un floriste s'y est laissé prendre. Ciest surtout en présence de ce groupe et de cette série de formes qui se relient les unes aux autres qu'on est forcé de reconnaitre la parfaite justesse de cette remarque de Vaucher: "Il est impossible, «après avoir observé de près toutes ces variations dans le développement des Prôles, " de ne pas concluie que les circonstances extérieures ont modifié et modifient"sans «cesse sous nos yeux leur type primordial (Mon. Prêl., p. 338).

En outre si l'on remarque que les deux Equisetum intermédiaires des autres groupes, pratense et littorale, sont ceux qui, tout en n'offrant qu'un petit nombre d'anomalies diverses, offrent un nombre incroyable d'individus sujets it des variations et affectés d'anomalies dans l'appareil reproducteur, on est tenté de se demander si ces plantes ne seraient pas des espèces plus récentes qui riont pas encore une forme définitive. Espèces qui, peut-être hybrides au point de départ, se comportent encore comme les hybrides obtenus arlificiellement, sont irrégulièrement fécondes, et, par des retours partiels vers leurs types parents, se balancent entre toutes les variations el toutes les anomalies possibles, jusqu'ì ce que, rencontrant des circonstances furorables, elles acquièrent définitivement une forme arrêtée et la vigueur nécessaire pour sc reproduire régulièrement el constamment (voy. Bull. Soc. bot. Fr., VII, p. 360).

$\Lambda$ côté du type spécifique le mieux déterminé se trouvent des variétés, des variations et des anomalies. Ces dernières, étant tout à fait individuelles, leur description ne doit point suivre celle de chaque espèce; il m'a paru suffisant de r'appeler brièvement celles auxquellcs charque espèce est sujette, soit ordinairement, soit dans telle cir- 
constance dommio, el d’indiquer les opinions que leur présence a fail naîlte. I.es raria-

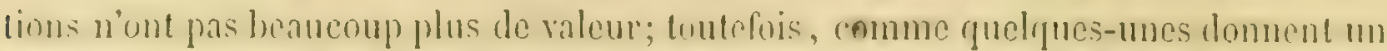
aspect particulier i certains individus, je citerai frierement les plus importantes, afin d'essayel de prévenir quelques erreurs.

Los varietes seules móritent une montion sérieuse. Eilles sont le risultat de l'action longlemps prolongéc dim meme ensemble de causes; elles peuvent durer indéfuiment arec la contimuitio de la mime action, et, apres la disparition partielle on totalte de ces causes, clles peuvent, par une sorte d'habitule congriblale, persister el meme.

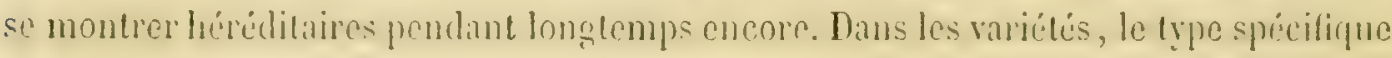
se conserve en ec qu'il a d'essentiel, et des modifications en plus ou en moins atteignent les formes de quelque organe secondaire. Or les vraies variétés sont rares

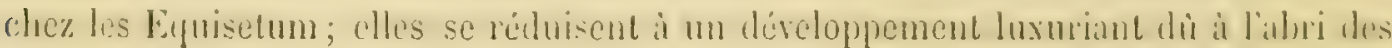

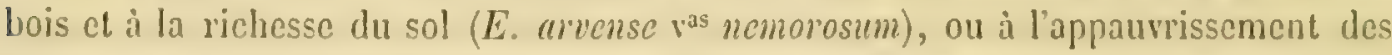
formes dans des terrains maigres durs et baltus (E. arvense vas decumbens etc.). La plupart des autres modifieations, mentionnérs comme variétés ${ }^{1}$, sout si indiviluelles, si accidentelles et si peu permanentes qu'clles n'affectent pas toutes les tiges d'un meme rhizome, et sourent ne se reproduisent pas deux anmées de suite dins le méme lien. Elles rentrent des lor's daus les variations; les mentionner minutieusement el les honorer d'un nom d'auteur serait tomber dans la liute que Linné a signalée en disant: "Conspurcavit magis Botanicen varietatum introductio, quam alia res ulla (Phil. bot., aphor. 259).

Pór rester rigoureusement fidele au principe de priorité, j’ai pris deux précautions: Jo j’ai conservi constamment le nom spécifune princeps; 20 jai fait surre co nom de la description qui l'accompagnait pu imitivenent el qui a servi a ćlablin l'espèce. Il me paraît regrettable qu'on n'ail pas continué à ciler la diagnose princeps

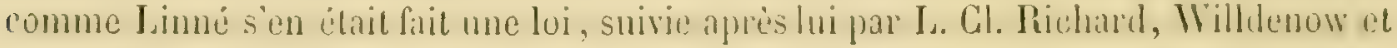
quelques autres. Celte citation permet de reconnaitre la valeur de la premiere description; et elle me paraît un moyen loyal et sûr de conserver à l'auteur princeps tous ses droils de priorite et de propriété, malgré les multiplications de gabres, réclamies quelquefois, il est vrai, par les justes nécessités de la science, mais trop sourent aussi imposées par de fantasques accès de mihimanic.

'Avec l'E. arrense on a fait pyramidale, comosum, elongalum, crespilosum etc. (roy. Pokorny, Eq. arv. "l liycin,, 0.9$)$. 


\title{
GHAPITRE III
}

\section{Clasșification et description}

\author{
$\S \mathcal{L}^{\mathrm{er}}$. Famille
}

EQUISETACEL DG.

Fulcum gen. Linn., Gen. plant., no 1169 , ct $\Lambda$. L. Juss., Gen.plant., p. 17. - Goxoprenides Willd., Grundr. d. Kïüul., el Sp.pl., V, p. xLi, xuv el 1. - Peltata Iloffm., Deulschl. M., II, p. i cl 2. - EQuisetace.e DG., $\boldsymbol{F l}$. frane., II, p. 580.

Diagnosis princeps. - Goxoptendes caule aphyllo articulato vaginato ramis verlicillatis instructo, quorum fructus indusio corniculato inclusi, receptaculis peltatis inserti el in formam spica dispositi. Willd., l. c.

Diagnosis nostra. - Sporic conformes, numerosissimx, spharicæ, elateribus binis spiraliter convolutis, ad apicem spathulatis, valde hygrometricis instructx; ad sporophymata genitales partes (antheridias et archegonia) nec non novos folus gerentia evadentes. Sporangia oblonga, latere interiore longiludinaliter fissa, in orlsem sub clypeolis stipitatis et in amento terminali verticillatim ordinatis disposita. Caulis simplex, nudus aut verticillatim ramosus, et, ut rami, ramuli et rhizoma, articulalus et ad articulos vagina dentata munitus.

Ordo juxta Filices collocandus.

\section{§2. Genre}

EQUISETUM Tourn. Inst. Ed. 3, ‥532. - Linn., Syst.nat., Ed. 1; el omn. auct seף.

Diagnosis princeps. - Spica fructificalionibus peltatis, basi dehiscentibus multivalvi. Linn., Syst. nat., Ed. 6a, no $937^{1}$.

Diugnosis $n^{\mathrm{n}}$ generis ac ordinis eadem.

\section{§. Division et classification des espèces}

Ire SEGTION. Tiges de deux époques et de deux formes: les spicifères plus prẻcoces; les stériles plus tardives, toujours rameuses, à rameaux sans cavité centrale.

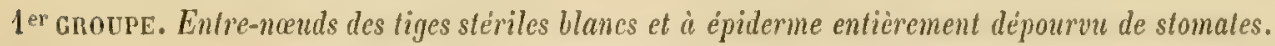

1. Divisions des gaînes et rameaux très-nombreux (vingt-cinq à quarante). Gaîne du premier entre-nœul des rameaux toute membraneuse, tris-longue, en massue.

E. Maxisuม Lam.

\footnotetext{
1 Linné et A. L. de Jussieu citent Tournefort comme auteur du genre Equisetum; mais la diannose de Tournefort est mot à mot copiée de Cesalpino (De plantis, lib. XVI, cap. 15, p. 598), lequel comprend plusieurs genres sous le nom Equisctum.
} 
2e Grourf. Entre-nunds des liges stériles plus on moins verls arec des stomales sur l'épiderme.

2. 'lige spicifire persistant après la sporose et produisant, comme les stëriles, des rameaux nombreus, filiformes, arqués et pendants, charges de ramuscules.

\&. svivitices L.

3. Tige spicifere persistant après la sporose, produisant des rameaux simples.

E. PRATFise Elirh.

4. Tige spicifere jamais verte, périssant après la sporose.

l. ARVENSE. L.

11. SECTYON. Tiges fertiles et stériles de même époque et de méme forme; les rameaux des tiges (quand ils existent) sont pourvus d'uue cavité centrale.

3e Grovre. Épi non apiculé; stomales épars dans les sillons; ostiole des stomates au niveau de l'épiderme.

5. Cavifé centrale égalant environ la moitić du diamètre total; gạines plus longues que. larges, premier entrc-noud des rameaux égalant ou dépassant à pcinc la gaîne caulinaire.

E. Littorale hïlıl.

6. Cavilé centrale très-grande, égalant au moins les trois quarts du diamètre; lacunes corlicales petites et transrersales an rayon manquant souvent; gaines aussi larges gue longues; premier entre-noud des rameaux un peu plus court que la gaine caulinaire.

E. Limosiy L.

7. Cavilẻ centrale petite; lacunes corticales très-rerandes, obosales el rayonnantes; gaînes deux fois plus longues que larges; premicr entrc-neud des rameaux n’alteignart pas ou atteignant à peine la moilie de la gaine caulinaire. E. p.tucstne I.

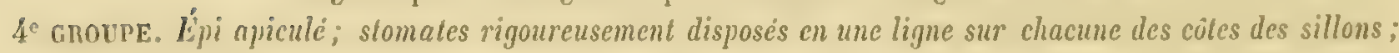
ostiole des stomates an fond d'une cavité ii bords irréguliers.

8. Tiges élevees, plus ou moins rameuses (ou formant des touffes dressies aprìs avoir élé fauchées); carité centrale trẻs-grande; arẻte des côtes non sillonnẻe; gaines longues et larges.

E. ramosissimum Desf.

9. Tiges très-grêles, presque couchées, très-nombreuses et formant gazon; cavité centrale pelite, côtes fortement sillonnćes; gaînes courtes, érasćes, ì dents persistantes.

E. vamegater Schleich.

10. Tigres un peu grêles, rigourcusement nues (ou ramenses aprís mutitation), ascendantes, formant des touffes; gaines appliquées, à dents colorécs et persistantes.

E. TR.ACHหonox Al. Iir.

11. Tiges robustes, très-rudes, raides, droites, isolées, rigourensement nues (saul les cas de mutilation); gaines appliquées, à dents colorées et finalement cadurues.

E. ITEMALE I.

\section{Description des espèces}

\section{Eevisetuy Maxigtex Lam.}

Diagnosis princeps. - Espèce remarquahle par sa grandeur, par la longueur de ses feuilles et par leur grand nombre à chaqque rerticille; ses liges stériles sont droiles, épaisses, garnies de heaucoup d'articulations pen ícarlies les unes des autres ef s'élèrent ì la hauteur de trois pieds; ses feuilles 


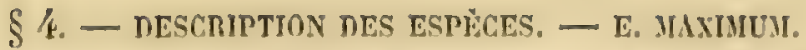

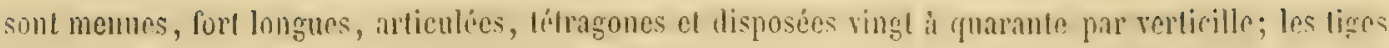
fleuries sont nues, épaisses, lautes d'un pied et naissent au printemps (Lam., Flor. franc., I, p. 7, $1778)^{1}$.

Diagnosis $n^{2}$ - Caulibus dimorphis, erectis ; spicigero nudo, pracoci, cito marcescente; sterili seriore valde ramoso; utroque per internodia nec sulcos, nee viridem colorem, nec stomata proferente.

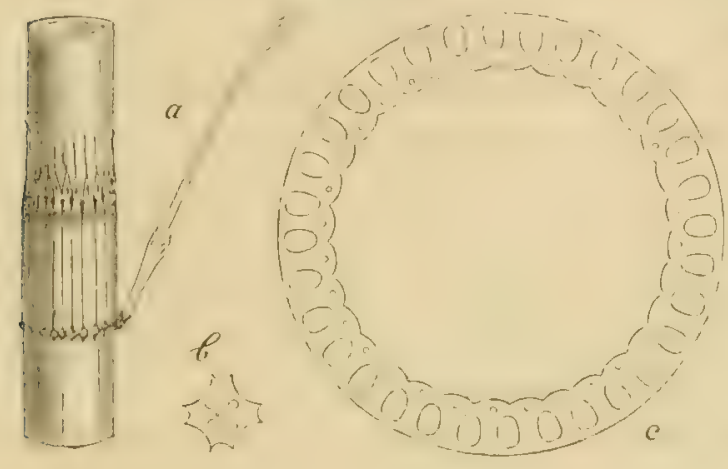

Fistula centrali vastissima $2 / 3 \mathrm{~s}, 4 / 5, c^{2}$. Vaginis in caule spicigero maximis, superne dilatatis, approximatis sxpissimeque internodia contegentihus, in sterili brevioribus valde distantibus, adpressis; in utroque divisionibus numerosis (2540), linearibus, subulatis. Ramis simplicibus, rarissime ramulosis, longis, gracillimis; quatuor sulcis et costis rursum sulcatis, sulcos octo menlientibus, $b$; raginis primi ramorum internodii brevissimi disparibus, amplis, longis, claviformibus, scariosis, membranaceis, ragina caulina brevioribus, $a$.

Description. - Les rhizomes rampent à une profondeur considérable, souvent même de 60 centimètres à 1 mètre, et ne produisent guère alors que des tiges stériles. Leur diamètre est de 5 à 10 millimètres; les entre-nœuds, d'abord d'un roux brun mat, puis plus tard noir's et luisants, sont très-longs, 10 à 22 centimètres, presque cylindriques; sept à neuf sôtes à peine saillantes, et rendues plus indistinctes encore par un tomentum épais qui les recouvre dans le jeune âge. L'épiderme a été décrit p. 18 et figuré pl. II, fig. 1 et 2 . Les gaines offrent des divisions lancéolées, fortes, épaisses, qui ne s’isolent pas, à leur partie supéricure, en dents régulières, mais se séparent presque entièrement sur deux ou trois points en deux ou trois lambeaux. Elles sont d'abord, comme les entre-nceuds, courertes d'un tomentum épais, qui disparait assez vite. Les nombreuses ouvertures que la cliute de ces fibrilles laisse sur les cellules épidermiques en déterminent rapidement la pourriture, et cela particulièrement sur de petites saillies qui se trouvent à la base des divisions des gaines et qui sont par cette destruction changées en petites fossettes (pl. I, fig. 4 bis dl). M. Ad. Brongniart a constaté celte circonstance dans les termes suivants: a Entre les protubérances (bourgeons de racines), et à la base de clacune des divisions

' La notoriété du nom d'Ehrhart nous fait un devoir de donner sa diagnose:

«E. Telmateia. Caulibus inæqualibus teretibus, lævibus, glabris; fructificante simplicissimo albo-rubello, marcescente; sterili ramoso, fistuloso-poroso, alho; ramis sulquadragonis (sic), octosulcatis ; sulcis alternis majoribus; dentibus vaginarum subulato-setaceis, scariosis; vaginularum quaternis, dorso sulcatis " (Ehrh., Ilannœv. Magus., st. 18, P. $287 ; 1783)$.

- Cette fraction indique le rapport de la cavité centrale au diamètre total. 
"de la gaine on observe dans les nouds les plus inférieurs des sorles de fossettes "rlliptignes entomées d'un rebord saillant, dont lit disposition est asse\% cinguliere, "mais dont les usages sont tout ả fait inconnus» (IIist. v'óg. foss., I, p. 100, pl. XI, fig. 10).

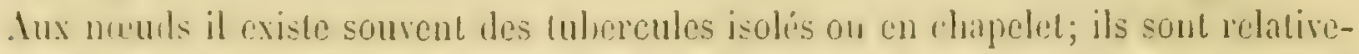
ment fort gros et nont pas moins de 20 millinitres en longueur sur un diandere de 1 ' millimitres (pl. I, firs. fi). Fiabord ovoüles, ils ont ensuite la forme d'une poire; très-atténués à leur extrémité d'adtrérence, très-renflés à l'autre extrémité avec un retrait central rayomant. Lecur surfiee, dabord tomenteuse, deviemt bientit glabre et d'un noir très-foncé. Leur tissu cellulaire est très-résistant, d'une incomparable régularité, mais spongieux et non rempli de granules amylacés comme dans les autres espèces ( $\mathrm{p}$. 位). Ils m’ont constamment présenté des vides très-irréguliers. Cies vides et l'absence de granules amylacés font que, par la dessiccation, ces tubercules si gros et si réguliers se déforment et se réduisent presqque ì rien.

La section transversale d'un entre-nœud ne laisse voir aucune trace de cavilé centrale (pl. V, figg. 10) el seulement sept à neuf lacunes; ce qui cst d'autant plus remarquable sur cette espece, qu'elle est précisément celle dont lis tiges présentent le plus de lacunes (vingt-cinq à quarante), et la plus grande cavité centrale, quatre cinquièmes. La partie souterraine de la tige, qui s'élève des rhizomes horizontaux, se modifie progressivement à mesure qu'elle se rapproche de la surface du sol; une cavité centrale commence i se montrer, le nombre des faees el des lacunes angmente a les entre-neruds deriennent plus nofis et glabres. Les lacmes corticales des thizomes principaux sont prodigieusement grandes, et súparies sculement par trois on quatre rangs de cellules presque aussi litrges que les pelites lacunes (pl. T, fig. 10 ). Ver's le tiers intérieur du rayon, la distinction des deux cylindres est marquéc par une guirlande de cellules plus petiles constituant un polygone à angles trés-inoussés et à faces rentrantes. Le cylindre interne est composé de cellules plus petites; ses lacunes ont de chaque côté un groupe de vaisseaux annulo-spiralés.

Les cellules des rhizomes, comme celles des tubercules, sont déporrvues de gramules anylacés ou n'en offrent que quelques-uns isolís et mal conformés. On remarquera lit coïncidence qui existe entre celte absenee el celle de la chlorophylle sur les entre-nouds des tiges.

Les tiges spicięres sont droites, grosses de 8 à 13 millimètres et hautes de 20 i 50 centimètres, avec des entre-nœuds d'un blane mat, lisses et sans sillons, nombreux, relativement courts et jusqu'après la sporose presque toujour's recouverts par les gaines. Colles-ci sont très-longues, 28 it 45 millimetres, larges el érasées vers le haut; leurs divisions très-nombreuses (vingt-cing ì trente-cinq) el trèsitroites, verditres sur leurs deux tiers inférieurs, sont i leur tiers supérieur colories 
en brun, et, soit isolément, soit encore réunies par deux ou trois, se détachent en lobes linéaires, très-aigus, sétacés, scarieux et fragiles. Sur les entre-nœuds, les cellules de l'épiderme sont toules conformes, sans stomates (pl. III, fig. 5 ). $\Lambda$ l'ćtat frais, les eòtes sont nulles; mais sur les gaines, les sillons très-étroits, profondément et bruscruement creusés a angle droil, ont a l'épiderne des cellules transversales, et les cuites, fortement dessinces, ont des cellules à chlorophylle et des stomates ver's les angles des sillons (pl. III, fig. 11 et 12). Sur le tiers inférieur, la surfice des cótes cst pline; un peu plus haut, elle se creuse en un sillon dorsal qui augmente de profondeur jusque vers la naissance des dents, où il expire.

Avant la sporose, la cavité centrale occupe environ la moitié du diamètre total (pl. VI, fig. 1); plus tard elle s’angmente aux dépens du lissu cellulaire qui la sépare des lacunes essentielles et elle finit par occuper les quatre cinquièmes du diamitre. Elle est souvent, ainsi que les lacunes, presque à moitić remplie d'eau. Lacunes corlicales ovales-rayonnantes; au-dessous de l'épiderme, deux ou trois rangs de cellules plus petites, très-allongées, à parois épaisses; vers le quart du rayon, une ligne ondulée de petites cellules sépare les deux cylindres. Vaisseaux du faiscean fibrovasculaire réduits à un ou deux. Lacunes essenticlles petites, arrondies. Dans les cùtes des gaines court un petit faisceau fibro-vasculaire.

Épi fusiforme, très-allongé, jusqu'à 8 centimètres, et atténué au sommet. Axe creux. Trente à trente-cinq verticilles de sporanges, composés vers le milieu d'une quinzaine de clypéoles. Sacs des sporanges très-allongés.

Tiges stériles droites, grosses de 7 à 12 millimètres, hautes de 50 centimètres à $1^{\mathrm{m}}, 80$ (2 mètres à Alger'); culre-nouds allongés, d'un blanc d'ivoire, les inférieurs tachelés de noir ou même entièrement d'un noir d'ébène, lisses, sans côtes, sans sillons, sans stomates (pl. III, fig. 6). Gaines cylindriques, étroitement appliquées, aussi longues que larges jusqu’à la naissance des dents, dès lor's beaucoup plus courtes que les gaînes des liges spicifères, mais semblables à elles par les côtes, les sillons, les stomates, la chlorophylle, le nombre et la forme des divisions.

Gavilé centrale occupant des deux tiers aux quatre cinquièmes du diamètre total (pl. VI, fig. 2). Lacunes corticales vingt-cinq à quarante, ovales-rayonnantes, également distantes et de l'épiderme el entre elles. Sous l'épiderme, point de cellụles à chlorophylle ni de faisceaux fibreux, mais seulement une couche uniforme et régulière de cinq ou six rangs de cellules allongées, étroites, ì parois assez épaisscs. La guirlande qui court entre les deux cylindres n'est séparéc des grandes lacunes que par un rang de cellules; le faisceau fibro-vasculaire a de chaque còté deux ou trois vaisseaux. $\Lambda$ son pourtour, la cavité centrale est assez régulièrement striée. La coupe d'une gaine offre les mèmes éléments et la même disposition que sur une tige spicifére: la chlorophylle en une bande concentrique et sö́largissant en spatule vers les extrémités qui 
correspondent aux lignes des stumates (pl. VI, figs. 1). Le plus sourent une lacune: tre̊s-petile existe au-dessous du faisceau fibro-vasculaire.

liameaux très-nombreux ${ }^{2}$ dressis on étnlés horizontalement, les infërieurs ruelqueluis arqués, asse\% longs et le plus sourent angmentant de longueur de la base au

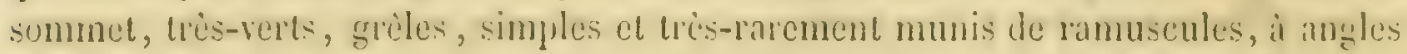
un peu rudes avee de petites asperités diriggés de bas en haut. Gaine basilaire peu oblique, relativement longue, incolore d'abord, puis d'un bean noir, arec le bord brun, it quatre dents larges el courtes; les cellules en sont figntées pl. III, figs. 21. Le premier entre-neend est tres-court et surmontí par une gaine membraneuse, longue de 7 a $\&$ millimètres, dilatie ef fusiforme, ordinairement à cing cùtes trésmarquées et a cinr dents acumines-sétacies et termines par de longues cellules hyalines (pl. YIII, fig. 13). Longtemps encore apres que le jeune ramean est sorti de la tigé, celte première gane est pour lui un organe protecteur et le recoure tout cntier; nulle autre espece ne présente de gaines semblables. Le premier entre-neud et sa gaine n'atteignent pas la naissance des dents de la gaine caulinaire. Les autres entre-nuruds sont tris-longs el leurs gaines ont une forme analogue i la preniere, mais aree de moindres dimensions el quatre dents sculement. Leur section montre qu'ils ont quatre larges sillons, très-rarement cinr, cl que leur's còtes ont clles-mèmes un large et profond sillon sur lour carène (pl. III, fig. 22, et pl. VI, fig. 3), ce qui simule huit sillons et a plus d'une fois induit en erreur. Ce sillon carénal se prolonge jusque sur les dents des gainnes. Chaque còte a un faisceau fibreux, cl suus chaque sillon est une grande lacune corticale. Le reste du cylindre extéricur est occupé par des cellules a chlorophylle. Le cylindre intérieur est nettement marqué par une ligne de cellules, rqui passe tont contre les lacunes corticales et s'arance en angles émoussés au-dessous de chaque cóte. Les quatre lacunes essentielies, avec leurs fuisceaux fibro-vasculaires, ocenpent tout le cylindre interne, qui ne mia jamais présenté de cavilé centrale.

Les deux ou trois derniers entre-nands d'une tige stérile bien complete ont, comme ceux des rameaux, de la chlorophylle et des stomates.

Variutions. - Les diflérences jue les tigges spieifëres présentent frërguenment dans leur taille, dans la longueur ainsi que dans la couleur plus on moins blanehe de leurs entre-nouds, confin dans la dilatation plus un moins considérable de leurs gaines, ne

\footnotetext{
1 La lige stérile que j'arais sous les jeux en faisant celte description, prise comme mojenne sur un trés-grand nombre, avait: de la surface du sol à la pointe de ses rameaux les plus élevés 1 in, 03 de hauteur; une circonférenee de 32 nillimètres, mesuréc à 18 centimètres au-dessus du sol; trente-neuf galines; les six inférieures nues; a la septiẻme commenraient les rameaux, dont le nombre par verticille, comptés en montant, était: 13, 27, 30, 33 à onze gaincs, $29,23,21,18,16,13,14,13,11,9,8,7,6,3$. Les quatre entre-nocuds supérieurs, furmant la pointe, étaient grèles, verts et nus comme un rancau; cn total 632 rameaux; les rancaux les plus linng avaicnt huit ou ncuf entre-nceuds.
} 
\$4. - DEscription DES ESPLCES. - E. MAximuy.

me paraissent meriter ni un nom particulier ni mème une courte description, parce qu'elles manquent de constance, qu'clles se trouvent presque toujours sur un même rhizome avec les formes les plus normales, el qu'elles ne modifient en rien la structure intérieure.

Les tiges stériles ne présentent guère que des dillërences analogues, consistant en ce que les. verticilles de rameaux commencent plus ou moins haut etc.

Je ne vois à noter que la variation suivante:

p. minor Joh. Lange, Pug.pl.hisp., p. 19. - Tiges spicifères beaucoup plus pelites, rappelant, par leur taille et l'éeartement de leurs gaines, les tiges spiciféres un peu fortes de l'E. arucrese. Tiges stériles moitié plus petites et plus grểes qu'à l'ordinaire. Nulle modification dans la structure. Faut-il rapporter à cette forme celles que mentionne M. Milde (Gof. Crypt. Schl., p. 427) : " 3 forma humile, tiges spiciferes», ct p. 429 : « $4 \mathrm{v}^{\text {as }}$ breve, tiges stériles»? Rien n’indique que les deux numéros se rapportent aux deux états d'une même plante.

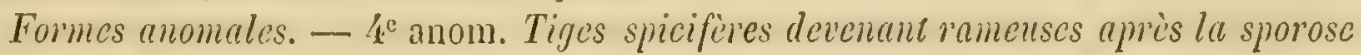
(voir p. 146).

$5^{\mathrm{e}}$ anom. Tiges stériles devcnant spiciferes (voir p. 147). L'épi est alors plus pelit el la tige moins élevée. Ordinairement aussi, mais non toujours, les deux gaines les plus rapprochées de l'épi, au lieu d'ètre étroitement appliquées contre l'entre-nœud, sont évasées, dilatées, plus colorées el sans rameaux. Quelquefois encore de petits épis terminent les rameaux des quatre à six rerticilles supéricurs; les rameaux du verticille spicifère le plus élevé sont très-courts el les épis presque contigus à la tige. Lauğmentation en longueur des autres est progressive. Il n'est pas rare de roir l'épi terminal et les épis des rameaux présenter les interruptions ou les terminaisons signalées aux anomalies 6 et 7 .

$6^{\mathrm{e}}$ anom. Épis interrompus; accompagnant assez, souvent la précédente.

$7^{\mathrm{e}}$ anom. Terminaison irrégulière des épis; mème observation.

$9^{\circ}$ anom. Pluralité des amneaux et anneaux incomplets. Assez fréquente, ainsi que des divisions de gaînes ou d'anneaux passant à l'état de sporanges.

$10^{\mathrm{e}}$ anom. Tubercules (pl. I, fig. 4). Très-beaux, mais très-difficiles à oblenir à cause de la profondeur à larjuelle pénètrent les rhizomes. J'en ai trouré exceptionnellement à 2 centimètres au-dessous de la surface du sol, à la base d'une tige spicifère qui s'élerait d'un rhizome rampant à 15 centimètres. C'est à tort que M. Ramey a cru rue sur cette espèce les tubercules ne se disposent point en chapelet (Tul. Eq.,

p. 210$)$.

$12^{c}$ anom. Gaines en spivale (voir p. 151).

$13^{\mathrm{e}}$ anom. Bifurcution des tiges (roir p. 152).

15e anom. Pluralité d'épis sur un épi mutile (voir p. 152, et pl. VIII, fig. 36). 
Ilabilut. - Celle belle espèce croit dans la zone tempéréc boréale, cn Europe, en Asie, en Amérique et dans l'Afrique française. Elle est répandue en Angleterre, en Écosse et en Irlande; elle manque en Suède, en Norwége et en Laponic. Sa limite boréale parait être le nord de l'Écosse, la partic méridionale du Danemark (Fries,

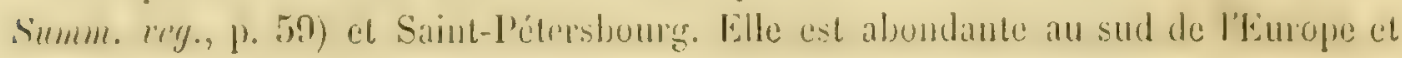
n'est pas rare sur le littoral de l'Nlgérie.

Limiles d'extension (non compris l'Amérique).

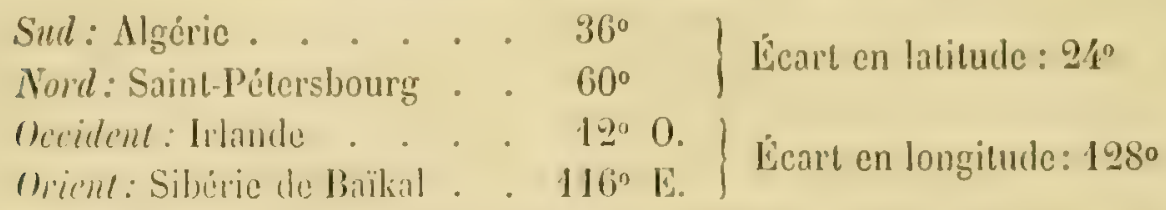

Carré d'expansion, 3072.

Station. - En général les sols argileux et calcaires, pourvu quu'ils soient constamment humides. Sa limite supérienre de végétition est en Suise de 1150 metres (Bernoulli, Gef. Kiypt. Schue. p. 49), de 850 metres pres du Dont-Blane (V. Payot, Catul.

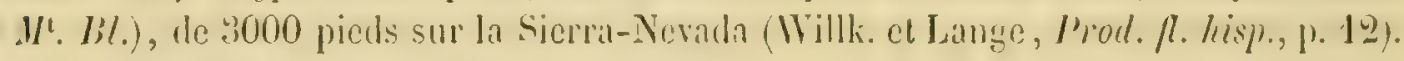

Localités françaises. - Répandu sur tout le territoire. Rare en Alsace; assez abondant en Lorraine; peu commun en Auvergne el dans l'Ouest; assez commun aux - environs de l'aris.

La forme minor est répandue aux environs de Grasse el de Marseille; l'anomalie $n^{\circ} 5$ près de Lisicux, el, en Alsace, près de Lampertsloch et de Lobsann.

Éporne de la sporose. - Les premiers jours du printemps. Les liges stériles pallaisond immédiatement apres les spiciferes; mais les épis anomanx qui terminent quelquefois les tiges stériles et rameuses sont plus tardifs et ne paraissent que vers l'été. Des liges spiciferes propres sortent parfois de terre dans les automnes chauds.

\section{EQUisetur SILVATICUM L.}

Diagnosis princeps. - L. caule spicato, frondibus compositis L. Sp. pl., Eid. 1n, p. 1061.

- Diannosis $n^{\mathrm{a}}$. - Caulibus dimorphis fere coxlaneis; spicigero sporoseos tempore nudo rel rudimentis ramorun brecibus instructo, denique sporis sparsis et spica marcescente ramos, ut sterili, et clam longiores frolerente el astatem perdurante. Stomatibus in lineas subregulares el subbiserialas dispositis. Fistula centrali modica, 1/3, c. Vaginis in caule spicigero maximis, remolis, dilatatis, ventricosis, scariosis; in sterili minoribus; divisionibus ipsarum 10-15 lineari-lancealatis, plerisque per

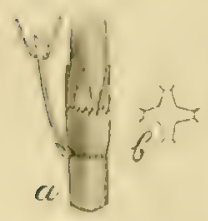
1-5 usque ad imum in 2-1ve lobos obtusos comntis. Kamis sterilium nec non et spicigerorum deuse verticillatis, gracillimis, arcuatonulantibus, rerlicillato-ranulosis, profunde 3 -1-sulcalis; $b$; raginis ipsorum adpressis, dentibus angustis longe acuminatis, fralentibus; primo in sterilibus internodio longo raginan caulinam adaguante vel valde superante, $a$, in spiciferis non adaequante. 
Description. - Les rhizomes principaux sont très-longs et ranpent à une profondeur de vingt-cinq à soixante-dix centimètres; leurs entre-nouds, d'un roux brun mat, deux et trois fois plus gros et plus longs que ceux des rhizomes verticaux qui supporteut les tiges, ont luuit à dix angles très-marqués; les plus élevés sont seuls tomenteux. Gnînes à dents étroites, aiguës, deux fois aussi longues que la partie vaginante.

Tubercules nombreux, sphériques aree un diamètre de 7 millimètres, ou ovoïdes avec un grand diamètre de 13 millimètres. Granules amylacés plus gros que sur aucune autre espèce, avec quelques petites inégalités à la surface. Entre-nouds sans cavité centrale (pl. V, figg. 11); seulement, sur les très-rieux rhizomes, les cellules du centre se déchirent très-irrégulièrement et se pourrissent. Lacunes corticales très-grandes. La guirlande de séparation des deux cylindres est presque aux deux tiers du rayon; elle reproduit assez exactement en petil le polygone extérieur; mais, ce que je n'ai vu que sur celte espèce, à l'intérieur du cercle des lacunes essentielles et séparće d'elles par deux rangs de cellules, existe une autre guirlande de cellules semblables, quelquefois colorées, décrivant un polygone à côtés très-concaves, et dont les angles s'avancent entre les petites lacunes vis-à-vis les faces du premier (pl. V, fig, 11). Les vaisseaux du pourtour des petites lacunes manquent souvent sur les vieux rhizomes.

Tiges spiciféres droites, hautes de 30 i 40 centimètres, aree un diamètre de 4 millimètres environ, persistant après la sporose. Entre-nœuds sept à dix, d’un rose pàle, rayés de vert; côtes lisses sur le dos, finement hérissées de chaqque côté; sillons trèslarges, peu profonds, ayant sur chaque côté une (rarement deux) ligne de stomates (pl. III, fig. 7). Gaines relativement graudes, longues de 10 à 25 millimètres, ventrues; leurs côtes sont très-prononcées, arrondies, sans aucun sillon carénal, mais marquées vers la base d'une ligne blanche répondant au faisceau fibreux, dont le retrait formera sur le sec un sillon carénal très-prononcé. Divisions neuf à onze, linéaires-lancéolées, soudées entre elles jusqu'au sommet et ne se déchirant jamais que sur deux ou trois points en lobes très-larges et obtus (pl. III, fig. 16, 17 et 18).

Sur une section transversale opérée à l'époque de la sporose (pl. VI, fig. 5), les sillons paraissent un peu plus prononcés par l'efiel des petiles aspérités horizontales qui en hérissent le bord. Ils ont environ trois fois la largeur des côtes. Le rayon de la cavité centrale occupe à peu près le tiers intérieur du rayon total, et les lacunes corticales assez grandes en occupent le tiers extérieur. Elles sont presque rondes et égales en largeur aux masses cellulaires qui les séparent. Suus les côtes est un large faisceau fibreux circonscrit par des cellules à chlorophylle, très-chargées de cette substance contre l'épiderme et sous les stomates, de moins en moins en s'avançant vers le centre. Un ou deux rangs de tissu fibreux sous les sillons. Lacunes essentielles à égale 


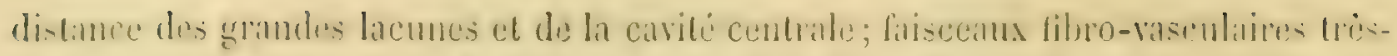

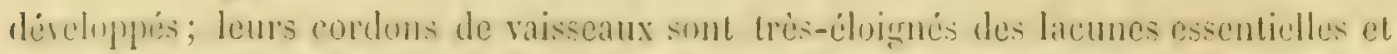

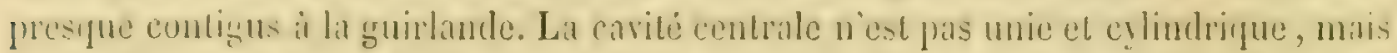
tunte sillonnice, el sa compe reproduit les contours de la seconde guirlande signalede dans le lissu des rhizomes.

Une section de la mime lige trés-développée oflee deux ligires dillérences: 1" he

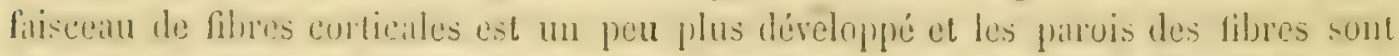

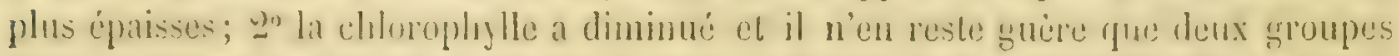
lateranx sons les stomates, li ou les cellules en étaient le plus chargées. Gette section est alor's identique à celle des tiges stériles.

P'ar la section transversale des gaînes on voit que, sur les deux sorles de liges, le dos des cotes cit vecupé par un liasceau fibreux au-dessous dequel sétend une conche de cellules a chlorophylle, yui savance contre l'épiderme vers les lignes des stomates, et s'en écarte ensuile pour pénétrer un peu sous les sillons ( $p l$. VI, fig. 7). La partic inferricure des ganoses est loujours beancoup plus riche en chlorophylle gue les cutre-

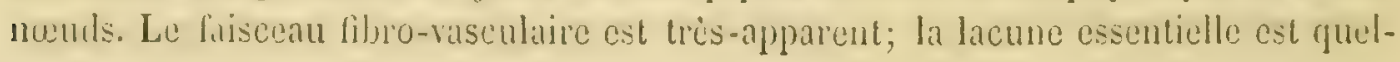
quefois très-réduite.

Dernicr entre-nuend (pédoncule) allongr; annean tres-saillant el contigu i l'épi. Épi linéaire, elliptique, long de 2 à 3 centimètres; axe plein; verticilles quatorze ì dix-huit, ayant vers la moitić douze à quinze clypéoles.

Lors de la sporose, les tiges spiciferres sont rarement tout à fait nues; le plus souvent elles ont i lours nouds supérieurs des rancaux naissants. Sur les tiges steriles, la ramificalion se fant de bas en haul; mais sur les liges spreiferres éest le contraire qui a licu, el les nouds supérieurs sont les premiers à produire des rameaux, dont le développement continue à ètre plus rapide et plus considérable quand noends inférieurs. Il en l'ésulte quapres le développenent complet des liges sturiles, leurs rameaux dicroisent en longueur depuis les verlicilles inférieurs jusquau sommel, yui se lelmine en pointe, et la forme de l'ensemble est plus ou moins pyranidale, tandis yue les tiges spiciferes, ayant en haut leurs rameaux les plus longs, offrent la forme inverse, c'est-ì-dire celle d'un cône renversé. Il semble qu'après la sporose toute la foree vitale s'est portie et condensée dans les rameaux des verticilles supúrieurs, qui deriennent beaucoup p'us longs, plus forts, plus branchus et plus rerts que ceux des

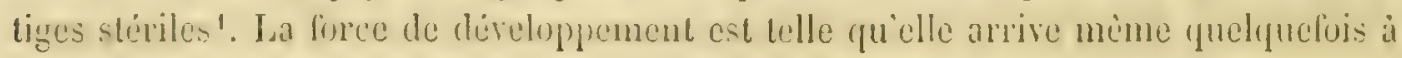

\footnotetext{
'En disant que eles rameaux des tiges stériles sont bien plus allongéz que cous des liges fertiles * (k).d. Fr., III, P. 641), M. Grenier the parait avoir pris pour l’état normal et général un état exceptionnel et abortif que Vaucher avait déja constaté en ces termes: asourcnt sur les tiges fertiles de cette espece, les rameaux des articulations supérieures

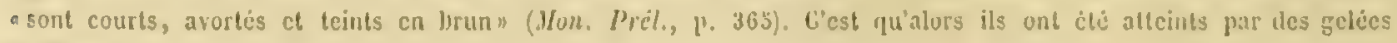
tarelises.
} 
\$ 4 . DESCRIPTTON DES ESPËCES. - E。 SYLVATICUM.

produire un verticille de rameaux sous l'anneau lui-mùme. Mrais néanmoins c'est à tort que .I. Bernoulli a fail de cette apparition anomale un caractere distinctif de cette espece, en disant: "pedunculus post maturitatem elongatus, ramis ex annulo oricna tibus ») (Gef. Krypt. Schw., p. 69). Il est bien viai que le dernier entre-noeud s'allonge après la sporose, mais l'anneau reste contigu à l'épi, même lorsque des rameaux naissent au-dessous de lui, ce qui, je le répète, est une exception rare, du moins en France, une anomalie, et non un caractère constant et spécifique.

Les tiges stériles ne diffërent des tiges spicifëres que par la forme générale ci-dessus mentionnée, par la dimension de leurs gainnes tonjours moitié plus petites, et par des entre-nœuds moins longs, moins gros, mais plus nombreux, douze à dix-huit.

Rameaux nombreux, commençant vers le cinquième nœud, très-grêles, longs, chargés de verticilles de ramuscules, étalés horizontalement ou arqués et pendants; ì quatre (rarement cinq) sillons profonds, à angles à peine un peu rudes. Gaine basilaire d'un brun fauve, allongéc, délicate et presque transparente, à dents très-aiguës; celle des ramuscules est plus petite et plus piile. Le premier entre-nœud dépasse la gaine caulinaire aux verticilles supérieurs; il l'égale aux verticilles inférieurs des tiges stériles; il est moins long qu'elle, et quelquefois même de moitié, sur les liges spicifères. Gaines des entre-nœuds rertes; partie vaginante très-courte, dents très-longues, vertes, étroites, un peu déjetées en dehors.

Une section transversale (pl. VI, fig. 6) montre que les faces des côtes sont trèsconvexes, que leur carène presque plane porte de chaque côté une rangée de petites saillies coniques et contient un faisceau fibreux, au-dessous duquel s'étendent les cellules à chlorophylle. Un autre faisceau fibreux très-petit se trouve au fond du sillon. Nulle trace de lacunes corticales. Le cylindre intéricur est nettement séparé par une guirlande de petites cellules, et montre les pelites lacunes arec un gros faisceau fibro-vasculaire. Point de cavité centrale.

Les ramuscules n'ont que trois côtes; leur organisation est identique.

Variations. - Gette espèce, la plus élégante de toutes, est en même temps la plus constante dans ses formes. Ses variations sc réduisent i ec que les tiges stériles sortent de terre toul à fail nues ou avec quelques rameaux, enfin à des différences de force, suivant qu'elle croît sur un sol gras ou maigre, découvert ou ombragé. C'est à des sujets grêtes, a rameaux très-fins, qu'il fiut rapporter l'E. capillare Ioffm. Ruprecht décrit ainsi cette dernic̀re forme: « forma gracilis, ramis et ramulis tenuioribus, lon"gioribus, non deflexis, sed angulo recto a stipite stricte divaricalis " (Distrib. Cryll. Ross., p. 23).

Formes anomales. $-5^{\mathrm{e}}$ anom. La tige spicifere, au lieu d'apparaitre sans rameaux et sans chlorophylle, sort do terre toute verte, avec des rameaux naissants et avec de petites gaines, conme les tiges stériles; ce qui simule la forme serotinum de l'E. urrense. Rare. 
9c anom. Pluralití des anncaux; anneaux incomplets. Rameaux sous l'anneau.

$10^{\circ}$ anom. Tubercules.

IIabitat. - Cette élégante espèce croît dans la zone tempérée boréale en Europe, en $\Lambda$ sie, en Amérique. Elle s'avance très-loin dans le Nord et jusque dans le Grœuland; au Midi elle parait s'arrêter vers le $40^{\circ}$ degré de latitude.

\section{Limiles d'extension.}

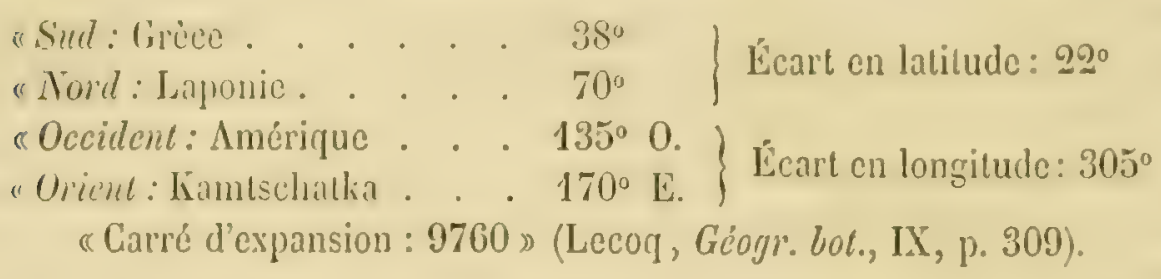

Sutution. - Au bord des bois el des prairies humides des montagnes, particulièrement dans la région des sapins. La limite supéricure de végétation parait être d'en-

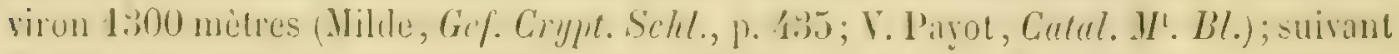
M. Bernoulli elle attcindrail 1650 mètres; 1200 mètres selon M. Lecoq.

Localités francaises. - Bord des bois et des prairies des Vosges (jusque dans la

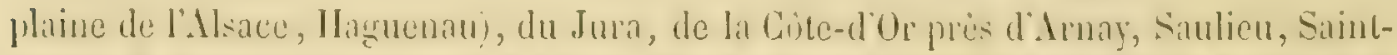

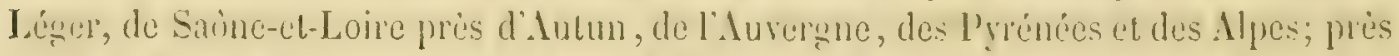
de Vire dans le Calsalos (Soc. Linn., $183($ i); a la lurüt de Lorge dans les Crites-duNord (Lloyd, Fl. Ouest, p. 567).

Énque de le sporose. - Au printemps., mais toujours en retard d'un mois sur celle de l'E. arvense.

\section{EQuisetur pratesse Ehrh.}

Diagnosis princeps. - Caules subrequales ramosi, fistulosi, sulcali, scaberrimi, subrirides. Rami subsenideni simplices quadrisulcali patentissimi steriles. Dentes vag̣inarum subulati scariosi acutissimi, vaginularum qualerni, dorso uninime sulcati. Afline L. arvensi, sed fructificalionem in fronde ramoso habet. Ab E. palustri el limoso malde diversum (Ehrhı, Beilr., 11I, p. 77).

Diagnosis $n^{\text {a }}$. - Caulibus dimorphis, fere cowtaneis; spicigero primum nudo vel rarius rudimenlis ramorum brevibus instructo, denique spica emarcida ramos, ut sterili, sed breviores emittente et wstatem perdurante. Stomatibus in lineas subregulares et subbiseriatas dispositis. Fistula centrali modica siepe parra. Vaginis in raule spicigero magnis, approximatis, apice dilatatis et scariosis, in sterili parvis et minus laxis; divisionibus ipsorun 12-18 lanceolatis, aculis, ad imum liberis. Ramis gracilibus, sxpe detlexis, simplicibus rariuste parce ramulosis, profunde trisulcatis. Vaginis ipsorum dilatatis, dentilus latis, breribus, adpressis; primo internodio brevi raginam caulinam vix adieguante.

Description. - Rhizomes principaux très-longs et rampant à 25 centimètres cuviron de profondeur. Rhizonts rerticaux i cutre-nemds sans tomentum, peu 
allongés, à sept ou huit angles très-marqués. Gaûnes à dents étroites, à peine plus longues que la partie vaginante, convertes d'un cipais tourentum el se détruisant rapidement.

La section transversale montre un épiderme atrès-dur et très-noir, dont les cellules sont petites et a parois épaisses (pl. II, fig. 6).

Tiges spicifères droites, haules de 15 à 20 centimètres, avec un diamètre d'environ 3 millimètres, persistantes. Entre-nœuds neứ à onze', d'un rose terne, faiblement rayés de blanc et de vert, peu profondément sillonnés. Côtes larges, doucement arrondies, sans sillon carénal, finement hérissées sur les angles (pl. III, fig. 8). Gaînes de longueur très-variable (5 à 13 millimètres), dilatées en entonnoir, ì côtes très-prononcécs, très-vertes, sans sillon carénal, si ce n'est sur le sec. Divisions douze à dix-lıuit, lancéolées, toutes séparées en dents raides, entièrement scarieuses el sphacélées, bordées d'une large membrane blanche arec une ligne plus foncée an milieu. Après dessiccation, les còtes de la partie vaginante portent un sillon carénal prononcé.

Sur une section transversale (pl. VI, figg. 7 bis), opéréo à l'époque de la sporose, on distingue aux bords des côtes de très-petiles aspérités. Le rayon de la cavité centrale occupe un peu moins du tier's du rayon entier et les lacunes corticales en occupent un autre tiers. Elles sont plutôt ovales-transversales que rondes, et dès lors plus larges que les masses cellulaires qui les séparent. Sous les côtes existe un très-petil faisceau fibreux circonscrit par les cellules ì chlorophylle. Celles-ci sont i peine vertes et s'avancent un peu vers le milieu du sillon, ct d'autre part assez profondément entre les grandes lacunes. Il n'y a que quelques fibres vers le milieu des sillons. Lacunes essentielles très-marquées, presque transversales, à mi-distance de la cavité centrale et des grandes lacuncs. A prìs la sporose, les faisceaux fibreux des còtes deviennent un peu plus forts; les sillons sont micux marqués; da couleur des cellules à chlorophylle est plus intense et s'avance encore davantage vers le centre. La partic vaginante des gaines est beaucoup plus riche en chlorophylle que les entre-nœuds, ainsi que cela se voit sur l'E. sylvaticum.

"Pédoncule» allongé. Épi linéaire elliptique, long de 1 à 2 centimẻtres et souvent plus court; axe plein; verticilles dix à quatorze, ayjant vers le milicu dix à douze clypéoles.

Lors de la sporose, les tiges spicifères se présentent sous trois aspects distincts: on tont à fait nues avet de grandes gaines (pl. IX, fig. $29 a$ ), ou avec des rudiments de rameaux, ou avec des rameaux assez développés et des gaines plus petites mais toute-

\footnotetext{
1 Il y a des tiges spicifères qui ont un plus grand nombre d'entre-nouds et des gânes moius évasées, mais alors leurs épis sont excessivement petits, et le nombre de leurs verticilles de sporangessemble s'ítre réduit en raison directe de l'augmentation de celui des entrc-nocuds
} 


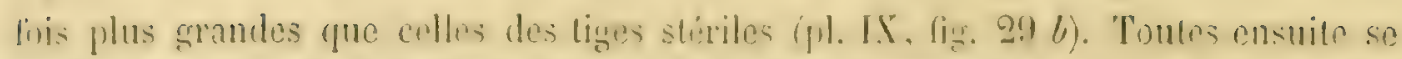

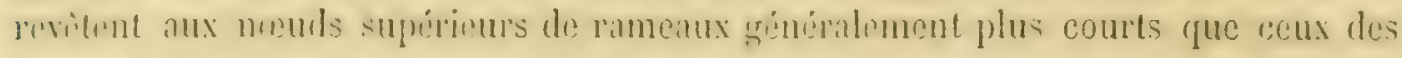
liges stériles; ils manquent aux trois ou quatre nouds inféricurs.

Tiges stiriles paraissant presque en mime tempes que les spicilieres, alteignant do Is it 40 centimetres de hautrur. I,es gaines sont moilie plus potites que sur les tigus

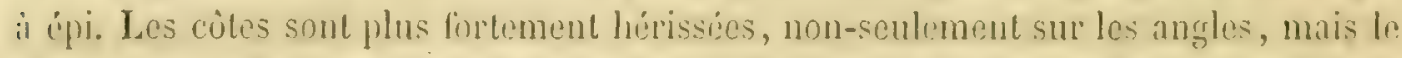
plus souvent jusque sur leur ligne dorsale. Rameaux grêles, mediocrement longs, simples ou rarement avec quelques ramuscules, étales horizontalement ou un peu arques en dehors, a trois (rarement quatre) sillons profouds, i angles a peine rudes. Gaine basilaire paile. Premier entre-nend plus court que la gante caulinairu sur les liges spicileres, el l'egalant à peu près sur les tiges stériles. Gátues les entre-norude vertes; partie vaginante très-dilatée, trois fois aussi longue que les dents larges, courtes, vertes, ave une grosse cote et une petite bordure membraneuso blanchr, non déjetées en dehors el même serrées contre l'entre-nocud. Ces gaînes par leur forme séloignent de toutes les autres et suffiraicut i elles seules pour firire reconnaître l'espèce.

Tariations et anomalies. - Les anomalies précidemment cilies, p. 149-152, ont été étudiẻes sur des spécimens provenant d'Angleterre ou de Prusse.

IInbitat. - Cet Eyuisetum parait essentiellement boréal; on en a constalé la prisence dans le Groulind, l’Imande, l’Écosse, la Laponic, la Russic, la Suede, la Norwerge, le Danemark (Fries, Summ., p. 59), la Prusse, les Ilpes de Suisse (Bern., G'f. Krypt. Schw., p. 70).

\section{Limites d'extension.}

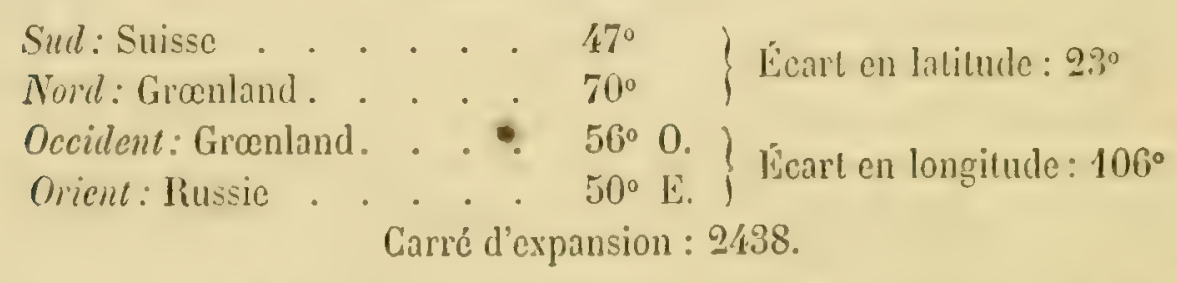

Station. - Au bord des bois, des prairies et quelquefois des champs liumides.

Locrelités franeruises. - Celte plante na point été mentionnée avec certitude sur le Lerritoire francais. De Lapeyrouse cita IF. umbrosum Willd. comme crobsant dans les Pyrénées (IIist. pl. Pyr., p. 619); II. Duby répéta celte mention sur l'autorité de

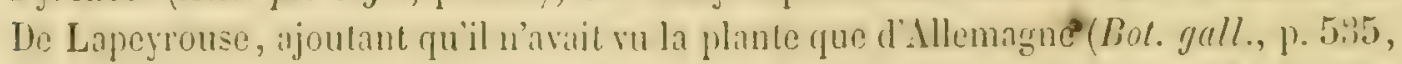
$n^{0}$ 4). Loiseleur-Deslongchamps ne la mentionna point, el avec raison, cal M. I). Clos a établi que les échantillons de l'herrice de De Lapeyrouse étiquetes E. umbrosum

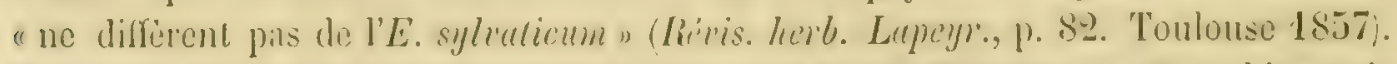
Mutel cite celle plante comme récolté par lui au bois de l'rémol prés firenoble; nais 
une note ajoutée dans son S'uppl. final, p. 180, inspire des doutes tels que MII. Girenice et Godron ont exclu cet Equisetum du nombre des plantes françaises $(F l$. d. Fr., III, p. 646).

II. V. Payot l'indique dans les "vallées de Chamounix, de Ferret et d'AlléeBlanche» (Cut. $21 \%$. Blune, p. 51); mais ce que ce naturaliste m'a enroyé sur ce nom est de l'E. palustre.

Époque de la sporose. - «Fructifie en été» (V. Payot, o. c.). Le même anteur dit de l'E. sylraticum qu'il "fructifie en arril et mai.) M. Newman dit qu'en Angleterre la sporose de l'E. pratense a lieu en avril (Brit. ferns, p. 63). M. Milde, signalant cette espèce comme abondante sur les rives de l'Oder et aux environs de Breslau, dit. aussi qu'elle afructific on même tomps que l'E. arvense, tandis que l'E. sylvuticum ne commence à fructifier qu'après que l'E. arvense a cessé de le faire) (Uelier d. Eq. Mctab., p. 202; Gef. Crypt. Schl., p. 439). Lassertion de II. V. Payot est loin de concorder aree ces indications précises; mais elle coïneide si bien avec l'époque de la sporose de l'E. palustre qu'elle ferait cesser tous mes doutes, s'il m'en restail encore sur la plante que ce naturaliste a nommée $E$. umbrosum.

Observation. - Cet Equisetum est, avec l'E. littorale, un de ceux qui fournissent le plus de sujets affectés d'anomalies et de petites irrégularités. Ét de même que l'E. littorale est intermédiaire entre les $E$. arvense el limosum, l'E. pratense par les deux aspects de ses tiges spicifères, par ses ramcaux simples ou branchus, par son élégance mème, est intermédiaire entre les E. sylvaticum ct arvense, mais toutcfois avec des formes plus constantes que l'E. littorale. J'en donne une figure (pl. IX, fig. 29) afin de prévenir de nouvelles erreurs sur l'indication de cette plante en France.

\section{EQuisetuir ARVENSE L.}

Diagnosis princeps. - E. scapo fructificante nudo, sterili frondosö L. Sp. pl., Ed. 1a, p. 1061.

Diagnosis $n^{\mathrm{a}}$. - Caulibus dimorphis; spicigero precoci, ciloque marcescente, erecto, nudo, fere asulco, nec viridem colorem, nec stomala per internodia proferente; sterili crecto vel decumbente, 6-12-sulcalo, ramoso, viridi, stomatum triplicem quadruplicemve seriem per utrumque sulcorum latus gerente. Fistula centrali parvat $1 / 3, c$. Vaginis in caule spicigero magnis, laxis, ventricosis, remotis; divisionibus ipsorum. 8-12 sxpe per duas aut tres usque ad imum
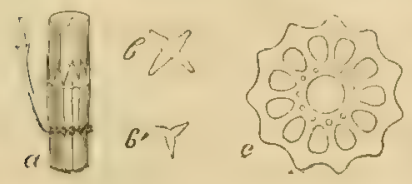
connatis; in sterili brevioribus et magis adpressis; divisionibus 9-12, lanceolatis, liberis. Ramis simplicibus aut ramulosis, valde et profunde guadrisulcatis, $b$; primo ipsorum internodio vaginam caulinam valde ct siepe duplo superante, a.

Description. - Rhizomes horizontaux rampant à une profondeur do 25 centimètres it $1^{\mathrm{m}}, 30$, d'un roux brun mat; entre-nouds très-longs (12 centimètres) et plus gros que ceux des rhizomes verticaux (pl. I, fig. 2); sept ì dix faces presque planes arec 
des angles tris-imoussis, i peine saillants; tomentum tress-abondaut sur les jetines

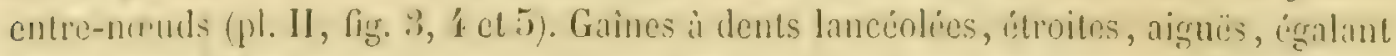
it peu pris la longueur de la partie vaginante, laguelte est couserte de tomentum. Tubercules asse\% fréguents, ovö̈des, phus ou moins allongés, petit liamitre 5 i 7 millimetres, grand diametre 10 a 15 millimètres; cellules épidermiques très-dures et tris-noires, présentant souvent vers leur milieu, apris la clute des fibrilles, des espaces transparents, i contour déchiré et lacinié, au travers desquels on distingue des cellules plus longues et ponctuées.

Cavité centrale nulle; cylindres partageant ígalement le rayon ; lacunes corticales éloignies du pourtour, médiocrement grandes, arrondies, lacunes essentielles relatirement grandes (pl. V, fig. 12).

Tiges spiciferes dressies, hautes de 15 ì 25 centimetres, d'un diametre de 3 i 5 millimètres, tris-caduques après la sporose; entre-nreuds cinq à luit, les inférieurs sourent très-courts et bruns; les supérieurs allongés, d'un rougo de chair, trèsmous et très-aqueux, sourent mème la cavité contrale et les lacunes sont remplies d'eau sur un quart ou sur un tiers de leur longueur; six a nouf côtes très-obtuses, it peine sensibles. Gaines augmentant en longueur de la base (8 millimetres) au sommet de la tige (20 millimetres), larges et lìches, renflies vers leur milien en forme de tonneau, vertes à leur liers inféricur, brunes au-dessus; sillons larges et profonds; còtes arrondies, lisses; divisions lancéolées-linéaires, aiguës, se séparant en dents isolies plus courtes que la partic vaginante, ou rarement soudées par deux ou trois, marquées aprìs dessiccation d'un sillon carinal. Gavité centrale petite, trisprofondément sillonnée, avec angles saillants répondant au milieu des faces ou des lacunes corticales, et en outre toute tapissic des lambeaux du tissu cellulaire qui s’est déchiré pendant la croissance (pl. VI, lig. 8). Lacunes corticales éloignées du bord, obovales, moins larges que les masses cellulaires qui les sćparent; point de fibres corticales. Lacunes essentielles très-petites, éloignées des petites cellules qui séparent les cylindres vers le milicu du rayon; faisceaux fibro-rasculaires très-gros, cordons de vaisseaux très-écartés. Une section sur le tiers infërieur de la gaine laisse voir au milieu des cótes un faisceau de fibres corticales plat et large, au-dessous de lui une large couche de cellules i chlorophylle el un firible faisceau fibro-vasculaire contre la lacune essentielle très-petite (pl. VI, fig. 11).

Dernier entre-numd (pedoncule) allongé, grèle; annean très-saillant. Épi ovölde oblong on lancéolé, long de 15 i 35 millimètres; axe plein; onze à dix-huit verticilles, avec onze à treize clypéoles vers le milieu de l'épi.

Les tiges stiriles sont très-variables, comme celia doit être de toute plante ubiquiste: droites, ascendantes, décombante: ou étalées en éventail, élancées, élevées et sans rameaux à la base, ou courtes et émettant aux cntre-nouds inféricurs des 
§ 4. - Description des ESpèCes. - E. ARvense.

rameaux branchus plus longs qu'elles-mèmes. Entre-ncuds courts et verts ou trèslongs et blanchâtres, diamètre do 2 a 6 millimètres; neuf à douze còtes saillantes arrondies; sillons profonds, ayant de chaqque côté quatre rangs de cellules i stomates. Gaînes longues de 7 à 8 millimètres, peu dilatées, à côtes et à sillons très-prononcés; divisions lancéolées, aiguës, se séparant en dents isolées plus courtes que la partic vaginante. Cavité centrale petite, égalant à peu près le tiers du diamètre total (pl. VI, fig. 9); lacunes corticales obovales, éloignées du pourtour extérieur; leur grand axe est égal au rayon de la cavité centrale; elles sont plus larges que les masses cellulaires qui les séparent. Le faisceau fibreux cortical des côtes est très-gros el circonscrit vers l'intérieur par unc bande scmi-circulaire de cellules à chlorophylle. Au milieu du sillon, un petit faisceau fibreux s'interpose entre les bandes de collules à chlorophylle. Les deux cylindres sont sćparés vers le milieu du rayon total par une guirlande toujours très-distincte et fortement ondulée. Lacunes essentielles assez grandes; faisceaux fibro-vasculaires très-peu larges; cordons de vaisseaux rapprochés.

Rameaux simples ou quelquefois portant des ramuscules; commençant dès le bas de la tige ou seulement au-dessus du tiers inférieur; dressés ou étalés, quelquefois même un peu arqués et pendants; les inféricurs toujours les plus longs. Gaine basilaire courte, d'un brun mat plus ou moins foncé. Le premier entre-nœud est, vers le bas de la tige, au moins égal à la gaine caulinaire; vers le milieu il est plus long qu'elle et sourent même du double. Les autres entre-nœuds sont plus longs que le premier, et, comme lui , à quatre, rarement cinq côtes très-prononcées, comprimées latéralement et très-étroites; sillons très-larges avec deux rangs de stomates de chaque còté. Gaînes peu dilatées; la partic engaînante, un peu membraneuse au fond des sinus, est à peu près égale aux dents étroites, aiguës, déjetées en dehors.

Par suite de l'étroitesse des côtes et de la largeur des sillons, la coupe transversale d'un rameau donne une croix à longs bras, qui est un des caractères saillants de celte espèce (pl. VI, fig. 10). Un faisceau fibrcux cortical occupe l'extrémité des còtes, dont le reste est rempli de cellules à chlorophylle. Un très-petit faisceau fibreux se voit au fond du sillon. Le cylindre interne est très-distinct; il présente ses faisceaux fibro-vasculaires et ses lacunes essentielles, mais point de cavité centrale.

Variations et v'ariélés. - Les variations el les variétés que présente celle espèce paraissent dépendre entièrement des circonstances locales.

La tige spicifẻre, constante dans sa structure intérieure, modifie un peu sa taille et sa grosseur selon les licux. Dans les sables de IIagnenau (Bas-Rhin), j’en ai trouvé quelques-unes dont les entre-nœuds inférieurs ne s'élaient pas allongés, et alor's l'entre-nœud supérieur ou "pédoncule» était à lui seul long de 6 à 9 centimètres,

La lige stérile, toujours identique à elle-même dans son organisation anatomique, offre les trois variétés suivantes: 
P nemorosum Al. Braun in Döll (Rhcin. Fl. p. 27, el Fl. Bad., I, p. 58). T'iges stériles

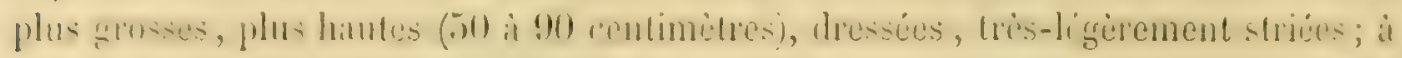

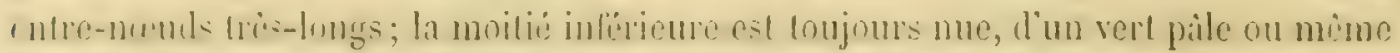
binche; les rameanx simples on quelquefois rantifies sont tris-longs, étolés ou un peu courbés en arc. Souvent les côtes des entre-nœuds inférieurs de la tige ont un sillon carénal très-marqué; celles des entre-nœuds du milieu n'ont qu'une strie légère, cl celles des entre-nocuds supérjeurs ont leur carène tout unie.

Colte varieté (je dirais plus volontiers cette firme) se prosente exclusivement dans

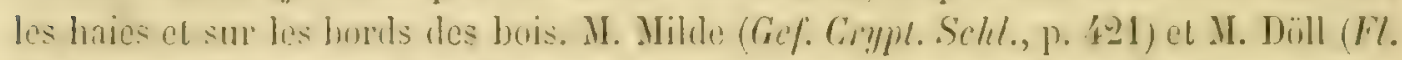
Bed., I, p. 59) avaient dit qu’elle ne produit jamais de tiges spiciferes. Il est raia qu' ell. en produit rarement; mais jen ai trouvé daus la furcit de IJaguenau; clles sont plus Inngues et plus grèles que les liges spicifures ordinaires. J'en ai adressé, en 1861, it M. Milde, qui a bien voulu Jes mentionner (Syst. Ey., p. 14:3). Dans un hosquet a Geispolsheim (Bas-Rhin), ou elle avait une taille el une beauté remarquables, je la récoltai trois années de suite toujours stérile. On coupa les arbustes; l'amée d'après elle était encore stérile, mais très-ríduite, et la seconde année je la trouvai au mème lieu arec equelgues tiges spicifires, un peu plus longues que celles des champs voisins.

i' decumbens Mcyer (Chlor. han., p. 666). Tigres presque entierement couchécs dans leur moitié inférieure, très-rameuses dès la base; les rameaux inféricurs sont presque aussi forts que la tige elle-mème, souvent plus longs et très-ramifics. Ordinairement les rameaux se déjettent de charpue còté de la tige et presque en éventail.

Celte forme, comme la précédente, offre rarcment des tiges spiciféres propres; e'est clle surtout qui porte des épis sur' ses tiges vertes et ramifiées ( $5^{e}$ anom.). Elle se trouve dans les terrains maigres et secs, sur les bords dénudés el durcis des chemins; sourent elle résulte du déreloppement d'un rameau de la base, après mutilition de la lige principale.

d. alpestre Wahlen]. (F/. lapp., p. 296). Tiges stériles décombantes, courtes; mmeaux peu nombreux, courts, rejetés sur les cotés ou seulement d'un cité. M. Ruprecht ajoute: "Rhizoma tubcribus onustum superficialibus, facile effodicndis" (Distrib. Crypt. Ross., p. 20).

Formes anomales. $-4^{\circ}$ anom. Frondescens. Tige spicifere devenan raneuse vers

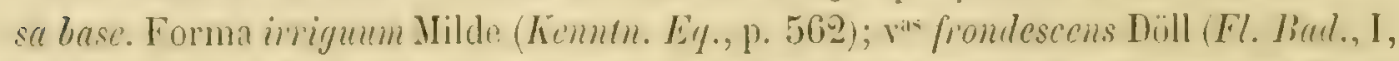

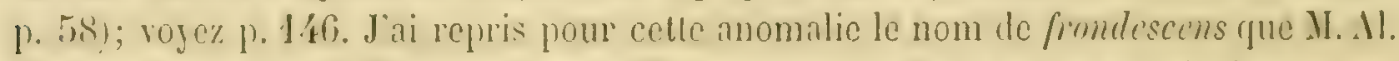

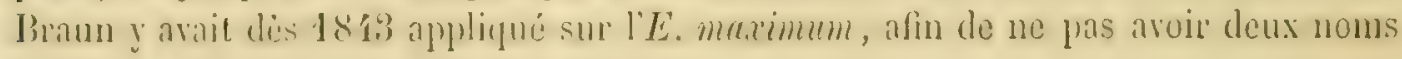
différents pour désigner la même anonalie.

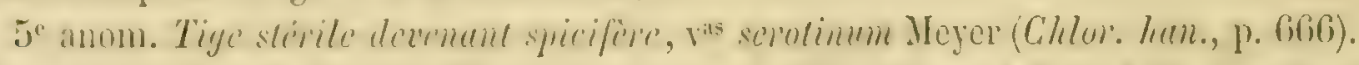

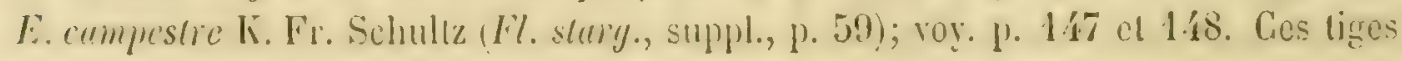


§. - DESGRIPTiON DES ESPËGES. - E. ARTENSE.

ramifiécs et spiciferes ont les deux ou trois gaines supéricures très-dilatées et un pou plus longues qu’à l'ordinaire, avec les cntre-nouds correspondants sans matière verte comme ceux des tiges spiciféres propres. Ces mêmes liges sont ou riches en rameaux ou plus rarement presque nues.

$9^{\circ}$ anom. Pluralité des ameaux; anneaux incomplets (pl. X, fig. 14).

$10^{\circ}$ anom. Tubercules. Dans nos contrées on en rencontre sur presque tous les rhizomes, quand on creuse assez profondément, mais toujours en petite quantité. Its paraissent plus abondants dans quelques contrées du Nord, car IIelwing dit quiils sont fort recherchés par les pores et les porchers (Flor. quasimod. Pruss., p. 31); voy. supra, p. 6 et 7. Smelowsky a proposé de les employer à la nourriture des porcs (.Mém. Acall. Pétersh., vol. I); cl I. Ruprecht mentionne que dans le nord de la Russie les tubercules se montrent sur les $v^{e ́ s} \beta$. borcalis et $\gamma$. alpestris en telle abondance et à si peu de profondeur qu'il pouvait à son grand étonnement les arracher avec les mains, «ipsis manibus cum surculo evulsa me in admirationem converterunt» (Distr. Crypt. Ross., p. 21).

En juillet 1861, i Bouxwiller (Bas-Rhin), je suivis les travaux d'une tranchée ouverte dans une butte d'argile compacte, certainement remuée pour' la première fois; l'E. areense en couvrait la surface de ses tiges, et ses rhizomes pénétraient la masse dans Lous les sens. Je ne vis aucun rhizome à direction franchement horizontale à une profondeur moindre de 35 centimètres; la plupart rampaient de 70 à 80 centimètres; quelques-uns seulement a $1 \mathrm{~m}, 30$. A celte profondeur une scule plante, Convolvulus arvensis L., disputait aux Equisetum la jouissance de ce terrain compact. Ces rhizomes étaient abondamment munis de tubercules offrant toutes les formes et toutes les combinaisons possiblos: gros, petits, sphériques, ovoïdes, déprimés, comprimés, en disque, en cocur, en verticilles simples, en chapelet, occupant la place des entrenœuds a la base, au milieu, vers le haut des rhizomes secondaires ou sur la longueur des rhizomes principaux. Il y en avail beaucoup de ces derniers qui avaient commencé à se développer dans une fissure et n'avaient pu continuer qu'en s'aplatissant, et alors ils remplacaient les entre-nœuds d'un rhizome par des chapelets plats de l'aspect le plus étrange.

$11^{\mathrm{e}}$ anom. Gaînes en crête.

$12^{\mathrm{e}}$ anom. Gaînes cn spirale.

$13^{\mathrm{e}}$ anom. Bifurcation des tiges.

$16^{\mathrm{e}}$ anom. Gracilité. Dans la forêt de IIaguenau et près de Strasbourg jả trouvé des liges spicifères robustes émettant de leur noud inférieur une petite tige grêle et filiforme, molle, aqueuse et colorée comme les tiges spicifères. Les deux ou trois cntre-nœuds inféricurs, développés et longs de 2 à 3 centimètres, supportaient une masse orö̈de pointue. En l'ourrant on ne troure qu'une masse de gaines on très- 
rarement au centro un épi rudimentaire, avec deux ou trois verticilles de sporanges

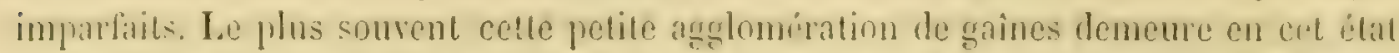

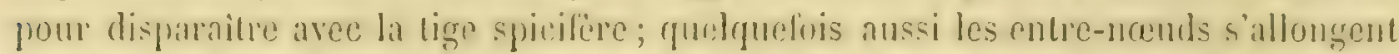
el lon voit une lige filiforme de 10 a 12 ecntimetres. Je n'ai jamais un le rudiment d'épi se développer et sortir des gaînes.

$17^{\mathrm{e}}$ anom. Infécondité des spores. Très-rare.

Ilubitut. - Cette espice croit dans loute la zone tempiréc horiale en Europr, en Asie, en Imérique. Elle s'avance au Nord jusque dans le Grumland, mas an Surl relle. ne parait pas sétendre beauconp au deli du $35^{\mathrm{e}}$ degré de latilude. Je ne l'ai pas vue en $\Lambda$ lgéric.

\section{Limiles d'extension.}

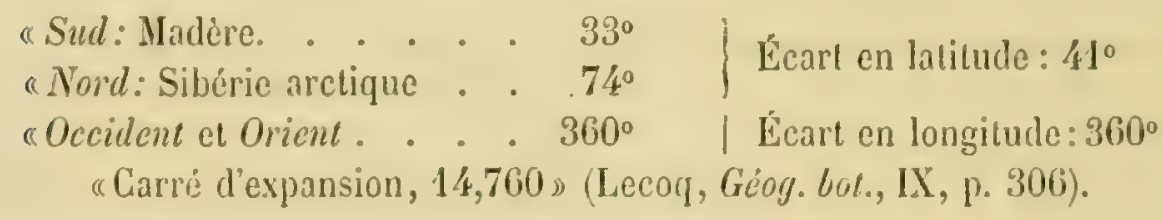

Station. - Champs cultivés ou incultes, bord des bois, prairies, marais, toulbieres, terrains caillonteux, sablonneux, siliceux, argileux ou calcaires, sables salins des embouchures du Rhòne, tout paraît bon à cette espece, qui s'élève jusqu'i 1300 mètres d'altitude, oủ elle devient rabougrie, $\delta$. alpestre.

Localités francaises. - Sur toute l'étendue du territoire. J'ai trouvé les anomalies $11^{\circ} 4$, frondescens, et no 5, serotinum, près de Schlestadt (Bas-Bhin), el près de Stratsbourg, dans l'Ile des Lipis. L'anomalie frondescens n’était pas rare dans cette demière. localité au printemps de 1861, après l'inondation de décembre 1860.

Éporne de la sporose. - Premiers jours du printemps. L'anomalie à liges rameuses devenant spicilères ne produit ses épis quen fum ma ou aux premiers jours de juin.

Comme les épis de celle espece sont déjĭ complétement formés au mois d’aont, it s'en montre quelquetois en automne si la température se mainticnt un pen devie.

Ubservation. - J'ai très-fréquemment observé sur cette espece le fait suirant. Les cntre-nauds sont sur toute leur longucur parfaitement réguliers et normaux, avec leur cavité centrale, leurs cylindres et leurs deux cercles de lacunes, mais vers le bas de ces entre-nouds, exactement au point qui correspond a la naissance des dents de lit gaîne, il y a un renflement notable, simulant presque un sccond noud. Lai cavilé centrale diminue; les lacunes du cylindre externe disparaissent, mais les lacunes cssentielles persistent. De ce point au diaphuragme du véritable noud, lépiderme cst d'aburd tris-blane ct plus tard roux brum. Cette particularite est tres-prononcée sur les tiges ascendantes; daus ce cas, les courbures n'ont point lieu sur la longueur des entreneuts, qui sont toujours tres-droits, ni aux nouds vis-il-vis du diaphragme, car 
eharge gaine continue exactenent la direction de l'entre-noud auquel elle est superposée, et la portion qu'elle enveloppe suit la même direction. Mais c'est au point signalé, i ce renflement ver's la naissance des dents, yu’a lieu la courbure. Je rappellerai que le développenent des entre-nouds a lieu a leur base et non a leur sommet, comme il a été dit p. 61 , et que dés lors la courbure doit naturellement se faire lá où les tissus plus tendres offrenl le moins de résistance.

Celte particularité peut se constater aussi sur quelques tiges couchées des E. maximum, sylvaticum, trachyodom ele., mais elle y est moins prononcée que sur l'E. areense; c'est pourquoi je ne la mentionne qu'ici.

\section{Equis fitui littorale Kühl.}

Diagnosis (scu potius descriptio) princeps. - In litore arenoso marino pr. Oranienbaum in societate Lathyri maritimi, Elymi et c. detexit et sub nomine all. communicavit cl. Kühlevein. Non sine studiis antecessis Equisetum hocce eximium pro planta sui juris commendare ausus sum; videmus quasi hiatum inter formas cognitas quasdam $\mathrm{E}$. arrensis ef E. fluviatile eo expleri et forsan evolutionem summam seriei parum adhuc notx, cujus exordium in E. arclico quxrendum. IIabitus formarum gracilium E. fluviatilis; sed ragin caulinx indolis alienissimx : dentes more E. arvensis sterilis latiores, albomarginati, plerumque per paria connati el c.; etiam spice longius pedunculati, brevissime ovatie, peltis senilibus densioribus parum hiantibus; ramuli nunquam pentagoni. Inter varietales E. arvensis cum nostro forsan comparari potest E. campestre ob surculos fertiles verticillato-ramosos, sed preter alias notas spica crassa el precipue vaginis atris longis, infundibuliformibus, inflatis discedit. Ex indole vaginarum juxta E. arcticum ponendum esset, sed reliqui caracteres abludunt. Surculi fertiles numerosi cxspitem constituentes pl. m. pedem longi sunt, $1 / 2-11 / 4$ linea crassi, erecti, ramis plerumque numerosis verticillatis quadrangularibus, strictis, erecto-patentibus, salis tenuibus vestiti. Nodi infra spicam 2-5 nudi; spica gracillima, 1-4 lin. longa, ad summum 1 1/2 lin. lata, primo sulssessilis, dein pedunculo 1/2-pollicari fulta. Initio Julii m. optimum, serius spica facile caduca, quo statu cum surculo sterili E. arvensis cujusdam stricti commutari posset (Ruprecht, Beitr. zur I'flanzenlunde d. mussischen Reichs, IV, P. 91, 1845)".

Diagnosis $n^{\mathrm{a}}$. - Spica obtusa, ovoidea, pedunculo normali elongalo. Caulibus superne et inferne (raro prorsus) nudis, in medio ramosis, leviter sulcatis, fistula centrali magna, 2/3-3/4; lacunis exterioribus 10-15 uratis. Vaginis infundibuliformibus, laxis, longioribus quam latioribus, superioribus campanulatis; divisionibus lanceolatis. Ramis erectis, parce elongatis, 4-sulcatis; primo ipsorun internodio raginam caulinam rix adequante, raro superante.

Description. - Rhizomes principaux rampant à 2 ou 3 décimètres au-dessous de la surface du sol ; extrèmement longs et formant un vrai réseau par leurs nombreuses divisions. Les entre-nœuds, d'un diamètre de 5 millimètres environ, el d'une longueur

\footnotetext{
- La première description donnćc par Lasch un an après it la mème plante, sous le nom d'L. inundafum, est plus répandue; on en trouve la traduction dans le Bull. Soc. bot. Fr., 1859, vol. VI, p. 602.
} 
de 3 i f centimetres, nont ni crites ni sillons distincts, mais huit ou neul' saillies longritudimales peu marquées, larges et arrondics. Lëpiderme est d'un brun chatain ou

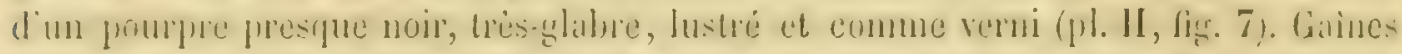
sins tomentum. Tuberentes liréquents, sunsent peu profonds, et disposés en chapelet far cing ou six; mime épiderme quiax entre-noruds, sculement un peu plus noir encore.

Sur une section transversale, la cavité centrate occupe à pen près le quart du dia-

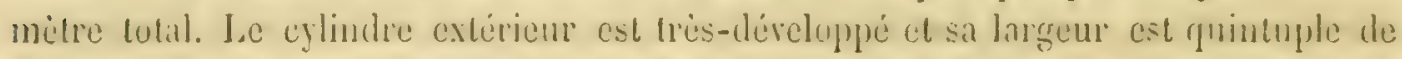
cului du cylindre intérieur (pl. V, figr. 13). Ses lacunes obovales sont plus grandes que la cavilé centrale, et comme elles ne sont síparies que par un tres-petit nombre de cellules, elles loceupent presque en entier. Tris-souvent mème la portion du tissu cellulaire qui leur est extérieure est repoussée vers le dehors, de sorte que celte région, qui correspond normalement an milicu des faces ou des sillons, simule ici des còtes arrondies, que la région normale des còtes est déprimée, et quainsi les lacunes corticiles paraissent placées vis-i-vis les còtes, contrairement a re qui a lieu sur toutes les espéces (pl. V, fig. 13). La guirlande de séparation est fortement marquice; ses ondulations suivent la direction normale et sont dis lor's en sens inverse de celles de la circonférence externe. Lacunes essentielles très-prononcées, transversales et tout entouries de tissu fibreux. Tout cet ensemble est très-distinct el mème trèséloigné de celui que présentent les coupes transversales de l'E. limosum et de l'E. arvense.

Tiges naissant très-rapprochées et formant de grosses touffes, droites, d'aspect raide, d'un rert clair; tris-variables en hauteur, 15 a 80 centimètres, el en diamilte, 2y it 5 millimetres'. Entre-nouds quinze it vingt, peu allongés ot au maximum de 5 centimeitres; les inférieurs el les supérieurs asse\% ordinarement sans lameaux; ils n'en sont tous dépourvus que rarement et sur les sujets les plus griêles. Cüles el sillons douze a scize, si peu sensibles que la surface parait plutit cylindrique que sillonnéc. Stomates disceminés sur tunte la largeur des sillons, à l'execption des entre-neuds les plus voisins de l'épi, où les deux ou trois rangs de cellules du milien du sillon sont dépourvus de stomates. Sur une section transversale (pl. VI, fig. 12), la cavilé centrale occupe les deux tiers on les trois quarts du diametre total. Lacunes corticales ovales-transterses ou presque rondes, séparies du pourtour extéricur, du pourtour intérieur el cutre colles par des masses cellulaires a peu près de mème largeur. Faisceanx de fibres corticales gros et semi-eylindriques; groupes de cellules a chlorophylle s'étendant sur toute lat largeur des sillons en un quadrilatère allongé dont les.

\footnotetext{
13. Milde, it qui jai communirqué l'E. litlorale cucilli ì Arles, fait commaitre "que la plante méridiunale cst'en

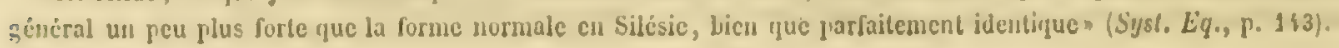


\$ 1. - DESCRIPTION DES ESPÈCES. - E. LITTORALE.

petits côtés suivent la limite du faisccau fibrcux, le côté extérieur suit la courbe de l'épiderme du sillon, et l'intérieur', qui est le plus grand, reproduit ì distance la courbe de la lacune corticale, en s'avançant sous le faisceau fibreux, mais très-rarement jusfru'i rejoindre les groupes des cellules vertes voisines et former une ligne continue. Telle est l'apparence normale el constante vers le milicu de la lige; mais vers lo bas, là oủ les stomates sont encore peu nombreux, comme vers le haut, lorsqu'il se trouve au milieu du sillon quelques lignes de cellules sans stomates, il apparait au-dessous de ces cellules un petit laiscean fibreux qui échancre la ligne extéricurc du groupe de cellules vertes (pl. VI, fig. 12), ou très-rarement le sépare en deux groupes. De semblables modifications se produisent d'ailleur's sur toutes les espèces. Les deux cylindres ne sont que très-confusément distingués; l'intérienr consiste en ume bande très-étroite, avec des ondulations intéricures opposées à celles de la surficc. Lacunes essentielles ovales-transverses; faisceaux fibro-vasculaires larges, ovalestransverses, s'avangant jusque entre les grandes lacunes. Les cordons de vaisseaux sont parallèles au contour de la lacune et non rayonnants comme dans les autres espèces.

Les gaînes sont presque appliquées sur la partie inféricure de la tige; mais au-dessus de la moitié, les dents commencent à s'écarter, puis les gaìnes elles-mêmes s'évasent en approchant de l'épi, et les plus élevées sont très-liches, campanuliformes el d'un blanc jaunàtre; la partic vaginante est plus longue que large el sa longueur est environ double de celle des dents. Divisions lancéolées, étroites, très-aiguës, souvent soudées deux à deux jusqu'à leur extrémité, d'un châtain plus ou moins foncé. $\Lambda$ l'état frais, ces divisions sont entièrement lisses et sans stries, mais après dessiccation le retrait des fibres corticales détermine quelquefois l’apparition d'un très-léger sillon carénal.

Rameaux en nombre très-variable et sourent répartis en verticilles incomplets; sur les tiges tout à fait stériles ils diminuent très-régulièrement de longueur jusqu'i la pointe de la tige åssez longuement effilée. Ils sont dressés ou un peu courbés en dehors, et ils ont, comme toute la plante, un aspect un peu raide. Gaîne basilaire assez longue, d'un blanc verdàtre ou d'un brun très-pàle. Le premier entre-nocud est plus court que la gaîne caulinaire, rarement il l'égale et plus rarement encore la dépasse aux verticilles supérieurs. Entre-nocuds presque deux fois aussi gros que sur I'E. limosum, à quatre ou cinq còtes très-prononcées, obtuses; gaines un peu évasées cu cloche, largement membraneuses ant-dessous du sinus; dents larges, aiguës, un peu déjetées en dehors. Cavité centrale réduite au cinquième du diamitre'; point de

\footnotetext{
1 M. Milde aftirme que cette cavité manque quelquefois (Sysl. Eq., p. 1 \$1 et 111). Pour moi, je l'ai toujours vue aux entre-nceuds inférieurs des rameaux. Elle se réluit, mais sans disparaitre tout it fait, aux entre-nocuds de l'extrémitč.
} 
lacunes corticales; fasceaux fitreux his-prononces sous les ciotes (pl. VI, fig. 1:3). Les cellules a chlorophylle forment un cerele contint et occupent presigue tout t: cylindre extérieur, lequel est nettement distine' du cylindre interne par une guirlande circulaire. Lacunes essentielles el faisceaux fibro-rasculaires très-marqués.

Lëpi normil est a 12 centimètres de la gaine la plus élerée; son "pédoncule o est d'une belle couleur rose de chair, mou, tress-eadue el tout à fait analogue a celui des tiges spiciferes du deuxime groupe, lien quil porte quelques rares stomales. Épi ovoïde, allongé, jaunatre, relativement petit, long de 7 i 15 millimètres sur un diamètre de 5 i 6 millinetres; treize a scize verticilles de sporanges ayant environ douze clypéoles. Axe avec une petite caviti centrale. Sporose s'eflectuant très-incomplétement; les sporanges, au lieu de sonvir et de s'écarter, demeurent lermés et serrés les uns contre les autres. Spores presque toutes incompletes et mal formées (pl. IX, fig. 9).

Variations. - $1^{\circ}$ Polystachyon; liges à rameaux supérieurs terminés par un épi (rallée de Barr).

20 Tiges de 35 i 50 conlimetres, garnies de rameaux assez longs el nombreux. $\lambda$ part la présence de l'épi, la grosseur des tiges et lear aspect plus lisse, cette forme rappelle les liges stériles les plus robustes de l'E. arvense. Elle est la plus ordinaire et croît sur les bords relevés des fossés, lì surtout oủ l'humus est mêlé d'argile.

$3^{\circ}$ Tiges plus élevées, plus grandes et plus rameuses encore, rappelant l'E. limosum. $\Lambda$ u bord des fossés, mais dans l'eau.

$4^{0}$ Tiges sans rameaux, grêles, courtes, dressées ou un peu couchées, d'un vert jaunâtre; forme appauvric des sols caillouteux et durs, très-pauvres en humus.

Telles sont les formes cxtrêmes unies par tous les intermédiaires possibles. Je les ai obtenues ì Arles sur un scul et même rlizome qui s'étendait du fond d'un fossé sons Ia terre du bord, puis, a un metre de distance, dins un sol caillouteux et dur quoique humide, à còté de petits individus de Chlura perfoliala nyant 3 centimètres de haut. Je ne peux done les considérer que comme des variations.

Formes anomales. - $6^{\mathrm{e}}$ anom. Épis interrompus. Très-fréquente à Arles.

$7^{\mathrm{e}}$ anom. Terminaison irréguliere des épis par la continuation de la lige. Rare à Arles.

Se anom. Anneanx au milicu des tiges. A Arles (voy. p. 149).

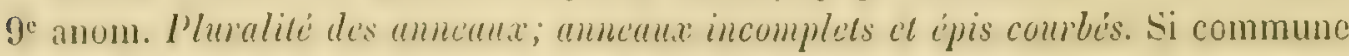
à Arles el dans le Bas-Rhin, qu'elle se troure au moins sur le tiers des liges spicifẻres.

$10^{\mathrm{e}}$ anom. Tubercules.

$16^{\mathrm{e}}$ anom. Gracilité.

$17^{\mathrm{c}}$ anom. Infécondilé des spores (roir p. 153). Cette anomalie est si ordinaire que 
§ 4. - DESCRIPTION DES ESPËGES. - E. LITTORALE.

M. Milde l'a signalée comme un caractère distinctif « sur lequel devra se porter d'abord l'altention des commençunts» (Rev. crit., p. 108). Elle existe en effet en Alsace et à Arles, comme en Silésie; cependant jai trouvé à Mrles quelques spores bien conformées, mais, malgré tous mes efforts, je dois répéter avec M. Milde "que les essais de germination, qui réussissent ailleur's si bien avec des spores friîches, sont restés ici sans résultat» (Eq. litt., p. 29, $1^{\mathrm{re}}$ col.).

On se ferait une ilée très-fausse si, pour apprécier combien cette plante est sujette aux anomalies, on ne considérait que le nombre des anomalies différentes qu'on peut y constater, car on n'en trouverait guère que quatre ou cinq de vraiment considérables. Mais, si on se rappelle que les anomalies 6,9 et 17, qui affectent toutes trois l'appareil reproducteur, se trouvent, les deux premières sur un tier's et la troisième sur la totalité des individus, on reconnaîtra que cet Equisetum est sans comparaison possible avec les autres pour le nombre des individus atteints d'anomalie.

Habitut. - Cette plante a été signalée d'abord en Russie, près de Saint-Pétersbourg, par M. de Kühlewein, puis dans la Neumark par M. Lasch; elle a été retrouvée aux environs de Breslau par M. Nilde, près de Ilambourg par M. Sonder, à Riga par M. Ilengel, à Brème par le docteur Koch, à lloyerswerda par. M. Jæmike, à Vienne par M. Juratzka, dans le duché de Bade, en Alsace et en Provence par moi, et il est probable que, mieux connue, clle se retrouvera dans d'autres contrées. Ce n'est qu'alors qu'il sera possible d'essayer de déterminer ses limites d'extension.

Station. - Je ne l'ai trouvée qu'au bord des eaux, mais sur tous les sols possibles. A Arles cl à Strasbourg sur un terrain argilcux el caillouteux, dans le duché de Bade et dans les Vosges sur du sable ou sur des fonds tourbeux etc. M. Milde a constaté de son côté que «cette plante se trouve sur tous les sols possibles, dans les plaines sablonneuses et stériles, comme dans les marécages, dans les terres en friche comme dans les champs de blé, et partout clle porte des épis, mais toutefois moins dans les marais que dans les sables» (Eq. lill., p. 28, $2^{\mathrm{e}} \mathrm{col}$.).

Localités francaises. - Cette plante u'avait pas encore été signalée sur le territoire français, lorsque je l'y trouvai, le 15 mai 1859, dans le département des Bouchesdu-Rhône, à deux lieues d'Arles, près de la station de Raphèle (chemin de fer de la Méditerranée). Elle courrait le fond d'un large fossé creusé dix ans aupararant pour fournir des matériaux de remblai, et y croissait en compagnie des Curex paniceu, $C$. Eideri, C. Psendo-Cyperus, G. stricta, Cladium IIariscus, Schœuns nigricans, Juncus acutus, Alisma ranunculoides, Anagallis tenella, Chlora perfoliata et d'autres plantes palustres. Elle s'y trourait en telle abondance que je pus la récolter pour les centuries de mon excellent ami M. Billot.

Je la retrouvai le 3 juillet suivant près de Strasbourg sur les bords du canal du 


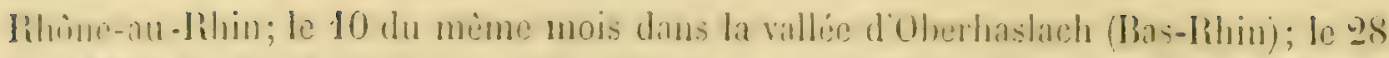

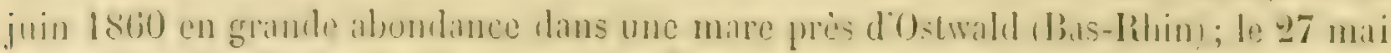

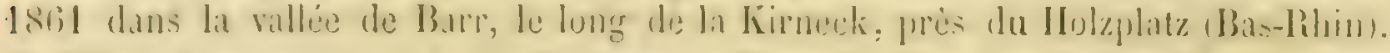

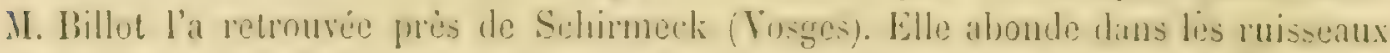
dirrigation pres d'oberkireh at entre Achern et Kappel (duehé de Bade). Dans ces

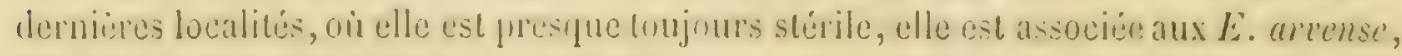
limosum, palustre; mais à Arles les E. limosum el arvense ne croissent pas dans la

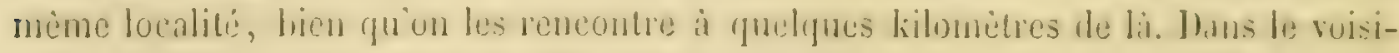

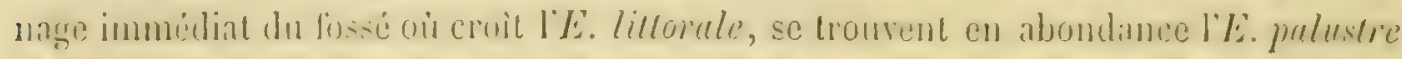

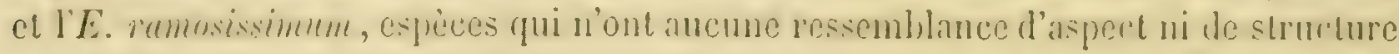
avec l'E. littoralc. J'ai fait de longues et vaines recherches pour trouver, dans la mème contrée, une seconde localité oủ crût l'E. littorale. Il n'y en a que dans ce fussé creusé en $18 \mathbf{8}$, et certainement la disposition des lieux el la nature du sol caillouteux de la Crau nauraient, arant cette épogue, permis a aueune espéce d'Liquisetum de végéter à celle place.

Époque de lu sporose. - Mai el juin. Un peu plus tardive que l'E. Limosum. A Arles, de nourelles tiges spiciferes se montrent abondamment $\mathrm{en} \mathrm{septembre,} \mathrm{si} \mathrm{les}$ premières ont été fauchées en août.

Obsernations. - En décrivant cette plante pour la premiere fois, N. Ruprecht, comme nous l'avons vu p. 189, fit observer qu'elle remplit on quelque sorte une lacune entre l'E. artense et l'E. Limosum; que quelques-unes de ses formes sont semHables a l'E. limosum, d'autres a l'E. arene et surtout a sa vare serotinum .leyer (E.cumpestre K. Fr. Schulty. M. Milde, dans les nombreux travaux qu'il a publiés sur celte plante (Bot. Zeit., 1851 , p. 705-71' ; Rer. crit., p. 109 de la trad.; Kenntn. Ey.,

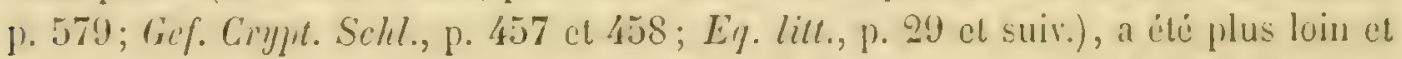
l'a constamment considerce comme un hybride de l'E. arense el de l'E. limosum. Dans le plus récent de ses travaux, après avoir exposé:

$1^{\circ}$ Que celte plante vient sur toute espèce de sol;

go Que son épi, par sa pelite cavité centrale, est intermédiaire entre l’épi creux de lE. limosum et l'épi sans cavité de lE. arrense; que les clypóoles restent scréés les uns contre les antres; que les sporanges mal conformés ne répandent jamais leur contenu consistant en spores abortives, sans élatères et infécondes;

8. Que la tige a des cutes comme l'E. arense, mais des stomates épars comme l'E. limosum;

$4^{\circ}$ Que les cavilés sont intermédiaires entre celles de ces deux espèces;

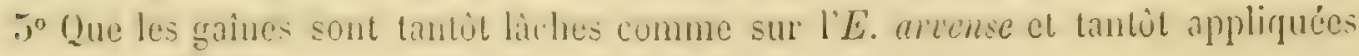
comme sur l'E. limosum;

6n (Gue la disposition des cellules à chlurophlylle n'est point constante, mais qu'elle 
varie en se rapprochant de celle de l'une ou de l'autre des espèces précitées";

$7^{\circ}$ Que les rameaux resscmblent à ceux de l'E. limosum;

$8^{\circ}$ Que les rhizomes portent des tubercules comme ceux de l'E. arvense,

Le savant botaniste de Breslau conclut en ces termes:

«Résultat:

(I. Notre plante se montre chaque amnée régulièrement dans les localités oủ elle a élé une fois observée, et n'est point, comme d'autres formes (par ex. E. arvense serotimum Neyer ou irrigumm Nilde), li conséquenec d'influences atmosphériques se produisant sans constance;

«II. Dans chaque localité et chaque année, elle produit des spores aljortives et des sporanges;

«III. Elle est une forme intermédiaire entre l'E. arvense et l'E. limosum.

«Elle rappelle l'E. arvense:

" $1^{0} \mathrm{Par}$ la disposition des côtes de la lige;

" $2^{\circ}$ Par l'existence constante de deux cercles de lacunes;

«3० Par la présence des lubercules;

« $\mathbf{4}^{\circ}$ Souvent par la forme et la couleur des gaines caulinaires;

« $5^{\circ}$ Enfin par la disposition des rameaux.

a Elle rappelle au contraire l'E. limosum:

« $\mathbb{1}^{\circ} \mathrm{Par}$ la répartition des stomates;

(20 Par l'absence de sillon carénal sur les dents des gaînes caulinaires;

« $3^{\circ} \mathrm{Par}$ la substance de la tige ne formant qu'un cylindre;

\& $4^{0}$ Très-souvent par la couleur et la forme des gaines de la tige et des rameaux;

« $5^{\circ} \mathrm{Par}$ la forme des masses cellulaires vertes.

«Les caractères qui tiennent le milieu entre les deux espèces sont les suivants:

« $u$. La forme des masses cellulaires vertes, qui se rapprochent tantòt de l'E. arvense et tantôt de l'E. limosum;

« $b$. La cavilé centrale, qui tient le milieu entre celle des espèces précitées;

«c. L'axe de l'épi, qui n'est ni creux comme sur l'E. limosum ni tout à fait plein comme sur l'E. arvense.

"IV. Cette plante doit done ètre regardée comme un hybride de l' $E$. artense et de l'E. limosum » (Eq. litt., p. 30).

M. J. W. Sturm ne partage pas l'opinion de M. Milde sur l'hybridité de cette plante (Flora, novembre 1857, p. 691).

- Je n'ai pu constater les différences considérables que cite M. Milde, si ce n'est sur des sections opérées ì des hauteurs différentes; or ces modifications se retrouvent sur toutes les espèces, sur l'E. limosum aussi bien que sur l'E. arcense (voir p. 162, 186 et 197). 


\section{Eousetur hiosum L.}

Diagnosis princeps. - E. FLUvintile caule striato, frondibus simplicibus. L. Sp. pl., Ed. 1a, p. 1062. E. Lisosus caule subnudo lecri L., o. c.
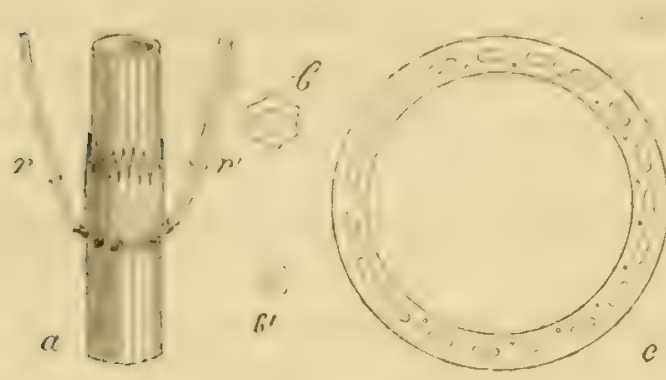

Diagnosis $n^{3}$ - - Spica obtusa, crassa, ovoidea, brevi, scrius clongata. Caulibus asulcis, lavissimis. Fistula centrali vastissima, $\$ / 5, c$; lacunis exterioribus 16-20 ovato-transwersalibus, siepe obliteratis. Yaginis adpressis, xque longis ac latis; divisionihus lineari-lanceolatis, rigidis, $a$. llamis aut mullis, aut paucis, aut numerosis, longis, gracilibus plerumque simpliciluus, leviter sulcatis, angulis $4-5$ oblusis, $b$; primo ipsorum internodio vaginam caulinam aut non aut vix adxquante.

Descriprion. - Rhizomes principaux rampant peu profondément dans la vase, sëtendant el se ramifiant tellement que toutes les tiges d'une mème mare ou d'un même fossé appartiennent le plus souvent à un mème rhizome, ainsi que Vaucher l'a déja fait remarquer (Mon. Prêl., p. 3931). Quelquefois ces mèmes rhizomes s'enfoncent. assez profondement sous la terre des bords du fossé, et alor's les tiges qui en naissent sont très-grêtes, presque toujours nues et stériles. Entre-nauds d'un diamètre de 6 a 10 millimètres, longs de 5 à 10 centimètres, cylindriques, quinze à dix-huit còtes a peine sensibles. Épiderme d'un beau pourpre noir, très-glabre, luisant el comme corné (pl. II, figg. 8 ct 9). Gaines sans tomentum, à dents lancéolées aiguës, trèsnoires, persistant longtemps sans pourrir. Cavité centrale très-grande et occupant au moins les deux tiers du diamètre total. Les deux cylindres sont intimement unis et non séparés par une guirlande de petites cellules (pl. V, fig. 14). Lacunes corticales très-éloignées de la circonférence, grandes, transversales, en demi-cercle dont l'arc est tourné vers le centre, et le diamitre, peu régulièrement concave, est parallële aux faces. Leur rayon est à peu près le lier's du tutal de la masse cellulaire. Cette forme est sans analoguc sur les autres espèces. Lacunes essentielles prononcées, tangentes iutérieurement a la mème circouférence que les lacunes corticales; faisceaux fibrovasculaires pénétrant profondément entre les lacunes corlicales, nettement limités et si paures en vaisseaux quion n'en voit ordinairement qu'un ou deux au pourtour des lacunes.

Tiges naissant un peu éloignées les unes des antres, très-droites et diaspect raidr, ordinairement très-rertes, hautes de 50 centimètres a $1{ }^{m}, 50$, d'un diamètre de 4 a 8 millimètres; les spicifëres ont de 20 a 30 entre-neuds; les stériles en ont sourent plus de quarante-cing, nombre à peu près égal à eclui des verticilles des tiges spici- 
ferres, en y comprenaut les verticilles de l'épi'. Ces entre-nouds sont ver's le milieu de la tige longs de 5 à 8 centimètres. La surface en est parfaitement lisse et unic, "caule levi » L.; les cûtes et les sillons (seize à vingt) ne sont distincts que par la coulcur de leurs tissus, les côtes par l'étroite ligne blanche de leur faisceau fibreux; les sillons par une bande verte six fois plus large, sur toute la surface de laquelle les stomales sont disséminés (pl. III, fig. 9). Une section transversale montre également que les côtes sont à peine marquées par de très-léģères saillies un peu déprimées au milicu (pl. VI, fig. 14). La cavité centrale n'occupe pas moins des quatre cinquièmes du diamètre. Lacunes corticales oblongues-transverses (grand axe parallèle à la circonférence), étroites, séparées slu pourtour extérieur par quelques cellules incolores et par une bande de cellules à chlorophylle, qui occupe uniformément toute la largeur des sillons entre les petits faiseaux fibreux des côtes. Comme l'ensemble des tissus a très-peu d'épaisseur, et que la bande de chlorophylle n'est pas interrompue au milieu du sillon, il arrive que, pour peu qu'elle se développe par suite de la grande quantité de stomates épars sur l'épiderme, elle oblitẻre entièrement les lacunes corticales; ces lacunes manquent donc très-souvent sur les tiges grêles et sur la partie supérieure de beaucoup de tiges. C'est donc à tort que Bischoff a affirmé, d'un còté, que les grandes lacunes manquent (toujours complétement» sur cette espèce (Krypt. Gew., p. 37), et que M. Döll a dit, d'un autre côté, que les lacunes qui manquent sont les lacunes “carénales» ou essentielles ${ }^{2}$; celles-ci ne manquent jamais. Eilles sont accompagnées d'un faisceau fibro-vasculaire très-gros, cylindrique, très-nettement circonscrit et s'avançant beaucoup entre les lacunes corticales, presque jusqu'à devenir contigu au faisceau fibreux cortical, qui est plus petit que lui et triangulaire. Sur le tiers supérieur de la tige, les sections offrent une cavité centrale relativement plus grande encore; vers le bas et sur la région submergée, les cellules à chlorophylle, ainsi que les stomates, sont distribués avec moins de régularité, et là oủ les stomates et les cellules à chlorophylle manquent tout à fait, il n'est pas rare de trouver de la chlorophylle dans les cellules de l'épiderme.

Gaines étroitement appliquées contre la tige, brillantes et comme vernies, tout à fait lisses et très-faiblement sillonnées; partie vaginante verte, courte et aussi large que longue. Divisions linéaires, étroites, se séparant régulièrement en dents aiguës, courtes, noirâtres, raides, très-persistantes, égales au tiers de la partie vaginante. Vers le bas de la tige elles sont un peu plus longues et plus étroites.

Rameaux en nombre très-variable, tantòt manquant entièrement, tantòt naissant

\footnotetext{
: Ce rapport d'égalité entre le nombre des verticilles des tiges stériles et le nombre total des verticilles foliaires et sporangifères des tiges à épi est un fait (je n’ose dire une loi) qui peut se constater sur toutes les espèces, en tenant compte du degré de force des sujets.

" "Kantenlücken (carenal cavities A. Braun) fehlen nur bei $E$. limusum * (Fl. Bad., p. 5i).
} 
en verticilles complets dis les numb inferinurs el decroissant rigulierenent en lon-

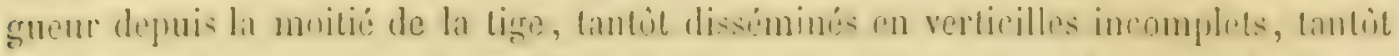
ne commeneant que vers la moitis de la lige et ensuite presque igaux en foncureur justuatu sommet; celle derniere dieposition ne se: montre guère rue sur les liges mutilies ou sur celles qui ont purte un épi. Les rameanx sont ators dreseres, tandis que le plus souvent ils sont étales horizontalement. La gaine basiliare est courte, dun brun pâle et mat. Le premier entre-nœud est très-court, et avec sa gaîne, assez. grande et dilatée, il n'atteint pas, ou, sur les verticilles superieurs, atteint i peine la naisance de la gaine caulinaire. Entre-nauds trés-gribles, il quatre on cing cotes obluses; faces i peine concaves. Gaines allungtes, ovoïdes, renllies an milien, verles, non-membranenses; dents vertes ou quelquefois avec la pointe noire, plus courles que la partie raginante, non étalées en hehors el plutit un pen inclinées en dedans. La coupe transversale des entre-nceuds pl. VI, fig. 14 bis) dome un carré ou un pentagone arec les angles arrondis et les cólés un peu rentrants; la cavilí centrale cst très-prononcée et occupe an moins la moitie du total, particulierement sur les rameaux a cinq còtes. Les faisccaux fibreux des còtes sont trìs-prononcis el quelquefois savancent presque juefuarux faisceaux fibro-rasculaires; la chlorophylle forme une ceinture non interrompue; point de lacunes corticales; les lacunes essenticlles sont petites, mais toujours apparente. Il est i remarquer qu'il y a fréquemment deux sortes de rameanx, et cela sur' un mème verticille. Les uns sont tels qu ils viennent d'ètre décrits, les autres, très-gros, sont de rraies tiges secondaires, souvent aussi forles, aussi longues et aussi rameuses que la tige principale, dont ils reproduisent exactement l'organisation. Leur gainne basilaire est très-large, et leurs autres gaînes ressemblent non a celles des rameaux, mais it celles de la tige. Il n'est pas rare de trouver sur les tiges spiciféres les rameaux des verticilles supérieurs spicifères eux-mêmes.

Epi normal brièvement pédoneulé et presque toujuurs entouré a sa base par la derniere gaine tres-dilatie, noir avant la sporose, cylindrique, gros, tris-obtus, triescourt et, ainsi que le pidoncule, sallongeant un peu pendant la sporose. Onze a quatorze rangs de sporanges, aree vingt clypéoles euviron. Axe creux. Sporose s'accomplissant de haut en bas, cl sur le còtí exposé au midi plutòt que sur l'autre, ce qui incline et déforme souvent les épis.

Variations. - Il n’y a point d’espèce qui varie autant dans le nombre ct létendue "de ses rameaux: quelquefois tout le verticille se développe, quelquefois une partie a seulement ou un seul rameau, et l'on distingue tris-bien sur la tige les points oủ les

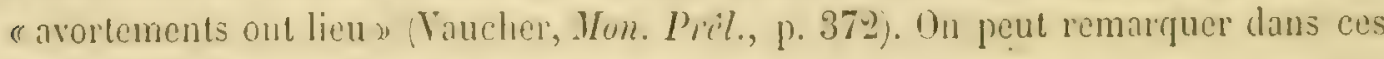
variations les quatre formes suivantes:

$1^{\circ}$ Tiges à rameaux supérieurs chargés d'épis fertiles; A. polyslachyon Vaucher (IIon. Prêl., p. 372 et 373). Dans les caux profondes el vaseuses. 
20 Tiges élevées, a rameaux nombreux; $E$. furiatile L. Dans les caux profondes.

$3^{0}$ liges élevées nues, E. limosum L.; plus particulierement sur le bord des fossés.

$4^{0}$ Tiges pelites, grìles, sans rameaux, E. uliginosum Mühllog. in Willd. Dins les tourbières el les prés humides couverts de mousses.

Formes anomales. - $6^{\mathrm{e}}$ anom. Epis interrompus par des verticilles de gaines ou par des anneaux plus ou moins réguliers; voir p. 149.

$9^{\circ}$ anom. Pluralité des anneaux el anneaux incomplets.

$11^{*}$ anom. Gaînes en crête. Cette anomalie est dans certaines annécs si fréquente sur cetle espece que plus d'une fois je l'ai vue sur presque tous les pieds d'un mème fossé (a Geispolsheim, près de Strashourg). En examinant ces tiges, jai ru que toutes avaient été attaquées dans leur première jeunesse. Vaucher avait dit: «Je n’ai pas «lieu de croire que les Prêles servent d'habitation à aucun insecte» (Mon. Prêl., p. 336); mais on trouve souvent, du moins en Alsace, les entre-nœuds de celle espice troués par des insectes qui dérorent les tissus du eylindre interne; les parois de la cavité centrale sont alors labourées et toutes noires.

$12^{\circ}$ anom. Gaines en spirale.

$16^{\circ}$ anom. Gracilité.

$17^{e}$ anom. Infécondité des spores; voir p. 153.

Ilabitat. - Tout le nord de l'Europe et de l'Asic. On la cite aussi dans le nord de l'Amérique. Limile supérieure de végétation: 700 mètres au pied du Mont-Blane (Payot), 5380 pieds en Suisse d'après M. Bernoulli. Je ne l'ai point vue en Algérie.

\section{Limites d'extension.}

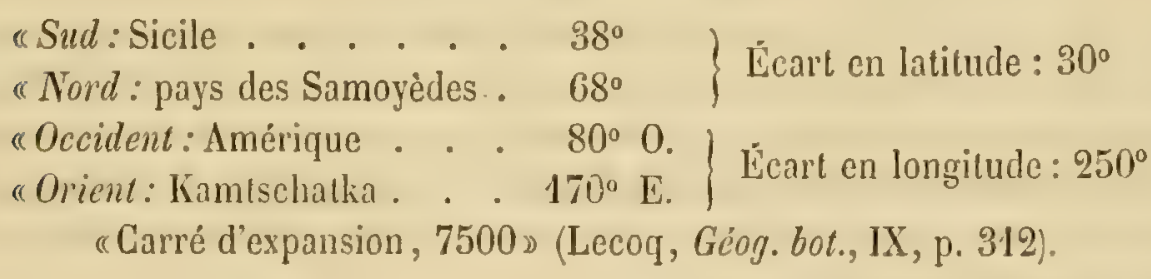

Station. - Les fossés, les mares, les canaux, les eaux peu profondes.

Lacalités françuises. - Dans tous les départements, mème les plus méridionaux, elle croil abondamment dans le moins profond des canaux de vidange qui ront de Tarascon à la mer. La $\mathbf{k}^{0}$ forme, uliginosum Mühllg., abonde dans les prés humides de l'Alsace, à Weiler pris de Wrissembourg, ì Geispolsheim prìs de Strasbourg ele.

Époque de la sporose. - Commence en fin arril, se prolonge jusqu'au mois d'aoùt dans la vallée du Rhin et recommence en septembre dans les canaux ả ArTes.

Observation. - Les deux formes principales de cette espèce ont été considérécs par Linné comme deux espèces, et sur ce point il y a encore quelques divergences d'opi- 
nion. Les uns pensent que chacune des plantes de Linue doil itre cilevée à la dirnnité l'espece; les autres n'en font que des varietis, et d'antres que des formes variables d'un mime type. Au nombre des premiers on trouve Wahlenterg qui, en 1820, dans son Flura upsuliensis, justifie la distinction en ces termes: " E. limosum sequenti " (fluviatili) utique valde affine, attamen non parum differt. Longe prius mense fronadescentix optime lecrendum; totum crassius sed minus durum, colore dilutiore, "dentibus tantum vaginarum nigris in caule, sed ramorum viridibus. \$ Il ajoute en parlant de son E. furiutile: "Exteris auctorilus non satis notum. 》ais en 1826, dans son Flora suecica, cet auteur réroque lui-mème on doute la validite de la prémiere distinetion, et il dit formellement: "E. limosum seruentis (E. flututilis) forsin "varietas pracocior, D p. 689. Watlroth ( $F \%$ crypt. Germ.) fait également de l'E. furiutile une espece distincte du limosum et du maximum. Fries (Siem. reg. Scand., p. 59 et 251) incline vers cette distinction et s'exprime ainsi : a E. Lryosux L. et "FLtriatile L. utique nimis affinia sunt, sed apud nos (circa Upsaliam vulgaria) " facile discernuntur el a nullo Bolanicorum Suecorum, al prisca contrahenda, "yuam nova distinguenda promptiorum, conjuncta. Ut pateat an nostra cum exte"rorum prorsus conveniant, utrumque dedimus in II. N. XI. » (p. 251). M. Grenier a bien voulu me permettre de consulter cette feuille de literbarium nomale de M. Fries, et il ne m’est resté aucun doute sur la réunion. M. Milłe dit également que liexamen des exemplaires originaux de l'IIerbarium normale de M. Fries a mis pour lui hors de tout doute possible la réunion des E. liniosum et furiatile L. (Gef. Crypt. Schl., p. 450 el 451 ).

A l'exemple de la plupart des auteurs, je rúunis ces deux formes sous un seul nom, et mème sans les qualifier de dénominations distinctes comme variétés, par la raison qu'elles n'ont pas de fixité, et que de plus on peut trouver que tous les intermédiaires adhèrent à un même rhizome, quand on prend la peine d'en arracher un de quelque longueur sur les bords d'une mare ou d'un fossé. Presque toujour's les tiges naissant hors de l'eau sont dépourvues de rameaux, tandis que les tiges immergées à leur baan. en sont richement pourvues.

\section{Eouisetum polutstie L.}

Diagnosis princeps. - E. caule angulato, frondibus simplicibus. L., $S p . p l .$, Ed. 1ª p. 1001.

Diagnosis $n^{\text {a }}$ - Spica obtusa, lineari-oblonga. Caulibus late el obscure sulcalis; fistula centrali ninima $1 / 6$, lacunas exteriores $6-8$ arato-radiantes adrequante, $c$. Vaginis

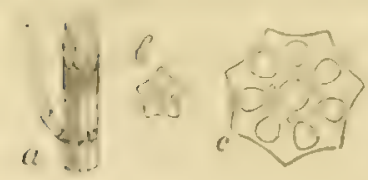
cylindricis duplo longioribus quam latioribus, $a$; divisionibus lanceolatis.

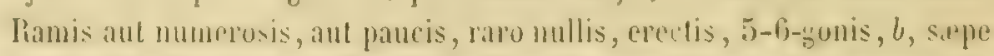
spicigeris ol caulem mentientibus. Primo ipsorum internodio varina canlinæ terliam aut raro dimidiam partem adæquante, $a$. Rhizomatibus profunde repentibus. 
Description. - Rhizomes principaux rampant très-profondément. Dans la crue de décembre 1860 , le Rhin coupa dans l'ile des Épis, près de Strashourg, toute la lisière d'un marécage oủ abondait celte espèce. Après le retrait des éux, je tronvai sur les bords du nouveau lit des rhizomes horizontaux déterrés à une profondeur de $1^{\mathrm{m}}, 50$. A celle profondeur, les entre-nouds sont très-longs et dépassent sourent 15 centimìtres avee un diamètre de 1 centimètre; les gaines sont pourries; à chaque nocud on trouve un bourrelet assez large, très-saillant, duquel sortent les rhizomes secondaires et les racines. Ce bourrelet est arrondi vers le bas et irrégulièrement déchiré à la place qu'occupait la base de la gaîne. $\Lambda$ ces bourrelets adhèrent de très-gros tubercules (diamètre, 10 millimètres; longueur, 18 millimètres), rarement isolés, mais disposés en chapelets verticillés (pl. I, fig. 5). Leur surface porte six ou sept còtes assez marquées séparant les faces glabres, mates et presque planes. Les rhizomes verticaux qui s'en élèvent et donnent naissance aux tiges sont beaucoup plus petits et quelquefois même tout à fait filiformes. Leurs entre-nœuds sont assez longs, d’abord jaunes, puis passant au rouge brun et au noir intense, toujours très-glabres, très-luisants et lustrés. L'épiderme a été décrit p. 19 et figuré pl. II, fig. 10, 11, 12. Còtes cinq à sept, toujours moins nombreuses que sur les tiges, peu saillantes; faces presque planes. Tubercules assez fréquents, ovoïles-allongés ou sphériques, noirs et luisants.

Cavité centrale nulle (pl. V, fig. 15). Cylindres très-marqués par une guirlande de petites cellules, si distincts et si facilement séparables, que si l'on coupe un rhizome et qu'on le laisse se dessécher un peu, le cylindre intérieur, se contractant moins que l'autre, s'en détache et fait saillie à l'extrémité. Lacunes corticales très-rapprochées du bord et entre elles, obovales, très-grandes; sur' les rhizomes principaux elles sont énormes et deux ou trois fois plus vastes que le cylindre intérieur. Lacunes essentielles assez grandes, très-marquées; faisceau fibro-vasculaire très-prononcé, assez riche en vaisseaux.

Tiges rapprochées et formant quelquefois des touffes, généralement droites et ramcuses, rarement nues ou un peu couchées, d'un vert clair et jaunâtre', sourent tachetées de brun; atteignant rarement 4 à 5 millimètres de diamètre et ne dépassant guère 50 centimètres de haut. Entre-nœuds neuf à treize sur les tiges spicifères, quinze à vingt sur les tiges stériles, presque tous de même longueur, 3 à 5 centime̊tres, prismatiques; six à neuf côtes émoussées, "caule angulato ๖ L.; sillons larges, peu profonds, à peine marqués șur le frais, avec des stomates disséminés sur toute leur largeur (pl. III, fig. 10). Cavité centrale très-petite, ả p̉eu près égale au sixième du diamètre total. Lacunes corticales très-grandes, larges, obovales ou presque rondes, très-rapprochées les unes des autres et du pourtour extérieur, plus

\footnotetext{
' Quand cette espèce, après avoir été coupée, reste exposée à l'humidité, elle jaunit très-vite.
} 


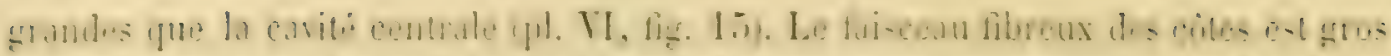

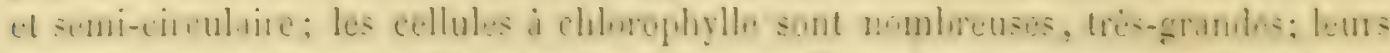

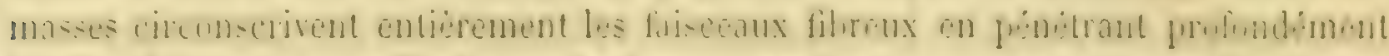

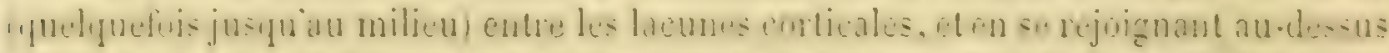
delles de manieve a former une ceinture continue tre-acidente. Cequendant il artive

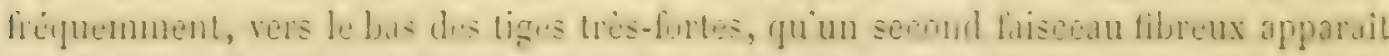
vers le milicu du sillon et savance jusqu aux lacunes, ou n en est sipare que par un rang de cellules i chlormphlte. Toute cette disposition est particuliemental propre a celle

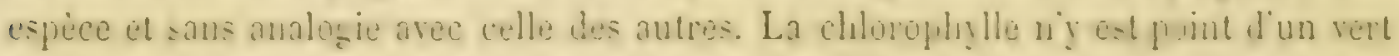
fonce, comme dans le reste du genre, mais diun rest junnitre. Ginirlan le scinant les

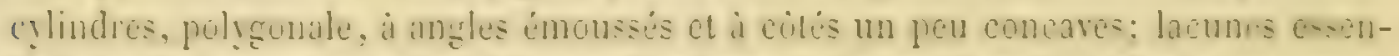

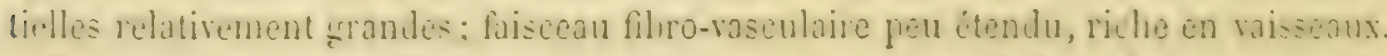

Ciaines prismatiques, lichement appliquecs, surtout vers le haut de la tice f partie vaginante deux fois aussi longue que large, a cites el sillons pen marqués; divisinns lanceulées, se sejparunt nettement en dents effilies. colorés, munies d'un sillon carénal el diune large membrane blanche, souvent moins longues que la moitie d: la partie raginante.

Rameaux simples, assez gros, dressis, tres-irregulièrement répartis: commencant dès le bas ou ver's la moitie, prar rerticilles complets, ou isolies, ou manquant tont i fait. Guine basilaire longue et large, noire et brillante, avee des dents plus fales et tondes de blanc. Entre-neuds a cing cuites émoussee, a sillons a peine concaves: le premier est tris-court et atteint à peine, arec sa gaine, le tiers ou rarement la moitie de la gaine caulinare. Les-autres gaines sont tres-longues, un peu dilates et ventrues: dents boun jate, avec un lord membraneux hlanc, larges, moitie moins lengues que la partie raginante, un peu inclinees en dedans. La section transwersale des cutre-nouds montre exactement la mème organisation que celle des tigeses, sur une échelle moindre; quelquelois seulement sur des sujets tres-qreles, leatremite d.s rameaux est télragone et les lacunes corticales sout ublitéres. Issz sument les ramean des verticilles supérieurs un mème de tous les verticilles se termineat frar un petit épi, aroide, et cela arec toules les modifications possibles de longueur a de position.

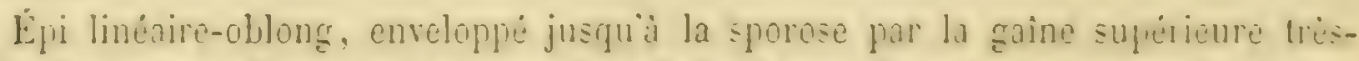

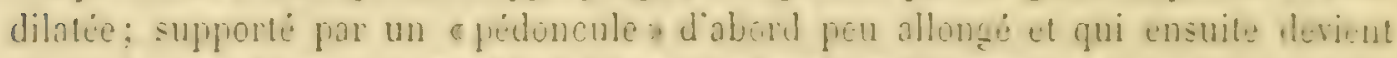

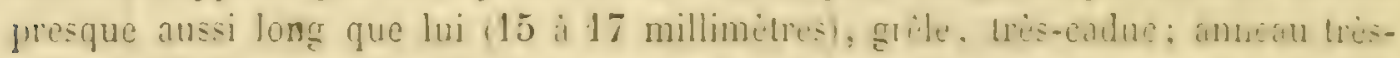

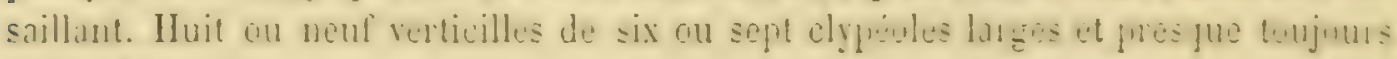
noirs. Axe creux.

Variations. - Les variations extrêmes de celle especce n’ont pas assez de permanence four itre cities comme variete: ulles ne persistent pas d'une ame a l'autre. 
et on les trouve quelquefois toutes sur un seul et mème rhizome. La forme polystitchyée est très-fréquente et affecte toutes les modifications possibles : $1^{0}$ des rameaux ¿ lous les nouds, et tous les rameaux sans exception portent des épis el alleigruent la mème hauteur; 20 rameaux sur la moitié supérieure el les plus élevés seuls spicifères; $3^{\circ}$ la tige, nue à la base, n’a de rameaux qu'au milieu; ils sont spiciferes, mais, aux noeuds les plus rapprochés de l’épi principal, les épis latéraux, très-nombreux, sont subsessiles.

On trouve dans les bois humides une grande forme stérile, très-rameuse, ayan presque un mètre de haut, et répondant exactement à la forme de l'E. arvense que M. Al. Braun a appeléc nemorosum. Entre celte forme robuste, la forme polystachyée et les formes grềles, amaigries et allongées, ou courtes et à demi-couchées (E. prostratum Hoppe) on trouve tous les intermédiaires possibles.

Formes anomales. - $7^{\mathrm{e}}$ anom. Terminaison irrégulière des épis. Épis des rameaux se continuant en entre-neuds; épis terminés par un acumen (voir ci-dessus p. 143 et 149). M. Cosson a signalé cette dernière anomalie comme «sous-variété mucionatum 》 (Fl. env. Paris, 2 e éd., p. 880; voy. Bull. Soc. bot. Fr., VIII, p. 297 el 368).

$10^{e}$ anom. Tubercules. Très-gros et nombreux sur les rhizomes horizontaux; petits el moius fréquents sur les rhizomes secondaires; toujours à épiderme d'un pourpre noir très-brillant.

$11^{\mathrm{e}}$ anom. Gaînes en crếce.

$12^{\circ}$ anom. Gaines cn spirale.

$13^{\mathrm{e}}$ anom. Bifurcation des tiges.

$16^{\mathrm{e}}$ anom. Gracilité des tiges. C'est ici qu'il faut rapporter les vés $\%$ mulum Duby (Bot. gall., p. 535), tenue Döll (Fl. Bad., I, p. 63) etc. Sans l'étude attentive de la struclure on est exposé à rapporter cette forme à l'E. variegatum (roy. E. Newman, Brit. ferns, p. 49).

$17^{e}$ anom. Infécondité des spores. Fréquente sur les sujets grêles à sporose tardive. Habitat. - Toute l'Europe el le nord de l'Amérique. Elle ne s’élève dans les Alpes qu’à 1500 mètres. Je ne l'ai point rencontrée en Algérie, et Desfontaines ne l'y menlionne point.

\section{Limites d'extension.}

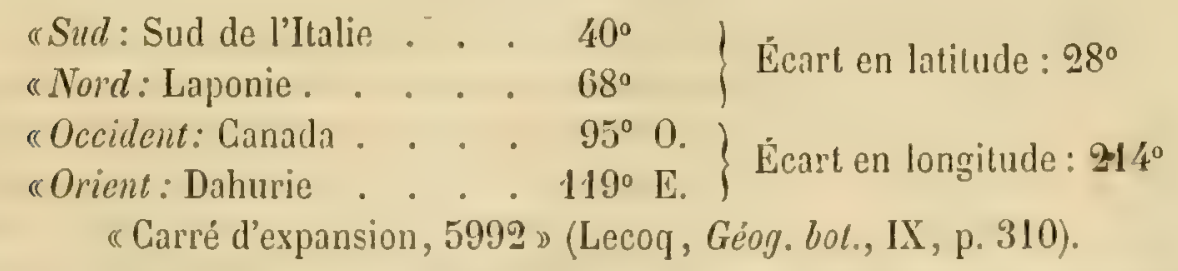

Station. - Tout terain lui parait bon, pourvu qu’il soit humide; champs labourés, prairies, marais, tourbières etc. 
Loculités francuises. - Partout oủ il y a des marécages.

E:porpue de la sponose. - Depuis le mois de mai jusqua a la fin de septembre.

Obsertation. - Quand on examine les formes polystachyées des E. limosum el pulustic, on remarque que jamais il napparait de rameaux spiciferes sur les tiges i sommet stérile; il ne s'en produit que sur les tiges spiciféres et, en général, après que l'épi terminal s"est flétri. Cela arrive surtout lorsque les épis de la première de ces espeeces se sont mal développés au printemps, soit que le froid les ail brülés, soil que des pluies continues les aient rapidement fait pourrir. On est alors porté i altribuer cette production à la coincidence d'un arrèt dans le développement en longueur de la tige principale et d'un excès de force dans la mème tige; et, sclon M. Milde, le développement de ces formes sur l'E. pulustre e est favorisé par la destruction précoce de l'épi principal ou par la muliation de la lige principale „ (Gef. Crymt. Schl., p. 462). En citant la forme polystachyie de l'E. palustic, M. Ed. Newman rappelle que Bolton l'avait attribucé ả la destruction de la sommité de la tige principale, el, d'accord avec M. Wood (Phytol., janvier 1843, p. 482), le même auteur fait observer qüil n'est pas nécessaire de rallacher la production des épis secondiares à la mutilation de la tige principale, "puisqu’on trouve un épi terminal sur les liges chargées de rameaux spiciferes» (Brit. ferns, p. 48). Cela est parfaitement exact, et, de plus, il arrive souvent qu'on voit déjà paraitre les épis à l'extrémité des rameaux avant que l'épi principal soil entièrement sorti de sa gaine, de sorte quil y a presque simultanéité. On remarque en outre que, sur les sujets polystachyés, les gaines des tiges et des rameanx sont plus dilatées, plus longuement dentées, et que les rameaux sont beancoup plus gros, ce qui indique une prédisposition antérieure à la destruction de lépi principal et à loute autre circonstance qui peut d'ailleurs très-bien favoriser le développement ultérieur des épis secondaires.

\section{Equisetum ramosissinum Desf.}

Diagnosis princeps. - E. caule striato, ramosissimo; ramis virgatis, strialis, erectis, rerticillatis, apice floriferis. Desf., $\mathbf{F l}$. all., II, p. 398.

Diagnosis $n^{\mathrm{a}}$. - Spica acuminata, lineari-ovoidea; pedunculo normali modice elongato (spicx sape aboriuntur et tunc nigræx, oroidex, sessiles). Caulibus subsolitariis, crectis, altissimis, validis (sen post mutilationem cxspiloso-aggregatis, reductis et tenuioril̨us), ad medium ramosis, raro nudis, late sulcatis. Fistula centrali mağna, 2/3; lacunis exterioribus 10-15 ovato-transwersalibus, c. Vaginis

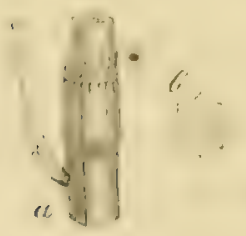

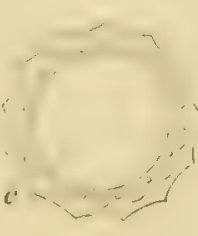

longissimis, admodum laxis, divisionibus lineari-lanceolatis, in dentes margine membranaceos persistentes rel hyeme caducos cradentibus. Ramis aut nullis, aut paucis et brevibus, aut numerosis ralidis et caules graciles mentientibus, $b$; primo ipsorum internodio quartam aut vix tertiam vaginx caulinx partem adxquante, $a$. Rhizomatibus profunde repenlibus. 
Description. - Rhizomes horizontaux rampant très-loin à une profondeur de 60 centimètres à $1^{\mathrm{m}}, 60$, el notablement plus gros que les rhizomes qui s'en élèvent verticalement et supportent les tiges. J'en ai trouvé de merveilleusement beaux, at 2 mètres de profondeur, dans les berges du canal de Craponne, it Rabet près d'Arles, oủ l'érosion des eaux les met à nu quclquefois sur une longueur de 6 mètres. M. Boreau attribue à celte espèce "de petits tubercules ovoïdes» (Fl. Cent.); les rhizomes ne m'en ont jamais offert, bien que souvent ils aient des entre-nœuds trèscourts et renflés. Leur diamètre le plus ordinaire est de 4 à 5 millimètres, mais il atteint jusqu à 7 millimètres à Arles et à $\Lambda$ ger. Les entre-nouds ont de 4 ì 8 centimètres de long, luit côtes et autant de faces peu marquées. Le plus souvent au-dessous du point d'émersion des rhizomes secondaires nait une forte racine, presque aussi grosse qu'cux et longue de 1 à 2 mètres. L'épiderme a été décrit p. 18 ct figuré pl. II, fig. 17. Les gaines dépassent souvent 18 millimètres; elles sont, ainsi que les entre-nœuds, d'un roux brun très-foncé et sans ces amas de tomentum qui revêtent les gaînes soutcraines de l'E. lyyemale et qu'on ne trouve ici que sur les racines. Ces gaines sont un peu molles et charnues, surtout à la base de leur's còtes; il en résulte qu'à ce point elles se détruisent plus facilement et qu'il se forme à la base de chaque gaine, sur le dos de ses côtes, de petites fossettes analogues à celles qu'on trouve sur l'E. maximum (p. 171). Les gaînes se détruisent d'ailleurs trèsrapidement et il est rare d'en trouver une complète sur les rhizomes un peu vieux. Lorsqu'elles sont tout à fait détruites, il n'existe plus vers les nœuds qu'un renflement crénelé et parfaitement régulier, dont les lobes arrondis répondent aux faces et les sinus aux côtes de l'entre-nœud (pl. I, fig. $6, b, c)$. Le fond de ces sinus a été préparé par la destruction des cellules de la petite fossette mentionnée ci-dessus. Cette élégante crénelure donne alors aux rhizomes un peu gros un aspect vraiment étrange. Cavilé centrale petite, égale an cinquième du diamètre total, toujours irrégulièrement ondulée aux bords. Le tier's extérieur du rayon est occupé par une masse continue de cellules colorées el très-chargées de grranules amylaccees; le tiers intérieur l'ost par le cylindre interne; les lacunes corticales occupent le tiers moyen et sont ainsi plus éloignées du pourlour extérieur que de l'intérieur (pl. V, figg. 16). Elles sont ovales ou presque rondes, leur plus grand diamètre est toujours transversal. Lacunes essentielles relativement très-grandes el presque aussi larges que les cluisons cellulaires qui séparent les grandes lacunes. Faisceau fibro-vasculaire très-petit, ne pénétrant point cntre les lacunes corticales; les cordons de vaisseaux sont très-marqués, rapprochés et rayonnants.

La partie souterraine de la tige offre la même coupe, mais la couche cellulaire qui sépare l'épiderme des lacunes corticales est de moins en moins épaisse.

Les tiges sont droites, en général isolées et mème éloignées les unes des autres; 
mais souvent anssi a la suite d'une mulilation vers la surface du sol, ha partie soutermaine des liges en produit des tonfes épaisses, toujours tres-droites. Eilles sont moins rudes au toucher que celles de l'E. hyemale et d'un ren't moins foncé. Leur hauleur normale est de 1 mitre euviron; mais elles varjent i linfini depuis 20 centimetres

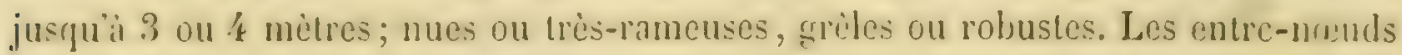
sont des lors aussi variables en grosseur et en longueur; sur les formes normales ils depassent presque toujours 10 centimetres vers le milieu de la tige; ils ont de dix i suize cotes un peu saillantes, i carene arrondie, obtuse, portant de chaque cúté un rang de petites saillies sur les sujets tris-forts et tris-vieux. Sillons tris-larges et peu profonds, avec une ligne de stomates de chaque còté . Cavite centrale srande, occupant environ les deux tiers du diametre total (pl. VI, fig. 17). Lacuncs corlicales i pen près également éloignées du pourtour extérieur, de lintéricur el entre elles, arrondies ou un peu ovales-transverses, souvent subquadrangulaires. Les deux cylindres sont plus distincls sur cette espèce que sur aucune autre du mème groupe; la guirlande de pelites cellules qui les sépare est extrêmement ondulée; elle passe a une égale distance entre les grandes lacunes el la cavité centrale, puis contourne a l'extéricur les faisceaux fibro-vasculaires et, pour cela, pénètre profondément entre les grandes lacunes et jusque vis-ì-vis leur bord extéricur. Les lacunes essentielles sont très-éloignées de la cavité centrale, et placées presque entièrement entre les grandes lacunes; elles sont plus larges vers l'intérieur; leur faisceau fibro-vasculaire est étroil avec des cordons de vaisseaux rapprochés, rayonnants el paralleles. Le faisceau fibreux des colcs est cunéiforme et pénètre profondẻment jusqu’i la séparation des grrandes lacunes et presque jusqu’au eylindre intéricur dont il n’est séparé que par quelques rangrs de cellules a chlorophylle. Un autre faiscenu fibreux, tıès-large el moins profond, sélend dans les sillons. Sur les especes du quatrieme groupe, la distribution des cellules i chlorophylle, quelque variéc qu’elle soit, oflie tonjours un caractère commun, dépendant de la disposition des stomates sur une seule ligne de chaque cite du sillon. Aiusi, sur toutes ces espèces, les groupes de cellules a chlorophylle ne touchent lépiderme yu'au point correspondant à la ligne de stomates; à partir de ce point leur numbre augmente vers l'intérieur, mais l'intensité de leur coloration diminue. Sur l'E. ramosissimum claque groupe se réunit an groupe roisin, et forme ainsi une conrome conlinue tris-fortement échancréc par les faisceatux fibreux (pl. VI, fig. 17). Elle est necessairement interrompue par ces mèmes faisceaux vers le bas des liges ou le développement de la chlorophylle est toujours moins prononcé.

Les gaines sont tris-longues et, y compris les dents, alteignent jusqu à 2́t milli-

\footnotetext{
' M. Döll mentionne l'existence accidentelle de deux lignes de stomates de chaque cútú du sillon sur une forme du and (Fl. Bad., p. 66).
} 
mètres; leur longneur moyenne est entre 12 et 18 millimètres, et, à diamètre égal, presque double de colles de l'E. liycmale. Étroitement appliquées sur leur tiers inléfricur, elles vont ensuite en sélargissant un peu en cloche. Llles sont d'abord tout a lait vertes el concolores avec un bord membraneux; très-rapidement les dents deviennent noires et se dessèchent, quelquefois mème elles tombent la seconde année sur les sujets qui ont passé l'hiver; la partie raginante qui reste présente alors un bord membraneux blanchàtre, encore longuement et inégalement dentelé, el non, comme l'E. lyycmale, des lobes courts d'un noir luisant, nettement arrondis, épais et comme charnus. Les divisions sont étroites, linéaires, et au delà de leur dilatation en cloche elles s'isolent et se réduisent à des dents filiformes sphacélées, largement mombraneuses aux bords. Les còtes ne different en rien de colles des entre-nœuds, dont elles sont la continuation; seulement vers le tiers supérieur clles semblent quelquefois avoir de petites carènes latérales; ce qui provient de ce que sur cette rígion le sillon commissural devient très-étroit et que sess bords coupés carrément sont un peu repoussés en dehors. Très-fréquemment, sur les sujets qui ont passé l'hiver, ou mème dès l'automne, les deux liers supérieurs de la partie vaginante prennent une conleur marron avec une bordure plus foncée en dessus el en dessous, ce qui donne à ces liges un aspect étrange.

Les rameaux varient beaucoup en nombre, en longueur, en position. Ils sont ordinairement dressés ou un peu étalés, quelquefois courbés en are, s’ils sont très-longs. Gaine basilaire courte, large, très-noire et luisante à la base, à quatre ou cinq dents courtes, obtuses, d'un brun mal et souvent bordées de blanc. Premier entre-nœud très-court, atteignant à peine, y compris ses dents, le quart, très-rarement le tiers de la gaîne caulinaire; les autres entre-nœuds sont souvent très-nombreux et trèslongs, à six ou huit côtes un peu plus prononcées que celles de la tige. Leur's gaines sont, sur de moindres dimensions, semblables à celles de la tige, mais beaucoup plus dilaties; la section transrersale offre les mèmes tissus semblablement disposés.

Épi apiculé linéaire-oblong; jusquà la sporose le «pédoncule» demeure si court que la base de l'épi est enreloppée par la gaine supérieure dilatée; a la sporose il s’allonge ct il est alors mou et d'une belle couleur rose. Douze à quatorze verticilles de sporanges, avec une dizaine de clypéoles, très-souvent noirs. Axe plein. Pendant la sporose, qui s'effectue de haut en bas, les épis sont d'un vert pâle et jaunàtre. Spores assez souvent abortives sur les épis des rameaux et des sujets grêles.

Variations. - «Celte espèce paraît être fortement influencée par la nature du " terrein dans lequel elle croit, car tantùt elle ne développe qu'un petit nombre de « tiges grêles et forl courtes, tantòt au contraire, et surtout lorsqu' elle sort d'un terrein " plus riche, on voit sortir de la touffe principale des tiges beaucoup plus grosses et plus « ramifiées qu'on croirait ne point appartenir à la mème espèce » (Vauch., .Ion. Prèl., 
1. :i33. Il semble dillicile de micux caractériser le protéisme de cel Equisetum, si justement nommé par le mème auteur E. multiforme. Il fiut cependant ajouter que ces diflérences de taille et d'aspect ne se trouvent pas seulement dans des terraing différents, mais quelles existent a côté les unes des autres et souvent sur un mème rhizome. De lì l'impossibilité absolue d"établir ces différenes en variétés dénommées, et la nécessite de les mentionner comme de simples formes presque individuelles et variables sous des influences qui sont loin d'être toujours appréciables.

La forme la plus vigoureuse el en mème temps la plus ordinaire en linance est trìsrameuse. Dans les haies, la moilié inféricure est nue, et les rameaux, commençant vers le milieu, vont jusquau sommet en diminuant de longueur. Souvent, dans les haies de Provence el d'Algérie, après que l'épi terminal s'est flétri el est tombé, des rameaux latéraux se développent avee vigueur, portent it leur tour des épis, puis des ramuscules qui se comportent de la mème maniere, el, au bout de deux ou trois ans, la plante, ainsi ramifice en tout sens, alteignant une dimension de 3 métres et poltant de soixante à quatre-vingts épis, justifie pleinement le nom imposé par Desfontaines et celte assertion de Mutel qui la dit "presque grimpante» ( Fl. d. Fr., IV, p. 169). Ciest particulièrenent sur cette grande forme que les gaines, fortement dilaties, perdent leurs lobes pendant lhiver et ensuite se colorent en marron sur la moitie ou les trois quarts supérieurs de leur longueur et donnent ainsi à la plante un aspect tout particulier. Vaucher avait fait de "ces belles gaines d'un brun clair o le caractere essentiellement distinetif de l'E. ramosissimum Desf. (Mon. Prêl., p. 369).

Dans les terres cultivées, les tiges sont en général moins élevées, grosses, rameuses dès la base el quelquefois mème si complétement rameuses à leur base que les rameaux constituent des groupes de tiges secondaires ayant la mème grosscur ct la même direction serticale que la tige principale. Cetle forme abonde en l'rovence daus les interstices des pierres formant les murs de soutènement des champs cultivés (Arles, Istres, Toulon, Grasse etc.).

Dans les prés, les licux déconverts, les sables el les graviers du bord des rivières, dans les terrinins salius des Bouches-du-Rhòne, elle a des formes plus grèles, plus élancées et plus nues; quelguefois mème elle est haute de plus de 75 centimètres, sans aroir un seul ramean.

Enfin lorsque dans ces mêmes lieux elle est coupée, ou foulée aux pieds, ou brnlée par le lioid, on mutile vers sa base diune lacon quelconque, elle produit i ses neuds une grande quantile de petits rameaux sonvent spicilëres; ces toufles cespiteuses ressemblent, à s'y méprendre, à celles de $I E$. variegatum et même plus d'une fois ont été prises pour clles. Elles s'en distinguent cependant facilement, mime il l'exléricur et sur le sec, par la longueur de leurs gannes, presque doubles de celles de l'E. variegatum, el aussi par ce que ce dernier porte un sillon carénal 
§ 4. - deschiption des eSPẺCES. - E. RAMOSISSIMUd.

prononcé sur les côtes des gaincs ainsi que sur celles des entre-nocuds à leur partic supérieure.

Formes anomales. - Les trois premières anomalies se présentent très-fréquemment sur celte espèce, oủ le déreloppement excessif des ramenux est presque l'état normal.

$9^{\circ}$ anom. Ameaux irréguliers. L’anneau est órdinairement très-saillant, et sur la plupart des sujels robustes celui de l'épi principal porte à ses lobes des sporanges et des spores, mais toujours imparfaits.

$17^{\mathrm{e}}$ anom. Infécondilé des spores. MI. Nilde dit de son E. clongutum, qui parait rentrer dans cette espèce: "Toutes les formes portent chez nous chaque année, comme je m'en suis convaincu, des spores incolores, stériles et des sporanges à cellules sans fibres spiralées » (Gef. Griypt. Schl., p. 468). Il n'en est pas de mème sur celte espèce en France, et des semis de spores m'ont donné chaque année de très-beaux sporophymes; mais néanmoins il n'est pas rare de la trouver telle que la mentionne M. Milde. Ainsi les épis dont la totalité ou la majeure partie des clypéoles sont très-noirs n'arrivent pas à sortir de la gaîne. Leur "pédoncule » rosé ne se développe point; ils demeurent petits et flasques, et, sur cette espèce et les suivantes, ne présentent alor's que des sporanges imparfaits. Au lieu de spores ils contiennent des masses informes, composées de quatre cellules agglutinées par une matière amorphe et renfermant quelques granulations verdatres. Ces épis paraissent avoir été frappés de mort avant leur complète évolution, car leur couleur noire intense est toujours celle que, sur les espèces de ce groupe, prennent les parties lésées ou ne fonctionnant plus.

Habitat. - Gette espèce parait s'arrêter au Nord sur une liggne partant de la vallée inférieure de la Loire, et s’avançant obliquement par Hayence, Halle, la Silésie, « la Lithuanie» (Lecoq, Géog. Got., IX, p. 313). En Suisse elle s'élève à 3000 pieds d'après M. Bernoulli, et au pied du Nont-Blane à 2000 mètres d'après M. V. Payot. Elle abonde dans le sud de l'Europe, le nord de l'Afrique et y remplace l'E. hyemale. Je l'ai vue d'Abyssinie dans le riche herbier de MI. Buchinger avec un Equiselum de l'île Naurice et un autre de divers points du cap de Bonne-Espérance qui doivent encore appartenir à celte espẻce, beaucoup plus répandue qu'on ne l'a cru jusqüici.

\section{Limites d'extension.}

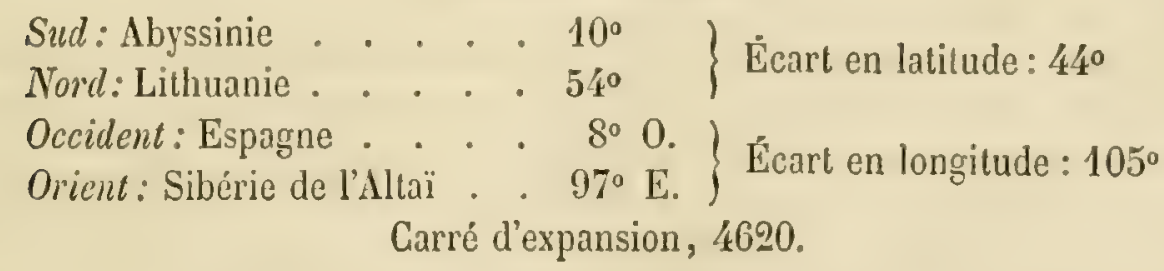

Station. - Je l'ai trouvée un peu dans toutes les positions; elle paraît toutefois affectionner les terrains sablonneux et un peu caillouteux. 
Loculitis fiengraises. - Vallée du Rhin, à Strasbourg, à Benfeld cle, dans les prai-

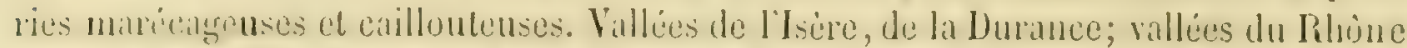
justue dans les sables salins des embouchures et dans le voisinage immédiat des salines a Rasuen, près d'Istres (Bonclies-du-Rhòne); bords de la Méditerranée, ou elle alleint son maximum de développenent; sables maritimes de la Gironde; vallée de la Dordusne jusquä Limeuil; vers l'embouchure de la Charente-Infurieure remonte fort loin dans la vallée de la Loire.

Époque de la sporose. - J'ai déjà dit plusieurs fois (p. 14, 208 elc.) que cetle espice ne résiste au froid que dans les endroits couverts et abrités, et que les liges périssent tous les hivers dans les lieux découverts en Provence et en Afrirue aussi bien que sur les bords da Rhin. Elle entre en végétation de bonne heure; dès le $1^{\text {er }}$ mai en l'rovence et ver's le 15 juin en $\Lambda$ sace, la sporose commence sur les épis principaux; clle est beancoup plus tardive sur les épis des rameaux et des tiges fuibles, ct quelquefois elle s'y produit encore en septembre.

Observation. - A l'état normal les tiges ne sont point « d'un vert blanchâtie » (Gren. el God., Fl. de Fr., III, p. 645); clles ne deviennent telles qu'en se desséchant, comme le fait sourent l'E. palustre. Elles sont an contraire d'un beau vert de jeunes fenilles d'oranger, tandis que l'E. hyemule adulte est d'un vert foncé el tirant sur le noir.

\section{Equisetum variegatum Schleich.}

Diagnosis princeps ab auctore denominationis nulla.

E. caule nudo; vaginarum dentibus lanceolato-subulatis. Fr. Weber et D. Mohr, Bot. Taschenb. a. d. Jalır 1807, 1) 60 .

E. variegatum. - Stirps gracilis tenella. Caules ex una radice plures, simplicissimi, erecli, nudi, glabri, amentiferi. Articuli longiusculi, sub-8-sulcati. Vaginis inferne nigricantes, ad dimidium usţue fiss $x$, dentibus lanceolato-subulatis, albidis, carina apiceque nigris, scariosis, suboctonis, vagin $x$ amentum cingentis maximis. Amentum terminale, parvum, oratum, nigricans. Receptacula partesque genitales ut in congeneribus. Obs. Caulibus simplicissimis, nudissimis, articulorum longitudine, raginarum colure, amenti denique forma Eq. Lyemali proximum, distinctissimum raginis ommibus dentatis, cxtera ut negligamus. Gum Eq. scirpoidi habitu tenello, colore, amento et articulorum ratione conrenit quidem, at vaginis in nostro multifidis, in illo trisetis ut labet cel. Michaux E. ejus interpres, toto colo differt. Weber et Molir, o. c., p. 447.

Diagnosis $n^{\mathrm{a}}$. - Spica acuminata, oroidea; pedunculo brevi. Caulibus numerosis, cxspitosis, atscendentibus aut decumbentibus, gracillimis, nudis, rarissime parce ramosis, valde sulcatis. Fistula centrali parva, 1/3; lacunis exterioribus 7-10 orato-rotundis, c. Vaginis paulo longioribus quam latioribus, dilatatis eliamque campanulatis; divisionibus lanceolatis in dentes membranaceos diaphanos, lanceolato-cuspidatos, persistentes cvadentibus. Ramis paucissimis, brevibus, cauli amulis; primo ipsorum internodio dimidiam circiter vagimc caulinx partem adxquante. Rhizomalibus fere ad superficiem repentibus. 
Description. - Les rhizomes rampent presque ì la surfice du sol et ne s'enfoncent guère au delà de 3 ou 4 centimètres. J'en ai obtenu qui dépassaient 3 mètres en longucur, et se ramifiaient tellement qu'ils formaient un véritable réseau sur le sol humide. Les entre-nœuds sont courts, 2 à 3 centimètres, irréguliers et tortueux, d'un diamètre qui ne dépasse guẻre 3 millimètres. Leur surface, très-noire et brillante, présente sept ou huit faces à peine marquées par des côtes très-obtuses. Les gaines sont assez longues, de 1 centimètre environ; la partic vaginante est étroitement appliquée, très-lisse, glabre el luisante comme les entre-nouds; les dents, qui occupent la moitié de la longueur totale, sont d'un brun moins foncé, fortement striées en long, linéaires-aiguës, souvent soudées par deux ou trois. Elles se détruisent assez vite, mais la partie vaginante persiste presque toujours, et, en tout cas, ne disparait jamais complétement en laissant un bourrelet régulier comme sur l'E. ramosissimun. Cavité centrale relativement grande et presque égale au tiers du diamètre total; lacunes corticales très-grandes, occupant plus de la moitié du rayon des tissus; larges, obovales, très-rapprochées de la cavité centrale, et à peu près également distantes de cette cavité et entre elles (pl. V, fig. 17). Lacunes essentielles relativement assez grandes, presque sur la même circonfẻrence que le bord intérieur des grandes lacunes. Faisceaux fibro-vasculaires très-petits, très-étroits, entièrement placés entre les lacunes corticales; cordons de vaisseaux très-apparents, très-rapprochés et rayonnants.

Tiges très-nombreuses et cespiteuses, couchées à la base, puis ascendantes. Les rhizomes partiels, qui se détachent obliquement du rhizome principal, se prolongent, suivant la même direction, en liges ascendantes, aux nœuds inférieurs desquelles naissent des tiges latérales tout à fait semblables aux tiges principales, sinon plus longues et plus fortes; el, l'année suivante, des nouds inférieur's de chacune de ces tiges il en naît de nouvelles formant des touffes épaisses assez rapprochées les unes des autres. $\Lambda$ u lieu de s'étaler en cercle autour de la première, toutes ces tiges secondaires sont presque parallèles et suivent la direction oblique du rhizome partiel auquel elles appartiennent. Elles sont très-grêles, presque lisses au toucher, simples ou très-rarement avec un ou deux rameaux. Leur hauteur normale est de 12 à 30 centimètres; moindre dans les lieux très-secs, elle atteint quelquefois 50 centimètres au bord des eaux. Entre-nœuds courts, de 15 à 30 millimètres, d'un diamètre de 2 à 3 millimètres; souvent arqués sur les sujets très-petils, ce qui fait paraitre les tiges tortueuses; sept à dix côtes saillantes, à carène plane ou un peu concave, bordées sur chaque còté de très-petites aspérités siliceuses. Sillons d'une largeur à peu près double, portant de chaque côté une ligne très-régulière de stomates. Cavité centrale dépassant à peine le tiers du diamètre total. Lacunes corticales presıue rondes, trèséloignées du pourtour extérieur, très-rapprochées de l'intéricur et entre elles (pl. VI* 
fig. 18\%. Cylindres indistincts. Lacunes essentielles très-petites, extéricurement tangrentes it la meme circonlërence que lus lacunes corticales; lissceau fibro-vasculaire saraneant entre les grandes lacunes, tries-ethoit, aree des cordons de raisseaux trèsmaripués, tres-rapprochés el presugue parallèles. Les faisceaux fibreux des còtes et des sillons sont à peu près de mème grosseur el de mème forme; très-larges et presque triangulaires, its s'araneent peu vers l'intérieur et forment sous l'épiderme une ceinture ondule qui n'est interrompue que sous les lignes de stomates. Les cellules à chlorophylle forment autour du fiisceau fibreux carénal une masse très-nettement circonscrite, subréniforme, bilobée, à lobes arrondis; elles manyuent sous le faisceau fibreux du milieu des sillons et ainsi se comportent à l'inverse de celles de l'E. liyemale qui, manquant vis-ì-vis des côtes, étendent leur masse sous les sillons (comparer les fig. 18 et 20 de la pl. VI).

Gaines courtes, 3 i 5 millimètres avec les dents, vertes et étroitement appliquées it la base (ce qui les fait paraitre encore plus courtes qu'elles ne le sont réellement), puis subitement dilatées en toupie sur les deux tiers supérieurs, surtout vers le haut des tiges spicifüres. Divisions lancéolées, avec un sillon carénal très-prononcé et se prolongeant sur les entre-nœuds. Dents persistantes, larges, très-aiguës, tout à fait membrancuses, blanches et un peu diaphanes. Celles des gaines les plus rapprochées de l'épi ont sur leur carène une ligne noire, el toutes présentent à leur base une large bande de la même couleur, qui contraste avec la blancheur de leurs bords membraneux, ct a valu à cetle espèce le nom que G. Bauhin lui a le premier appliqué et qu'elle a conservé depuis.

Rameaux nuls ou accidentels et alors entièrement conformes aux tiges. La gaîne basilaire est courte et trìs-large, d'un noir brillant; le premier entre-nœud est trèscourt el, arec la gaine, atteint ou dépasse ì peine la moilié de la gaîne caulinaire.

Épi apiculé ovoïde, long de 8 à 10 millimètres, d’abord presque sphérique et sessile dans la dernière gaine très-dilatée et presque en soucoupe, puis s'allongeant lor's de la sporose, ainsi que son "pédoncule» rosé, qui ne s’élève pourtant jamais beaucoup au-dessus de la gaine. Ameau très-large; six à neuf verticilles de six à huit clypéoles. Axe creux. Pendant la sporose, qui s'effectue de haut en bas, les épis normaux sont d'une belle couleur orange. Spores très-rarement abortives, si ce n'est dans les sporanges supéricurs dont le clypéole est très-noir.

Variutions et formes anomales. - Cette espèce présente deux rariations dans ses dimensions extrêmes. Lorsqu'elle croil sur le bord d'une eau courante, dans un terrain bon quoique caillouteux, elle pousse de longs rhizomes sur lesquels sont espacécs de belles tiges, longues de 50 centimètres, droites, presque solitaires, quelquefois un peu rameuses, el ayant jusqu'i treize ou quatorze còles; c'est la ve Wilsoni Ed. Newm. (Brit. ferns, p. $38 \mathrm{et} 39$ arec fig.) et la ve clatum Düll (Fl. Baul, I, p. 71). Elle est très- 
§ 4. - DESCRIPTION DES ESPËCES. - E. VARIEGATUM.

abondante le long de quelques fossés de la citadelle de Strasbourg. Lorsqu'au contraire l'E. variegatum croit dans des lieux très-découverts, sur un sol très-maiggre, trèscaillouteux et très-sec, ses rhizomes courts, tortueux, prorluisent de nombreuses touffes de tiges cespiteuses, petites, de 10 à 15 centimètres, grêles, couchées, ascendantes ou courbées ou tortueuses, n'ayant plus quelquefois quue quatre ou cinq côtes. Ce sont les formes extrêmes de la vé ecespitosum Döll (Fl. Bad., I, p. 71). Si je ne considère pas cette forme comme variété, non plus que la précédente, c'est parce que, si on arrache avec précaution les rhizomes de celle-ci, on constate qu'en dehors de l'eau et dans le sol maigre ils portent à l'autre extrémité des touffes de la petite forme cespileuse.

$3^{\circ}$ anom. Tiges rameuses après mutilation. Ne se produit guère que sur les sujets très-robustes.

$17^{\circ}$ anom. Infécondité des spores. Assez rare; j'ai même trouvé des spores bien conformées dans des sporanges dont la plupart des cellules étaient dépourvucs de fibres spiralées.

IIabitat. - Celte espèce aurait sa limite boréale en Norwége, d'après M. Fries (Summ. veg., p. 59); selon M. Milde elle atteindrait la Finlande et la Laponie (Ger. Crypt. Schl., p. 470). Elle croît assez fréquemment dans les trois parties de l'Angleterre, et se retrouve le long du Rhin depuis Mayence jusqu'aux Alpes de la Suisse, oủ elle s'élève à 7000 pieds selon M. Bernoulli. M. V. Payot l'a signaléc au pied du Mont-Blanc, à une altitude de 1100 à 1300 mètres; M. Zelterstedt, dans les Pyrénées, à 2194 mètres.

\section{Limites d'extension.}

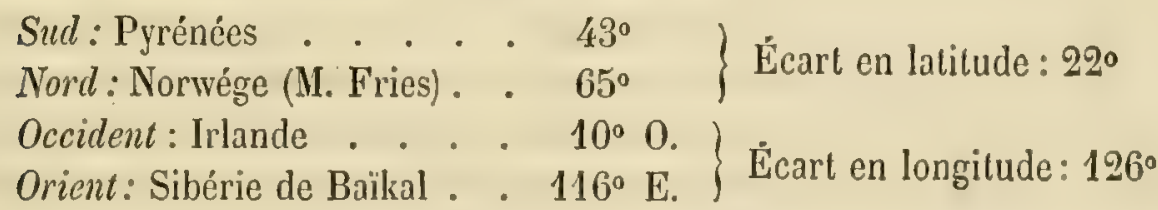

Carré d'expansion, 2772.

Station. - Voisinage des eaux, dans des licux caillouteux ou dans le gros gravier. Localités françaises. - Le long du Rhin en Alsace. Abonde à Strasbourg, à Seltz etc. Vallée de Chamounix (V. Payot). Pyrénées, à Tourmalet, entre Rencluse et la Maladetta (Zetterstedt). Les Mlpes, d'oủ cette espèce peut et doit sans doute descendre dans les vallées du Rhône et de la Durance; mais je n'ai pu l'y rencontrer, et ce que j'y ai vu, comme ce que j'ai, sous ce nom, reçu de ces vallẹes, se rapporte aux formes cespileuses de l'E. ramosissimum. M. Boreau la cile comme rare dans les alluvions de la Loire et de l'Allier ( $F l$. Cent., $3^{\circ}$ éd., II, p. 748). 
Ejpargu de la sporose. - Les tiges de cetie espèce sont, dans les licux trís-deconverts, humides et un peu gras, brûlées par les premières gelées, et elles courrent le sol de leurs articles siparés el hlanchis. Mais au pied des arlorisscaux, ou a la faveur d'un abri quelconque, elles persistent. Sur les graviers découverts, les tigges courtes grêtes et cespiteuses résistenl davantage; la plupart même échappent et poussent aussitot que cessent les gelées. J'en ai trouvé le 27 février $1859 \mathrm{cn}$ pleine sporose; les cellules des sporanges étaient pour la plupart sans fibres spiralies, mais les spores étaient bien conslituées et se développèrent en beaux sporophymes. J'ai mème trouvé en janvier, au moment de la fonte des neiges, des épis en sporose; mais les sporanges et les spores étaient incomplétement formés. Cette espèce semble ainsi pouvoir être toute l'année en ítat de vérgétation et de sporose, bien qu'elle ne soit pas celle qui résiste le mieux au froid. L'époque où la sporose est le plus abondante et le plus générale est en avril, pour les tiges qui ont passé l'hiver; puis en août et septembre, il y en a une seconde presque aussi forte, c'est celle des tiges de l'année.

\section{Equisetum tracilyodon Al. Br.}

Diagnosis princeps. - .....Durch die persistenten, schmalen und rauhen Scheidenzähne als eigene Form sehr kenntlich..... Flora, 1839, p. 305.

Diagnosis $n^{a}$. - Spica acuminata, oroidea, subsessili, plerumque abortiva. Caulibus numerosis,

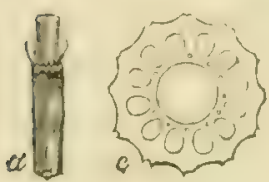
crespilosis, adscendentibus, gracilibus, nudis (post mutilationem aut rulnera ramosis), asperis. Fistula centrali parva, $1 / 4-1 / 3$; lacunis exterioribus $10-12$ ovato-radiantibus, c. Yaginis stricie adpressis; divisionibus linearibus, dento subulato, membranacco, spliacelato, persistente terminatis, a. Ilizumatibus haud profunde repentibus.

Description. - Rhizomes s'enfonçant irréguliẻrement; je n’ai jamais pu en obtenir de trìs-longs; ils mont paru constamment ou pourris ou desséchés et flasques, it ¿quelque distance des nombreuses tiges qui s'en élèrent obliquement en formant des touffes étalées. Leurs entre-nœuds sont moins gros (3 millimètres) et tout aussi courts que ceux de l'E. liyemale, à huit ou neuf faces d'un roux brun et sans tomentum. Les gaines sont asse\% longues; dents fortement strices, se pourrissant vite; partie vaginante assez longue, glabre ou quelquefois revêtue contre les dents d'un large el épais bourrelet de tomentum, qui devient moindre ou nul sur les vieilles souches ou sur celles qui ont été exposées à l'air.

La cavité centrale est petite, et n'occupe pas le quart du diamètre tolal; lacunes corticales obovales, petites, à peine un peu plus rapprochées de la cavité centrale que du pourtour extérieur (pl. V, fig. 18). Lacunes essenticlles très-petites, langentes il l'intérieur de la circonférence, à laquelle les grandes lacunes le sont extérieurement. 
Faisceau fibro-vasculaire très-petit, s'avanģant entre les grandes lacumes; cordons de vaisseaux très-rapprochés.

Tiges nombreuses, ascendantes et le plus souvent disposćes en touffes étalées, moins rudes, moins grosses et moitié moins longues que celles de l' $E$. hycmale; d'un vert pâle souvent lavé de rougc; nues ou portant un ou deux rameaux après mutilation. Entre-nœuds dix à douze, à peu près tous de même longucur et alteignant au plus 5 centimètres, sur un diamètre de 2 millimètres, rarement tout à fait droits, mais reproduisant la courbure des tiges ascendantes. Toutefois, les grandes inflexions ont lieu dans les gaines au-dessus du noud (royez p. 188 et 189). Dix à douze côtes très-marquées, quoique peu en relief, à carène plane bordée latéralement d’aspérités siliceuses transversales; sillons d'une largeur presque triple, peu profonds, avec une ligne de stomates de chaque côté. Gavité centrale petite, occupant le tiers du diamètre total; lacunes corticales obovales, rayonnantes, plus éloignécs du pourlour extérieur que de l'intérieur et entre elles (pl. VI, fig. 19). Lacunes essentielles très-petites, tangentes à l'intérieur de la circonférence, à laquelle les grandes lacunes le sont extérieurement. Faisceau fibro-vasculaire étroit et pénétrant un peu entre les lacunes corticales. Cordons vasculaires rapprochés, rayonnants, presque parallèles. Les deux cylindres sont généralement marqués par une guirlande très-ondulée, qui contourne à l'extérieur les faisceaux fibro-vaseulaires et passe entre les grandes lacunes el la cavité centrale. Les fibres corticales forment, sous les côtes et sous le milieu des sillons, des faisceaux triangulaires qui pénètrent profondément vers le centre et s'interposent entre les cellules à chlorophylle; celles-ci sont disposées au-dessous de chaque ligne de stomates en groupes arrondis vers l'extérieur et se prolongeant un peu de chaque côté des grandes lacunes.

Gaines une fois plus longues que larges, appliquées; divisions linéaires peu convexes, très-nettement marquées d'un sillon carénal qui se prolonge jusqu'au milieu de l'entre-noud, et de deux sillons latéraux presque aussi prononcés; dents très-aiguës, noires sur la ligne médiane, bordées d'une large membrane blanche, très-persistantes ct ne tombant que par lambeaux après le premier ou après le second hiver ${ }^{1}$. Une large bande, d'un noir très-intense à ses bords, règne sur la moitié supéricure de la partie vaginante, laquelle est bien plus persistante que sur l'E. hyemale.

Rameaux nuls ou succédant à une mutilation; même structure, mêmes gaînes que sur les tiges. Six ou sept côtes assez saillantes. Gaîne basilaire d'un nuir brillant; premier entre-nœud très-court, et avec la gaine atteignant environ le tiers de la gaine caulinaire.

\footnotetext{
' Je ne mentionne point ici les aspérités qui existent sur la lignne dorsale des dents et qui ont valu à celte plante le

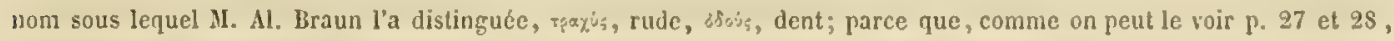
ces aspérités se montrent à celte région sur la plupart des espèces et sur toutes celles de ce groupe.
} 
Ejpi apirulé, ovoïle, long de 7 a 10 millimètres, sescile dans la dernière gaine, qui est tris-dilatée et l'enveloppe presque entirement. Sept ou huil verticilles de six ou sept clypules noirs. Axe creux; cavité trés-petite. Sporose s'accomplissant mal ou pas du tout. Spores presque toujours abortives.

Formes unomules. - S" anom. Tiges derenant rameuses après mutilation, comme sur l'E. liyemale. Au printemps de 1861, c'est-i-dire aprés linondation de décembre 1860 , les sujets rameux et id plusicurs épis se rencontraient frérquemment sur les digues du Rhin, à Strasbourg.

$17^{\circ}$ anom. Infécondite des spores (voir p. 153). "Tandis que les êpis de l'E. hycmale répandent librement leur graine ou leur pollen, je n'ai dans aucun cas vu ceux de l'E. Muclinii en faire autant. Ils paraissent mûrir sans sortir entièrement de la gaine qui les enveloppe. (Brichan, Descr. Eq., p. 374).

I. Milde dit "n'aroir jamais ru sur cet Equisetum de spores bien constituées et fécondes. " (E. litt., p. 29; Gef. Crypt. Schl., p. 405, et Syst. Eq., p. 146). J'en ai rencontré plusieurs fois, mais toujours en tres-petit nombre, une ou deux sur des milliers de stériles. Ordinairement le sac membraneux de cette plante offie des cellules sans fitres en spirale el renferme des spores irrégulieres, de diverses grosscurs, mais toujours plus petites qu'une spore normale (pl. IX, fig. 10, 11). Elles paraissent incolores et se composent d'une mombrane extéricure ct d'une autre membrane enveloppant une masse intéricure toule remplic de pelits grains très-légèrement verditres. Quelques-uns, en tris-petit nombre, ont presque la grosseur normale et présentent vers leur éfquateur un commencement de fissure en spirale. Le tout est accompagné d'une matière mucilagincuse, à demi-visqueuse, qui se dissout lentement dans l'cau el pas du tout dans la potasse caustique. Létude microscopique de ces spores (roir p. 9't permet de reconmaitre qu'elles ont étí arrêties vers l'avant-dernière phase de leur évolution.

Ilablitut. - Ir. Ed. Newman signale celte plante dans le nord de l'Irlande et en Écosse; II. Mrilde, en Silésie; M. Döll, dans le grand-duché de Bade. Elle cst assez fréquente sur les digues du Rhin en $\Lambda$ lsace. M. V. Payot la mentionne au pied du Hont-Blane, à une altitude de 1200 i 1300 mètres; M. Willkomm, en Espagne. Je ne possiede pas assez de domées certaines pour essayer de déterminer les limites d'extension de celte plante.

Station. - Sur les bords des rivières et particulièrement sur leurs digues.

Localités fiunguise's. - Sur les dignes du Rhin, sur les remblais du chemin de fer fres du Rhin, it Strabbourg; très-abondante entre Seltz (Bas-Rhin) et le Rhin; II. (irenier (Fl. de Fr.) la mentionne dans l'Ouest, sur l'autorité de M. Boreau, lequel

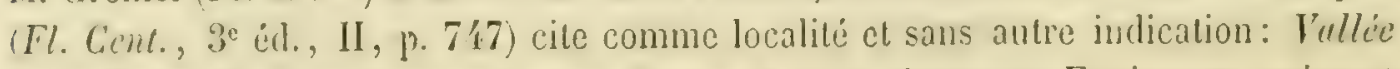
de le Luire. Mr. Lluyd rapporte, arec donte, a cette plante un Equisetum croissant 
dans les lieux sablonneux de Pierre-Percée, dans la vallée de la Loire ( $F l$. Onesl, p. 547).

Époque de la sporose. - Comme celle de l'E. hycmale, cl même un peu plus tardive. Les sporanges semblent au mois d'août à leur maximum de développement, et ce n'est qu’à celte époque que j'ai trouvé quelques spores bien conformées. Je n'en ai jamais vu une seule dans les épis dont l'hiver avail retardé l'évolution jusqu'au printemps.

Cette plante supporte les froids de l'hiver absolument comme l'E. hyemale, bien qu'elle croisse constamment dans des endroits tout à fait décourerts. C'est à peine si les plus grands froids d'Alsace (de — 20 à — 23 degrés) donnent à ses tiges une couleur un peu rougeâtre. Dès les premiers jours du printemps, le développement recommence, et il est alors très-facile de reconnaitre à la base de chaque entre-nœud la partie nouvellement formée. Elle est d'un vert clair et tendre, tandis que la partie supérieure est d'un vert très-foncé ou souvent rougie par le froid (voir p. 61).

M. Milde dit qu'en Silésie « cette plante périt tous les ans dès le mois d'octobre, et produit en mai de jeunes pousses qui fructifient encore la même année, en juillet » (Gef. Grypt. Schl., p. 465). Est-ce bien la même plante?

Obscrvation. - A l'exception de M. V. Payot, qui assigne pour station à celte plante "les licux stériles et sablonneux très-secs », tous les auteurs mentionnent sa présence sur les bords et particulièrement sur les digues des rivières. Noi-même je n’ai pu la trouver' en Alsace que sur les digues du Rhin, ou sur des terrains remués pour l'établissement de ces digues, ou sur les remblais du chemin de fer tout près du Rhin et toujours en des lieux déconverts el sur des terrains maigres et cailloutenx. Cette double circonstance, à laquelle se joignaient l'imperfection ordinaire des spores et la croissance en touffes assez ordinaire sur les rhizomes coupés, m’a porté plus d'une fois à douter de la valeur de cette plante comme espèce, et à penser que des fragments de rhizomes d'E. liyemale coupés et transportés dans les terres du bord des rivières ou arec les matériaux des digues, pourraient bien avoir produit ces nombreuses tiges avec leurs formes grêles el leurs sporanges imparfaits. D'autant que sur ces mêmes digues d'Alsace il est très-rare, pour ne pas dire impossible, de trouver un pied d'E. hycmale normal, tandis que dans les bois traversés par les digues, là où le sol, plus riche en humus, n'a pas été remué, on trouve en abondance les belles et fortes tiges de l'E. hyemale, et cela à un mètre du talus des digues couvertes d'E. trachyodon ${ }^{1}$. Mais, d'une part, les différences indiquées plus haut dans la disposition des cellules à chlorophylle, quelque légères qu'elles soient, et, d'autre part, l'autorité

\footnotetext{
' Cette hypothèse est corroborée par l'observation suivante de M. Birchan: a In no instance have I seen $E$. hyemale * assiociated with either of the other two, although $E$. Mackaii grows in is immediate vicinitys (Descr. Equis., p. 371).
} 
imposante de l'auteur de l'eppice, M. A1. Braun, de MIM. Birchan (Deser. Eq. p. 369 it 377 ) et Ld. Newman (Brit. ferns, p. 29), gui la considerent comme une espece distincle, de .1. Milde qui l'établit comme sous-espèce (Cíf. Crypt. Schl., p. 466), de M. Döll, rui en fait une variélé ( $\mathrm{F}$. Bad., p. 69), ne me permettent pas de rester sans quelyues serupules dins mon opinion el de réduire cetle plante à n’ètre qu'une variation de l'E. hyemule, variation due à des circonstances locales et qui ne se prolongerait que par la permanence des mèmes circonstances.

Voici en quels termes M. Al. Braun s’est exprimé sur cette plante, dans la réunion des naturalistes et médecins allemands ì Fribourg en Brisgau, le 25 septembre 1838 :

"D'un còté, cel Equisetum est très-reconnaissable comme forme particulière par ses gaines a dents persistantes, étroiles el rudes; mais, d'un autre côté, par de nombreux rapports et surtont par la présence de sillons carénaux distincts, il lient tellement le milicu cntre l'E. higemale et l'L. v'ariegatum qu'on ne peut s'empececher de recomnaitre une liaison plus étroite entre ces espices, et de les regarder comme des sous-espèces d'une espèce principale. En partant de l'E. hycmale genuimum, les E. trachyodon et veriegatum constituent une séric de formes, dans laquelle les sillons carénaux se montrent toujours de plus en plus distincts et qui aboutit à l'E. scirpoides, l'espèce la plus nettement tranchée, sur laguelle les sillons carénaux sont presque aussi prononcés que les sillons commissuraux de la tige. Dans un sens opposé vient se rattacher à l'E. hyemale genuinum, également à titre de sous-espèce, un autre Equisetum très-répandu et trop sourent méconnu, riche en formes diverses et en synonymie, l'E. ramosun (rumosissimum Desf., clongatum Willu., proccrum, Pollini, illyricum IIoppe), sur lequel les sillons carínaux ont complétement disparu. La première série (E. hyemale genuinum, trachyodon, variegatum, scirpoides) parait appartenir davantage au Nord; la seconde (E. ramosum et varielates) paraît plus propre au Sud, commenȩant dans le sud de l'Allemagne el s'étendant i travers tous les pays transalpins de l'Europe, peut-être même à travers toute l'A ririque. Toutes les especes ou sous-espèces dont nous parlons ici constitucnt une division propre, celle des Equiseta hyemalia, di-tincte par ses rejetons verts en liver, ne mûrissant leur fructification que la scconde année, ayant un pédoncule court, un épi acuminé et les stomates rangés en ligne » (Flora, 1839 , p. 305 et 306).

II. Nilde affirme que M. Al. Braun ne regarde plus celte plante que comme une forme de l'E. lingemale.... "Selbst von dem Entdecker als frorm von E. liyemale betrachtet..... ) (Syst. Eq., p. 184). 


\section{Eguisetum iryemale L.}

Diagnosis princeps. - E. caule nudo scabro basi subramoso L., Sp. pl., Ed.1 a, p. 1062.

Diagnosis $n^{\text {a }}$ - - Spica acuminata, oroidea, subsessili.

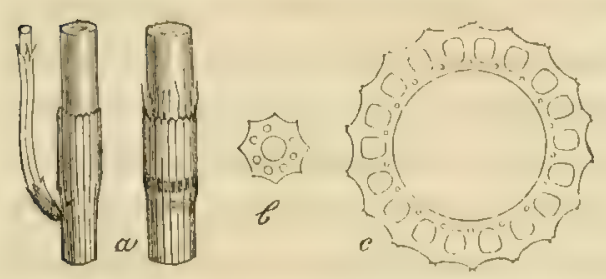
Caulibus solitariis aut paucis, erectis, altissimis, rigidis, nudis (mutilati aut vulnerati evadunt parce ramosi), asperrimis. Fistula centrali vasta, 2/3; lacunis exterioribus 18-24 ovato-radiantibus, $c$. Vaginis longis stricte adpressis; divisionibus linearibus, dente membranaceo, sphacelato, subulato, seu persistente, seu caduco et tunc lobo brevi, spisso, gibboso, nigerrimo terminalis, a. Rhizomatibus profunde repentibus.

Description. - Les rhizomes s'étendent parallèlement à la surface du sol de 3 à 7 décimètres de profondeur; ils sont très-longs, très-peu ramifićs, et j’en ai obtenu qui dépassaient 4 mètres en longueur et dont je n'avais ni le commencement ni la fin, car ils étaient d'une grosscur uniforme et cassés par moi aux deux bouts. Plus ils sont profonds, plus ils sont gros. Les rhizomes verticaux égalent à peine la grosseur du bas des tiges qu'ils supportent (3 à 4 millimètres). Ils s'élèvent isolés, à des distances très-inégales, quelquefois d'un demi-mètre, souvent beaucoup plus considérables. Leurs entre-nœuds sont d'autant plus courts qu'ils sont plus rapprochés de la surface du sol, oủ ils n'ont plus guère que 2 à 3 centimètres. Leurs lix à douze faces, à peine distinctes, sont d'un noir mat et couvertes d'un tomentum très-caduc. L'épiderme a été décril p. 18, 20 et 21 et figuré pl. II, fig. 13, 14, 15, 16. Gaines relalivement peu allongées, 12 centimètres environ; partic vaginante très-courte, d'abord tomenteuse, puis bientôt glabre; dents longues, étroites, très-aiguës, souvent soudées par deux ou par trois; se détruisant assez vite, ainsi que le reste de la gaîne, qui laisse une cicatrice irrégulière. Cavité centrale petite, occupant le quart du diamètre total. Lacunes corticales obovales, très-petites; leur grand diamètre est à peine égal à l'épaisseur des masses cellulaires qui les séparent du pourtour extérieur et du pourtour intérieur, el au rayon de la cavité centrale (pl. V, fig. 19, 20). Leur largeur est à peu près égale à celle de leurs cloisons de séparation. Lacunes essentielles trèsprononcées, rondes, très-éloignées de la cavité centrale et tangentes à l'intéricur de la circonférence, à laquelle les grandes lacunes sont extérieurement tangentes. Faisceau fibro-vasculaire très-petit, placé entre les grandes lacunes vers leur partie la plus étroite. Cordons de vaisseaux très-rapprochés et se touchant presque par leur bord extérieur.

Tiges isolées, très-élevées ( 1 à $\left.1^{\mathrm{m}}, 30\right)$, droiles, fortes et raides, d'un vert intense, trèsraboteuses au toucher, absolument simples et ne produisant quelques rameaux qu'après 
mutilation "; ce n’est également qu'après mutilation de la tige principale qu'un même neud du rhizome vertical donne naissance i deux ou trois tiges rapprochées comme celles de l'E. trachyodon. Entre-nauds quinze ì dix-huit très-longs, dépassant souvent 1 decimètre vers le milieu de la tige, sur un diamètre de 5 i 6 millimètres, parfaitement droits; dix-huit à ringt-quatre còtes trìs-marquées, mais peu saillantes, à carène plane et bordée d'aspérités siliceuses transversales; sillons d'une lirgeur presque triple, peu profonds, ayant de chaque côté une ligne régulière de stomates séparés par des intervalles égaux ì leur longueur ( $\mathrm{pl}$. V, fig. 4). Ces liggnes sont visibles à l'ceil nu ou arec un faible grossissement, sous forme de deux lignes de points blanchâtres. Cavité centrale grande, égalant ou dépassant un peu les deux tiers du diamètre total; lacunes corticales arrondies ou obovales, un peu quadrangulaires, rayomnantes, occupant la majeure partic de l'espace dans l'ensemble des lissus; séparées de chacgue pourtour extéricur et intérieur et entre clles par des bandes cellulaires érgales el très-étroites (pl. VI, fig. 20, 21). Lacunes essentielles très-petites; leur centre est placé sur la circonférence, à laquelle les lacunes corticales sont tangentes extérieurement. Faisceau fibro-vasculaire très-étroit et pénétrant très-avant dans la cloison qui sćpare les grandes lacunes. Cordons vasculaires très-apparents, très-rapprochés, presque parallètes, rayonnants. Sur un grand nombre dindividus, la distinction des deux cylindres est marquée par une guirlande de petites cellules, qui passe i égale distance entre les lacunes corticales et la cavité centrale, et pénètre dans les cloisons de séparation en contournant le faisceau fibro-vasculaire; sur d'autres on ne trouve que les traces de cette guirlande qui se confond facilement avec les faisecaux fibro-vasculaires. Les fibres corticales forment sous l'épiderme une ceinture qui n'est interrompue que sous les lignes de stomates; réduites à un ou deux rangs sous les sillons, elles forment sous les côtes un faisceau triangulaire très-étroit, mais très-allongé et qui pénètre entre les cellules corticales presque jusqu’à la rencontre du faisceau fibro-vasculaire. Les cellules à chlorophylle entourent les grandes lacunes d'une bande verte semi-circulaire s'élargissant vers les stomates et les côtes; ce qui leur donne un contour extérieur quadiangulaire, avec des angles arrondis et des faces un peu concaves.

Gaînes longues, itroitement appliquées, paraissant un peu dilatées à leur partie supérieure, parec que les nouds sont toujours beaucoup plus étroits que le milieu des entre-nouds. Divisions linéaires tout i fait semblables vers leur base aux cótes qu'clles continuent; puis, sur les deux tiers supérieurs, elles deviennent planes, sépa-

\footnotetext{
- Yoy. ci-dessus p. 143, 30 anomalie. A l'état normal, cetle espèce est alısolument sans rameaux, et c'est encorc aller trop loin que de dire avec $\mathrm{M}$. Grenier $(\mathrm{Fl}$. de Fr., IIl, p. 645): tiges nues et rarement subrameuses; avec A. Bernoulli (Gef. Krypt. Schw., p. 75): "caules simplices vel rarius parce ramosi:" ct enfin arec M. Milde (Gef. Crypl. Schl., p. 462): Etengel astlos, selten mit zerstreuten .Esten. "Koclı avail fort bien remarqué qu'elle ne devient rameuse qu'après mutilation (Sym., Ku. 3a, p. 725).
} 
rées par un sillon commissural très-étroit ct marquées d'un sillon carénal; enfin elles se déchirent en dents très-étroites, subulées, noires, déjà sphacélées dans le bourgeon et très-rapidement caduques, ou persistant quelque temps sous forme de soies noires. Après leur chute, qui arrive tôt ou tard, les divisions sont ì leur extrémité arrondies, épaissies et rejelées en dehors, comme charnues et d'un noir brillant; ce qui a fait dire que la gaîne est dépourvue de dents et se termine par de petits lobes arrondis, noirs, épais et charnus, aspect tout à fait particulier à cette espèce (voir p. 58 et 59). Très-souvent les gaînes ont, à leur bord et vers leur milieu, des bandes noires plus ou moins larges; sur les tiges de seconde ou de troisième année il n'est pas rare de les voir d'abord devenir blanches, puis tomber par fragments. Vers le haut de la tige les divisions des gaines sont moins planes et plus semblables aux côtes qu'elles surmontent. La gaîne qqui est immédiatement sous l'épi est dilatée, à demi-membraneuse, et conserve ses dents, même sur les tiges oủ les dents des autres gaînes sont le plus caduques.

Rameaux nuls, ou paraissant seulement après une mutilation. Leur structure est absolument celle des tiges, sauf les dimensions; huit à dix côtes saillantes. Gaîne basilaire d'un noir brillant; premier entre-noud excessivement court, se réduisant presque à sa gaîne, et avec elle égalant à peine le tiers de la gaîne caulinaire; ce qui a fait dire à II. Milde que, sur cette espèce, «la base de chaque rameau est entourée par deux petites gaines basilaires, et non par une seule, comme sur les autres Equisetum » (Rev. crit., p. 111, trad.; Gef. Crypt. Schl., p. 463).

Épi apiculé, ovoïde, de 10 à 12 millimètres de long, strié longitudinalement par l'impression des saillies intérieures de la dernière gaîne, dans laquelle il est presque sessile. "Pédoncule » court et conique; huit à dix verticilles de sept ou huit clypéoles; axe creux; cavité très-petite; sporose s'accomplissant de haut en bas, mais lentement et difficilement; les épis sont alors verdâtres; les clypéoles se séparent très-peu. Spores souvent abortives sur les épis arrêtés par l'hiver dans leur développement; les clypéoles sont alors noirs.

Variations et formes anomales. - Cetle espèce est sans contredit la moins variable dans sa forme générale ainsi que dans la forme de ses parties; et elle ne présente que deux anomalies, les plus faibles de toutes.

$3^{\mathrm{e}}$ anom. Tiges devenant rameuses après mutilation. L'examen de très-nombreux individus récoltés aux environs de Strasbourg, où cette plante abonde au point d'y être un objet de commerce, m'a permis de constater que ses tiges ne portent des ramcaux latéraux qu'après une mutilation, et ce, en très-petit nombre et seulement aux nœuds les plus rapprochés de la mutilation. Par suite de la parfaite nudité et de la durée des tiges de cette espèce, cette anomalie s'y montre plus saillante que sur les tiges des premiers groupes, lesquelles sont détruites par les gelées d'automne avant que les 
bourgens aient pu se développer à la suite d'une mutilation. Quelfuefois des épis dija formés en automne sont alleints par les froids ou rendus stériles par une autre cause; des rameaux naissent alors au-dessous de la gaine qui les enveloppe on de celle qui la précède, et ordinairement ces rameaux sont cux-mèmes spicifëres; souvent mème ils sont si courts que les épis paraissent sessiles et groupés au-dessous de l'épi principal devenu stérile, comme les épis d’un Scirpus; particularité que Vaucher avait déji signaléc et figurée: "Sourent les sommités se terminent par un épi noir el coma pact, enveloppé dans sa gaine et entouré d'épis plus courts, qui donnent à la "plante l'apparence d'un Schoin » (Mon. Prêl., p. 37/, pl. IX, fig. 1). Aprés l'inondation de décembre 1860 , les sujets rameux et portant plusieurs épis étaient trèsfréquents dans les bois du bord du Rhin, au printemps de 1861.

$17^{\mathrm{e}}$ anom. Infécondilé de's spores. Assez fréquente, quoique affectant rarement tout un épi.

Ilabitat. - Gette espèce se rencontre dans tout le nord de l'Amérique et de l'Europe, en Laponic, en Russie, en Suede, en Norwége, en Anglelerre, en Prusse, dans les Alpes de Suisse et dans une grande partic de la France.

\section{Limites d'extension.}

$$
\begin{aligned}
& \left.\begin{array}{l}
\text { Sud: dép'. des Basses-Alpes. } \quad 44^{\circ} \\
\text { Norl: Laponic . . . . } 70^{\circ}
\end{array}\right\} \text { Écart en latitude: } 26^{\circ}
\end{aligned}
$$

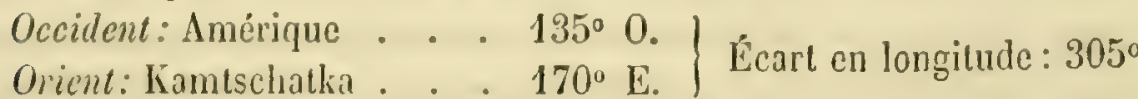

$$
\begin{aligned}
& \text { Carré d'expansion, } 7930 .
\end{aligned}
$$

Station. - Les bois humides el les marais à fond caillouteux. Sa limite supéricure de régétation parait ètre entre 1000 à 1200 mètres dans le Cantal (Lecoq).

Luculités francraises. - Bois et prairies marécageuses de la plaine de l'Alsace, des Basses-Alpes de Provence (a Vergons), de la Savoie (i Chambéry) ctc.. Eille parait manquer entièrement dans la basse Provence el dans le Languedoc maritime, et y être remplacée par l'E. ramosissimum, lequel est mentionné sous le nom d'E. hycmale dans les anciennes flores méridionales. Eille reparait dans le Lot, le Cantal, le Puyde-Döme (Lecoq). Eille est dissémincée el rare dans le centre; on l'a rencontréc près d'Autun, dinns la Haute-Loire, dans l'Allier, dans la Nièvre, dans le Cher, dans le Loiret etc. (Boreani). MI. Cosson la cite aux environs de Paris, Etang-Neuf près Iloudan (Dänen) et au bois de Valvin (Woods); Ouest (Boreau).

Éporue de le sprorose. - Les liges nouvelles de celte espice sont les plus tardives de toutes a se montrer. Vers le mois de septembre elles apparaissent sous forme de 
bourgeons. De cette époque à la fin du mois de novembre, elles atteignent une longueur de 2 centimètres au plus, ct elles restent en cet état an-dessous de la surface du sol jusqu'à la mi-avril; c'est alors seulement el quelquefois plus tard qu'elles sortent de terre. Elles se développent assez rapidement, et depuis les premiers jours d'août jusqu'à l'automne on pent trouver sur ces tiges de l'année des épis bien conformés et mème en état de sporose. Mais sur cette espèce, comme sur l'E. trachyodon, cet acte n'atteint point tous les verticilles de l'épi avec la simultanéité ou la rapide succession qu'on voit aux autres espèces; l'émission des spores a lieu avec lenteur, d'une manière imparfaite, sur quelques verticilles seulement, ou d'un scul côté; ce même il n'est pas rare de trouver des sporanges bien conformés, renfermant des spores tout à fait normales, et qui demeurent cependant sans s'ouvrir et sans répandre leurs spores. Les premiers froids de l'hiver viennent interrompre cette tardive émission, ainsi que l'évolution des épis les plus en relard. C'est là à peu près le scul effet de l'hiver sur cette plante, à laquelle une température de - 20 degrés ne paraît pas faire subir de modification appréciable. Les épis dont le froid a arrêté l'évolution deviennent quelquefois mous, flasques et abortifs, mais le plus souvent ils persistent et se développent alors au printemps. Assez constamment, pour ne pas dire toujours, sur ces épis arrêtés par l'hiver, le sac des sporanges esî sans fibres spiralées et les spores sont sans matière verte. Contrairement à l'opinion de plusieurs auteurs, et peut-être à la signification de son nom linnéen, celte espèce ne «fructifie» point pendant l'hiver. Ses épis persistent seulement pendant cette saison, et comme alors l'absence de feuilles dans les bois où elle croît, permet de mieux l'y voir et de mieux l'y récolter, lì oủ elle est un objet de commerce, cette circonstance aura sans doute induit en erreur. A Strasbourg, sur d'énormes quantilés de fagots que j’ai vu récolter à celte époque, je n’ai jamais pu trouver un seul épi véritablement en état de sporose; il n’y avait que les restes fanés des épis d'automne ou les épis à demi-développés et dont l'évolution étail suspendue.

Les différences dans l'époque de croissance et de sporose suffiraient pour distingucr comme espèces les E. hycmale, variegatum et ramosissimum. Ce dernier a ses premières tiges en pleine sporose au nois de mai, alor's que celles de l'E. hyemale commencent à peine à sortir de terre, pour' ne porter épi qu'en été et plus tard. L'E. variegatum est toute l'année en état de végétation el de sporose. C'est donc aller trop loin que de dire arec M. Al. Braun: «Le groupe des Equisela hyemalia a pour caractère distinctif des tiges vertes en hiver et qui ne mûrissent leur fructification que la seconde année» (Flora, 1839, p. 306).

Obscrvations. - J'ai dit plus haut que les tiges nouvelles se montrent déjả sous forme de bourgeons en septembre, mais qu'elles restent en cet état jusque vers la fin d'avril. Si on les examine pendant ce temps, on remarquera ce qui suit: 
10 Ces bourgeons, coupes longitulinalement, offrent deja i peu pres autant de gaines el de nauds (guen auront plus tard les tiges arlultes (de treize à dix-sept).

go Sur celle espéce, comme sur les autres, toutes les gaines sont, pendant le jeune igge, continues et non lobulées i leur terminaison; la division en lobes n’a lieu qune par un déchirement mécanipue, résultant de la pousséc que font les entre-nouds supericurs pour sortir de la gaine enveloppante des cutre-neuds inféricurs, ainsi que je l'ai décrit précédemment (p. 12 el 58).

$3^{\circ}$ Enfin ces bourgeons sonl enduits d'une substance incolore, d'aspect gílatineux, el assez abondante pour qu'on puisse en détacher des grumeaux de 1 i 2 millimètres de diamétre. Apres vingt-quatre heures de sejour dans l’eau, ces grumeaux ne m’ont présenté aucune modification appréciable; ils sont immediatement solubles par ibullition dans une solution de potasse caustique, et la liqueur, versée dans l'eau ordinaire, précipite en flocons blanchâtres.

L'examen, mème le plus superficiel, d'une jeune gaîne d'E. lyyemale permet de constater que la moilié inférieure est, comme l'entre-nœud qu'clle couronne, épaisse, verte et munie de lignes régulières de stomates. Vers le milieu, les côtes se renflent en un épaississement de couleur brune, d'oủ résulte un anneau coloré qui divise la gaîne en deux régions. La région inférieure doit persister et constituer la gaine proprement dite. La région supéricure qui, en se déchirant, doit former les dents, est membraneuse, très-mince dans les sillons, plus ćpaisse sur les côtes el toujours de couleur brune. Elle est de plus trés-étroitement contractée en une pointe pyramidale, anguleuse, cxactement semblable (sauf les dimensions) à l'acumen qui termine les épis du groupe des Ityemalia, et l'acumen de chaque gaine pénètre dans la base de celui que forme la gaîne superposée.

Si l'on suit avec quelque attention l'évolution des tiges, on trouve que, par rapport à celte partie supérieure de la gaîne, il arrive de trois choses l'une:

$1{ }^{\circ}$ Ou bien elle se déchire, par la poussée des entre-nauds supérieurs, en lanières ou dents extrêmement délicates, qui persistent en continuation des côtes de la gaîne et au-dessus de leur épaississement brun, se dessèchent très-vite, se crispent el s'enroulent même assez souvent. Or, comme on avait attribué à l'E. hyemale le caractère spécifique de gaines tronquées sans lobes, on fit avec les sujets qui portaient des lobes paliacés une espèce ou une variété, sous les noms d'E. palcacenm ou d'E. hyemale $\mathrm{v}^{\mathrm{as}}$ paleaceum.

20 Ou bien, après le déchirement en dents, ces dernières parties se dessèchent tout de suite, se síparent tout a fait et tombent. On a alors le type le plus ordinaire de l'E. hyemale'.

\footnotetext{
- C'est le type de Linné. Sur l'cxeniplaire original du Species pluntarum, conservé à Londres arec l'herbier de
} 
$3^{\circ} \mathrm{Ou}$ bien encore, quand le bourgeon arrive à l'air libre, l'enveloppe conique

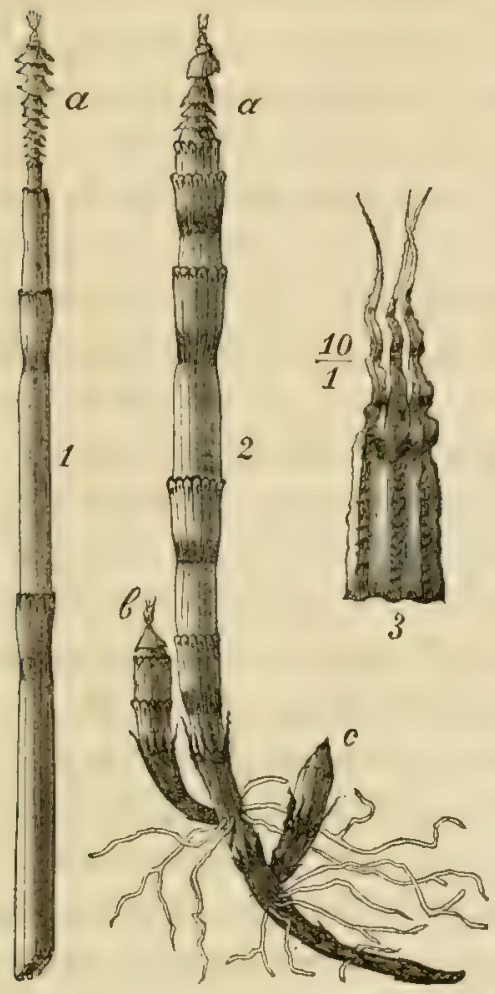

En 1842, M. Birchan a mentionné, sans l'expliquer, ce singulier mode de terminaison de quelques tiges. Je crois devoir donner la traduction du passage entier: $₫ \mathrm{Au}$ sommet de quelques liges de l'E. hyemale j'observai une pile de gaînes (a pile of sheaths) petiles, d'un brun foncé, membraneuses, élastiques, coniques, renversées, de la même substance que les dents de la gaîne qui enveloppe l'épi, augmentant de largeur en remontant et s'enveloppant si étroitement les unes les autres qu'on ne voit que le bord de chacune, à l'exception de la plus haute et la plus large qui donne au sommet de la pile une forme conique. Bien qu'ayant à peine un pouce de long, cette pile ressemble à un avortement de la plante en sens inverse. La gaine qui en constitue l'extrémité inférieure est d'abord placée sur la plus haute gaine et recouverte de plusieurs petites gaines d'une texture semblable à la sienne, placées les unes dans les autres. Elle est ensuite tout à fait poussée en avant peu à peu, et elle se détache en

l'auteur, on lit sur le feuillet intercalé en regard de la p. 1517 , vis-à-vis du no 6 E. hyemale, les mots suirants de la main de Linné : Caulis viridis scaber radens vagince pallide basi maryinisque denticulis obsoletis atris gibbis. On lit aussi dans le Mlantissa, II, p. 50 : Caulis viridis, scaber, radens. Vagince arliculorum pallide, basi margineque atris, denticulis obsoletis. 


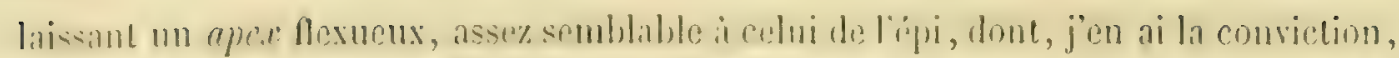
elle precide la formation. Lin diséguant le sommet de plusieurs tiges, despuelles la

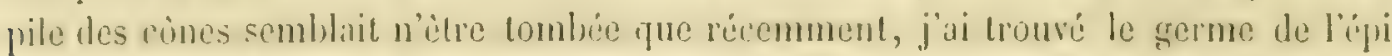
complétement enfurmé dinss sa gaine, dnut les dents, non encore séparées, forment par leur rémion l'apex pui parait lorsque la pile tombe de dessus. Une dissection, tontefois, faite lorigue les cines sont présents, semble souvent décourrir un germe alurtif (un ubortire yerm). Le nombre des gaines ainsi renverstes sur une de ces piles "st environ de douze. Quelle en est la fonction particuliere? je ne puis le conjecturer" (The plyylologist, Lond. 1812, 2 e part., p. 369; se troure '́ralement dans E. Newman, Brit. forns, 184, p. 23).

De ce qui précède, il me semble qu'on peut tirer les conclusions suivantes:

$1^{\circ}$ Les « piles de gaines» n'ont point de fonction particulière; clles ne sont que laceumulation des régrions supéricures de gaines, séparées du reste sans sëtre divisées en dents.

Q La présence de dents persistantes sur les gaines de l'E. laycmale n'est point suffisante pour constituer une espèce, non plus que pour établir une variété on mème une simple variation, puisqu'on trouve, sur une même lige ou sur les liges d'un mème rhizome, des gañes munies ou privées de cet appendice qui existe loujours dans le jeune àge.

L'emploi de ce caraclère pour établir son E. paleacenn induisit plus tard en erreur Schleicher lui-mème, ct, d'aprìs II. Bernoulli, il confondit dans ses exrsiccula celte variété arec l'E. remosissimum, "et il finit mème par ne plus distinguer son E. paleatceum. Dans son herbier il le mêla avec l'E. hyemale, et ce qu'il envoya à Vaucher sous ce nom est de l'E. ramosum devenu très-rameux par suite de la mutilation de la partie supérieure de la tiges (Gef. Krypt. Schw., p. 76, note).

II. Poliorny a done eu raison de n'accorder qu'une importance secondaire «aux caractères tirés de la persistance ou de la caducité des cxtrémilés foliaires (dents) de la gaine, mais il me semble quil leur altribue trop de valeur encore en s'en servant pour établir sa ve $\beta$ palcaceum (Eq. arv. et hyem., p. 137).

\section{CHAPITRE IV}

\section{Historique et synonymie}

\section{$\$ 1^{\mathrm{er}}$ Genre}

Essayer de díterminer avec quelque certitude à quelles plantes répondent les noms, les phrases on les deseriptions des anciens auteurs sorail un travail long et difficite, peut-être même impossible et dans tous les cas inutile. Avant la nomenclature 
moderne, les botanistes ont le plus souvent sous une mème dénomination confondu ou réuni des espèces distinctes, comme sous un même norn ils réunissaient, en les confondant, des genres différents. Dans le Critica botanica, it l'appui des raisons qu'il avance pour la justification el l'explication de l'aphorisme 21 \& du Fundamenta botanica: "Quæcumque plantæ genere differunt, diverso nomine generico designandx "sunt, Linué cite précisément, comme excmple, le nom générique EQuisetu». « Si "quis diceret se observasse singularem Equiseti speciem foliis circa caulem positis, "bifurcatis, levissimis spinis obsitis, ego mox revocarem in memoriam species « Equiseti secundum genus 777', nec somniarem de Charce alicujus specie 801 .

« Veterum

« Equisetum palustre = Equisetum.

" $"$ fotidum $=$ Chara.

(" $)$ polyspermum $=$ Hippuris.»

Il aurait pu ajouter:

Equiselum palustre etc. $=$ Elatine;

„sub aqua etc. $=$ Ceratophyllum;

» montanum etc. = Ephedra ${ }^{2}$.

G. Bauhin essaya le premier de mettre quelque ordre dans ces dénominations diverses; en 1623 dans son Pinax, p. 15 et 16, et plus tard en 1658 dans son Theatrum botanicum, p. 239-254, il rangea les plantes appelées Equisetum sous treize numéros. Mais comme son $n^{\circ}$ I répond à l'Elatine Alsinastrum L., son $n^{0}$ IV à l'Hippuris vulgaris $L$, et son $n^{0}$ XIII au genre Chara, il reste dix numéros que l'on peut rapporter, mais non sans beaucoup de doutes, à nos espèces européennes. Ses types sont à peine caractérisés, et «les restes de l'herbier de ce grand homme sont extrê"mement défectueux à cet égard» (Mon. Prél., p. 330). Ginquante ans plus tard, Tournefort, en ses Institutiones (1700), établit le genre Equisetum «ex lege artis » (Linn., Phil. bot., p. 139 et 14k), et Dillenius en sépara nellement les Chara (Dillen., Nov.plant.gen., p. 88, et Dissert.epist., p. 58).

\section{§. Espèces}

Dans les deux éditions du Species plantarum, Linné décrit six espèces européennes, savoir:

\footnotetext{
- Ce numéro el le suivant sont ceux des genres Equisetum et Ghara dans la 1ra édition (1737) du Genera plantarum.

2Equisetum palustre, linarix scoparix fulio C. Buuh., Pin. $15=$ Elatine Alsinastrum L. Sp., pl., Ed. 2n, 527.

Equisetum sub aqua repens, foliis bifurcis Los., Pruss., 67, t. 12 = Ceratophyllum demersum L., Sp. pl., Ed. 2a, 1403

Equisetum montanum creticum $\mathrm{Alp}$, De pl. exol., $14 \mathrm{t}=$ Ephedra fragilis Desf., Il. allant., II, p. 372.
} 
1. E. sylvaticum,

9. E. arrense,

3. E. palustre,

4. E. 1luvialile,

5. E. limosum,

6. E. hyemale.

Lesquelles se réduisent en réalité à cinq, comme nous le verrons ci-après, par l'identité des E. fluviatile et limosum.

L'extrùme concision à laquelle aspirait constamment ce grand naturaliste, a jeté trop souvent de l'obscurité dans ses phrases descriptives, et nulle part peut-être ce défaut n’est plus sensible que dans celles des espéces de ce genre. J'ai cru devoir dans la synonymie citer in extenso le lexte de la deuxième édition (20 vol., 1763), afin que l'on puisse mieux roir à quels types antérieurs Limné fait allusion, quelles figures il cite, el combien avec ce texte évidemment insuffisant, j'oserais presque dire néglige é les noms linnéens. Aussi y a-t-il eu de nombreuses indécisions, de longues el pen profitables discussions.

Pour essayer d'arriver à quelque certitude sur la distinction des types linnéens, plusieurs auteurs ont regardé comme indispensable de recourir à l'herbier de Linné et de combler par l'étude des spécimens les lacunes que laissait un texte insuffisant. Je crois a propos de reproduire ici, en le traduisant, le travail que M. Ed. Newman a publiti sur ce sujet d'abord dans The plinglologist, Lond., arril 18.43, p. $5.30 \mathrm{ct}$ suir., et plus tard à la fin de son beau volume British ferns, p. $412 \mathrm{et} \mathrm{suiv.} \mathrm{(1844).}$

๔ On sait, je crois, assez généralement que l’herbier de Linné fut achelé par J. E. Smith, el plus tard par la Société linnéenne de Londres ${ }^{3}$, dont il est demeuré la propriété. Les spécimens sont fixés sur une demi-feuille de papier ministre; les noms sont de Linné lui-même el écrils de sa main; on y troure aussi les remarques que J. E. Smith a mises partout oủ il lui a paru nẻcessaire ou avantageux d'ajonter une note explicaliwe. Onelgues étiqueltes, arec des notes manuscrites, y sunt aussi collexes, mais je ne sais

- Le Flara lapponica fut publié en 1737 , c'est-ì-dire seize ans arant l'impression du Species plantarum, dans lequel linné fit l'application de ses nomina specifica trivialia ou de sa nomenclature binaire. Or, dans le Flora lapponica, les espèces sus-mentionnées (moins l'É. limosum qui n’y fiğure pas) ont déji les noms linnéens du noureau sys-

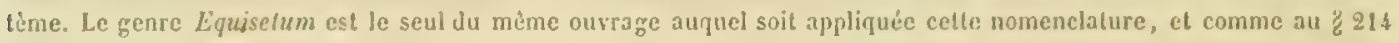
de son Crilica Lolanica, publié également en 1737, Linné cite ce mème ğcure Equiselum comme exemple de nomenclature confuse, il y a lả un rapprochement curieux, et il est permis de se demander si ce n'est pas sur ce genre que Linné a fait le premier essai de sa nomenclature.

- Linné lui-mème les énumère avec une grande némligence (Mon. Prél., p. 330).

s En mars 1829, pour le prix de 3000 guinces (78,000 fr. environ).

On trouvera sur l'herbier de Linné des délails intéressants dans: Fée, J'ie de Linné, p. 319 à 326 ; If. E. Richter, Cod. Lot. linn., p. xxv et xxru, el surtout Lassigne, Mhs. bol. de J. I). Delessert, p. $339 \mathrm{et}$ suiv. 
pas avec certitude qui en est l'auteur. Les Equisetum sont renfermées dans un fascicule de neuf feuilles portant sur le dos: 1169. Equisetum, de la main de Limné

«Dans le même local sont conservés les exemplaires de la $1^{\text {re }}$ et de la ge édition du Species plantarum ayant appartenu à l'auteur. Sur le premier, toutes les espéces possédées par l'auteur sont désignées par une marque particulière, et le deuxième est enrichi de notes originales inédites. Je vais maintenant essajer de réunir les renseignements puisés à chacune de ces sources, en ne citant les caractères déja publiés que lorsqu'il y aura nécessité el en ajoutant mes propres remarques sur chaque spécimen.

«Linne. - 1. sylraticum.

FOLIO 1.

«E. N. - Un seul échantillon jeune de l'E. sylfaticum de Smith, avec un épi parfuit.

FoLIo 2, altaché par Linné avec le précédent.

«E. N. - Deux échantillons mûrs de l'E. sylvaticum de Smith, sans frucification.

«Linné, - 2. arvense.

FOLIO 3.

«Anonym. - 1061. Equisetum setis ramosis. Equisetum verticillis ad folia numerosis. Hall. Stirp. IIclv., 144. Equiselum sylvaticum tab., p. $253^{2}$.

«E. N. - Deux échantillons; celui de droite est un échantillon fertile de l'E. arvense de Smith, avec un épi parfail; celui de gauche un échantillon mùr de l'E. sylvaticum de Smilh, sans fructification.

FOLio 4, attaché par Linné avec le précédent.

"Linné. - Ilispania, 713. Loft. ${ }^{3}$.

«E. N. - Trois échantillons sans fructification, tous provenant de sujets éliolés et altérés; ils appartiennent probablement à l'E. arvense de Smith. La note de Linné est au revers de la feuille.

«Limné. - 3. palustre.

FOLIO 5.

(Smith. - ?

«Anonym. - 1060. Equisetum selis simplicilus. Equisetum minus terrestre. J. B. I., p. 730.

«E. N. - Deux échantillons sans fructification et récoltés en très-mauvais élat; celui de droite me parait être l'E. arvense de Smith, celui de gauche est peut-être l'E. palustre de Smith.

FOLIO 6.

«Linné. - 4. fluviatile.

«Smith. - limosum? ccrte.

«E. N. - Quatre échantillons, tous avec des épis, ct identiques à l'E. limosum de Smilh. Il n'y a point d'échantillon destiné à représenter l'E. limosum de Linné, et l'exemplaire du Spec. plant., $1^{\text {re éd., }}$ n'indique point que Linné l'ait possédé. L'exemplaire de la deuxième édition porte, sur le feuillet blanc intercalé, vis-à-vis le passage de Haller cité (p. 1517, lig. 3) dans la description de l'E. fluviatile, la

\footnotetext{
- Ce no 1169 est celui du Syst. nat., Ed. 12a; du Gen. plant., Ed. Ga et 7a; du Syst. vegel., Ed. 13a, 14a, 15a.

- Citation fautive de Tabernamontanus, ic. 253.

${ }^{3}$ Ne faut-il pas lire 1753, Lœll, ; comme indication de l'année oủ l'infortuné Loening avait recueilli ces échantillons en Espagne (voir Lœil., Il. hisp., p. 6 š et 288)?
} 
note manuscrite suirante de la main de Limné: «hoc caules proliferos a sterilibus definter profert "Hall', , et plus bas cette autre: "forte merat rarietas prioris (E. palustre) ex solo aqua profundioris. " Et vis-h-vis la description de l'E. limosum le même exemplaire offre celle addition: «Hallerus laane « facit varietatem E. palustris. $\$$

«Il est clair que, pour la dislinclion de son L̇. limosum, Linné se fie à l'autorité de Ray, puisqu’il cite la ligure que donne cet auteur (Syn., t. V, fig. 2, a, b), laquelle représente évidemment la forme sans branches de l'E. limosum de Smilh. Il semble dis lors que l'E. Iluviatile de Linné est la forme rameuse et son E. limosum la forme non ramense de l'E. limosum de Smith, el que l'E. fluviatile de Smith (L. maximum Lam.) n'a de représentant ni dans l'lerbier ni dans les ourrages de Limné. Quant à l'obscrvation précitée de Linné, suirant laqquelle llaller ferail de l'E. limosun une variélé de l'E. palustre, elle manque de justesse. Haller cite la fig. 3 de Ray comme variété de l'E. palustre, et, selon moi, avec raison; landis que Linné cite la fig. 2 qui est éridemment l'E. limosum de Smith. Une preure positive que l'E. lluviatile de Linné n'était pas l'E. fluviatile de Smith se troure dans ce fait que Limé a essayé de rendre comple de sa grande taille comme d'une altération due à sa croissance sur le fond d'une eau profonde; ce qui est bien le cas pour l'L. limosum de Smith, mais jamais par l'E. lluviatile du mème, qui au conitraire croît de jréférence dans des lieux découverts graveleux el salilonneux.

\section{FoLIO 7.}

«Limé, - EEdific : lyemale.

«E. N. - Un simple échantillon de l'L. lıyemale.

"Sur le feuillet intercalé dans l'exemplaire du Sp. plant., on lit vis-i-ris de celte espéce ces mots de la main de Linné : «quiselum caule simplici aspero raginis non laciniatis. Iall. Ilclv, 113. Caulis "riridis scaber, radiis (?) vagine pallidx basi marginisque denliculis obsolelis atris gibbis ${ }^{3}$, »

\section{FOLIO 8.}

«linné. - Tourelle.

(Smilh. - Asperrimum, Dick., variegatum, Jacף. H. B. - J. E. S.

« Anonym. - Equisetum basiliense no 1678, Haller. An species distincla apud cl. Linn.? In liorto cullum.

(E. N. - Un simple échantillon de l'E. variegalum. Linné a répondu à la question touchant la dislinction de l'espéce en donnant comme synonyme à son E. ligemale la pllırase de Bauhin: Eq. nudum minus variegatum basiliense Pin. Le nom de Tourelle écrit par Limé indique probablement l'habitat.

FoLio 9, altaché par Linné avec le précédent.

«Limé. - Suec.

(E. N. - Un simple échantillon de l'E. variegatum, beancoup plus gréle que le précédent. La note manuscrite de Linné indique évidemment qu'il provient de la Suede.

\section{Dans les Acta acal. scient. de Stockholm, 1849 et 1851, M. Ch. Ilartmin a}

\footnotetext{
'Le texte de Haller est: a Caulis floriger videtur a folioso remotus o (Enum. slirp. I/clv, P. 144).

2Il cst probable qu’il faut lire arlific, dans cette abbréviation de Linné. Cette plante est en eftet citéc sous le no $83 \$$ dans le Florn aeconomica, conme servant à l'industrie (Amoen. Acad., I, p. 385).

${ }^{3}$ Le vrai texte est: "Caulis viridis, scaber, radens. Vagina pallidie, Jasi margineque atris, denticulis ubsoletis

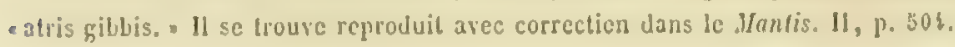


publié: Annutationes de plantis Scandinaricis herbarii Linnerani, in Musce socict. linneance Londin. asservati, et dans ce nouvel examen de l'herbier de Linné on lit (p. 260 et 261 du tirage à part) les observations suivantes sur les Equisetum:

\section{«Equisetum}

a sylvaticum 1 (man. prop. Linnxi).

«arvense 2 (man. prop.) (Smith addidit:)?

"palustre 3 (man. prop.) (manu Smith)?

« Est Equisetum pratense recentiorum.

( hyemale (man. prop.) atque prope specimen: artific. (?)

a(Sine nomine, man. prop. tantum) 6 (et) suec.

(Est E. scirpoidles auct. »)

On roit par ce rqui précède combien l'herbier de Linné était un guide peu sûr pour arriver à la fixation certaine de ses types spécifiques '.

Les descriptions de IIaller précédèrent et suivirent celles de Limné; elles sont vraiment plus satisfaisantes. Leers, Pollich, Roth donnèrent des détails plus exacts encore, et qui concoururent puissamment à la distinction des espèces.

Jusqu’à la IOnographice des Prêles par Vaucher (1822) il n'y cut aucune classification entre les espèces; on les mettait les unes après les autres, sans l'énoncé du principe de distinction et de coordination. Vaucher, comme tant d'autres après lui, s'inspirant des divisions établies par De Lamarck et par De Candolle dans les « méthodes analyliques» qui précèdent leurs Flores françaises, coordonna les caractères distinctifs qu'il employa, et classa les espèces françaises qu'il décrivit dans l'ordre suivant:

\section{«TABLEAU SYNOPTIQUE ET ANALYTIQUE DES ESPËCES}

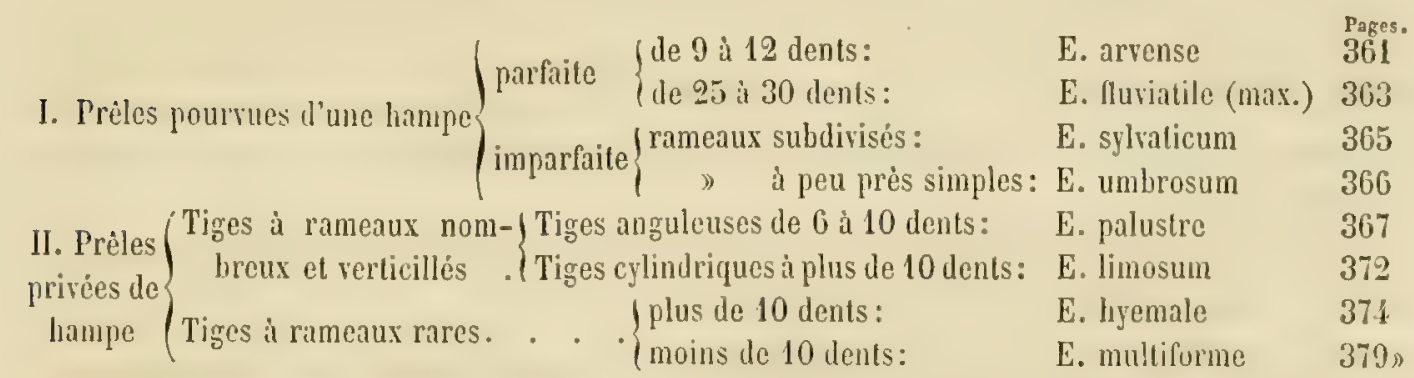

(Extrait de la Mon. Prél., p. 359).

\footnotetext{
- IIerbaricu Linneanum sape fallax, patris senescentis filique Linnoi dubiis per quindecim et quod excurrit annos ab culmine Linnæano obnoxium, sxpe specierum originalia non continct,... : corumque loco sxpius fursitan fovet serius missa ab aliis excmplaria, male delerminata et raptim a Linnais ineptiore loco seposita, - vel prarsus careb plantis vulgaribus, quas ob it ipsum forsan nondum collegerat et exsiccaverat herbarii posscssor .... (II. E. Iichter, Cod. bot. linnaranus, p. xxiI).
} 
Si lon remarque que cet auteur comprendl'E. reniegutum dans les rariétés de son E. mullifurme, on roil qu'il ne manque it sa liste que l'E. lillorele Kühlewein el l'E. trachyodon $\Lambda$ l. Braun.

Comme lindique ce tablean, el comme l'auteur l'explique longuement p. 35. et suiv., le caractere qu'il place au premier rang est l'existence a d'une hampe et d'une "lige stérile siparées; le sceond est le nombre des dents de la gaine. Le troivieme "caractire est celui de la lige qui est tantôt cylindrique, tautôt plus ou moins angu"lcuse, tantôt lisse el unie, tantòt rude ct raboteuse.

a Le quatrième est celui de la régularité des rameaux.....

"Le dernier caractere est celui des glandes corticales (stomates)'; el indépendam"ment de ces caracteres principaux il en est d'autres accessoires qu'on ne doit pas " entierement négliger. Tel est celui des tubes intérieurs, qui varient pour le nombre " des rangs et pour la forme; tel est le caractere des rameaux qui sont cylindrigues "ou anguleux; tel est celui de la consistance de la tige, et tels sont enfin ceux du port, a de la hauteur, du diamètre des liges el de leur durée.

a Par rapport au nombre des rameaux, à la forme des dents, aux appendices "transparents qui les terminent, à l'amplitude des collerettes (gaines), aux coulcurs ( dont clles sont teintes, et autres caractires quont souvent employés les botanistes, " il liuut s'en défier beaucoup, parce qüils dépendent, en grande partic, de la saison " de l'année où l'on observe la plante, du terrein sur lequel elle a crù, de la quantité a d'épis dont elle est chargée et d'autres circonstances faciles à apprécier.»

En 1828, Bischoff (Krypt. Gew., p. 56) reprit la première grande division de Vaucher, cn substituant de grands mots grees, Scapocarpi, Canlocripi, aux simples phrases françaises du botaniste de Genève.

Dix ans plus tard, dans la rémion des naturalistes et docteurs médecins qui cut licu à Fribourg en Brisgau (1838), .I. Al. Braun donna la division suivante des esjéces européennes:

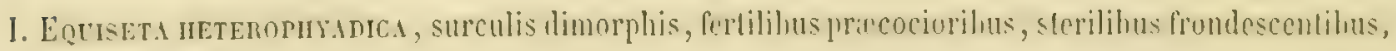
serolinis.

A. Ametabola, surculis fertilibus non frondescentibus, cito pereuntibus (E. vernalia).

1. E. arvense.

2. E. eluurneum.

B. Metabola, surculis fertilibus ramulos serotinos frondosos proferentibus persistentibus $(E$. subernalia).

\footnotetext{
1. Les especes différent beaucoup entre elles par le nombre et la disposition de ces mèmes glandes. Les unes, comme "la Prèle des bois, des ombrages et celle des fleures en sont presque dépourvues. Les autres, comme celle des marais, - en sont plus abondamment fournies; dans certaines especces, elles sont éparses; dans d'autres elles sont arrangées - dans un ordre régulier " $(p, 3+4)$.
} 
3. E. sylvaticum.

4. E. umbrosum.

II. E. Hoмopryadica, surculis uniformibus, frondescentibus, sterilibus fertilibusrque similibus, aut coxtaneis, aut omnibus ferlilibus.

A. Surculis annuis, hieme pereuntibus (E. cestivalia).

5. E. limosum.

6. E. palustre.

B. Surculis hiemem perdurantibus, sempervirentibus (E. hiemalia).

7. E. hiemale, cujus subspecies:

1. E. ramosum.

2. E. hiemale (genuinum).

3. E. trachyodon.

4. E. variegatum.

8. E. scirpoides》 (Flora, $22^{\circ}$ année, 1839, n²0, p. 307).

Le plus simple coup d'oil permet de roir que la division donnée par M. Al. Braun est encore identiquement celle de Vaucher.

En 1843 le même botaniste publia dans le Silliman's American Joumal of science and arts (numéros d'octobre et de décembre) un nouveau principe de division tiré de la disposition des stomates sur les tiges, où ils sont: soil épars (Vaucher) sPeiropona (Al. Braun), soil arrangés dans un ordie régulier (Vaucher) sticropona (MI. Braun). Comme on le voit encore, e'était l'application d'un principe de division déjà soigneusement indiqué et figuré par l'auteur de la Monographie des Prêles, p. 343, 344, 355 , pl. I, II, III, IV, V, VI, VII, VIII, IX, X, XI, XII et XIII (voir la note de la page précédente et les p. 34 et 35).

Ces divisions, au moins dans leurs principaux groupes, ont été reproduites par les auteurs modernes, MM. Koch, Grenier et Godron, Bernoulli et Döll, par ces deux derniers avec quelques critiques (Bern., Gef. Krypt. Schw., p. 65); MI. Döll (Fl. Bad., I, p. $56 \mathrm{el} \mathrm{65)}$ substitue même à la dénomination des denx grandes divisions speiropora et stichopora, les noms de malacocaulon el sclerocanlon, tirés de la rudesse et de la dureté des tiges ou de leur mollesse et du poli de leur surface. M. Milde fait observer avec raison que ces noms et ces caractères sont faits pour induire en erreur, «altendu que les tiges stériles des $E$. sylvaticum et pratense et les rameaux de l'E. Telmateia ont des aspérités siliceuses qui en rendent la surface très-rude, bien que ces espèces appartiennent à la section Malacocaulon» (Syst. Eq., p. 140). On pourrait ajouter que c'est également à tort que M. Döll, dans sa division, attribue à l'E. ramosissimum des tiges « annua et non perennantia » (Fl. Bad., I, p. 65 et 67 ).

MM. Grenier et Bernoulli, at l'exemple de De Candolle (Fl. fr., suppl., p. 245), ont tiré de la terminaison de l'épi, obtuse ou acuminée, un caractère de division facile, mais qui à lui seul n'est pas suffisant. 
Lin 18.8 , je lus it la Societe botanique de France, dans sa réunion a strasbourers, une Xulesur les Equisclum de France, dins larpuelle je proposais diajouter aux caraclieres dija adoptés pour la distinction des especes: 10 la relation de lonsueur entre les gaines de la lige et le premier entre-neud des rameaux adjacents; 20 la coupe transversale des rameaux; $3^{\circ}$ la coupe transwersale des tiges. Les deux premicrs élaient nouveaux; le dernier, déja proposé, mais non appliqué par Vaucher, avail élé appliqué partiellement par quelques auteurs modernes. Ils ont été employés depuis par M. Cosson (Fl. enu. Perris, ac éd., p. 876 et suiv.) et par M. Milde (Sigst. Eq., p. 1/1 ct suiv.).

Hais en mème temps II. Milde, repoussant par les raisons exposées ci-dessus les grandes divisions et les noms de MII. Al. Braun el Döll, et s'appuyant, comme il le dit, sur la différence constatée par M. C. Sanio entre les stomates des premiers groupes et ceux des IIyemalia, établit deux grands groupes: $1^{\circ}$ Praxeropon, espèces dont les stomates sont de niveau avec l'épiderme; go Cinpropons, especes dont lis stomates sont cachés aut-dessous de l'épiderme et au fond d'une cavité transversale à bords irreguliers. Pour le premier groupe il reprend ensuite la division de M. II. Braun en heterophyadica ct homophiyudica, et les heterophyadica se subdivisent en anomopora et stichopora (Syst. Eq., p. 138 et suiv.).

J'ai consacré le chap. II de cette partie a l'exposition des principes que j'ai suivis dans la distinction et la classification de nos espèces.

Des onze Equisetum signalés comme cruissant en France, six sont très-répandus et ont été dès lors très-anciennement connus el distingués, savoir: E. maximum Lam., syleaticum L., areense L., limosum L., pulustre L., hyemale L La distinclion des autres est plus récente, comme nous le verrons dans l'histoire el la synonymic qui suivent.

\section{E. yaxinum Lam.}

Cette belle espèce a été très-anciennement distinguée, et il cst facile de la reconnaître dans les descriptions de J. Bauhin et dans celles de IIaller. Il l'est moins de se fixer sur le nom qu'elle doit porter.

Elle recoit te plus souvent le nom d'E. Telmateia Ehtht.; je dis le plus souvent, car on troure encore dans des ourrages très-récents celui d'E. Aluviatile L. à clle donné, soit directement, soit en șynonyme. Eu mime temps, d'autres auteur's excluent absolument de la synonymie de cette plante l'E. fluriutile L., ct appliquent ce nom, les uns a une espèce distincte, les autres il'E. limesum directement ou par șnonymic.

Jexaminerai successivement l'opinion des anteurs qui roicut dans notre espice IE. furintile L., et les raisons de ceux qui prétendent rue ce nom ne doit point lui être appliqué. 
Quant aux premiers, faisons d'abord remarquer que, si le nom $E$. puviatile L. appartient à celte espèce, comme il est le plus ancicu, il y aurail justice à l'y appliquer, non en seconde place et en synonyme, mais directement et comme nom princeps. Ceci me semble trop évident et trop juste pour insister. Ce qui a porté ces auteurs à croire que le nom liméen $E$. fuviatile convenait à la plante nommée plus tard E. Telmateia par Ehrhart, a élé d'abord la présomption que Linné ne pourait pas ne pas aroir connu et mentionné celte espèce si répandue, si caractérisée, la plus grande et la plus belle des Equisetum européens. Ils ont ensuite été induits en erreur en trouvant que daus le Flora suecica, le Flora lapponica ct le Species plantarum, Linné cite en synonymie la phrase de G. Bauhin : E. palustre longioribus setis, et cello de Ilaller: E. caule non sulcato latissimo, verticillis densissimis, qui toutes deux se doivent rapporter à notre plante. Bien plus, dans son Mantissa $I^{\text {a }}$, p. 504, après la description de l'E. Ruviatile, Linné a ajouté la citation suivante: "Caulcs poriferi a "sterilibus distincti, ut $\mathrm{E}$. arvensis. Haller.» Et, comme il est de toute évidence que celte citation et la synonymie qui la précède s'appliquent à notre espèce, les anciens botanistes, Leers (Fl. herb., p. 223) et Smith (Fl. brit., p. 110\%), onl été jusqu'à un certain point autorisés à voir dans l'E. fuviatile de Linné la plante de G. Bauhin, celle de Haller, une espèce à tiges spicifères distinctes des tiges stériles, en un mot l'E. Telmateia d'Ehrhart. Vaucher, ayant donné à notre espèce le nom d'E. fluviatile, qu'il croyait être le nom linnéen, en justifie l'application par ces termes singuliers: ( Linné a décrit la Prêle des limons (E. limosum) sous le nom de Fluviatile, el elle "existe sous cette dénomination dans son herbier avec la phrase suivante: Eq quisctum "caule striato, frondibus subsimplicibus, Sp.pl., 1517, Fl. lapp., 393. La Telmateya " est dans une autre feuille, sous le nom de Flwviatile, provenant de l'herbier de "Miller. Ces détails m’ont été fournis par M. De Candolle» (Mon. Prêl., p. 373). Je reviendrai tout à l'heure sur ce point.

Voici maintenant quelques-unes des raisons qui ont fait penser à d'antres auteurs que l'Equisetum 836 du Flora suecica, E. furiatile du Flora lapponica et du Species plantarum, n'a rien de commun avec la plante de IIaller, et que, si Linné a emprunté la synonymie de ce botaniste, c'est parce qu'il n'avait pas vu l'espèce décrite par lui.

L'E. Telmateia Ehrh. ne croît ni en Suède ni en Laponie, ainsi que l'altestent J. E. Wickström (Ann. de l'Acad. suéd., 1837-184'4, p. 177), Wahlenberğ (Fl. succica, p. 689: «Nostra planta (E. limosum) differt ab E. Telmatcia Ehrh. in Suecia «non observato), » et M. E. Fries (Sum. veg., p. 59). Or, dans le Fl. lapponica, Linné indique son $E$. fluriatile "ad ripas lacuum el fluviorum passin copiosissime; il le cite comme pouvant fournir aux rennes, qui le mangent avec avidité, la nourriture d'hiver, si difficile à se procurer (p. 310); dans le Fl. succica il mentionne l'usage que les Suédois en font pour la nourriture des vaches, il donne huil des noms popu- 
laires qu'il a reens en suede et dans le Nord (p. 368), en un mot Linné parle de son L. Anrulite comme d'une plante répandue en Sucde, rue et possédée par lui. Mais lexamen de son herbier, fait par .I. L. Newman et par II. Ch. Hartman, démontre, ainsi

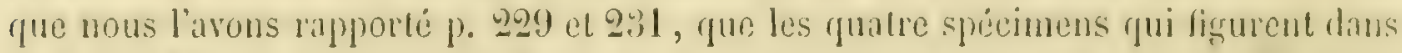
lis feuille 6 pour représenter l'E. furiatile, ajpartiemment tous les quatre à la forme rameuse de l'E. limosum et rue l'E. Telmateia n'a aucun représentant dans l'herbier de Linne̊.

Si Limé, dans son Mantisse $I^{4}$, et dans les notes de son herbjer, attribue à son E. Anvictile, sur l'autorite de Inaller, des tiges florifires distincles des tiges fertiles, d'autre part, dans la deuxiẻme édition de son Flora suecica, c'est-à-dire dans un ourrage ou il consigne ses propres observations, il ajoute à la description de lE. limosum: "precedenti (E. fluviatili) nimis affinis et interdum frondes parciores "assumens» (p. 368). Ajoutons que dans son Fl. lapponica, p. 310, Limné rapporte à son E. furiatile l'E. mudum Lind. Wilisb., 11. Or', si I'E. furiatile de Limé était réellement l'E. Telmatcia Ehrh., la comparaison el la synonymie qui précédent eussent été impossibles, non pas sculement pour Linné, mais pour un novice en botanique; et en mème temps il est inadmissible que, dans une Flore de Suède, Limné compare son E. limosum à une plante qui ne croit pas dans ce pays, tandis que l'E. fluviulile L. rapporté à la forme rameuse de l'E. limosum y eroît abondamment, el justifie entierement cette comparaison ainsi que ce qui a été cité plus haut sur l'abondance de cette plante, sur ses usages en Suẻde et sur ses usages possibles on Laponie.

Enfin, il est indubitahle que si Limné avait vu seulement une fois l'E. Telmateir Elrh., ce grand descripteur n'aurait pas laissé à son E. arvense la phrase : E. scepo fructificunte undo, sterili frondoso (Sp. pl., p. 1517), laquelle convient a l'un et i l'autre.

Ainsi done, il parait certain que cette espèce n'a jamais été connue de Linné, qu’aucun de ses noms spécifiques ne peut la désigner, el que ce n'est que par suite d'une confusion que Linné a rapporté ì son $L$. furiatile la synonymie de G. Bauhin, celle de IIaller et la citation de ce dernier.

En 1783, Fr. Ehthart imposa ì notre plante (Hamöv. Mragaz., Stück 18, p. 987) te nom de Telmatcia, dont la signification Tétua, Tíluatos, boue, et cía, herbe, mancque d'exactitude pour désigner la station de celte belle espéce (roy. Bull. soc. Lot. Frence, t. IX, p. 393 et 925 ). En 1797, Roth, dans ses Cialul. botan., fise. I, p. 128 , la nomma E. cburneum, exprimant ainsi avec justesse un caractire physiologigue très-important ct tout at fait particulicr à celte espece, satroir l'absence de stomates, ct des lors de chlorophylle, sur les entre-neuds, ce qui occasiomne leur belle couleur d'un blanc d'iroire. Mais, au lieu de se rapporter au type de l'espece, la description 
de Roth et de Schrelier concerne la forme frondescens, sur laquelle les tiges spiciferes, au lieu de se faner et de disparaître après la sporose, persistent et produisent des rameaux ${ }^{\mathbf{1}}$ C'est ce qui ressort clairement de la diagnose de Roth: « E. scapo fructi«ficante nudo denum elongato in frondem verticillato-ramosam.... ») (Tent. R.gcrm., vol. IV, p. 4), non moins que de sa description: «.....Semine disperso et spica marcesa cente, demum corrugata vel decidua, scapus elongatur in frondem verticillato(ramosam, sxpius ultra tripedalem, erectam, strictam» (ibid., p. 5). Quant à l'identité de l'espèce elle est altestée par Roth lui-mème en ces termes: "Observationibus " edoctus ill. Proses de Schreber in litteris me fecit certiorem Equisctum suum cbura neum eamdem esse plantan cum Equiseto Tolmateju Ehrlı., et hoc in alterum cotate «transire» (ibid., p. 5).

Ainsi le nom d'Ehrhart a plus d'extension et est plus ancien que celui de Roth, el, à ce double titre, il a été généralement adopté. Cependant il n'est pas le nom le plus ancien, le nom princeps. Il a été imposé en 1783. Or, dans la première édition de la Flore frangrise du chevalier De Lamarck, qui est de 1778 , c'est-à-dire antéricure de cing ans au Hannöversche Magazin, on trouve, à la p. 7 du t. I er, celte espèce décrite sous le nom d'E. maximum. Il est vrai qu'on trouve en synonyme $E$. fluviatile I., mais le texte ne peut laisser aucun doute. En effet, cetle espèce, avec l' $E$. arvense, forme le «III Groupe, Tiges fleuries mues et les stériles feuillées, » et la description dit formellement: «Les tiges fleuries sont nues, épaisses, hautes d'un “ pied et naissent au printemps. D'autre part, Poiret, continuateur de De Lamarck, en reprenant, quoiqu'avec doute, le nom linnéen $E$. fluviatile comme antérieur, a cité en synonymic les noms imposés par De Lamarck et par Ehrhart (Enc. méth. bot., V, p. 614). Mais ce qui pourrait inspirer quelques doules, c'est que De Candolle, également continualeur et en quelque sorte collaborateur de De Lamarck, n'ait pas repris le nom imposé par ce botaniste. Dans sa Flore française, 1805, t. II, p. 581, De Candolle mentionne et décrit sur le no 1454 l'E. Telmateya Ehrh., sans autre synonymie que l'E. eburneum Rolh, rapporté seulement à la variété $\beta$; et, à la page

\footnotetext{
"Vaucher, qui ne connaissait pas cette forme frondescente, se scandalise du caractère que Roth assigne à son $E$. eburneum, et dit avec humeur: "c'cst une erreur d'imaginer, comme l'a fait Roth, que la hampe fructifère pousse "des feuilles après la chute de l'épi; il n'y a point de rapport entre l'organisation de la tige stérile et celle de la "hampe, Cette dernière meurt constamment après avoir répandu ses graines " (Ion. Prêl., p. 36i). Le très-consciencieux et très-clairroyant Roth n'avait rien imaginé; il avait décrit ce qu'il avait vu, seulement il avait pris un accident pour la forme constante. Il parait que cette forme frondescente est si répandue dans quelques contrées de l'Allemagne que Bischoff' (Krypt. Gew., p. $46 \mathrm{et} \mathrm{47)} \mathrm{la} \mathrm{regarde} \mathrm{presque} \mathrm{comme} \mathrm{essenticlle} \mathrm{it} \mathrm{l'espèce.} \mathrm{Lc} \mathrm{mème} \mathrm{auteur} \mathrm{dit} \mathrm{ailleurs:}$ - La frondescence de la hampe fertile est un fait si constant aux environs de Heidelberg et entre Junich et Schæeflarn, que j’aurais dû regarder cette frondescence comme la règle et son absence comme l'exception; mais il parait que la proportion entre les deux sories de tiges peut varier selon les localités (Bemerl. Eq., p. 108). M. Milde aftirme que celte forme ne se rencontre qu'exceptionnellement en Silésie (Kenntn. Eq., p. 587, et Gef. Krypl. Schl., p. k9S cl suiv.). Nous avons vu plus haut qu'elle est égalcment très-rare en France.
} 


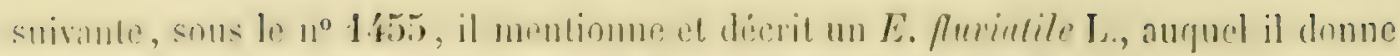
en synonymic E. maximum Lam. el E. heleocharin Ehrh. Mais ces doutes dispa-

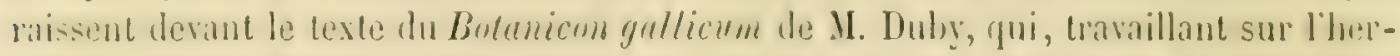
biel de De Candolle, identife les deux numéros et les deux noms, en les rapportant à une seule et même espèce; ct, comme ce dernicr auteur croyait aussi que celte plante avait éte connue de Iinne, il la nomme, comme l'oirel, par droil de priorité

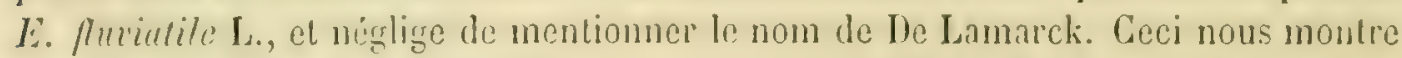
que De Candolle ne se rendait pas un comple exact de la distinction de ces deux espices', et nous explique la contradietion apparente qui existe entre les renseignements quil a fournis a Taucher sur l'herbier de Limué et les témoignages plus précis et plus modernes de M.I. E. Newman et Ch. IIariman.

Done, pusisue le nom d'E. furiutile L. ne peut comvenir à notre espece, et que celui de De Lamarck a la priorité sur celui d'Ehrhart, il y a justice à reprendre le nom E. maximum Lam. comme nom princeps, et nous avons ainsi fait?

1625. II. E. palustre longioribus setis C. Bauhin, Pin., p. 15; Thealr., p. 211, cum icon. pessima. 105I. E. palustre teruissimis et longissimis selis J. Bauhin, Ilist. pl., III, p. 730 (et forte confusum cum $\mathrm{E}$. arrense, ras nemorosum?).

1742. E. caule non sulcato, latissimo, verticillis densissimis Hall., Enum. stirp. Helv., p. 144).

1768. E. caule florigero nudo, sterili rerticillato, radiorum quadraginta ILall., Hist. slirp. Helv., $n^{\circ} \mathbf{1 6 7 5 .}$

1775. E. Amialile Leers, Fl. herb., p. 293 (non L.).

1778. E. saxisu Lam., Fl. fr., I, p. 7 (exclud. syn. L.).

1783. E. Tclmatcia Ehrhart, IIannö̀. Magaz., St. 18, p. 287.

1788. E. Telmaleia Ehrhart, Beitr., II, p. 159 ct 160.

1804. E. fluvialile el E. macrostachyon Poirel, Encycl. mélh., V, p. 614.

1801. E. fluvialile Smith, Fl. brit., p. 110 (non L.).

1805. E. Telmatcia DC., M. Fr., 1I, p. 581 ; et E. fluviatile (I. c., cx. auct. Duby).

1807. E. Telmatcia Lois. Desl., Fl. gall., Ed. 1ª, p. 700 (cum syn. Rothii); el E. fluviatile ejusden, ก. 701 .

1810. E. fluviatile Willd., Sp.pl., V, p. 2 (excl. syn. L.).

\footnotetext{
- Dans les deux éditions du Flora gallica, Loiseleur-Deslongchamps a reproduit le mème double emploi. Après avoir lécrit l'F. Telmaleia Eltrh. sous son no 2, l'E. arvense sous son $n^{\circ} 3$, il décrit de nouveau notre espèce sous Ic no 4 , E. fuviatile, mentionnant sa double forme fertile et stérile, ses gaìnes grandes et rapprochées, le synonyme de Smith, la figure de bolton ctc. Cependant sil'on consulte l'herbier de cet auteur, on trouve que la feuille éliquetée $E^{\prime}$. Telmateir contient plusicurs spécimens ćliquetés $E$. fuvialile, mais aussi que la fenille spécialement étiquetée $\boldsymbol{E}$. furvialile ne contient que deux tiges fertiles et très-ramenses de l'E. limosum. De là incertitude complète. Le genre Equiselum est très-mal représenté dans cel herbier, lequel, achetẻ par Esprit liequien et légué au musée de la ville d'dvignon, y pourit misćrablement dans un grenier ouvert à tous les vents.

"Comme je ne traite quue des Equiselum de la France, j’ai dù me borner ì citer, apris le nom princeps, la synongnic des flores générales de cette contrie. Toute autre citation est motivée par la valeur particulière de la description ou des renseimnements qui l'accompagnent. Le simne! indingue les autears que l'on consultera avec le plus de fruit.
} 
!1822. E. fluviatile Vaucher, Mon. Prêh, p. 363 (non L. et exel. syn. L. helencharis Ehrh.).

!1828. E. fluvialile At. Brongniart, Ilist. veig. foss., I, p. 99 et suiv.

1828. E. fluviatile Duby, Bot. gall., I, p. 535.

1835. E. fluviatile Mutel, Fl. fr., IV, p. 166 (non L.).

1844. E. Telmateia Newman, Brit. ferns, p. 67.

1845. E. Telmateia Koch, Syn., Ed. 2a, p. 964.

1 1852. E. Telmateia Milde, Kennin. Eq., p. 585.

1850. E. Telmateia Gr. el God., Fl. d. Fr., III, p. 643.

!1857. E. Telmateia Döll, Fl. Bad., 1, p. 59.

!1857. E. Telmateia Bernoulli, Gef. Krypt. Schw., p. 67.

! 1858. E. Telmateia Nilde, Gef. Crypt. Schl., p. 425.

!1861. E. Telmateia Cosson el Germain, Fl. env. Paris, 2e éd., p. 877.

Vario $\beta$ minus.

1860. E. Telmateia ${ }^{\text {as }}$ minor Joa. Lange, Pug. pl. hisp., 19; et Willk. et Lange, Prod. f. hisp., p. 12.

$4^{e}$ anom. frondescens Al. Braun.

1.1797. R. eburneum Roth, Catalect. botan., fusc. I, p. 128; Tent. n. germ., IV, p. 4.

1843. E. Telmateia C frondescens Al. Braun, in Sill. Amer. Journ., vol. XLVI, p. 84.

$5^{e}$ anom. serolinum Al. Braun.

18.13. E. Telmateia vas serotinum Al. Braun, in Sill. Amer. Journ., vol. XLVI, p. 84.

\section{E. syluaticuir L.}

La slation de cette espèce, son port tout parliculier, l'ont fait facilement distinguer. Elle n'a done point de synonymie proprement dite; et le nom d'E. capillar'e, donné par Iloffmann à des sujels grêles, n'a pas élé par l'autcur lui-même appliqué en synonyme à toule l'espèce (roy. Deutschl. Flor. Crypt., 1795, p. 3, et ci-dessus p. 179). Des individus peu développés ont été pris par Lapeyrouse et par Mutel pour I'E. umbrosum Heyer (voy. Rúvision de l'herb. de Lapeyrouse, par MI. Ie docteur Clos, 1857, p. 82).

1588. E. sylvaticum Tabern., Lröut., 569, ic. 253.

1650. VII. E. sylvaticum tenuissimis setis C. Bauh., Pin., p. 16; Theatr., p. 245.

1651. E. sive Hippuris tenuissima non aspera J. Bauh., Hist. pl., III, p. 730.

1742. E. verticillis ad folia mumerosis IIall., Enum. stirp. Helv., p. 144.

1753. E. sylyaticuir L., Sp.pl., Ed. 1a, p. 1061.

Texte de Linné (Sp.pl., Ed. 2a, p. 1516).

sylvaticum. 1. Equisetum caule spicato, frondibus compositis. Fl. suec., 834; 927.

Equisetum selis ramosis internodio multoties longioribus. Roy. lagdb., 496.

Equisetum sylvaticum Fl. lapp., 391. Tabern. hist., 562.

Equisetum sylraticum, tenuissimis setis. Bauh. pin., 16. thealr., 245. 
1768. E. Foliis repelito-ramosis, vaginis laxissimis Hall., Hist. stirp. Helv., 1680.

! 17.-. I. sylualicum Pollich, llist. pl. Pulal., III, . . 5.

1778. R. sylualicum Lam., Fl. fr., 1, p. 8.

1800. E. syluaticum lioh, Tent. Fl. germ., IV, p. 3.

1805. R. sylvaticum DG., Fl. fr., lom. II, p. 583.

1807. E. syluaticum Lois. I)esl., Fl. gall., kid. 17, II, p. 700.

1813. E. umbrosum Lapeyr., Dyr., p. 619 (non .Veyer).

!1822. K. syluaticum Vaucher, Mon. 1'vêl., p. 365.

1828. E. syluaticum Duby, Bot.gall., p. 535 ; ct E. umbrosum, 1. c., fide Lapeyrouse (non llejer).

1835. E. sylvaticum Xlulel, Fl. Fr., IV, p. 166; et E. unbrosum, o. c., p. 167 (quoad specimina gallica).

!18.14. E. sylvalicum Newman, Brit. ferns, p. 59.

18.5\%. K. sylvaticum Kioch, Syn., Ed. 2a, p. 96.4.

1853. E. syluaticum Hilde, Kenntn. Eq., p. 597.

1850. F. syluaticum Gr. et God., Fl. d. Fr., III, p. 643.

! 1857. E. syluaticum Düll, Fl. Bad., I, p. 61.

!1857. K. syluaticum Bernoulli, Gef. Krypt. Schw., p. 69.

!1858. E. syluaticum Milde, Gef. Grypt. Schl., p. 431.

1861. E. syluaticum Cosson et Germain, Fl. env. Paris, 2e éd., p. 878.

3. E. pratexse Ehrh. (non Rolh, non Sprengel, non Schlechtendal).

Celle espèce a été pour la première fois signaléc el décrite sous ce nom en $\mathbf{1 7 8 3 ,}$ dans le IIanüurersche Maga:in, par Fr. Ehrhart, qui l'avait découverte dans des prés de la principauté de Blaukenbourg. Le mème botaniste la décrivit de nouveau en 1788 (Beiträge, JII, p. 77). G. F. Ioffmann crut d'abord que celle plante n'était qu'une forme de l'E. arvense (Deutschl. Fl., II , p. 3, n 5 , 1795); d'autre part Roth décrivit sous ce nom la variété nemorosum de l'E. artense (Tent. f. germ., III, p. 6, 1800), en citaut des figures qui n'ont aucun rapport avec la plante d'Ehrhart. Mais, en 1803, Hoflmann, averti par B. Boclihausen (1/onog. Crypt. Gew. in Römer's Archiv., I, nº 3, p. 5 et 6,1798 ) revint sur son opinion; il reconnut la plante comme une bonne espèce, et, d'après les échantillons originaux d'Ehrhart, en donna une description cxacte et une bonne figure colorice dans ses Phytographische Blülter (I, n ${ }^{\circ}$ viI, p. 117, tab. 2); il combaltit en mème temps l'erreur de Roth. Malheureusement les "Feuilles phytographiques s de IIollmann manqquèrent de publicité et ne furent mèrue pas continuées; il en résulta qu'apres les crreurs de Ioffmann et de Roth, l'altention des botanistes fut complétement détournée de ba plante d'Ehrhart, et dans l'Encyc. mith. Lot., V, p. 613, 1804, Poirct ne la cite que comme une variété de l'E. arvense.

En 1809, c'est-i-dire vingl-quatre ans apres la premiere publication d'Ehrhart, un officier francais, Bory de Saint-Vincent, faisant la guerre en Prusse, y trouva celle 
plante et la communiqua à Neyer età Willdenow. Ce dernier la crut nouvelle et la décrivit en 1809 sous le nom d'E. umbrosum, dans son Enumer. plant. horti Berolinensis; ct l'année suirante il la caractérisa mieux encore dans le Spec. plant., lout en y mentionnant l'E. pratense d'Ehrhart, mais comme plante à lui inconnue, admise sur l'autorité d'Ehrhart, en citant la synonymie erronée de Rolh, mais non les figures de Ioffmann. En même temps, c'est-à-dire en 1809 , Retz retrouvait cette plante en Scandinavie et la publiait comme nouvelle sons le nom d'E. amphibolium. $\Lambda$ partir de ce moment, la plante connue sous les noms de Willdenow et de Relz fut retrouvée en plusicurs licux; mais son premier nom d' $E$. protense avait presque complétement disparu. Vaucher décrivit el figura l'E. umbrosum de Willdenow, tout en conservant l'E. pratense Ehrh., mais, ainsi que Willdenow, à titre de plante inconnue, sur l'autorité d'Ehrhart, et en citant toujours la fàcheuse synonymie de Roth, mais non les figures de Iloffmann. En 1830, Sir W. Hooker décrivit encore cet Equisetum comme plante nourelle en la dédiant à Th. Drummond qui l'avait trouvée dans le Forfarshire. L'emploi fait par Willdenow et Vaucher de deux noms différents pour désigner une même plante, avait après eux porté la plupart des botanistes à chercher sous ces noms deux plantes distincles, el avait par cela occasionné quelques erreurs (Schlechtendal, Flor. Berol., II, p. 3 ct 259, 1824; E. Fries, Novitice fl. succ., Ed. 2a, p. 297, 1828, et 1lant., I, p. 21, 1832; Reichenbach, Flor:excurs., p. 154 ct 155, 1830 ; Homann, Flor. Pomer., III, p. 87 et 89,1835 etc.). M. de Schlechtendal nous a fait plus tard conuaitre lui-même que son E. pratense n'était qu'une forme du palustre. L'E. pratense de Sprengel (Fl. Hal., Ed. 2a, p. 435) était une forme de l'E. ramosissimum; enfin la confusion était extrême lorsqu'en mai 1836, M. Hampe, de Blankenbourg, après aroir consulté les exemplaires originaux dans l'herbier d'Ehrhart conservé à Göttingen par G. F. W. Meyer, écrivit au directeur du Flora une lettre où il rétablissait lidentité de l'E. pratense Ehrh. ct de l'E. umbrosum Willd. (Flora, 1836, p. 268 et suiv.); et dans le numéro suivant du même journal, M. de Schlechtendal inséra un très-savant mémoire, oủ il retraçait l'histoire complète de celte plante et de ses noms divers, et rendait ainsi impossible à l'avenir toute erreur à cel égard (Flora, 1836, p. 273 el suiv.).

1783. E. PRAtense Ehrh. Hannöv. Mlagaz., st. 18.

1788. E. pratense Ehrh., Beitr., III, P. 77, no 36.

1803. E. pratense Iloflmann, Phylograph. Blätt, I, no vil, P. 117, pl. II.

1809. E. amphibolium Retz., Fl. scand., suppl. 2, p. 12.

1809. E. umbrosum Willd., Enum. pl. hort. Berol., p. 1065.

:1810. E. umbrosum Willd., Spec.pl., V, p. 3.

1810. E. pratense Willd., Spec. pl., V, p. 6 (Exclud. syn. Roth).

1822. E. umbrosum Vauch., Mon. Prêl., p. 366.

1822. E. pratense Vauch., Mon. Prêl., p. 387 (Exclud. syn.). 
1826. E. syluaticum 3 minus Wahlenib., Fl. suec, Eil. 1, p. 689.

1831. E. Drummondii Hooker, Engl. Bol., suppl. 2777, ct Bril. flor., p. 454.

1836. E. Ehrharti G. F. Mejer, Chlor. Ilanov., p.666.

18.13. E. Drummondii Babingt., Man. brit. bol., p. 380.

! 18.15. E. umbrosum kioch, Syn. R. germ., Ed. 2a, p. 965.

!1844. E. umbrosum Kewm., Brit. ferns, p. 63.

11857. E. pratense Bernoul., Gef. Kirypt. Schw., p. 70.

!1858. E. pratense Vilde, Gef. Crypt. Schl., p. 437.

\section{Eouisetum anvense L.}

A parler exactement, cette espece est sans synonymie, le type ayant constamment conservé la dénomination linnéenne.

La grande forme nemorosum parail avoir été prise par Roth pour l'E. pralense Ehrh.; elle aurait élé ensuite, selon II. Bernoulli (Gef. Krypt. Schw., p. 66), prise par De Candolle pour l'E. fuviatile L.

Une forme très-grềle el très-réduite, avec ramification anomale à la base de la tige spicifere, serait, selon M. Milde (Ge\%. Crypt. Schl., p. 417 et 419 , et Syst. Eq., p. 142), l'E. riparium Fries.

L'anomalie de liges rameuses terminées par un épi a élé décrite comme espèce par K. F. Schultz, sous le nom d'E. cempestre. Vaucher ne mentionne point celle anomalie; il dit au contraire tris-expressément: « Je n'ai jamais ru dans celte espece les tigges a slériles porter des fleurs » (Mon. Prêl., p. 362).

Cet auteur ne mentionne pas davantage l'anomalie de tiges spicifires propres rameuses it la base; mais .1. Bernoulli nous fait connaitre (Gef. Krypl. Sclue., p. 66) que l'herbier de Vaucher (appartenant actuellement a $I$. . $\Lambda l_{p}$ h. De Candolle) renferme cette anomalie noté $\boldsymbol{E}$. pulustre, arec unc hampe purticulière. On trouve en effet dans la Mon. des Prêles une variété de l'E. palustre ainsi mentionnée:

a $B$. Equisctum nudum vaginis dilatatis, p. 367 .

a Il y a une seconde variété oủ la lige qui porte l'épi est couverte de gaines dilatées a dépourrues de rameaux. Daus cet étit elle a des rapports aree les prèles à hampe p (p. 368). Il est tres-probable que ces termes se rapportent, comme la plante de therbier de Vaucher, a l'anomalie précitie de l'E. areense, au moment ou elle offre à peine des rameaux naissants, et que le savant auteur de la Monographie se sera lui-même induit ę erreur en donnant au caractire fondamental de sa division des especes une valeur absolue (voir ci-dessus p. 165).

1552. Hippuris minima 'Tragus, De stirp., p. 693.

1586. Equisetum alterum sive minus Dalech., Hist., 1070, fig. 1, 2.

1625. IX. E. arrense longioribus selis C. Baulı., Pin., p. 16; Thealr., p. 2.47 (icon ad dextram bona). 
1651. E. mimus terrestre J. Bauh., Hist. pl., III, p. 730.

1742. E. caulibus foriferis a foliosis diversis Ilall., Enum. stirp. Ilelv., p. 144.

1753. E. arvense L., Sp. pl., Ed. 1a, p. 1061.

Texte de Linne̊ (Sp. pl., Ed. 2a, p. 1516).

arvense. 2. EQuisetur scapo fruclificante mudo, sterili frondoso.

Equisetum scapo fructificante nudo, caule sterili ramis compositis. $\mathrm{Fl}_{\text {. }}$ suec., 833; 928. Mat. med., 479. Dalib. paris., 308.

Equisetum setis quadrangularibus internodio longioribus, caulibus reptantibus. Guett. stamp., I, p. 201.

Equisetum selis simplicilus internodio multoties longioribus. Roy. lugdb., 496.

Equisclum arvense. Fl. lapp., 390. Hort. cliff., 471. Gron. virg., 123.

Equisetum arvense longioribus selis. Bauh. pin., 16.

Equisetum minus. Fuchs, hist., 323.

1768. E. caule forigero nudo, sterili verticillalo, radiorum duodecim Hall., Hisl. stirp. Helv., nº 1676.

11777. E. arvense Pollich, Hist.pl. Palat., III, p. 6.

1778. E. arvense Lam., Fl. fr., I, p. 6.

!1800. E. artense Rolh, Tent. f. germ., IV, p. 5.

1805. E. arvense DG., Fl. fr., II, p. 581 .

1807. E. arvense Lois. Desl., Fl. gall., Ed. 1a, Il, p. 700.

1810. E. arvense Willd., Sp.pl., V, p. 1.

!1822. E. arvense Vauch., Mon. Prêl., p. 361 (exclud. syn. E. amphibolium Relz.).

1828. E. arvense Duly, Bot.gall., I, p. 534.

1835. E. arvense Nutel, Fl. fr., IV, p. 166.

!1844. E. arvense Newman, Brit. ferns, p. 77.

!1845. E. arvense Koch, Syn., Ed. 2a, 1. 964.

1 1853. E. arvense et v'tes Milde, Kenntn. Eq., p. 560 et suiv.

1856. E. arvense Gr. et God., Fl. de Fr., III, p. 043.

!1857. E. arvense Döll, Fl. Bad., I, p. 59.

!1857. E. arvense Bernoul., Gef. Krypt. Schw., p. 65.

!1858. E. arvense Milde, Gef. Crypt. Schl., p. 417.

!1861. E. arvense Cosson et Germain, Fl. env. Paris, 2e éd., p. 877.

\section{$\beta$ nemorosum Al. Braun.}

1620. ? VI E. palustre tenuissimis et longissimis setis C. Bauh., Prod. p. 24. Pin., p. 16. Theatr., p. 245 (an confusum cum E. maximo el limoso?).

! 1800. E. pratense Roth, Tent. f. germ., IV, p. 6 (non Ehrh.).

1805. E. fluviatile DC., Fl. fr., II, p. 583 (ut rult Bernoulli; ralde dubito).

!1843. E. arvense $\beta$ nemorosum Al. Braun in Döll, Rhein. Fl., p. 27.

1857. E. antense $\beta$ nemorosum Bernoul., Gef. Krypt. Schw., p. 66.

$\gamma$ decumbens Meyer.

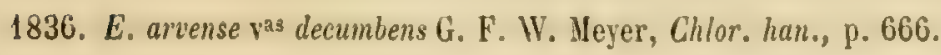


ò alpestre Wahlenberg.

1812. E. arvense $\beta$ alpestre Wahlbg., Fl. lapp., p. 290.

4c anom. frondescens.

1822. E. palustre vas B. Vauch., Mon. Prêl., p. 367.

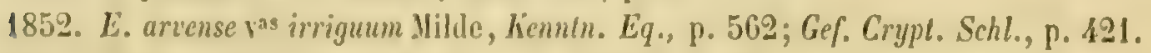

1857. L. arvense ras frondescens Düll, Fl. Bad., I, p. 58.

1842. E. ripurium Fries, Nov., Mant., III, 1). 167 et Summ.veg. Scand., p. 251, est hæc anomalia tenuis et gracilis, ex auct. Milde, Syst. Eq., p. 142.

$5^{\circ}$ anom. serotinum Neyer.

1836. E. arvense $\mathrm{r}^{33}$ serotinum G. F. W. Neyer, Chlor. han., p. 666.

1819. E. campestre K. Fr. Schultz, Flor, starg., suppl., p. 59.

\section{Equisetum littorale Kühl. el Rupr.}

Cetle plante a été décrite pour la première fois en 1845 , par M. Ruprecht, sur des exemplaires que M. de Kü̈hlewein avait recueillis aux environs de Saint-Pétersbourg, et elle a reçu le nom d'E. littorale Kühlewein et Ruprecht (Beitr.z. P/anzenk.d.russ. Reichs, IV, p. 91). Ciest sous ce nom qu'elle figure sans synonymie dans le Flora rossica de Ledebour, IV, p. 487.

En 1846, M. Lasch, qui avait trouvé cet Equisetum dans la Neumark, le décrivit sous le nom d'E. inundatum Lasch dans le Bot. Centralblull de M. Rabenhorst, ${ }^{\circ}{ }^{2}$, p. 28. Deux ans aprés, M. Rabenhorst le ramena en variété à l'E. artense el le men-

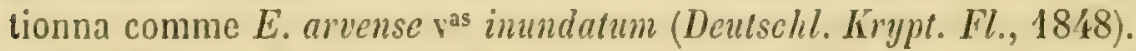

En 1853, G. Böckel reçul cetle plante de II. Koch, qui l'avait trouvée près d'U $\mathrm{U}_{p}$ jever, et, la croyant nouvelle, il la décrivit comme telle, mais d'une manière pen satisfaisante, et la nomma E. Kochianum. (Auf:ëhl.u. Beschr. Brem. Crypt. etc.).

Dans ses Crypt. r'ascul. Europer, 2e fasc., $1^{\circ} 4 \mathbf{4}$, M. Rabenhorst a donné l'E. lillorale kühl. sous ce nom, avec la synonymie d'E. immulatum Lasch; les exemplaires étaient recueillis par .II. Milde et Lasch; mais dans le $3^{\mathrm{e}}$ fascicule, $n^{0} 68$, 1860, la même plante reparait sous le nom d'E. arvensi $\times$ limosum Lasch, avec une note de M. Lasch, exprimant l'opinion que cette plante n'est qu'un hybride. C'est aussi l'opinion de M. Milde.

M. Düll identifie celle plante avee lE. artense formu serutinum ou E. campestre K. F. Schultz (Fl. Bad., I, p. 58).

En 1858, M. Milde publia dans le Bonplandia, p. 26 et suiv, une très-ḩonne nolice historique sur cet Equisclun, après s'ètre assuré par l'étude d'échantillons originanx de l'identitẻ des plantes décrites sons ces noms divers. 
Cet Equiselum n'avait point été mentionné en France lorsque je l'y trouvai sur trois points différents au printemps de 1859. Cette rencontre fut signalée par moi dans le Bull. de la Soc. bot. de France cn aoùt 1859, vol. VI, p. 602, et par M. Billot dans ses Ainnot. à la Flore de Fr. et d'Allem., p. 196, 1859.

1 1845. E. Litorale Kiuhlewein el Ruprecht in Beitr.z. Planzenk. d. russ. Reichs, IV, p. 91.

1846. E. inundatum Lasch., Bot. Centralblatt, p. 28, traduit dans le Bull. de la Soc. bot, aout 1859.

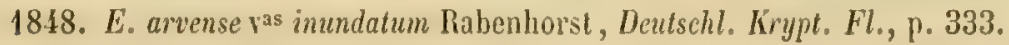

!1852. E. inundalum Milde, Kenntn. Eq., p. 573.

1853. E. litorale Ledebour, Flor. ross., IV, p. 487.

!1853. E. inundatum Milde, Rev. crit. Eq., p. 190.

1853. E. Kochianum Bückel, Aufz. und Beschr. Brem. Crypt. Gefässpfl.

!1858: E. littorale Vilde Bonplandia, 1858, no 2, p. 26 et suiv.

!1858. E. littorale Milde, Gef. Crypt. Schl., p. 451.

1859. E. inundatum J. Duw. J., Bull. Soc. bot., août 1859; tom. VI, p. 602.

1860. E. arvensi $\times$ limosum Lasch. in Crypt. vascul. Europa Rabenhorst, $3^{\bullet}$ fascicul., no 68, arec une note de Lasch.

\section{EQujsetum limosum L.}

L'adoption de ce nom linnéen a été si générale qu'elle semble exclure toute synonymie. Toutefois il y a encore quelques divergences d'opinion. En effet, alors même qu'on est d'accord pour reconnaitre que l'E. fluviatile $\mathrm{L}$. n'a rien de commun avec l'E. maximum Lam., et pour admettre que l'E. fluviatile et l'E. limosum de Linné ne sont que des formes d'un même type, il reste à déterminer lequel des deux noms doit devenir celui du type.

M. Ed. Newman (Phylologist, 1843, p. 534, ct Brit. ferns, p. 415), s'appujant sur cette considération que l'herbier de Linné contient sous le nom de fluviatile quatre spécimens en bon état, et qu'il ne renferme point de limosum, propose de reprendre fluviatile pour le nom du type, ainsi qu'il suit:

«E. fluviatile L. $=E$. limosum Smith.

". $\quad \beta$ limosum, with the stem quite simple, $=E$. limosum L.»

Comme il s'agit du même auteur, et que l'adoption de l'un quelconque des deux noms ne blesse aucun droit de priorité, j’ai préféré conserver à cette espèce le nom de limosum qu'elle porte dans presque tous les ouvrages modernes.

1552. Hippuris altera major Tragus, De stirp., p. 691.

1625. VIII. E. pratense longissimis setis C. Bauh., Pin., p. 16. Theatr., p. 246 (cum icone pessima).

1651. E. majus aquaticum J. Bauh., Hist. pl., III, p. 728. (Descript. ad alias species etiam spectat.)

1690. E. nudum locvius nostras Ray, Syn., 131, t. 5, f. 2.

1742. E. sulcis in caule plurimis, verticillis densissimis Hall., Enum. stirp. Helv., p. 143. 
1753. E. Lusosty L., Sp.pl., Ed. 1a, p. 1062. = Forma subnuda.

1753. E. Fluviatue L., $S p . p l .$, Ed. 1a, p. 1062. = Forma frondosa.

Texte de Linne (Sp.pl., Ed. 2a, p. 1517).

limosum 5. Equisetur caule subnudo levi. Fl. swec., 837; 931. Dalib. paris., 308.

Equiselum scapo nudo simplicissimo. loy. lugdl., 496.

Equisetum nudum licvius nostras. Maj. angl., 3, p. 131, 1. 5, f. 2.

nuriatile 4. Equisetus caule striato, frondibus subsimplicibus.

Eqquisetum caule non sulcaio lalissimo, verticillis densissimis. Ilall. helv., 144. Fl. suec., 836; 930. Dalib. paris, 307.

E. fluriatile. Fl. lapp., 393.

E. palustre, longioribus selis. Bauh. pin., 15. theatr., 212.

1768. E. caule sulcato, ramis mullifloris, foliis indirisis, $\beta$ planta in aquosis mascens, caule nudo latiori et numerosis sulcis distincto Ifall, Hist. stirp. Helv., no 1677.

11777. E. furiatile Pollich, Iist.pl. Palat., III, p. 8 (Exclud. syn. Halleri).

1778. E. palustre $\mathrm{r}^{\text {as }}$ y Lam., Fl. fro, I, p. 7.

! 1780. E. limosum Koth, Tent. /. germ., IV, p.9.

1783. E. Ileleocharis Elırh., IIannöv. Mag., St. 18, p. 286, no 30.

1805. E. limosum DC., Fl. Pr., II, 582.

1807. E. limosum Lois. Desl., Fl. gall., Ed. 1ª, II, p. 701.

1810. E. uliginosum Willd., $S_{p}$. pl., tom. V, p. 4 (forma subnuda et debilis secund. Al. Braun in litt., ex Ledebour).

1810. E. limosum Willd, Sp.pl., tom. V, p. 4.

1822. E. limosum Yauch., Mon. Prêl., p. 372.

1828. E. limosum Duby, Bot. gall., p. 535.

1835. E. limosum Mulel, Fl. Fr., IV, p. 168.

!1844. E. fuviatile Jewman, Brit. forns, p. 51.

!1845. E. limosum Koch, Syn., Ed. 2a, p. 965.

! 1852. E. limosum Milde, Kenntn. Eq., 601.

1856. E. limosum Gr. el God., Fl. de Fr., III, p. 644.

! 1857. E. limosum Döll, Fl. Bad., I, p. 63.

1857. E. limosum Bernoul., Gef. Krypt. Schw., p. 79.

11858. E. limosum Vilde, Gef. Crypt. Schl., p. 446.

!1861. L. limosum Cosson et Germain, Fl. env. Paris, 20 éd., p. 880.

\section{EQuisetum palustre L.}

A part lïllusion que fit à De Candolle un sujet dont le rhizome était garni de tubercules, celle espèce n'olfre aucune synonymie.

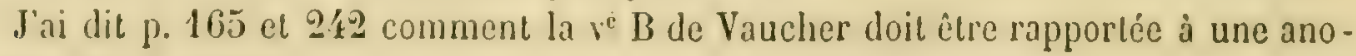
malie de l'E. arvense.

«Les exemplaires avec rejelons sans branches constituent l'E. pratense $\beta$ simplicis- 
simum Al. Braun, que cet illustre auteur a dans le Silliman's Journal (décembre 1843 , p. 85) caractérisé par ces mots: Stems without branches » (Döll, Fl. Bacl., I, p. 63).

Dans beaucoup d'herbiers, comme dans celui de Linné, les liges stériles de celle espèce sont confondues avec les liges stériles de l'E. arvense.

1552. Hippuris minor altera Trag., De stirp., p. 693.

1625. III. Equiselum palustre brevioribus setis C. Bauh., Pin., p. 15. Theatr., p. 241 (sine icone).

1742. E. multiflorum caule tetragono verticillis rarioribus Hall., Enum. stirp. Helv., p. 143 (ad duas vares spectat).

1753. E. ralustre L., Sp.pl., Ed. 1, p. 1061.

T'exle de Linné (Sp.pl., Ed. 2a, p. 1516).

palustre. 3. EQuisetur caulc angulato, frondibus simplicibus.

Equișetum setis simplicibus internodia vix superantibus. Roy.lugdb., 496. Fl. suec., 835; 929. Dalib. paris., 307.

Equisetum palustre $\mathrm{Fl}$. lapp., 392.

Equisetum palustre brevioribus selis. Bauh. pin., 15; theatr., 242.

$\beta$ Equisetum palustre minus polystacliyon. Bauh.pin., 16; prodr., 24. Raj. angl., 3, P. 131, t. 5, f. 3 .

1768. E. caule sulcato, ramis mullifloris, foliiz indivisis. $\alpha_{.}$planla terrestris, caule angustiori, tetragono, pentagono, sulcato Hall., Hist. slirp. Helv., no 1677.

1777. E. palustre Pollich, Hist. pl. Palat., III, p. 7.

1778. E. palustre Lam., Fl. fro, I, p. 7 (exclud. ras $\gamma$ ).

11800. E. palustre Roth, Tent. M. germ., IV, p. 7.

1805. E. palustre DC., $F l$. fr., tom. II, p. 583, ! et rol. VI, p. 244.

1807. E. palusire Lois. Desl., Fl. gall., Ed. 1a, II, p. 701.

1815. E. tuberosum DG., Fl. fi., suppl., p. 245 sec. Duby, Bot. gall., p. 535 (Exclud. syn. Willd.).

11822. E. palustre Vaucher, Mon. Prêl., p. 367 (Exclud. $\mathrm{v}^{\text {as }}$ B).

1828. E. palustre Duby, Bot. gall., p. 535.

1830. E. pratense Rclib., Fl. germ. excurs., p. 155, n० 939 (non Ehrh.).

1835. E. palustre Nutel, Fl. fr., IV, p. 167.

!1844. E. palustre Newman, Phytol., a. 1842, p. 529. Brit. ferns, p. 43.

!1845. E. palustre Koch, Syn., Ed. 2a, p. 965.

1852. E. palustre Vilde, Kennin. Eq., p. 600.

1856. E. palustre Gr. et God., Fl. de Fr., III, p. 644.

!1857. E. palustre Döll, Fl. Bad., I, p. 62 , cum ve tenue.

1857. E. palustre Bernoul., Gef. Krypt. Schw., p. 71.

!1858. E. palustre Vilde, Gef. Crypt. Schl., p. 459.

1861. E. palustre Cosson el Germain, Fl. env. Paris, 2 èd., p. 879.

Forma polystacliyon.

1625. V. Equisetum palustre minus polystachyon C. Bauh., Prod., p. 24. Pin., p. 16. Theatr., p. 245.

1690. E. palustre minus polystachyon Dill., in Ray Syn., 131, 1.5, f. 3. 
1768. E. II. Var. Ramis mullis, quorum quisque spica florigera lerminalur Hall., Hist. stirp. Helv., n० 1677.

E. palustic vas polystachyon om. aucl. sup. cilat.

Forma tentuis.

E. prostratum Hoppe secund. lioch, Syu., Ed. 24, p. 965, Döll, Fl. Bad., I, p. 62, et Nilde, Ger. Crypt. Schl., p. $\$ 59$.

1828. E. palusire ras y nudum. Duby, Bot. gall., p. 535.

1813. E. pratense ${ }^{\text {as }} \beta$ simplicissimum A1. Braun, Sill. Journ., déc. 18.13, p. 85 (sceund. Düll., 1. c.).

1857. E. palustre ras temue Düll, Fl. Bad., I, p. 63, et Vilde, Gef. Crypt. Schl., p. 461.

1863. E. albo-marginalum Kï., Acr. Inng., p. 265.

\section{Eouisetum Rayosissiyum Desf.}

Par suite de la multiplicité de ses formes diverses, celte especee est celle qui a la synonymie la plus variée.

Abondamment répandue dans toute l'Europe méridionale, clle était, dés 1715 , mentionne par Garidel (llist. plunt. ent. d'.lix) et rapporte par lui a l'E. fuliis

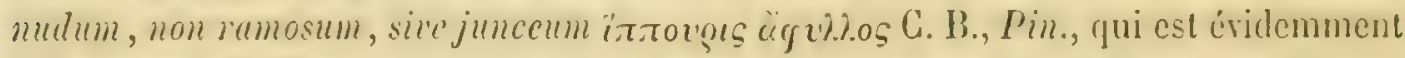

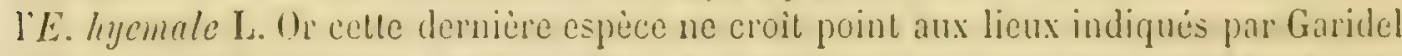
"Bois de la Dagleleine, à Barguiasso; i la Beauroisine et le long de l'Are». Jai souvent visité ces localites, et j’y ai trouvé en abondance la présente espèce, mais nulle trace de l'E. haycmale, que je n’ai d'ailleurs vu mulle part en Basse-Provence. Gérard (Flor. gall. jror., p. 46) et Gouan (Flor. monspel., p. 438) confondirent cette plante arec IE. liyemale L., ou plutút ils suivirent l'exemple de Linné qui, dans sa synonymie, arait éridemment réuni à son $E$. hyemale des régrions du Nord celte plante déjả mentionnéc peut-être par G. Bauhin sous son $n^{\circ} \mathrm{XI}$.

Ce n’est qu'en l'an VI de la République qu’elle fut par Desfontaines établie comme cspece distincte et nommée E. ramosissimum. En l'an XII, Poiret, tout en conservant l'espèce de Desfontaines, donna le nom d'É. campanulatum à une forme récoltie sur les bords de l'Lveaune, it IInseille, el y rapporta i tort en stnonymie la phrase que G. Bauhin consacre a l'E. veriegutum. En 1806 De Candolle décrivit la mème espèce comme nouvelle el la nomma E. ramosum (Synopsis, p. 118, $1^{\circ} 1457^{\circ}$ ). Cest done doublement i tort quon appelle cette plante E. ramosum Schleicher; car, d'une part, le nom de Desfontaines, ayant la priorilé, doit être conservé, el, d'autre part, le nom I'E. rumosum n’appartient pas it Schleicher, mais bien à De Candolle. Le premier Catalogue de Schleicher, sans dite (1800?), n'en fait aucune mention; il ne parait gue dans le Catalogue de 1807, postérieur d'un an au Synopsis, el I'S qui suil fait a l'initiale du Synopsis ou de Sehleicher une allusion ambiguë que De Candolle luimème, dans son supplément de 1815 , fit tourner au profit de Schleicher, en cilant 
par erreur la p. 21 du Catal. pl. IIclv. après le nom d'E. ramosum qui ne s'y trouve pas. Sur l'autorité de De Candolle l'erreur s'est propagée sans contradicteurs. Celte espèce fut d'ailleurs si mal distinguée par Schleicher que, d'après M. Bernoulli \{Gef. Krypt. Schw., p. 74), ce collecteur la distribua mêlée à l'E. hyemale vas paleaceum. Comment De Candolle; en son Supplément de 1815, rapporta-t-il à cette plante \&l'E. limosum All. ped., $\mathrm{n}^{\circ}$ 23 s (citation fautive)? c’est ce que je n'ai pu m'expliquer.

La forme simple devint en 1810, d'une part, l'E. pamonicum de Willdenow (d’après les témoignages de MMI. $\Lambda$. Braun in Koch, Syn., Ed. 2a, p. 966; Döll, Fl. Bad., I, p. 65 ; Milde, Kemtn. Eq., p. 600, fondés sur la vue des exemplaires originaux de Willdenow), d'autre part, l'E. elongatum du même, "nom sous lequel, dit De Candolle, l'auteur paraît avoir réuni des plantes différentes» $(F l$. fr., Suppl., VI, p. 244). Le protéisme de celte espèce a été caractérisé par le nom si juste d' $E$. multiforme que Vaucher lui appliqua. Enfin, en 1839, M. Al. Braun la ramena en variété à l'E. hyemale L.

Lorsque les liges de cette espèce ont été coupées contre terre, elles produisent une multitude de petits rameaux grêles; et trop souvent cet aspect cespiteux lui a valu dans les herbiers le nom d'E. variegatum.

M. Ch. Desmoulins fait remarquer qu'en Périgord « la plante entière, très-glauque «el blanchâtre, présente un aspect extrêmement pâle el qui n'est pas habitucl dans (le genre; ) et re savant botaniste ajoute: «Je me demande si ce ne serait pas là l'E. "pallidum Bory, Exp. de Morće, p. 282, cité sous le $n^{\circ}(9)$ entre parenthèses par le « Sylloge de Nyman avec ces seules indications de localités françaises: Gall. (Garonne, "Nontpell.), puis en Espagne et en Grèce » (Gatal. phanérog. Dordogne, suppl. fin., p. 387).

? 1625. XI. Equiselum foliis nudum ramosum C. Bauh., Pin., p. 16. Theatr., p. 249 (cum icone rix bona).

1753. E. hyemale L. (ex parte sec. syn. Bauh.).

1765. E. hyemale Gouan., Flor. monsp., p. 438 (non L.).

! an VI. E. ramosissimum Desf., Fl. all., II, p. 398.

an XII. E. campanulalum Poiret, Enc. méth., V, p. 613 (exclud. syn.); et E. ramosissimum Poiret, o.c., p. 612.

1806. E. ramosum DC., Syn., p. 118, et $1815 \mathrm{Fl}$. (r., supp. VI, p. 245 (Exclud. syn. Ail.).

1807. E. campanulatum Lois. Desl., Fl. gall., Ed. 1ª, II, p. 701.

1807. E. ramosum Lois. Desl., Fl. gall., Ed. 1a, II, p. 701.

!1810. E. elongatum Willd., Sp.pl., tom. V, p. 8 (Exclud.pl. ins. Borbon.?).

!1822. E. ramosissimum Vaucher, IIon. Prêl, p. 369.

!1822. E. multiforme $\beta$ ramosum, y paleaccum, s campanulatum Vaucher, Mon. Prél, p. 379 et 381 (Exclud. syn. E. tenue Iloppe).

1828. E. multiforme ${ }^{\text {as }} \beta$ ramosum et $y$ campanulalum Duby, Bol. gall., p. 535. 
1828." E. campanulatum Lois. Jesl., Fl. gull., Li. 2a, II, p. 358.

1828. E. ramosum Lois. Desl., $\mathrm{Fl}$. gall., Ed. 24, II, p. 353 (cum? aucloris).

?1832. E. pallidum Bory, Expéd. I/or., p. 282.

:1835. E. clongatum Iul, $F \%$ fro, N, p. 169.

1839. E. hyemale 1 ramosum Al. Braun, in Flora, 1839, p. 308.

18.15. E. ramosum Koch, Syy., Ed. 2a, p. 966.

1856. E. ramosum Gr. el God., Fl. de Fr., III, 13. 6.15.

! 1857. E. elongalum Iöll, Fl. Bad., I, p. 65.

:1857. E. ramosum Bernoul., Gef. Krypt. Schw., p. 74.

1857. E. elongatum Pokorny, Eq. arv. el hyem. p. 9.

! 1858. E. elongatum Milde, Gef. Crypt. Schl., p. 466 (ut subspecies E. hyemalis L.) (cum syn. E. procerum Pollini, Plant.veron., p. 28, el $\boldsymbol{E}$. incanum Vaucher, Mon. Prêl., p. 382; synunymia valde dubia!).

\section{E. variegatum Schleich.}

Celle espèce, évidemment distinguée et mentionnée par G. Bauhin, Parkinson et Haller, fut comprise par Linné dans son $E$. hycmale, arec lequel elle resta longtemps confondue. Elle existe dans son herbier annotée par J. E. Smith: «E. variegatum Jac(q. II. B.» En 1800, Schleicher, sans la décrire, la nomma "E. v'aricyalum All.; » cette mention d'Allioni me parait inexplicable. En 1807, F. Weber et II. Mohr en donnèrent une diagnose el une très-bonne description, que je crois les premieres, et ru'à ce titre j’ai cilées p. 210. Willdenow mentionna celle espèce, en lui donnant pour synonyme E. limosum All., no 238'4; c'est une erreur évidente que Poiret a reproduite (Enc. méth., suppl. IV, p. 548).

Cet Equisctum a continué d'être distingué sous le nom de v'ariegatum, soit comme espèce propre, soit comme variété de l'hyemalc L., du multiforme Vauch., du rep̧tans Whlbg.

Une grande forme du bord des caux a été dédiée par M. Ed. Newman a M. Wilson; M. Milde affrme, d’après les échantillons originaux de l'herbier de M. Al. Braun, que ce n'est pas même une variété (Gef. Crypt. Schl., p. 470).

! 1623. Equiselum mudum minus variegalum Basileense C. Bauh. Pin., p. 16; Theutr, p. 250 (deser. opt.).

1640. E. nudum, mimus, variegatum Park. Theatr., 1202.

1742. E. caule simplicissimo, vaginis profunde sectis Ilall., Enum. stirp. Ilelv., p. 143.

1753. E. hyemale L. (ex parle).

1768. E. cuule sulcato, submudo, vaginis aristatis Hall., Hist. stirp. Helv., $\mathrm{n}^{\circ} 1678$.

1800. E. variegatur Schleich, Cat.pl. Helv., p. 21; Ed. 1807, p. 27; Ed.1815, p. 32; Ed. 1821 , p. 38 (sine descript.).

1807. E. caricyalum Web. u. Nohr, Boi. Tasch. a. d. Jehr 180\%, p. 60 et $\$ 17$ (cum descr. princ. et opt.). 
1810. E. variegalum Willd., Sp.pl., V, p. 7 (Exclud. syn. All.).

:1812. E. reptans $\beta$ variegatum Wahlbg., Fl. lapp., p. 298.

1815. E. variegatum DG., Fl. fr., suppl. VI, p. 244.

1822." E. multiforme a variegalum et $\delta$ tenue Vauclıer, Mon. Prêl., p. 379 et 381.

1828. E. multiforme a variegalum Duby, Bot. gall., p. 535.

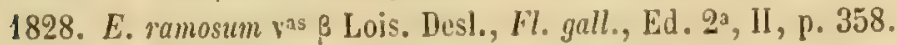

1835. E. variegalum Mut., Fl. fr., IV, r. 168.

!1842. E. hyemale q variegalum Newm., Phylol., a. 18.12, p. 337.

:1842. E. variegatum J. B. Brichan, Descr. Eq., in Phytol., a. 1842, p. 370 et seq.

!1843. E. variegalum Koch, Syn., Ed. 2a, p. 967.

1844. E. variegatum Newm., Brit. ferns, p. 31, et (form. elat.) vas Wilsoni, p. 39.

1856. E. variegalum Gr. el God., Fl. de Fr., III, p. 646.

!1857. E. variegatum Döll, Fl. Bad., I, p. 71.

1857. E. hyemale vas e variegalum Kirschl., Fl. d'Als., II, p. 379.

!1857. E. variegatum Bernoul., Gef. Krypt. Schw., p. 77.

!1858. E. variegatum Milde, Gef. Crypt. Schl., p. 469.

\section{Equisetum trachyodon Al. $\mathrm{Br}$.}

Cet Equisetum, nous apprend M. Ed. Newman (Brit. ferns., p. 26), a été observé pour la première fois, en 1833, dans le nord de l'Irlande, par M. J. F. Mackay, « the «well know author of the Flora hibernica) (Dublin 1836). G'est donc a ce bolaniste que M. Newman le dédia, en 1842, comme variété de l'E. hyemale (The Phytol., 1842, p. 306). Mais déjà M. Al. Braun avait décrit cette plante dans le Flora de 1839 ,

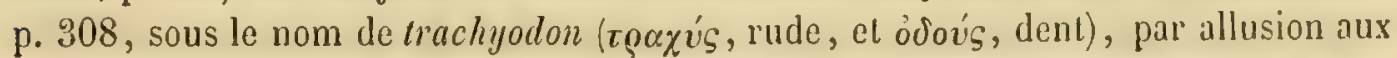
aspérités qui hérissent les dents des gaînes de cette plante dans son jeune âge. Depuis, cette plante a été constamment distinguée, soit comme espèce (Brichan, Desc. Eq., p. 369-377; E. Newm., Brit. ferns., p. 25 et 411 ; Borcau, Fl. Cent.), soit comme variété de l'E. hyyemalc. M. Pokorny $(E$. arv. et hyem., p. 10) en fait même une variété de l'E. clongatum Willd. (E. ramosissimum Desf.); mais comme le même auteur fail d'abord de celte plante une variété de l'E. hyemale et quill ne rapporte en variété à l'E. elongatum que des sujets récoltés à Carlsruhe, la description qu'il en donne m'a fait penser, peut-être à tort, qu'il a pu prendre pour de l'E. trachyodon des sujets très-grêles de l'E. ramosissimum.

L'E. trachyodon a été signalé pour la première fois en France par M. Boreau, dans sa Flore du Centre.

!1839. E. Tnacirodon Al. Braun, Flor., 1839, p. 308.

!1242. E. hyemale vas $\beta$ Mackaï Newman, Phytol., a. 1842, p. 305. Brit. ferns, p. 25.

!1842. E. Mackaï J. B. Brichan, Descr. Eq., in The Phytologist., a. 1842, p. 369 el suir 
!1813. E. trachyodon Koch, Syn., Ed. 2a, p. 967.

18.9. E. trachyodon Boreau, Fl. Centr. Fr., 2e èd., II, p. 625.

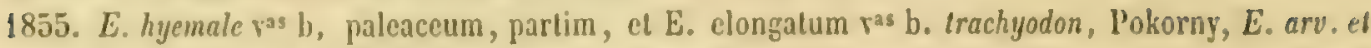
liyem., p. 9 et 10.

1856. E. traclyodon Gr. el God., $F l$. de Fr., III, p. 615.

1857. E. hyemale ras d trachjodon Kirschl., Fl. d'Als., II, p. 379.

1857. E. hyemale ras c trachyodon Düll, Fl. Bad., I, p. 69.

! 1858. E. trachyodon Jilde, Gef. Crypt. Schl., P. 46.1 (ut subspecies E. hyemalis L.).

\section{Equisetuy Heyale L.}

La nudité, la rudesse et la pérennité des tiges de cette espèce l'ont toujours fait distinguer non-seulement des botanistes, mais encore de tous les ouvriers qui l'employaient. Elle n'a done point de synonymic. Nais l'imperfection des figures el des descriptions des anciens et des modernes a sourent trompé les botanistes, et ils ont appliqué trop facilement le nom d'E. hyemale à plusieurs formes de l'E. ramosissimum el de l'E. v'ariegatum, à l'exemple de Linné, qui réunissait à celle cspèce l'E. variegatum et l'E. ramosissimum, ainsi que l'indique pour tous les deux sa citation de la synonymie de Bauhin, et son herbier pour l'E. variegatum.

1552. Hippuris major Tragus, De stirp., p. 691.

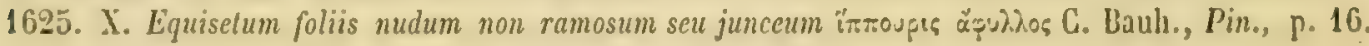

Theatr., p. 2.18 (cum icone pessima, et potius E. limosum referente).

1690. E. nudum Ray., Syn。, 131.

1742. E. caule simplicissimo, aspero, vaginis non laciniatis IIall., Enum. stirp. Helv., p. 143.

1753. E. uyedale L. (ex parte sec. syn. Bauh.), Sp. pl., Ed. 1a, p. 1062.

Texte de Linné (Sp. pl., Ed. 2a, p. 1517).

hyemale. 7. EQusetcrs caule nudo scabro basi subramoso. Fl. suec., 838; 931. Dalib. paris, 308.

Eqquisetum scapo nudo simplicissimo. Roy. lugdb., 496. Gron. viry., 196.

Equisetum liyemale. Fl. lapp., 394.

Eqquisetum foliis nudum ramosum. Bauh. pin., 16.

Equisclum nudum minus variegatum basiliense. Bauh. pin., 16. prodr., 25.

Equisetum foliis nudum non ramosum. Bauh. pin., 16.

Eqquisctum. Cam. epil., 770, f. \.

1768. E. caule subnudo, asperrimo, vaginis caulinis indivisis, rameis cilialis. Hall., Hist. stirp. Helv., $n^{\circ} 1679$ (nonne ad E. ramosum etiam speclat?).

!1777. E. liyemale Pollich., Hist. pl. Palat., III, p. 9.

1778. E. hyemale Lam., $N$. fr., I, p. 6.

!1800. E. hyemale Rolls, Tent. R. germ., IV, p. 10. 
1805. E. hyemale DG., Fl. fr., II, p. 580 .

1807. E. hyemale Lois. Desl., Fl. gall., Ed. 1a, II, p. 701.

1810. E. hyemale Willd., Sp. pl., tom. V, p. 8.

!1822. E. hycmale Vaucher, Mon. Prél., p. 374.

1828. E. hyemale Duby, Bot. gall., p. 535.

1835. E. hyemale Mutel, $F l$. fr. , IV, p. 168.

11812. E. hyemale Newman, Phytol, a. 1812, p. 272; Brit. ferns, p. 17.

118.2. E. hyemale J. B. Brichan, Descr. Eq. in The Phytologist, a. 1842, p. 369 et suiv.

!1845. E. hycmale Koch, Syn., Ed. 2a, p. 966.

!1852. E. hyemale Milde, Kenntn. Eq., p. 603.

1856. E. hycmale Gr. et God., Fl. de Fr., III, p. 644.

!1857. E. hyemale Döll, Fl. Bad., I, p. 67.

1857. E. hyemale Bernoul., Gef. Krypt. Schw., p. 75.

! 1858. E. hyemale Nilde, Gef. Crypt. Schl., p. 462 (ex parte).

1861. E. hyemale Cosson et Germain, Fl. env. Paris, $2^{\circ}$ éd., p. 881.

\section{G H A I T RE V}

\section{Iconographie}

Les figures des auteurs anciens sont en général si peu fidèles et dès lors si pen utiles pour la distinction exacte des espèces, que je n'ai pas voulu grossir de leur's citations les listes suivantes. J'ai mentionné seulement celles qui sont reconnaissables; mais toutefois j’ai voulu citer scrupuleusement celles auxquelles Linné a référé ses espèces, et pour les distinguer, je les ai fait précéder du signe $(\mathrm{L})$.

Les meilleures figures modernes sont celles du Flora danica, de Schkuhr, de Bischoff, de Newman, de Hofmeister et de Nilde. On les trouvera citées, chacune en leur lieu; un ! indique celles que l'on pourra consulter avec le plus de fruit. Le signe de la division (:) signalera les figures analytiques; le signe de Vénus (q) celles des organes de reproduction; un $\mathrm{R}$ renversé, celles des irrégularités ou monstruosités.

\section{E. MAXIMUM Lam.}

(L) 1658. C. Bauhin, Theatr., p. 241 (pessima).

! 1790. Bolton, Fil. brit., lab. 36 et 37.

! 1790. Smith, Engl. Bot., 2022.

! 1845. Flor. danic. IX. 1469 (forma serotina).

!! 1798. Schkuhr, Kirypt. Gew., tab. 168.

1822. Vaucher, Mon. Prêl., pl. II, fig. 1-2, 3-4 (\%), 5-6 (:); ê pl. II, A (y).

1823. Vaucher, Fruct. Prêli, pl. XXVII. 
!(:) 1828. Bischoff, Lirypt. Gev., tab. 4, fig. 26-27; tab. 5, fig. 45.

: 1828. Ad. Brong. Hist. vég. foss., I, pl. XI, fig. 1-10, ot pl. Xll, fig. 6-10.

! 1840. Dietrich, Deulschl. Krypt. Gow., pl. I.

! 184. Sewn., Brit. ferns, p. 67 et 76 (:).

1852. Milde, Kenntn. Eq., tab. 55, fig. 21-22, 24-39 (נ) , tab. 56, fig. 23, 40-42 (घ).

(8) 1852. Milde, Arch. Eq. Tclm., pl. V'II, fig. a, b, c; Botan. Zeit., 1852, p. 539.

1854. liamey, Tub. Eq., pl.1.

(:) 1858. J. Duv. J. Bull. Soc. bot. Fr., V, p. 515.

(11) 1858. Reinsch, in Flora, 1858, no 5, tab. 2, fig. 3-1.

\section{E. SYLVATICUM L.}

(L) 1658. C. Baul., Thealr, p. 245 (bona).

! 1790. Bolton, Fil. Urit., lab. 32 et 33.

! 1790. Smith, Eng. Bot., $187 \%$.

! 1794. Flor. danica, VII, tab. 1182.

(:8) 1798. Hedwig, Theor. gener., Ed. 23, tab. 1.

! 1798. Schkulir, hrypt.Gew., tab. 166. (\$) mala.

! 1829. Vaucher, IIon: Prêl., pl. III, fir. 1-3, 4 (:).

(:) 1828. bischoff, hrypt. Gew., tab. 3, fig. 3-4.

! 1844. Nerm., Brit. perns, p. 59, 62 et 50 (:).

1853. Milde, Ueber d. Eq. metab., pl. I, fig. 1-6 (y).

(₹) 1853. Bischoff, Bot. Zcit., 1853, tab. 2, fig. 3, reproduit dans les Ann. sc. nat., Illo série bot., tom. 19 , pl. IX, lig. 3.

1858. Milde, Gef. Crypt. Schlo, tab. 32, fig. 29-30 (u); tab. 34, fir. 36-39 (u); tab. 35, fig. 48 el 50 (y).

(:) 1858. C. Sanio, Epid. u. Spalt. Eq., pl. 11I, fig. 22-26.

(:) 1858. J. Duv. J., Bull. Soc. bot. Fr., V, p. 516.

\section{E. PRATENSE Ehrh.}

! 1803. Iloffmann, Phylograph. Blïlt., I, n. vur, pl. II.

! 1822. Yaucher, Mon. Prêl., pl. IV, fig. 1-4.

! 1823. Flor. danica, X, fasc. 30 , lab. 1770 , sub: E. umbrosum Willd.

! 1829. Flor.danica, XI , fasc. 33, p. 5, tab. 1943, sub: E. arrense var. \%.

! 1831. Hooker, Engl. Bol., suppl. 2777.

! 1840. Dictrich, Deulschl. Krypt. Geu., pl. V.

! 1844. Newm., Brit. ferns, p. 63, el p. 81 et 82 .

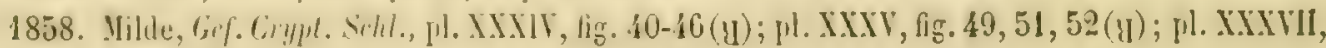
fig. 63 (:).

(:) 1858. Sanio, Epid. u. Spall. Eq., fig. 19-21. 


\section{E. ARVENSE L.}

De toutes les anciennes figures, Linné ne cite que celle de Fuchs, p. 323, qui représente à peu près une lige stérile de celle espèce; Tragus (éd. de 1552) donne à la p. 692 (à droite) une réduction renversée de la p. 323 de Fuchs, en la distinguant de l'espèce figurée p. 694, qui est pourtant la même, mais bien mal représentée.

(L) 1515. Fuchs, IIist. stirp., p. 323.

1552. Tragus, De stirp. (Ed. 1552), p. 694 et 692 (dextra).

1658. C. Baulı., Theatr., p. 247 (subhona).

1700. Tournefort, Inst., lab. 307.

1790. Bolton, Fil. brit., tab. 34.

! 1798. Schkuhr, kimpt. Gew., lab. 167, cum (:) mala.

(:) an IX. Nirbel, Anat. Prèl., pl. I, fig. 16, nos 1-4; pl. II, fig. 1 (mala).

! 1813. Smilh, Engl. Bot., 2020.

(:) 1815. Mirbel, Phys. végét., pl. LXIV, fig. 5, A, B, C (malæ).

! 1817. Gurtis, $\mathrm{Fl}$. lond., pl. CCLXXXV et LXIV.

1822. Vaucher, Mon. Prêl., pl. I, fig. 1-2, 3-4 (:), 5-6 (१).

() 1823. Agardh, Germ. Prêl., pl. XIII, fiğ. 1-4.

!(:) 1828. Bischoff, Kiypt. Gew., lab. 3, fig. 2; tab. 4, fig. 6-19, 27-28; tab. 5, fig. 29-41.

1829. Flor. danica, XI, tab. 1942, vas; XII, lab. 2001.

(:) 1833. Krocker, De plant. epid., pl. I, fig. 5 et 6.

1844. Newm. Brit. ferns, p. 77, 81 (:) et 82.

!(\&) 1851. Hofinst., Vergl. Unters., tah. 18, fig. 7-13 et 20, fig. 32-62.

!(?)1851. Hofmst., Kenntn. Gef. Krypt., tab. 17, 18 et 19.

! 1852. Milde, Kenntn. Eq., lab. 54, fig. 1-7 (y), 8 (:); lab. 57, fig. 1-36; tab. 58, fig. 37-47; tab. 59, fig. $47 b-56$; tab. 60 , fig. 57-58 (\%).

(:) 1853. Pringsheim, Bot. Zeit, pl. VI, fig. 1-4.

1854. Ramey, Tub. Eq., pl. II.

1858. Nilde, Gef..Crypt. Schl., tah. 31, fig. 10-18 (y), tab. 32, fig. 19-25 (y); fig. 26-28

(II) et div. forma); lab. 33, fig. 31-34 (div. forma); tab. 34, fig. 35 et fig. 47 (div. forma).

(:) 1858. Sanio, Epid. u. Spalt. Eq., pl. III, fig. 17-18.

(:) 1858. J. Duv. J., Bull. Soc. bot. Fr., V, p. 515.

(२) 1859, J. Duv. J., Bull. Soc. bot. Fr., VI, pl. II.

\section{E. littorale Kühlw.}

1852. Nilde, Kennin. ${ }^{5}$ Eq., tab. 54! fig. 10-13 (:); tab. 55 , fig. 14 (:), 15-20 (४).

1858. Nilde, Gef. Crypt. Schl., tab. 35 , fig. 536 ; tab. 36 , fig. 53 a, 54, 56 type. 


\section{E. Limosuy L.}

1658. C. Bauh., Thealr., p. 216 (pessima).

(L) 1690. Iay, Sym. angl., p. 131, tab. 5, fig. 2, a, b.

1790. Bolton, Fil. brit., tab. 38.

! 1797. Flor. dan., VII, 118.4.

!! 1798. Schliuhr, Krypt. Gew., tab. 171. 1800. Smith, Engl. Bol., XIII, 929.

(:) an IX. Mirbel, Anut. Prế., pl. II, fiğ. 2, nos 1-7 (mala).

1829. Vaucher, Mon. Prếl, pl. VIII, fiğ. 1-3, 4-5 (क), 6-7 (:).

() 1823. Agardh, Germ. Prêl., pl. XIII, fig. 6-9.

(:) 1828. Bischoff, Krypt. Gow., tab. 4, fig. 24; tab. 5, fig. 42.

! 1840. Dichrich, Deutschl. Krypt. Gew., pl. I.

! 184. Newm., Brit. ferns, p. 51 et 50 (:).

(\$) 1851. G. Thurel, Zoosp. alg., pl. XV, fig. 1, 2.

1851. Ilofmst., Vergl. Unters., tab. 19, fig. 1-24, et 20, fig. 1-31.

1859. Milde, Licnnln. Eq., tab. 54, fig. 9 (:); lab.56, fig. 45 (1).

1858. Milde, Gef. Crypt. Schl., lab. 36, figg. 55 (y) (tige spiralée), et fig. 58 (y).

(:) 1858. G. Sanio, Epid. U. Spalt. Eq., pl. 11I, fig. 1-11.

(:) 1858. J. Dur. J., Bull. Soc. bot. Fr., Y, p. 517.

\section{E. palustre L.}

On ne peut ciler aucune ancienne figure de celle espèce ayant quelque valcur; Gerorde (Herb. Em., 1113) a copié De Lobel (Ic., 795). La figure de Roy, la scule que cile Linné, ne se rapporte qu'à la variété polystachyon.

1581. Lobel, Icon., p. 795, fig. dext.

(L) 1690. Ray, Syn. anyl., tab. 5 , fig. 3 (vas polystacliyon).

1790. Bollon, Fil. bril., tab. 35.

! 1797. Flor. danica, VII, tab. 1183.

! 1798. Schkulir, Krypt. Gew., tab. 169; vas polystachyon, tab. 170.

1810. Smith, Engl. Bot., 2021.

1822. Vaucher, Mon. Prèl., pl. V, fig. 1-2, 3-4 (:), 5-6 (q),

(१) 1823. Agardh, Gcrm. Prêlı, pl. Xill, f. 5.

1828. Bischoff, hrypt.Gew., tab. 3, fig. 1; tab.5, fig. 46-61 (₹).

(\$) 1828. Bischofl, Entw. Eq., tab. 5 \%.

! 18.4. Newm., Brit. forns, p. 43 et 50 (:); ras polystachyon, p. 47 ; ras nudum, p. 49.

1852. Ifilde, Kemnln. Eq., tab. 5f, fig. 41 (

(\$) 1853. Bischuff, Bemerk. Eq., tab. 2, fig. 1-2; reproduil dans les Ann. sc. nat., IIIo série Bot., tom. $19, \mathrm{pl} . \mathrm{IX}, \mathrm{fig} .1-2$.

1858. Slilde, Gef. Crypt. Schl., tab. 36, fig. 57 (y); tab. 37, fig. 61 (:). 
(:) 1858. Sanio, Epid. u. Spalt. Eq., pl. III, tig. 12-16.

(:) 1858. J. Duv. J., Bull. Soc. bot. Fr., V, p. 516.

\section{E. ramosissimum Desf.}

1563. Camerarius, Epit.Natth., 772 (subbona).

1658. G. Bauh., Theat., p. 250; copic de celle de Camerarius.

! 1798. Schliuhr, Krypt. Giew., tab. 172 b., dextra.

1822. Vaucher, Mon. Prêl., pl. VI, fig. 1, 2 (:); pl. XII, fiğ. 3, 1 (:) sul) : L. campanulatum.

(:) 1828. Bisclıofl, Krypt. Gew., tab. 4, fig. 23; tab. 5, lig. 4 4.

(:) 1858. J. Duv. J., Bull. Sac. bot. Fr., V, p. 517.

\section{E. variegatuin Schleich.}

! 1798. Schkuhr, lirypt. Gicw., tab. 172 b, sinistra. 1810. Smith, Eng. Bot., 1987.

! 1822. Vaucher, Mon. Prể., pl. XII, fig. 1, 2 (:).

! 1828. Bischoff, Krypt. Gew., tab. 3, fig. 5.

! 1844. Newm., Brit. ferns, p. 31, 37, 39, 40 et 30 (:).

1845. Flor. danica, XIV, 2490.

1851. IIofmst., Vergl. Unters., tab. 18, fig. 1-6.

1858. Milde, Gef. Grypt. Schl., tab. 36, fig. 59 (J); tab. 37, fig. 62 (:).

(:) 1858. J. Duv. J., Bull. Soc. bot. Fr., V, p. 517.

\section{E. traciryodon Al. Br.}

1844. Newm., Brit. ferns, p. 25, 29 et 30 (:).

(:) 1858. J. Duv. J., Bull. Soc. bot. Fr., V, p. 518.

\section{E. IIYeMale L.}

Les anciennes figures sont plus propres à induire en erreur qu’a éclairer. Si Tragus, dans la figure qu'il en donne (De Stirp., éd. de 1552, p. 692, i gauche), n'avait eu la précaution de dessiner à côté un de ces anneaux (Schaftheukrïuze) que l'on fait en Alsace arec cette espèce pour nettoyer les ustensiles de cuisine, il serait impossible de deviner si cette figure d'aspect étrange a la prétention de représenter l'E. hygemale plutôt que l'E. limosum ou l'Itippuris vulyaris, on tonte autre chose. II faut en dire exactement autant de la figure de G. Bauhin (Theat., p. 248), que Linné a eu raison de ne pas citer; clle est détestable. La description qui l'aecompagne et l'habitat assigné démontrent que G. Bauhin a confondu cette espèce avee l'E. limosum vas mudum. La figure de Camerarius (Epit. Matth., 770, ), la seule que Limné ail citée directement, représente assez bien l'E. hyemale'. Dalechamp (Ilist., I, 1071) donne une copie en sens inverse de la figure de Tragus. Celles de Iratthiole (2,375), de Gerarde (Iterb. Em., 1113), auxquelles renvoie Bauhin (Pin., 16, cité par Linné), se rapportent plutòt it l'E. variegutum. Nous avons vu d'ailleurs daus lia synonymie que Linné n'avait pas distingué ces deux plantes. 
1530. Brunf, Herb. viv. cic., HI, p. 14!.

(L) 1563. Camer., Epit. Multh., 770, lig. $\Lambda$.

1790. Bolton, Fil. brit., tab. 39.

! 1798. Schkulır, kirypt. Gew., tab. 172 «.

1800. Smill, Engl. Bot., tab. 915.

! 1810. Fl. denica, Vlli, tals. 1409.

1822. Vaucher, Mon. Prêl., pl. IX, fig. 1-2, 3-5 (:).

! 1828. Hoolier, Fl. lond., V, 101.

(:) 1828. Ad. Brong., Ilist. vég. foss., I, pl. XII, fig. 1, 2, 4, 5.

(:) 1828. Bischolf, kirypl. Gew., tab. 4, fig. 20-22; tah. 5, fig. 43.

(:) 1837. Meyen, Neues Syst. d. Iflanzenplyssiologie, 11. V, fig. 15-17.

! 1844. Newm., Brit. ferus, p. 17, 21 et 30 (:).

1858. Nilde, Gef. Crypt. Schl., tab. 36, fig. 60 (y).

(:) 1858. Sanio, Epid. u. Spall. Eq., pl. III, fig. 27-3..

(:) 1858. J. Juv. J., Bull. Soc. bol. Fr., V, 1. 518.

1860. J. Duv. J., Bull. Soc. bot. Fr, VII, p. 166.

\section{CHAPITRE GOMPLÉMENTAIRE}

\section{Noms; propriétés et usages; composition chimique}

$$
\S 1 \text { ro . Noms }
$$

C'est dans Dioscoride que se troure, sinon la premiere mention, au moins la plus

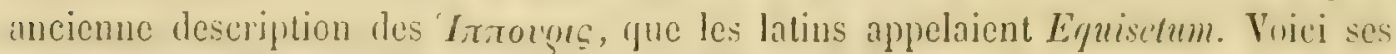
expressions: "Prima in aquosis locis ct fossis nascitur; cauliculos edit inanes, (rubescentes, suljasperos, solidos, geniculis invicem insertis dissectos, ac circum "ipsos folia juncea crebra tenuiaque. In sublime autem attollitur, vicinos scandens rathormm caudices, ex iisque dependet, circumfusa ramis multis, nigris, ut est equi "cauda: radix lignosa ac dura. Allere caule est recto, ápuali, cubito majore, fuodam"modo inani, comis yer intervalla brerioribus et mollioribus candidioribusque» (Mater. med., lib. IV, cap.4k-45, Ed. 1529; cap. 38, Ed. 1549). Le commencentent de la première description pourrait à la rigueur se rapporter à un Equisetum; la fun s'en éloigne alssolument. La secunde description s'en rapproche un peu plus.

Pline est cncore moins clair. Il mentionne une première plante sans la décrire: "Equisetum, Mippuris a Gruess dieta, el in pratis vituperata nobis (est autem pilus "terre equine selie simitis)....; p puis il en mentionne une seconde: " alii pinus foliis "similem nigricantem, codem nomine appellant ${ }^{1} . .$. ; et aussitút après une troisième,

\footnotetext{
' Dans son commentaire Dalechamp rapporto celle description aux Chetra.
} 
\$1. - voss.

qu'il dil: « juxta arbores nasci et scandentem eos dependere ramis junceis multis nigris, «ut est equorum cauda, geniculatis ramulis, folia habere pauca, exigua, semen (rolundum, simile Coriandro....) (Lib. XXVI, cap. 83 (xui), vol. 7, part. 2, p. 755, Ed. Lemaire, cur. L. Desfontaines; 1830). Ainsi que l'ont déjà fait remarifuer J. Bauhin (Ilist.plant., III, p. 728) et G. Bauhin (Theat., p. 233, 234 et 940), il est de toute évidence qu'il ne s'agit plus ici d'Equisetum, non plus que dans la première description de Dioscoride que Pline a empruntée à la même source, sans en donner le commencement. Ensuite l'auteur latin revient à une quatrième plante: a Faciunt « et aliam Ilippurim, brevioribus et mollioribus ramis, candidioribusque» (l. c.), qui est la scconde de Dioscoride.

On trouve enfin une autre description de l'llippuris des Grecs, dans l'écrivain de

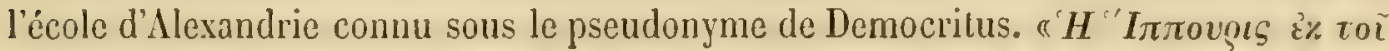

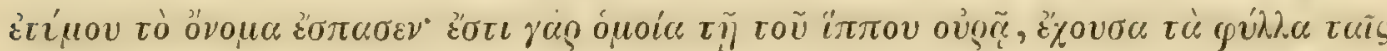

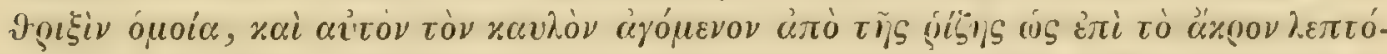

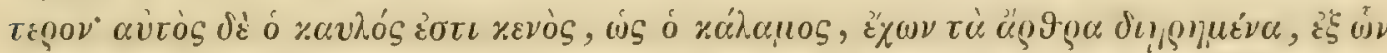

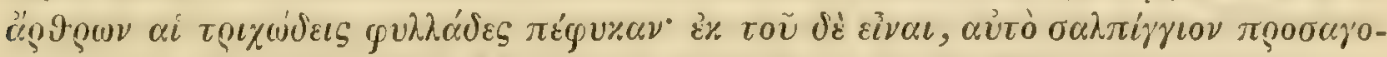

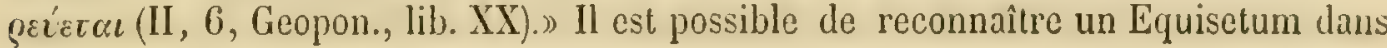
cette description, bien que la dernière phrase déroute un peu.

Quoi qu'il en soit, si on admet que parmi ces plantes il y en ait quelqu'une qui réponde réellement aux Equisetum, il faut admettre en même temps que ces auteurs n'ont pu parler que d'espèces spontanées en Grèce et en Italie, et assez rameuses

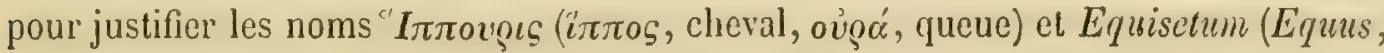
cheval, seta, crin' $\left.{ }^{2}\right)$. Or, dans le nord de l'Italie, l'E. arvense est très-commun et porte encore les noms de Coda caballina, Coda equina, Coda di caballo; les E. maximum, limosum et palustre y sont répandus aussi, et il se peut que les noms anciens aient été appliqués à ces plantes. L'E. sylvalicum ne se trouve que rarement au nord de l'Italie et dans les bois des plus hautes montagnes; il est donc évident que c'est i Lort que Sprengel se demande si les $E$. sylvaticum et pratense ne répondent pas aux plantes de Dioscoride (Hist. rei herb., I, p. 192; 1807). Il est vrai que plus tard, dans son édition commentée de Dioscoride (II, p. 593, in Mcel. grecc. op., vol. XXV et XXVI; 1830), le même botaniste crut reconnaître dans la première plante de l'auteur grec l'E. fluviatile (maximum), ct dans la seconde l'E. limosum. On peut rapporter un Equisetum à la description du pseudonyme Democritus, citće plus haut et par Sprengel; mais il est difficile d'en voir un dans la plante grimpante de Dioscoride.

Jusqu'à Tournefort et Dillenius, les deux noms IIipperis et Equisetum furcnt

\footnotetext{
' Linnó place le nom Eqquiselum parmi les noms génériques qui " perversa ex erronea lectione veterım, vario meta. morphosin insignem subierunt. EQUisexuy pro Equisclis. s Phil. botan., 1. 175, Ed. princ.
} 
presurue indifferemment cmployés par tous les botanistes pour désigner nos Eunu. setum el tout ce qui pourail y ressembler; ces deux autcurs n'employèrent gue te nom Equisetum et rappelèrent celui d'llippuris en synonymie. Enfin Linné appliqua ce dernier à un genre de phanérogames, dont le port rappelle celui des Equisctum.

Le nom gree et le nom latin faisaient allusion a l'aspect général de la plante; te nom franerais Asprílt: se rapporte à la rudesse, àl'aspreté de ses tiges qui les rendait redoutaliles aux faucheurs ${ }^{2}$, ou les fiasait rechercher des ourriers pour polir le bois, et des ménagères pour nettoyer les planchers et la vaisselle. Lorzque le mot aspre a cessé de s'écrire avec un s, l'Asperelle', Asprelle, Aspreslè a subi le mème changement

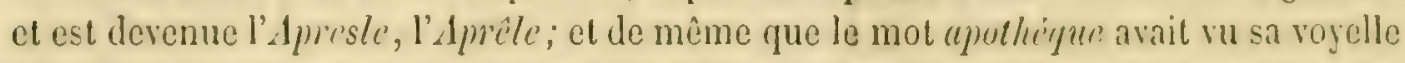
initiale se transformer en article et étail devenu la boutique, de même l'A presle devint le l'reslí. Cependant la plupart des dietionnaires (Boiste etc.) conservent les anciens noms Asprite, Apréle, qui sont encore employés en Dauphiné el en Savoie ${ }^{3}$. Toutefois remarquons que G. Bauhin, qui rapproche el cite dans la même ligne Asprelle, Esprelle et Presle, trouve plus simple de faire venir ce dernier mot de peutiná, net, propre (Theat., p. 2.40). Il est permis de douter que nos ménagères, nos lourneur's et nos faucheurs soient allés demander au gree le nom de la plante epui émoussait leurs faux, polissait leur bois ou netloyait leurs ustensiles.

Les Equisetum portent en Provence le nom de Caousaonda, Cousaodo, Coussauda, Cassaouda, Cassauda, Gassouda (IIonnorat, Dict.prov'.). Quand on voil l'enthousiasme avec lerguel le botaniste provençal Garidel et les auteurs latins vantent les Efquisclum comme plantes vulnéraires, on est porté à faire dériver le nom provengal du latin consolido, souder. Nais en remarquant que dans les noms anciens et modernes ILippuris, Equisetum, Coda di caballo, Coda di mula, IIorse-tail, Rossschwanz, Katzenwadel elc., l'ilée de queue se rencontre constamment, on trowe que le nom provencal pourrait bion avoir la même signification, cl être composé de cou, cau, coun, queue,

1 Equisctum, aliis Asprella dicitur \$ (Tragus, De slirp., p. 692;1552). - nquiscli genera, quam vulgo Asprellam evocant.... (Cesalpino, De plant., lib. XVI, cap. 15; 1582). - Unum Equiseti genus longius quod nonnulli nostra - etatis herbarii ab asperitate quam obtinet Asprellam vocant. Hinc est quod materiarii, fabri..... (I'uchsius, Hist. stirp., p. $321 ; 1542)$.

" Queuë de cheval a prins cc nom ì cause que son lierbe ressemble aucunement le poil a la quenë d'un cheval. - Aucuns nomment ceste herbe, uspresie, nour sa grande aspiete et rudesse du unanier, dont les ourricrs imagers, - peigners et autres faisant choses délicates, se servent pour polir leur ourrage l'en froltant. II y en a encore de deux - especes, l'une plus grande que l'autre" (Olivicr de Serres, Théat. d'agric., liv. VI, ch. 20, p. 511; édit. de Genève, 1651).

* Pantagruélion est plus abliorré ct haï des Larrons, plus leur est contraire, que la Presle aux Fauscheurs, que le Xénufar et Nympliéa aux ribaulx Moines ete. "(Rabelais, Pantag., liv. II, ch. 49, p. 519, édit. de Leduchat, 17.11).

s Apréle est le nom populaire cn Savoic, ct M. V. l'ayot donne Aprela ct Aprailas comme les noms patois it Chamounix (Calal. M4. Bl., p. 49, 51, 53). M. Bernoulli donne Sprella, Asparella, liasparella comme les noms populaires en Italic (Gef. Lirypt. Schw., p. 76). 
et sauda, seta, crin. Celte signification primitive du nom générique me sourirait d'autant plus qu'on trouve dans Festus loca candata pour désigner des endroits couverts du crin des Equisetum (roir Dalechamp, éd. de Pline; Ljon 1587, lib. 26 , cap. 83 (xIII).

\section{\$2. Propriétés et usages}

On pourrait toul résumer dans celte phrase de Vaucher: «On dit, mais sans “l'assurer, que les Prêles ne sont pas inutiles ả la médecine; quoi qu’il en soit, clles «ne serveni, au moins jusqu’à présent, qu'aux ouvriers en bois et en métal» (IIon. Prêtl., p. 336). Mais si c'était dire tout ce qu'il y a peut-être de rrai, ce ne serail pas suffisamment faire connaitre toutes les idées qui ont eu cours sur les vertus de ces plantes.

Ingénicux à altribuer aux plantes tant de qualités merveilleuses que chaque végétal devenait presque une panacée universelle, les anciens ne devaient pas oublier les Equisctum. Aussi Dioscoride attribue-t-il de nombreuses vertus à ses deux Hippuris: "12 Adstringit herba; ob quam causam sanguinis ex naribus eruptiones sistit succus " ejus. Bibitus idem cum vino utiliter contra intestinorum tormina, et urinam cit. "Glutinant cruenta vulnera trita et inspersa folia ejus; succurrit radix et herba

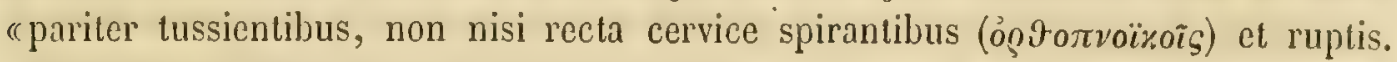
"Quin etiam intestinorum divisiones, resicam dissectam et intestinorum ramices "glutinare feruntur folia cum aqua pota. 2a Altera concisa ex aceto eadem potestate «vulneribus medetur» (Mat. med., lib. IV, cap. 44, 45).

Pline parle deux fois des Equisetum. Dans le premier passage il les nomme "Equiselis, ) et les comprend au nombre des plantes nuisibles aux prés. «Invisa et “ Equiselis est, a similitudine equinæ setæ» (lib. XVIII, cap. 67 (xxvI), vol. 6, p. 320, ed. cit.). Dans le second, après avoir fait allusion à ce qu'il en avait dit d'abord, «in "pratis vituperata nobis, ) il expose les vertus de la première sorte d'Equisetum: "Lienes cursorum exstinguit decocta fictili noro ad tertias, quantum vas capiat, et "per triduum heminis pota: unctis esculentis ante diem unum interdicitur. Greco«rum varia circa hanc opinio.» Puis il cite les vertus de la seconde, qui n'est probablement pas un Equisetum: "Alii pinus foliis similem, nigricantem, codem nomine «appellant, vim ejus admirabilem tradentes, sanguinis profluvia vel tacto tantum (homine sisti ) (lib. XXVI, cap. 83 (xIII), vol. VII, p. 755-756, ed. cit.). Je n'ai point à citer les merveilleuses propriétés qu'il attribue à l'autre Equisetum (le premicr de Dioscoride) ì tiges grimpantes et ì graincs globuleuses, attendu quili répète encore ce qu'en dit Dioscoride; toutefois je les énumérerai rapidement, parce que la plupart des anciens médecins botanistes, sans rechercher si sous l'identité de nom il y avait 
identité de plantes, les ont attribuées indistinctement i tous les Erpuisetum possibles': "Il arrête le saignement de nez, le flux de ventre, la dysenterie, provoque les urines, gucirit la toux, l'orthopnée, les ruptures, les ulcères. Le suc des feuilles est bon pour les maladies des intestins, de la ressie el aussi pour les hernies.) Enfin il cite la derniere espece: alrevioribus et mollioribus comis, perquam utilem ischiadicis, ct vul"neribus ex aceto impositam, propter sanguinem sistendum»(1. c.).

Galien va plus loin: "Vulnera maxima conglutinat, etiamsi presectos esse nerros "contingal; preterca intestinorum ramices cogit" (De simpl. medic. facult., lib. IV, cap. 101).

Natthiole, dans les commentaires dont il accompagne la traduction de Dioscoride, rappelle toutes les propriétés altribuées par les anciens et indique un autre emploi de nos plantes: "IIoc rura nostra ${ }^{2}$ vulgo vocant Paltrufalo, in cilumgrue assumunt, (jejunii quadragesimalis tempore. Etenim illud prius clixant, mox farina circum"spergunt et oleo in sartagine frigunt manduntque piscium vice. Ex quo ciho alvus "quandogue corum adeo constringilur et constipatur, ut in coli cruciatus facile incur“rant» (Matth. in Diosc., cap. 42, p. 515, Ed. 1540).

Fallope nous apprend aussi que: "Equiseti cauliculus tenerrimus dum crumpit, "pro asparago ad mensas venit» (De simpl. med. purg., Op. omn., I, p. 125, Eal. 1600).

Olivier de Serres, en mentionnant l'usage qu'on en faisail de son temps, nous prouve qu'on attribuait alors a nos plantes toutes les qualitis dont Dioscoride et Pline avaient gratifié leur Equisetum «grimpant»: «Son jus mis dans le ne\%, cstanche (le sang qui en sort; de mème fail des menstrues des fommes mis en pessaire. La a décoction faite en caue ou on vin guérit la dysenterie, provoque l'urine. Ses feuilles " Iroyées, appliquées sur les playes, les consolide tresbien. Son herbe el sa racine "soulagent ceux qui sont travaillez de la toux» (Théat. d'agr., VI, ch. 15, p. 5't, éd. de Genève, 1651).

G. Bauhin, énumérant les qualités des Equisetum, rappelle qu’Aricenne les tient pour froids au premier degré et secs au second degré, mais que Brasarola les prítend anssi froids an premier degré, mais secs au troisième degrï; que Galien les cstimait astringents, amers et siccatifs. Quant à leurs vertus (vires), le même autcur reproduit d'ahord tontes les propriétés attribuces par Dioscoride, par I'line, par Galien et par tous les médecins du moyen îge, puis il rappelle, en l'appuyant de l'antorite de Petronius (De vict. Roman., lib). 3, cap. 3), l'emploi culinaire des Equisetum: "Etiam plebs romana tempore quadragesimo esitare solet, quibus Pesse del centlo avocatur, sed usus periculosus cst. Sunt qui asparagum siccum anmum asservant,

\footnotetext{
- Nous avons vu p. 227 qque sous le nom ginérique Equiselum on comprenait, arec quelques Ẽquisctum, l'llippuris, Jes Chara, un Elatine, des Ceratophyllum, des Ephedra.

: Matthole ćtait talien, et il habita longtemps la petite ville de Trente.
} 
a quo ad dysenterias utuntur, aqua calida per noctem macerantes corfuunt, ct in cibo "magno successu exhibent» (Theat., p. 252-25.3). Apres quoi le même auteur énumère une infunité de nouvelles propriétés reconnues par les modernes à son Equisctum $n^{\circ}$ IX (E. arvense). Il est efficace contre le crachement de sang, les ulcires des poumons, la phthisie, la pierre, la rétention d'urine, le diabétés, les maux de gorge, les ulères des parties, les liémorrhoïdes, les maladies du foie, l'érysipiele, les pustules malignes, l'engorgement des testicules, l'hydropisic, les contusions; il guírit eneore les maladies des chevaux, ce qui, suivant Brasavola, lui a valu son nom d'Equisetum. Enfin «a mulicribus cxpetitur, ad ustensilia culinaria extergendiı, vasa proscrtim "stannea et omnis generis supellectilia, quare non temere Asprellu nominatur" (Theat., p. 254).

J'ai cité de préférence ce très-savant auteur, parce cu’il est plus complet que tout autre et qu'il contient tout ce qui a été dit par ses prédécesseurs. Après lui le nombre des vertus attribuées aux Equisetum va toujours en diminuant, bien que Garidel parle encore arec enthousiasme de leurs "propriétés vulnéraires el astringentes " (IIist. plant. de Provence, p. 159-160; 1715).

Dans son Materia medica, Linné, voulant résumer les vertus altribuées à ces plantes, ne cite qu'une espèce, et trois lignes suffisent à l'énumération de ses propriétés:

«E. arvense $\mathbf{L}$.

aUd. sicca.

Exoleta, eximia.

a VIs. adstringens! diuretica!

"Usus. Ilematuria! Gonorrhoea benigna, Profluria, Phthișis " (I/ar. med., p. 271. ed. Schreber).

Bergius s'exprime ainsi :

(E. lyyemale L.

"Vintus. Leniter adstringens.

"Infusum aquos. lutescens velut potus There, odore fere infusi Thex similis, " sapore in eandem Theam incidente; vitriolo martis fuscescit.

" $E$. arvense L. in omnibus cum $E$. hyemali convenit» (Hat. mol., Ed. 2a, II, p. $896 ; 1782)$.

J. $\Lambda$. Murray expose comme il suit les diverses opinions qui araient cours de son temps: «Profuit Equisetum aqua decoctum ac calide cxtrinsecus pubis regioni impo"situm, ejusque fomentum in connubio decocti pro potu (Riedtrists in IIevcir. Oper, «p. 253). Gum quibus haud facile comparari potest aliorum laus in micu cruento "sedando (Comm. Nor., 1733, p. 286). In nephiretide calculoso decoctum herhe a Equiseti ex cerevisia alba adjecto butyro el melle tanquam domesticum, sed non "spernendum remedium proponitur (IIorrs., Med. syst., tom. 4, P. 2, p. 386). Dum 
"dissuadet in calculo Ilorenes (.1ct. nat. curios. Cemt., I, 2, p. 127) ex casu unico "Equisetum, non de co sulo, sed comlinato cum beceis Juniperi in pulvere loquitur. "Dissensus hi tinto nocentiores esse possent, nisi in hodierna praxi medica rarissi«mum esset Equiscti nomen 》 (J. A. Murray, Alparal. medicam., V, p. 484-485; 1790). Soit par suite de ces contradictions, soit à cause de leur inefficacité, les Equisctum, comme le disait Murray, étaient done tombés dans un profond oulji, lorsqu'en 1826 le docteur Lenhossek fit arec eux de nombreuses expérienees et constata leur propriété diurétique. D’après ce savant, l'intensité de celte propriété varie selon les espieces; faible dans les E. arenense, variegatum, ramosum et palustro, elle est si développée dans les $E$. hyemale et limosum, que souvent elle détermine l'hématurie. $\Lambda$ la plante fraiche, qui est trop active, on doit préférer la plante siche; il convient de l'alministrer en décoction de 8 a 12 grammes sur 500 grammes d'eau. On en donne toutes les deux heures une ou deux cuilleríes aux enfants, 100 a 200 grammes aux adultes. L'action n'en a rien d'irritant pour les voies digestives el n'apporte ancun trouble dans lit circulation. C'est surtout contre l'iecumulation de scerosití par atonie, et à la suite d’allections exanthématiqques, guiril en conscille l'usaçe; il le proscrit au contraire toutes les fois qu'il y a inflammation (Beob. . Abhandl. pratit. IIrilli. von Doct. und Prof. d. Uniu. . Wion, V, 1826, p. 392-108. -Voy. aussi: Edliull. med.a. Surg. Journ. Janu., 1827; Arch. gén. de méd., XVI, p. 456; Joum. de chim.

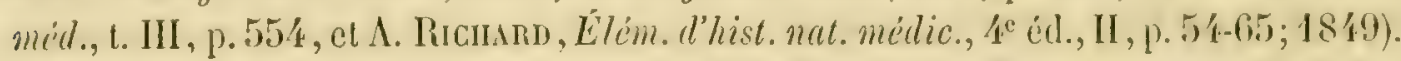

Malgré tout cela, les Equisetum ont été laissés de cité; ct ces plantes, si vantíes autrefois, sont anjourdhui complétement délaissées. Le Codex ne les mentionne plus; Soubeiran fait de même dans son Traité de pharmacie, et M. Dorvault (Offic., p. 507; 1858) se contente de dire: "Prèle, estimée diurétique; inusitée.) M. Schnizlcin, professcur à Erlangen, dans sa Rerue de botenique médicale et pluermaceutique, Erlangen 1860, dit sculement: "E. arrense, diureticums (1). 72). La médecine ne les emploie done plus; elles sont exclues des pharmacies; et c'est a peine si en $\Lambda$ sace et en Provence elles sont quelquefois, sur leur vicille réputation, demandées aux herboristes de ces contrées. Comme on peut le eroire, je n’ai pu vérifier par moi-même ce qu'elles méritent réellement, de l'ancienne confiance ou de l'oubli présent. Nais il n’en a pas été de même de leur emploi culinaire. Comme IIaller nous indique très-cxpressiment que son $11^{\circ} 1675$ (E. maximum) "hoc fuerit Equisctum "quod a plebe romana in cibum recipitur» (IIist. stirp. ILelv', III, p. 1), j’ai roulu en essayer. Or, avec la meilleure volonté du monde, il m'est impossible d’admettre que, mème en carème, par esprit de mortification el de pénitence, on puisse manger des beignets de jemnes Equiselum. Cela n’a aucun mauvais ggoût, ct lépi a mème unc saveur amere, ngréahle; mais les gaines et les entre-nends de la tige spicifere ressemblent par trop ì ce ru'on obtiendrait avee des handes létoupe saupoudrées de 
sable fin. Je croirais plus volonticrs qu'il s'agit des turions de quelque plante au fenillage fin (peut-être de l'Asparagus acutifolius), qui se mange en Provence. Il cst d'ailleurs à remarquer que Cesalpino (De plent., lib. XVI, cap. 15, p. 598, Ed. 1583), G. Bauhin (Thecte, p. 240) et tous les auteurs intermédiaires donnent le nom d'Asparagus aux jeunes tiges spicifères d'Equisetum, ce qui peut avoir facilité la confusion.

Si on a attribué aux Equisetum tant de propriétés bienfaisantes pour l'homme, il n'en a pas été de même, et tant s'en faut, de leurs propriétés dans l'économie rurale. Pline avait débuté par les aceuser «invisa Equisetis.... in pratis vituperala «nobis »... Longtemps oubliée, celte accusation fut relevéc par Ilaller, qui y joignit ses plaintes personnelles. Il dit, en effet, de l'E. palustre: \& IIoc equisetum paulo ( minus quam 1676 (E. arvense) tamen et ipsum pecori nocet et dentium facit in ( bobus et vaccis vacillationem tum diarrhoam. Cum seductus pulchritudine Trifolii «Equiscto 1676 inquinati, famulus, qui boum meorum curam gerebat, semcl aut «iterum raccam nuper vitulum enixam hac pestilente herba aluisset, ex diarrhoa «immedicabili eadem periit. Quare magnis pecuniis nostri arcanum redimerent, quo "prata infaustissima herbarum liberarent. Mihi neque aratrum, neque fimus, neque «alia cura profuit. Equis non nocet, neque ovibus et rangiferis. Porci nostrates recu"sant, cum in Suecia non detrectent. Radicibus tamen glandium simile aliquid sxpe "adhæret, quod porcos credas requirere.» La mauvaise humeur du propriétaire semble même réagir sur le médecin, qui ajoute: "Vires medicas vix satis certas «autumo. Aquosa planta est, parum acris; ci adstringentes vircs tribucrunt in diar"rhœa, in hæmoptoe efficaces. Nocuit tamen, Ioyero teste, ventriculo et vesicre. "Serio vix credo adhiberi 》 (IIist. stirp. IIelv., IH, p. 2).

Linné est mieux disposé en faveur des Equisetum; et si un blâme se joint à ses éloges, ce n'est qu'en cas d'excès. Son Flora lapponica contient ce qui suit: Rangi" feri, Lapponum pecora, fenum per hyemem non adsumunt facile, hine Lappo "nocles diesque eos per sylvas ducere tenetur. Obtuli circa autumnum redeuntibus «cx longo itinere Rangiferis fasciculum foeni, el observavi cos hanc plantam ( $E$. fuviatile id est limosum) seligere et adsumere, relicua fere intacta relinquerc. " Annon itaque hocce Equisctum majorem œconomix lapponicie usum afferre posset, (incolis judicandum relinquo» (p. 310).

Dans son Flora xconomica il établit que:

" $\mathrm{N}^{\circ} 833^{\prime}$ (arvense). Equisetum ab ovibus prægnantibus nimium comestum abortum "producit.

«No 836 (limosim). Equisetum vaccis utilissimum foenum.

' Ces numiros répondent it ceux du Flora suecica, Ed, 1", 1745. 
" $\mathrm{N}^{\circ} 838$ (hyemal'). Fabri loc ipso pectines, manubria, scipiones, melalla lieriagant et expolliunt. ( $F l$. acon., in $\Lambda$ mœn. acad., p. 385).

Enfin, dans le Flora succica, Linné est plus explicite encore:

"E. sylraticum. Equis primarium inter omnes plantas pabulum Jemtiis.

- E. urrense. Bohus intactum persistil, nisi perestini vel fame coacti edant, uli ex a Frisia ultrajecti, tum vero ca consumuntur (Commel. holl.).

"E. limesum. Vissecatur in pabulum boum, ut varce lac copiosius probeant. Equis " minus acceptum. Rangiferi qui foenum recusant, hoc tamen assumunt.

"E. liyemale. Eqquis salutare. Vaccis noxium, que dentes amittunt; herbam ore "sapius dejiciunt. Oves abortiunt et abhorrent a pabulo (Leys.) ) $\{$ Fl. suec., Ed. 2", p. 367-368).

Adanson et Ventenat, peut-être sur l'autorité de Linné, affirment e gu'on Laponie " les bu'uf's et les rennes recherchent l'E. limosum L. D (Tubl. régn. vig., II, p. 7I).

J. A. Murray (Appar. medicam., V, p. 48 t) rapporte, sans les disculer, les témoignages suivants: a Ex Equiseto copioso inter pauca gramina crescente demesso ol in horreum " illatn, quo vacrée el oves per hyemem saginate, paucissima absque abortu crase"runt (J. A. Schulae in Act. nat. curios., vol. I, p. 521). Similia de E. luyemali nar"rantur, vacce pratcrea inde jacturam dentium pati (Gotrscuen, Fl. prus.s., 1. 66). "In armentis mictum cruentum quoque creat (IIoll. Matatschepp, ?, p. 159). .)

Lightfoot répète que l'E. hyjemale fait tomber les dents des raches (Fl. scot., p. 6.jl).

Le Joumal of a naturalist de II. Kunapp mentionne que l'E. limosum est recherché par les rats d'eau (p. 143).

Smelowsky, en signalant la présence des tubercules sur les rhizomes de l'E. arrense, propose d'en tirer parti pour engraisser les porcs, attendu que ces animaux en sont friands et les recherchent avidement (Mém. Aculd. Pétersh, I, 1803-1806); Bischoff, en citant cet auteur, fait remarquer "que la diffeulté d'obtenir en assez grand nombre ces tubercules disséminés et profonds n'en rendrait pas l'emploi économique. et profitable» (Krypt. Gew., p. 50).

Sir W. Ilooker donne crédit a la réprobation qui frappe l'E. hyrmale, en disant que "bien qu'il soit prouré qu'il est nuisible aux moutons et qu'il fait tomber les dents des raches, il est tout it fait salutaire pour les chevaux" (Fl. Lond., p. 161). M. E. Newman traite d'imaginaires les propriétés midicinales attribuécs ì la même espece (Brit. forns, p. 21), et il ue justifie l'E. Telmatcia (p. 70) el lE. arrense (p. 79) qu'en supposant aux bestiaux trop d'instinct pour en manger, sauf les cas de faim extrême.

Bischoff, après avoir dit que "dans quelques contrées du Nord les E. syltaticum et palustre sont donnés cn nourriture aux chevaux, tandis que l'E. urense est considéré comme leur útant très-nuisible ainsi qu’aux hîtes à cornes, "ajoute: "sur ce 
point les opinions les plus contradictoires ont igalement cours" (Kirypt. Gew., p. 50).

Enfin, dans le journal La Frence agricole (numúro du 26 janvier 1860, p. 56), M. E. Paltard, secrétaire du Comice agricole de Bazas, reproduit, mais non d’après son expérience personnelle, toutes les accusations qui ont été lancées contre l'E. palustre. «Mêlée aux foins cl aux fourrages, la Prêle des marais exerce une action "si nuisible sur les animaux et principalement sur ceux de l'espèce bovine qu'on "peut la considérer comme un rrai poison pour eux. » Ce cultivateur indique comme moyen de destruction le défonçage, le drainage el l'ensemencement du terrain avee. du Ray-Grass et du Trèfle de IIollande; et à l'appui de ses expériences il cite celles de M. de Marignan, secrétaire du Comice agricole de Bayonne.

En présence de ces assertions contradictoires, il fallait avoir recours à l'expérience. Vaucher avait bien fait remarquer que «tous les jours les bêtes à cornes consomment "des Prêles vertes ou sèches, sans qu'il en résulte d'accident» (Mon. Prêl., p. 336); mais je voulais des expériences directes. J'étais bien placé pour cela; car les prairies de l'Alsace ct du duché de Bade produisent une telle quantité d'Equisetum qu'il est vraiment impossible d'en trouver d'avantage ailleurs. Or, dans les réunions des Comices agricoles, je me suis adressé aux propriétaires de bestiaux; j’ai consulté M. Imlin, ancien élève de l'école d'Alfort, très-habile vétérinaire de Strasbourg, et dont la vaste clientèle s'étend dans toute la vallée de l'Alsace et du pays de Bade, et les réponses que j’ai obtenues peuvent, sans aucune exception, se résumer ainsi qu’il suit: Il est incontestable que les Equisetum ne fournissent pas un bon fourrage; ces plantes sont dures, sans parfum et sans saveur; les animaux ne les mangent pas volonliers; si elles sont consommées, elles nourrissent très-peu, mais elles ne font aucun mal. La place qu'elles occupent dans les prés est une place perdue; mais voilà tout. J'objectais à M. Imlin le «dentium facit in bobus et vaccis vacillationem » de IIaller et des autres; il me fut répondu que sans doute Haller avait été trompé par un valet de ferme qui voulait faire attribuer aux Equiselum plutôt qqu'à sa négligence le mauvais état des bestiaux; que les incisives des ruminants sont toujours mobiles, même dans l'élat de santé parfaite, et que le grand physiologiste suisse ne pouvait pas ignorer ce que les entremettcurs juifs de l'Alsace connaissent parfaitement, et exploitent de même, pour obtenir à meilleur marché des vaches qu'ils prétendent très-malades, attendu, disent-ils, que les dents leur branlent.

Dans son Nowveau système de climic organique M. Raspail lance un nouveau chef d'accusation:

"3393. Influence des paturages. On a remarqué que la Prêle furiutile donne au "lait de vache une couleur plombée et bleuitre, et le prive de sa portion crímeuse. II ( cst probable que ce lail est acide, que l'albumine s'y trouve par conséquent en moins 
"grande quantite, vu rue les glandes mammaires n'auront pas assez recu de menstrue a alcalin pour en enlever au sang qu'elles élaborent. En conséquence le lait se trono vera plus on moins réduit i l'état de petit-lait dont il a la couleurs (t. III, p. 1 1́6, el aussi \$3357, ed. de 1838). Cette accusation est difficile ì concilier avec l'assertion de Limné que celte plante donne aux vaches de Laponie un lait plus abondant; est-clle mieux fondíe? Il est ficheux que le savant chimiste n'ait pas dit qui a remarqui le fait dont sa théoric offre l'explication, car les proprictaires sont d'accord pour dire qu'ils n'ont rien constaté de semblable.

Il est donc permis de croire que les propriétés malfaisantes attribuces aux Equisetum ne leur appartiennent guère plus que les vertus merveilleuses dont les douait la crédulité d'un autre âge,

\section{et đqu'ils n'ont mérité \\ Ni cet excès d'honneur ni cette indignité.}

En définitive, l'emploi qui paraît le plus incontestable est le polissage. M. E. Newman atteste que, pour cet usage, l'Angleterre recoit (sous le nom de Dutch Rush) de grandes quantités d'E. hycmele qui croit en abondance sur les digues de la Ilollande (Brit. ferns, p. 21).

Dans nos départements méridionaux, en particulier dans celui des Bouches-duRhoine, l'E. ramoxissimum (quelquefois aussi l'E. palustre) est récaltí en abondance; on le tortille en gros anneaux (appelés Fretalour, Escurete, Escurrt) qui scrvent it netloyer la vaisselle et les petits pavés rouges. Dans sa Statistique de's Bouche's-duRhine, M. de Villeneuve comprend la "Préle au nombre des produits naturels du "dipartement rui domnent un revenu régulier; ) il estime «qu'il s'en récolte 30,000 "douzaines de bolles, évaluées à 10,000 fr.) (I, p. 65f; 1821). De nombreux renscignements, recueillis dans ce département, mont démontré que cette évaluation, qui me paraissait d’abord exagérée, cst au-dessous de la vérité. La douzaine d’anneaux se vend en etí 5 cent., en hiver 10 cent., et il y a peu de ménages qui n'en usent deux douzaines par mois. En réduisant la dépense annuelle à une moyenne de 1 fr., la ville de Marseille, ì clle seule, a plus de 10,000 ménages qui emploient ces anneaux à nettoyer la vaisselle el les pavés.

En Alsace on recueille I'E. hyemale pour le mème usage et on en fait également des anneaux (Schaftheulirinze), que Tragus a figurés ì côté de son Ilippuris major (De stirp., p. 692). Les deux Bauhin ont aussi donné un dessin de ces amneaux.

A Strasbourg, comme cu IIollande, l'E. hyjemale' est récolté en abondance pendant l'automne et l'hiver, el expédié à Paris, ou les tourneurs et les tabletiers l'emploient pour polir les menus ouvrages de bois, de corne, d'ivoire ete. Mais chaque année les commandes diminuent, el le papier-rerre remplace l'A mide. Ainsi celte plante que les hotinistes regardent comme un reste précieux de la végétation d'une autre période 
géologique, ectle plante si vantée par l’ancienne médecine, que l'rignts pouvilt en dire "utrisque corporis partibus utilissima" (De stip., p. 693), est maintenant, si non méconnue, au moins inconnue en médecine el relégućc dans nos arrière-cuisines au plus vil des usages: Sic transit gloria mundi.

\section{§3. Composition climique}

Pour compléter ce qui précède je crois devoir faire connaître ce qqui a été publié sur la composition chimique des Equisetum.

La plus ancienne mention sur ce point se trouve dans Tournefort: "La Presle est (d'un goût salé, détersif, et ne rougit presque pas le papier bleu : il y a beaucoup) "d'apparence que le sel de cette plante est semblable au sel de Corail; mais il y est "mêlé avec un peu de sel ammoniac et de soufre. Par l'analyse chimique on tire de la "Presle plusicurs liqueurs acides, peu d'huile, beaucoup de terre, point de sel volatil "concret, mais quelque peu d'esprit urineux: le sel fixe de cetle plante ne se résout "pas facilement à l'air et ne rend pas la solution de sublimé corrosif rouge orangé. "Extrait des registres de l'Acad. roy. des sciences» (IIist. des plant. cuv. Paris, $2^{\circ}$ éd., II, p. 46).

Bischoff s'exprime comme il suit, sans faire connaître à qui sont dues les analyses:

"La souche de la plupart des Equisetum est particulièrement riche en fécule et en gluten. Outre cela, ils contiennent encore un sirop brun el sucré (sucre incristallisable); et la cendre de la tige brûlée contient plus de moitié de silice (sur 100 grammes de cendres l'E. palustre donne $53^{\text {gr }}, 75$ de silice et l'E. liyemale 62gr 90 ). En outre on trouve dans la cendre: sulfate de chaux, carbonate, chlorure et phosphate potassiques, chlorure et phosphate sodiques, phosphate de chaux, phosphates de fer et de magnésie, chaux et au plus charbon 1/2 》 (Krypt. Gew., p. 50).

M. E. Newman dit à son tour: « Sir Ilumphrey Davy a constaté dans la tige de cette plante (E. hycmale) la présence d'une quantité extraordinaire de silice; c'est celte substance qui donne à la tige sa rudesse et en fait ainsi un article de commerce. La silice se montre sous forme de petits cristaux, arrangés avec une régularité parfiate. Dans le volume sur l'optique (Lardner's Cabinet Gyclopedia), le docteur Brewster constate qu'il a trouvé les molécules de silice douées d'un axe de dubble réfraction. Nous arons entendu dire à des botanistes que la quantité de silice est si grande cl que les molécules en sont si étroitement unies que toute la sulsstance végétale peut ètre enlevée par macération sans altérer la forme de la plante»(Brit. ferns, p. 22).

MI. Kirschleger évalue «à 95 pour cent la quantité de silice que l'on rencontre dans les cendres des Préles» (Fl. Als., II, p. 375); mais il ne cite ni l'auteur de l'analyse ni l'espèce analysée. 


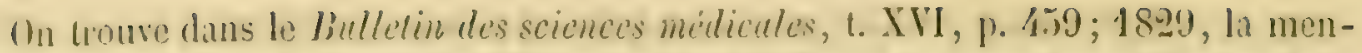
tion suivante d'une analyse de lE. hyemale: "M. Dichold a trouve que l'herbe sielse a de cetle plante contient les principes chiminues suivants: de la maticre colorinte "rienense (chlorophlylle), de la cire, une matière extractive jaune, de la fécule, du " sallate de chaux, du sucre, de l'acide sorbigue (malique), de la fibre vétritale, de e ligydrochiorale el du carbonate de polasse, du sulfite, du carbonate el du phosphate "de chaux et de l'oxide de fer. Il parail que l'auteur n'a point cherehie la silice que (1I. Pictet, aimsi que M. Julu, ont trouvée en assez grande quantité dans la Prête. "Le principe de cette plante, employée comme diurétique, parait itre la matière "résineuse àcre, qui y existe en quantité prélominante. o L'analyse de M. Diebold est contenue dans Buchner, Repertor. f. Pharm., XXVIII, p. 366; 1828.

Ėn cette mème annéc 1898, Bracomol faisait paraitre dans les Anneles de chimie et de physique (XXXIX, P. 1 i 24) des Recherche's chimiques sur le structure des Prêles; ce qui suit en est extrait:

«Résumé de l'analyse de 500 grammes de l'E. Tclmatcia.

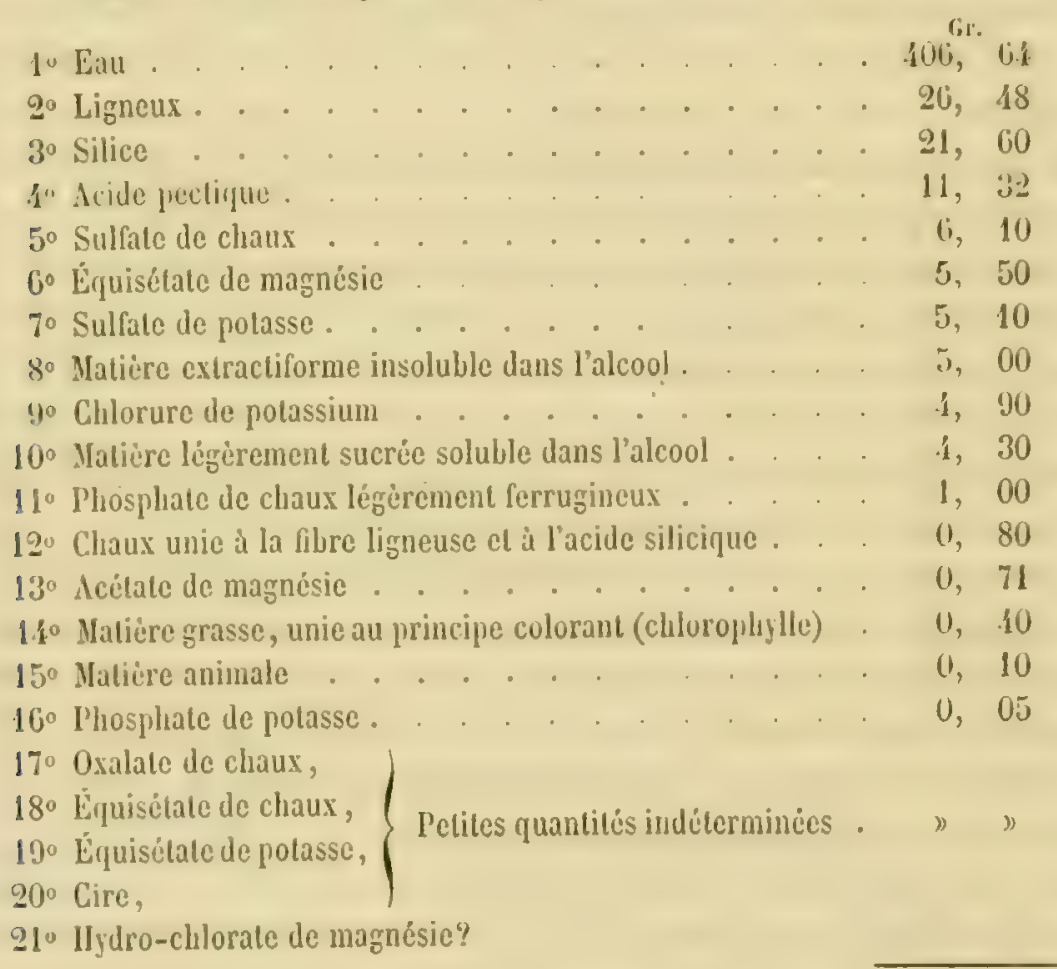

Tolal ... 500,00

"Incinération de l'E. T'elmatcia.

"Cinfuante grammes de cette plante fraiche, brùlés dans un creuset d'irrent, ont 
§3. - COMPOSITION CIMMLUE.

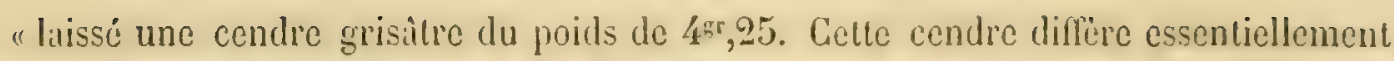
"par sa composition de toutes celles des autres régétaux. Sia lessive, mème concen"trée, ne contient point de sous-carbonate de potasse et ne change nullement le "papier rougi par le tournesol. Éaporée en grande partie, elle a laissé déposer du «sulfate de chaux cristallisé en fines aiguilles; il pesail $0^{\text {gr }}, 1$ après sa calcination. En "continuant l'évaporation, on a obtenu un résidu salin, lequel, chauflé fortement, "pesait 1 gramme; il était entièrement formé de sulfate et de muriate de potasse sans "aucun indice de sulfate de magnésie. Redissous dans l'eau, le nitrate de baryte y a " formé un précipité du poids de $0^{\mathrm{ar}}, 67$ correspondant à $0^{\mathrm{gr}}, 51$ de sulfate de potasse. "Celle quantité soustraite de 1 gramme laisse $0^{\mathrm{gr}}, 49$ pour le chlorure de potassium. - La portion insoluble de la cendre sur laquelle on a versé de l'acide hydrochlorique "affaibli, a produit une légère effervescence. On a délayé le tout avec un demi-litre « d'eau distillée, ct après l'avoir fait bouillir et filtréc à travers un papier-joseph préala" blement lavé à l'acide hydrochlorique, il est resté une grande quantité de silice; elle "pesail 2gr,11. La liqueur acide, évaporée à siccité, el le résidu repris par un peu "d'eau, a laissé du sulfate de chanx; lavé avec de l'eau alcoolisée et chaufié au rougse, " son poids était de $0^{\mathrm{gr}}, 51$. Pour m'assurer si ce sel ne contenait point de silice, on l'a "mis en ébullition avec de l'eau acidulée par l'acide hydrochlorique, tout s'est dis"sous, mais la liqueur est restéc louche. Filtrée, il est resté une matière gélatineuse, "laquelle desséchée, était pulvérulente et légère; c'était en effet de la silice. Fonduc "au chalumeau arec un peu de carbonate de soude, elle a donné un verre limpide, "d'une belle couleur jaune de topaze, due sans doute à la présence de quelques traces "de sulfate de chaux converti en sulfure. Le liquide séparé des 0 ar 50 de sulfate de "chaux, après avoir été acidulé par un peu d’acide hydrochlorique, a été précipité "par l'ammoniaque, qui en a séparé $0^{g r}, \mathbf{I}$ de phosphate de chaux légèenenent ferru"gineux; on y a ver'sé ensuite du carbonate de potasse el on l'a évaporé à siccité. La "masse saline, lavée avec de l'eau, a abandonné le earbonate de magnésie el le car"bonate de chaux. Ces sels saturés par l'acide sulfurique et chaullés au rouge, on a "obtenu $0^{\text {gr }}, 71$ de matière. Une dissolution salurée de sulfite de chaux en a dissous " 0 gr 35 de sulfate de magnésie, correspondant a $0^{\text {gr }}, 22$ de magnésie, et il est resté ( 0 gr, 36 de sulfate de chaux, équivalant à 0 gr, 26 de carbonate de chaux.

\section{«Examen de la cendre de l'E. hyemale?.}

«Cent grammes d'E. hyemale, brûlés dans un creuset d’argent, ont exigé beau"coup de temps pour leur parfaite incinération. Il est resté près de 12 grammes

\footnotetext{
- "On sait que cetle espèce est employée de préférence ì toute autre pour les arts, ct que, pour l'employer à polir ales métaux, on introduit dans la cavité de sa lige à cannelures très-rudes un fił de fer de mème diamétre qu'elle, qui "permet de l'appliquer sans la briser cuntre les objets à polir. »
} 
- d'une cemle qui a oflert des caracteres assez particulier's. Bien lave avec de l'eau, ra lessive alealine, concentrée par léraporation, a pris une cunsistance nucilagi- neuse, et il s'est forme a la surfice une pellicule fiagrile et diaphane comme du - verre. Ce résidn épais ayant élé redissous dans un peu d'ean, on y a versé de lacide - acélique qui a donné naissance à un magma gélatineux abondant, en ne produisant - qu une lérgère effervescence. Le mélange, évaporé presque i sec el lavé avec de "l'eau, a laisse une poudre, larpuetle, chanflée au rouge, était tres-hlanche, acide el

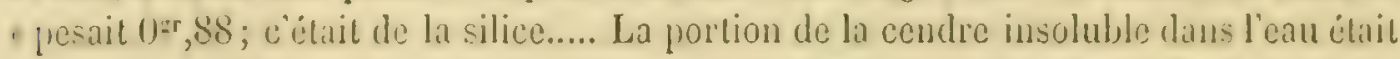
¿ presque entierement forméc de silice.... L'ammoniague en a sépare $0=5,6$ de phos"phate de claux ferrugineux.....

"Je terminerai ce mémoire en présentant le tableau résultant de l'analyse de lil x cendre des espéces de prêles que j’ai examinées:

\begin{tabular}{|c|c|c|c|c|c|c|c|c|c|}
\hline \multirow{2}{*}{$\begin{array}{c}\text { MONS } \\
\text { DES PHELES. }\end{array}$} & \multirow{2}{*}{$\begin{array}{c}\text { CENones } \\
\text { gournics } \\
\text { frar } \\
100 \text { partice } \\
\text { do } \\
\text { flamat.... }\end{array}$} & \multicolumn{8}{|c|}{ COMPOSITION DE I $A$ CENIDRE. } \\
\hline & & SHLICE. & $\begin{array}{c}\text { stheste } \\
\text { do } \\
\text { chaux. }\end{array}$ & $\begin{array}{l}\text { stzacate } \\
\text { de } \\
\text { potasse. }\end{array}$ & $\begin{array}{l}\text { Curbantrite } \\
\text { de } \\
\text { potassium. }\end{array}$ & $\begin{array}{c}\text { carnowate } \\
\text { de } \\
\text { chaus. }\end{array}$ & MAGXX์SIE. & $\begin{array}{c}\text { PHospuAte } \\
\text { de chans } \\
\text { ferrugineux. }\end{array}$ & $\begin{array}{l}\text { Potasse } \\
\text { en partie } \\
\text { unic } \\
\text { a l'acide } \\
\text { silicique. }\end{array}$ \\
\hline E. Telmateia. & 23,61 & 12,00 & 3,39 & 2,83 & 2,72 & 1,40 & 0,66 & 0,55 & 0 \\
\hline E. lyemale. & 11,81 & 8,75 & 0 & 0,33 & 0,28 & 0,93 & 0 & 0,80 & 0,72 \\
\hline E. arvense . & $13,8.1$ & 6,38 & 0 & 0,37 & 0,22 & 5,51 & 0,10 & $\begin{array}{l}\text { Quantité } \\
\text { industerin. }\end{array}$ & 0,30 \\
\hline E. limosum . & 15,50 & 6,50 & 3,30 & 2,20 & 1,20 & 1,50 & 0,30 & $\begin{array}{l}\text { indelerin. } \\
\text { ld. }\end{array}$ & Indices. \\
\hline
\end{tabular}

«Nancy, le 20 août 1828.

\section{(Bracoswot.)}

En $1836 \mathrm{el}$ dans les mimes Annules. I. V. Reguault sounit à l'analyse l'acide rinuisétique de braconnot el reconnut que cet acide présente la mème composition et la

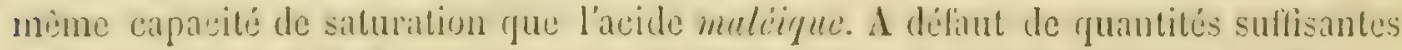

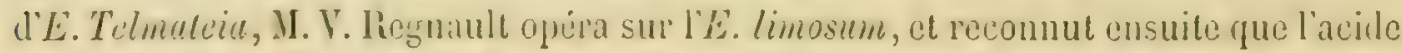
de cette espeice est identigue à celui de l'autre. Voici les résultats de cette analyse:

«Gent parties d'équisétate renferment donc:

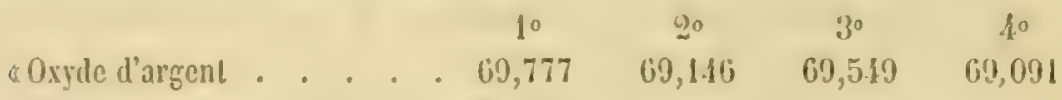

$$
\begin{aligned}
& \text { e. Moyenne . . . . . 69,391 }
\end{aligned}
$$


«D'un autre côté:

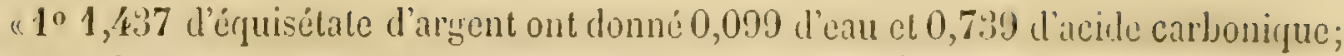

" 201,087 ont donné 0,075 d'eau et 0,576 d'acide carbonique;

« $3^{\circ} 1,173$ ont donné 0,092 d'eau el 0,621 d’acide carbonique.

"On déduit de là:

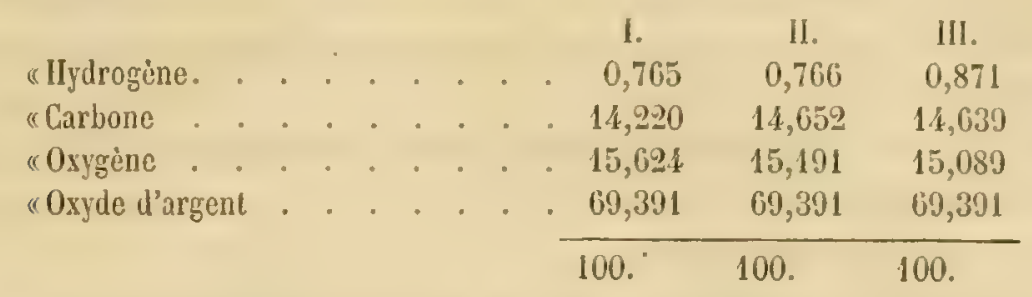

"Ces nombres s'accordent aussi bien fqu'on peut le désirer aree ccux que donnent "le maléate et le paramaléate d'argent. En effet, ces derniers sels sont composés de " la maniẻre suivante:

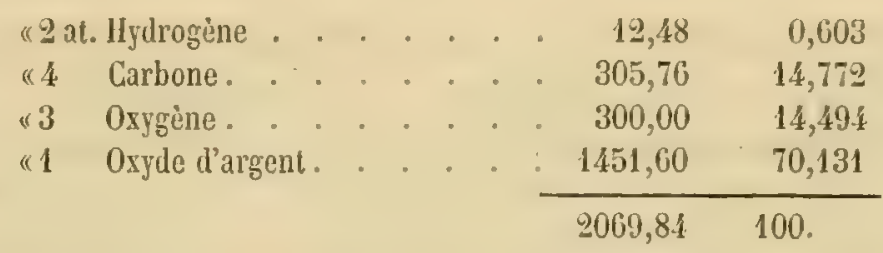

"La composition de l'acide équisétique cristallisć correspond aussi parfaitement à " celle de l'acide maléique. En effet:

" 0,495 d'acide équisétique cristallisé, desséché dans l'ail sec, ont donné 0,180 "d'eau et 0,740 d'acide carbonique; d'oú l'on tire pour la composition de l'acide:

$$
\begin{aligned}
& \text { «ydrogine . . . . . . . 4,040 } \\
& \text { "Carlsone. . . . . . } 41,338 \\
& \text { "Oxygène. . . . . 54,022 } \\
& 100 .
\end{aligned}
$$

(La composition de l'acide maléique cristallisé est:

$$
\begin{aligned}
& \text { «f at. Hydrogène . . . . . . . } 24,96 \quad 3,416 \\
& \text { «4 Carbone . . . . . . 305,75 41,843 } \\
& \text { « Oxggène . . . . . 400,00 54,741 } \\
& 《 C^{4} I^{3} 0^{3}+I^{2} 0=730,71 \quad 100 .
\end{aligned}
$$

"D'ailleurs l'acide équisétique est très-soluble dans l'eau et se distingue par cela (de l'acide paramaléique; ainsi, d'après ce qui précède, il ne peut rester aucun doute « sur l'identité de l'acide extrait de l'E. limosum avec l'acide maléique.

«Reste maintenant à faire voir que l'acide cstritit de l'E. limusem est bien le mème 
"que celui trouve par M. Braconnot dins l'E. Telmalciu. Jai traiti la petite quantite "de cetle dernière plante que jai pu me procurer par la mème série d’opérations: "que j’avais suivie pour extraire l'acide de l'E. limosum. J'ai obtenu un acide iden"lique par ses propriélís extérieures arec le premier. Cet acide transformé on scl diar« gent a donné les résultats suivants:

" $0,8,49$ de sel d'arsent ont donné $0,5,5$ d'argent mútallique correspondant i oxyde «d'argenl 0,58532 .

« $1, \mathbf{1 8 6}$ ont donné 0,103 d'eau el 0,805 d'acide carbonique. V'où l'on tire frutr lit a composition du sel:

\begin{tabular}{|c|c|}
\hline $\begin{array}{l}\text { "Mydrogene. } \\
\text { "Curtone. } \\
\text { "Oxyene. } \\
\text { "Oxyde d'argent. }\end{array}$ & $\begin{array}{l}0,770 \\
1.1,178 \\
15,301 \\
68,942\end{array}$ \\
\hline & \\
\hline
\end{tabular}

* Celte analyse s'accorde avec les précédentes; ainsi il est démontré que l'acide "érquisétique de M. Braconnot n'est autre chose que l'acide maléique, et que cet acide "se trouve dans d'autres espèces de Prềes que dans la Prìle fluviatile (E. Telmutéiu). "Ces plantes renferment même une quantité assez considérable d'acide maléinue "pour qu'il y ait peut-être avantage à s'en servir pour la préparation de cel acide" (Extrait des Amneles de chimie et de physique, LXII, p. 208-214; 1836). Voyez aussi V. Regnault, Cours élém. chimic, $3^{\circ}$ éd., tom. IV, p. 285.

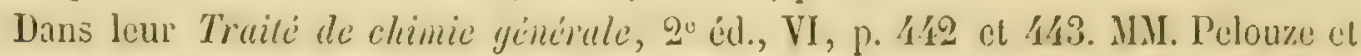
fremy rapportent les analyses que braconnot arail faites de l'E. muximum, des cendres de celte espèce et de celles des E. liyemale, arvense ct limosum. 


\section{DES GITATIONS}

ODs. Dans cette table ne sont point mentionnés les ouvrages de botanique descriptive les plus répandus; les abréviations ordinaires les feront suffisamment reconnaitre.

Agardin, Germ. Prêl. = C. Agardh, Olsservalions sur la gocrmination des Prềles, dans les Mém. du Mus. d'hist. nat., tom. IX, p. 283-292. - Le Mémoire fut livré par l'auteur en 1822.

Alp., De pl, ex. = Ex. Apinus, De plantis exoticis, lib. II; 1629.

Bensull., Gef. Krypt. Schw. = C. Hermoulli, Die Gefisshryptogamen der Schweiz; 1857.

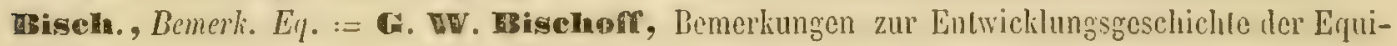
seten, in Bot. Zeitung, Feb. 1853, p. 97-109, avec fig. - Reproduit on parlie, aver les figures; dans les Ann. sc. nat, 3e série, bot., tom. XIX, p. 232 et suiv.

Biseln., Entw. $E_{\eta} .=$ G. Bisehor, Ucber die Entwicklung der Equiseteen, inshesondere des Equisetum palustre, aus den Sporen, in Nov. Acta Academice Cacs. Leop. Carol, maturce currios., tom. XIV, part. 2, p. 779-798; 1828; avec une planche.

Bbiseh., Frypt. Few. = G. B. Biselnoff, Die liryntogamischen Gewiichse mit besonderer Berücksichligung der Flora Deutschlands und der Schweiz; 1828; avec planches.

Bisch., Lehrb. Bot. = G. Iv. Bisehofr, Lehrbuch der allgemeinen und speciellen Botanik; 1834-1839.

Erac., Chim. Prêl. = X. IBracommot, Recherches chimiques sur la nature des Prêles, dans les Ann. de chim. et de phys., tom. XXXIX, p. 1-24; 1828.

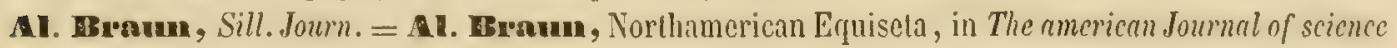
and arts, by Silliman, vol. XLVI; oct.-déc. 1843 , p. 81 ct suiv.

Briehan, Descr. Eq. = . T. Mrichan, Description of Equisetum hycmale, Mackaii and rariegatum, in The Phytologist; nov. 1812, p. 369-377.

Ad. Brong., Hist. vég. foss. = Au. Brongniaxt, Ilistoire des vézélaux fossiles, ou recherches botaniques et géologiques etc.; 1828-1837. - Equiselacées, vol. I, p. 99-120.

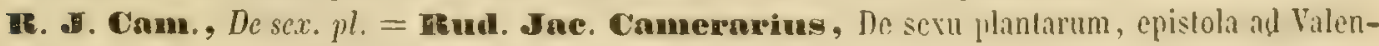
tinum. Tubingen, 1695, et in Polychresta exol. Valentini; 1700.

Cesalp., De $p l .=$ And. Cesalpino, De plantis, lib. XVI; 1583. 


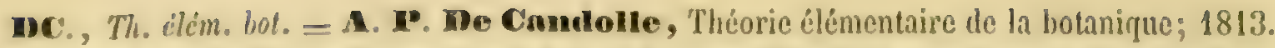

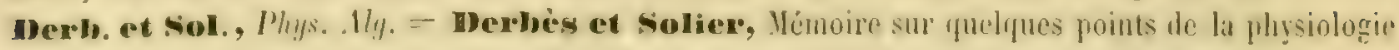
des Algues, dans le Suppl. aux comples rendus des séances de l'Académie des sciences, lom. Ier.

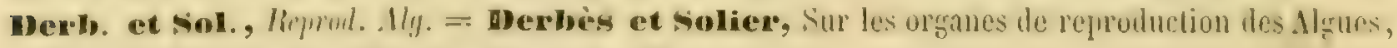
dans les Ann. se, nat., 3e série, bot., tom. XIV.

Desf., $F$. allant. = Desfontaines, Flora atlantica; 1798-1800.

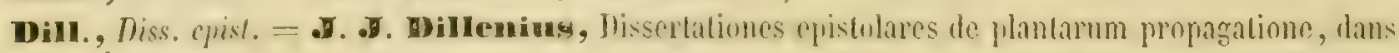
les Éphémérides de l'Acad. des curieux de le nature, année 1717, centurie V at VI, append., p. 45-95.

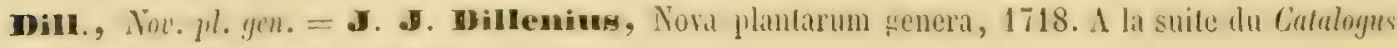
plantarum sponle circa Gissam crescentium.

Däll, Fl.Bad. = J. Ch. Döll, Flora des Grossherzogthums Baden, I; 1857.

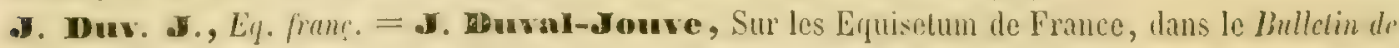
la Soc. Wot. de France, tom. V, p. 512 et suiv.; 1858; arec figures dans le texte.

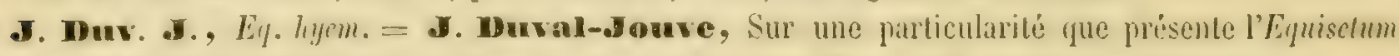
hyemale, dans le Bulletin de la Soc. bot. de France, tom. VII, p. 16.8; 1860; avec figures.

\. Duv. छ., Org. reprod. Eq. arv.= \$. Duwal-Jouve, Sur les organes de reproduction de l'Equisetum arvense, dans le Bulletin de la Soc. bot. de France, tom. VI, p. 699 et suiv.; 1859; avec une planche.

J. Denv. J., Pétiol. foun. = J. Duvol-ðouve, litudes sur le pétiole des Fougìres, dans les Annotations ì la flore de France et d'Allemagne, publiées par C. Billot; Ilaguenau, 1856.

Duvern., Salu. mat. = A. T. Duvernoy, De salrinia natante, cum aliquibus alis plantis comparata; dissert. inaug. bot.; Tubingen, 1825.

Ehx.h., Beitr. = F. Ehrohart, Beitrige zur Naturhunde, liesonders der Botanik cte., 7 rol.; 1787-1792.

Frane., Ancl. brit. ferns. = C. Franeis, An analysis of the british ferns and their allies, 2.e éd.; 1843.

Garr., Form. stom. = Garreau, Mémoire sur la formation des stomates dans l'épiderme des fenilles de l'Ĺpuť̉ẻne des jandiss dans les Ann. sc. nat., 4º sér. bot., I, p. 213-219; 1854.

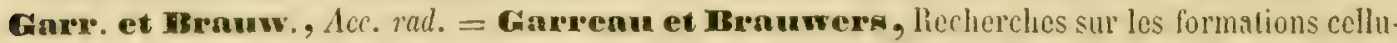
laires, l'accroissement et l'exfoliation des extrémités radiculaires, dans les $A n n$. sc. nut., fe sér. bot., X, p. 180-192; 1858 .

Caspare., Suce. rad. $=$ G. Gasparrini, Riccrehe sulla matura dei succiateri et la secrezione delle radici. Naples 1856.

Gleielı., Neuest. Unters. = Gleichen, Das Neueste aus dem Reiche der Pnanzen oder microscopische Untersuchungen elc., 1764. - Traduit en français par Isenflamm: Découverles les plus récenles dans le règne végétal ou observations microscopiques sur les parties de la génération des plantes etc.; 1770.

Grew, Anat. $p l=\mathbf{x}$. Grew, The anatomy of plants with an idea of a philosophical history of plants; 1682.

Medw., Funl. muse. = Joh. Medwig, Fundamentum hist. nat. muscorum frondosorum, concernens corum llores, fruclus, seminalem propagationem clc.; 1782.

Melw., Th. gen.= Joh. Hedwig, Theoria generationis el fruclificationis plantarum cryptoga-

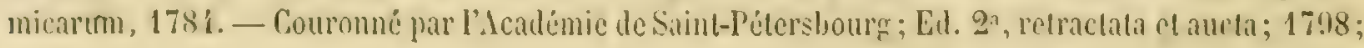
avec planches. 
IClw., Flor. quesimod. $=$ F. M. Melwing, lilora upuasimodo genita, sive cnumeratio aliquot plantarum indigenarum in Prussia etc.; 1712.

Hend., Reprol. Eq. = Jos. Henderson, On the reproductive Organs of Equisetum, in Transuctions of the Linnean Society of London, vol. XVIII, p. $566 \mathrm{et}$ suiv.; 1842; arec planches.

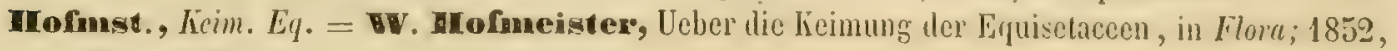
$\mathrm{n}^{0} 25$, p. 385 et suiv.

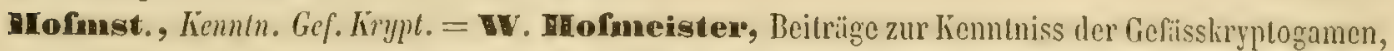
in Abhandlungen der mathemalisch-phlysischen Classe der königlich sëchsischen Gesellschuft der Wissenschaften, vol. IX; 1852 ; avec planches.

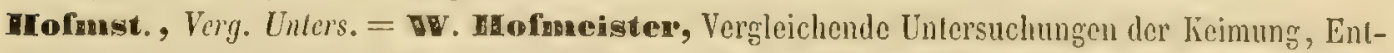
faltung und Fruchtbildung höherer Iriyptogamen (Moose, Farrn, Equiselaceen, Rhizocarneen und Lycopodiaceen) und der Saamenbildung der Coniferen; 1851; avec planches. - Equisetum de la p. 89 à 102; pl. XVIII, XIX et XX.

A. 1. Juss., Élém. bot. = Arrien de Jussien, Cours élém. de hotanique; 1848.

Ixirehh., Lab. org. = AIf. Irisehleor, De labiatarum organis vegetativis commentarium; 1861 .

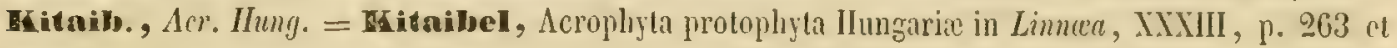
suiv. - Notes posthumes.

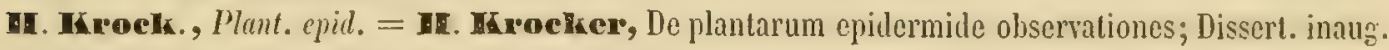
phytotomica. Breslau 1833; avec 3 planches.

Tange, Pug. 1 l. Misp. = \$olh. dange, Pugillus plantarum imprimis hispanicarum, in Naturhist. Foren. vidensk. Meddelelser. Copenhague 1860.

Gecou, Géog. bot. = E⿱一𫝀口. Lecou, Études sur la géographie botanique de l'Europe ctc., 9 tom.; 1854-1858.

Lindl., Introd. nat. syst. = Folnm Hisadley, An introduction to the natural system of botany; 1830.

Cindl., Nat. syst. bot. = Jolu Cindley, $\Lambda$ natural system of botany; 1836.

Ciuk, Elem. phil. bot. = T. F. Gink, Elementa philosophix botanicx; 1824.

L., Pral. ord. nut. = Eimuć, Prolectiones in ordines naturales plantarum, Edidit. P. D. Giselic; 1792.

Loes., Pruss. = Jos. Loeselius, Flora prussica etc., cure. J. Gottsched; 1703.

MaIp., Anat. $p l .=$ M. Malpighi, Anatome plantarum, 2 vol.; 1675, London. - Je n'ai cu ì ma disposition que l'édition de Leyde; 1687.

Mett., Seitenkn. Fum.=G. Mettenius, Ueber Seitenknospen bei Farnen, in Abhandl. d. math.phys. Classe der lioniglich sëchsischen Gesellschaft der Wissenschaften, vol. VII, p. 610-628; 1860.

Meyen, $P /$. phys. = F. उ. F. Mreyen, Nenes System der Pflamzenplyysiologic; 1837-1839.

Milde, Arch. Eq. Telm. = J. Milde, Des Auftreten der Archegonien am Vorlicime von Eq. Telmateia Ehŕh., in Flora; 1852, no 32, p. 497, avec planche. - Reproduit en partie dans la Botanische Zeitung, no 32; août 1852, p. 537 et suiv.; avec figures.

Milde, Entw. Eq. = J. Milde, Zur Entwicklungsgeschichte der Equiscten, in Nor. Act. Academiur Caes. Leop. Carol. naturce curiosorum, tom. XX, part. 2, p. 613-630; avec un supplément, p. 637641 ; 1852; avec planches. - Communiqué le 25 août 1851.

Mnilde, Eq. litt. = \$. IHilde, Ueber Equisetum littorale Kühlw., in Bonplandia, férrier 1858 . p. 26-31.

Milde, Gef. Grypt. Schl. = J. Mrilde, Dic Gefïss-Cryptogamen in Schlesien preussischen und üsterrei- 
chischen Antheils, in Nov. Act. Academie Gies. Leop. Carol. nelure curiosomun, tom. XXYI, part. 2 , P. 412-\$76; 1857; arec planclies. - II y a cu un tirage at part, mais j’ai cité de préférence la paginalion des Pora Acta squi se trourent plus fréqquenment dans les bibliothèques publiques.

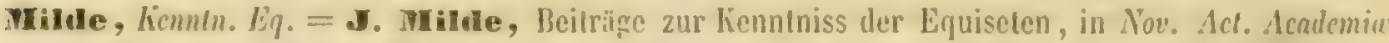
Cires. Lcop. Car. nalure curiosorum, tom. XXIl1, part. 2, p. 557-612; 1853; arec planclies.

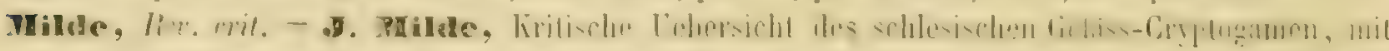
lesonderer Berüclisichtigung der Equiseten, in Denkschrift sur Feier ihres fünfaigjührïgen Beslchens, herausyegcbẹn ron der schlesischen Gesellschaft für valerlëndische Kultur, p. 183-197; 1853. - Ce qui se rapporte aux Equisetum a été traduit sous le titre de: Revue rritique des Équisctum de lu Silésie, dans les Archives de flore, p. 100 el suiv.; 1855. llagnenau.

Milde, Spor. Eq. = \$. Milde, De Sporarum Equiselorum germinatione, Dissert. inaug. bot. Breslau, 6 nov. 1850; avec deux planches.

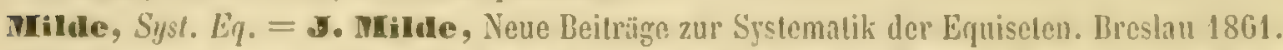

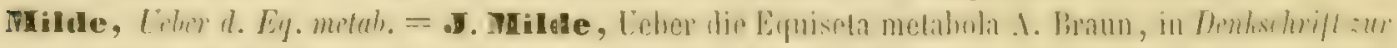
Feier ihres fünfzigjührigen Bestehens, heransgegeben von der schlesischen Gesellschaft für valerlïndische Kultur, p. 199-201; 1853; avec une planche.

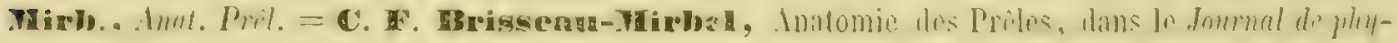
sique; prairial an $\mathrm{IX}$, tom 52 . p. 444 i 449.

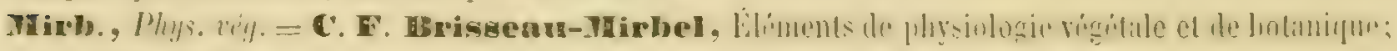
1815; arec allas.

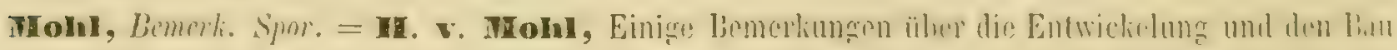
des Sporen der liryptogamischen Gewïchse, in Flora; 1833, p. 33-16, und in Vermischle Schriflen ele., p. 67-83.

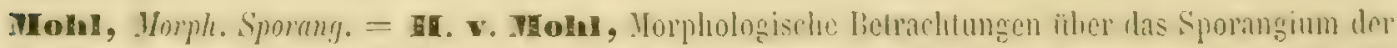
mit Gefissen rersehenen liryptogamen; dissert. inaug.; 1837, und in Vermischte Schriften elc., p. $94-107$.

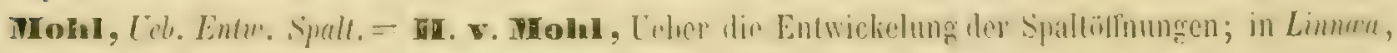
1838, p. 541-5.18; und in Vormischte Schriften, p. 252-259.

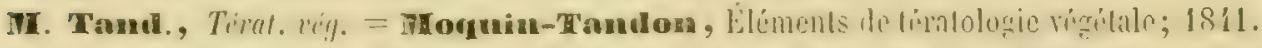

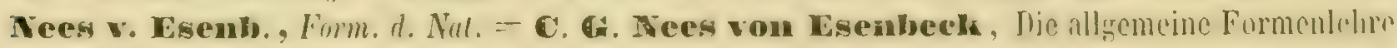
der Natur. Breslau 1861.

E. Newma., Bril. ferns = Ealw. Newman, A History of Rritish Forns and allied Plants, ㄹ. inl.; London 1844; arec d'excellentes grarures sur bois.

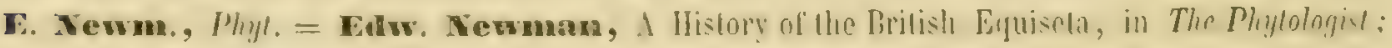
$1842,11.273,305,337,529$.

G. Oyjis., struet. fern. = Oyilvie, On the Forms and Strueture of Forn-Slems, in The Annak and Hagazine of Natural IIislory, décembre 1859; p. 101-411, arec figures.

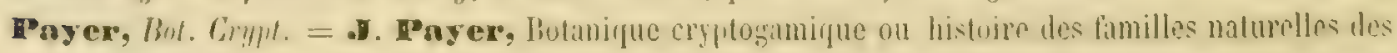
plantes inférieures; 1850.

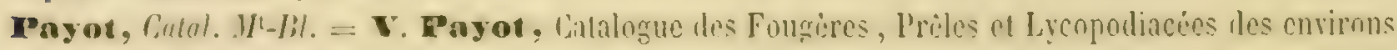
du Nont-Blanc; 1860.

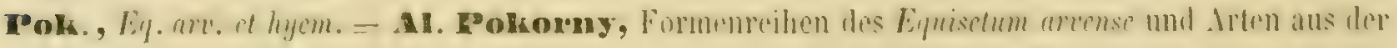
Gruppe iler Equisela hyemalia, in Verhandl. des soolog.tiol. Vereins in Wien, tom. VII, p. 9-10; 1857. - Analysé dans le Bull. de la Soc. bot. de France, tom. V, p. 137-138. 


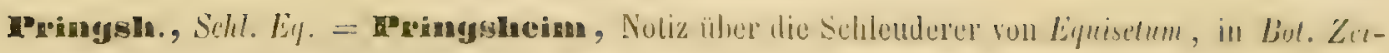
tung, 8 arril 1853, 11. 241-24.4.

Meaj., Mcth.pl. = Foln HEay, Methodus plantarum emendata el aucta; 1711.

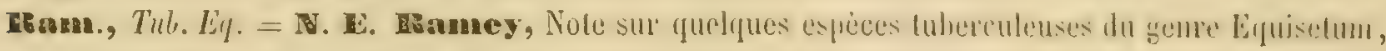
dans les Mém. de la Société des se. phys. et nat. de Bordeaux; 185.1, p. 209-219; avec deux planches.

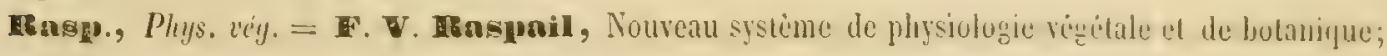
1837.

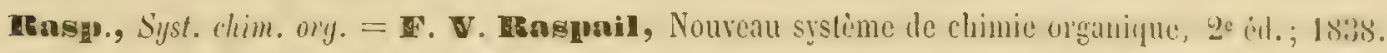

IRegn., Acid. Equis. = v. Izegnault, Idenlité de l'Acide Equisélique de II. Braconnul aree l'Acide Maléique, dans les Ann. de Chim. et de Phys., tom. LXI, p. 208-214; 1836.

rtichared, Élém. bot. et phys. = Ach. Beichand, Nouveaux éléments de butinique el de physiulogie végélale, 7 e éd.; 1840.

IRoth, Tent. /l. germ. = ERoth, Tentamen floræ germanicx; 1788-1800.

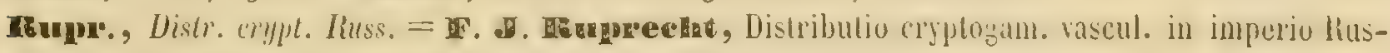
sico, in Beiträgez. Pflanzenkunde des russischen Reichs, 3't Lief.; 1845.

Sanio, Epid. u. syalt. Ey. = Caro Sanio, Lntersuchungen üher die Epidermis und dic splaltöfhungszellen der Equisetaceen, in Linnæa, vol. 29, p. 385-416; 1858; avec une belle planche. - Tirage à part de 32 pages. J'ai cité la pagination du Linnceea, qui est plus rẻpandu dans les bibliothèques.

Sehacht, Pfansenselle $=$ 聠. Sehaehat, Die Pftanzenzelle, der innere bau und das Luben der Gewächse; 1852; avec planches.

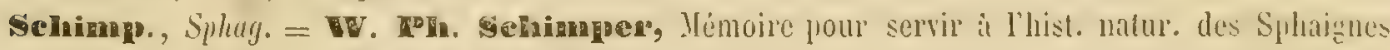
(Sphagnum), dans les Mémoires présentés par divers savanls à l'Académie des sciences, tom. XV; 1857.

Struve, Silic. = A. Atruve, De silicia in plantis nomnullis. Dissert. inaug. chem.; 1835; avec deux planches.

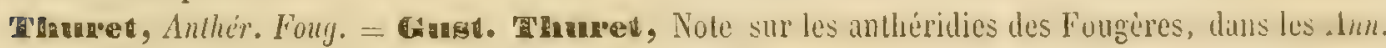
sc. nal., 3e série, bot., tom. XI, p. 9 et $10 ; 1849$.

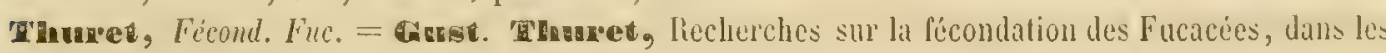
Ann. se. nat., $4^{\circ}$ série, Jot., tom. II, p. 197-214; 1854.

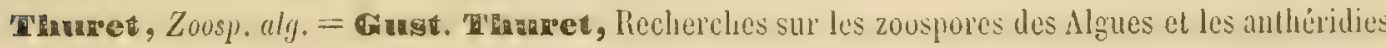
des cryptogames, dans les Ann. sc. nat., $3^{e}$ série, bot., tom. XVI, p. 77 et suiv.; 1851 ; arec planche.

Tréén, Form. second. rell. = A. Tréeul, Mémoire sur lé furmations secondiries dans les cellules végétales et sur les formations spirales, annulaires et réticulées, dans les Ann. sc. nat., $4^{\circledR e}$ séric, bot., tom. II, p. 273-356; 1854 .

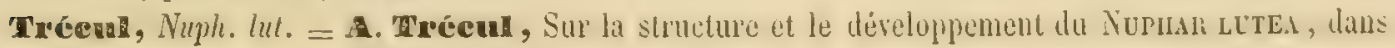
les $A n n$. sc. nat., 4e sér., bot., IV, p. 286-345; 1845.

Trécul, Or. rac. $=$. Trécul, Recherches sur l'origine des racines, dans les Am. sc.nat., 3e sér., bot., VI, p. 303-345; 1846.

Wnger, Acc. entren.= Ex. Unger, Sur l'accroissement des entre-nouds, extrait du Bot. Zeitung; 1844, p. 489 et suiv. - Traduit dans les Am. sc. nat., 3 série, bot., IV, p. 193-209.

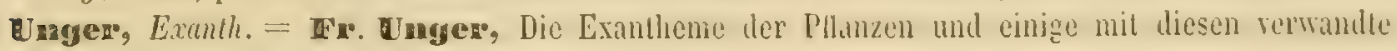
Krankheiten der Gewächse; 1833; arec planches. 


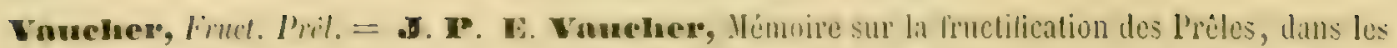
Mém. du Muscum d'hist. nat., tom X, p. 129-434; 1823; arec planche.

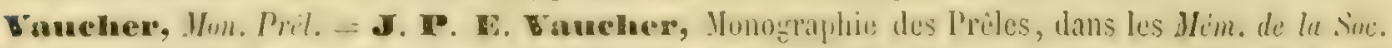
de physique el d'hist. nat. de Genève, Iom. I, 2c part., p. 329-391; 1822; avec planches. - Une uote initiale mentionne que cel important ourrage fut lu a la Société en férriel 1818. Tirage à part de

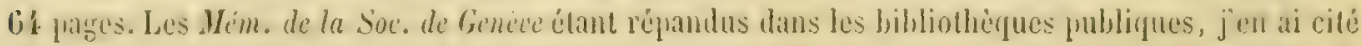
la paginalion, de préférence à celle du tirage a part. $\Lambda$ la fin de quelques exemplaires de ce tirage se trouve une note de deux pages d'impression ajoulie en 1820 ; elle est lort imprortante. Je l'ai cilce sous l'abróviation suivante.

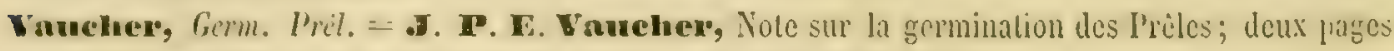
ajoutées en 1820 après la $64^{\circ}$ page du tirage à part de la Mon. Prêl. 


\section{PLANCHES}

ViT

\section{EXPLICATION DES PLANGHES}




\section{PLANGHE I}

\section{Rhizomes}

1. Eunisetum arvenke. Rhizome verlical. $a$ liourgeon de tige spicifirc. - b bourgeon de tige stérile. - $c$ Rhizome latéral ayant commencé par un tubercule. - d' Tubercules en chapelet. c lbourgeon terminal du dernier tubereule.

2. E. mrevense. lilizome.

a Fragment de rhizome horizontal, - 6 Entrenreul renfé en tubercule. - c Gainc basilairc du rhizome seconlaire.

3. C. arvemse. Coupe d'un gros tubercule adliétent a un rlizome horizontal trés-profond.

4. C. moximum. liragment de thizome arec tulsercules.

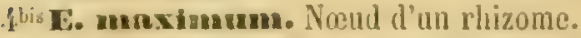
dl Finsette entre les racines.

5. E. ponlustre. Fragment d'un rlizome horizontal et d'un rhizome vertical avec tubercules.

6. E. nomossissimum. Fragnent d'un rhizome horizontal.

$a$ liameau vert commengant th pousser sur un rhizome mis à nu. - b liourrelet crénelé ì la base d'une gaine détruite. - c l'ragment de waine encore subsistant; le bourrelet est jeu saillant. "l Fossclte.

7. E. arvense. Coupe du noud d'un thizome, ducfuel naissent un rlizome lateral et une racine, 20 diamelres. lig.

"Tissu cellulaire central. - b Cellules courtes du diaphragme. $-c$ taisceaux fibro-vasculaires. 1 Groupes de cellules courtes et rayées répondant aux faisceaux fibro-vasculaires, $-e$ 'Tissu it grandes cellules séparant les lacuncs corticales. - f Lacunes corticales. - $y$ Saillic au sommet te la lacune corticale. - h Vaiscaux ile haciuc. $i$ l'artie de l'épiderme soulevée par le rhizome latéral et par la racisıc. - li Eipilerme. - I Gainc coupée au fond du sillon. - $m$ Gaine basilaire du rhizome latéral. - $n$ Premier nneu du rhizome maissant.

8. E. Iimosmm. Un des nouds inféricurs de la tige montrant la posilion des bourgeous de rameaux (a) el de racines (b) à l'élat expectant.

9. E. Ly emale. Granules amylacés du rhizome, 350 d.

10. E. nxwense. Granules amylacés des tuber- cules, $350 \mathrm{~d}$.

11. E. arvense. Les mêmes éclairís par la lumière polarisée, 350 d.

12. E. monximume. Granules amylacés d'un. très-jeunc inbercule, $350 \mathrm{~d}$.

13. F. arvense. $a$ Celluiss d'un tubercule arec quelques-unes des granules amylacés qui les remplissent. 6 Vaisscaux, $1.12 \mathrm{~d}$. 


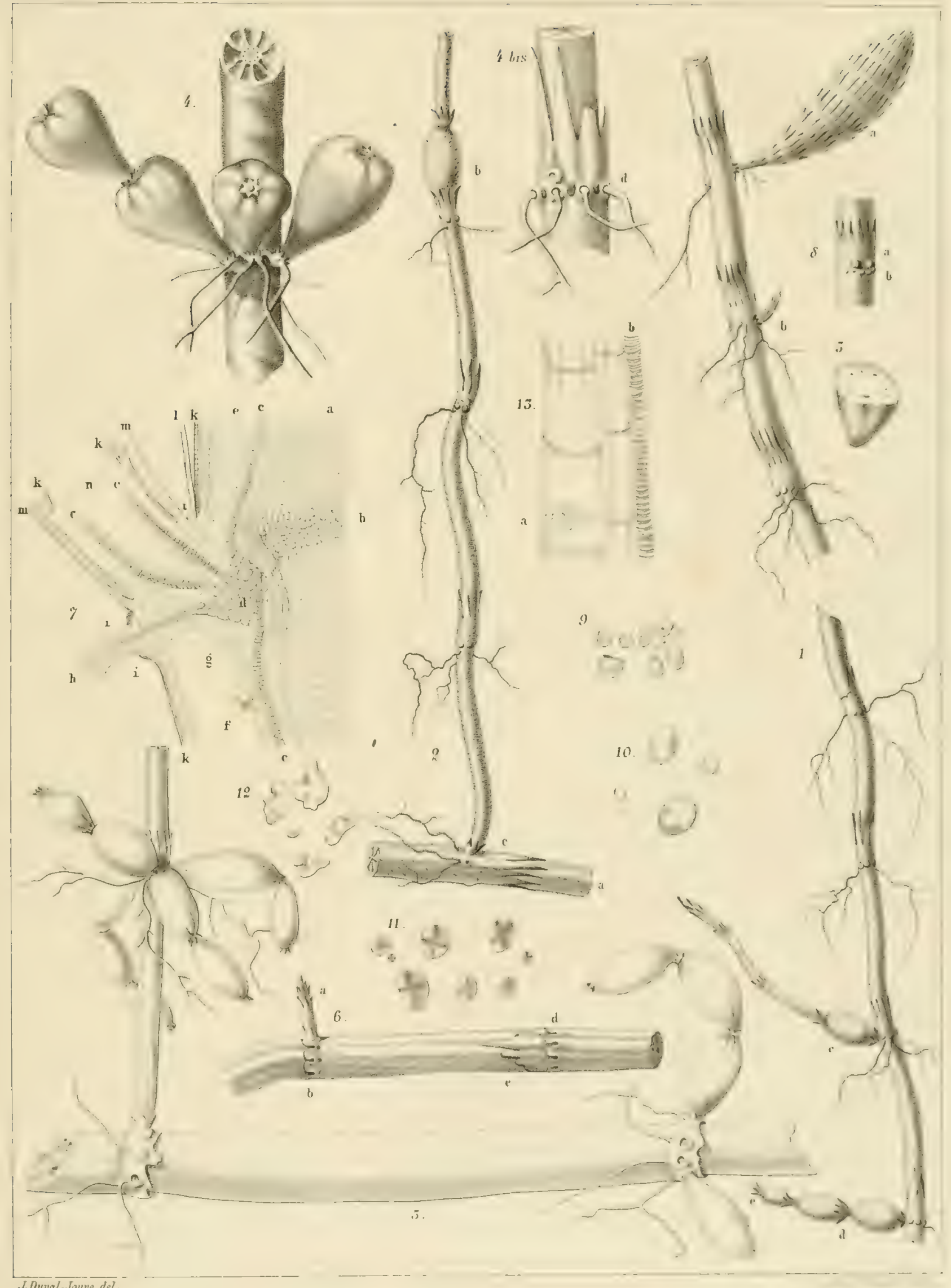

RHIZOMES 




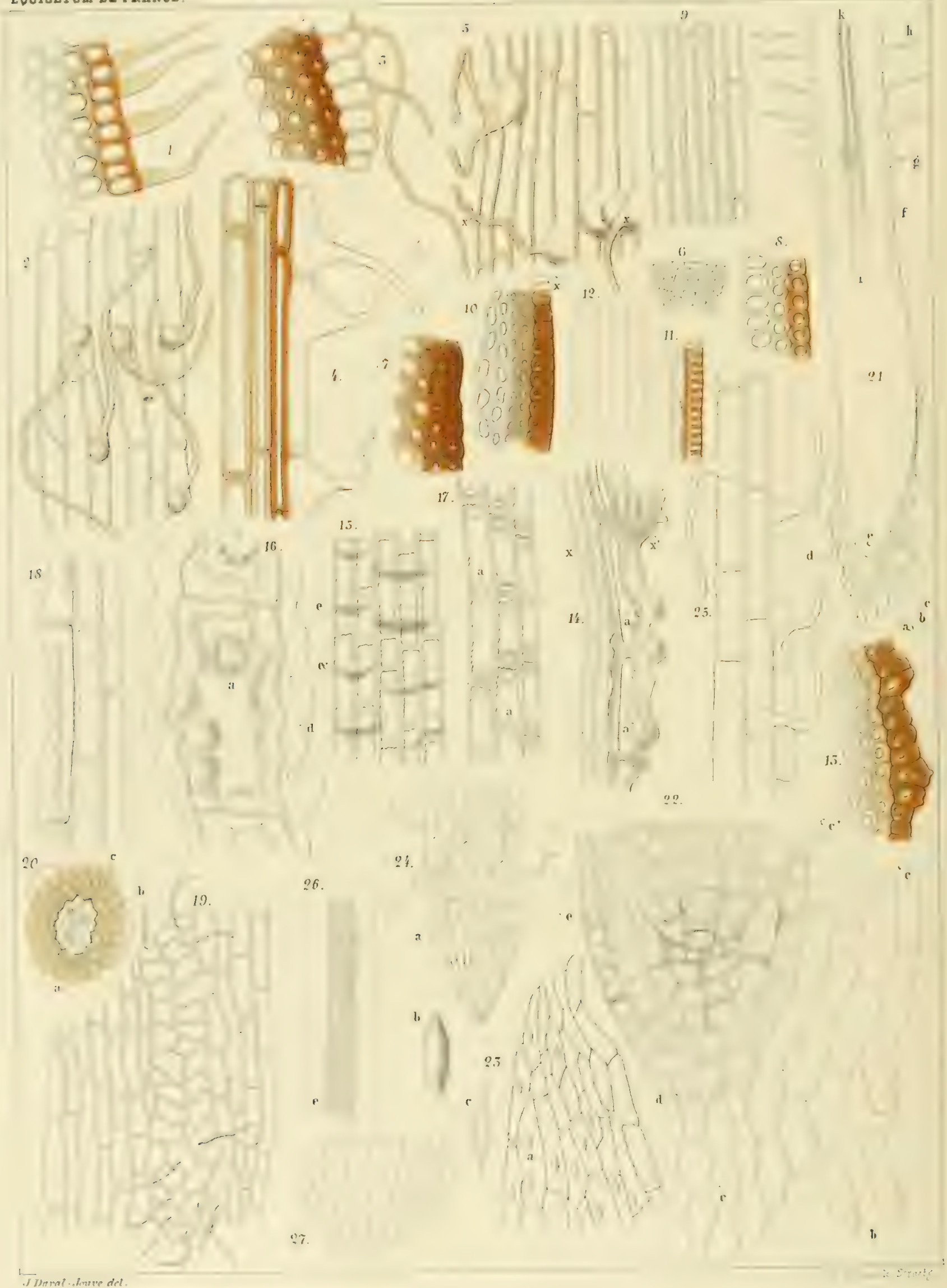

EPIDERME DES RHIZOMES.- RACINES. 


\section{PLANGHE II}

\section{Épiderme des rhizomes. Racines}

ligig.

1. Equisetum maximum. Coupe transrersale de l'épiderme des rhizomes, 112 diamètres.

2. E. maxinuma. Surface extérieurc du même épiderme, $142 \mathrm{~d}$.

3. E. arvense. Coune transversale de l'épiderme des rhizomes, suivant la ligne $x x^{\prime} \mathrm{de}$ la fig. 5, $142 \mathrm{~d}$.

-1. E. arvense. Coupe longitudinale du mêmc épiderme, $142 \mathrm{~d}$

5. E. sirvense. Surface extéricure du même épiderme, $142 \mathrm{~d}$

6. E. prontense. Coupe transwersale de l'épiderme des rhizomes, 142 d.

7. E. Iittorale. Coupe transversale de l'épiderme des rhizomes, $142 \mathrm{~d}$.

8. E. Limoswun. Coupe transversale de l'ẻpiderme des rhizomes, $142 \mathrm{~d}$.

9. E. limosum. Surface extérieture du mêne épiderme, $142 \mathrm{~d}$.

10. E. palustre. Coupe transversale de l'épiderme des rhizomes, $142 \mathrm{~d}$.

11. E. palustre. Coupe longitudinale du même épiderme, $142 \mathrm{~d}$.

12. E. pallustre. Surface extérieure du même épiderme, $142 \mathrm{~d}$.

13. E. Inyemaie. Coupe transversale de l'épiderme d'un gros et vieus rlizome, suivant la ligne $x x^{\prime}$ de la fig. 14, $142 \mathrm{~d}$.

14. E. Hyemale. Coupe longitudinale du même épiderme, $142 \mathrm{~d}$. a Dilatation de la cavité des cellules.

15. E. hyemale. Surface extérietrre du même épiderme, $142 \mathrm{~d}$.

e Rugosités siliceuses transversales s’étendant J'une cellule sur l'autre.

16. E. Iyemale. Une cellule du même épiderme, $670 \mathrm{~d}$.

a. Espaces plus transparents répondant aux dilatations de la cavité des cellules.

17. E. xamosissimutun. Surface extérieure de l'épiderme d'un jeune rhizome, $142 \mathrm{~d}$. a Espaces circulaires plus transparents.
Fig.

18. E. variegatum. Coupe longitudinale de l'épiderme d'un gros rlizome, montrant les inégalités de la cavité des cellules, $142 \mathrm{~d}$.

19. E. hyemale. Eniderme des gaines d'un rhizome, $110 \mathrm{~d}$.

20. E. arvense. Coupe transtersale d'une petite racine, $68 \mathrm{~d}$.

$a$ Cellules extérieures. $-b$ Couches de cellules a apparence médullaire. - $c$ Faisceau central fibro-vasculaire.

21. E. arvense. Coupe longitudinale de l'extrémile d'une racine plus grosse, $30 d$.

a Mucilage visqueux dans lequel sont des cellules exfolices. - b Cellules exfoličes. - c Piléorhize. - $c^{\prime}$ Extrémité supérieure de l'exfoliation de la piléorhize. - $-d$ liangs de cellules conecntriques i Ia surface. - e Cellules disposées on rangs non concentriques à la surface. - $f$ Cellules de l'ćpiderme se renflant en fibrilles radicellaires. - g Les mêmes plus avancées. - $l$ Les mèmes plus avancées encore. - $i$ Faisceau fibro-vasculairc en voic de formation. - $k$ Faisceau libro-vasculaire tout $i$ fait développé.

22. E. arvense. Extrẻmité de la racine de la figure précédente sans le mucilage, $255 \mathrm{~d}$. $c$ et $c^{2}$ Cellules de la piléorhize; elles ne sont pas toutes dans le mème plan "que les cellules suivantes. - $d$ Cellules concentriques i la surface de l'extrémité de la racine. - e Cellules disposées en sens inverse.

23. E. anvense. Cellules extérieures de la piléorhize prises au point d'exfoliation le plus éloigné de la pointe, $142 \mathrm{~d}$.

$a$ Petits renflements verdatres contre la paroi des cellules. - $b$ Un de ces rentlements, 600 d. c Le méme vu de profil, $600 \mathrm{~d}$.

24. E. arvense. Extrémité d'une racine ne s'accroissant plus et ayant perdu la pilćorhize, 68 i.

a Fibrilles radicellaires naissantes.

25. E. limosum. Epiderme d'une racine; les fibrilles radicellaires sont rares, mais trislongues, $142 \mathrm{~d}$.

26. C. Limostum. Cellules tris-longues et vaisseaux de la même racine, $142 \mathrm{~d}$.

27. Extrémilé de racine, extraite de Ilofmeister, Vergl. Unters., pl. XIX, fig. 14. 


\section{PLANCHE III}

\section{Épiderme des tiges et des rameaux}

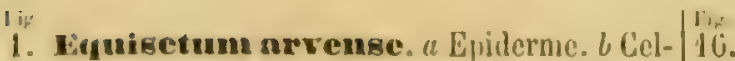
lules sous-jacentes d'une tige spicilëre, 142 diamètres.

¿. L. novense. Surface extéricure du mêne épiderme, $142 \mathrm{~d}$.

3. E. пуелне. Eucroûtement siliceux pris sur une lire slérile, $182 \mathrm{~d}$.

1. E. arvense. Surface extéricure de l'épiderme d'une tige stérile, $1.42 \mathrm{~d}$.

j. E. maximun. Surface extérieure de l'épiderme d'une lige spicifere, $142 \mathrm{~d}$.

6. L. maximum. Surface extéricure de l'épiderme d'une tigne stérile très-adulte, $142 \mathrm{~d}$.

7. L. sylvatiemm. Surface exlérieure de l'épiderme d'une tige spicifere jeune, $142 \mathrm{~d}$.

8. E. pratense. Coupe longitudinale d'une cellule courte des côtes avec sa papille, 240 d.

9. E. limosmm. Surface extéricure de l'épiderme d'une lige, $142 \mathrm{~d}$.

10. E. palustre. Fragment d'épiderme d'une lige, pour montrer la répartition des stomates, $70 \mathrm{~d}$.

a Milieu des cóles. - b Milieu des sillons. e Aspérités siliceuses des coles.

$10^{\text {his }}$ E. ramosimsimanem. Coupe longitudinale de l'épiderme el des fíbres corticales sur la côle d'une tige encore jeune, $210 \mathrm{~d}$.

11. E. maximum. Extrémité supérieure de deux lobes encore unis de la gaine d'une tige spicifère très-jeune, $4 \mathrm{~d}$.

12. E. maximumu. Fragment d'un de ces lobes, 142 d.

13. E. orvense. Lobe de la gaine d'une tige spicifere très-jeune, $4 \mathrm{~d}$.

1.4. E. srvense. I'ragment du même lobe sur te sillon commissural, $10 \mathrm{~d}$.

15. C. nxense. l'ragment de l'épiderme du mêne lobe, 112 d.
16. N. gylvaticum. Qualre lobes unis d'une gaine de la tige spicifiere, $2 \mathrm{~d}$.

17. E. mylvaticum. l'ragment d'un de ces lolies sur le sillon commissural, $20 \mathrm{~d}$.

18. E. sylvaticum. Fragment du même lobe sur la ligne de stomates, $142 \mathrm{~d}$.

19. E. arvense. Coupe longitudinale sur la ligne dorsale du lobe d une gaine de rameau trèsjeune, $142 \mathrm{~d}$.

a Epiderme. - $b$ Tibres corticales. - $c$ Grandes cellules. - $d$ Faisceau filuro vasculairc. - $e$ Cellules de la surface interne de la gaine.

20. E. arvense. l'ragment d'une gaine basilaire, $20 \mathrm{~d}$.

21. E. maximum. Cellules de la gaine basilaire; celles de la couche interne sont figurées par des points, $335 \mathrm{~d}$.

29. L. msximum. Coupe d'un rameau, 10 d. a Sillon. - i Arêtes quí séparent les sillons des cûtes. - c Sillon sur la lígne dorsale des cúles.

23. E. maxinum. Épiderme du même rameau, $50 \mathrm{~d}$.

$a$ Cellules du sillon. $-b$ Cellules des arétes qui séparent les sillons des cûtes. - $c$ Cellules du silLon de la ligne dorsale des cúles.

2.4. E. survense. Coupe longitudinale des cellules épidermiques sur la ligne dorsale des côles des rameaux, $142 \mathrm{~d}$.

y. E. sylvatieum. Epiderme des rameaux, $70 \mathrm{~d}$.

20. E. Iimosum. Surface extéricure des cellules épidermiques du clypéole, $310 \mathrm{~d}$.

27. C. limostun. Coupe des mêmes cellules, $310 \mathrm{~d}$.

28. E. maximum. Cellules épidermiques du clypéole, $310 \mathrm{~d}$.

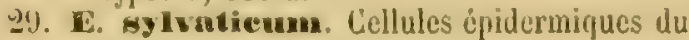
clypéole, $310 \mathrm{~d}$.

30 C. Vฉriegatum. Cellules épidermiques du clypéole, $310 \mathrm{~d}$. 


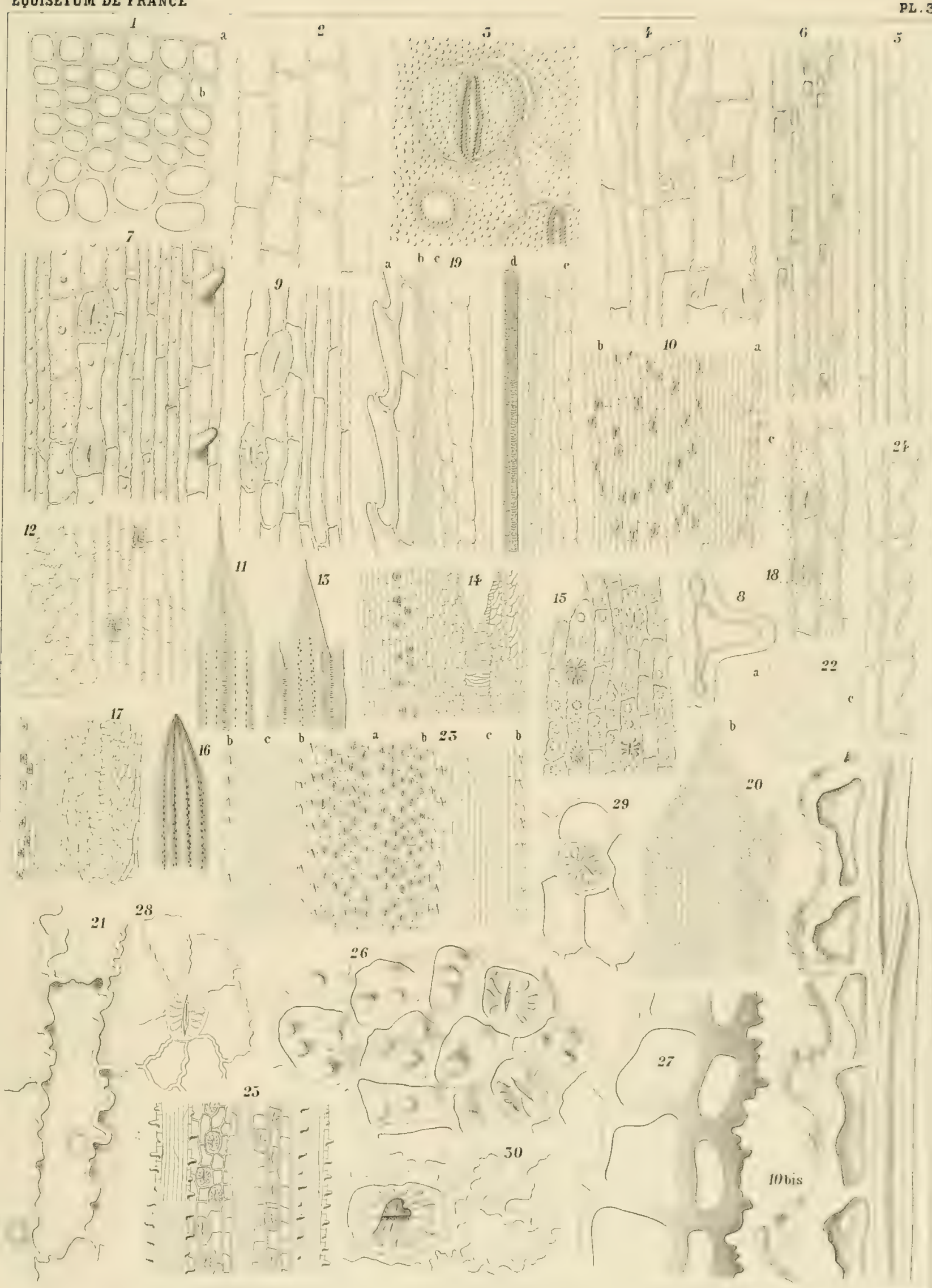






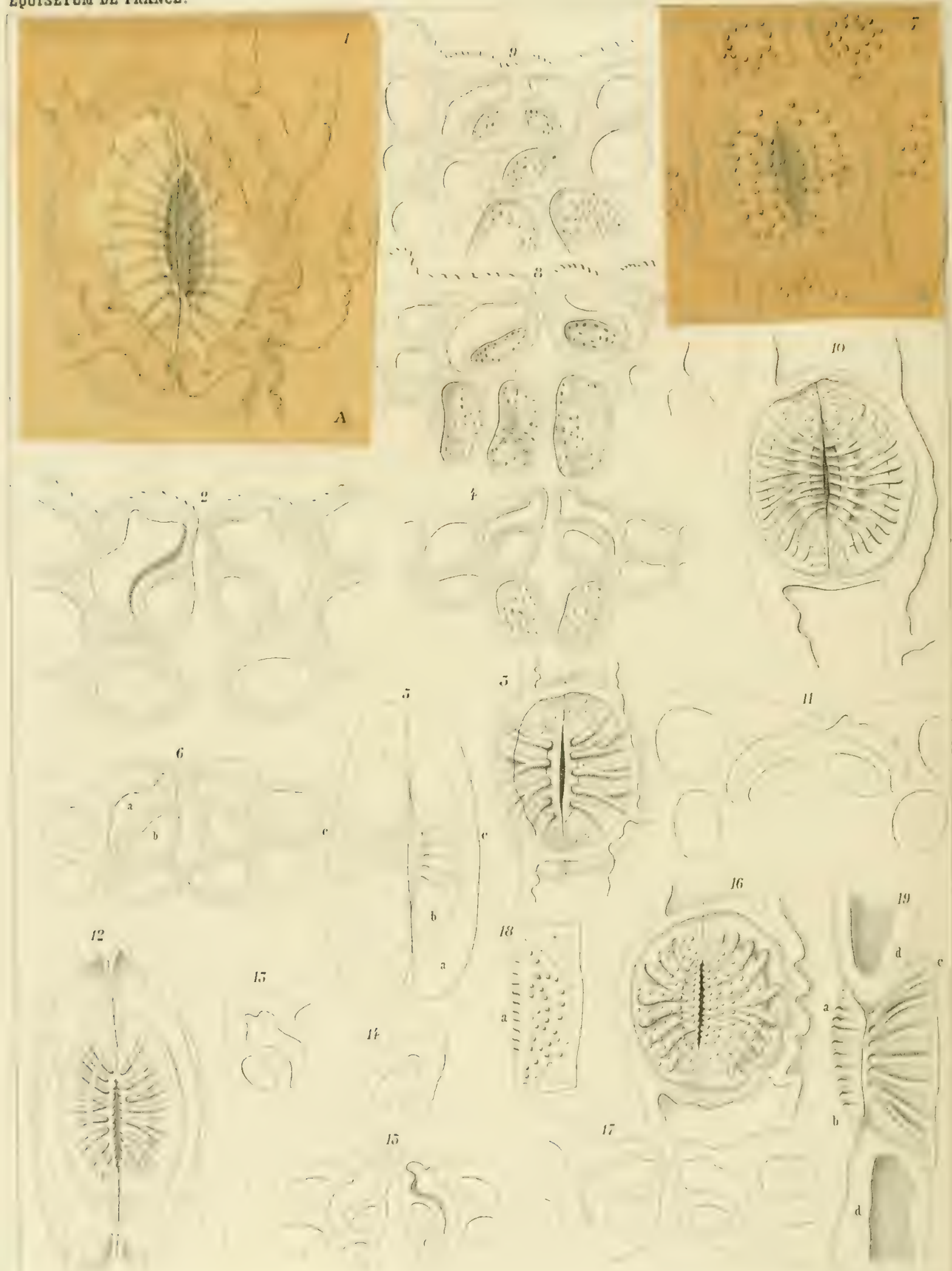




\section{PLANGHE IV}

\section{Stomates}

rif

1. Crunigetam moximnm. Stomate d'une gaîne de tige spicifere, 670 diamètres. A Cellules de l'ćpiderne. - B Paire de cellules extérieures. - G Paire de collules intérieures.

2. m. maximum. Coupe transversale sur to milieu du même stomate, 670 d. $\Lambda$ la paroi inféricure de la cellule supérieure de gauche on voil ombrée une stric rayonnante.

3. E. sylvaticam. Stomate d'un entre-noeud de la tige stérile, $670 \mathrm{~d}$.

1. E. Eylvaticum. Coupe transversale sur le milieu du même stomate, $670 \mathrm{~d}$.

5. E. ruratense. Gellules d'un stomate de la tige, au trait, sans l'encroûtement siliceux et sans les cellules contiguës, $670 \mathrm{~d}$.

6. E. pratemse. Coune transversale sur le milieu du même stomate, 670 d.

7. E. arvense. Stomate d'un entre-nocud de la tige stérile, $670 \mathrm{~d}$.

A Eneroûtement siliceux. - B Cellules de l'épiderme. - C Paire de cellules extéricures. - D Paire de cellules intérieures.

8. T. arvense. Coupe transversale sur le milieu du même stomate, $670 \mathrm{~d}$.

9. E. arvense. Coupe transversale du même stomate vers une de ses extrémités; la cavité n’est plus ouverte, $670 \mathrm{~d}$.

10. C. Iittorale. Stomate d'un entre-nocud de la lige, $670 \mathrm{~d}$.

11. E. littorale. Coupe transversale sur le milieu du même slomate, 670 d.
12. E. Immosmun. Slomate d'un entre-nnemil d'une lige tris-grosse, 670 d. Les points de l'encroûlement siliceux n'ont pas été dessinés.

13. L. Limeswma. Coupe transversale sur le milieu du même stomate, 670 d.

14. C. Iimosem. Coupe transversale rers une des extrémités du même stomate, $670 \mathrm{~d}$.

15. C. limosum. Coupe transrersale d'un stomate pris sur une lige plus jeune et plus petite. Au-dessous de la cavité de la cellule supérieure de droite on voit ombrée une strie rayonnante, $670 \mathrm{~d}$.

16. E. malustre. Stomate d'un entre-nocud de la tige, $670 \mathrm{~d}$.

17. E. palustre. Coupe transversale sur le milieu du même stomate, 670 d.

18. E. palustre. Une moitié de l'encroûlement siliceux superposé à un stomate, $670 \mathrm{~d}$. $a$ Petites saillies se prolongeant sur les côtés de l'ostiole.

19. E. malustre. Une moitie longitudinale du même stomate vue de l'intérieur, 670 d. La cellule inférieure a élé enlevée pour laisser voir les stries de la cellule supérieure.

a Petites saillies bacillaires que l'encroûtement siliceux projette sur les lèvres de l'ostiole. $-b \mathrm{Li}$ mite de la cavité de la cellule supéricure. - $e$ Stries de la paroi inférieure de cette même cellule. d Cellules de l'épiderme. 


\section{PLAINGHE V}

\section{Stomates. Coupes de rhizomes}

Fig. Equisetum xาmosissinum. Stomate d'un entre-nœud do la tige, 670 diametres. A Eneroûtement siliceux. - B Cellules de l'épiderme. - Ci l'aire de cellules extérieures. I) laire de cellules intéricures.

2. E. romosinfimum. Coupe Iranstersale du même stomate au delí de l'ouverture de l'encroûtement siliceux, 670 d.

guis E. Tromosiswimamm. Coupe de deux stomates suivant la ligne de l'ostiole, sur une tige encore très-jeune. Sur celui d'en haul la petite cellule intérieure a été laisséc; sur colui d'en las, elle a élé enlerée, 210 d.

3. E: voxiegntums. Stomale d'un entre-nocud de la tige, arec les cellules épidermiques supérieure el inférieure, 170 d.

4. E. hyemsle. Fragment d'épiderme pour montrer la disposition das stomales, 70 d. $a$ Milieu du sillon. - $b$ Milicu de la côte couvert de grosses aspériltís siliceuses.

j. E. Lyemale. Coune transversale d'un stomate d'une très-forte lige; clle est opércie au delia du milieu et laisse voir un peu en retrait l'arancement de la croutte siliceuse, $670 \mathrm{~d}$.

6-92. hyemnle. Coupes transtersales des stomales pour montrer les variations de la carité de la cellule extérieure, $670 \mathrm{~d}$.

10. E. maximumu. Coupe transversale d'un

\section{Fir.}

rhizome horizontal rampant it 50 cenlimètres de profondeur, $10 \mathrm{~d}$.

11. E. Ey Ivaticum. Coune d'un rlizome it 10 centimètres de profontleur, $20 \mathrm{~d}$.

12. E. sivense, Coupe d'un rlizome à $50 \mathrm{cen}$ timètres de profondeur, 20 il.

13. E. Iittornle. Coupe d'un rlizome is $25 \mathrm{cen}$ timétres de profondeur, $20 \mathrm{~d}$.

14 E. limosmm. Coupe d'un rhizome horizontal, $10 \mathrm{~d}$.

15. E. Ealustre. Coune d'un rhizome rerlical i 10 centimètres de profondenr, $20 \mathrm{~d}$.

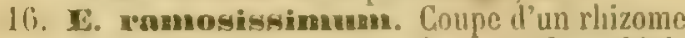
vertical à 60 cenlimètres de profondeur, $20 \mathrm{~d}$.

17. C. varegatum. Coupe d'un rhizome, $20 \mathrm{~d}$.

18. E. trachyolon. Coupe d'un rhizome, 20 d.

19. E. Inyemale. Coune d'un rhizome à 50 centimètres de profondeur, $20 \mathrm{~d}$.

20. E. Iny emale. Coupes transiersale el longitudinale d'un rhizome, $68 \mathrm{~d}$.

a Ėiderme: le mème se voit plus prossi pl. II, fig. $13,14,15,16 .-Z$ Cellules sous-jacentes ì l'épiterme; les mèmes so voient plus grossies pl. VII, fir. 1. - c. Cellules séparant les grandes lacunes. - d Grandes lacunes. - e libres des faisceaux fibro-vasculaires; les mèmes se voient plus grossics pl. vil, fig. 2. $-f$ Vaisseaux; les mèmes se voient jlus grossis pl. YHI, fign. 3. g Gros vaisscau du pourtour des petites lacunes; le mime se voit plus erossi pl. VII, fio. h. $h$ l'etites lacunes. $-i$ Cellules séparant les petites lacunes de la cavité centrale. - j Cavité centrale. 


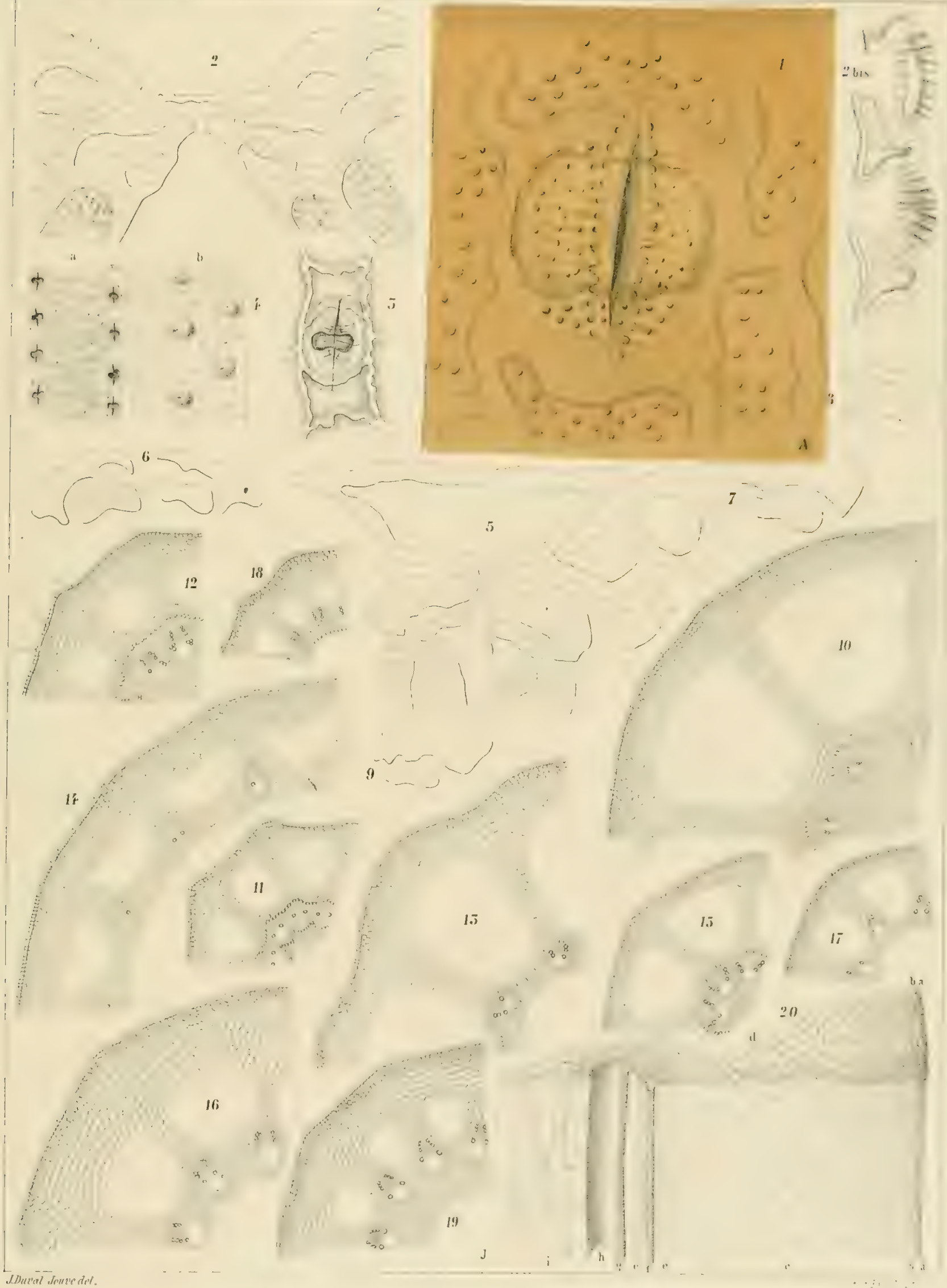

STOMATES. COUPES DE RHIZOMES 




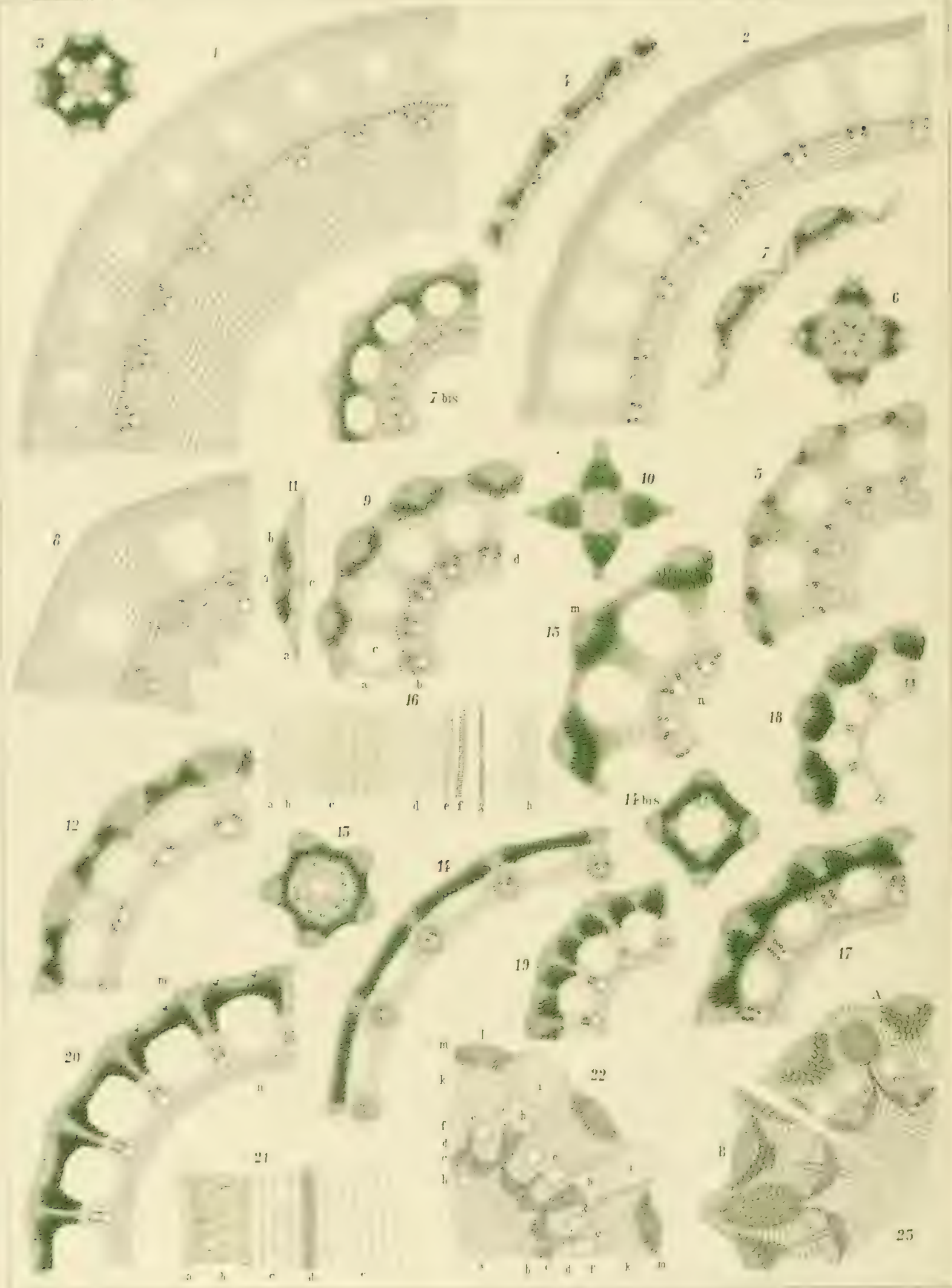




\section{PLANGHE VI}

\section{Coupes de tiges et de rameaux}

Fig.

1. Eutuisetum maximumu. Coupe transversale d'une lige spicifere propre, au moment de la sporose des verticilles inférieurs, 20 diamètres.

2. E. Imaxisamm. Coupe transversale du $6^{\circ}$ entre-nocud d'une tige stérile, $20 \mathrm{~d}$.

3. E. maximount. Coupe transversale du $2^{\circ}$ entre-noud d'un ramcau, 10 d.

4. E. maraximuma. Coup̣e d'unc gaîne de lige stérile, $20 \mathrm{~d}$.

5. C. sylvatiexne. Coupe d'une tige spicifère au moment de la sporose, $20 \mathrm{dl}$.

6. S. sy Iratienm. Coupe transwersale du Qe entre-nocud d'un rameau, $40 \mathrm{~d}$.

7. E. sylvatieurar. Coupe d'une gaine de tige adulte, $20 \mathrm{~d}$.

$7^{\text {bis }}$ C. preatense. Coupe d'une tige spicifère un peu après la sporose, $20 \mathrm{~d}$.

8. E. Coupe d'une tige spicifere propre, $20 \mathrm{~d}$.

9. E. arvense. Coupe d'une lige stérile au $6^{\circ}$ entre-nould, 20 d.

10. E. arvense. Coupe transversale dn $2^{\circ}$ entrenocud d'un rameau, 20 d.

11. C. revense. Coupe d'une gaîne de la lige spicifẻre propre, $25 \mathrm{~d}$.

$a$ Fibres corticales. $-b$ Cellules à chlorophylle. - $c$ Vaisceau fihro-vasculaire.

12. E. littorale. Goupe de la tige au $6^{\circ}$ cntrenoud, $20 \mathrm{~d}$.

13. E. Iittorare. Coupe transversale du $2^{\circ} \mathrm{entre-}$ nooud d'un rameau pris sur un sujet de moyenne grandeur, $20 \mathrm{~d}$.

14. E. Imogum. Coupe d'une tige de movenne grosseur an $6^{\circ}$ entre-nceld, $20 \mathrm{~d}$.

14bí E. limostme. Coupe au $2^{\circ}$ entre-nocud d'un rameau pris sur un des sujets les plus forts, $20 \mathrm{~d}$.

15. E. palustre. Coupe transversale d'une tige all $6^{\circ}$ entre-nocud, $20 \mathrm{~d}$.

16. E. palmstre. Coupe longiludinale d'une tige all $6^{\circ}$ entre-nocud, suivant la ligne $m n$ de la fig. 15, $40 \mathrm{~d}$.

$a$ Epiderme. $-b$ Fibres corticalcs. $-c$ Cellules transversales à chlorophylle. - $d$ Cellules inco-
Fig.

lores. $-e$ Guirlande de cellules séparant les deux cylindres. - $f$ Vaisceau fibro-vasculairc. - g Gros vaisseau au pourtour des petites lacunes, - $l \mathrm{Cel}$ lales da pourtour de la cavité centrale.

17. E. namogissimmm. Coupe transversalo sur le $6^{\ominus}$ entre-noud d'une tige de moyenne grosseur, $20 \mathrm{~d}$.

18. r. vaniegatum. Coupe d'une tige au Ge entre-nocud, $20 \mathrm{~d}$.

19. E. trachyoulom. Coupe d'une lige au 6 e entre-nocud, $20 \mathrm{~d}$.

20. C. Inyemale. Coupe transversale d'une tige 2il $6^{\circ}$ entre-nocud, $20 \mathrm{~d}$.

21. E. Inyerase. Coupe longiludinale d'une time au $6^{\circ}$ entre-noud, suivant la ligne $n n$ de la fig. $20,40 \mathrm{~d}$.

$a$ Epiderme. - b Cellules transversales à chlarophylle. - $e$ Cellules incolores séparant les grandes lacunes. - d Faisceau fibro-vasculaire. - e firandes cellules du nourtour de la cavité centrale.

22. E. arvense, Coupe d'un noend, au point d'origine des rameaux, sur une lige adulte, $20 \mathrm{~d}$.

$a$ Cellules centrales du diaphrame. -6 Restes des lacunes essenticlles presque oblitérées. c Groupes de cellules rayées ou de vaisseaux trèscourts en avant des lacunes essentielles. - $d$ Groupes de petites cellules ou de fibres courtes. - e Cordon vasculaire se rendant ì la gaîne. - f Tiestes de la lacune corticale qui est comme bipartie à son sommet par la saillie que produit le passage des vaisseaux de la gaîne. - g Cordon vasculaire allant s'épanouir dans le rameau. Sa direction est ascendante et il a été coupé par la section. $h$ Fibres allant s'ćpanouir dans le rameau. $i$ Grandes cellules et épiderme lu rameau coupés obliquement par la section horizontale. $-k$ Grandes ccllules du cylindre extéricur. - $l$ Fibres corticales ct chloropliylle. $-m$ Eniderme de la tige.

23. E. arvense. Coupe d'un noud de lige stérile jeune, au moment de l'érolution des rameaux, 20 d.

A. Coupe alors que le bourgeon à rameau était très-jeuno et que le faisceau vasculaire n’allait pas jusqu'it lui.

b. Mème coupe sur un nœud plus avancé, où lc faisccau vasculaire attcignait Ic jcune bourgeon. Le couteau a presque entièrement enlevé le faisceau vasculaire qui se rend de la petite lacune dans la gainc. 


\section{PLANGHE VII \\ Tissus. Évolution des organes}

1. Euro. Exetum hyemale. Cellules à parois épaisses, sous-jacentes it l'épiderme des rhizomes, 310 diamétres.

a Pores le communication fermés par une membrane. $-b$ Les męmes ouverts. $-c$ Granules amylacés. - le Pores vus par le bout.

2. E. Inyemnle. Fibres des faisceaux fibro'vasculaires d'un rhizome, $310 \mathrm{~d}$.

3. E. Iyemale. Vaisseaux des mêmes faisceaux, $482 \mathrm{~d}$.

1. E. Iyemnle. Gros raisscau du pourtour des lacunes cssentielles, $482 \mathrm{~d}$.

i. E. hyemsle. Très-pelit raisseau déroulable au pourlour des mêmes lacunes, $482 \mathrm{~d}$.

ij. E. arvense. Coupe longitudinale de l'extrémilé d'une fibre corticale, $510 \mathrm{~d}$.

7. C. arvense. Coupe transversale de fibres corticales prises loin de l'épiderme, $670 \mathrm{~d}$.

8. E. palustre. Fibres corticales prises tout contre l'épiderme, $670 \mathrm{~d}$.

$a$ Vues de hout. $-b$ Vucs de profil et montrant une double spirc plus claire et des jores jariétaux.

9. E. polnstre. Gros vaisseau du pourtour d'une lacune essenticlle de la tige, $510 \mathrm{~d}$.

10. E. ramosisgimum. Vaisseau du faisceau fibro-rasculaire d'une tige, $510 \mathrm{~d}$.

11. E. smense. Conpe longitudinale d'un nocud de la tige spicifere, $12 \mathrm{~d}$.

a Coune de la gaine vers sa ligne múliane. 1) Cordon de vaisseanx du faisceau fibro-vasculaire. - c Vaisseau du pourtour de la lacune es. sentielle. - $l$ Groupe de cellules courtes et rayées formant un cercle d'un blanc opaque. $-e$ Cellules de la guirlande séparant les deux cylindres.

12. E. Limosum. Groupes de cellules à parois unies, qui, dans les nœuds, correspondent aux fibres du cylindre interne, $670 \mathrm{~d}$.

13. E. arvense (lige spicifëre propre). Groupe de cellules striées qui, dans les noeuds, correspondent aux cordons vasculaires, $142 \mathrm{~d}$.

14. E. limosum. Deux des mêmes cellules, $670 \mathrm{~d}$.

15. E. paluatre. Moitié d'une long̣e cellule prise entre les groupes d'un nocud of les vaisseaux, $210 \mathrm{~d}$.
Fig.

16. C. Myemale. Hoilie d'une longue cellule prise au mẻme point, $9.40 \mathrm{~d}$.

17. E. hyemale. Parlic d'un vaisseau court pris conire un noeud, $730 \mathrm{~d}$.

18. E. arvense. Coupe longitudinale d'un pédicelle at d'un clypéole de sporanges, $12 \mathrm{~d}$.

a Faisceau central fibro-vasculaire. - $b$ Sporanges. $-c$ Clspéole.

19. E. arvease. Portion de la membrane formant le sac des sporanges, $500 \mathrm{~d}$.

30. E. nxrense. Fragment d'une spirale des cellules du sac des sporanges avec sa cavité, $1100 \mathrm{~d}$. Le chaque côté on voil un petit reste de la membrane qui unit ces tours de spire indéroulables.

21 C. axvense. Coune longiludinale de l'extrémite d'un rejeton naissant à la base d'une lige stérile, $68 \mathrm{~d}$.

$a$ Masse cellulaire terminale. - $b$ faines des nouds inférieurs. - $c$ Origine de la plus jeune gaine. - d Diaphragmes. - e Vaisseaux.

22. E. arvense. Cellule terminale et première division de la cellule infraposée en quatre cellules, 212 d.

23. Coupe schématique de la $1^{\text {ro }}$ division des quatre mêmes cellules.

24. Coupe schématique de la 2 c division des mẻmes.

2.4bis Jeunes gaines à trois degreis successifs de développement.

25. E. variegatum. Bourgeon terminal non coupé, au moment de la division des cellulesmères de la gaine, $212 \mathrm{~d}$.

a Cellule terminale avec deux nucléus. - b Division des cellules-méres de la gaine.

26. E. voriegatum. Nucléus des cellules supérieures du même bourgcon expulsés par compression. Un est vu de profil, $420 \mathrm{~d}$.

27. C. Limosum. Extrémité d'un ramenu naissant, non coupé, mais débarrassé de ses enveloppes, $212 \mathrm{~d}$.

28. E. maximum. Longues cellules diaphanes it la pointe d'une des dirisions de la gaine Ires-jeune, 212 d. 


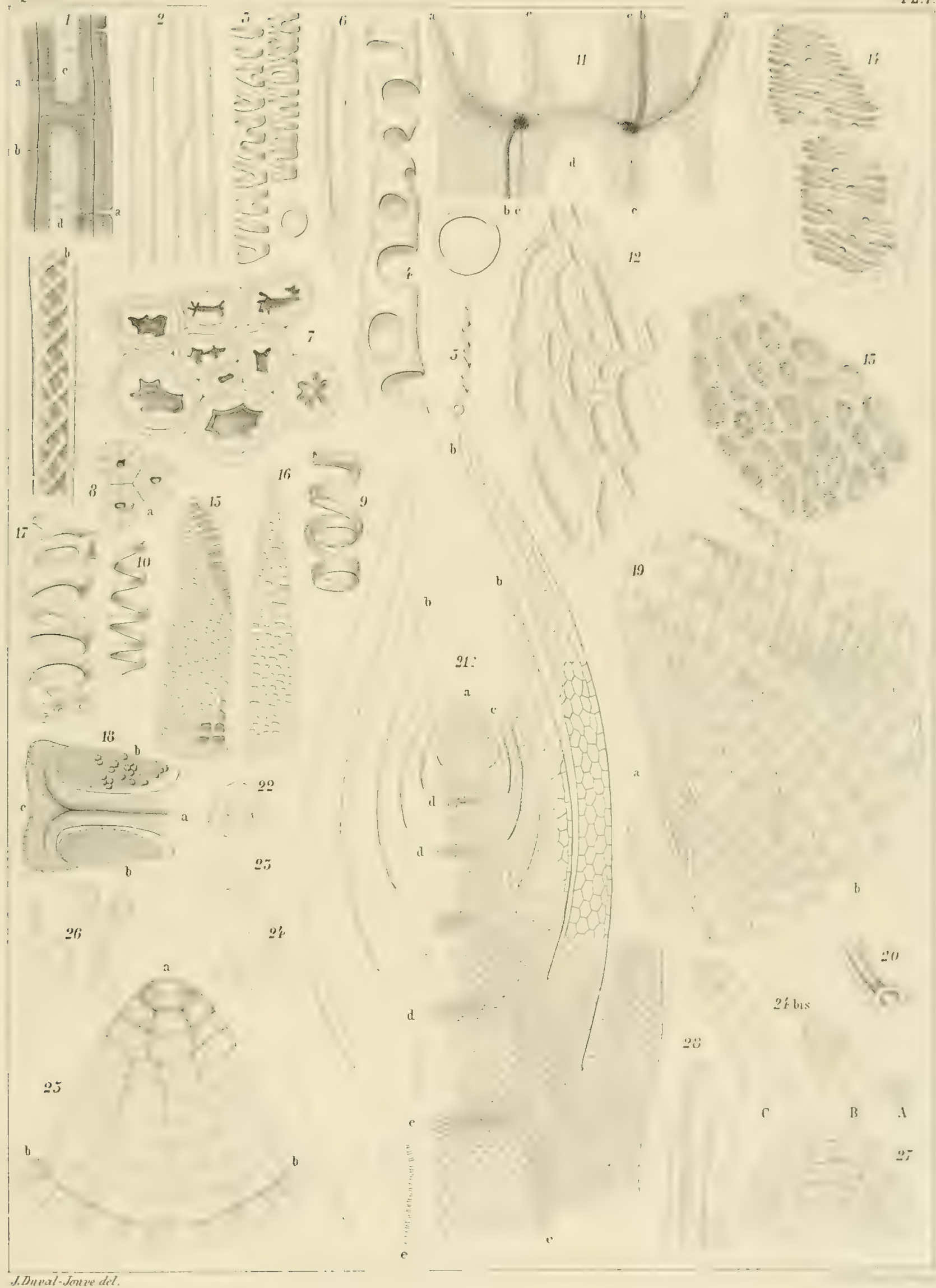

IIFIIE EVOLUTION DES ORGANES 



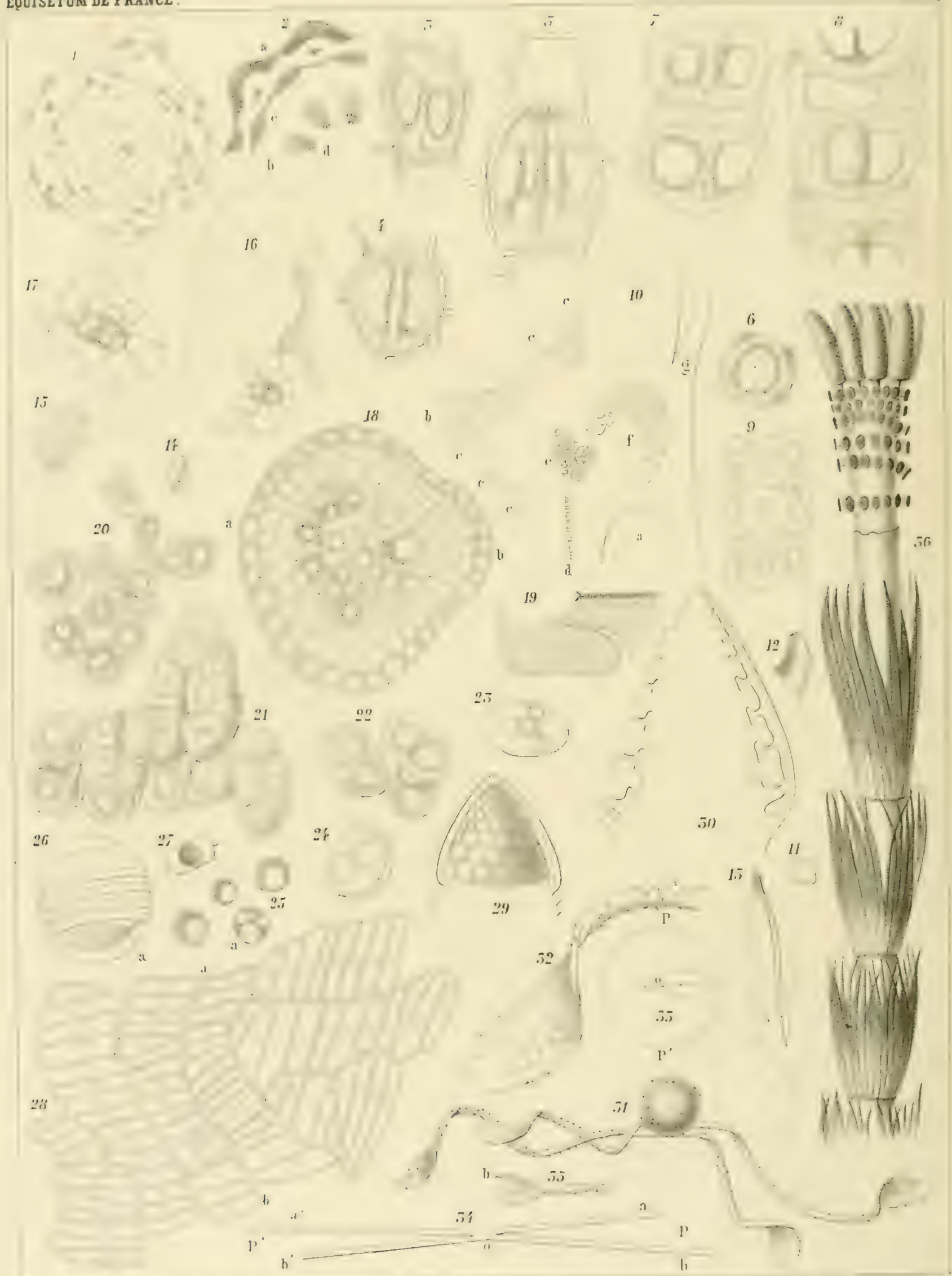




\section{PLANGHE VIII \\ Évolution des organes}

1. Cunisetum anvense. Coupe transversalc de l'extrémité d'une tige en étal de développement. $\Lambda$ centre une très-jeune galne forme bourrelet á la base du bourgeon ferminal coupé, 40 diamétres.

2. C. arvense. Coupe vers l'extrémite d'une tris-jeune lige, $\mathbf{4 0} \mathrm{d}$.

$a$ Gaine. - $b$ Tige. - $c$ Fibres corticales. $d$ laisceaux fibro-vasculaires. - 11 n'y a point encore de chloropliylle dans lit lige, et tout ce qui n'est pas occuní par' les fibres corticales et par les faisceaux fibro-vasculaires, l'est par du tissu incolore ì grandes cellules.

3. C. Cimosmm. Cellule-mere d'un stomate, avec deux nucléus latéraux, prise à la base d'un entre-noud encore jeune, $670 \mathrm{~d}$.

4. C. limosam. Cellule-mère plus avancée el prise à un demi-millimèfre plus haut. Les granules s'accumulent sur la ligne médiane it lit place de la future cloison, $670 \mathrm{~d}$.

5. E. Iimosam. La cellule-mère s'est diviséc en denx cellules latérales, dans chacune desquelles on voit vers l'exterieur un grand uucléus et vers l'intérieur un plus petit; prise à un millimètre plus haut que celle de la fig. 4, $670 \mathrm{~d}$.

Comparer les fig. $3,4,5$ arec la fig. 12 , pl. IV, nour apprécier les dillírences de grandeur.

6. E. 1อฐmosissimumm. Cellule-mère d'un stomate en son premier ćlal, vue de l'extéricur, $670 \mathrm{~d}$.

7. C. ranosissimum. Deux cellules-mères de stomates a leur second élat; entre clles est la cellule qui les sépare, 670 d.

8. C. manasiseimmm. Cellules-méres de stomales à lcur second élal plus avancé, $670 \mathrm{~d}$.

On voit encore une grande vésicule nucléaire dans une des celiules de.séparation.

Comparer les fig. 7 et 8 avcc la fig. 1, pl. V, pour apprécier le développement uhtéricur.

9. E. arvense. Premierre forme d'une cellulemère d'un bourgeon, vue de face, $300 \mathrm{~d}$.

10. E. hyemale. Bourgeon à l'état cxpectant sur une jeune lige de 20 centimetres de litut, $35 \mathrm{~d}$.

$a$ Grande lacune. - $b$ Cellules du diaphragne - Cellules médullaires a divers ctats. - $d$ Position des vaisseaux. - e l'osition des cellules rayées. - f Bourgcon recuuvert de sa gaine basilaire. $y$ Gaine.
11. Disprosition des qualre saillies annoncant l'aphparition des dents de la gaine basilaire.

12. C. Limosem. Bourgeon de rameaux de 1 millimétre de long, couvert de sa gâne basilaire, $10 \mathrm{~d}$.

13. E. maximame. Gaîne basilaire el première gaine d'un rameau, 3 d.

1. . E. limosam. I'remier état du développement d'un verticille de sporanges, 212 d.

15. Idem. Ilem.

16. E. Iimosam. I'remicr développencnt d'un sporange, $212 d$.

17. E. Iimaserm. Ieuxic̀me division da la première cellule intéricure d'un sporange, $250 \mathrm{~d}$.

18. E. Timosmma. Coupe transversale d'un sporange au moment qui précede la résorplius. des cellules internes du sic, $212 \mathrm{~d}$. a Cólé externe. - b couté interne.

19. E. Limosam. Forme du sporange apres l'isolement des cellules intérienres, $20 \mathrm{~d}$.

20. E. Iimosmm. Cellules isolées au même moment, $310 \mathrm{~d}$.

21. E. limosmm. Les mêmes cellules groupécs par qualre au même moment, 310 d.

2). L. Limosum. Les memes cellules arec deux grands nucléus, $310 \mathrm{~d}$.

23. E. limosmm. Les mêmes divisées en deux nouvelles cellules avece des nucléus disposés en croix vers leurs extrémités, 310 d.

24. E. Iimosum. Les mêmes beaucoup plus avancées, $310 \mathrm{~d}$.

25. E. limosrum. Spores trés-jeunes atrec espaces splieriques sans gramules, $212 \mathrm{~d}$.

26. E. Iimosmm. Spores at moment de la lormation des élatères, $482 \mathrm{~d}$.

7. Figure citée de M. Ilenderson.

28. E. Iimosman. Cellules du sac des sporanges au moment de l'isolement des cellules-mères des spores, $212 \mathrm{~d}$.

a $b$ Ligne de déhiscence.

29. E, limosam. Epi jeunc (10 avil), $20 \mathrm{~d}$.

30. L. nnvemse. Coupe d'un épi jeune (12 juillct), $20 \mathrm{~d}$.

31. Ee nxvense. Spore avec ses élateres, 310 u.

32. E. nx-vense. Estrémité d'un élatère, $670 \mathrm{~d}$.

33. Figure théorique.

3-1. Figure théorique.

35. Copie d'unc figure de $\mathrm{V}$. I'ringshein.

úli. L. mensimann. $15^{\circ}$ anomalie; pluralili d'épis sur un épi mutilé. 


\section{PLANGHE IX}

\section{Spores. Sporophymes. Anthéridies. Spermatozoïdes}

Fig. Equisetum srvense. Spore après ćbul- $\mid \begin{aligned} & F_{i g} \\ & 18\end{aligned}$ lition dans la solution polassique, 225 diamètres.

3. L. arvense. Spore dont la membrane interieure s'est brisée, $225 \mathrm{~d}$.

4. E. arvenne. Spore dont la membrane cxtérieurc s'est brisće, $2205 \mathrm{~d}$.

5. L. servense. Spore abortive aprés le mémo traitement, $225 \mathrm{~d}$.

6. E. arvense. Spore trailée par l'acide sulfurique, $225 \mathrm{~d}$.

7. E. sarvense. Spore abortive avec dépression de la membrane intérieure, $225 \mathrm{~d}$.

8. E. prinstre. Spore prise dans un sporange avant la déhiscence, $482 \mathrm{~d}$.

9. E. littorale. Spores abortives, $482 \mathrm{~d}$.

10. E. trachyodon. Spores abortives, $482 \mathrm{~d}$.

11. E. Irachyodon. Une des cellules se trouvant parmi les spores abortives, $482 \mathrm{~d}$.

12. C. sxvense. Spore après quarante heures de semis sur terre, $140 \mathrm{~d}$.

13. E. arvense. Suore semée le 13 avril; sporophyme dessine le 18.

14. E. pahustre. Spores semées sur terre le 8 septembre; sporophymes dessinés le 12 , $1.40 \mathrm{~d}$.

14t Sporophyme venu sur l'eau (mêmes dates); on voil ả côté des fragments d'élutères et de sporoderme.

15. E. palustre. Spore seméc sur terre to 8 septembre; sporophyme dessiné le 15. $150 \mathrm{~d}$.

16. E. palustre. Spore semée sur l'eau le 8 septembre; sporophyme dessiné le 15 , $150 \mathrm{~d}$.

17. L. Iimosum. Sporophyme dessiné le 16 juin; semis sur terre du ter mai, $68 \mathrm{~d}$.

A. Deux cellules terminales, $212 \mathrm{~d}$.

18. L. Iimosum. Sporoplyme dessine te 10 juin; semis sur l'cau du jer mail, fi8 d.

19. E. palustae. Grains de chlorophylle arec plasieurs granules verts, pris vers la base d'un sporophyme ùgé de 20 jours, $1100 \mathrm{~d}$.

20. E. palustre. l'remier's développements d'une anthéridie, $112 \mathrm{~d}$.

$a$ Ler état; $a^{\prime}$ coupe transversale. $\quad b$ qo état; $b^{\prime}$ coupe transversale. - c 30 état; $c^{\prime}$ coupe trans. versale.

21. E. paluatre. Coupe d'une anthéridie après l'apparition de cloisuns parallèles à la jériphérie, $112 \mathrm{~d}$.

22. E. palustre. Coupe d'une anthéridie au moment de la division en pelites cellules culiquues, $112 \mathrm{~d}$.

23. E. arvense. Anthéridies dessinées le 20 juin; semis du 16 avril; $112 \mathrm{~d}$.

a Anthéridic encore fermée. - b Anthérillic ouvertc. - $c$ Anthéridic expulsant les spermatozoiides.

24. E. pahustre. Anthéridies dessinées le 26 juillet; semis du 22 juin, $112 \mathrm{~d}$.

a Anthéridie encore ferméc. - c Anthéridic cxpulsant les spermatozoides.

25. E. palustre. Spermatozoïdes avec leur's cellules, au moment de leur sortie, $1100 \mathrm{~d}$.

26. E. limosum. Formes de spermatozoïdes dessinés par II. G. Thurel (Ann.sc.nat., 29e série, bol., tom. XVI, pl. 15, fig. 2).

27. Spermatozoüdes dessinés par II. Hofmeister (Kennt. Gef. Krypt., tab. XVII, fig. 1 i 6. )

28. E. arvenue. Formes de spermatozoides vues par moi, $500 \mathrm{~d}$. a Spermatozoide altaché par son extrémité à un corps étranger. - $b$ Spermatozoüle à demi sorti de sa cellule-mére.

\section{E. protense Ehrls.}

$a$ Tige spiciferce, saus rameaux, avec de grandes gaines.

b 'Tige spicifère, avec des rameaux cl des gaines de grandcur moyenue. 


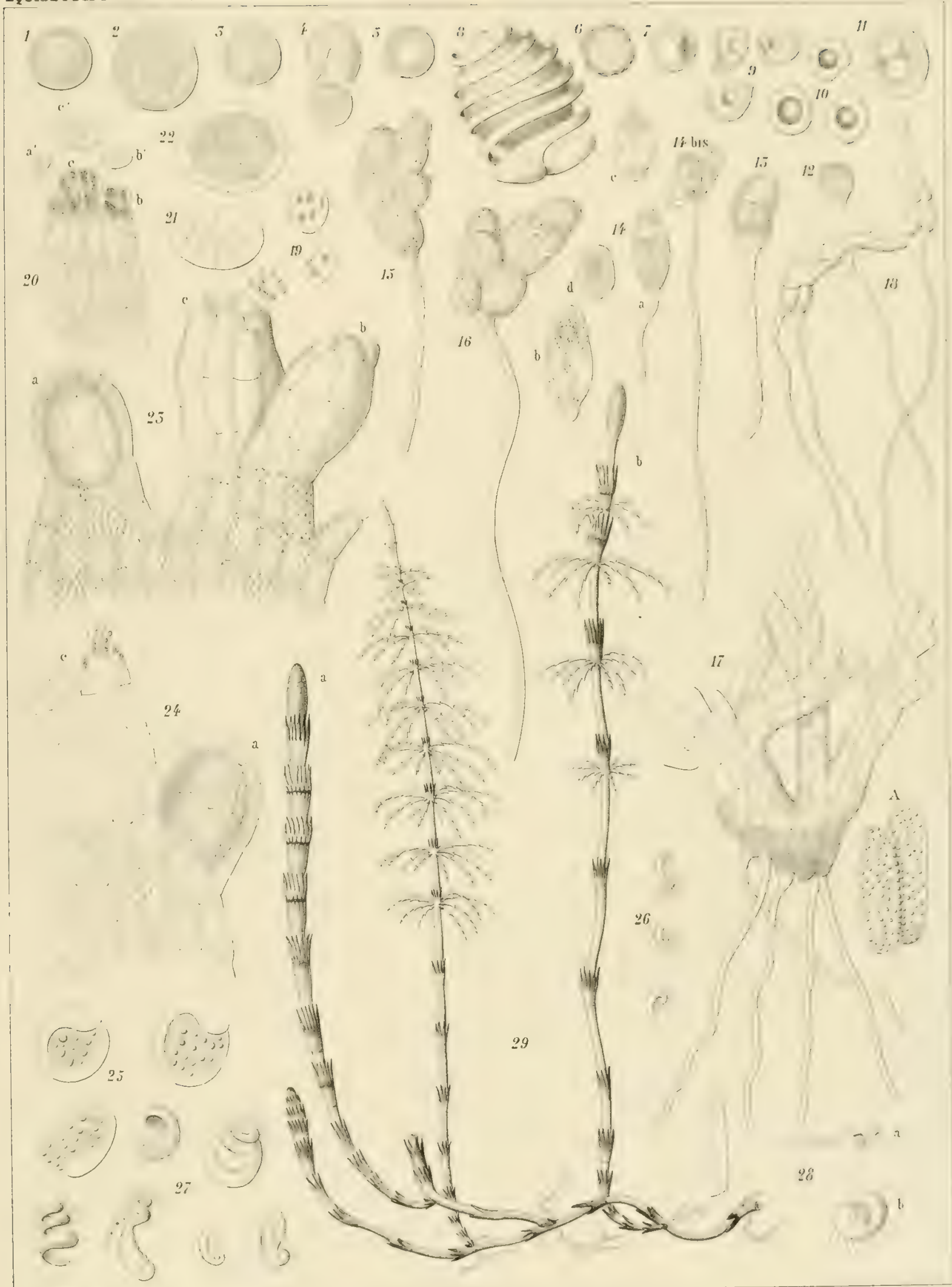





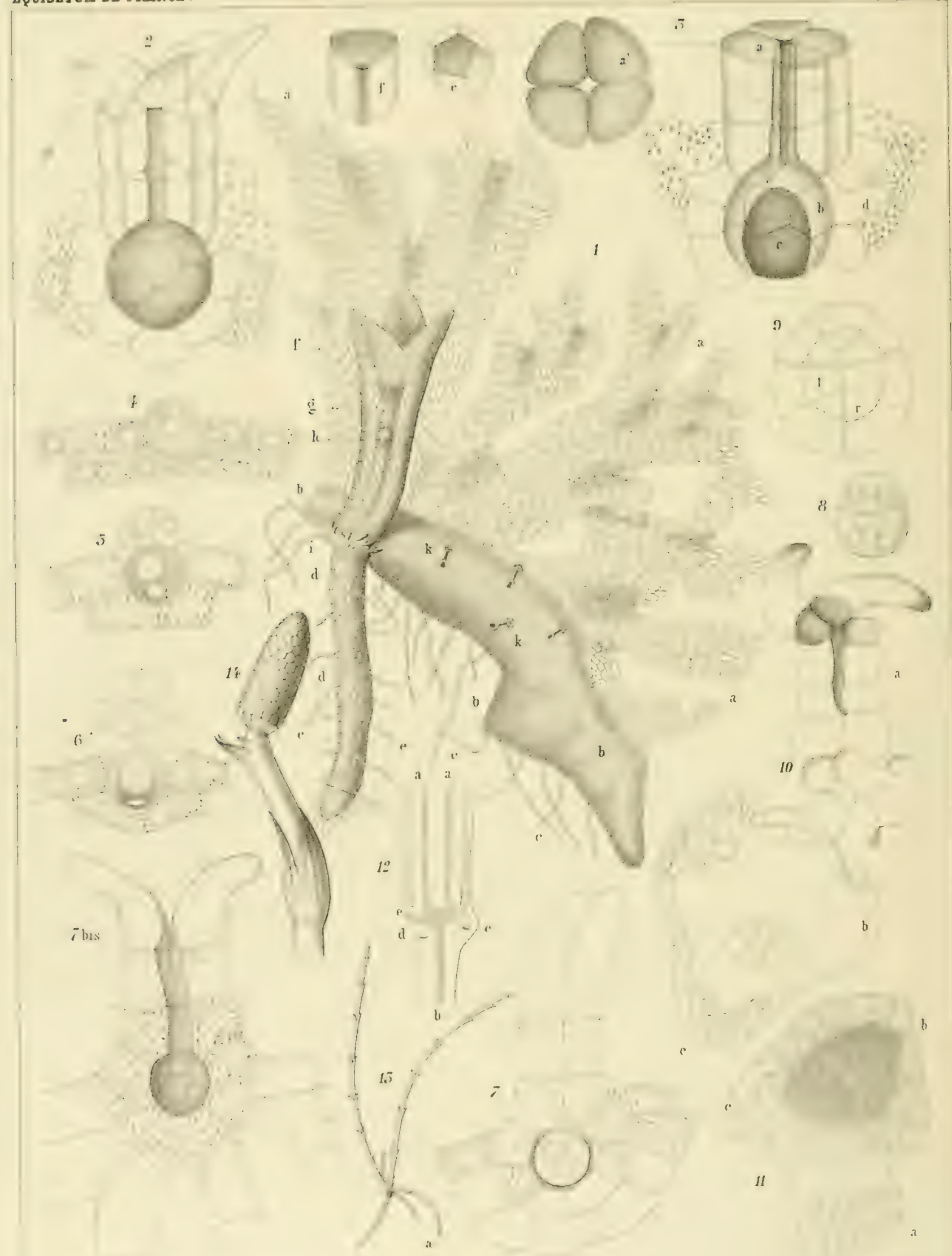




\section{PLANGHE X}

\section{Archégones. Pseudembryon. Jeune plante}

Fir. Eunisetum arvense. Moitié d'un sporophyme portant une jeune plante, 20 diamètres.

$a$ Lobes et Iobules du sporophyme. $-b$ Région charnue, dans le tissu de laquelle apparaissent les archégones. - c Radicelles du sporophyme. d Racines de la jeune plante. - e Fibres radicellaires de cette racine. - f Gaîne basilaire de la nouvelle plante. - g Premier entre-nocul. $h$ Second entre-noud commençant à se former. i Restes des cellules enveloppant le pseudembryon. - $k$ Archémones non fécondés et fortement colorés.

2. E. arvense. Archégone avec ses cellules terminales, $300 \mathrm{~d}$.

3. E. arvense. Archégone sans ses cellules terminales, $300 \mathrm{~d}$. $a$ Rosace quadrilobée; $a^{3}$ la même vue de face. $-b$ Ventricule. $-c$ P'seudembryon nort et fortement coloré. $-d$ Petites cellules à chlorophylle. $-e$ Cellule du ventricule. - f Cellule du col.

4. C. palustre. Cellule-mère d'un archégone, $237 \mathrm{~d}$.

5. E. palustre. Jeune archégone après la première division de la cellule du col, $237 \mathrm{~d}$.

6. E. polustre. Jeune archégone au moment de la 2 e division; on n'a dessiné que la moitiẻ latérale, $237 \mathrm{~d}$.

7. E. palustre. Le même un peu plus avancé, $237 \mathrm{~d}$.

This E. palustre. Moitie d'un archẻgone non fécondé coupé longitudinalement. Le pseudembryon est tombé, $237 \mathrm{~d}$.

Nota. Les fig. 4, 5, 6, 7 et 7 bis ont cété dessinées du 28 juillet au 12 aoùt sur des semis du 22 juin.
8. E. arvense. Première division du pseudembryon, $237 \mathrm{~d}$.

9. Fiğure sclećmatique, indiquant par $=\ldots$ ... la succession des divisions du pseudembryon.

t Cellule qui donnera naissance is la nouvelle petite tige. $-v$ Cellule de la division de laquelle proviendra la premic̀re racine.

10. E. anvense. Axe secondaire ou nouvelle plante très-jeune; elle montre en 6 le commencement des dents de la première gaine, en $c$ le commencement de la racine. Audessus est le col de l'archẻgone brisé $a$, 142 d. J'ai dessiné seulement les cellules que j'ai pu voir parfaitement distinctes sur cette masse non coupéc et d'un vert intense.

11. E arvense. Axe secondaire plus jeune encore, 142.

a Riestes du pseudembryon rejeté de côlé. $b$ Pointe de l'axe secondaire. - $c$ Commencement de la racine.

12. E. nrvense. Coupe de la partie inférieurc d'une jeune plante, $25 \mathrm{~d}$.

$a$ Vaisseaux de la tige. - $b$ Vaisseaux de la racine. $\rightarrow$ Bourgeon d'une seconde tige. $-d$ Bourgeon d'une seconde racine. - $e$ Masse de cellules rayées et spiralées.

13. E. nvense. Jeunc tige dessinẻe le 13 août; semis dit 16 avril.

a Tige descendante ou rhizome.

14. E. arvense. 9e anomalie; lobes de l'anneau devenant des sporanges. 



\section{TABLE DES MATIERES}

PITISACE.

AnPPotet de M. Ad. Ironguiart.

\section{PREMIÉRE PARTIE. Anatomie. Reproduction}

Coup d'œeil préliminaire sur le genre Equisetum et sur les espèces franģaises .

Caractères du genre, 1. Liste des espèces françaises, 3

\section{CIIA PITIE PIEnIGR. Deseription extérieure d'une plante adulte}

$\$ 1 \mathrm{er}$. Du rhizome el de ses appendices

Articles, 3. Verticilles de rhizomes latéraux, 4. Bacines, 5. Tomentum, bे. T'ubercules, 6. Profondeur des rhizomes, 8. Rapports des rhizomes et des tiges, 9.

52. De la tige stérile

beux sortes de liges, 10. Articles et gaines, 11. Divisions de la gạine, 12. Dcux cylindres, 12. Rameaux, 13. Durée des liges, 14.

S 3. De la tige spicifère et de l’appareil de reproduction . . . . . . . . . . . . . . . . Aspect des tiges spiciferes propres, 15. Des autres liges spiciferes, 15. Du pédoncule, 16. De l'anneau, 16. De l'épi, des pédicelles et des clypéoles, 16. Des sporanges, 17. Des spores ct des élatères, 17. Des sporophymes, 17.

\section{CIA P. II. Deseription analytique d'une plante adulte.}

S ler. De l'épiderme.

Épiderme des rhizomes, 18. Fibrilles du tomentum, 18. Modification selon les espices, 18. Épiderme des tubercules, 21. Épiderme des liges, 21. Encroûtement siliceux, 21. Épiderme des liges spicifères propres, 24. Ĺpiderme des tiges stériles de l'E. maximum, 25. Épiderme des tiges de l'E. arconse et des autres espèces, 20. Épiderme des gaines, 27. Liderme des r'ameaux, 29: Épiderme des épis, 30. lies stomates, 30. Leur position, 31. Leur structure, 31. Ilistorique des travaux sur les stomates des Equisetum, $3 \mathbf{4}$.

\$2. Des tissus du rhisome. . . . . . . . . . . . . . . . . . . . . . . . .

Deux cylindres, 37. Tissus du cylindre cortical, 37. Tissus du cylindre interne, 38. Tissus des tubercules, 40. Granules amylacés, 41 . Tissus des racines, 41.

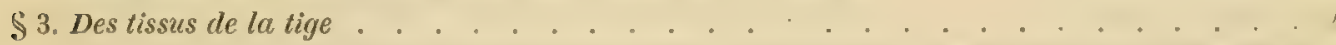

Comparaison avec les tissus des rhizomes, 42. Fibres corticales, 43. Chlorophylle, 44. Tissus des tiges spicifères propres, 46 . Tissus des gaines, 47. Analyse des nœuds et direction des faisceaux fibro-vasculaires, 48. Tissus des rameaux, 49. Gaine basilaire, 50.

$\$$ 4. De l'appareil de reproduction.

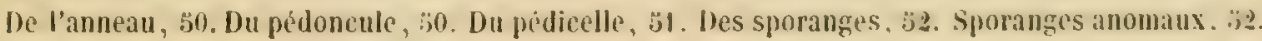




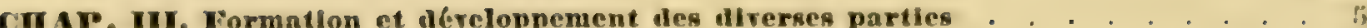

S ter. De la lige el des gaínes . . . . . . . . . . . . . . . . . . . . 5.

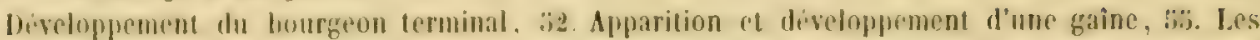
gaines se développent par leur base, 58. Apparition des vaisseaux, 59. Les cntre-nocuds sc développent par leur base, 61. Disposition primitive des vaisseaux, 62. Apparition des rameaux, 62. Apparition et développement de l'épiderme et des stomates, 62. Apparition de la chlorophylle, 64. Apparition de la cavité centrale et des lacunes, 65.

\$. Des rameaux, des rejetons, des rhizomes et des tubercules . . . . . . . . . . . Développement des rameatix, 63. De la gaine basilaire, 67. Diveloppement des rhizomes, 69. Développement des tubercules, 69.

S 3. Des racines . . . .

Déreloppement des racines, 70 .

S 4. Des spores. . . . . . . . . . . . . . . . . . . . . . . Liportues favorables pour cette étude, 7f. De l'anneau, 77. Apparition des verticilles sporangifères, 77. Formation des sporanges, 77. Apparition des spores, 80. Apparition des élatères, 80. Apparition des cellules fibro-spiralées, 81. Sporose, 81. Corrélation entre le déreloppement des sporanges et celui des spores, 82.

S5. Anatomie des spores.

Deux datires, 8ł. De leur adhérence entre eux et a la spore, 8ł. De leur apparente inégalití, 86. Ils ne se croisent pas a leur point d'union, 87. Des granules et des rides de leur surface, 87. Opinion de M. Pringsheim, 88. Forme des spores, 89. Du stigmate de Hedwig, 90. Des deux membranes des spores, 90. ve leur contenu, 91. Des spores abortives, 93.

CuAP, Iv. Meproduction.

\$1. Déreloppement des spores en sporophymes

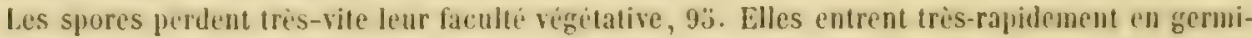
nation, 96. Apparition des premières cellules, des radicelles el de la chlorophylle, 96. Les sporophymes des Eųuisetum n'ont point une forme syntirique, 97.

S2. Des antheridies et des spermatozoüdes.

Apurition d'une antheridie, 99. Son développement, 99. l.es antheridies ne sont point entouries d'un anneau, 100. Formation des spermatozoides, 100. Leur émission, 101. Leurs apparences, 101. IIs ont un mouvement propre, 104.

S 3. Des archégones

I.eur position, 10j. Jeur premiere apparition, 106. Leur developpement, 106. Ibirric ordinaire des sporophymes, 107. Moyens de transport des spermatozoides, 108. Modifications qui suivent la fécondation, 109. Archégones non fécondés, 109.

\$ 4. Du pseudembryon et de la jeune plante . . . . . . . . . . . . . . . . .

bécloppement du pseudembryon, 110. Apparition du premier axe secondaire qui sera la nonvelle plante, 111. Apparition de la première racine, 111. Apparition des vaisseaux, 112. Rapports des vaisseaux de la jeune lige à ceux de la racine, 112 Conséquences, 113. Des gaines des jeunes tiges, 116. Disposition des liges qui suivent la premiere, 117. Apparition de tubercules, 117. Ëpoques de déreloppement, 118. Direction descendante des rhizomes, 118.

S 3 . Mode de semis.

S6. Hislorique

Cesalpino, 121. Camerarius, 122. Tournefort, 122. J. Ray, 122. Dillenius, 122. Linné, 123 Haller, 123. Adanson, 124. Gleichen, 124. Kölreuter, 124. De Iamarck, 124. J. Iledvig, 124. A. L. de Jussicu, 125. Loth, 125. Willdenow, 125. De Candolle, 126. Mirbel, 126. Vaucher (1822) fait germer des spores, 126. C. A. Agardh, 127. Vaucher (1823) obtient par semis une 
nouvelle plante, 128. Link, 129. J. L. Duvernoy, 129. liequien (1826) trouve le premier de. jeunes Equisetum spontanés, 129. De Candolle, 130. Bischoff, 130. Ad. Brongniart, 131. L. Reichenbach, 131. II. Mohı, 131. Raspail, 131. G. Thuret (1848) découvre les anthéridies et Ies spermatozoüdes des Lquisetum, 132. Milde. 132. Hofmeister, 133. Hofmeister (1852) décrit les archégones des Equisetum, 133. J. Nilde (1852) les figure, 133. Hofmeister (1852) décrit les premiers développements de la jeune plante, 134. Bischoff, 134.

CIrAP. V. De quelques opinions morphologiques et des anomalies.

S 1er. Morphologie. . . . . . . . . . . . . . . . . . . . . . . . 13

De Ia gaine; opinion de Mirbel, 134. Opinion de Düll, 133. Des rameaux; opinion de Döll, 137.

De l'anneau, 138. Des pédicelles sporangifères, 141.

\$2. Anomalies.

DEUXIĖME PARTIE. Glassification. Description.

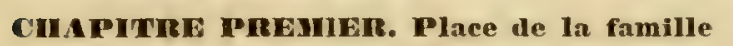

G. Bauhin, 155. Tournefort et J. Ray, 15i. Dillenius, 155. Linne, 156. Schreber, 156. Willdenow, 156. Iloflmann, 156. Adanson, 156. A. L. de Jussieu, 157. De Candolle, 157. Sprengel, 157. Oken, 157. El. Fries, 157. Bischoff, 157. Lindley, 157. L. Reichenbach, 157. Ilenderson, 157. Ad. Brongniart, 158. Grenier, 458. Ilofmeister, 158. Richard, 158. Bernoulli, 158. Döll, 158. J. Milde, 158.

Rapports des Equisetum et des Fougères, 158.

CHAI. II. Examen des caractères spécifiques

Division en deux grandes sections, 161. Division de charue section en deux groupes, 161. Modification des tiges après la sporose, 162. Coupe transversale des tiges et des rameaux, 162. Rapport entre la longueur du premier entre-noud des rameaux et la gaine basilaire, 162. Forme des gaines, 162. Nombre des divisions des gaines, 162. Valeur des caractìres fournis par la coupe transversale des tiges, 163. Importance des caractères fournis par la coupe transversale des rameaux, 164. Aucun caractère, réduit à lui seul, n'a une valeur absolue, 165. Du degré de spéciéité des Equisetum français, 166. Des variétés, des variations, des anomalies, 168. Observation sur les noms d'espèces, 168.

\section{CILAP. HI. Classification et description}

\$ ler. Famille

S. Genre.

\$ 3. Division et classification des espèces . . . . . . . . . . . . . . . . . . . . . . . . . 169

$\$$ 4. Description des espèces.

1 er Groupe. E. maximum, 170.

$2^{2}$ Groupe. E. sylvaticum, $176 ; E$. pratense, $180 ; E$. arvense, 183.

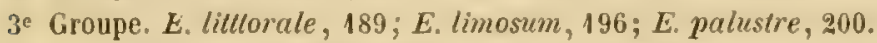

$4^{\circ}$ Groupe. E. ramosissimum, 204; E. variegatum, 210; E. trachyodon, 214; E. lyemale, 219.

CIIA IP. IV. Historique et synonymie

Des espèces de Linné, 227. Des types de l'herbier de Linné, 228. Des divisions de Vaucher, 231. Des divisions postérieures, 232. E. maximum, historique, 23ł; synonymie, 238. E. syluaticum, historique, 239; synonymie, 239. E. pratense, historique, 240 ; synonymie, 241. E. arcense, historique, $242 ;$ synonymie, 242. E. lillorale, historique, 244; synonymie, 245. E. limosum, historique, 245 ; synonymie, 245 . E. palustre, historique, 246 ; synonymie, 247. 
E. ramosissimum, historique, 248 ; synonymie, 249. F. eariegatum, historique, 230 ; synonymic, 230. E. Irachyodon, historique, 231 ; synonymic, 251 . L. hyemale, historique, 252 ; synonymic, $23 \mathrm{z} z$.

CII IP. V. Iconographie

F. maximum, 253. E. syleaticum, 25\%. E. pratense, 23\%. E. ariense, 255. F. liltorale, 25:5

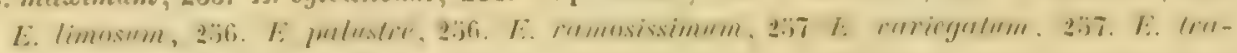
chyodon, 257. E. hyemale, 2507.

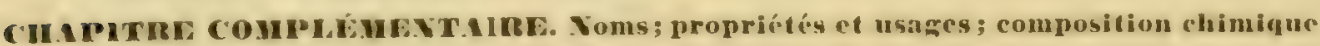

S fer. Noms

I-xวนคเร, 238. Equisetum, 258. Isprête, 260.

\$. Propriétés et usages.

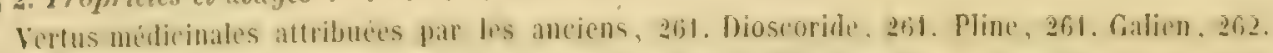
Matthiole, 262. Fallope, 262. Olivier de Serres, 262. G. Bauhin, 262. Garidel, 263 Linné, 26.3. Bergius, 263. Murray, 263. Lenhossek, 26k. Soubciran, 26k: Dorvault, 26k. Schnizlein, $26 k$. Emploi culinaire, 265. Propriétés dans l'économie rurale, 265. Pline, 265. Llaller, 265 . Linn 6. 265. Alauson, 266. Ventenat, 266. Murray, 266. Lightfoot, 266. Kinapp, 266. Smelowsky. 266. W. Hooker, 266. Newman, 267. Bischof, 267. Paltard, 267. Comices agricoles d'Alsace, 267. Imlin, 207. Raspail, 268. Usages actuels, 268.

\$3. Composition chimique

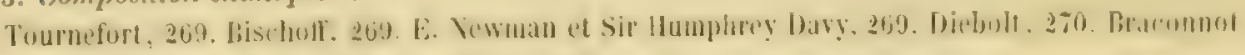
270. V. Regnault, 272. Pelouze el Fremy, 27\%.

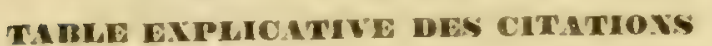

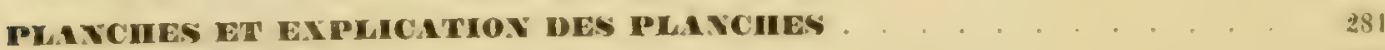

Planche I. Rhizomes. . . . . . . . . . . . . . . . . . . . . . . 282

Planche II. Épiderme des rhizomes. Racines. . . . . . . . . . . . . . . . . 283

Planche III. Épidermes lles tiges et des rameaux . . . . . . . . . . . . . . . . 284

Planche IV. Stomates . . . . . . . . . . . . . . . . . . . . . . . . 285

Panche V. Stomates, Coupes de rhizomes . . . . . . . . . . . . . . . . . . 286,

Planche VI Coupes de tiges et de rameaux . . . . . . . . . . . . . . . . . . . 287

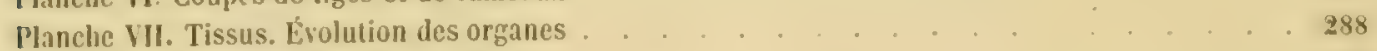

Planche VIll. Livolution des organes . . . . . . . . . . . . . . . . . . . . 289

Planche IX. Spores. Sporophymes. Anthéridies. Spermatozoïdes . . . . . . . . . . . 290

Panche X. Archigones. Pseudembryon. Jeune plante. . . . . . . . . . . . ע9।

FIN. 



$$
\text { I }
$$




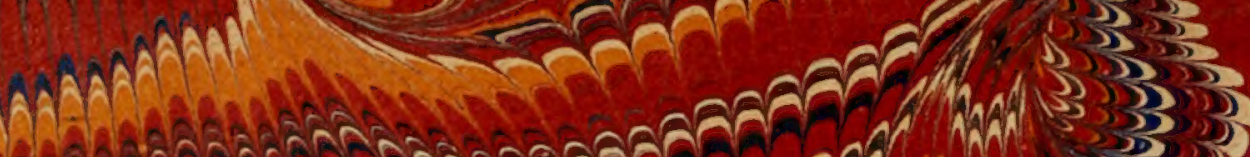

$50 \mathrm{Mm}$ a

N

-

N

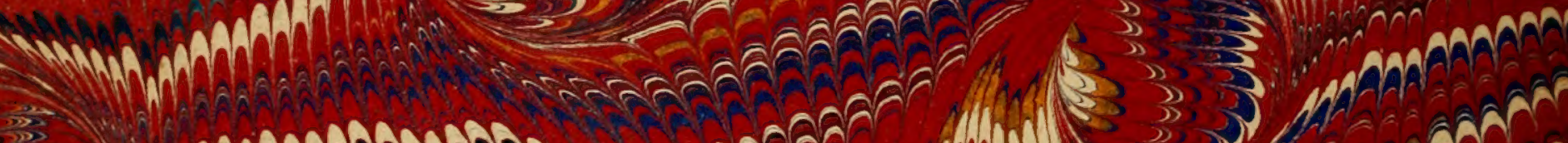

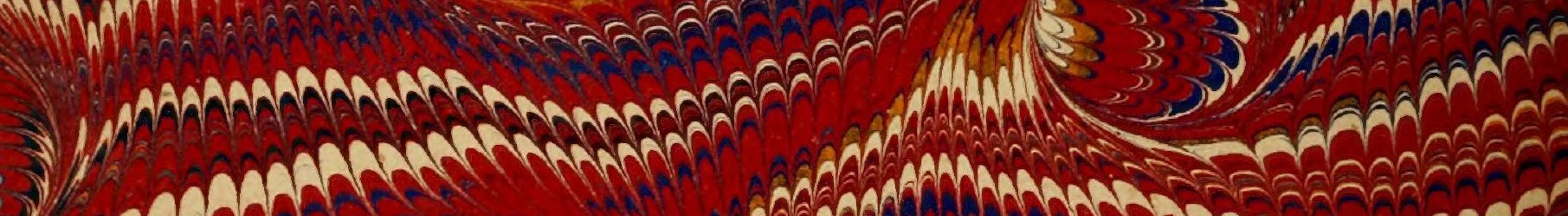

工 (1)

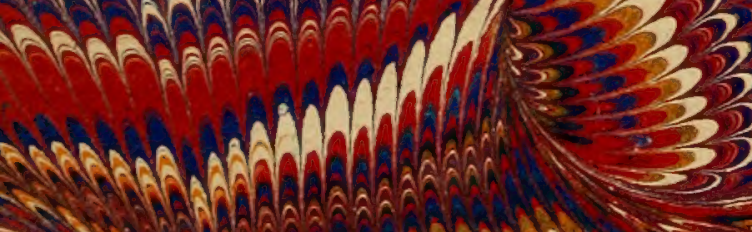

manin -Whantâna

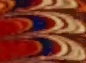

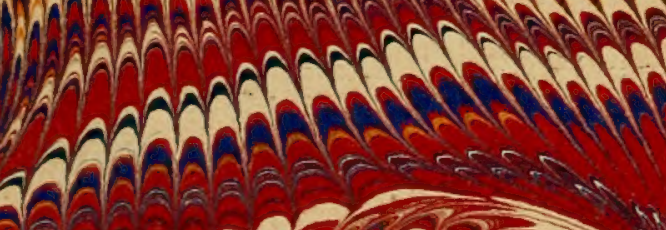
(
3)
a

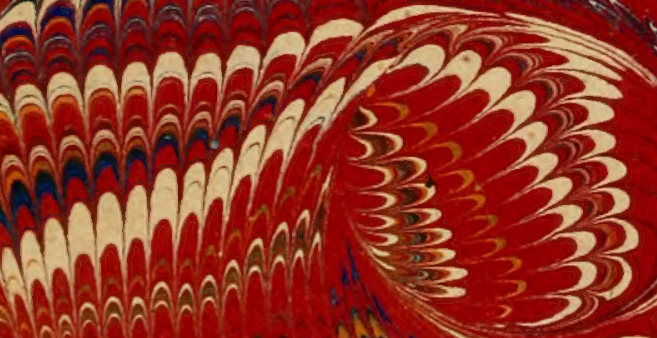

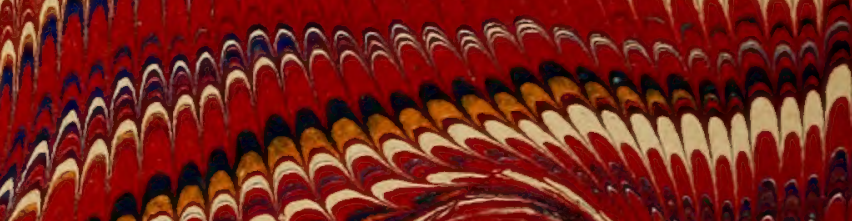

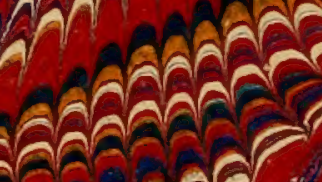

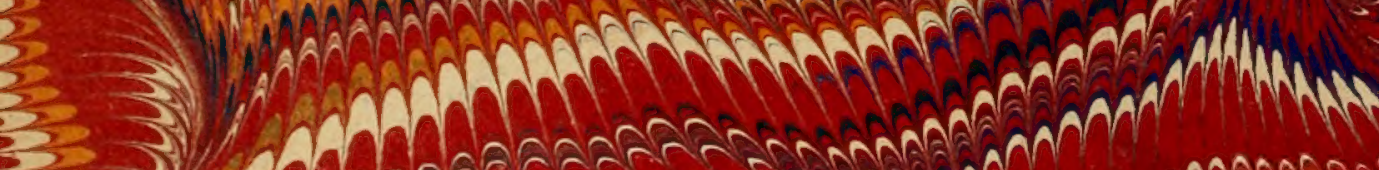




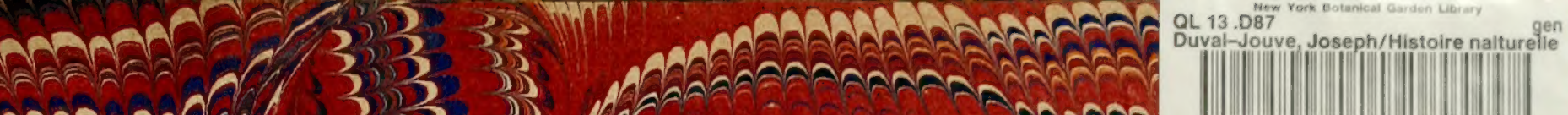
manc 3,3, the

3518500063

WNA

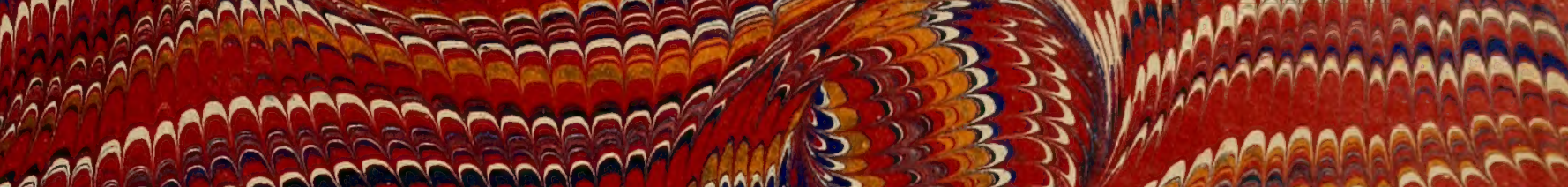

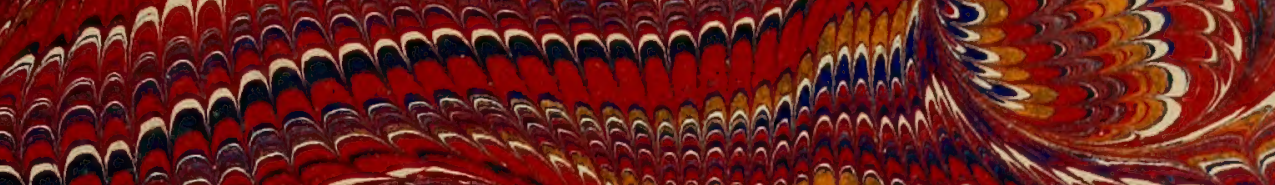

mant

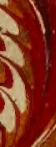

inâh

1Antand

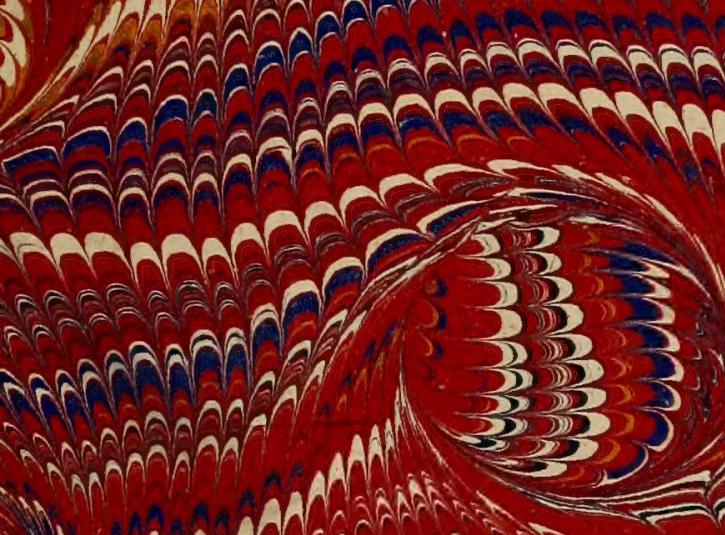

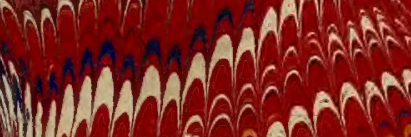

ลิลิว

nimmonh $3 \frac{3}{3}$ )

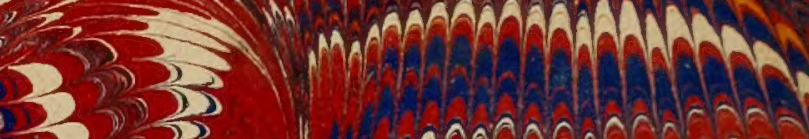

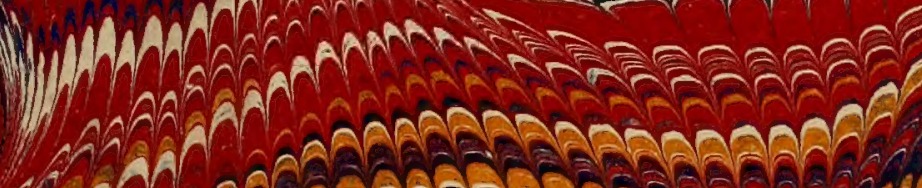

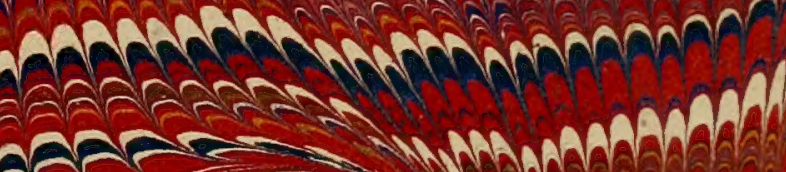

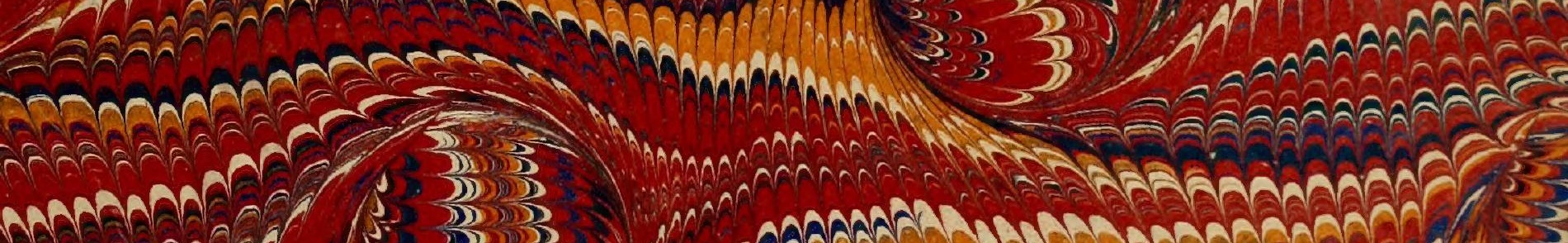

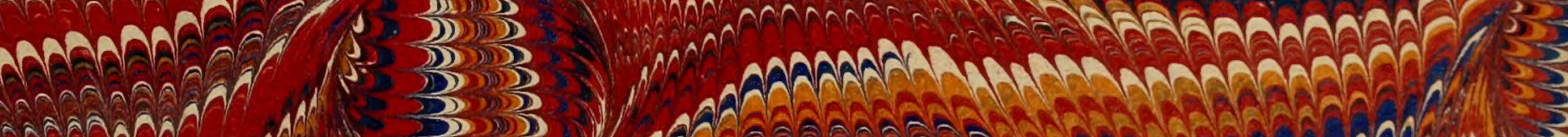

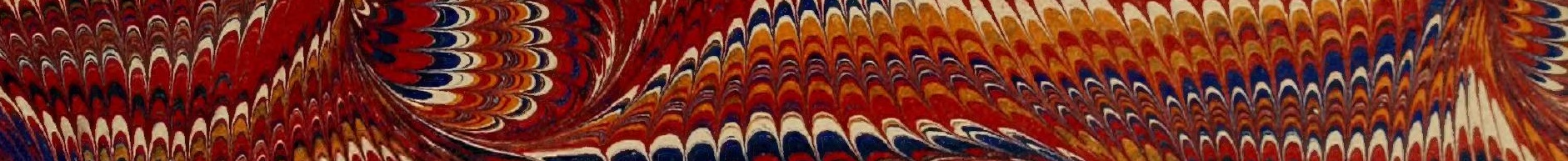

- Mâी

)

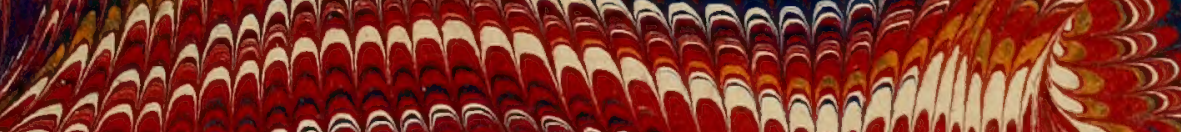

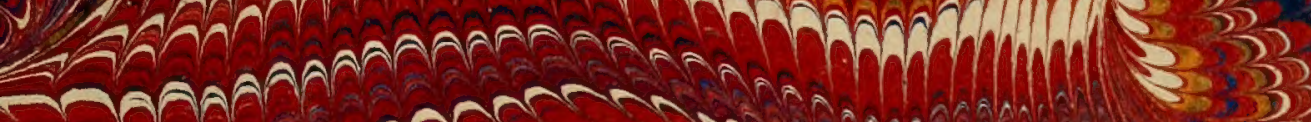

âितिकित 
